\title{
Fuel-Element Failures in Hanford Single-Pass Reactors 1944-1971
}

Hanford Environmental Dose Reconstruction Project

S. P. Gydesen

July 1993

Letter report prepared for the Technical Steering Panel and the Centers for Disease Control and Prevention under Contract Number 200-92-0503(CDC)/18620 (BNW)

Battelle

Pacific Northwest Laboratories

Richland, Washington 99352 


\section{Preface}

The Hanford Environmental Dose Reconstruction (HEDR) Project was prompted by mounting concern about possible health effects to the public from more than $\mathbf{4 0}$ years of nuclear operations at the Hanford Site. The primary objective of the HEDR Project is to estimate the radiation dose (with descriptions of the uncertainties inherent in such estimates) that individuals could have received as a result of emissions since 1944 from the U.S. Department of Energy's (DOE) Hanford Site near Richland, Washington. An independent Technical Steering Panel (TSP) directs the work on the project which is conducted by Battelle, Pacific Northwest Laboratories (BNW) under contract with the Centers for Disease Control and Prevention.

The HEDR Project work is conducted under several technical and administrative tasks, among which are the Source Terms Task and the Information Resources Task. Under the Source Terms Task, estimates of radioactive emissions from Hanford facilities since 1944 are developed. These estimates (source terms) are based on historical measurements and production information. The Information Resources Task staff identify, search for, retrieve, declassify (if necessary), and make available to HEDR Project staff and the public those documents essential to meet projest information needs such as the historical measurements and production information necessary to develop the source terms.

To estimate the radioactive emissions from Hanford facilities, the sources of emission must be identified. One source of emissions was fuel containers that failed. Whenever a leak developed in the fuel container, radioactive emissions to the Columbia River were likely to increase. These leaks were known as fuel-element failures.

The Information Resources Task staff searched for and retrieved those documents that recorded the fuel-element failures in single-pass reactors. The purpose of this letter report is to make available to the public copies of the original documents which recorded the fuel-element failures. The data in these documents will be used by the staff of the Source Terms Task to determine the contribution of fuel-element failures in single-pass reactors to the radioactivity of the reactor effluent. 


\section{Summary}

The primary objective of the Hanford Environmental Dose Reconstruction (HEDR) Project is to estimate the radiation dose that individuals could have received as a result of emissions since 1944 from the U.S. Department of Energy's (DOE) Hanford Site near Richland, Washington. To estimate the doses, the staff of the Source Terms Task use operating information from historical documents to approximate the radioactive emissions. These historical documents are identified, searched for, retrieved, declassified (if necessary), and made available by the Information Resources Task staff.

\section{Scope of Work}

One source of radioactive emissions to the Columbia River came from leaks in the aluminum cladding of the uranium metal fuel elements in single-pass reactors. The purpose of this letter report is to provide photocopies of the documents that recorded these failures. The data from these documents will be used by the Source Terms Task to determine the contribution of single-pass reactor fuel-element failures to the radioactivity of the reactor effluent from 1944 through 1971 . Each referenced fuel-element failure occurring in the Hanford single-pass reactors is addressed. The first recorded failure was in 1948, the last in 1970. No records of fuel-element failures were found in documents prior to 1948 . Data on the approximately 2000 failures which occurred during the 28 years (1944-1971) of Hanford single-pass reactor operations are provided in this report.

\section{Technical Approach}

The search tree method (Shipler 1993, p. 12.8) was used to find the fuel-element failure information. Once the documents were identified, the fuel-element failure data were extracted and provided to the staff of the Source Terms Task. The Source Terms Task staff then electronically recorded the data in the Source Term fuel-element failure file for use in estimating the Columbia River source term.

\section{Results}

All recorded, applicable fuel-element failures have been identified. The information presented in this report is available to the public and for use by the Source Terms Task staff to estimate the radiation emitted to the Columbia River. 


\section{Glossary}

Only those definitions for the data needed by the Source Terms Task are defined here. For example, fuel-element lot identification and component codes will not be used by the Source Terms

Task, so those terms are not defined in this report. Such definitions may be found in Newell (1969a). Any other terms not defined in Newell or this report may be found in the cited report from which the data were extracted.

\section{General Terms}

Days in Pile

GPM

KW

MWD/T

Slug Rupture

\section{Type of Metal}

I\&E E

I\&E EB

I\&E ES

I\&E N

I\&E NB
Days in reactor

Gallons per minute

Kilowatts

Megawatt days per ton

Term used interchangeably with fuel-element failure

Symbol for internally and externally cooled reactor fuel elements, enriched in uranium-235

Symbol for internally and externally cooled reactor fuel elements which are enriched with uranium-235, have projections (bumpers), and are irradiated in ribbed process tubes

Symbol for internally and externally cooled reactor fuel elements which are enriched with uranium-235, have projections (bumpers), and are irradiated in ribless process tubes

Symbol for internally anó externally cooled natural uranium reactor fuel elements

Symbol for internally and externally cooled natural uranium reactor fuel elements which have projections (bumpers) and are irradiated in ribbed process tubes 
I\&E NS

Thoria

\section{Type of Failure}

Symbol for internally and externally cooled natural uranium reactor fuel clements which have projections (bumpers) and are irradiated in ribless process tubes

Solid element containing thorium oxide target material.
1. Terms Used to Identify the Location of the Failure
C Core
Cleavage or growth failure
E End
Failure at the end of the fuel element
H Hole
Failure on the internal surface of the I\&E fuel element
S Side
Failure on the side of the fuel element
U Unknown
Location of failure unknown

\section{Terms Used to Identify the Reason for the Failure}

M Defect

E Examined Not Found

G Groove Corrosion

H Hot Spot

L Ledge Corrosion

D Mechanical Damage

N Not Examined

P Parallel Split

T Transverse Split

U Unclassified
Failure caused by a defect in fuel fabrication

Suspected failure pieces examined but no failures found. Other detector systems, such as Panellit, gamma monitor, etc., however, provided conclusive proof of failure.

Failure caused by groove-pitting corrosion attack

Failure caused by accelerated high-temperature corrosion attack

Failure caused by ledge or other near-uniform corrosion atiack

Failure caused by mechanical damage to the fuel element

Failure not examined

Failure caused by cleavage of the core in a direction parallel to the axis of the fuel element

Failure caused by cleavage of the core in a direction transverse to the axis of the fuel element

No other character logically applies to the failure 


\section{Contents}

Preface $\ldots \ldots \ldots \ldots \ldots \ldots \ldots \ldots \ldots \ldots \ldots \ldots \ldots \ldots \ldots \ldots \ldots$ iii

Summary $\ldots \ldots \ldots \ldots \ldots \ldots \ldots \ldots \ldots \ldots \ldots \ldots \ldots \ldots \ldots \ldots$

Glossary ............................ vii

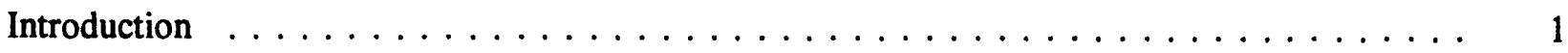

Quality Assurance and Data Quality Objectives .................. 3

Fuel-Element Failure Data $\ldots \ldots \ldots \ldots \ldots \ldots \ldots \ldots \ldots$

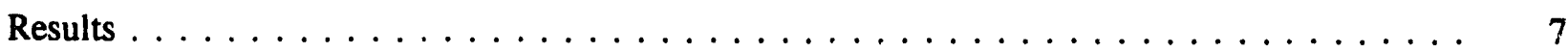

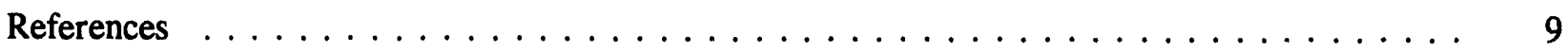

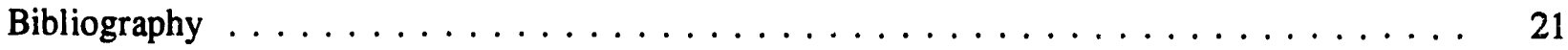

APPENDIX A - Fuel-Element Failure Data for $1948-1951 \ldots \ldots \ldots \ldots \ldots \ldots$

APPENDIX B - Fuel-Element Failure Data for $1952 \ldots \ldots \ldots \ldots \ldots \ldots \ldots$

APPENDIX C - Fuel-Element Failure Data for $1953 \ldots \ldots \ldots \ldots \ldots \ldots \ldots$. . . . . . .

APPENDIX D - Fuel-Element Failure Data for $1954 \ldots \ldots \ldots \ldots \ldots \ldots \ldots$

APPENDIX E - Fuel-Element Failure Data for $1955 \ldots \ldots \ldots \ldots \ldots \ldots \ldots$

APPENDIX F - Fuel-Element Failure Data for $1956 \ldots \ldots \ldots \ldots \ldots \ldots \ldots$

APPENDIX G - Fuel-Element Failure Data for $1957 \ldots \ldots \ldots \ldots \ldots \ldots \ldots$

APPENDIX H - Fuel-Element Failure Data for $1958 \ldots \ldots \ldots \ldots \ldots \ldots \ldots$

APPENDIX I - Fuel-Element Failure Data for $1959 \ldots \ldots \ldots \ldots \ldots \ldots \ldots$. . . . . . . . . .

APPENDIX $J$ - Fuel-Element Failure Data for $1960 \ldots \ldots \ldots \ldots \ldots \ldots \ldots$

APPENDIX K - Fuel-Element Failure Data for $1961 \ldots \ldots \ldots \ldots \ldots \ldots \ldots$

APPENDIX L - Fuel-Element Failure Data for $1962 \ldots \ldots \ldots \ldots \ldots \ldots \ldots \ldots$

APPENDIX M - Fuel-Element Failure Data for $1963 \ldots \ldots \ldots \ldots \ldots \ldots \ldots$ 
APPENDIX N - Fuel-Element Failure Data for 1964

N. 1

APPENDIX O - Fuel-Element Failure Data for 1965

0.1

APPENDIX P - Fuel-Element Failure Data for 1966

P. 1

APPENDIX Q - Fuel-Element Failure Data for 1967-1970

Q.1 


\section{Introduction}

The first step in determining radiation doses from Hanford Site operations is to calculate the source terms - the radionuclides that were released to the environment. The quantities of radionuclides available for release at any time are a function of the irradiation history of the fuel, the time elapsed since the fuel was removed from the reactor, and the amount of irradiated fuel being dissolved. Fuel-element failures fall under the category of fuel irradiation. Historically, once the fuel was sealed inside a container, it was irradiated. Whenever a leak developed in the fuel container, the radioactivity of emissions to the Columbia River was likely to increase. These leaks are known as fuel-element failures. The data recorded when fuel-element failures occurred in single-pass reactors are necessary to estimate the source term for the Columbia River pathway.

The purpose of this report is to provide the fuel-element failure data for the period of concern to the Hanford Environmental Dose Reconstruction (HEDR) Project: 1944-1971. In Gydesen (1992a and 1992b), the start-up and shut-down dates for the Hanford Site single-pass reactors are recorded. These are the dates that determine the period of study for the HEDR Project. The first fuel-element failure was not recorded until 1948. That was when the U. S. Government decided to increase the production of plutonium. To do this, the power levels were raised. The increased heat that resulted caused fuel-element failures. The last failure was recorded in 1970. Shortly after, in 1971, the last single-pass reactor was shut down. 


\section{Quality Assurance and Data Quality Objectives}

The data quality objective established for the Information Resources Task is completeness (Shipler 1993). Completeness is necessary as a data quality objective to ensure that all available 1) operating information needed for dose reconstruction and 2) monitoring information indicating concentrations of radioactive materials in the environment will be found. Completeness ensures that discovery of any additional information would not alter the HEDR Project reconstructed results. The approach to achieve completeness is to use the search tree process to review currently available subject, author, periodical, and serial indices for documents of interest to the HEDR Project, which in this report are the fuel-element failure documents.

The search for the fuel-element failure documents was performed using the search tree method (Shipler 1993, p. 12.8). Internal peer reviews of this process were performed by the author's line manager and the Source Term Task leader. The line manager's review ensures that the development of the search trees was sufficient for this particular activity. The Source Term Task leader's review ensures that the information necessary to reconstruct radioactive releases has been provided.

Additionally, a surveillance was performed by the quality engineer to verify 1) that the internal reviews performed were sufficient and 2) that for those fuel-element failure documents that are not available, it is indeed because they have been destroyed. These activities ensure that all applicable (single-pass reactor) fuel-element failures have been identified and the corresponding data provided to the Source Terms Task staff. 


\section{Fuel-Element Failure Data}

Photocopies of the data on the approximately 2000 fuel-element failures which occurred during the 28 years (1944-1971) of Hanford single-pass reactor operations are provided in this report. The data reproduced here include:

\section{Date of failure}

2. Tube number and reactor in which failure occurred

3. Days in pile or date fuel was loaded

4. Fuel (slug) power in kilowatts (kw)

5. Type of failure

Only fuel-element failures from single-pass reactors are addressed because it is only the releases from single-pass reactors that could have an effect on the source term. The name, single-pass reactor, refers to the fact that the cooling water passed through the reactor core only once before being released to the retention basin and thence to the Columbia River. When a fuel-element failure occurred, the releases that resulted went directly to the river with the holdup time provided by the retention basins being less than 4 hours. Therefore, it is important to calculate the source terms for the single-pass reactor releases that resulted from fuel-element failures.

This contrasts with the $\mathrm{N}$ reactor which recirculated the primary cooling water through the reactor continually during operation. Because the $\mathrm{N}$ reactor recirculated its cooling water, no discharge went directly from it into the Columbia River. When a fuel-element failure occurred in the $\mathrm{N}$ reactor, the recirculating water was flushed out and stored in tanks or plastic-lined ponds. The only way radioactivity from fuel-element failures in the $\mathbf{N}$ reactor could have reached the Columbia River would have been by leakage from these tanks and ponds through the soil into shallow springs that eventually fed into the river. Such releases were indirect and much lower than those from the Hanford single-pass reactors. Therefore, fuel-element failures that occurred as a result of $\mathrm{N}$ reactor operations are not addressed in this report nor will they be used in the calculation of source terms.

The vast majority of the fuel-element failures involved either natural or enriched uranium fuels. The other failures involved thorium (metal), thoria (thorium oxide), lithium targets (variously referred to as $\mathrm{P}-10$ or mint), and enriched uranium-aluminum driver fuel (C slugs or $\mathrm{J}$ slugs). In the early 1950s a small number of failures occurred in the lithium target fuels. In 1965 a thoria program was initiated for the production of uranium-233 from the irradiation of thorium or thorium-oxide target elements. During this program, which was completed in 1968, a total of 27 failures occurred. A brief history of the program and the first 25 failures are reported by Bowles and Schmidt (1966). These other types of fuel-element failures did not significantly contribute to the radioactivity of the reactor effluent. 
Appendixes A-Q present photocopies of the fuel-element failure data. Only that information needed for source term calculations (as determined by the Source Terms Task Leader) were extracted for each fuel-element failure and included in this report. The Source Terms Task will use 1) date, 2) burnup, and 3) weight loss for the few failures for which weight-loss data are available. The weight-loss data are not provided in this report but will be referenced in the forthcoming report on key reactor releases to the Columbia River.

Because fuel-fabrication information (e.g., vendor and fabrication history, core geometry, aluminum-component history and canning-serial history) will not be used by the Source Terms Task, any acronyms found in the appendixes concerning fuel fabrication are not defined in this report. Should the reader need these definitions, they may be found in the referenced report from which the tables were extracted.

Every effort was made by the Information Resources staff to locate the most consistent data among the historical documents and make that information available to the Source Terms staff. It should be noted, however, that there were numerous organizations working at the Hanford Site from 1944-1971. Their fuel-element failure documents did not always report the same types of data or even the same number of failures for the same month. This was due to the fact that, depending on the date a report was written, a suspected failure may or may not have been verified by that time as indeed being a failure. Whenever there was a reading on a monitor that implied a failure, that information was recorded as a failure until it was proven otherwise. Those that proved to be failures were assigned a number. It is the data from these numbered fuel-element failures that the Source Terms staff will use to calculate emissions. The entries without numbers are those that turned out not to be failures after all. That information is, of course, of no interest to the HEDR Project.

Because the fuel-element failure information in this report was photocopied directly from the historical documents, legibility varies depending on the quality of the original copy. Nonetheless, to avoid introduction of errors when working with large collections of numbers, the tables have not been retyped. A glossary of selected initialisms has been developed to aid the reader. A bibliography of selected topical reports on fuel-element failures is also included for additional reading. 


\section{Results}

All recorded, applicable fuel-element failures in single-pass reactors have been identified. The information presented in this report is available for use by the Source Terms Task staff to estimate the radiation emitted to the Columbia River which resulted from fuel-element failures. The results of that study will be reported in a future document on Columbia River radionuclides.

All documents relating to this report have been placed in the U. S. Department of Energy Richland Operations Office Public Reading Room and sent to the Office of Scientific and Technical Information (OSTI) in Oak Ridge, Tennessee. OSTI abstracts, indexes and adds the documents to energy databases which can be electronically searched on several commercial systems. 


\section{References}

Anderson, J. K. 1953a. Ruptured Slug Data and Comparative Rupture Rates for May-June 1953. HW-28675, General Electric C.o., Hanford Atomic Products Operation, Richland, Washington.

Anderson, J. K. 1953b. Ruptured Slug Data and Comparative Rupture Rates July Through September 1953. HW-29629, General Electric Co., Hanford Atomic Products Operation, Richland, Washington.

Anderson, J. K. 1954a. Ruftured Slug Data and Comparative Rupture Rates October Through December 1953. HW-30703, General Electric. Co., Hanford Atomic Products Operation, Richland, Washingtun.

Anderson, J. K. 1954b. Ruptured Slug Data and Comparative Rupture Rates January Through March 1954. HW-31282, General Electric Co., Hanford Atomic Products Operation, Richland, Washington.

Anderson, J. K., and R. J. Bartlett. 1955a. Ruptured Slug Data - February 1955. HW-35826, General Electric Co., Hanford Atomic Products Operation, Richland, Washington.

Anderson, J. K., and R. J. Bartlett. 1955b. Ruptured Slug Data-March 1955. HW-36188, General Electric Co., Hanford Atomic Products Operation, Richland, Washington.

Anderson, J. K., and R. J. Bartlett. 1955c. Ruptured Slug Data-April 1955. HW-36707, General Electric Co., Hanford Atomic Products Operation, Richland, Washington.

Anderson, J. K., and L. W. Hartse. 1956a. Ruptured Slug Data-January 1956. HW-42367, Hanford Atomic Products Operation, General Electric Co., Richland, Washington.

Bartlett, R. J., and L. W. Lang. 1952a. Ruptured Slug Data. HW-24718, General Electric Co., Hanford Works, Richland, Washington.

Bartlett, R. J., and L. W. Lang. 1952b. Ruptured Slug Data for June 19.52. HW-24857, General Electric Co., Hanford Works, Richland, Washington.

Bartlett, R. J., and L. W. Lang. 1952c. Ruptured Slug Data. HW-25293, General Electric Co., Hanford Works, Richland, Washington.

Bartlett, R. J., and L. W. Lang. 1952d. Ruptured Slug Data. HW-25499, General Electric Company, Hanford Works, Richland, Washington.

Bartlet, R. J., and L. W. Lang. 1952e. Ruptured Slug Data. HW-25784, General Electric Company, Hanford Works, Richland, Washington. 
Bloomstrand, R. R. 1956a. Ruptured Slug Data - July and August 1956. HW-45144, General Electric Co., Hanford Atomic Products Operation, Richland, Washington.

Bloomstrand, R. R. 1956b. Ruptured Slug Data-October 1956. HW-46680, General Electric Co., Hanford Atomic Products Operation, Richland, Washington.

Bloomstrand, R. R. 1956c. Ruptured Slug Data-November 1956. HW-47572, General Electric Co., Hanford Atomic Products Operation, Richland, Washington.

Bloomstrand, R. R. 1957a. Ruptured Slug Data-December, 1956. HW-47940, General Electric Co., Hanford Atomic Pisducts Operation, Richland, Washington.

Bloomstrand, R. R. 1957b. Ruptured Slug Data-January 1957. HW-48501, Genera Electric Co., Hanford Atomic Products Operation, Richland, Washington.

Bloomstrand, R. R. 1957c. Ruptured Slug Data-February 1957. HW-49575, General Electric Co., Hanford Atomic Products Operation, Richland, Washington.

Bloomstrand, R. R. 1957d. Ruptured Slug Data-March and April, 1957. HW-50182, General Electric Co., Hanford Atomic Products Operation, Richland, Washington.

Bloomstrand, R. R. 1957e. Ruptured Slug Data-May and June, 1957. HW-51443, General Electric Co., Hanford Atomic Products Operation, Richland, Washington.

Bloomstrand, R. R. 1957f. Ruptured Slug Data - July 2, 1957 Through August 24, 1957. HW53483, General Electric Co., Hanford Atomic Products Operation, Richland, Washington.

Bloomstrand, R. R. 1957g. Ruptured Slug Data-August 26, 1957 Through September 11, 1957. HW-53482, General Electric Co., Hanford Atomic Products Operation, Richland, Washington.

Bloomstrand, R. R. 1957h. Ruptured Slug Data-September 12, Thru October 17, 1957. HW53954, General Electric Co., Hanford Atomic Products Operation, Richland, Washington.

Bloomstrand, R. R. 1958a. Ruptured Slug Data October 18, Thru December 4, 1957. HW-54658, General Electric Co., Hanford Atomic Products Operation, Richland, Washington.

Bloomstrand, R. R. 1958b. Ruptured Slug Data December 5, Thru December 31, 1957. HW54659, General Electric Co., Hanford Atomic Products Operation, Richland, Washington.

Bowles, K. C., and J. P. Schmidt. 1966. Thoria Target Element Failures. DUN-1010, Douglas United Nuclear, Inc., Richland, Washington.

DeMers, A. E., and E. C. Wood. 1951. Ruptured Slug Data. HW-22687, General Electric Co., Hanford Works, Richland, Washington. 
DeNeal, D. L. 1970. Historical Events - Single Pass Reactors and Fuels Fabrication. DUN-6888, Douglas United Nuclear Inc., Richland, Washington.

Gydesen, S. P. 1992a. Letter Report: Title Listing of Daily Operating Data on Hanford Single-Pass Reactors, 1944-1971. PNL-7998 HEDR, Pacific Northwest Laboratory, Richland, Washington.

Gydesen, S. P. 1992b. Selected Hanford Reactor and Separations Operating Data for 1960-1964.

PNh D-2017 HEDR. Battelle, Pacific Northwest Laboratories, Richland, Washington.

HAPO - Hanford Atomic Products Operation. 1952a. Hanford Works Monthly Report for January 1952. HW-23437 DEL, General Electric Co., Richland, Washington.

HAPO - Hanford Atomic Products Operation. 1952b. Hanford Works Monthly Report for February 1952. HW-23698 DEL, General Electric Co., Richland, Washington.

HAPO - Hanford Atomic Products Operation. 1955a. Monthly Report Hanford Atomic Products Operation for May 1955. HW-36928 DEL, General Electric Co., Richland, Washington.

HAPO - Hanford Atomic Products Operation. 1955b. Monthly Report Hanford Atomic Products Operation for June 1955. HW-37658 DEL, General Electric Co., Richland, Washington.

HAPO - Hanford Atomic Products Operation. 1955c. Monthly Report Hanford Atomic Products Operation for July 1955. HW-38375 DEL, General Electric Co., Richland, Washington.

HAPO - Hanford Atomic Products Operation. 1955d. Monthly Report Hanford Atomic Products Operation for August 1955. HW-38828 DEL, General Electric Co., Richland, Washington.

HAPO - Hanford Atomic Products Operation. 1955e. Monthly Report Hanford Atomic Products Operation for September 1955. HW-39260 DEL, General Electric Co., Richland, Washington.

HAPO - Hanford Atomic Products Operation. 1955f. Monthly Report Hanford Atomic Products Operation for October 1955. HW-39751 DEL, General Electric Co., Richland, Washington.

HAPO - Hanford Atomic Products Operation. 1955g. Monthly Report Hanford Atomic Products Operation for November 1955. HW-40182 DEL, General Electric Co., Richland, Washington.

HAPO - Hanford Atomic Products Operation. 1956a. Monthly Report Hanford Atomic Products Operation for December 1955. HW-40692 DEL, General Electric Co., Richland, Washington.

HAPO - Hanford Atomic Products Operation. 1956b. Monthly Report Hanford Atomic Products Operation for January 1956. HW-41205 DEL, General Electric Co., Richland, Washington.

HAPO - Hanford Atomic Products Operation. 1956c. Monthly Report Hanford Atomic Products Operation for February 1956. HW-41702 DEL, General Electric Co., Richland, Washington. 
HAPO - Hanford Atomic Products Operation. 1956d. Monthly Report Hanford Atomic Products Operation for April 1956. HW-42626 DEL, General Electric Co., Richland, Washington.

HAPO - Hanford Atomic Products Operation. 1956e. Monthly Report Hanford Atomic Products Operation for May 1956. HW-43137 DEL, General Electric Co., Richland, Washington.

HAPO - Hanford Atomic Products Operation. 1956f. Monthly Report Hanford Atomic Products Operation for July 1956. HW-44580 DEL, General Electric Co., Richland, Washington.

HAPO - Hanford Atomic Products Operation. 1956g. Monthly Report Hanford Atomic Products Operation for August 1956. HW-45115 DEL, General Electric Co., Richland, Washington.

HAPO - Hanford Atonic Products Operation. 1956h. Irradiation Processing Department Monthly Record Report for September 1956. HW-45717 DEL, General Electric Co., Richland, Washington.

HAPO - Hanford Atomic Products Operation. 1956i. Monthly Record Report Irradiation Processing Department October 1956. HW-46349 DEL, General Electric Co., Richland, Washington.

HAPO - Hanford Atomic Products Operation. 1956j. Monthly Record Report Irradiation Processing Department November, 1956. HW-47021 DEL, General Electric Co., Richland, Washington.

HAPO - Hanford Atomic Products Operation. 1957a. Monthly Record Report Irradiation Processing Department December, 1956. HW-47615 DEL, General Electric Co., Richland, Washington.

HAPO - Hanford Atomic Products Operation. 1957b. Monthly Record Report Irradiation Processing Department Januiry, 1957. HW-48046 DEL, General Electric Co., Richland, Washington.

HAPO - Hanford Atomic Products Operation. 1957c. Monthly Record Report Irradiation Processing Department February, 1957. HW-48769 DEL, General Electric Co., Richland, Washington.

HAPO - Hanford Atomic Products Operation. 1957d. Monthly Record Report Irradiation Processing Def xrtment March, 1957. HW-49205 DEL, General Electric Co., Richland, Washington.

HAPO - Hanford Atomic Products Operation. 1957e. Monthly Record Report Irradiation Processing Department April, 1957. HW-49826, General Electric Co., Richland, Washington.

HAPO - Hanford Atomic Products Operation. 1957f. Monthly Record Report Irradiation Processing Department May, 1957. HW-50368 DEL, General Electric Co., Richland, Washington.

HAPO - Hanford Atomic Products Operation. 1957g. Monthly Record Report Irradiation Processing Department June, 1957. HW-51067 DEL, General Electric Co., Richland, Washington.

HAPO - Hanford Atomic Products Operation. 1957h. Monthly Record Report Irradiation Processing Department July, 1957. HW-51703, General Electric Co., Richland, Washington. 
HAPO - Hanford Atomic Products Operation. 1957i. Monthly Record Report Irradiation Processing Department August, 1957. HW-52053, General Electric Co., Richland, Washington.

HAPO - Hanford Atomic Products Operation. 1957j. Monthly Record Report Irradiation Processing Department September, 1957. HW-52809 DEL, General Electric Co., Richland, Washington.

HAPO - Hanford Atomic Products Operation. 1957k. Monthly Record Report Irradiation Processing Department October, 1957. HW-53355 DEL, General Electric Co., Richland, Washington.

HAPO - Hanford Atomic Products Operation. 19571. Monthly Record Report Irradiation Processing Department November, 1957. HW-53719 DEL, General Electric Co., Richland, Washington.

HAPO - Hanford Atomic Products Operation. 1958a. Monthly Record Report Irradiation Processing Department December, 1957. HW-54291 DEL, General Electric Co., Richland, Washington.

HAPO - Hanford Atomic Products Operation. 1958b. Monthly Record Report Irradiation Processing Department January, 1958. HW-54689 DEL, General Electric Co., Richland, Washington.

HAPO - Hanford Atomic Products Operation. 1958c. Monthly Record Report Irradiation Processing Department February, 1958. HW-55101 DEL, General Electric Co., Richland, Washington.

HAPO - Hanford Atomic Products Operation. 1958d. Monthly Record Report Irradiation Processing Department March, 1958. HW-55462, General Electric Co., Richland, Washington.

HAPO - Hanford Atomic Products Operation. 1958e. Monthly Record Report Irradiation Processing Department April, 1958. HW-55870 DEL, General Electric Co., Richland, Washington.

HAPO - Hanford Atomic Products Operation. 1958f. Monthly Record Report Irradiation Processing Department May, 1958. HW-56184 DEL, General Electric Co., Richland, Washington.

HAPO - Hanford Atomic Products Operation. 1958g. Monthly Record Report Irradiation Processing Department June, 1958. HW-56527 DEL, General Electric Co., Richland, Washington.

HAPO - Hanford Atomic Products Operation. 1958h. Monthly Record Report Irradiation Processing Department July, 1958. HW-56911 DEL, General Electric Co., Richland, Washington.

HAPO - Hanford Atomic Products Operation. 1958i. Monthly Record Report Irradiation Processing Department August, 1958. HW-57278 DEL, General Electric Co., Richland, Washington.

HAPO - Hanford Atomic Products Operation. 1958j. Monthly Record Report Irradiation Processing Department September, 1958. HW-57597 DEL, General Electric Co., Richland, Washington.

HAPO - Hanford Atc nic Products Operation. 1958k. Monthly Record Report Irradiation Processing Department Octot:i, 1958. HW-57840 DEL, General Electric Co., Richland, Washington. 
HAPO - Hanford Atomic Products Operation. 19581. Monthly Record Report Irradiation Processing Department November, 1958. HW-58360, General Electric Co., Richland, Washington.

HAPO - Hanford Atomic Products Operation. 1959a. Monthly Record Report Irradiation Processing Department December, 1958. HW.58686, General Electric Co., Richland, Washington.

HAPO - Hanford Atomic Products Operation. 1959b. Monthly Record Report Irradiation Processing Department January, 1959. HW-59041, General Electric Co., Richland, Washington.

HAPO - Hanford Atomic Products Operation. 1959c. Monthly Record Report Irradiation Processing Department February, 1959. HW-59456, General Electric Co., Richland, Washington.

HAPO - Hanford Atomic Products Operation. 1959d. Monthly Record Report Irradiation Processing Department March, 1959. HW-59840 DEL, General Electric Co., Richland, Washington.

HAPO - Hanford Atomic Products Operation. 1959e. Monthly Record Report Irradiation Processing Department April, 195y. HW-60246, General Electric Co., Richland, Washington.

HAPO - Hanford Atomic Products Operation. 1959f. Monthly Record Report Irradiation Processing Department May, 1959. HW-60594, General Electric Co., Richland, Washington.

HAPO - Hanford Atomic Products Operation. 1959g. Monthly Record Report Irradiation Processing Department June, 1959. HW-60918, General Electric Co., Richland, Washington.

HAPO - Hanford Atomic Products Operation. 1959h. Monthly Record Report Irradiation Processing Department July, 1959. HW-61399, General Electric Co., Richland, Washington.

HAPO - Hanford Atomic Products Operation. 1959i. Monthly Record Report Irradiation Processing Department August, 1959. HW-61789, General Electric Co., Richland, Washington.

HAPO - Hanford Atomic Products Operation. 1959j. Monthly Record Report Irradiation Processing Department September, 1959. HW-62184, General Electric Co., Richland, Washington.

HAPO - Hanford Atomic Products Operation. 1959k. Irradiation Processing Department Monthly Record Report October, 1959. HW-62599, General Electric Co., Richland, Washington.

HAPO - Hanford Atomic Products Operation. 19591. Irradiation Processing Department Monthly Record Report November, 1959. HW-62900, General Electric Co., Richland, Washington.

HAPO - Hanford Atomic Products Operation. 1960a. Irradiation Processing Department Monthly Record Report December, 1959. HW-63317 DEL, General Electric Co., Richland, Washington.

HAPO - Hanford Atomic Products Operation. 1960b. Irradiation Processing Department Monthly Record Report January, 1960. HW-63753 DEL, General Electric Co., Richland, Washington. 
HAPO - Hanford Atomic Products Operation. 1960c. Irradiation Processing Department Monthly Record Report February, 1960. HW-64122, General Electric Co., Richland, Washington.

HAPO - Hanford Atomic Products Operation. 1960d. Irradiation Processing Department Monthly Recorá Report March, 1960. HW-64555 DEL, General Electric Co., Richland, Washington.

HAPO - Hanford Atomic Products Operation. 1960e. Irradiation Processing Department Monthly Report April, 1960. HW-65013, General Electric Co., Richland, Washington.

HAPO - Hanford Atomic Products Operation. 1960f. Irradiation Processing Department Monthly Report May, 1960. HW-65527, General Electric Co., Richland, Washington.

HAPO - Hanford Atomic Products Operation. 1960g. Irradiation Processing Department Monthly Report June, 1960. HW-65924, General Flectric Co., Richland, Washington.

HAPO - Hanford Atomic Products Operation. 1960h. Irradiation Processing Department Monthly Report July, 1960. HW-66296, General Electric Co., Richland, Washington.

HAPO - Hanford Atomic Products Operation. 1960i. Irradiation Processing Department Monthly Report August, 1960. HW-66685, General Electric Co., Richland, Washington.

HAPO - Hanford Atomic Products Operation. 1960j. Irradiation Processing Department Monthly Report September, 1960. HW-66970, General Electric Co., Richland, Washington.

HAPO - Hanford Atomic Products Operation. 1960k. Irradiation Processing Department Monthly Report October, 1960. HW-67286 DEL, General Electric Co., Richland, Washington.

HAPO - Hanford Atomic Products Operation. 19601. Irradiation Processing Department Monthly Report November, 1960. HW-67681, General Electric Co., Richland, Washington.

HAPO - Hanford Atomic Products Operation. 1961a. Irradiation Processing Department Monthly Report December, 1960. HW-68039, General Electric Co., Richland, Washington.

HAPO - Hanford Atomic Products Operation. 1961b. Irradiation Processing Department Monthly Report January, 1961. HW-68343, General Electric Co., Richland, Washington.

HAPO - Hanford Atomic Products Operation. 1961c. Irradiation Processing Department Monthly Report February, 1961. HW-68718 DEL, General Electric Co., Richland, Washington.

HAPO - Hanford Atomic Products Operation. 1961d. Irradiation Processing Department Monthly Report March, 1961. HW-69080, General Electric Co., Richland, Washington.

HAPO - Hanford Atomic Products Operation. 1961e. Irradiation Processing Department Monthly Report April, 1961. HW-69491, General Electric Co., Richland, Washington. 
HAPO - Hanford Atomic Products Operation. 1961f. Irradiation Processing Department Monthly Report May, 1961. HW-69835, General Electric Co., Richland, Washington.

HAPO - Hanford Atomic Products Operation. 1961g. Irradiation Processing Department Monthly Report June, 1961. HW-70211, General Electric Co., Richland, Washington.

HAPO - Hanford Atomic Products Operation. 1961h. Irradiation Processing Department Monthly Report July, 1961. HW-70625, General Electric Co., Richland, Washington.

HAPO - Hanford Atomic Products Operation. 1961i. Irradiation Processing Department Monthly Report August, 1961. HW-70952, General Electric Co., Richland, Washington.

HAPO - Hanford Atomic Products Operation. 1961j. Irradiation Processing Department Monthly Report September, 1961. HW-71230, General Electric Co., Richland, Washington.

HAPO - Hanford Atomic Products Operation. 1961k. Irradiation Processing Department Monthly Report October, 1961. HW-71584, General Electric Co., Richland, Washington.

HAPO - Hanford Atomic Products Operation. 19611. Irradiation Processing Department Monthly Report November, 1961. HW-71910, General Electric Co., Richland, Washington.

HAPO - Hanford Atomic Products Operation. 1962a. Irradiation Processing Department Monthly Report December, 1961. HW-72179, General Electric Co., Richland, Washington.

HAPO - Hanford Atomic Products Operation. 1962b. Irradiation Processing Department Monthly Report January, 1962. HW-72567, General Electric Co., Richland, Washington.

HAPO - Hanford Atomic Products Operation. 1962c. Irradiation Processing Department Monthly Report February, 1962. HW-72813, General Electric Co., Richland, Washington.

HAPO - Hanford Atomic Products Operation. 1962d. Irradiation Processing Department Monthly Report March, 1962. HW-73153, General Electric Co., Richland, Washington.

HAPO - Hanford Atomic Products Operation. 1962e. Irradiation Processing Department Monthly Report April, 1962. HW-73473, General Electric Co., Richland, Washington.

Lang, L. W. 1952a. Ruptured Slug Data. HW-24020, General Electric Co., Hanford Works, Richland, Washington.

Lang, L. W. 1952b. Ruptured Slug Data. HW-24379, General Electric Co., Hanford Works, Richland, Washington.

Lang, L. W., and J. K. Andersion. 1952c. Rupture Slug Data and Comparative Rupture Rates for October, 1952. HW-26060, Gerieral Electric Co., Hanford Works, Richland, Washington. 
Lang, L. W., and J. K. Anderson. 1952d. Rupture Slug Data and Comparative Rupture Rates for November, 1952. HW-26385, General Electric Co., Hanford Works, Richland, Washington.

Lang, L. W., and J. K. Anderson. 1953a. Rupture Slug Data and Comparative Rupture Rates for December, 1952. HW-26646, General Electric Co., Hanford Works Richland, Washington.

Lang, L. W., and J. K. Anderson. 1953b. Rupture Slug Data and Comparative Rupture Rates for January, 1953. HW-26980, General Electric Co., Hanford Atomic Products Operation, Richland, Washington.

Lang, L. W., and J. K. Anderson. 1953c. Rupture Slug Data and Comparative Rupture Rates for February, 1953. HW-27266, General Electric Co., Hanford Atomic Products Operation, Richland, Washington.

Lang, L. W., and J. K. Anderson. 1953d. Ruptured Slug Data and Comparative Rupture Rates for March, 1953. HW-27616, General Electric Co., Hanford Atomic Products Operation, Richland, Washington.

Lang, L. W., and J. K. Anderson. 1953e. Ruptured Slug Data and Comparative Rupture Rates for April, 1953. HW-28027, General Electric Co., Hanford Atomic Products Operation, Richland, Washington.

Neef, W. I. 1958a. Ruptured Slug Data January 1, Thru March 31, 1958. HW-55823, Hanford Atomic Products Operation, General Electric Co., Richland, Washington.

Neef, W. I. 1958b. Ruptured Slug Data April I, .Thru April 13, 1958. HW-56140, Hanford Atomic Products Operation, General Electric Co., Richland, Washington.

Neef, W. I. 1958c. Ruptured Slug Data April 11 Thru July 6, 1958. HW-57255, Hanford Atomic Products Operation, General Electric Co., Richland, Washington.

Neef, W. I. 1958d. Ruptured Slug Data July 7 Through August 4, 1958. HW-57592, Hanford Atomic Products Operation, General Electric Co., Richland, Washington.

Neef, W. I. 1958e. Ruptured Slug Data August 5, Through August 14, 1958. HW-57648, Hanford Atomic Products Operation, General Electric Co., Richland, Washington.

Neef, W. 1. 1958f. Ruptured Slug Data August 15 Through September 30, 1958. HW-57946, Hanford Atomic Products Operation, General Electric Co., Richland, Washington.

Neef, W. I. 1959a. Ruptured Slug Data October 1, 1958 Through December 11, 1958. HW-58965, Hanford Atomic Products Operation, General Electric Co., Richland, Washington.

Neef, W. I. 1959b. Ruptured Slug Data December 12, 1958, Through March 6, 1959. HW-59823, Hanford Atomic Products Operation, General Electric Co., Richland, Washington. 
Newell, L. J. 1962. Ruptured Slug Data May 1, 1962, Through August 31, 1962. HW-74967, Hanford Atomic Products Operation, General Electric Co., Richland, Washington.

Newell, L. J. 1963a. Ruptured Slug Data September 1, 1962, Through December 31, 1962. HW76198, Hanford Atomic Products Operation, General Electric Co., Richland, Washington.

Newell, L. J. 1963b. Ruptured Slug Data January 1, 1963, Through April 30, 1963. HW-77333, Hanford Atomic Products Operation, General Electric Co., Richland, Washington.

Newell, L. J. 196'3c. Ruptured Slug Data May 1, 1963, Through August 31, 1963. HW-79031, Hanford Atomic Products Operation, General Electric Co., Richland, Washington.

Newell, L. J. 1964a. Ruptured Slug Data September 1, 1963, Through December 31, 196j. HiW80481, Hanford Atomic Products Operation, General Electric Co., Richland, Washington.

Newell, L. J. 1964b. Ruptured Slug Data January 1, 1964, Through April 30, 1964. HW-83588, Hanford Atomic Products Operation, General Electric Co., Richland, Washington.

Newell, L. J. 1964c. Ruptured Slug Data May 1, 1964, Through August 31, 1964. HW-84069, Hanford Atomic Products Operation, General Electric Co., Richland, Washington.

Newell, L. J. 1965a. Ruptured Slug Data September 1, 1964 Through December 31, 1964. RLREA-545, Hanford Atomic Products Operation, General Electric Co., Richland, Washington.

Newell, L. J. 1965b. Ruptured Slug Data January ', 1965 Through April 30, 1965. RL-REA-2076, Hanford Atomic Products Operation, General Electric Co., Richland, Washington.

Newell, L. J. 1965c. Ruptured Slug Data May 1, 1965 Through August 31, 1965. RL-REA-2557, Hanford Atomic Products Operation, General Electric Co., Richland, Washington.

Newell, L. J. 1966a. Fuel Failure Data September 1, 1965 Through December 31, 1965. DUN729, Douglas United Nuclear Inc., Richland, Washington.

Newell, L. J. 1966b. Fuel and Target Failure Data January 1, 1966 Through April 30, 1966.

DUN-1067, Douglas United Nuclear Inc., Richland, Washing:on.

Newell, L. J. 1967. Fuel and Target Failure Data May 1, 1966, Through December 31, 1966.

DUN-1972, Douglas United Nuclear Inc., Richland, Washington.

Newell, L. J. 1968. Fuel and Target Failure Data January 1, 1967 Through December 31, 1967. DUN-3857, Douglas United Nuclear Inc., Richland, Washington.

Newell, L. J. 1969a. Fuel and Target Failure Data January 1, 1968 Through December 31, 1969. DUN-5488, Douglas United Nuclear, Richland, Washington. 
Newell, L. J. 1969b. Fuel Failure Reports-1968-1969. DUN-5558 RD, Douglas United Nuclear Inc., Richland, Washington.

Paulovich, K. F. 1955. 100 and 300 Areas Monthly Reports - 1954. HAN-54065 DEL, U.S. Atomic Energy Commission, Richland Operations Office, Richland, Washington.

Paulovich, K. F. 1955. 100 and 300 Areas Monthly Reports for January through August, 1955. HAN-58378 DEL, U.S. Atomic Energy Commission, Richland Operations Office, Richland, Washington.

Shipler, D. B. 1993. Integrated Task Plans for the Hanford Environmental Dose Reconstruction Project, FY 1992 through May 1994. PIWD-2020 HEDR, Battelle, Pacific Northwest Laboratories, Richland, Washington.

Sowards, J. W. 1956a. Ruptured Slug Data - February 1956. HW-42654, General Electric Co., Hanford Atomic Products Operation, Richland, Washington.

Sowards, J. W. 1956b. Ruptured Slug Data-March 1956. HW-42962, General Electric Co., Hanford Atomic Products Operation, Richland, Washington.

Sowards, J. W. and L. W. Hartse 1956c. Ruptured Slug Data-April 1956. HW-43065, Genural Electric Co., Hanford Atomic Products Operation, Richland, Washington.

Sowards, J. W. and L. W. Hartse 1956d. Ruptured Slug Data - May 1956. HW-43838, General Electric co., Hanford Atomic Products Operation, Richland, Washington.

Sov ards, J. W., and L. W. Hartse. 1956e. Ruptured Slug Data - June 1956. HW-44261, Hanford A. J Jmic Products Operation, General Electric Co., Richland, Washington.

Wood, E. C. 1951. Ruptured Slug Data. HW-22984, General Electric Co., Hanford Works, Richland, Washington.

Wood, E. C. 1952. Ruptured Slug Data. HW-23185, General Electric Co., Hanford Works, Richland, Washington. 


\section{Bibliography}

Anderson, J. K. 1954. Slug Rupture Outbreak at H Pile. HW-33645, General Electric Co., Hanford Atomic Products Operation, Richland, Washington.

Anderson, J. K. 1955. 1954 Slug Rupture Experience at Hanford. HW-35221, General Zlectric Co., Hanford Atomic Products Operation, Richland, Washington.

Bloomstrand, R. 1954. Effects of Tube Power and Exposure on Rupture Rates of 8" Metal at C Reactor. HW-32004, General Electric Co., Hanford Atomic Products Operation, Richland, Washington.

Bloomstr and, R. 1956. Raising K Reactor Power Levels: Ruptured Slug Aspects. HW-46447, General Electric Co., Hanford Atomic Products Operation, Richland, Washington.

Bloomstrand, R. R., and W. I. Neef. 1958. Analyses and Correlations of HAPO Rupture Experience with Natural Uranium Material. HW-55377, General Electric Co., Hanford Atomic Finducts Operation, Richland, Washington.

Borgmier, B. J. 1951. Bibliography on Slug Failures Occurring in the Hanford Production Reactors. HW-21199, General Electric Co., Hanford Works, Richland, Washington.

Cell, R. F. 1951. Summary of Results to Date of Statistical Investigation of Slug Failures. HW22762, General Electric Co., Hanford Works, Richland, Washington.

Cawley, W. F. 1955. Possible Slug Rupture Mechanism. HW-33685-Rev, General Electric Co., Hanford Atomic Products Operation, Richland, Washington.

Crane, P. W., and M. H. Smith. 1944. Detection of Slug Failure by Means of the Activity in Discharge Water. HW-3-0588, E. I. Du Pont de Nemours and Company, Hanford Engineer Works, Richland, Washington.

Evans, E. A. 1956. Discussion of Failure Rates. HW-56352-RD, General Electric Co., Hanford Atomic Products Operation, Richland, Washington.

Hall, R. B. 1963. Environmental Effects of a Fuel Element Failure. HW-79073, General Electric Co., Hanford Atomic Products Operation, Richland, Washington.

Healy, J. W. 1953a. Single Tube Failures. HW-29555, General Electric Co., Hanford Atomic Products Operation, Richland, Washington.

Healy, J. W. 1953b. Single Tube Failures. HW-29907, General Electric Co., Hanford Atomic Products Operation, Richland, Washington. 
Healy, J. W. 1954. Single Tube Failures. HW-31127, General Electric Co., Hanford Atomic Products Operation, Richland, Washington.

Jacky, G. F. 1955. "Low Exposure" Slug Failures: Associated Factors and Hypothesized Causes. HW-38629, General Electric Co., Hanford Atomic Products Operation, Richland, Washington.

Jerman, P. C., W. N. Koop, and F. E. Owen. 1965. Release of Radioactivity to the Columbia River from Irradiated Fuel Element Ruptures. RL-REA-2160, General Electric Co., Hanford Atomic Products Operation, Richland, Washington.

Jordan, W. E. 1944. Exit Water Monitoring for Slug Failure - Operation of System in 105B. HW-31121, E. I. Du Pont de Nemours and Company, Hanford Engineer Works, Richland, Washington.

Lang, L. W. 1952a. Comparison of Rupture Rates in Group 7 and Group 8 Material. HW-24247, General Electric Co., Hanford Works, Richland, Washington.

Lang, L. W. 1952b. Analysis of Ruptured Slug Data. HW-25177, General Electric Co., Hanford Works, Richland, Washington.

Mars, H. L. 1951. Detection of Slug Failure by Monitoring the Activity of the Discharged Water. HW-20017, General Electric Co., Hanford Works, Richland, Washington.

McCormack, J. D., and L. C. Schwendiman. 1959a. Significance of Rupture Debris in the Columbia River. HW-61325, General Electric Co., Hanford Atomic Products Operation, Richland, Washington.

McCormack, J. D., and L. C. Schwendiman. 1959b. Calibration of Rupture Monitors. HW-61326, General Electric Co., Hanford Atomic Products Operation, Richland, Washington.

Mehan, R. O. 1951. Ruptured Slug Data. HW-22730, General Electric Co., Hanford Works, Richland, Washington.

Nelson, S. L. 1954. High Rupture Rate at 100-H During October 1954. HW-33945, General Electric Co., Hanford Atomic Products Operation, Richland, Washington.

O'Connor, J. J. 1945. Exit Water Monitoring for Slug Jacket Failure. HW-3-1913, E. I. Du Pont de Nemours and Company, Hanford Engineer Works, Richland, Washington.

O'Keefe, D. P. 1954. Macro-Examination of Enriched Uranium Slug Ruptures. HW-31044, General Electric Co., Hanford Atomic Products Operation, Richland, Washington.

Pearl, W. L., and C. A. Pursel. 1954. Design Considerations Regarding Slug Ruptures in the Intermediate Power Level Reactor. HW-33535, General Electric Co., Hanford Atomic Products Operation, Richland, Washington. 
Perkins, R. W. 1962. Effect of a Reactor Fuel Element Failure on the Columbia River Radionuclide Concentrations at Pasco, Washington. HW-76153, General Electric Co., Hanford Atomic Products Operation, Richland, Washington.

Spencer, H. G. 1951. Negative Correla ion of Ruptured Slugs with Control Rod Locations. HW21304, General Electric Co., Hanford Works, Richland, Washington.

Walkup, P. C. 1957. H Reactor Startup Causing Fuel Element Ruptures. HW-53481, General Electric Co., Hanford Atomic Products Operation, Richland, Washington.

Warren, J. H. 1952. Production Loss Due to Ruptured Uranium Slugs - Year 1951. HW-22813, General Electric Co., Hanford Works, Richland, Washington.

West, J. M. 1946. Detection of Slug Failure by Means of the Activity of the Discharged Water (Final Report). HW-7-4671, E. I. Du Pont de Nemours and Company, Hanford Engineer Works, Richland, Washington.

Young, J. R. 1956. Analysis of Reactor Section Rupture Experience in Regular Metal Exposed to High Concentration. HW-44773, General Electric Co., Hanford Atomic Products Operation, Richland, Washington. 
APPENDIX A

FUEL-ELEN̂IENT FAILURE DATA FOR 1948-1951 
๙

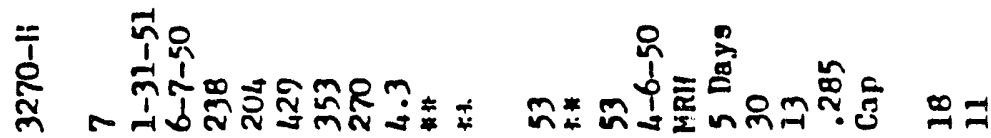

至昏

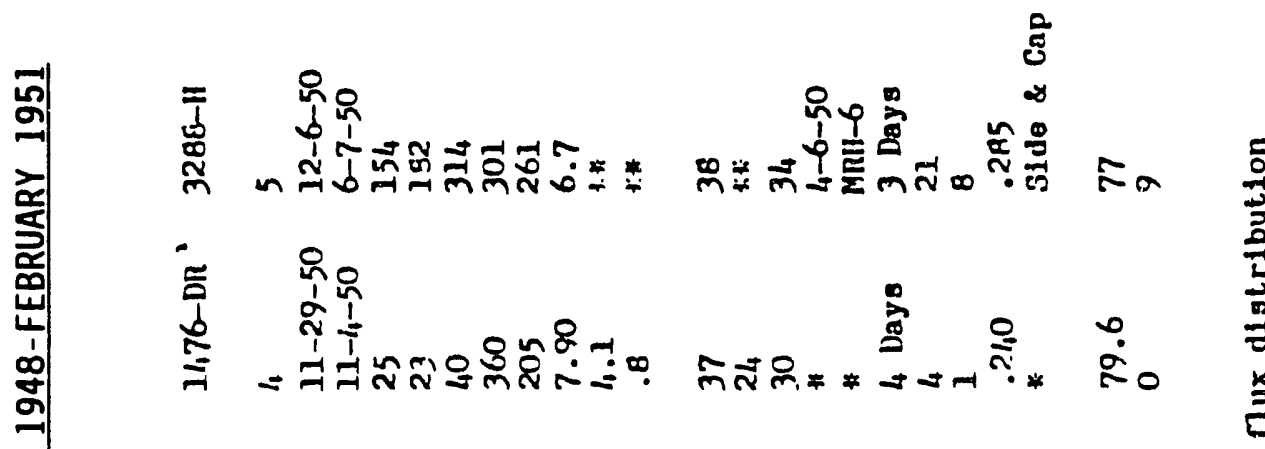

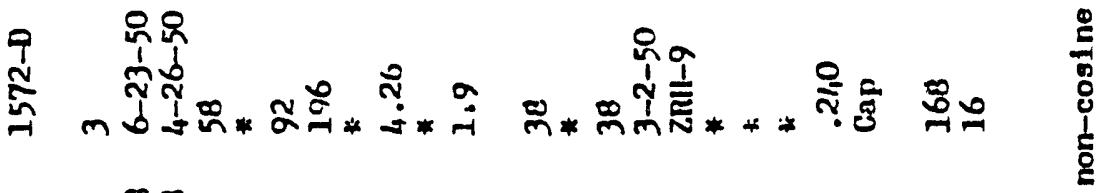

- $\quad \sum_{i}^{\infty} \stackrel{0}{I}$

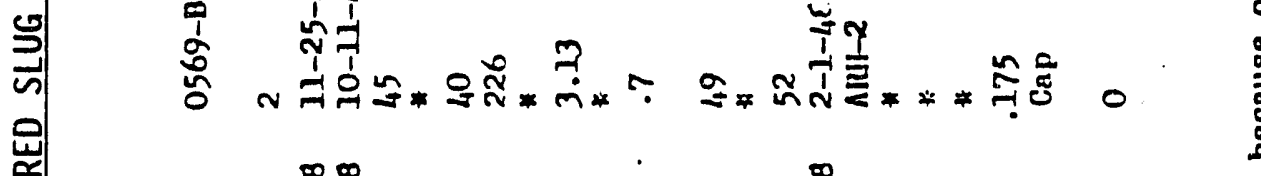

을

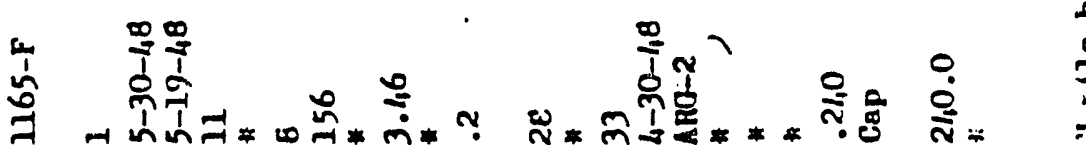

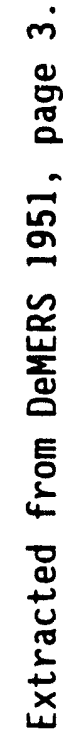

A. 1 


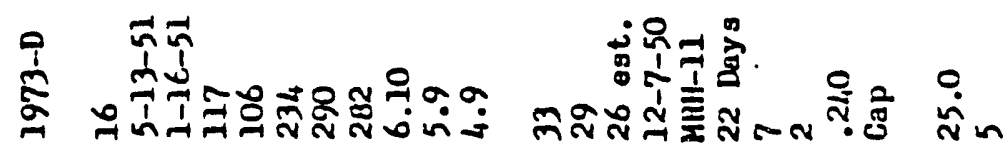

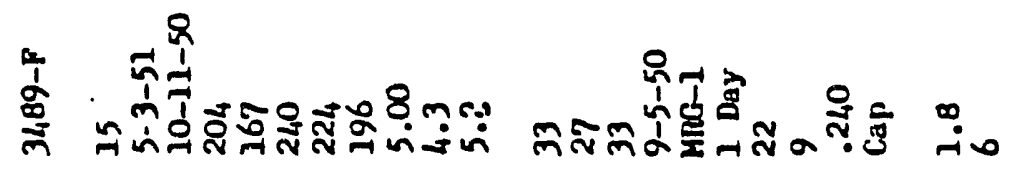

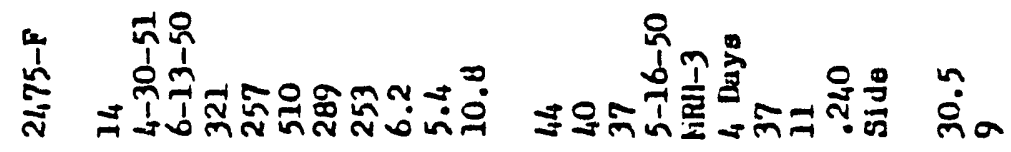

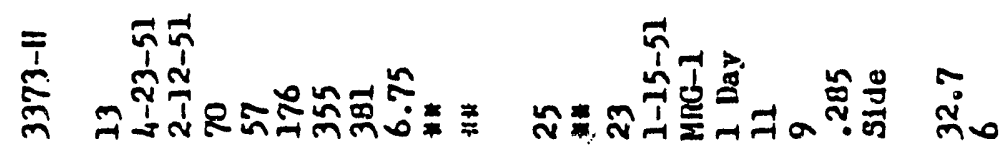

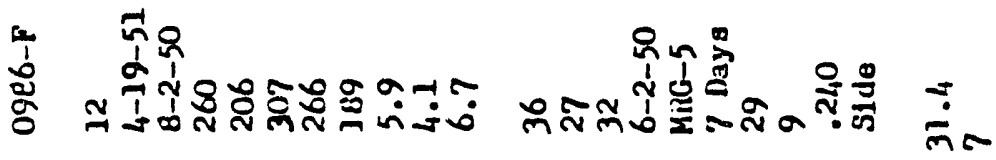

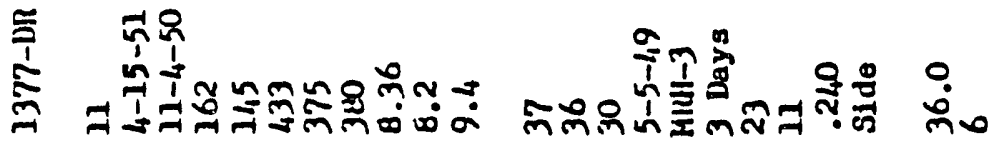

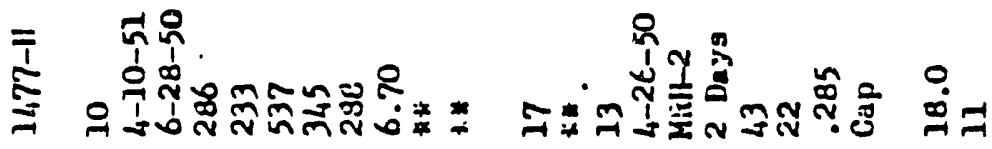

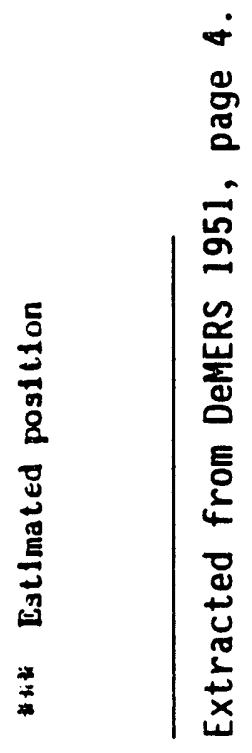




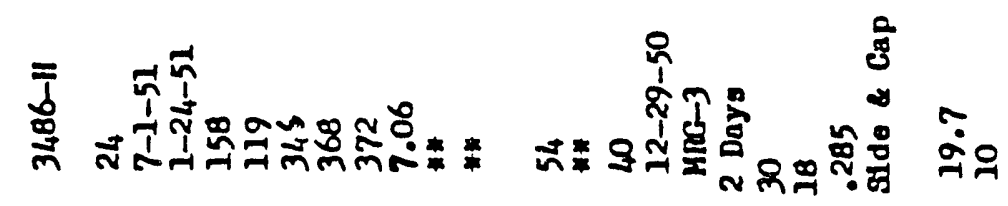

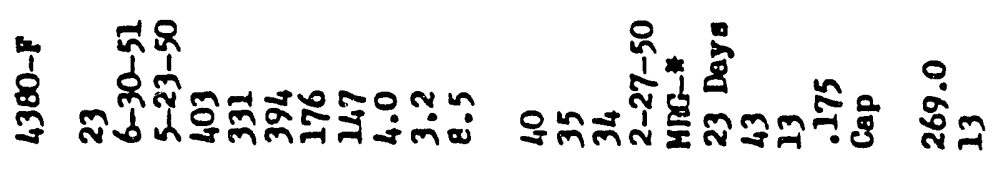

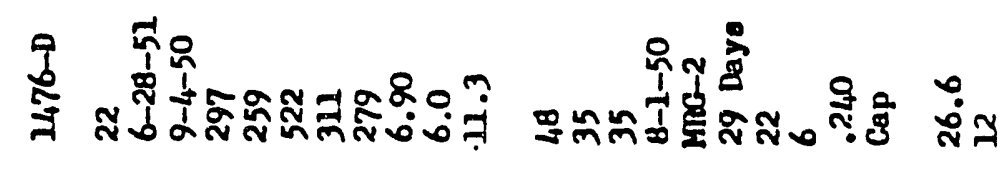

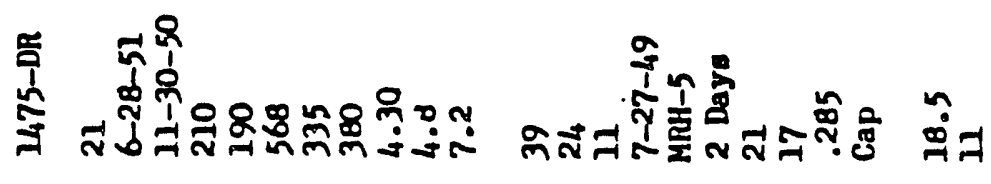

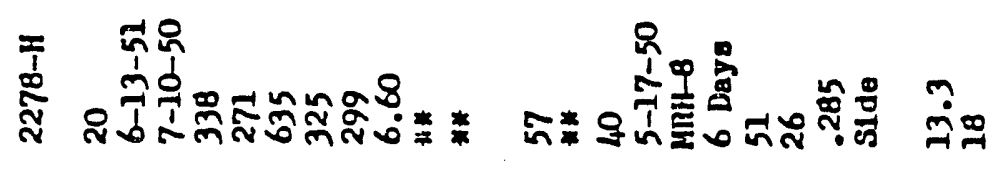

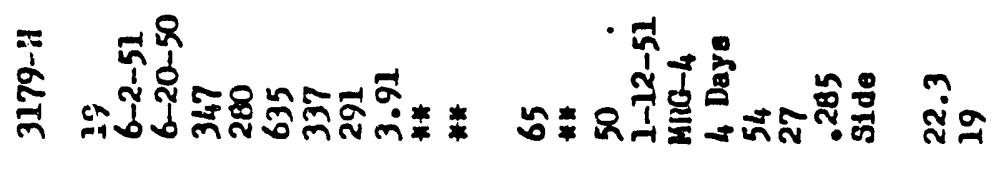

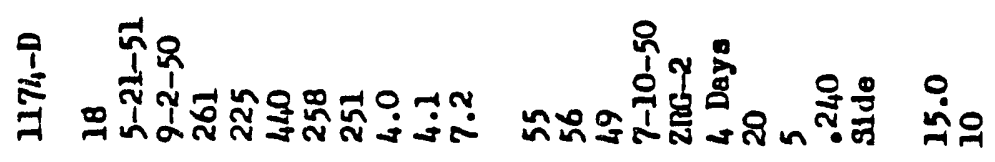

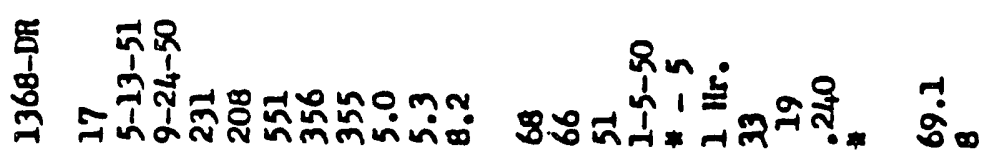




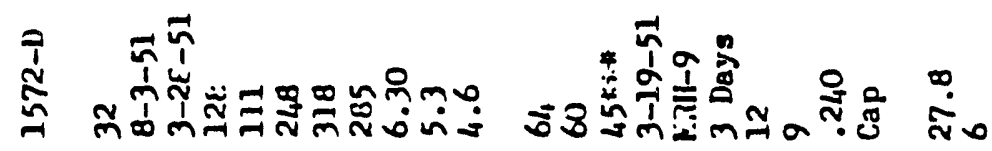

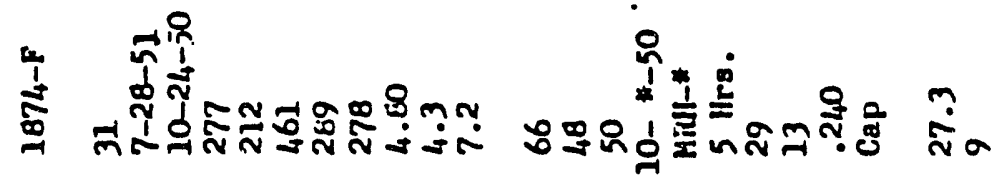

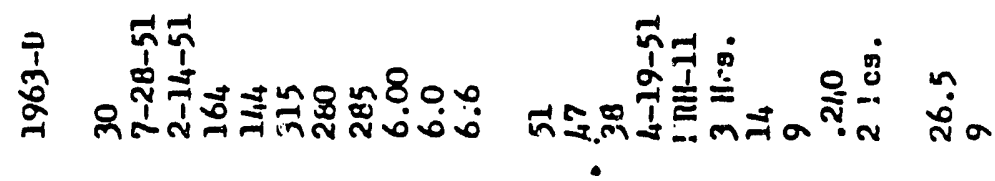

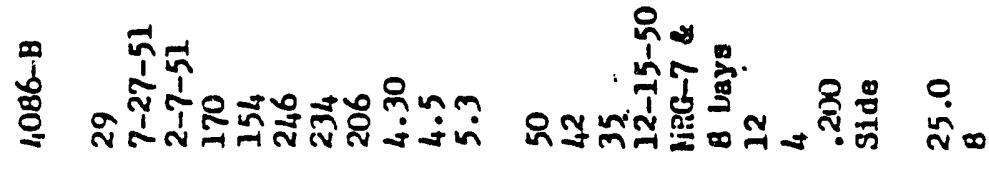

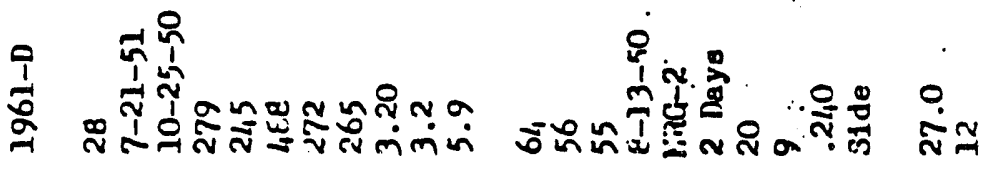

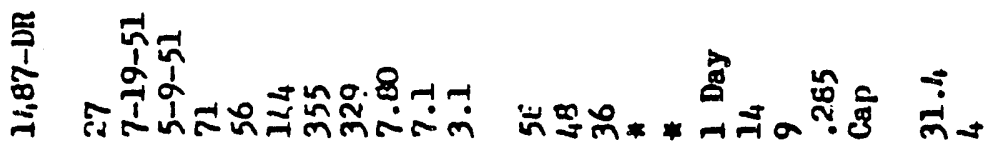

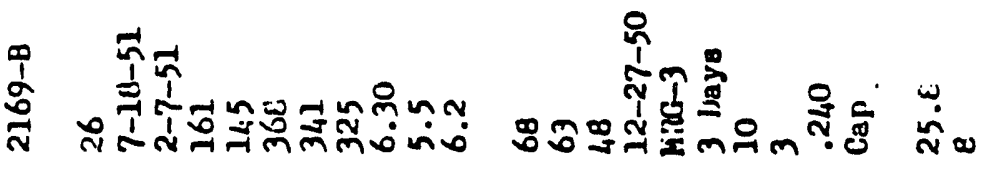

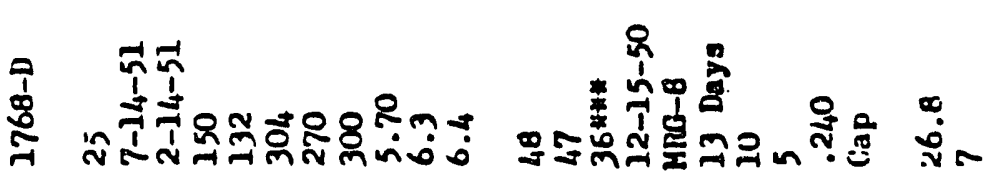

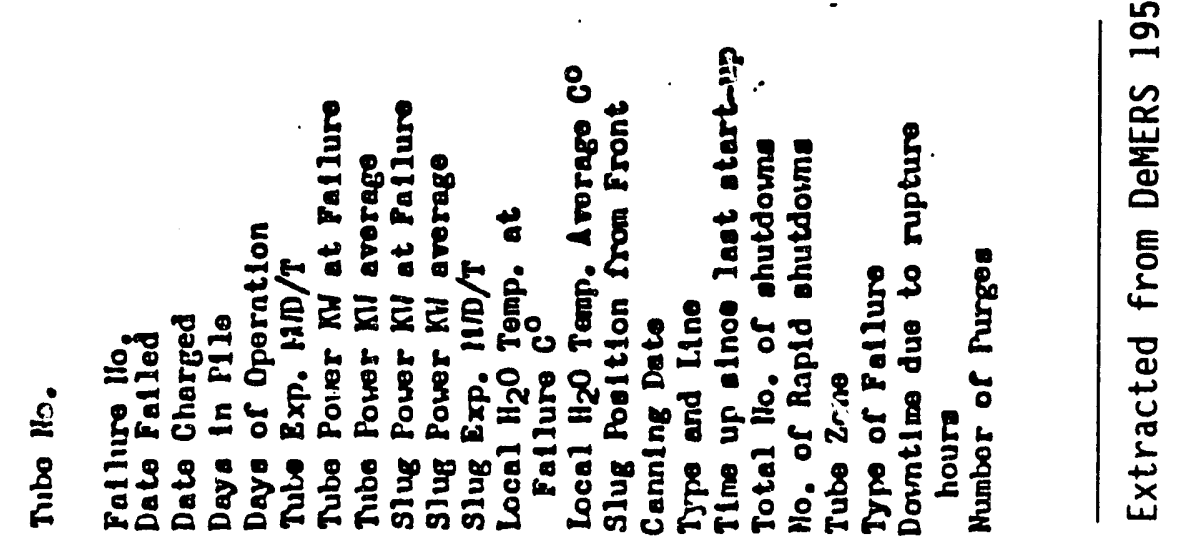




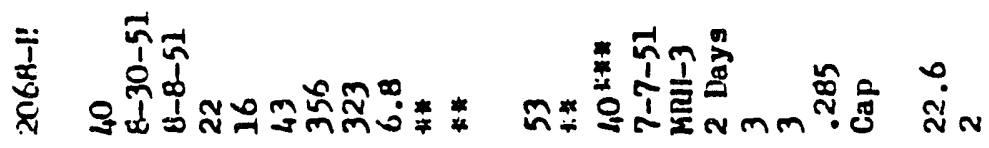

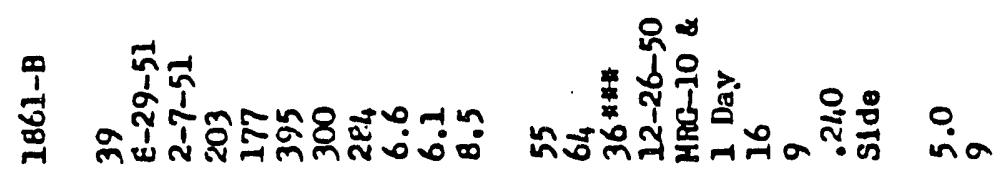

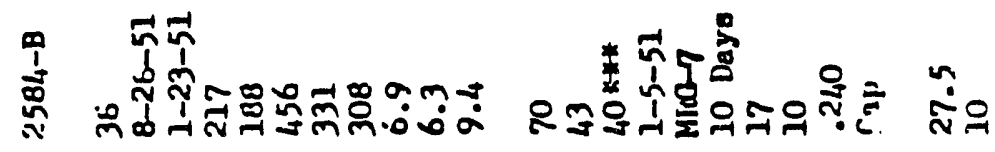

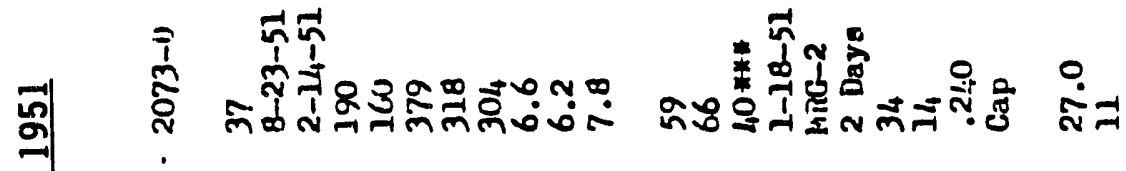

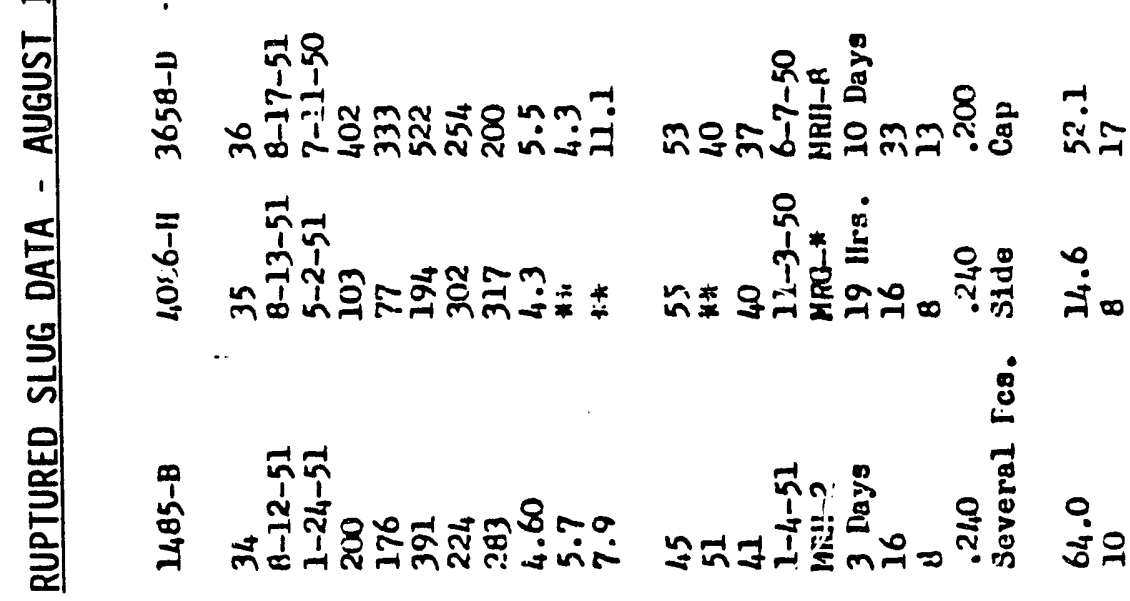
竞

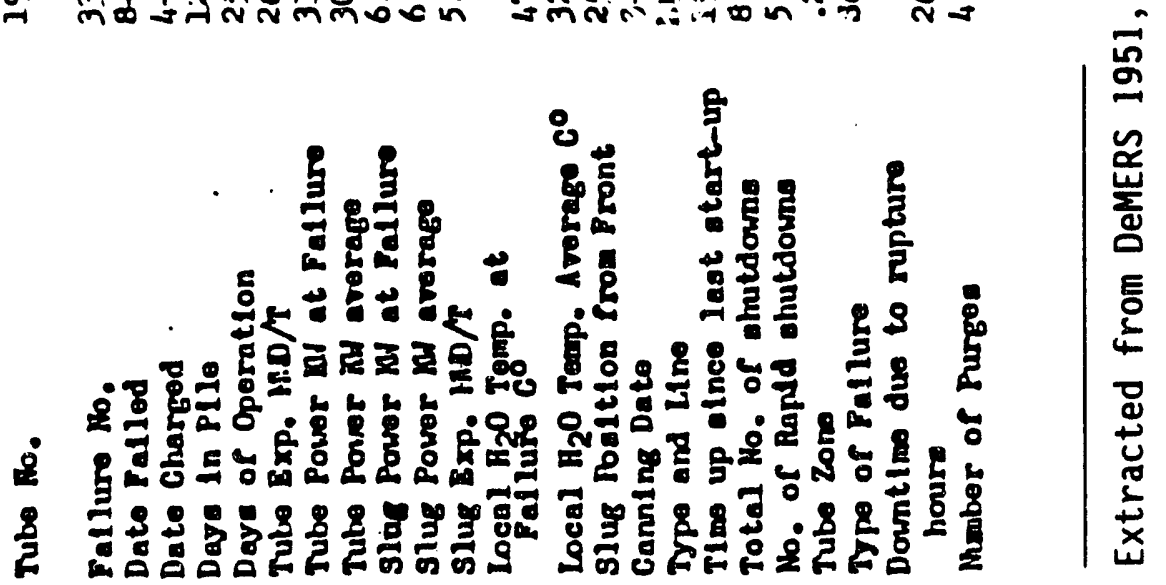




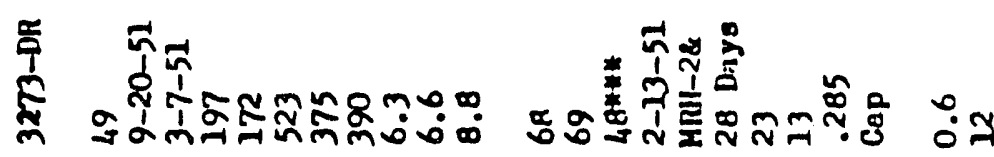

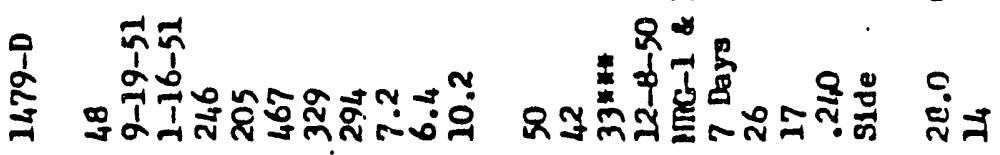

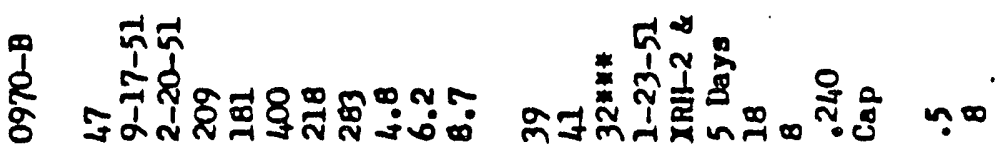

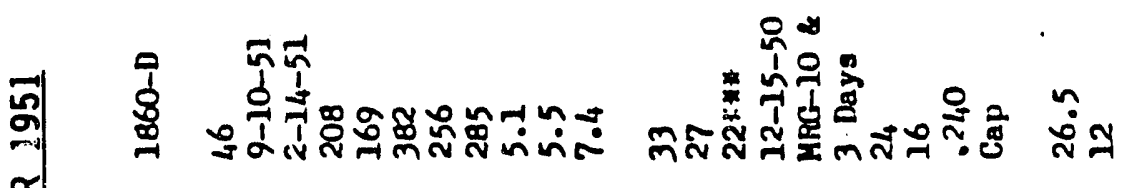

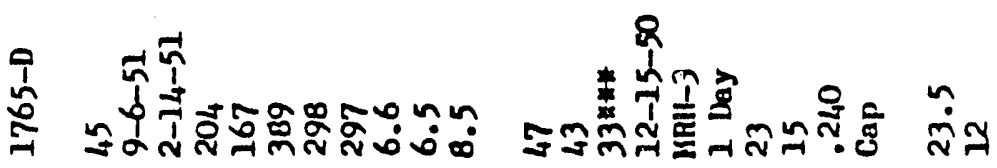

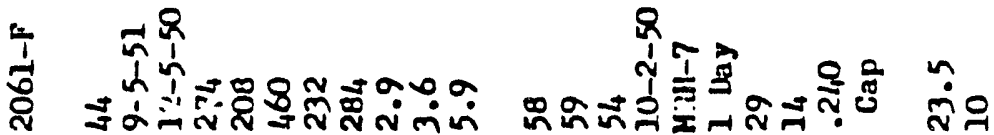

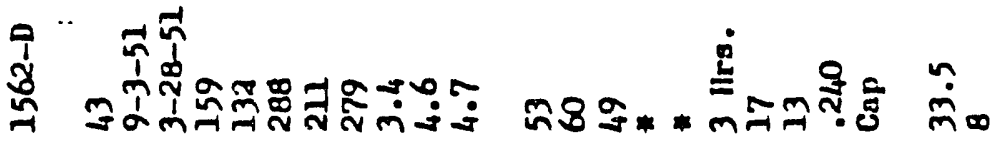

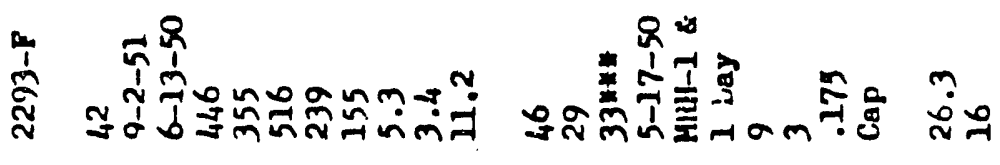

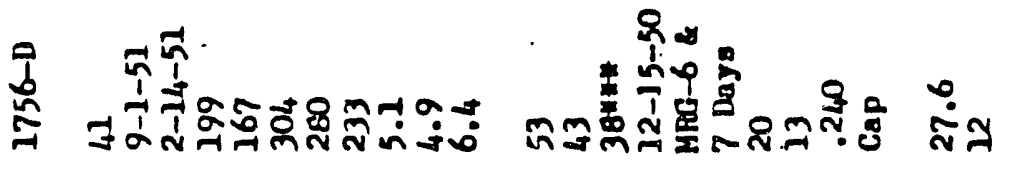

$\infty$

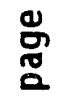

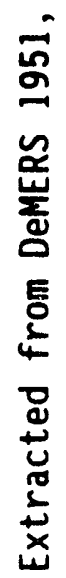




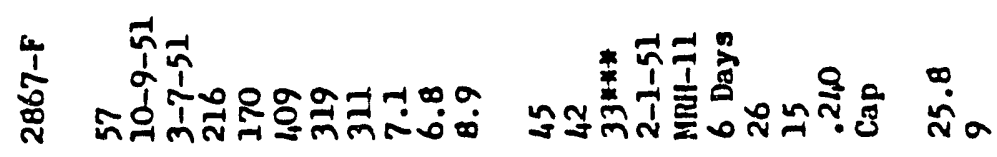

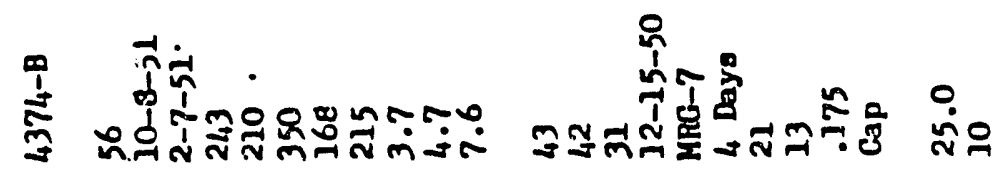

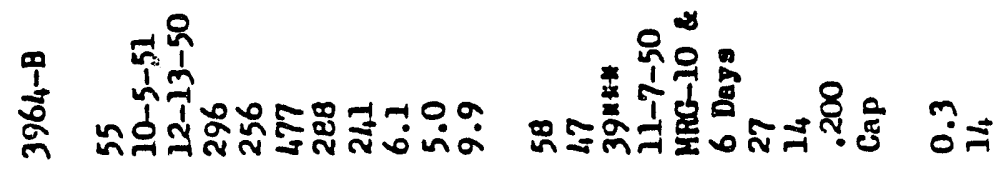

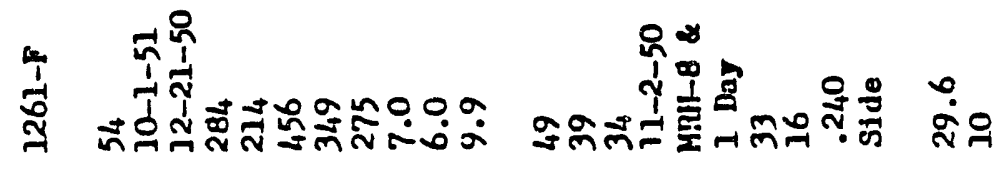

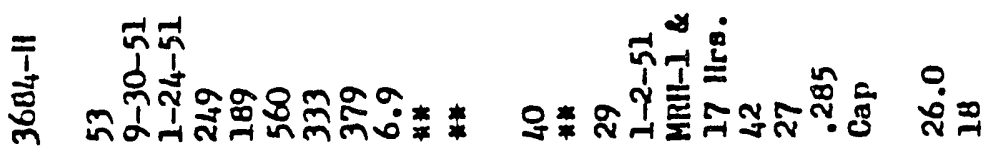

容

亭

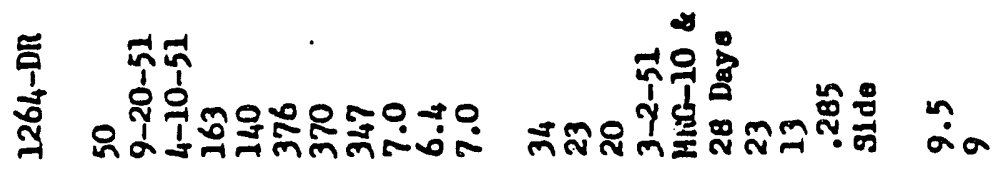

iे

号

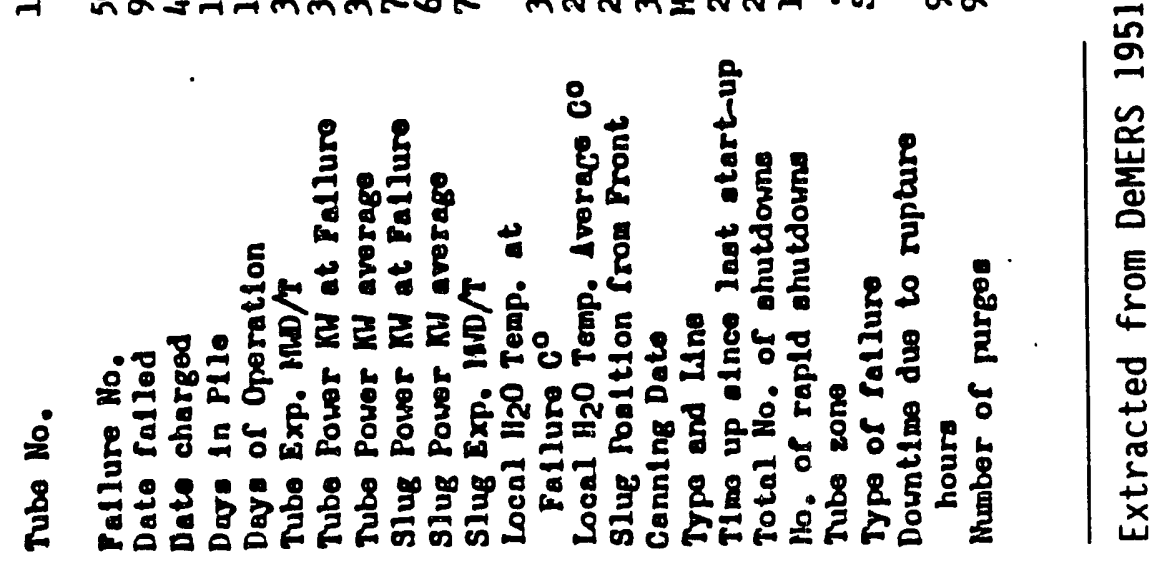

A. 7 


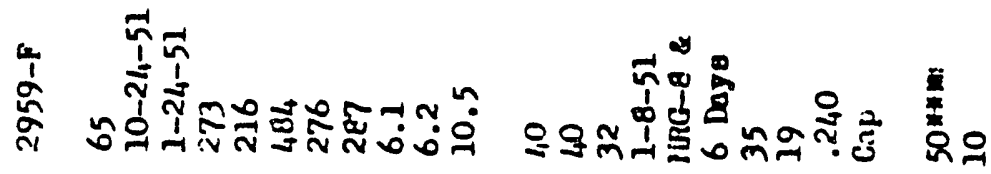

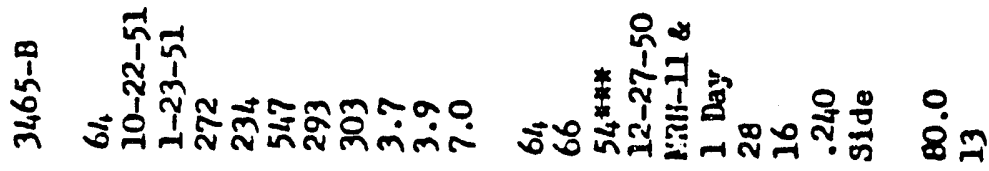

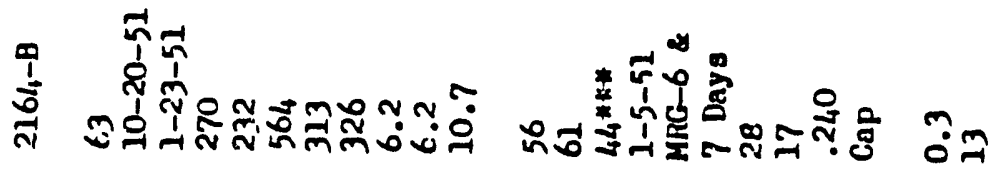

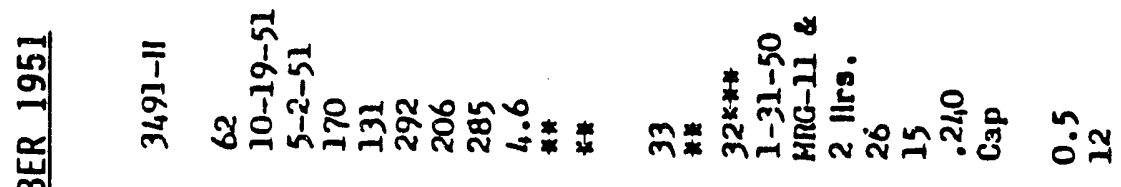

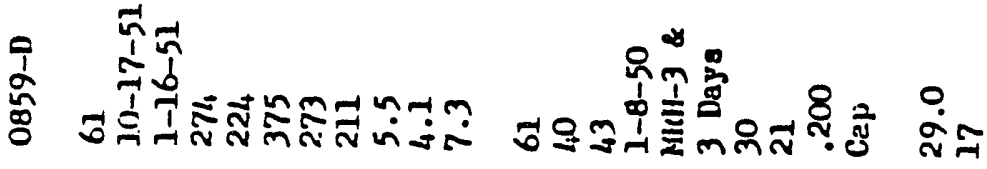

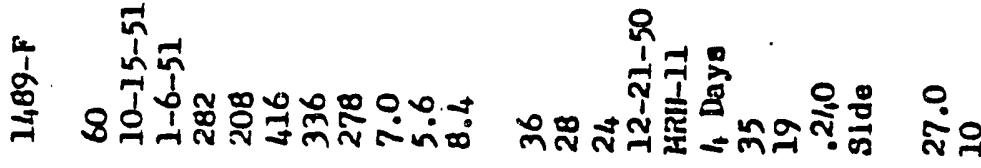

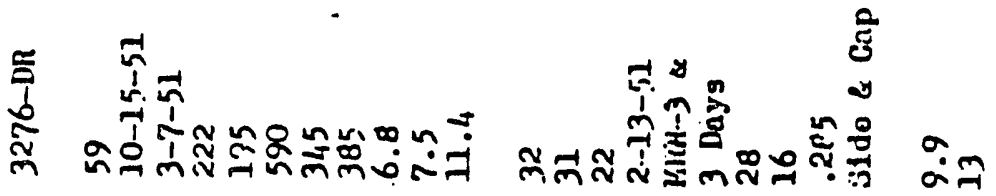

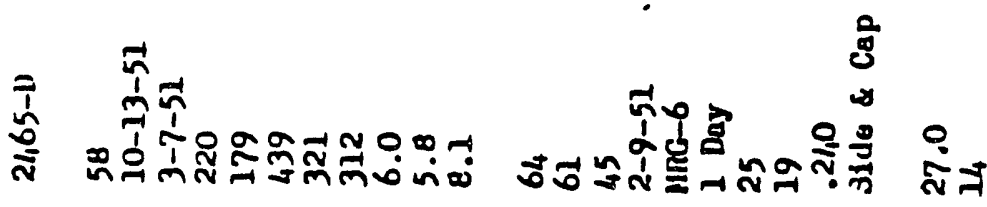

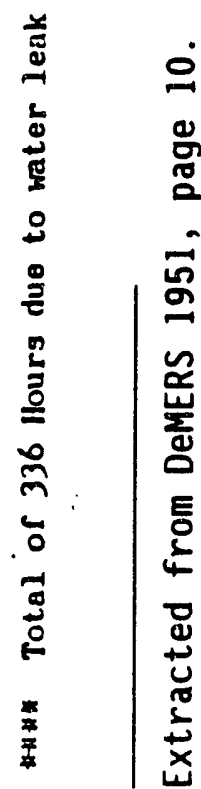




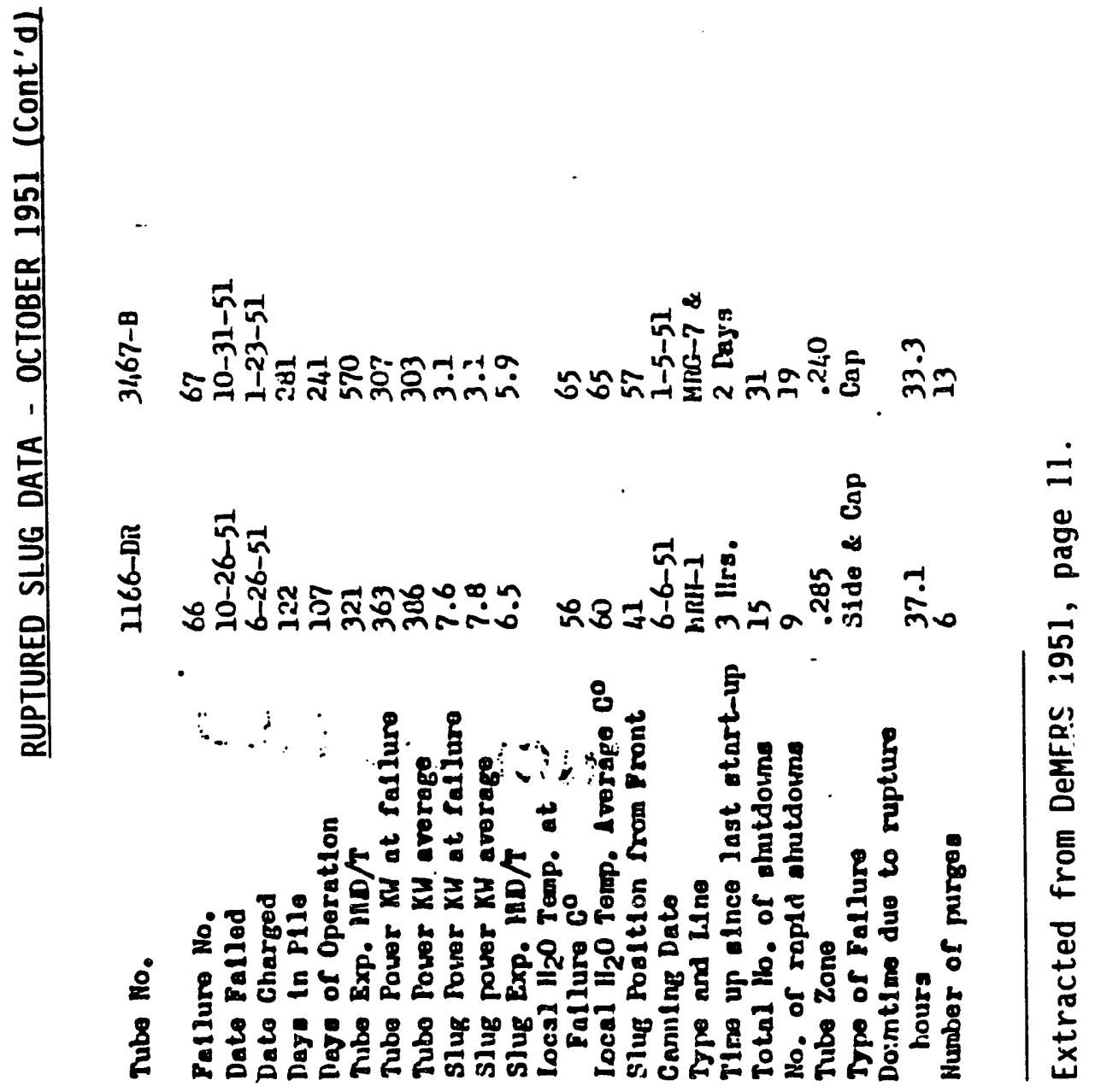




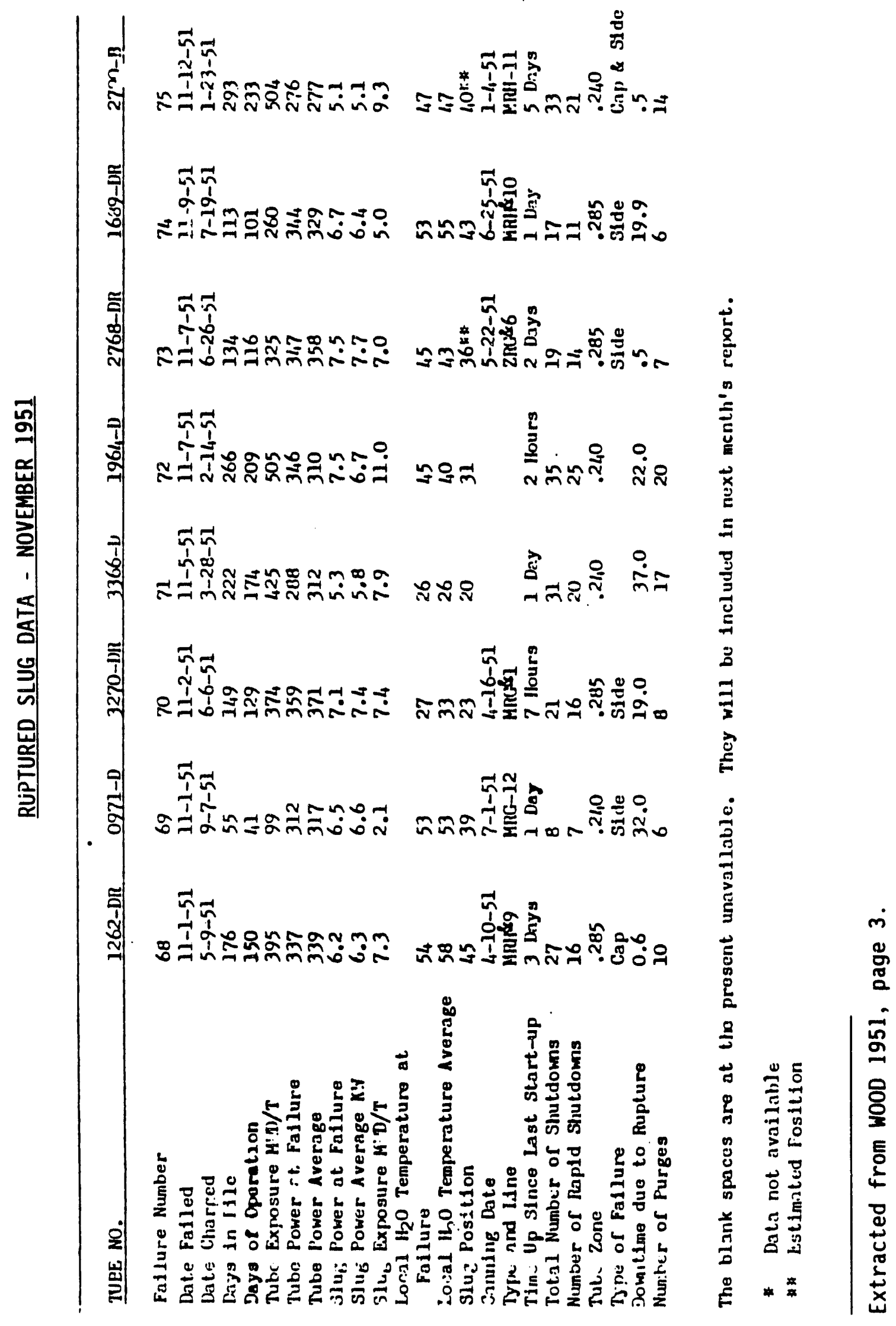




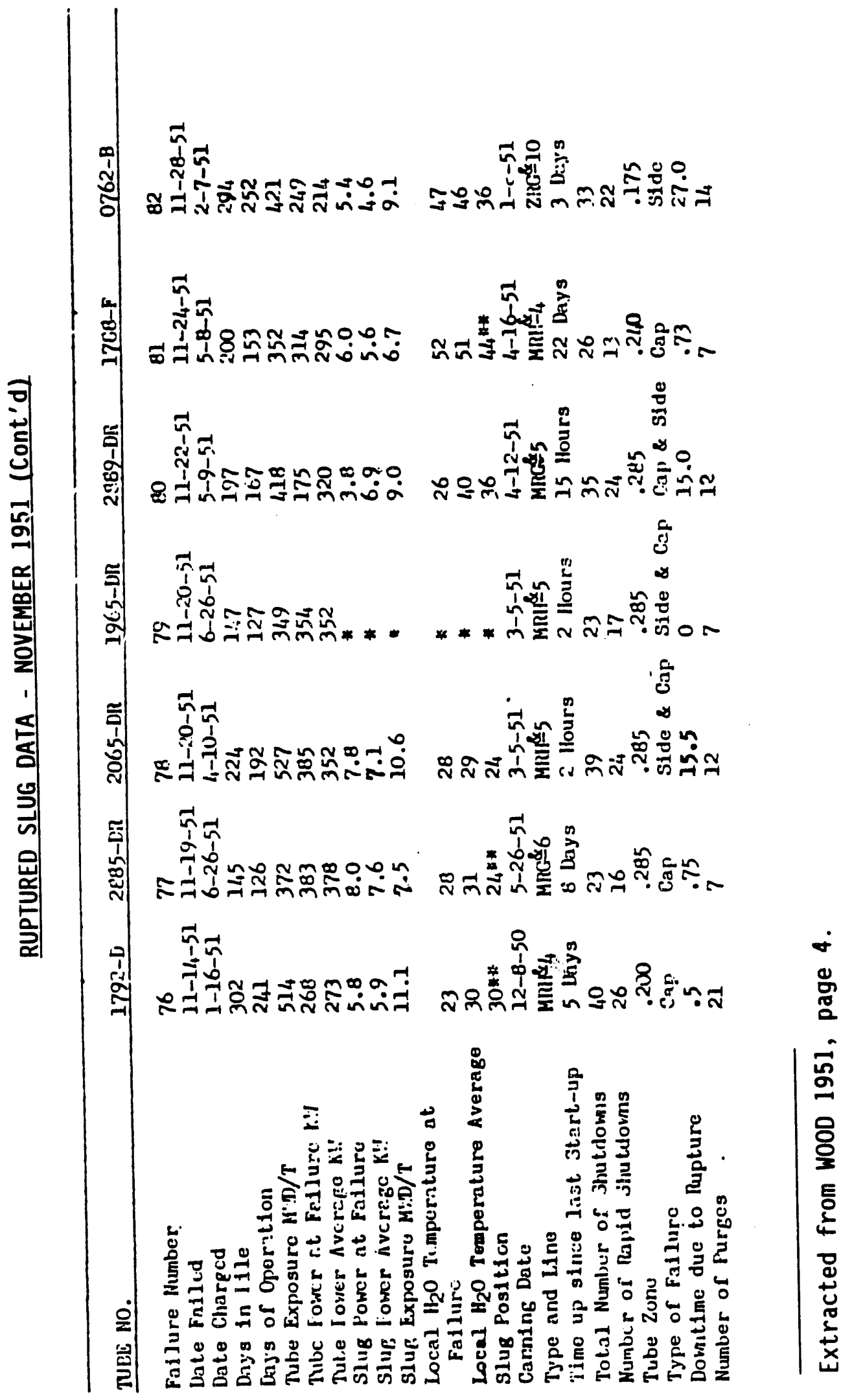




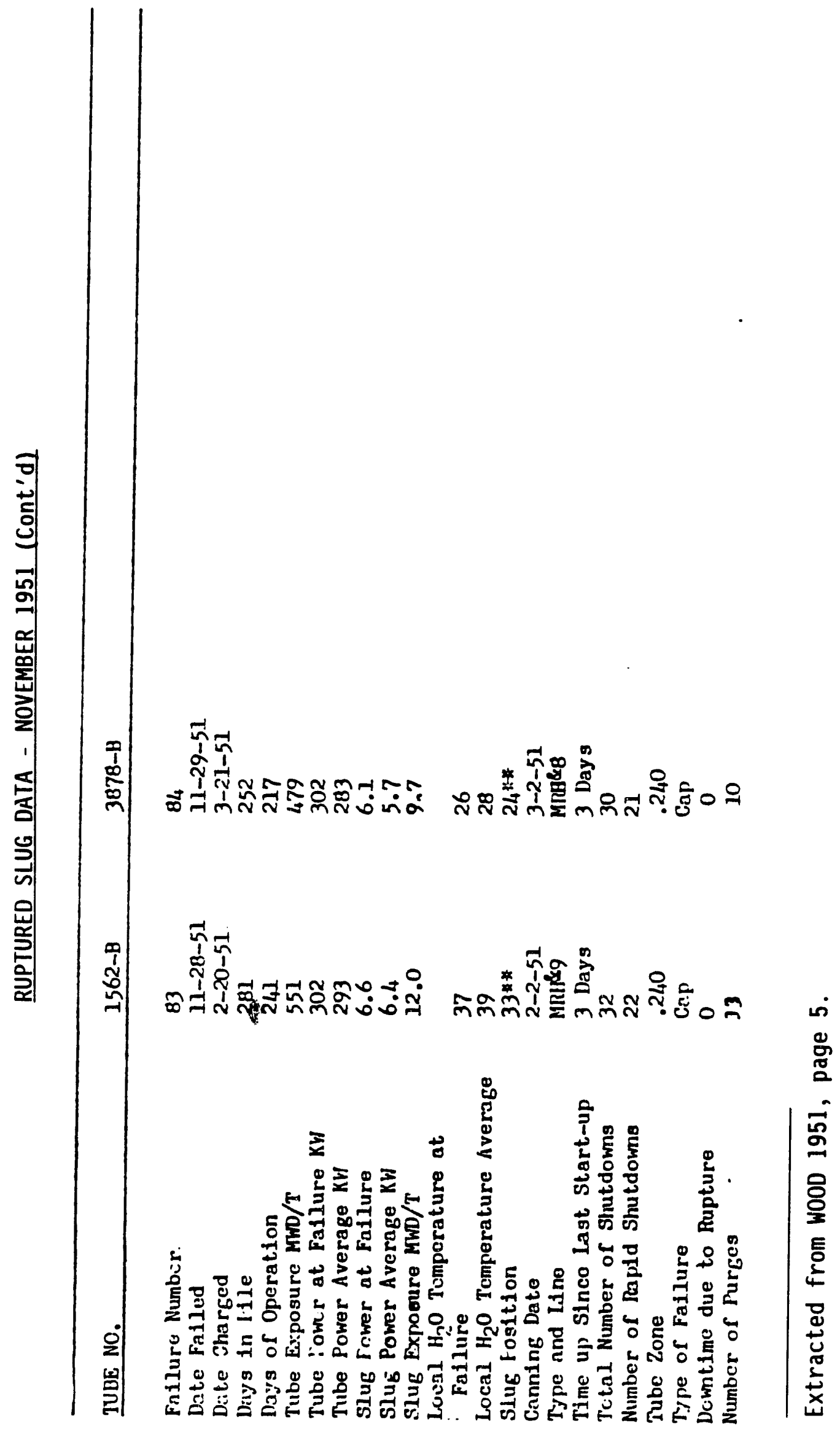




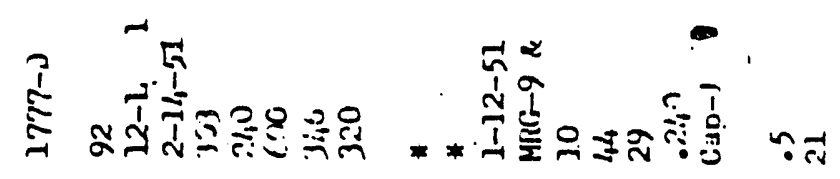

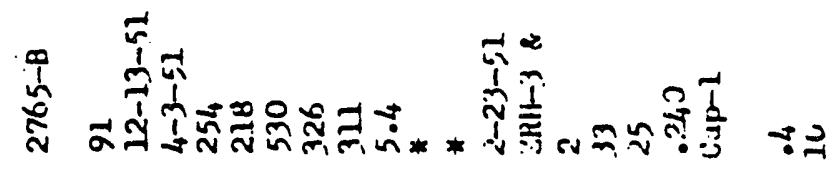

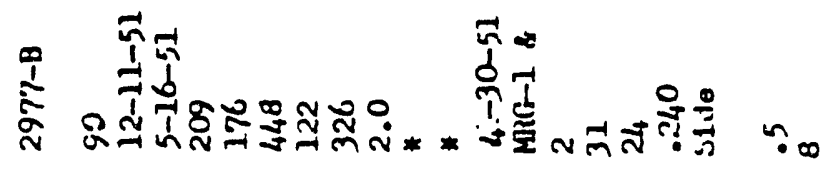

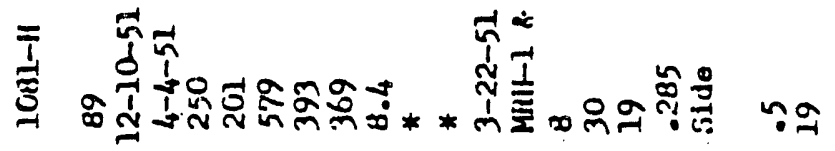

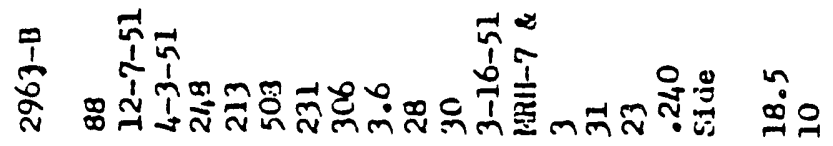

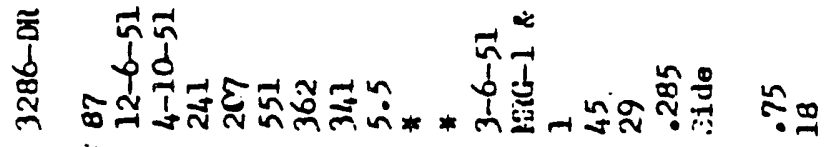

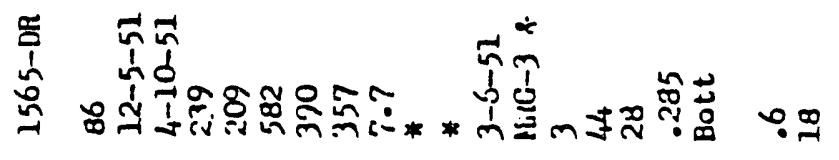

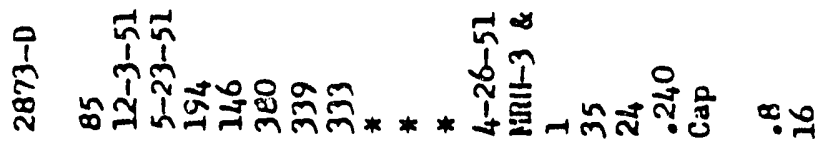

$\underset{\mathscr{g}}{\stackrel{g}{0}}$

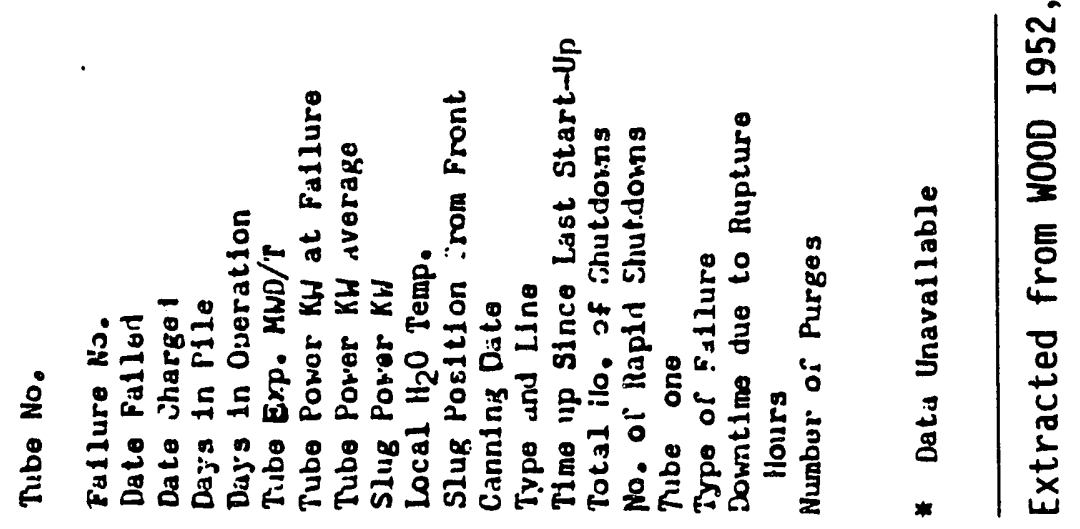




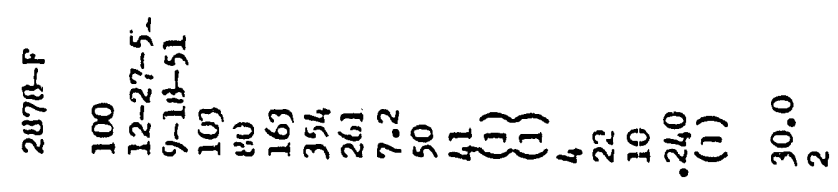

ำ

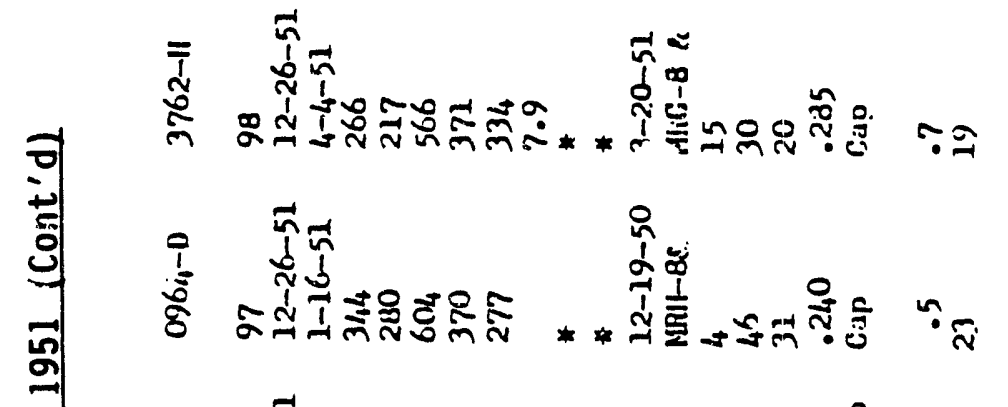

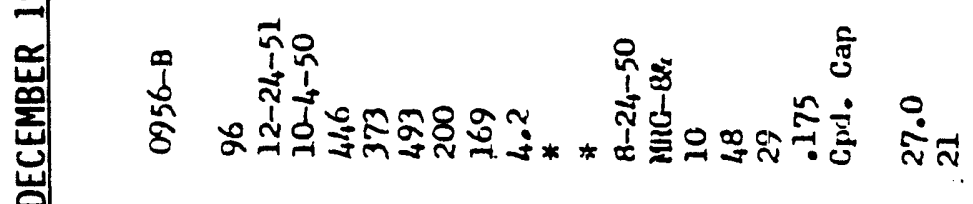

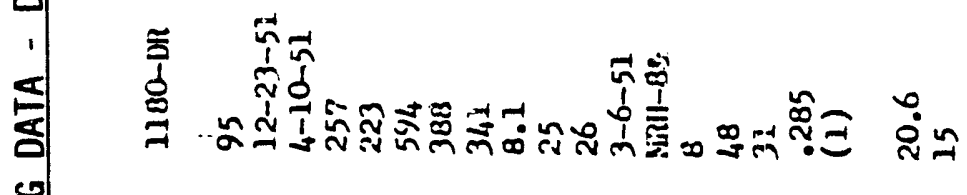

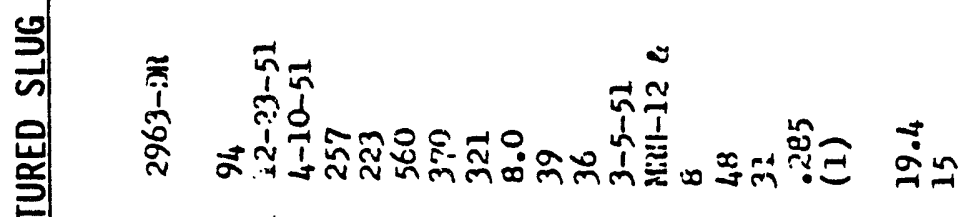

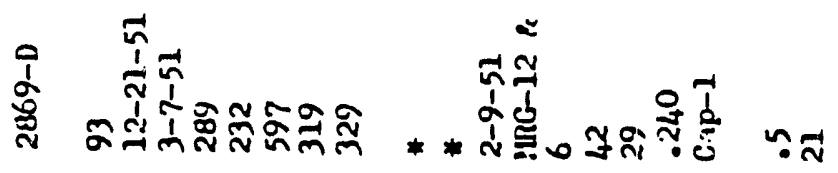




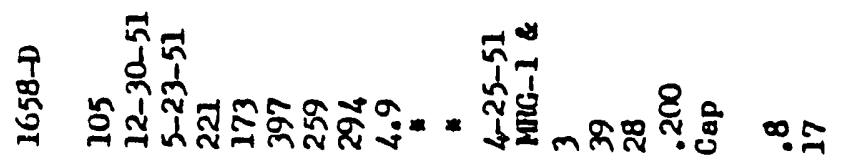

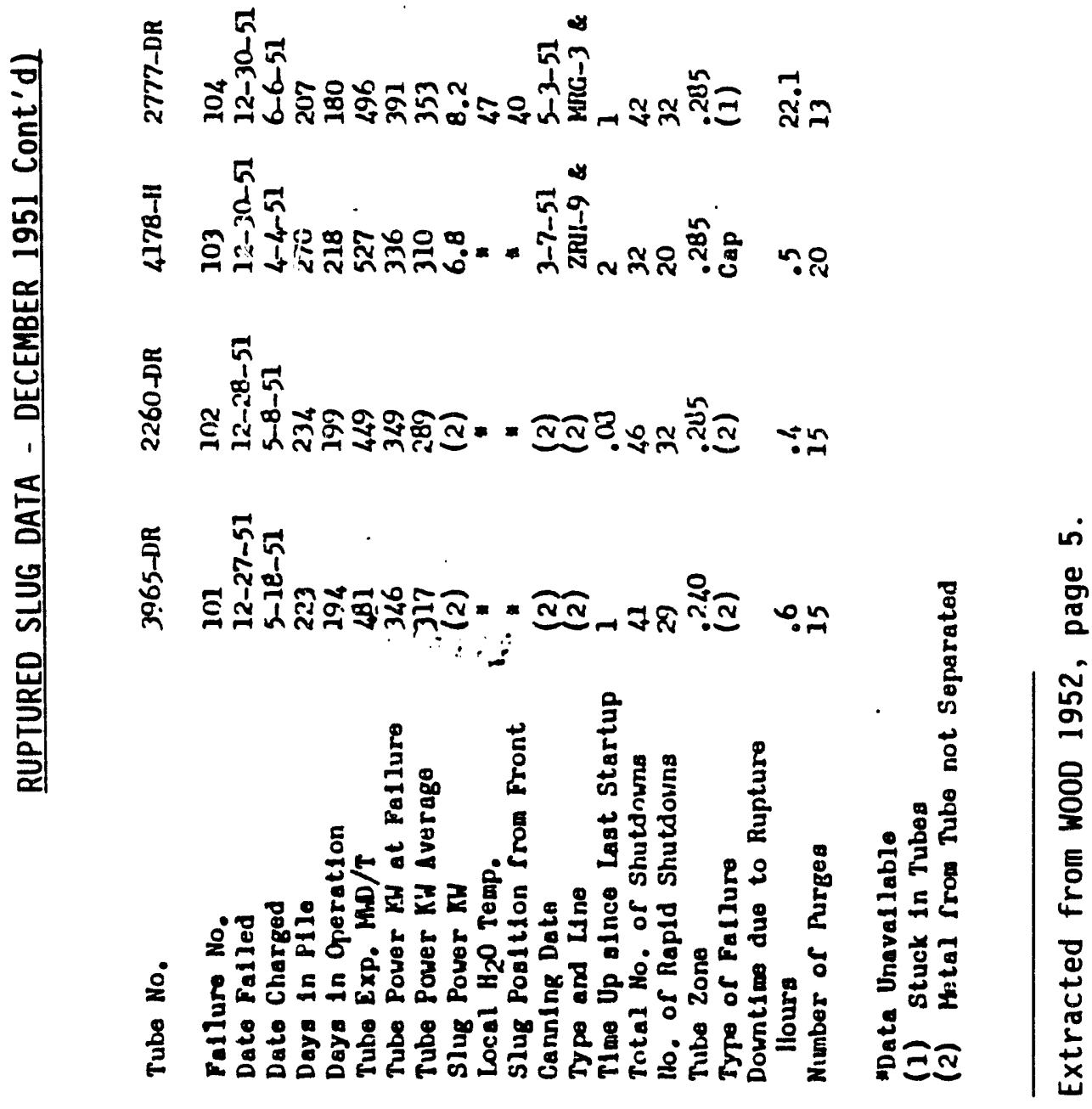




\section{APPENDIX B}

FUEL-ELEMENT FAILURE DATA FOR 1952 
RUPTURED SLUG DATA - JANUARY 1952

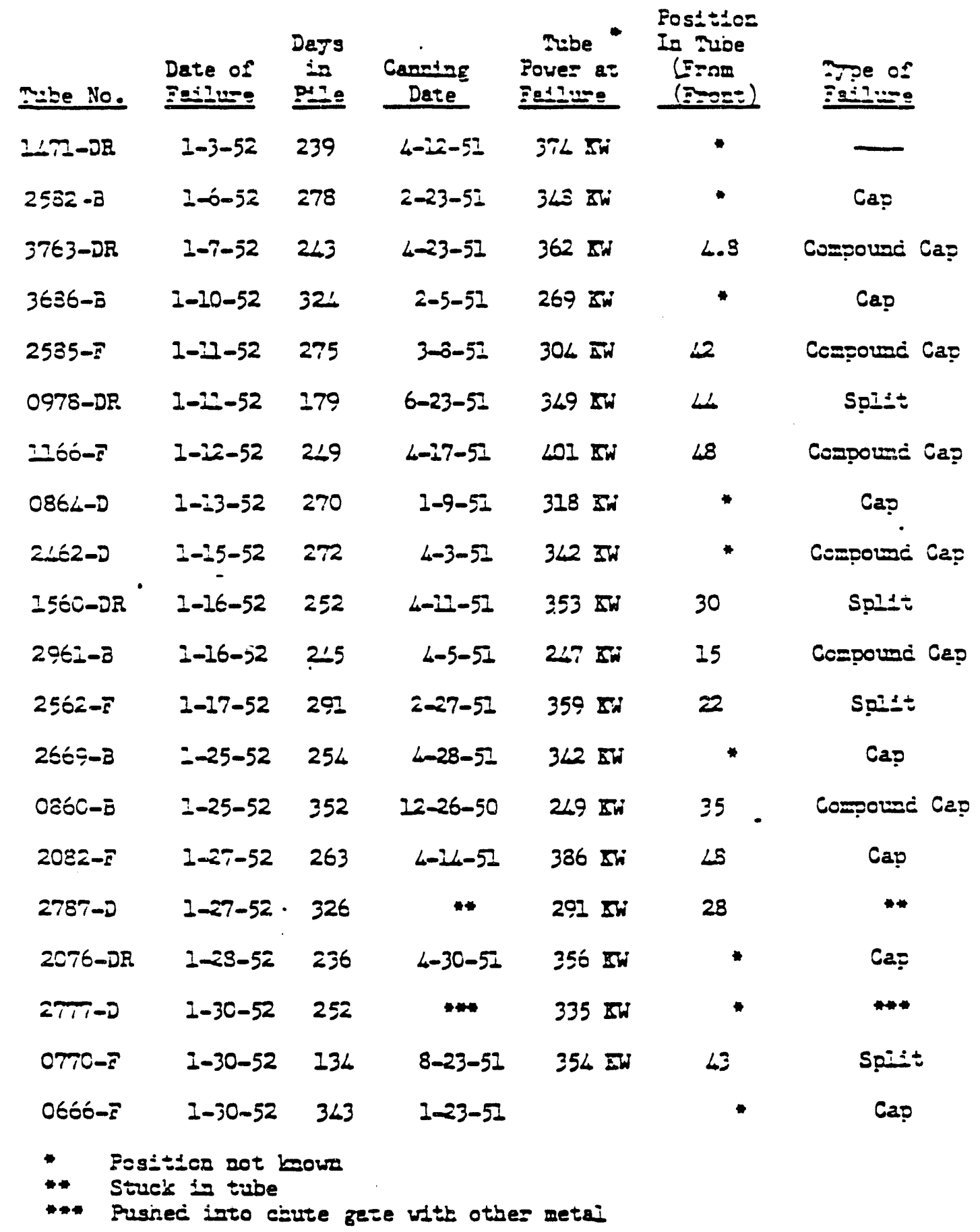

Extracted from HAPO 1952a, page 84.

B. 1 


\section{RUPTURED SLUG DATA - FEBRUARY 1952}

\begin{tabular}{|c|c|c|c|c|c|c|c|}
\hline Tube :is. & $\begin{array}{l}\text { DE: of } \\
\text { Failuse }\end{array}$ & 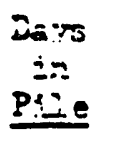 & 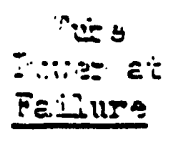 & 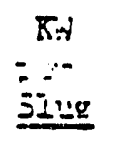 & 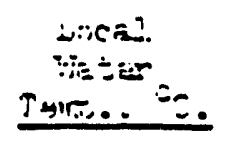 & $\begin{array}{c}\text { Caraing } \\
\text { Dat.n } \\
\end{array}$ & 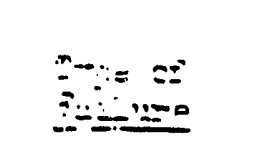 \\
\hline IT7!!-DR & $2-2-52$ & 2117 & $346 \mathrm{Kr}$ & $\ldots$ & $4 i$ & $4-j 00-5:=$ & se:- \\
\hline 218Bj-H & $2-2-52$ & $17 \hat{i}$ & . & $\therefore=$ & if: & $7-0-5=$ & ي \\
\hline $105,2-8$ & $2-3-52$ & EEP? & $255 \mathrm{KN}$ & : & 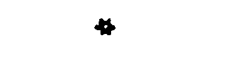 & $£-20-51$ & $C_{\Xi}:$ \\
\hline IE.75-JR & $2-7-52$ & $2 ! \cdot \varepsilon$ & 351.65 & ن & $3:-$ & $5-3-52$ & Sitis \\
\hline $1286-B$ & $2-12-52$ & 272 & 2ณ โล & 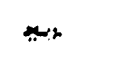 & * & $11-5-5 i$ & Ge:" \\
\hline $1871-2 \Omega$ & $2-11-52$ & 253 & $477 \quad 5=1$ & $? \therefore$ & * & $5-5-51$ & Ca: \\
\hline $1056-D R$ & $2-15-52$ & 12.3 & $290 \mathrm{INH}$ & 5.5 & 15 & $4-5-52$ & SoIft \\
\hline $3465-\ddot{5}$ & $2-15-52$ & 191 & $390 \mathrm{KW}$ & 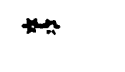 & * & $0-6-51$ & Cạ̣ \\
\hline$I 6^{\prime} \therefore=-F$ & $2-15-52$ & 169 & $36=\operatorname{Iin}$ & 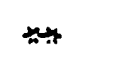 & * & $7-27-51$ & Split \\
\hline$\vdots 2: 3-B$ & $\hat{z}-16-5 \hat{c}$ & 276 & جיك & $\therefore \therefore$ & $\star$ & $1 !-2^{\circ}-51$ & Compurse Vas \\
\hline $0: 72-F$ & $2-16-52$ & 284 & LOL SFi & rist & $*$ & $11-26-52$ & Compound $C=p$ \\
\hline $3690-F$ & $\hat{c}-1 \hat{i}-52$ & 252 & $2815 \times$ & 6.2 & $2 ?$ & $5-30-5$ & Solit \\
\hline $1057-D$ & $2-16-52$ & 348 & jol & $\Xi$. & 28 & $1-29-51$ & Split \\
\hline $2 \times 64-D ?$ & $2-21-52$ & 240 & $3998: ?$ & $? \therefore$ & 28 & $5-29-51$ & SpIEt \\
\hline I $860-D R$ & $2-25-52$ & 292 & 342 एक & $\because \because$ & $\leq 4$ & $4-2-51$ & Split \\
\hline $1886-F$ & $3-2 T-5 Z$ & 295 & irols $\mathrm{SW}$ & س & * & $1-211=I$ & Cap \\
\hline $3: T 3-5$ & $2-28-52$ & $16 j$ & 35. IN & $6 . ?$ & 33 & $5-27-59$ & Split \\
\hline * Si: rir & $r c=\{\vdots i c s$ & Wranown & & & & & \\
\hline * Da $\div 2$ & unavailoci & & & & & & \\
\hline
\end{tabular}

Extracted from HAPO 1952b, page 86. 


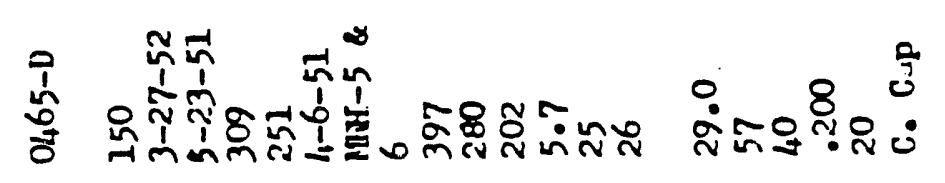

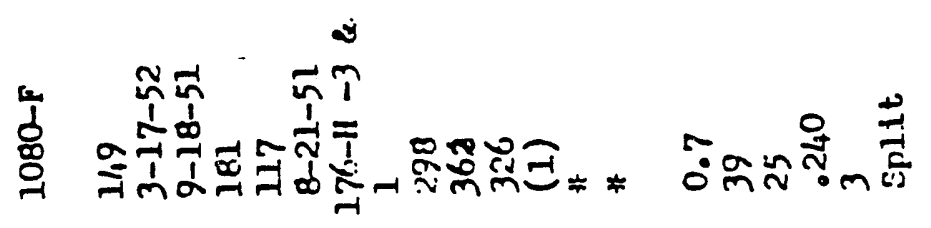

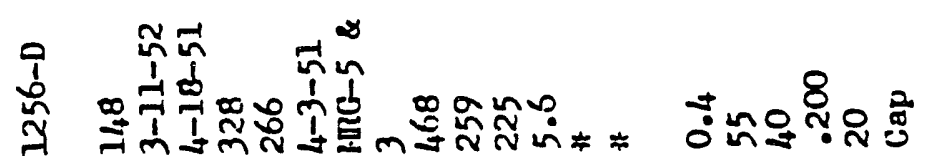

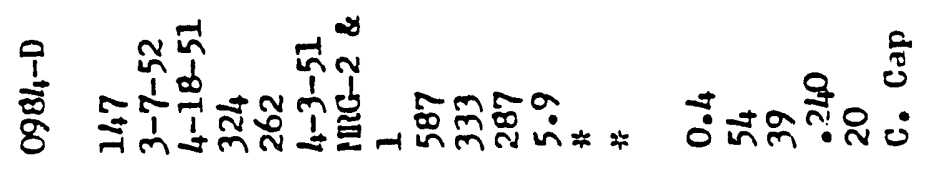

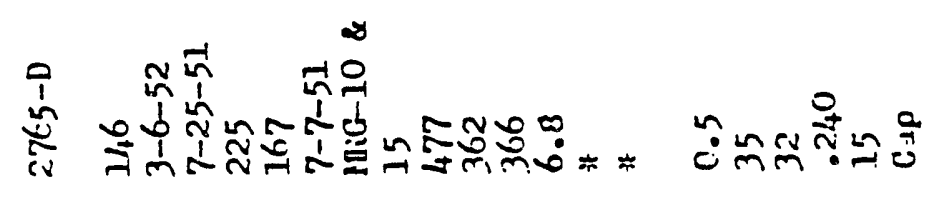

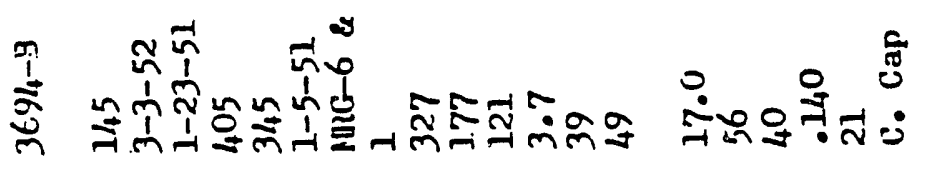

胥

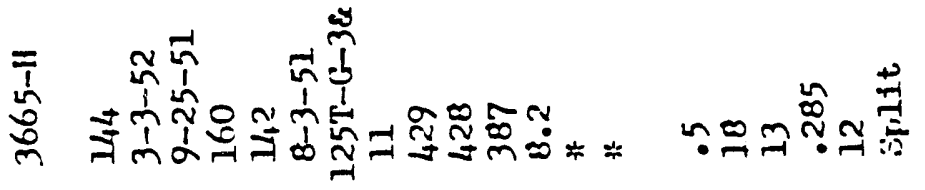

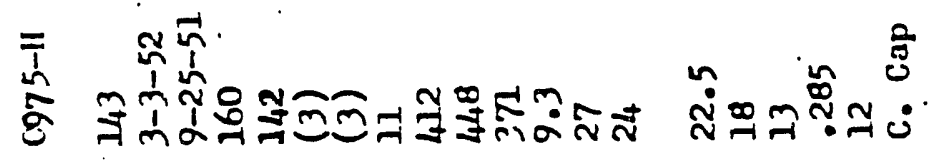

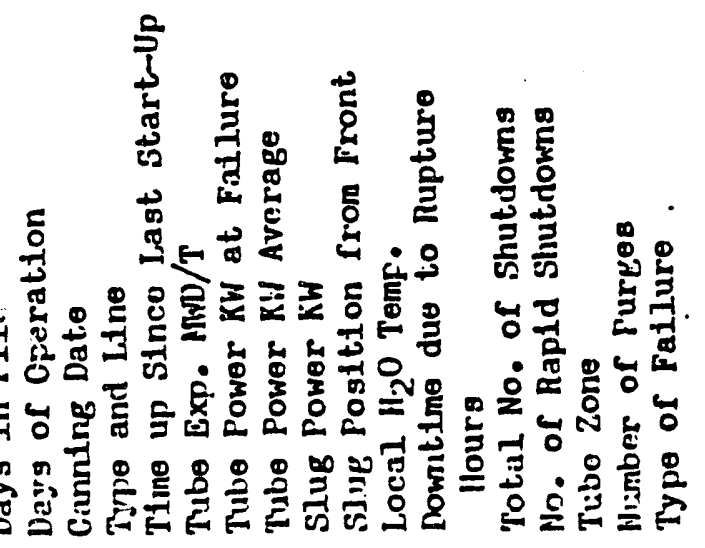




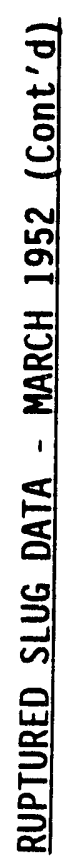

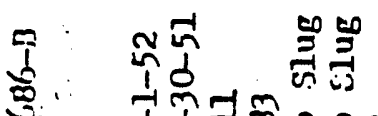

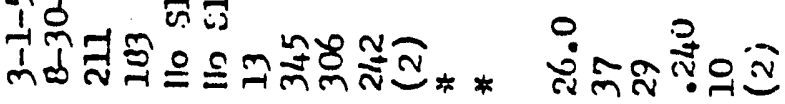

要

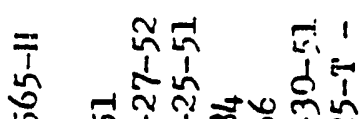

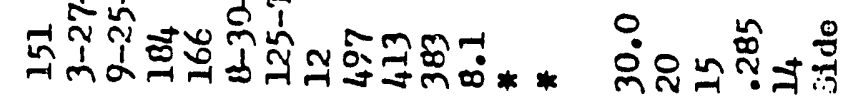

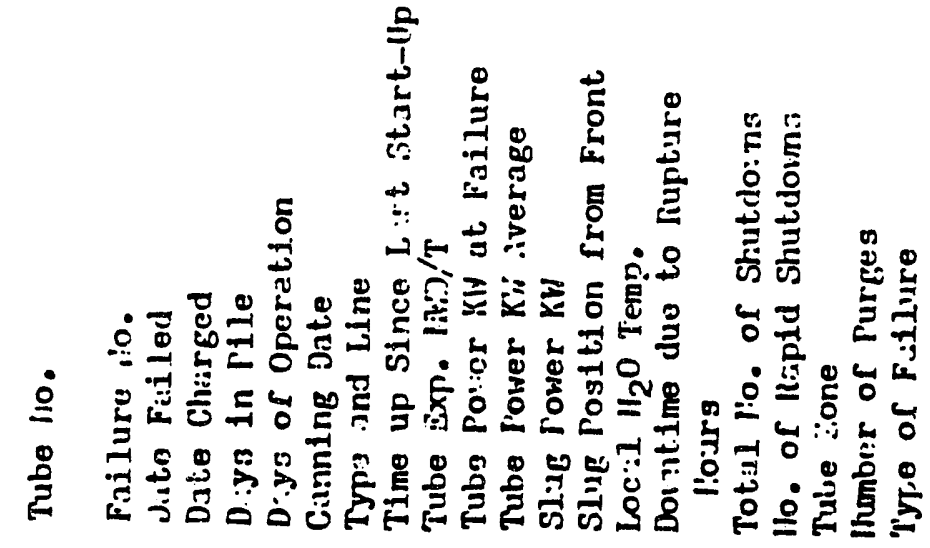

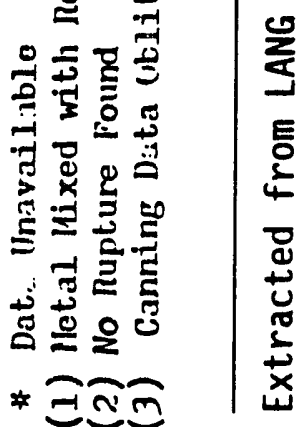




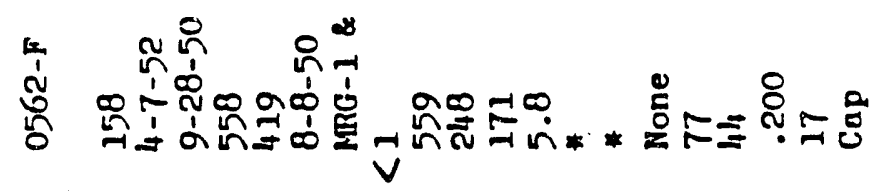

家

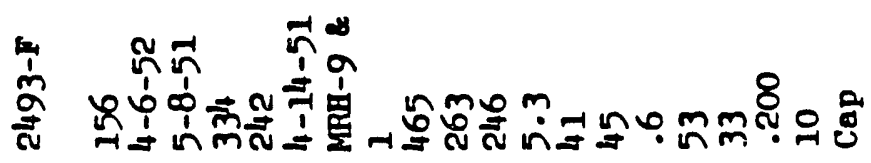

:

密

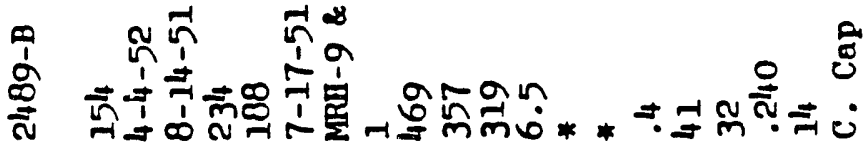

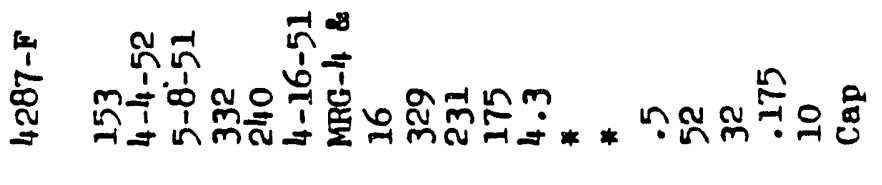

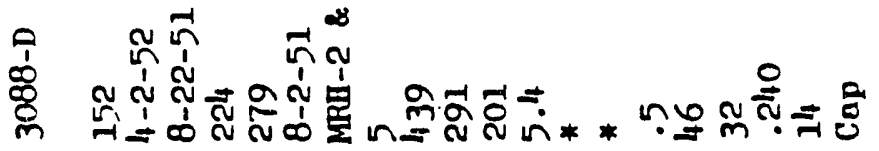

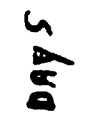

ซ

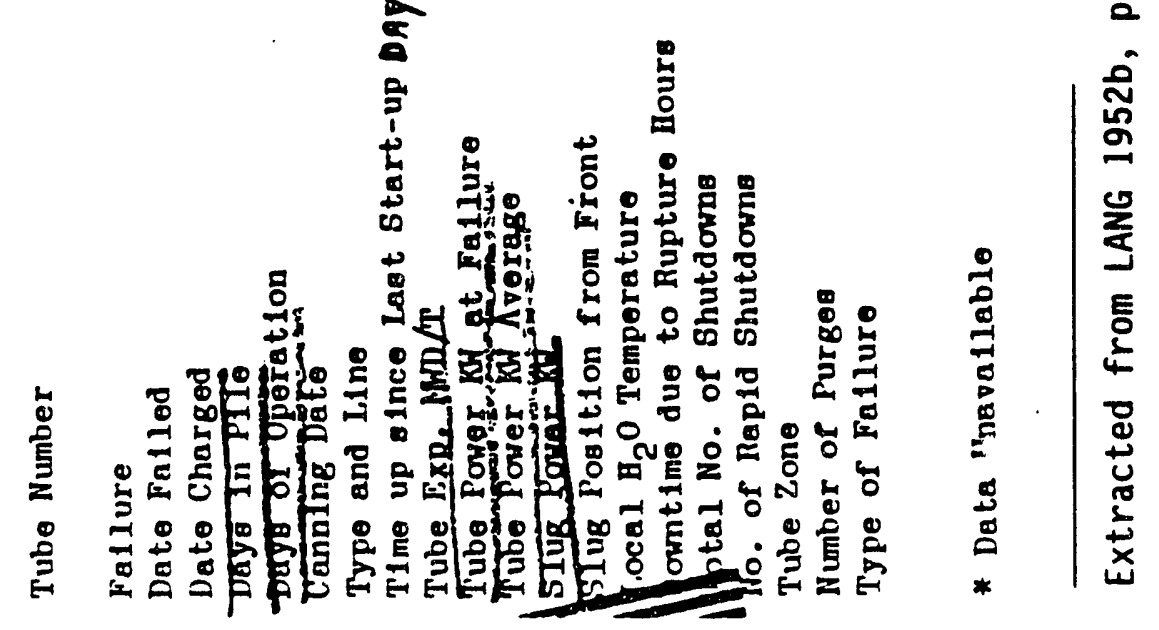

B. 5 


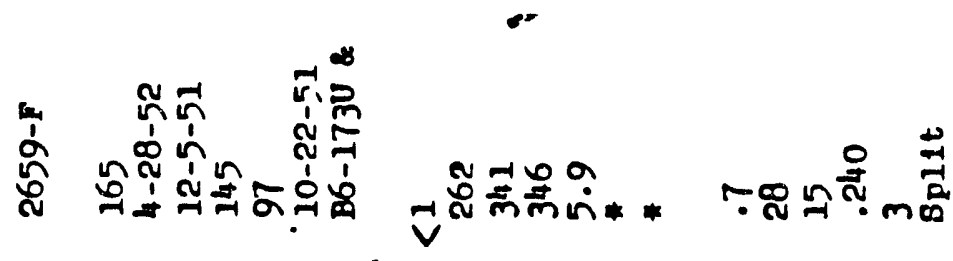

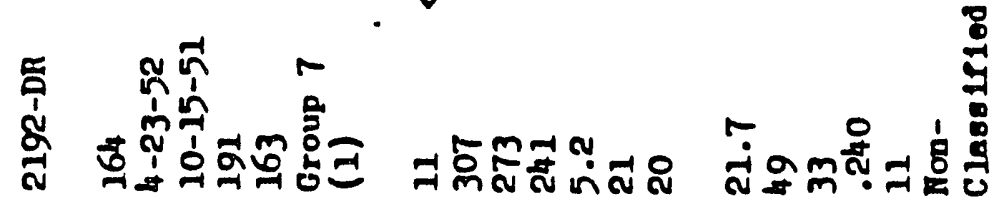

개

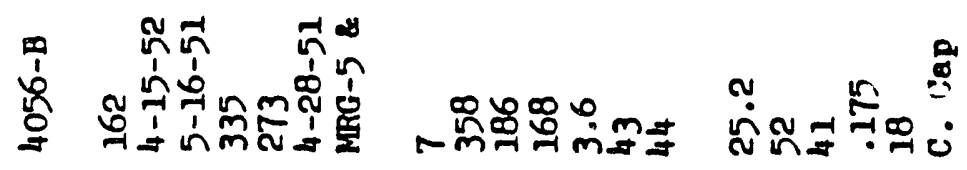

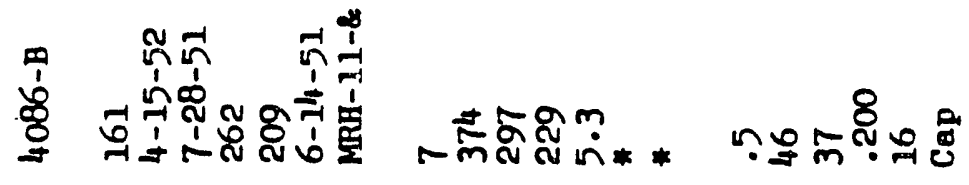

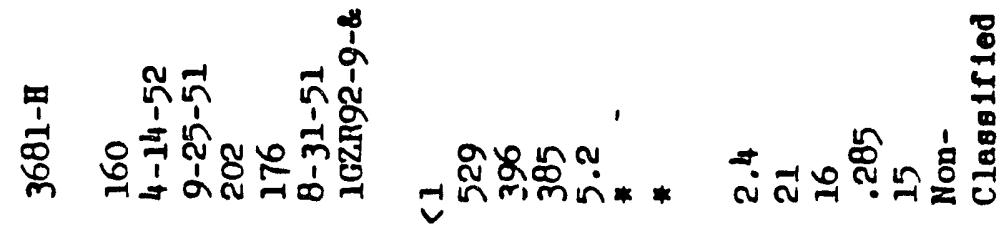

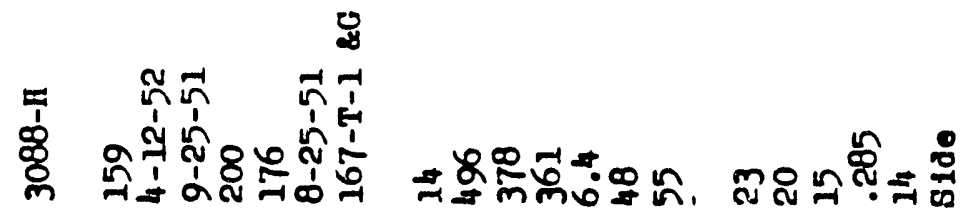

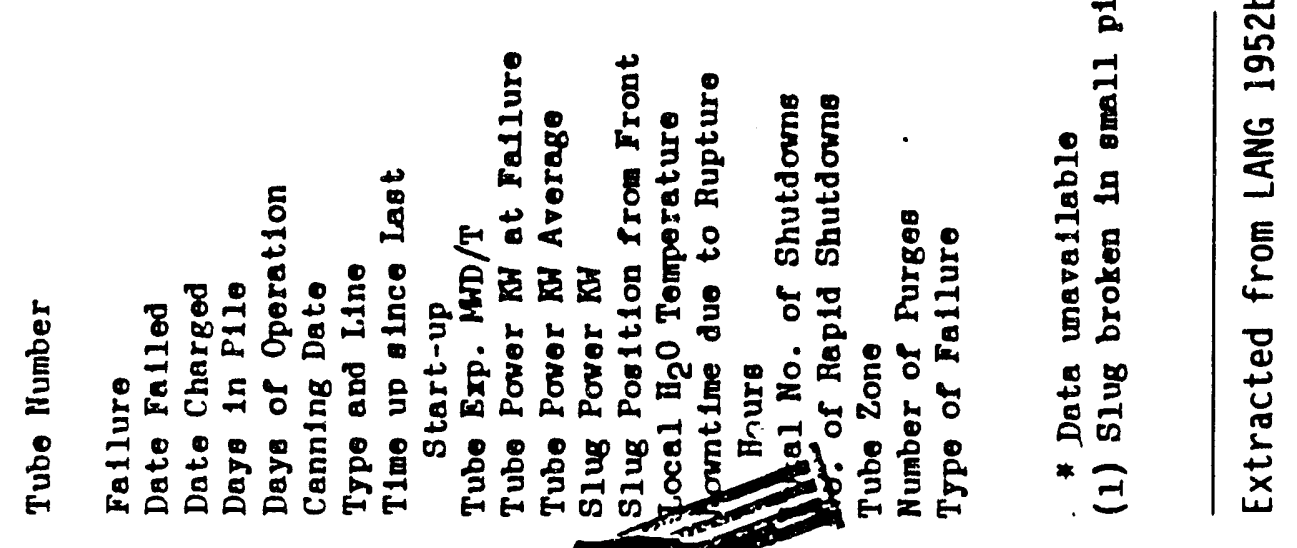




\section{RUPTURED SLUG DATA - APRIL 1952 (Cont'd)}

\begin{tabular}{|c|c|c|}
\hline Tube Irumber & $0386-B$ & $4060-D$ \\
\hline 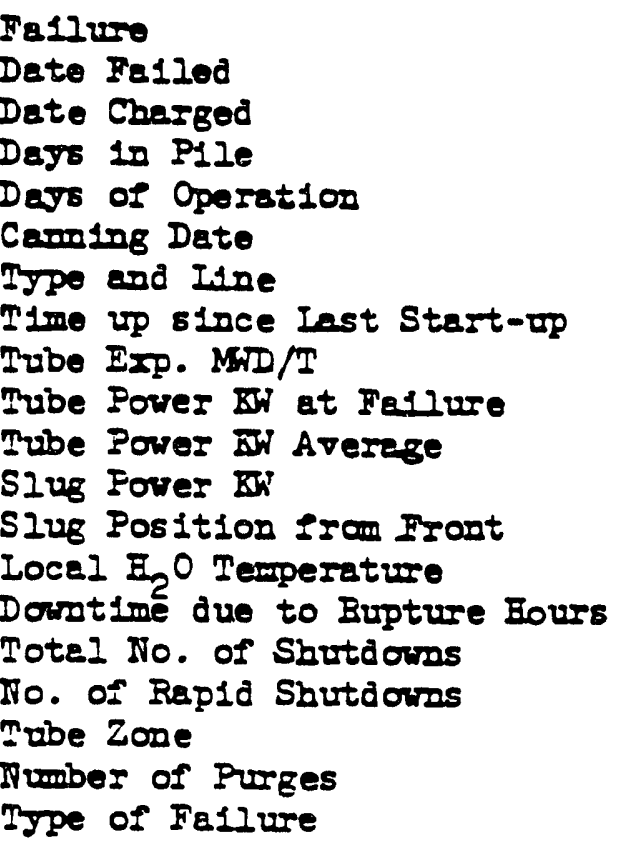 & $\begin{array}{l}166 \\
4-29-52 \\
1-23-51 \\
462 \\
383 \\
1-5-51 \\
1-5-2 \& \\
4 \\
385 \\
157 \\
129 \\
3.1 \\
* \\
* \\
.5 \\
62 \\
45 \\
.140 \\
26 \\
\text { C. Cap }\end{array}$ & $\begin{array}{l}167 \\
4-29-52 \\
3-7-51 \\
419 \\
348 \\
2-12-51 \\
\text { MRG-6 } \\
19 \\
617 \\
268 \\
227 \\
2.9 \\
\text { * } \\
.3 \\
65 \\
45 \\
.200 \\
25 \\
\text { C. Cep }\end{array}$ \\
\hline * Deta meveilablo & & \\
\hline
\end{tabular}

B. 7 


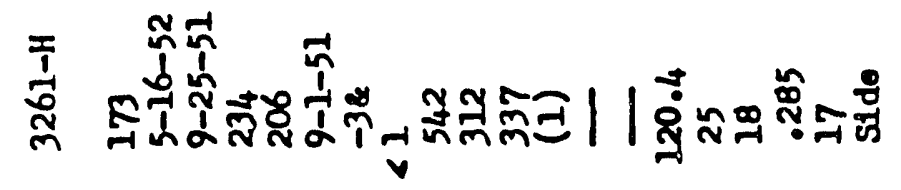

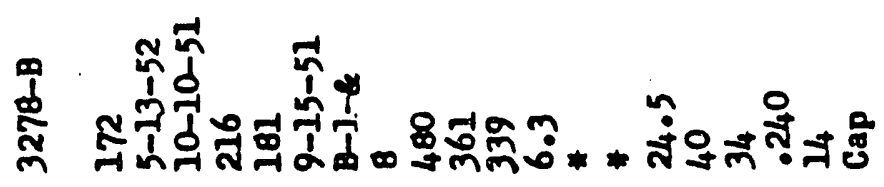

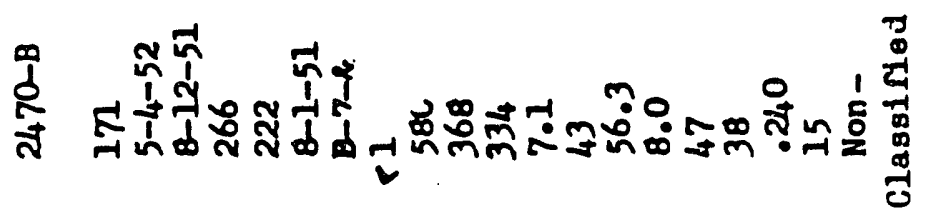

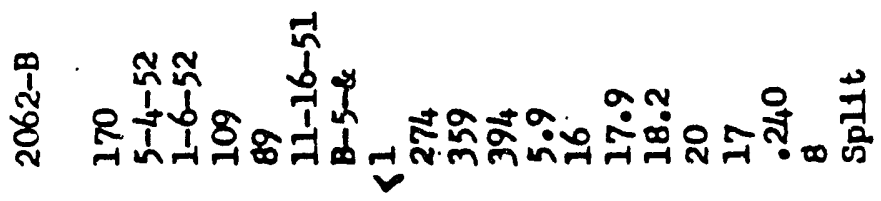

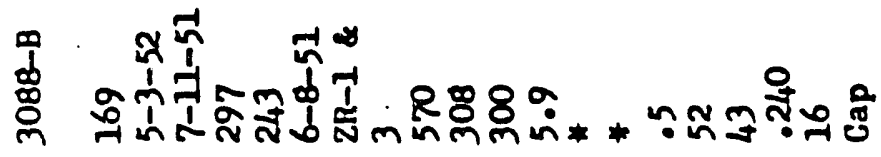

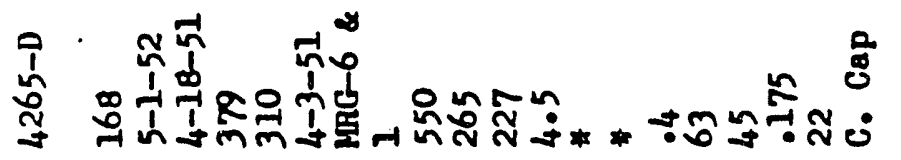

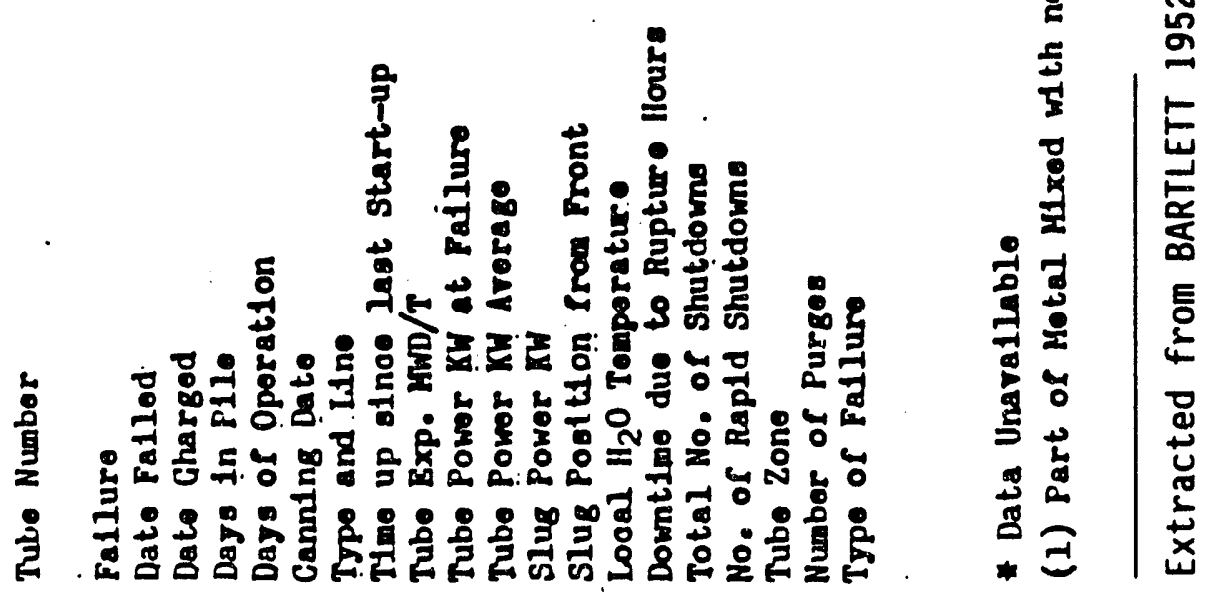




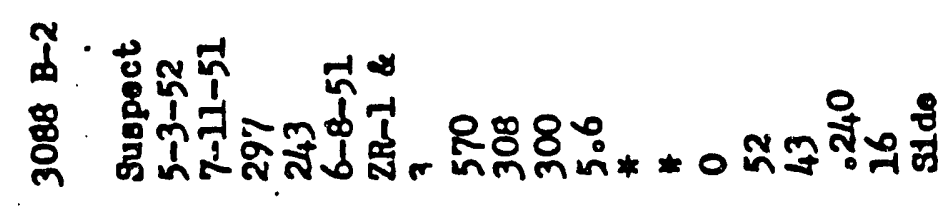

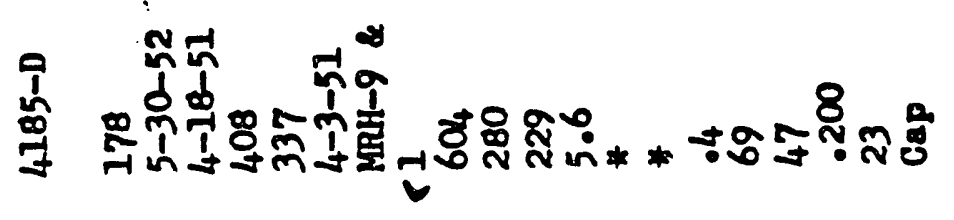

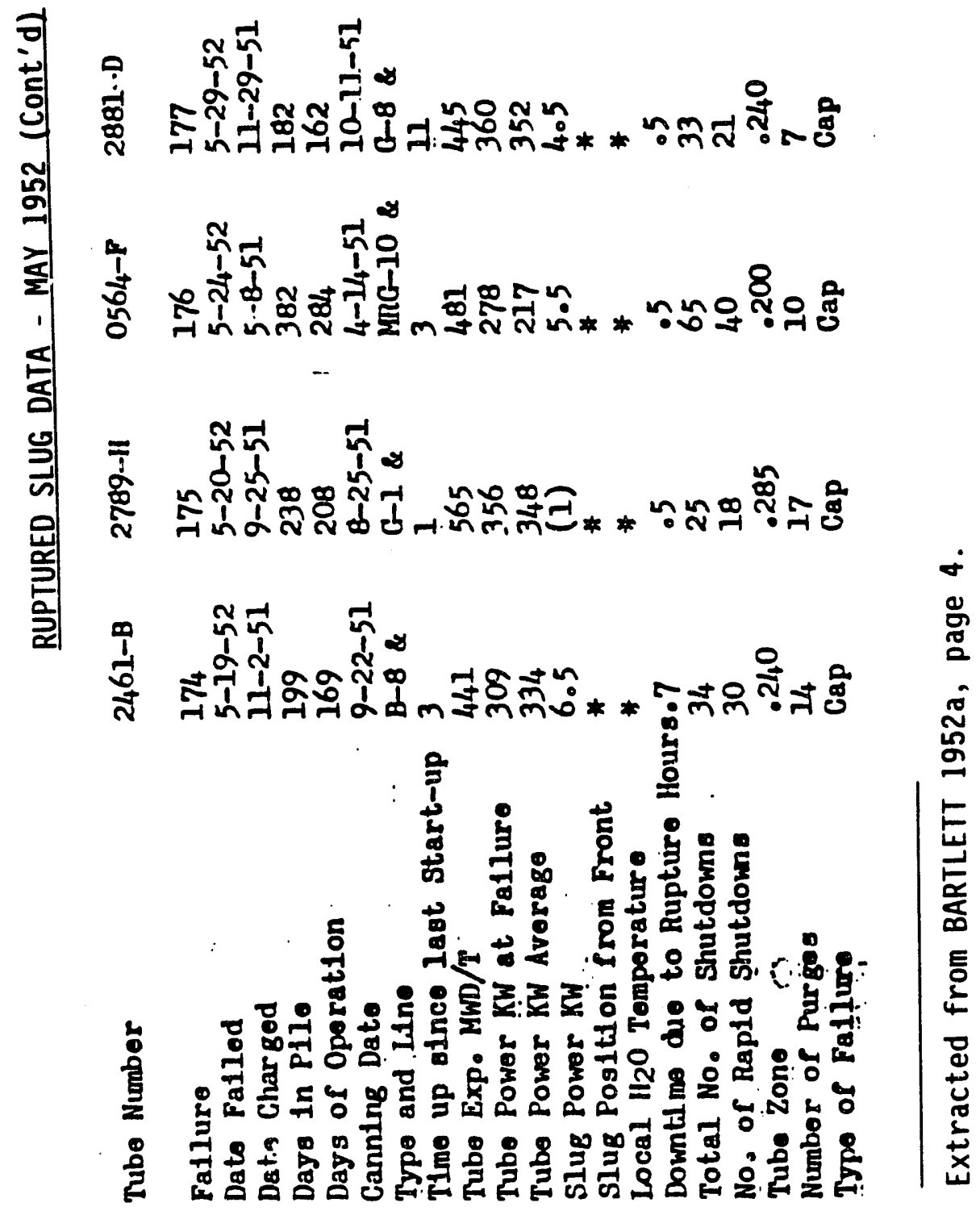

B. 9 


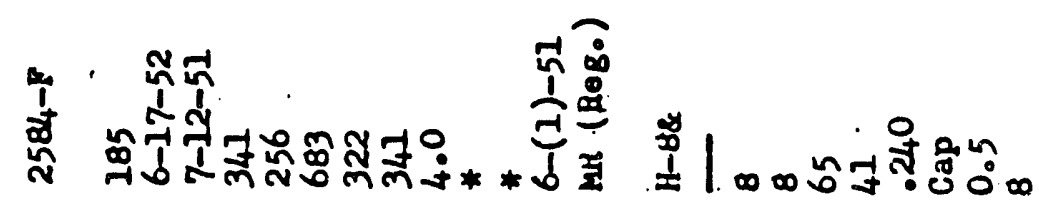

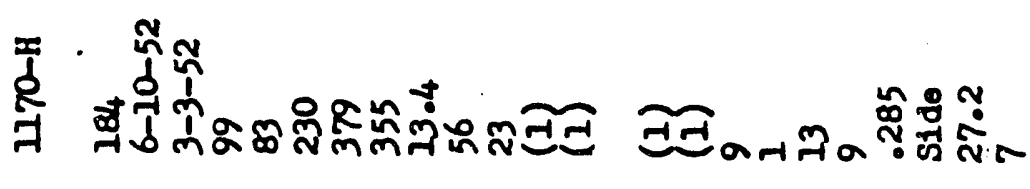

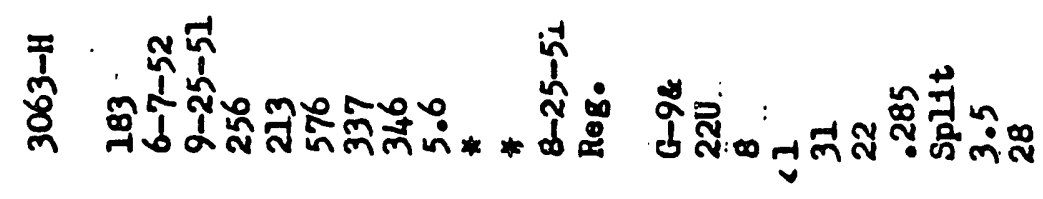

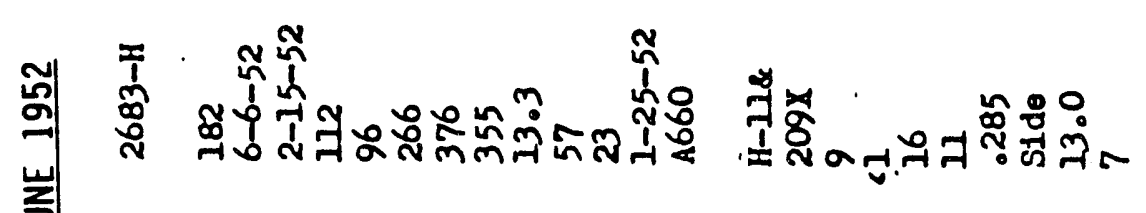

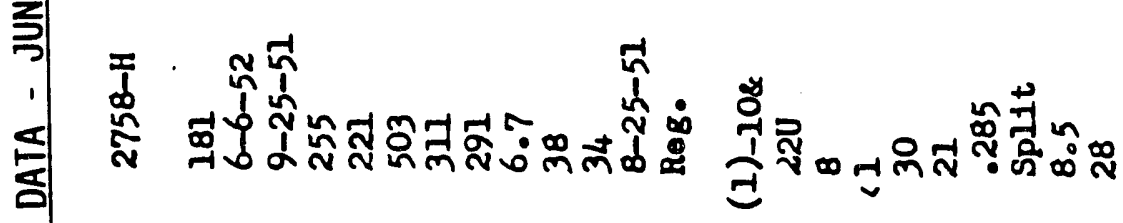

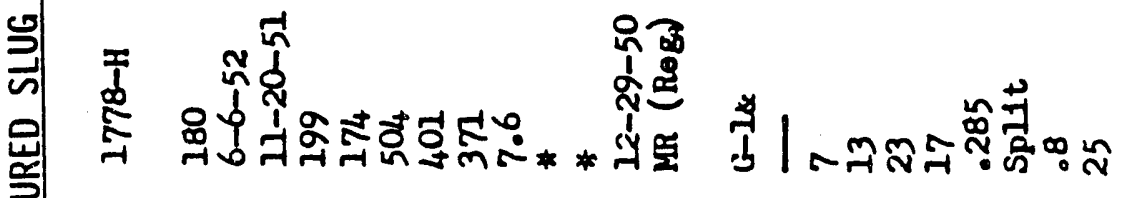

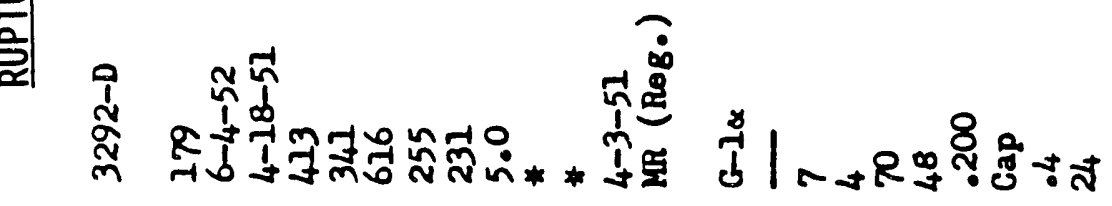

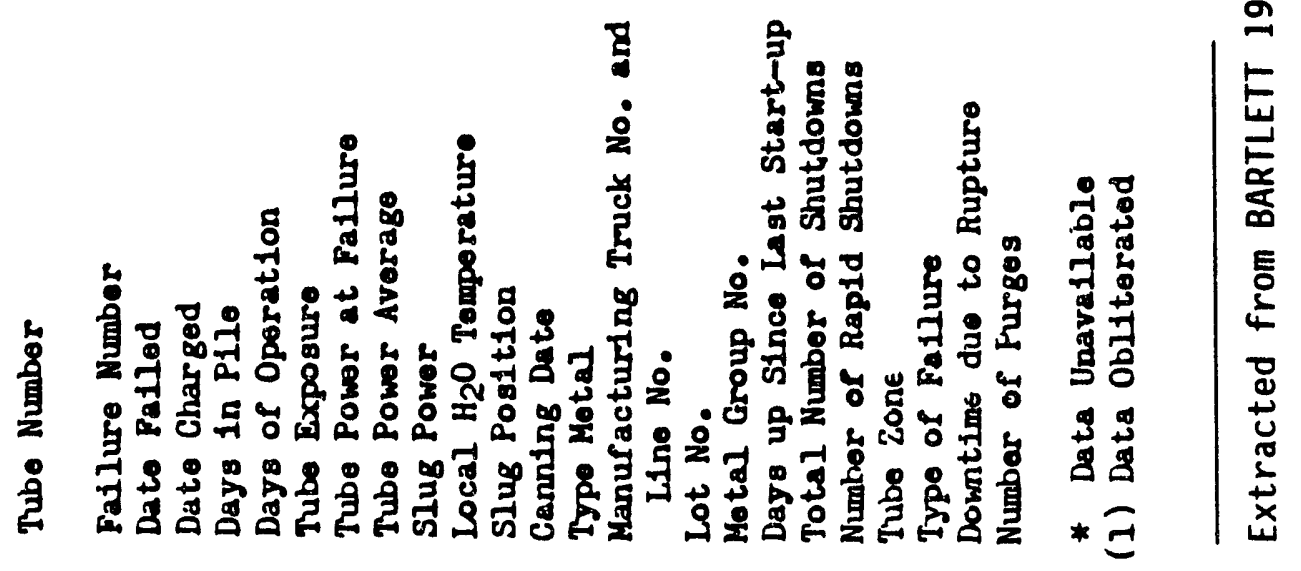




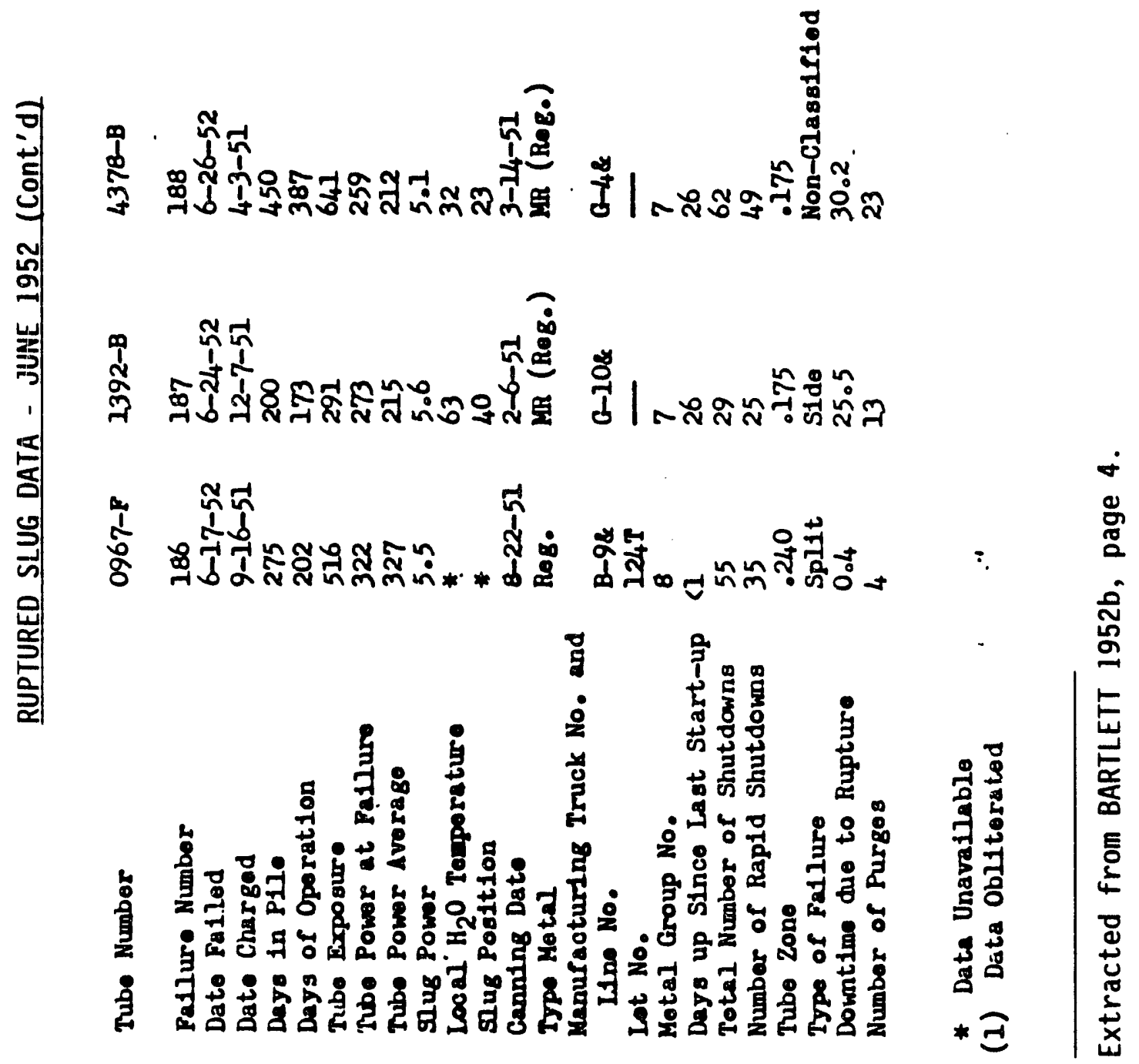




\section{RUPTURED SLUG DATA}

- The following table contairs the data for those slugs which ruptured in July 1952. Date Sources

1. Tube number - Reacior Section ruptured slug reports.

2. Failure number - Chronoiogical order of failures from Reactor Section daily repcr sheets.

3. Date charged - Reacter Section f1le of charge cards.

4. Dags in pile - Caieslated from charge dates and faiiure dates.

5. Days of operation - Days in the file minus day down.

6. Tube exposure-Reactor section froduction schedulting.

7. Tube power at failure - $(.264)(\Delta T)$ (flow)

8. Tube power average - (Exposure) (Charge Weight)

Q. Days of Operztion

9. SIug power - (.94) (Tube Power) (Radiation level of rapture) Sum of ha ation Letels

10. Locai $\mathrm{H}_{2} \mathrm{O}$ temperatire - nssumed cosine flux distizibution reported for exact position only because of error in estimated slug position.

11. SIug position - Oris exact siug position reported. Slug position estimated fror wasel data were found to be in error as mach as 30 per cest due to pile flottening and non-cosine flux distributicn.

12. Canning daite - Visusi inspection of piece.

13. Type metal - Regulariy macinined pieces, or ro-processed rejects from slug markings and intai preparztion section.

14. Vanufact fing truck noo and Ifne no..- Obtained Irom slug marinings and MetaI Preparation Section.

15. Lot No. - Netai from wiich siug manufactured. Obtained from slug mericings and Metal Freparaition Section.

16. Netal group no. - From manufacturing date, Hí-24099.

17. Days p since Iasi sta-tuf-Reactor Section daily report sheets.

18. Total mumer of shuticwns - Reactor Section daily report sheets.

19. Number of rapid siutiowns - Reactor Section daily report sheets.

20. Tube zone - Tscinical Section temperature maps.

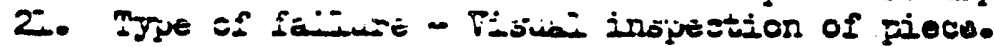

22. Downtime dise tc rirtine - Regotor Sention rupture slug reports.

23. Nurber of purges - Reactor Section pressure drop seport shoets.

DCsEibis E=2E in Data

1. Tube power $t 5 \%$ because fiow nct certain.

2. Siug vewez I $10 \%$ besause of tube fores exror.

3. Lose $\mathrm{H}_{2} \mathrm{O}$ tenperature $\frac{1}{2} 1 \mathrm{CF}$ because of non-cosine flux distzibution.

Extracted from BARTLETT 1952c, pages 6-7 
夏

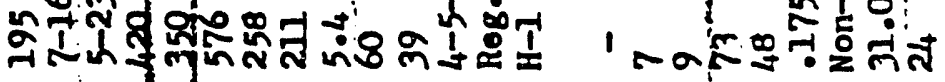

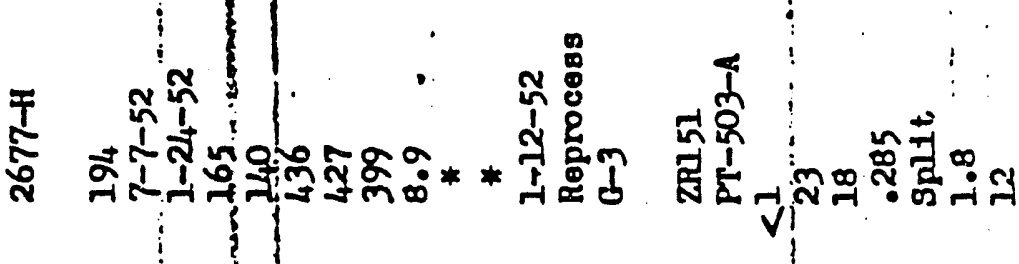

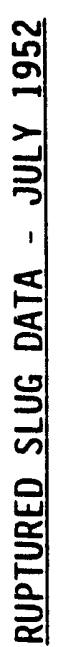

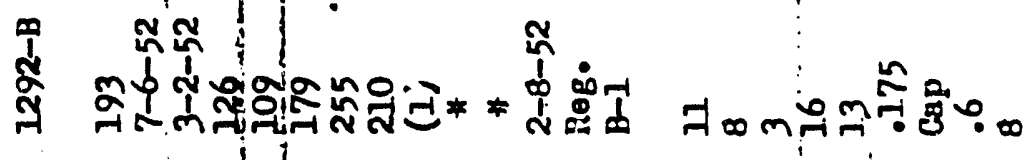

ฮั

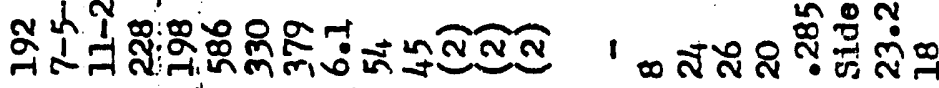

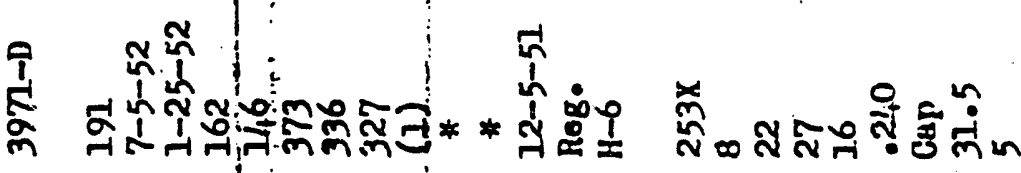

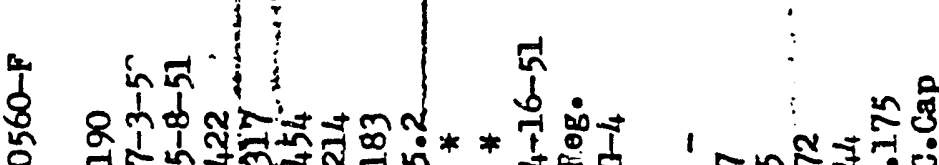

क तूप

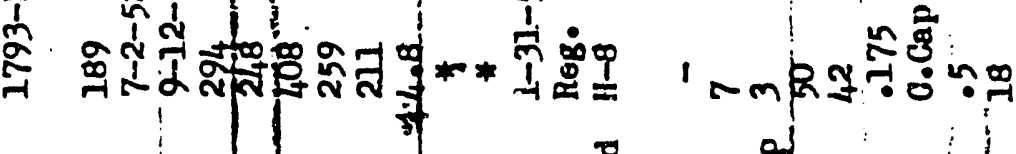

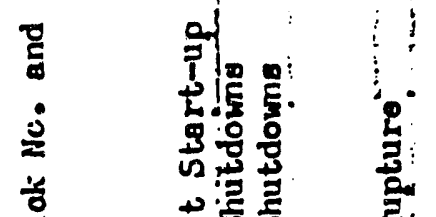

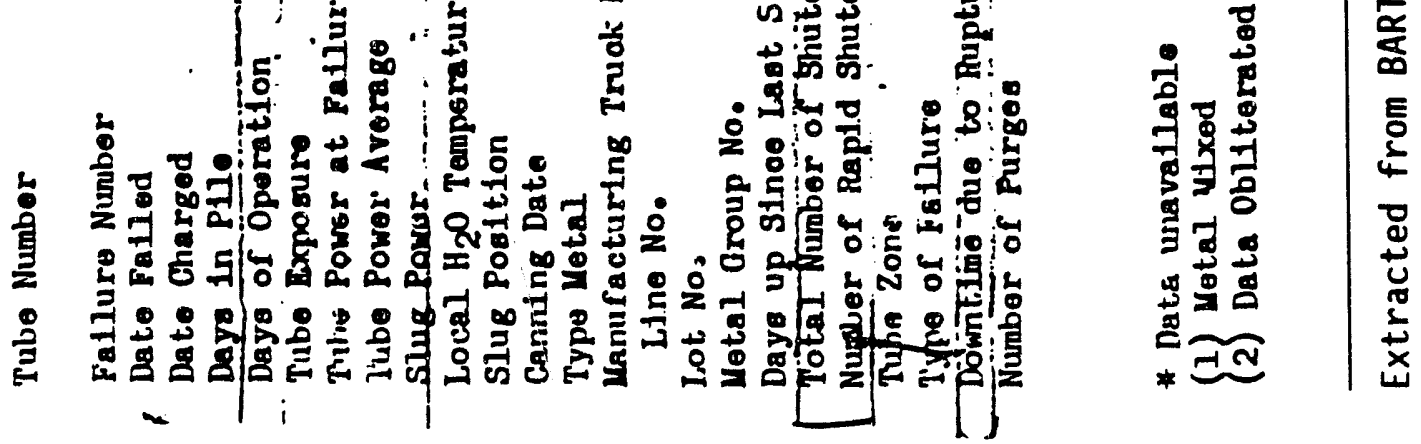




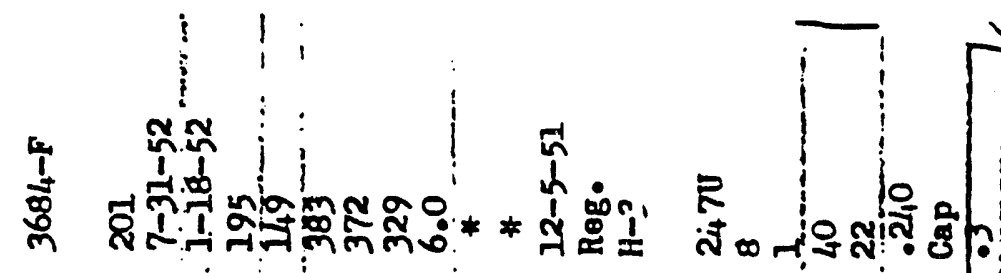

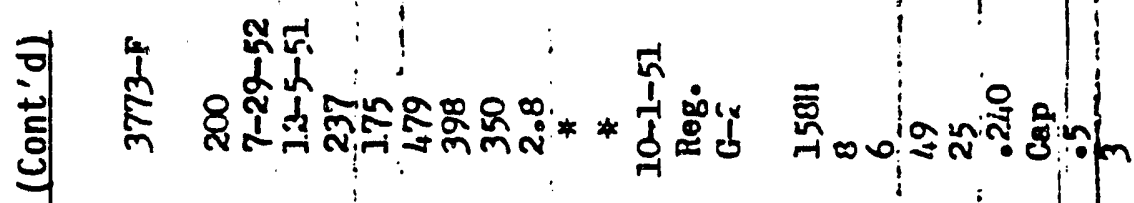

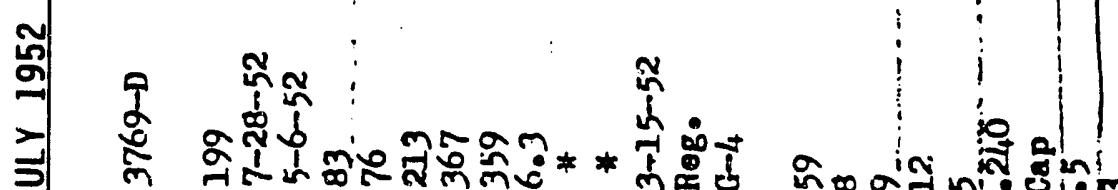

5) m नำ

호을

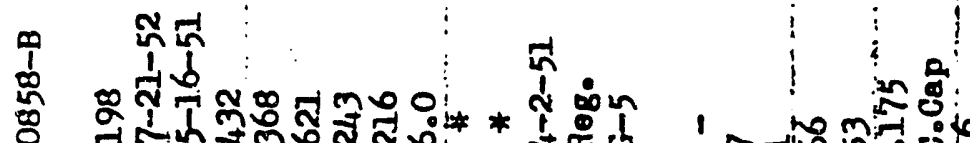

-

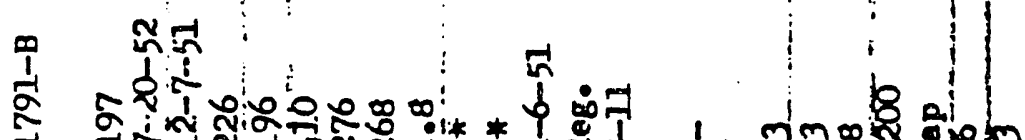

$\sum_{\infty}$

$\sigma^{\circ}$

(1) 


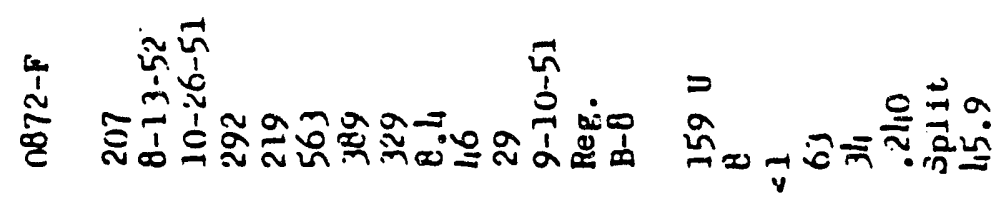

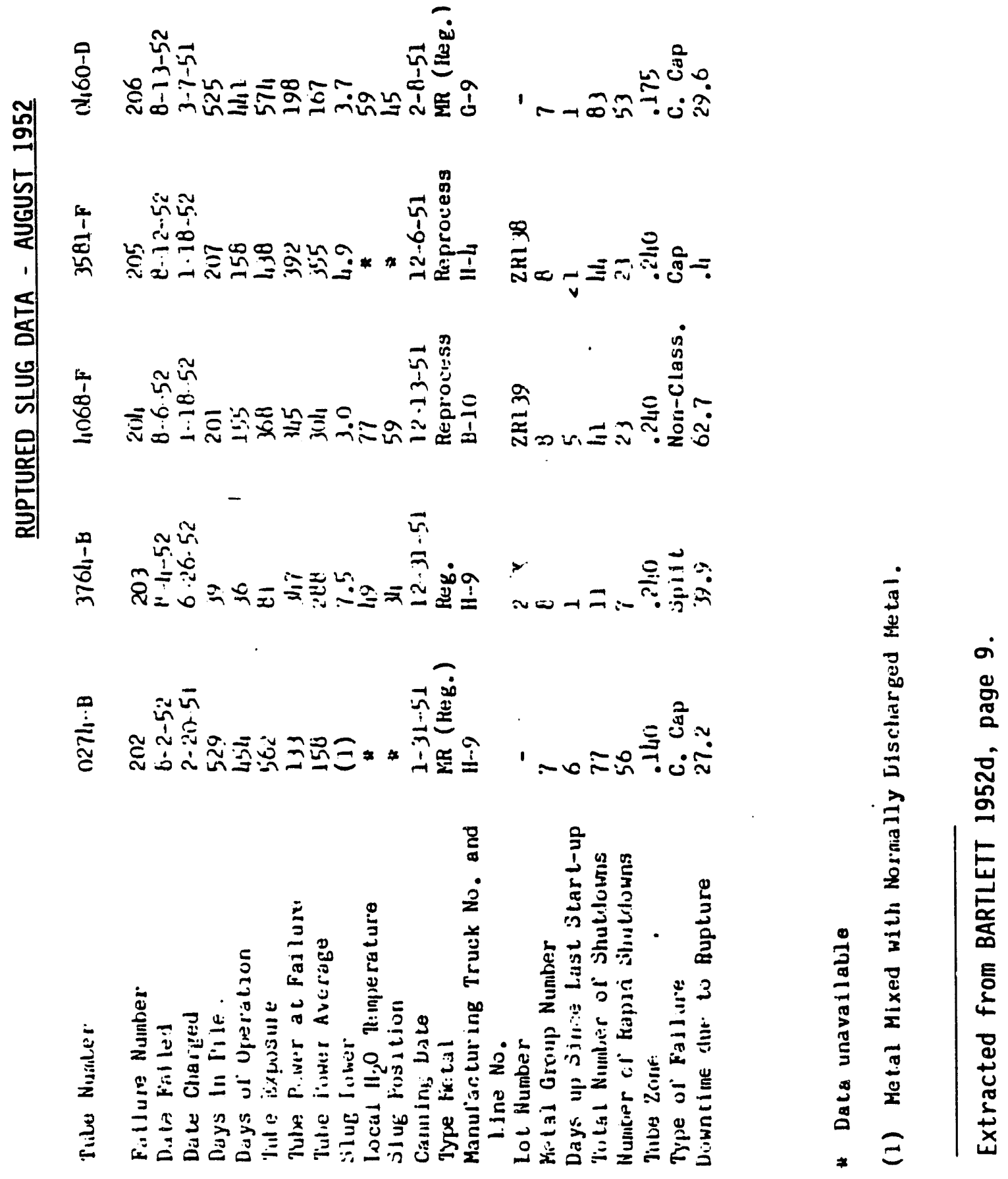




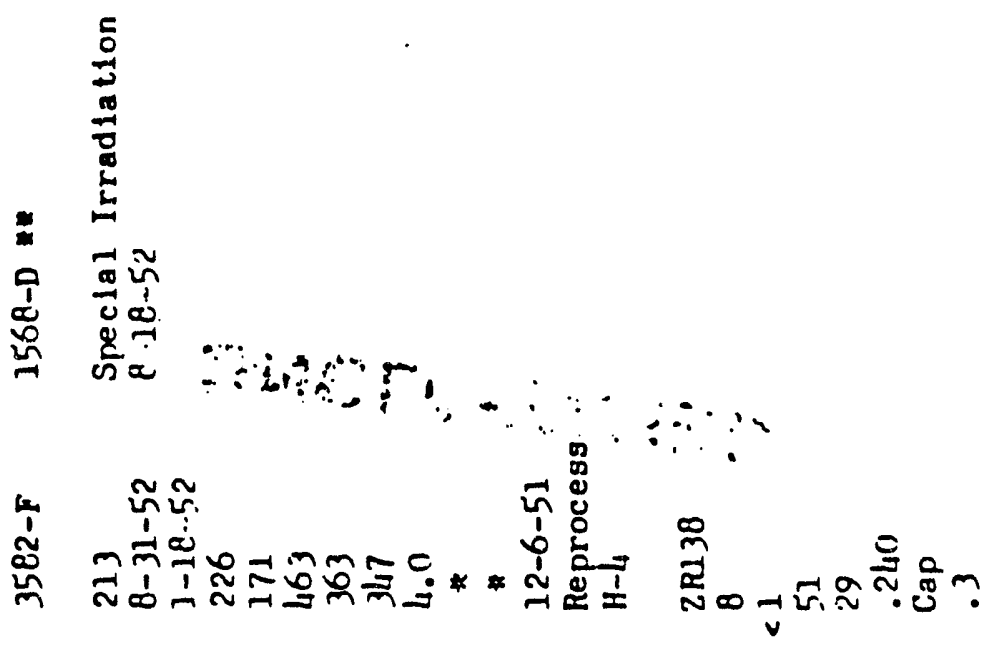

苟

至

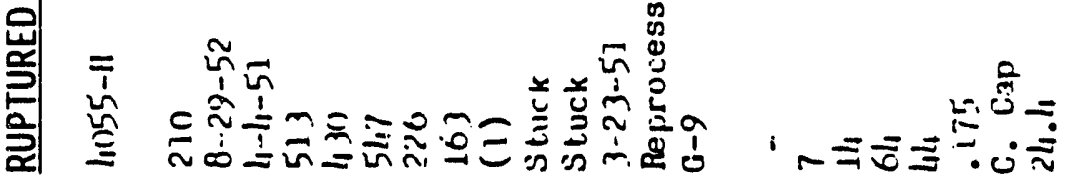

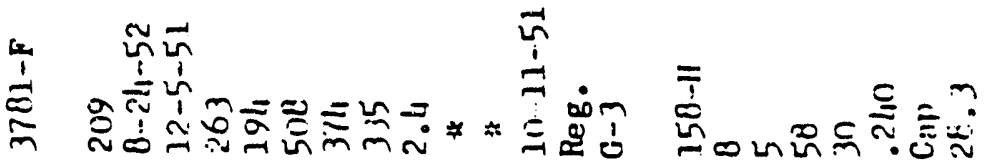

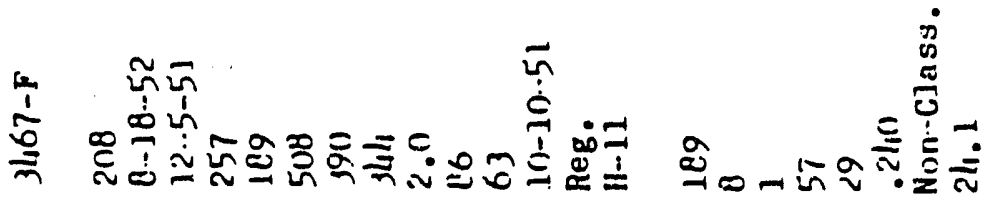

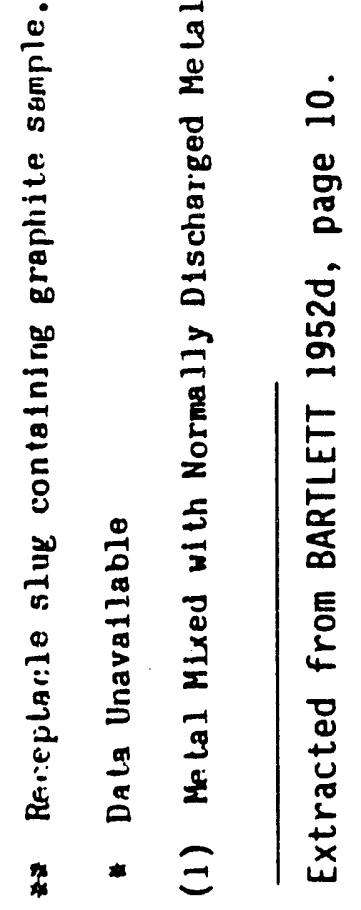




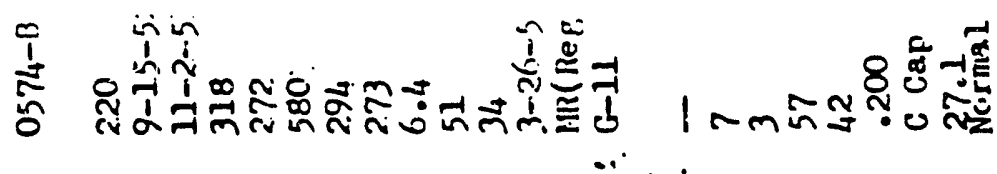

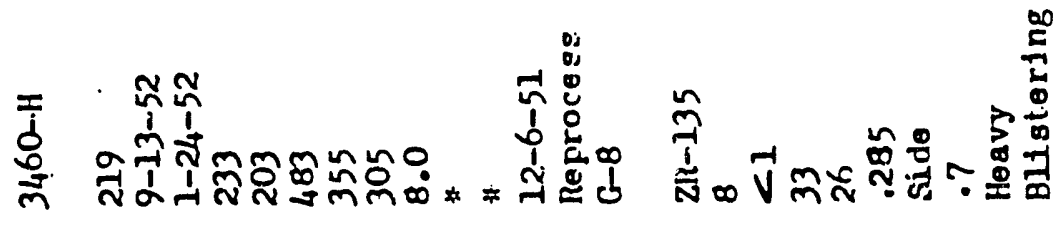

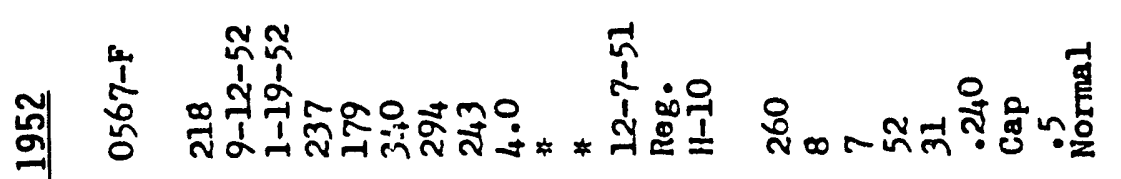

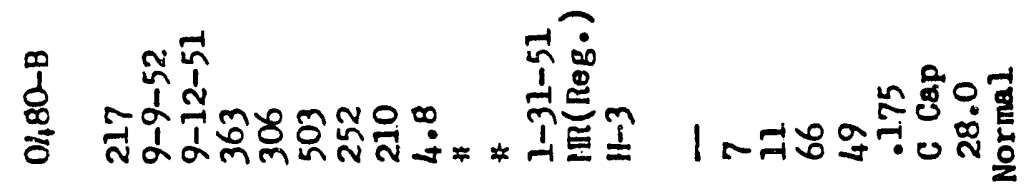

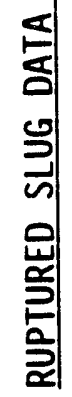

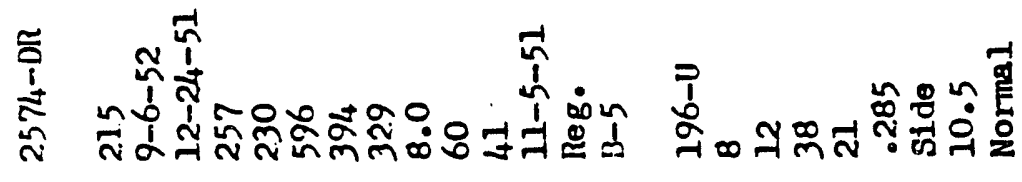

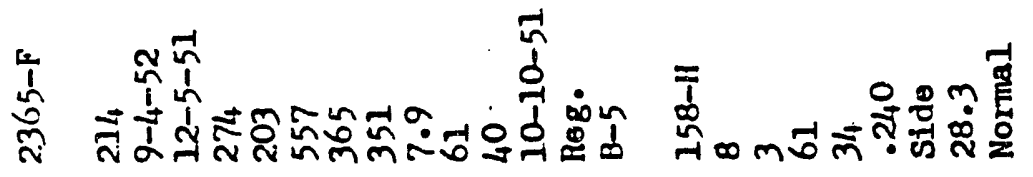

$\dot{s}$

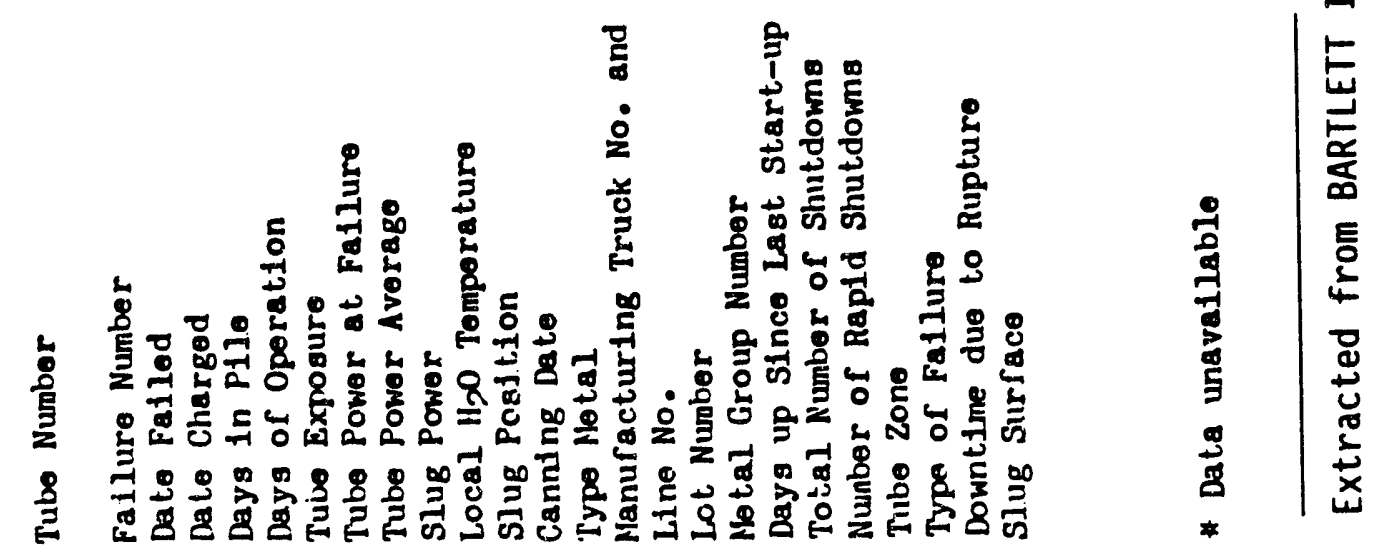




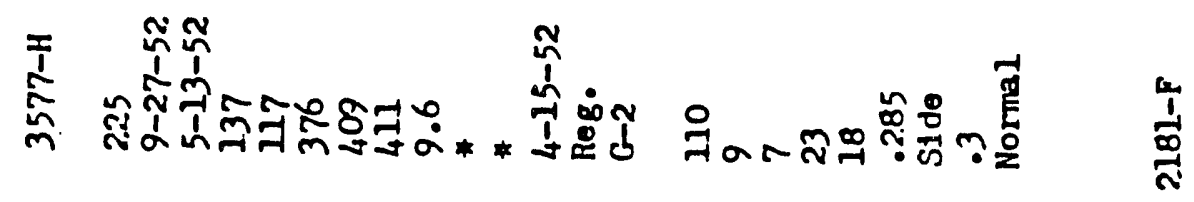

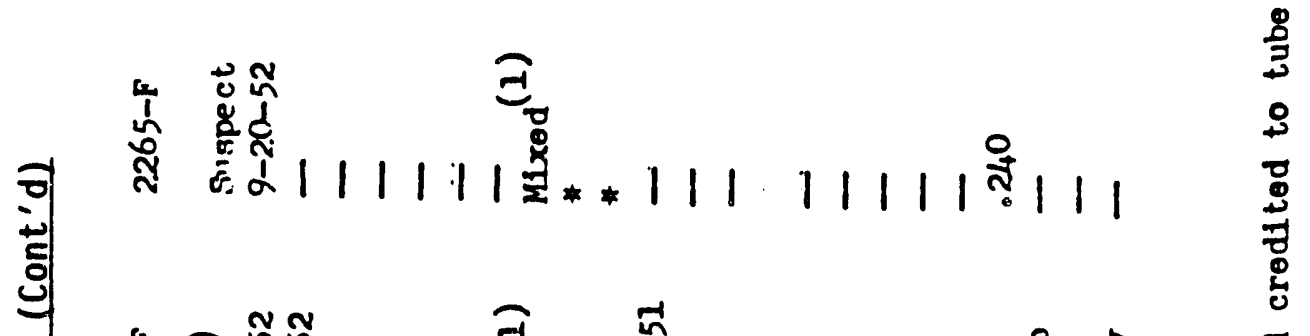

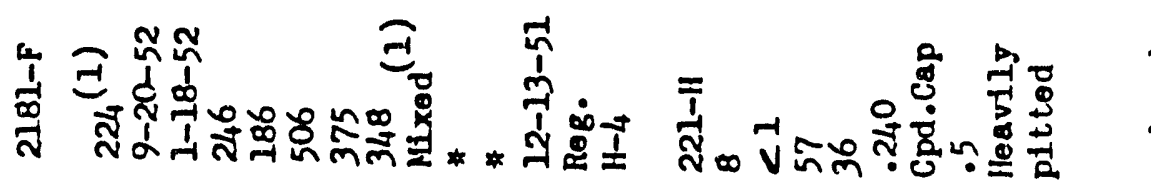

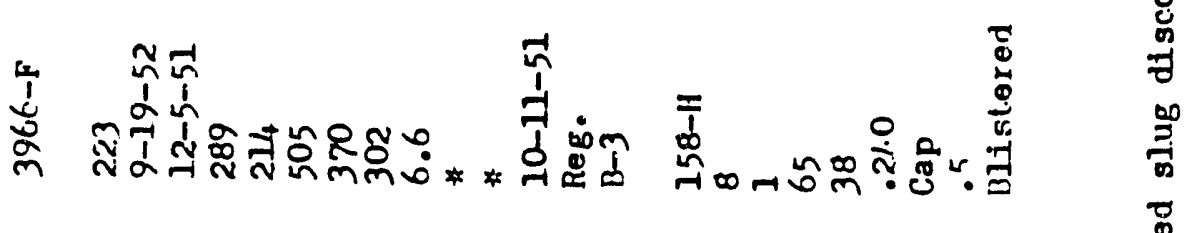

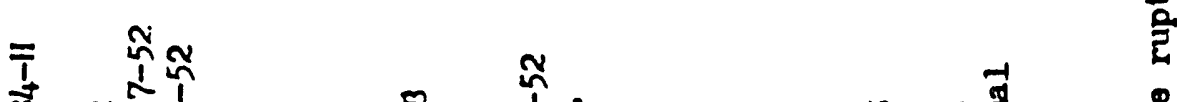

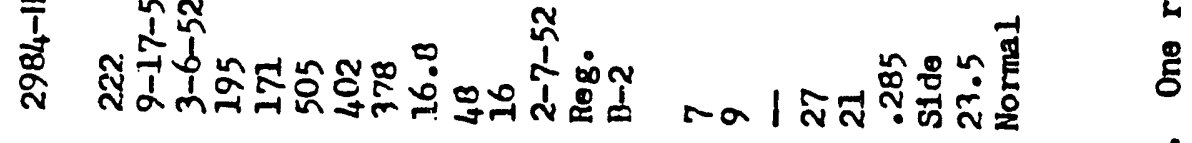

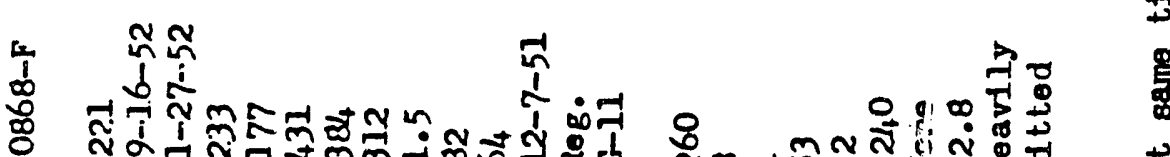

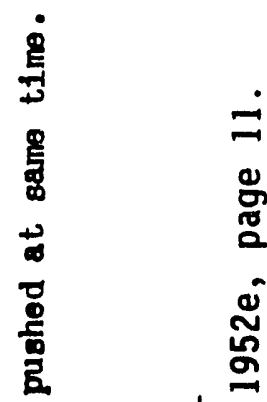

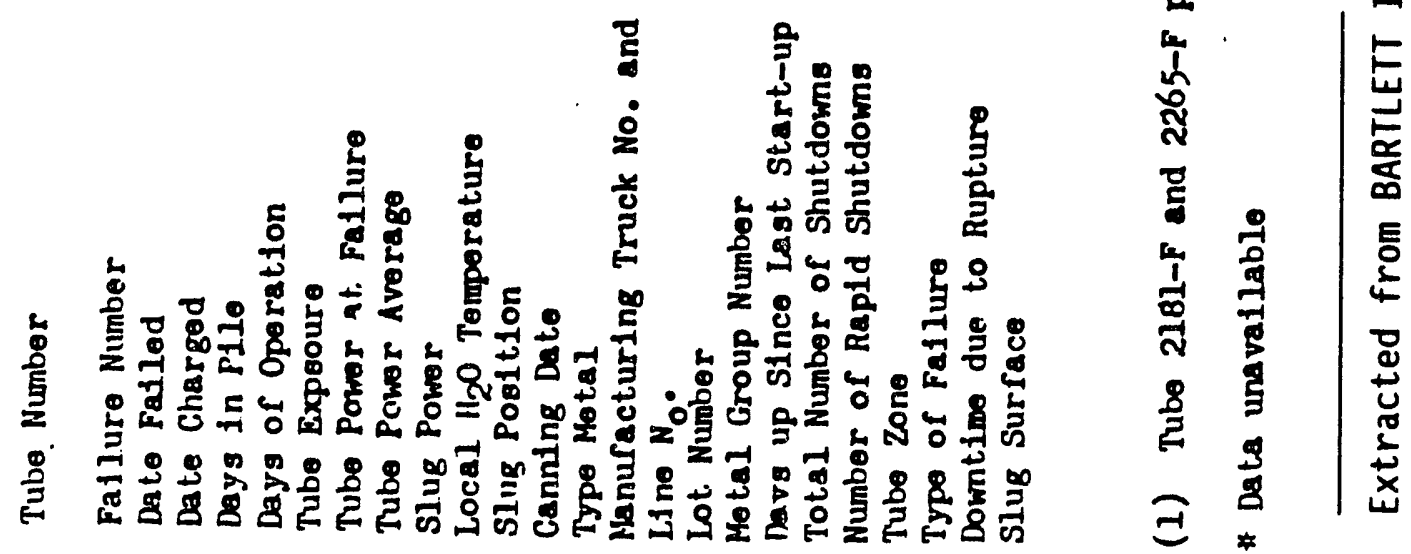




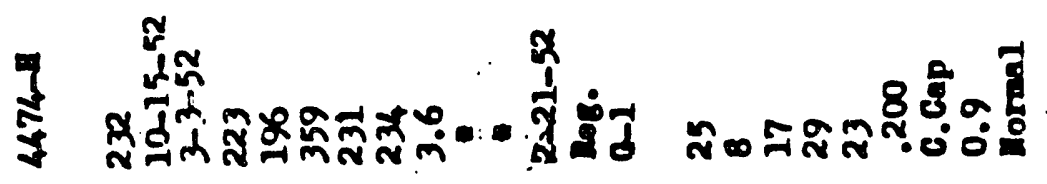

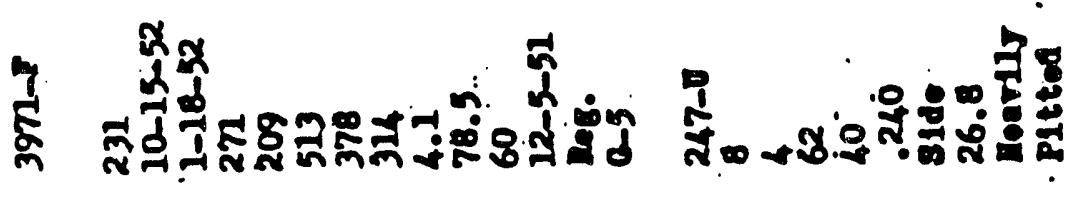

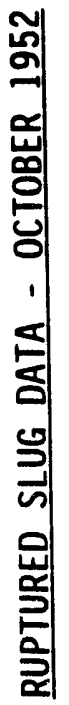

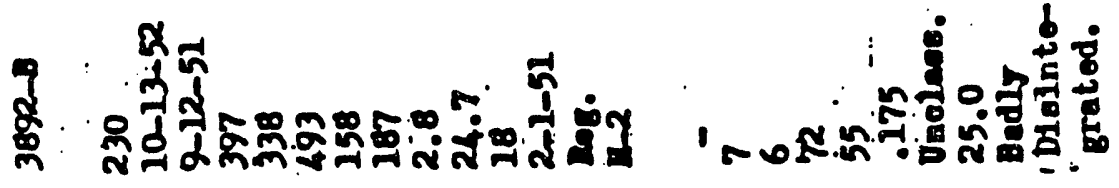

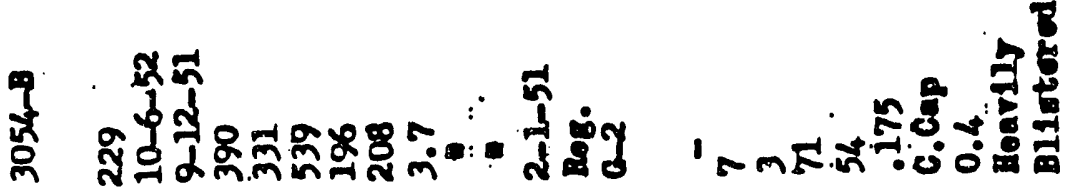

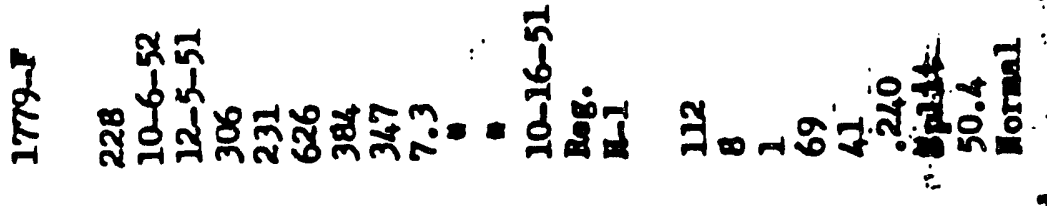

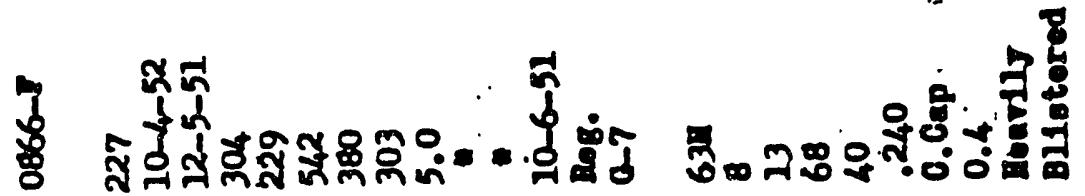

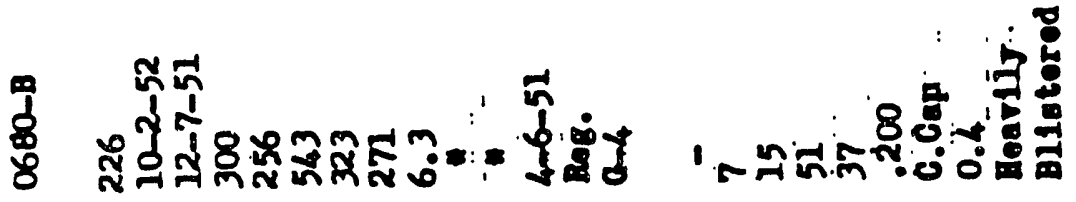

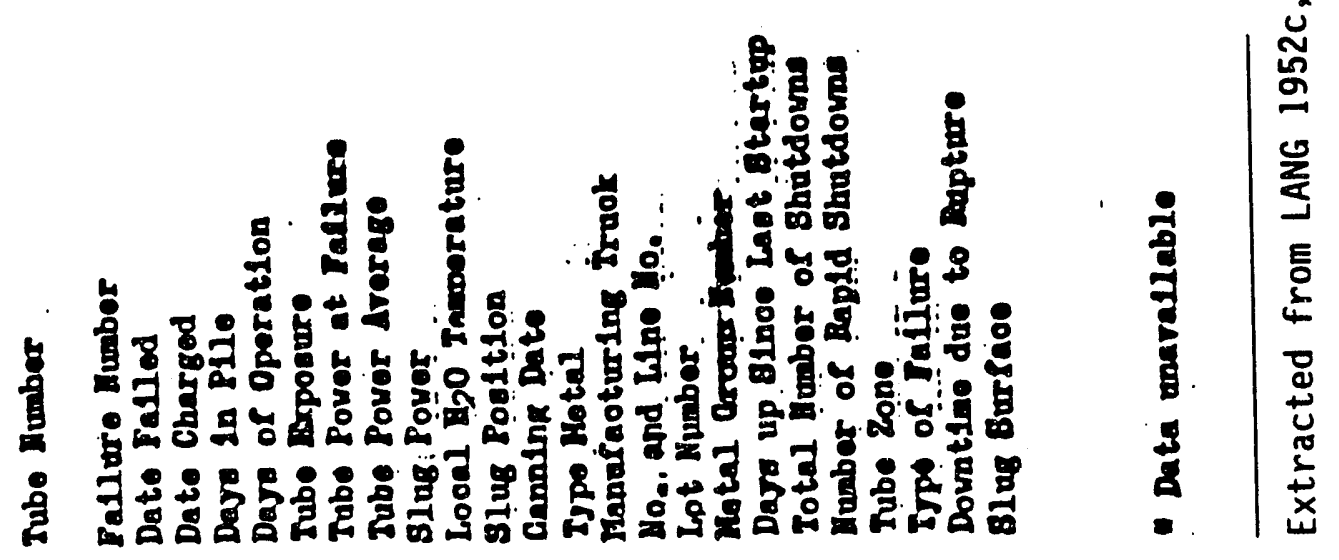




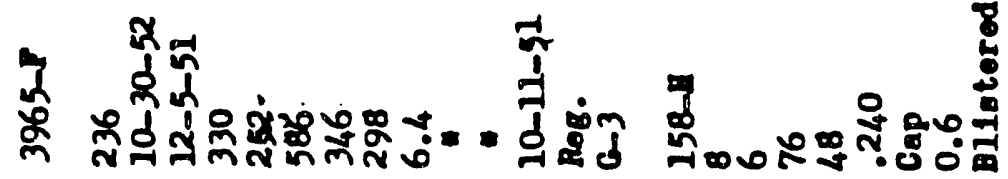

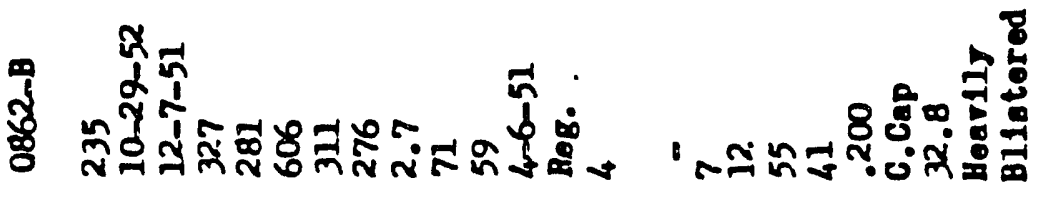

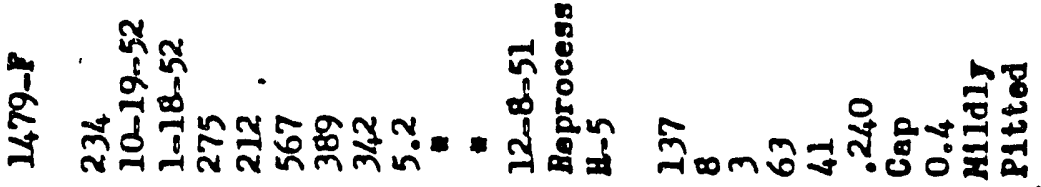

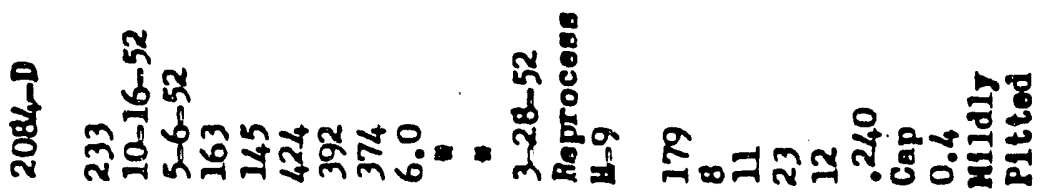

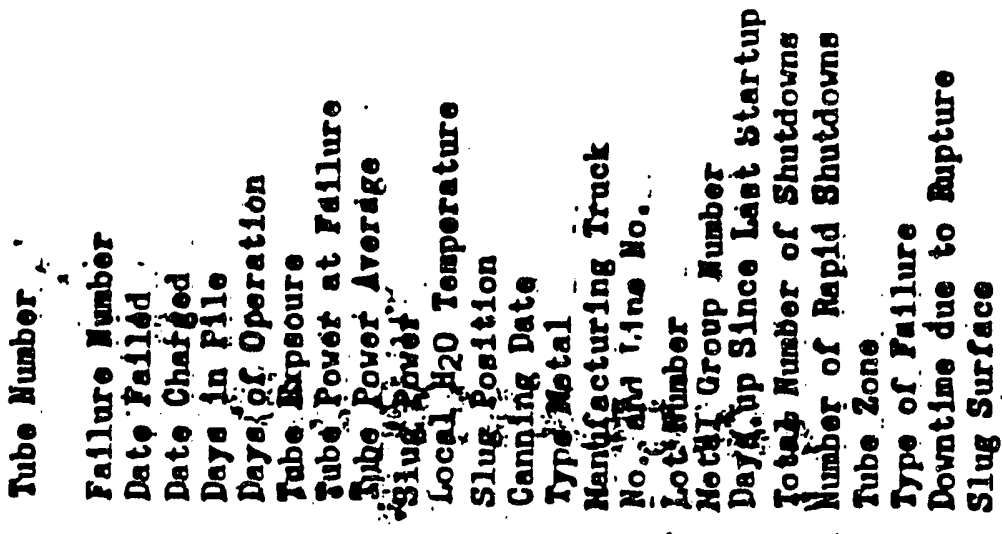




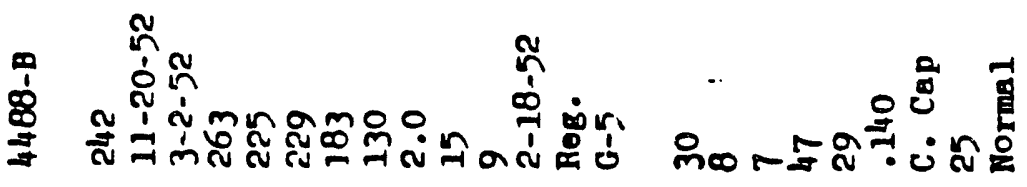

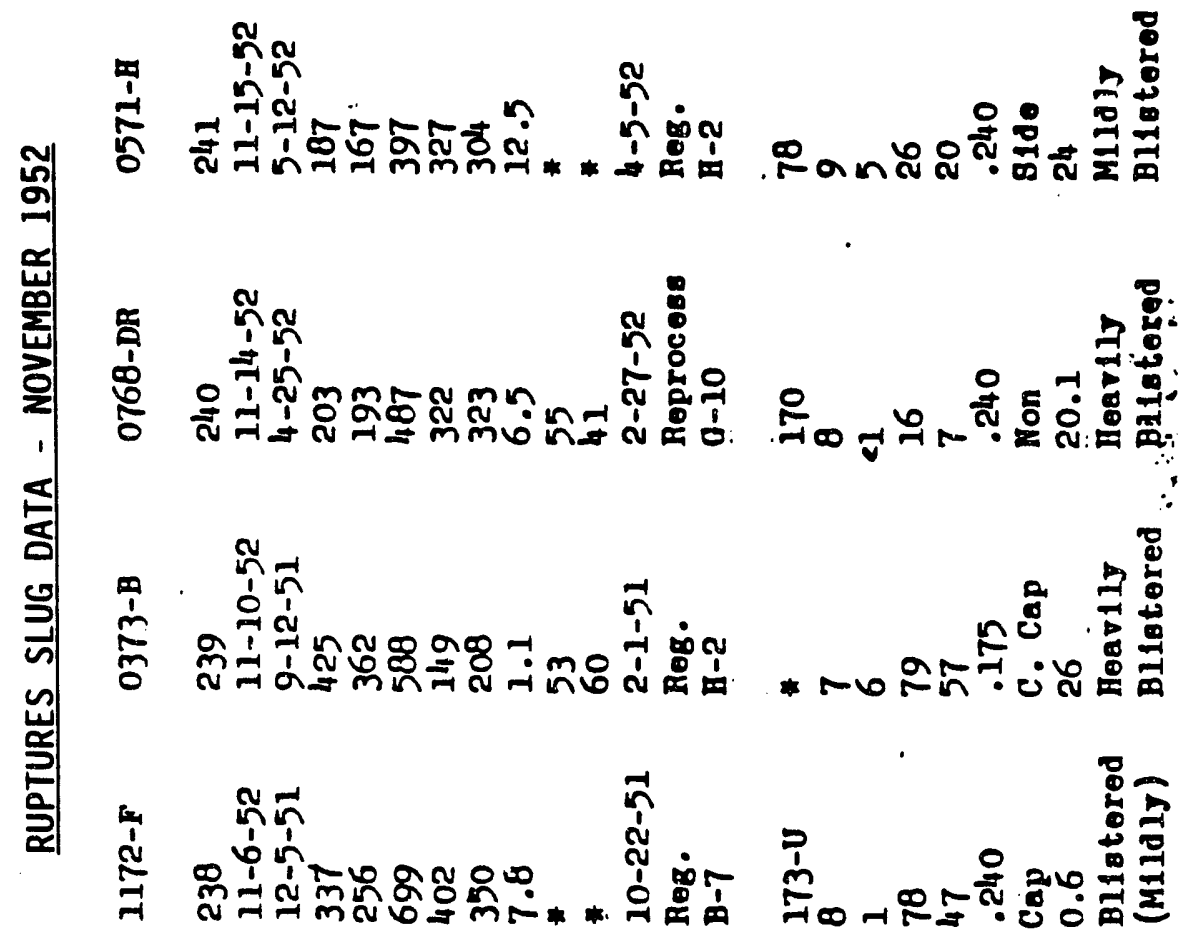

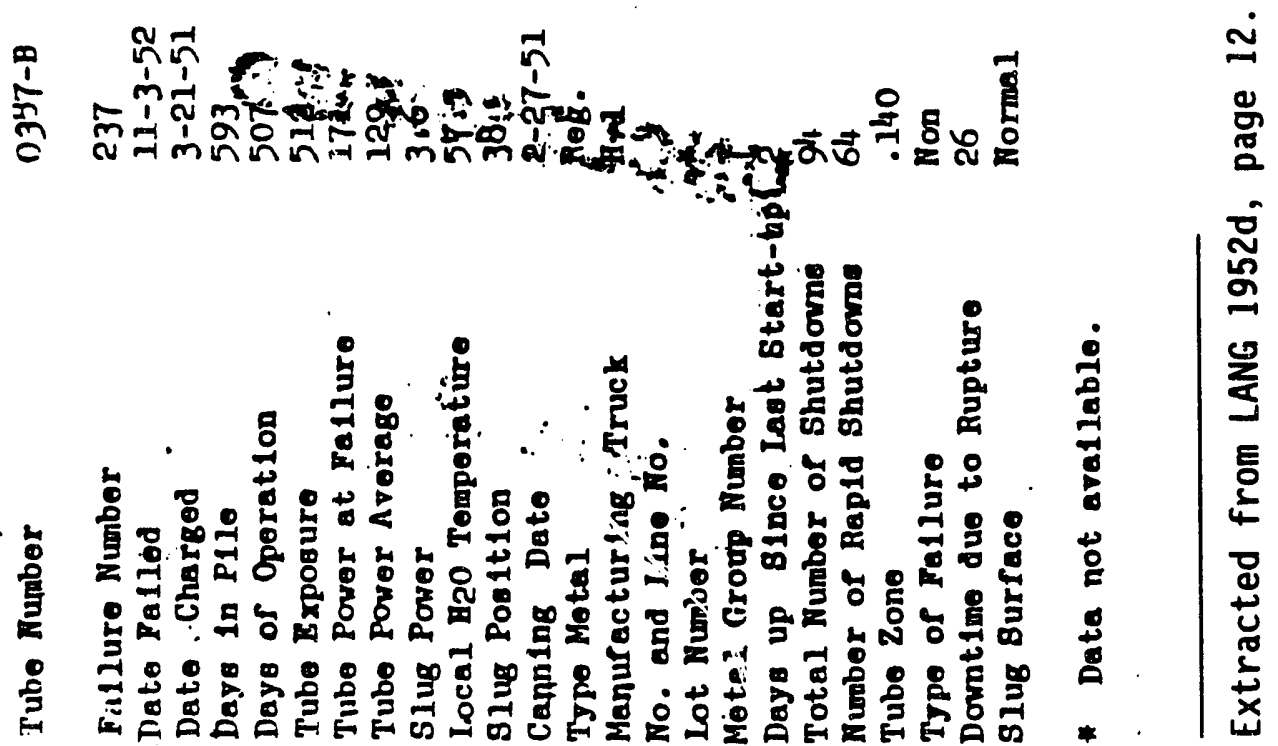




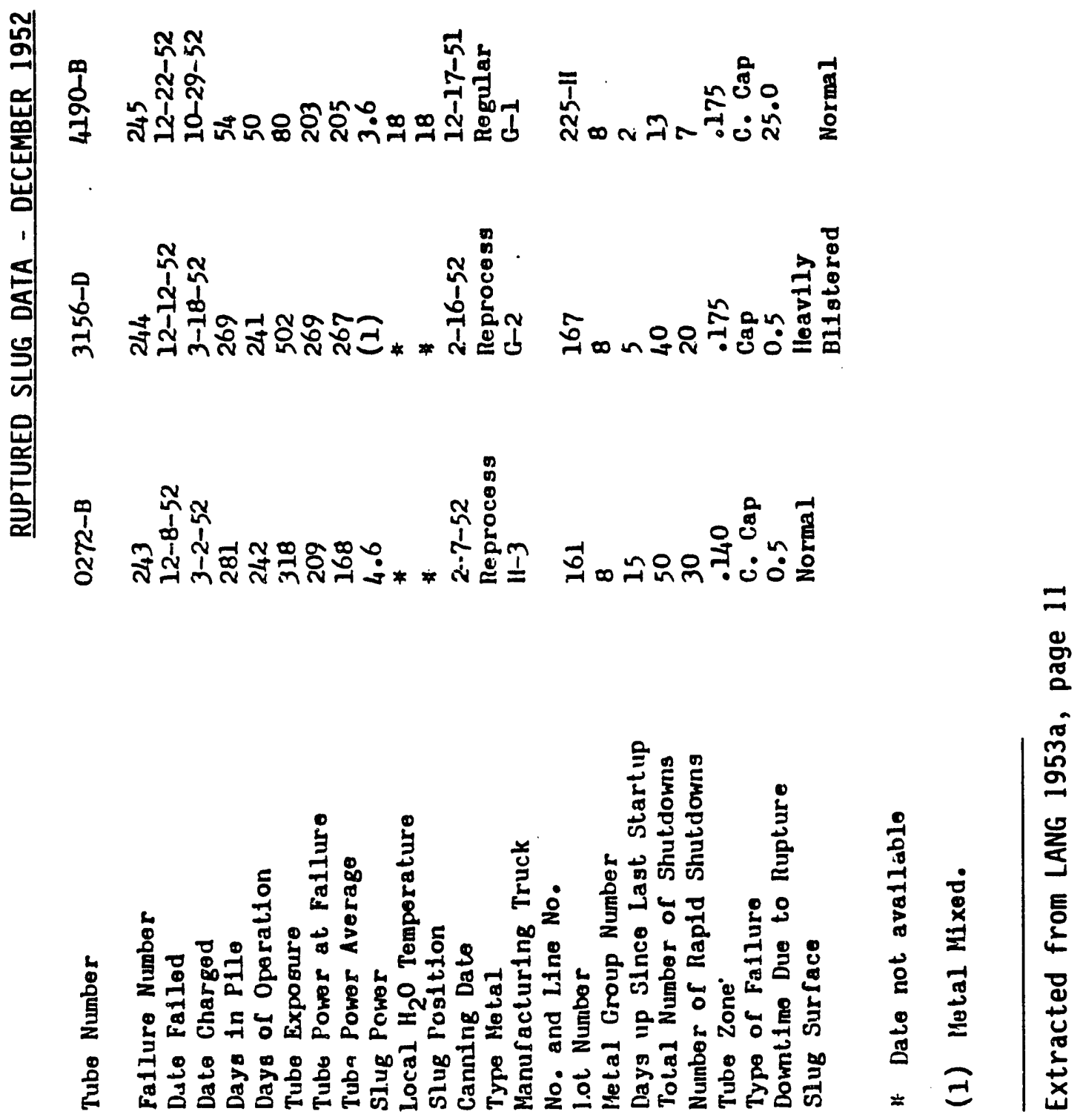


APPENDIX C

FUEL-ELEMENT FAILURE DATA FOR 1953 


\section{RUPTURED SLUG DATA - JANUARY 1953}

Trbe Number

Fallaro Number

Deto Fallod

Deto Chargod

Dejo in P1lo

Deje of Operation

Tabe Expo: ure

Tube Porrcr at Fa1luro

Tube Porrer Arerage.

Slus Power

Iocel $\mathrm{B}_{2} \mathrm{O}$ Tomperaturo

Slus PoB1tion

Cannlin, Dato

Type Motel

Nanurectaring Truck

IIrmber and IIne Irumber

Lot Irmber

Notal Group Number

Dajo Up alnce Last Start-Op

Total Number of Shatdown

Irumber of Rap1d Shutdorns

Tube Zone

TJpe of Falloro

Downt Ime Dae to Rupturo

Slas Surfece

* Data not arailablo.

Extracted from LANG 1953b, page 12.

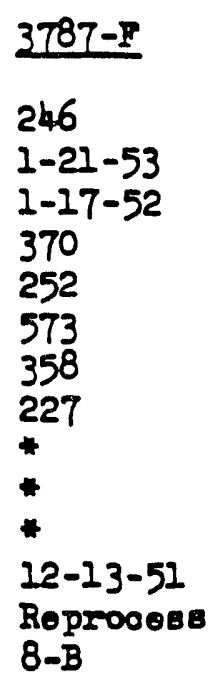

2RR-139

8

1

78

47

.240

sido

27

Hoar11y

Bllatered.
$2487-F$

247

$1-29-53$

4-7-52

297

206

550

390

267

4.3

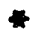

*

3-11-52

Reprocese

$2-G$

ZRR-175

8

6

64

38

.240

Cap

0.5

Tormal 
RUPTURED SLUG DATA - FEBRUARY 1953

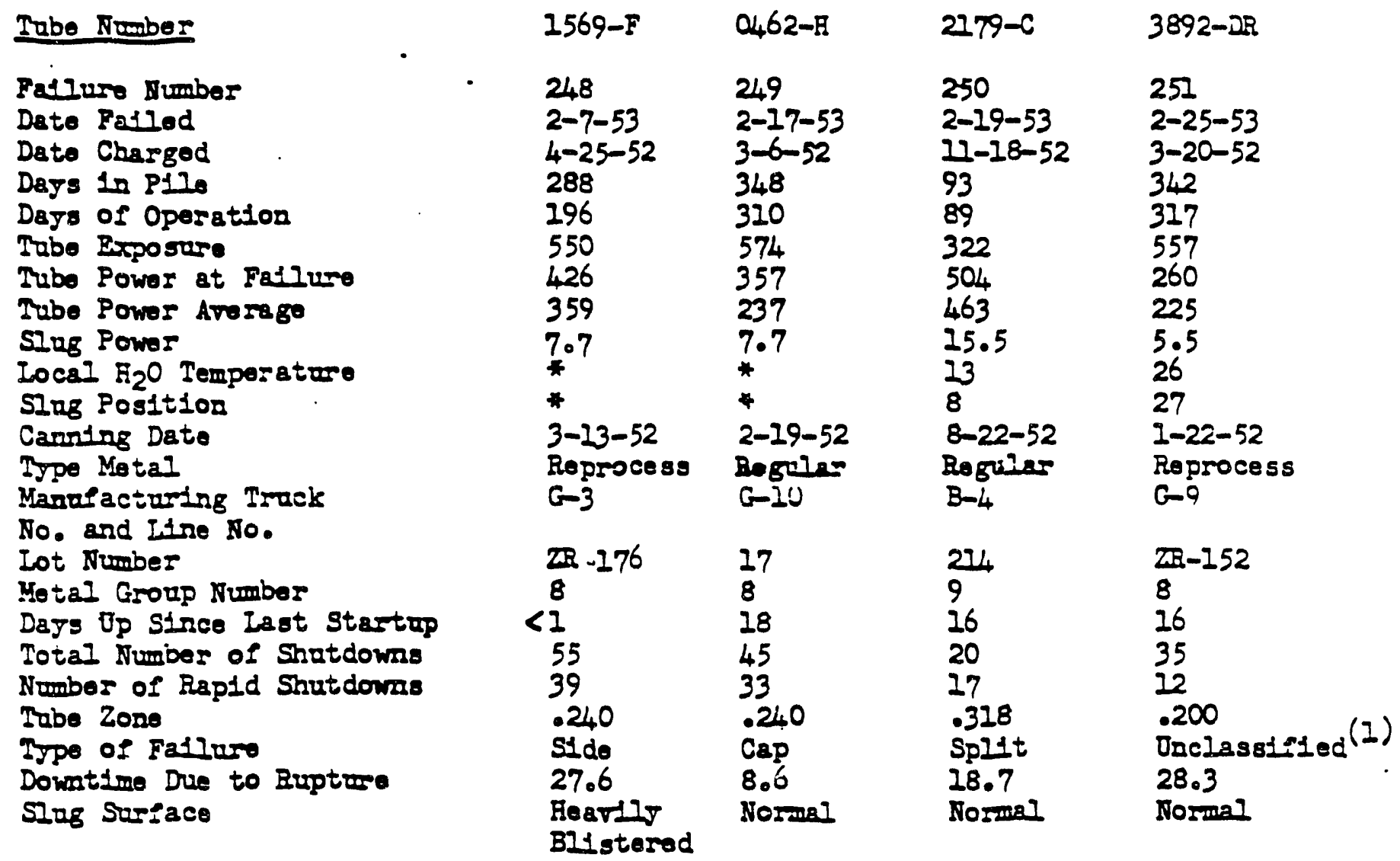

* Data Unarajlable

(I) Slug broken lato several pleces.

Extracted from LANG 1953c, page 11. 


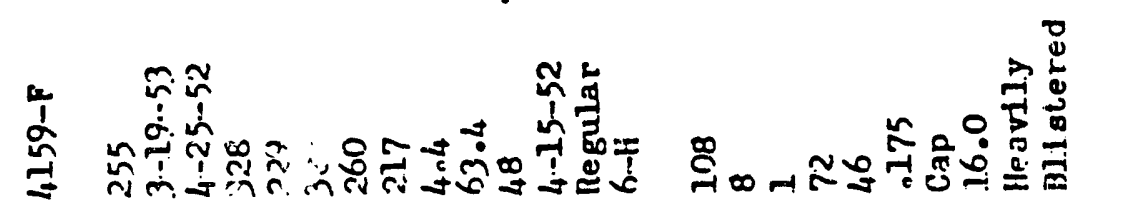

$\stackrel{\oplus}{E}$

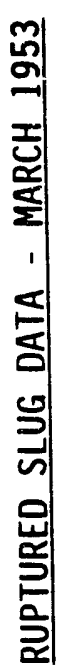

i.

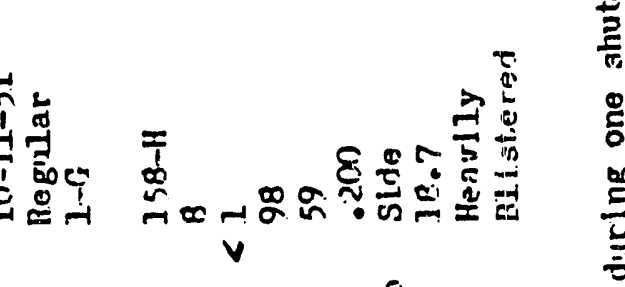

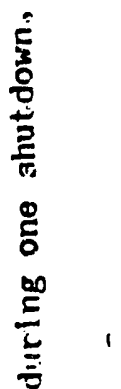

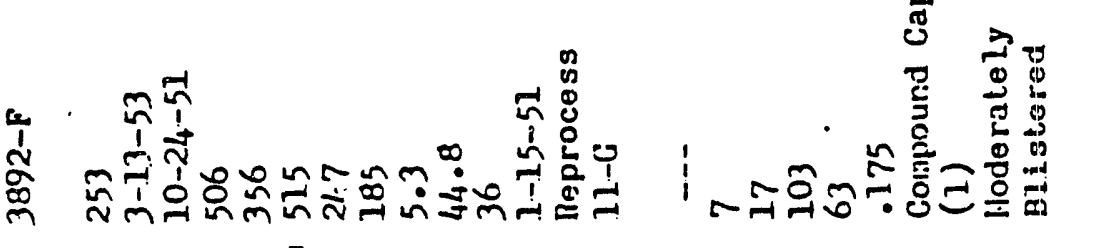

.

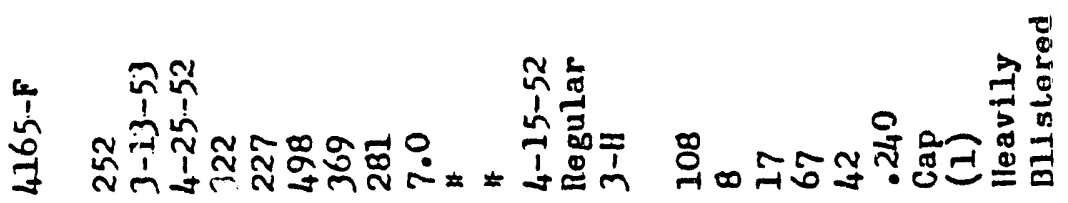

๙ุำ

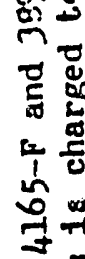

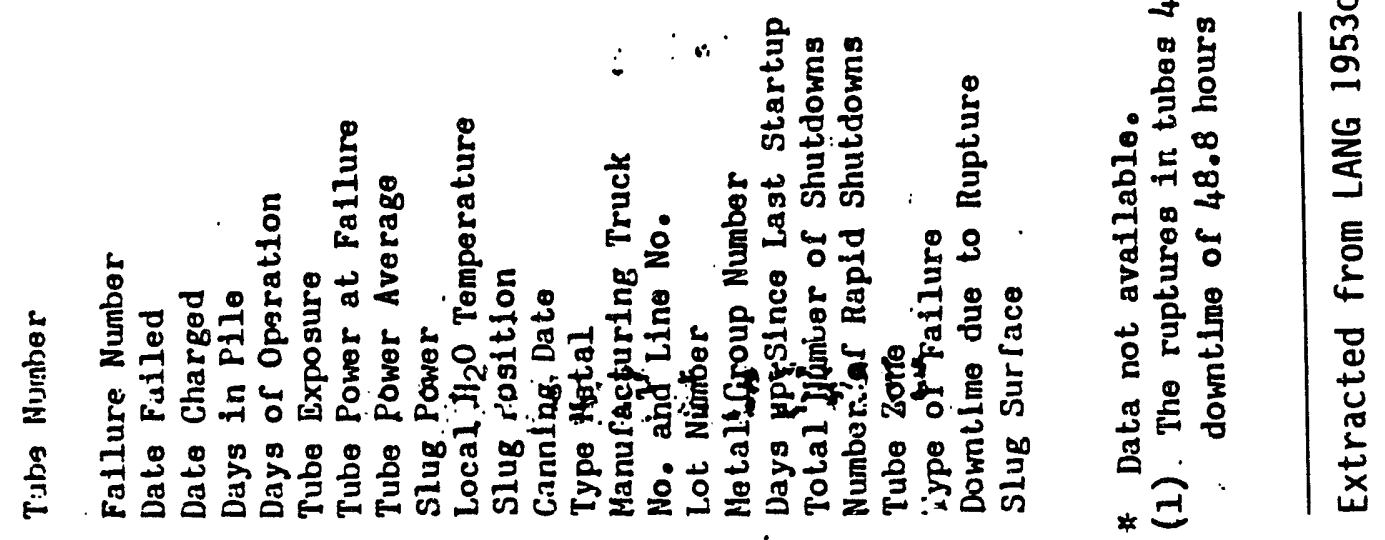




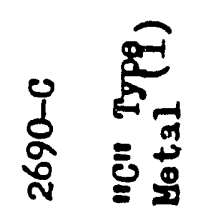

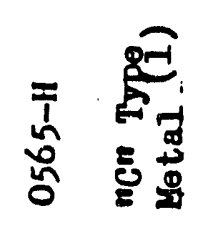

等

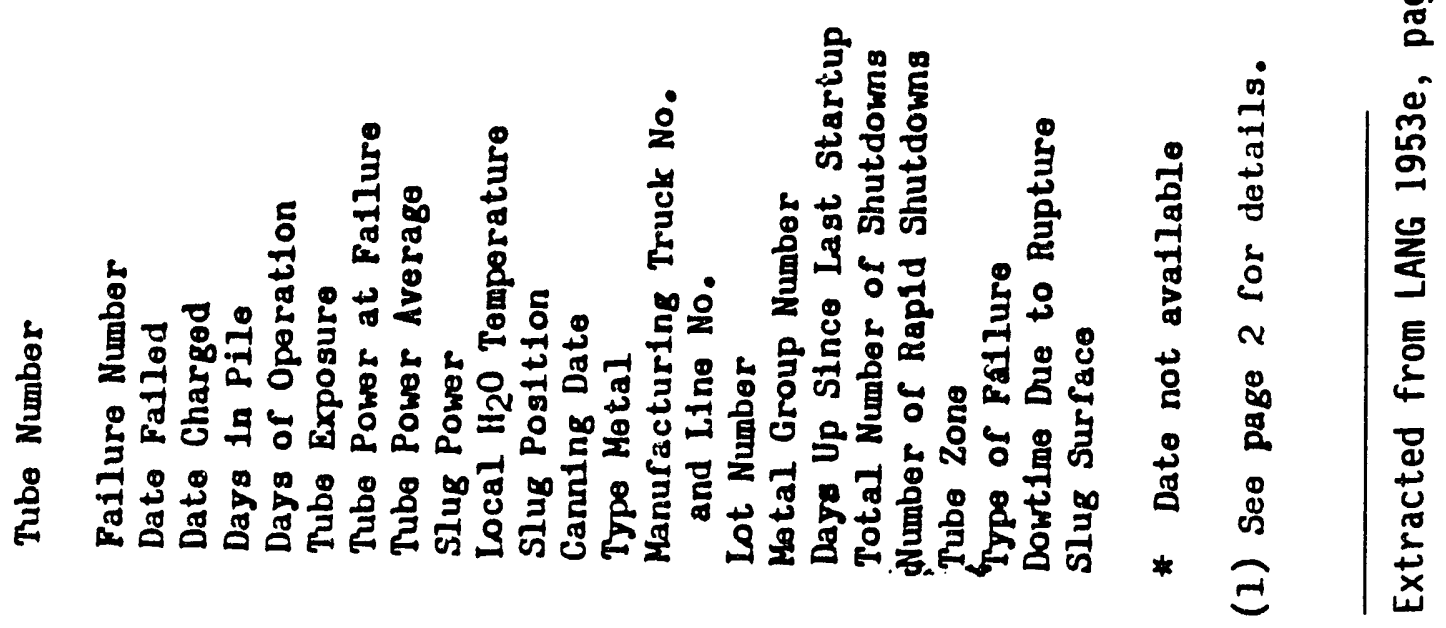




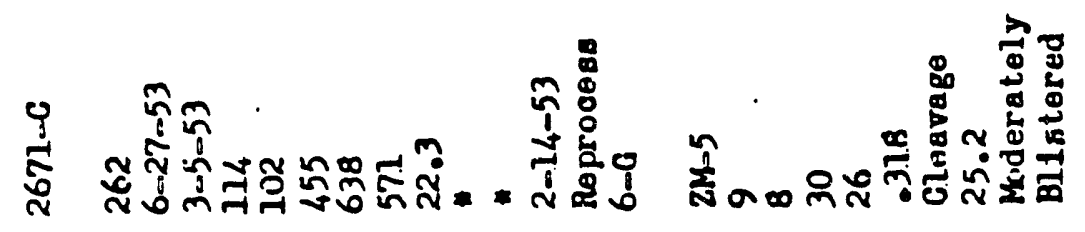

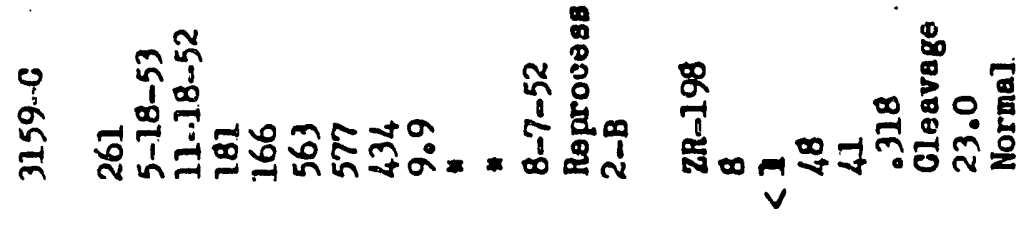

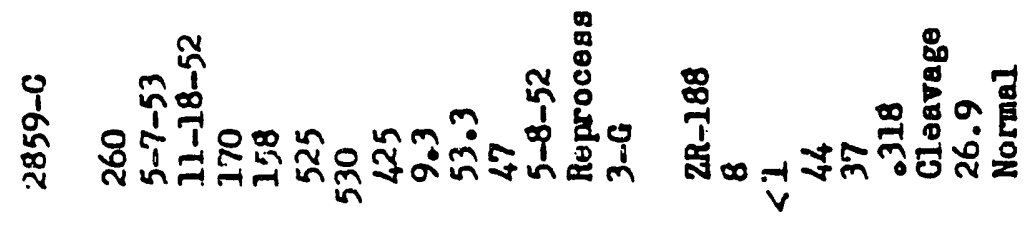

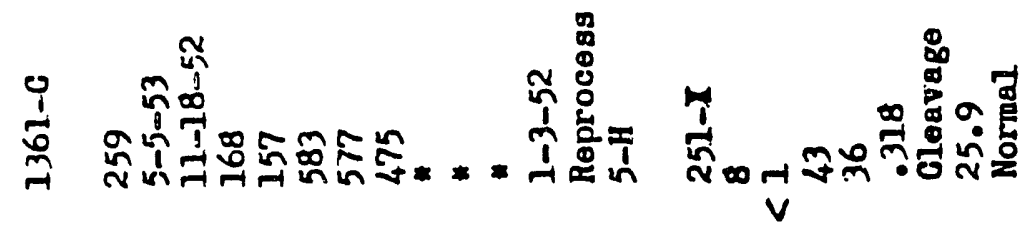

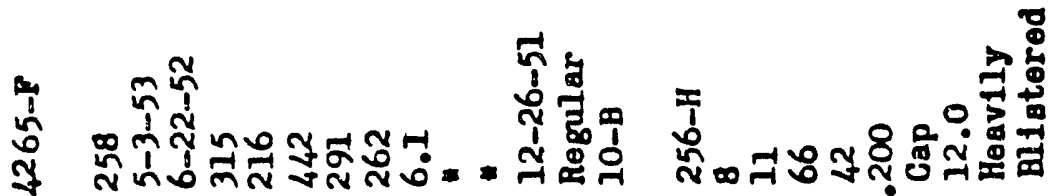

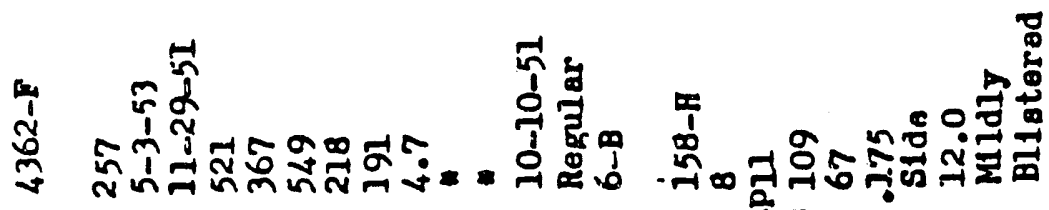

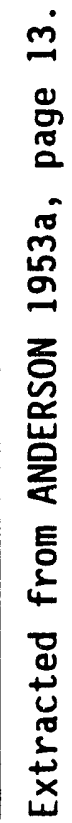




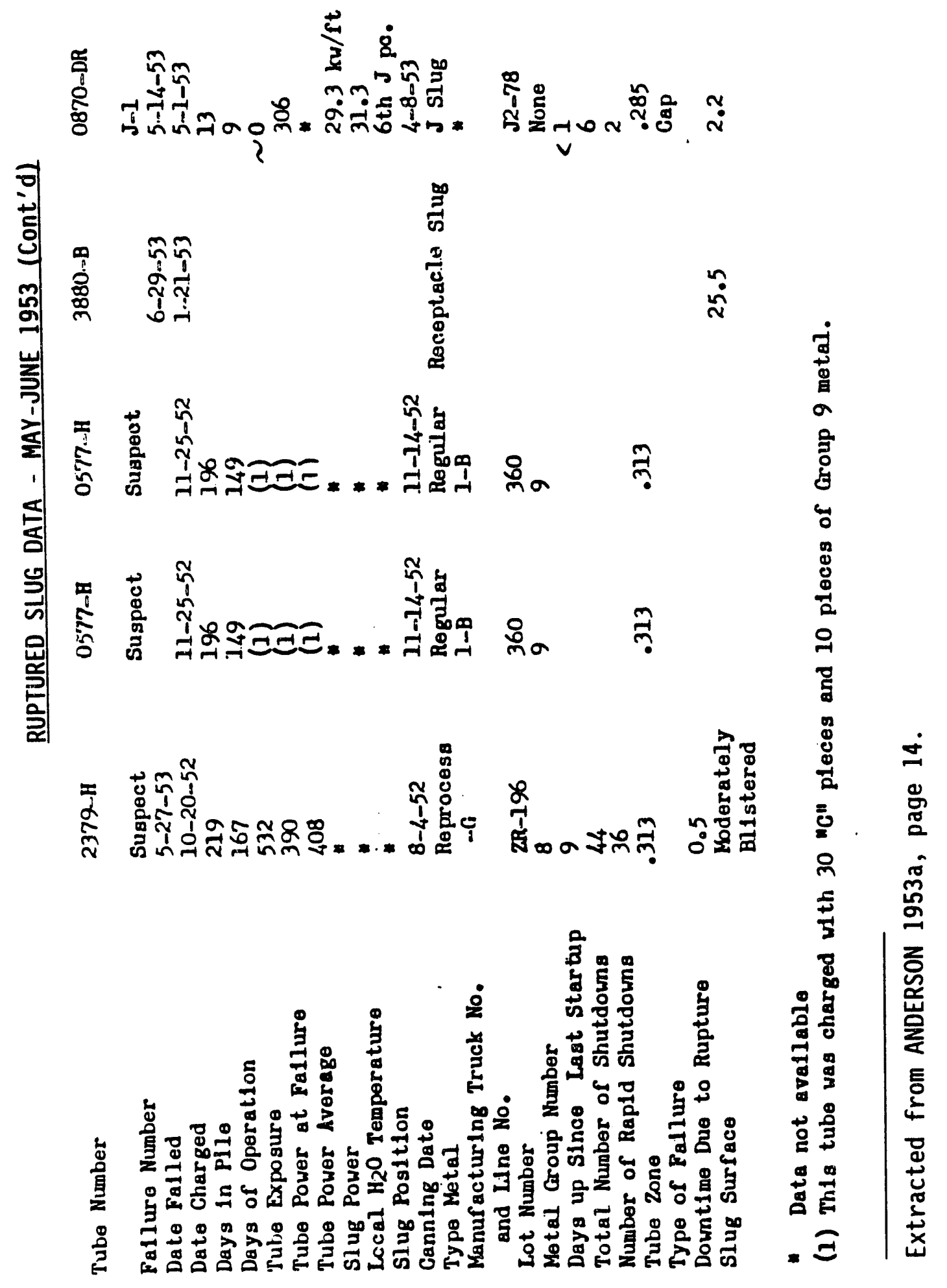

C. 6 


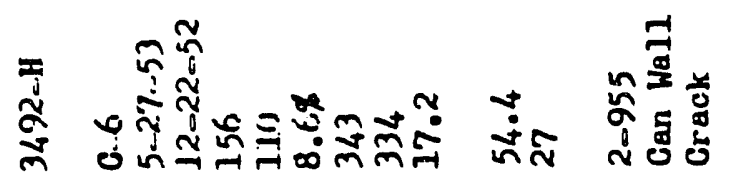

๙

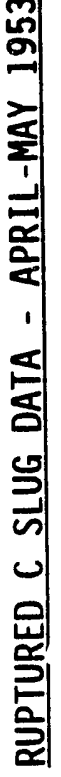

๙

\&

ठำ

:

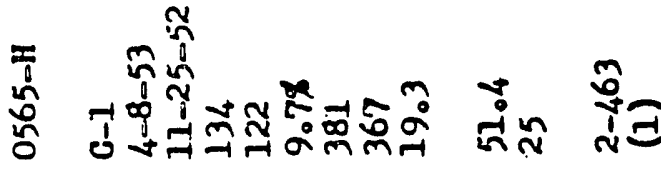

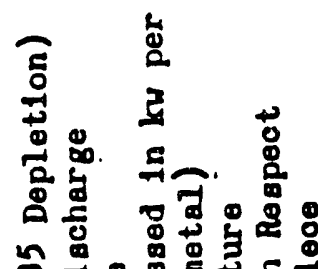

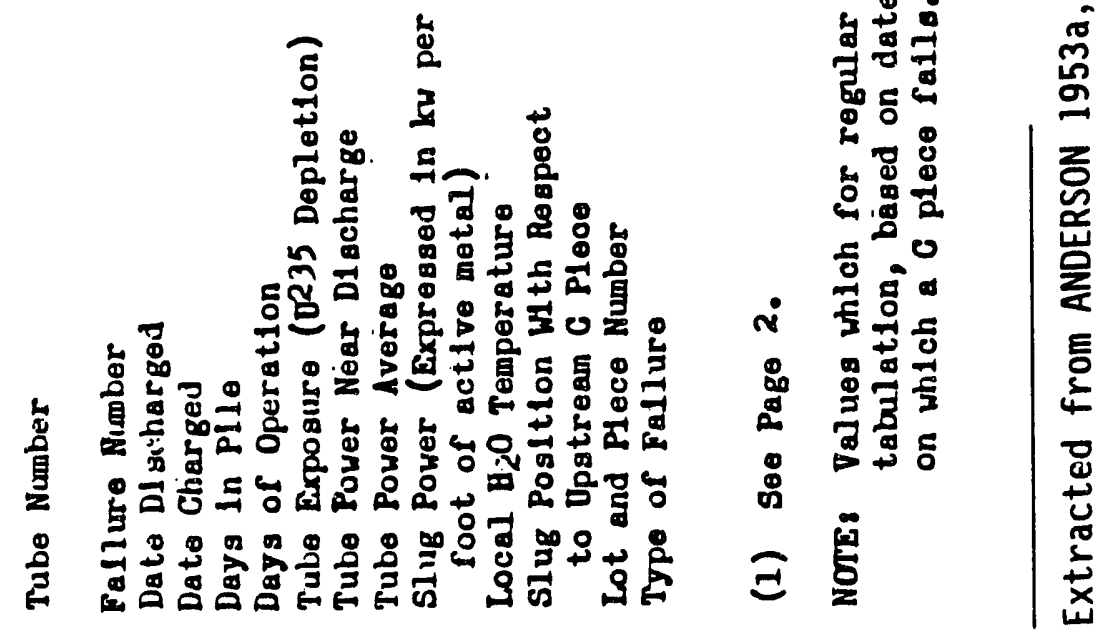

焉兽兽 


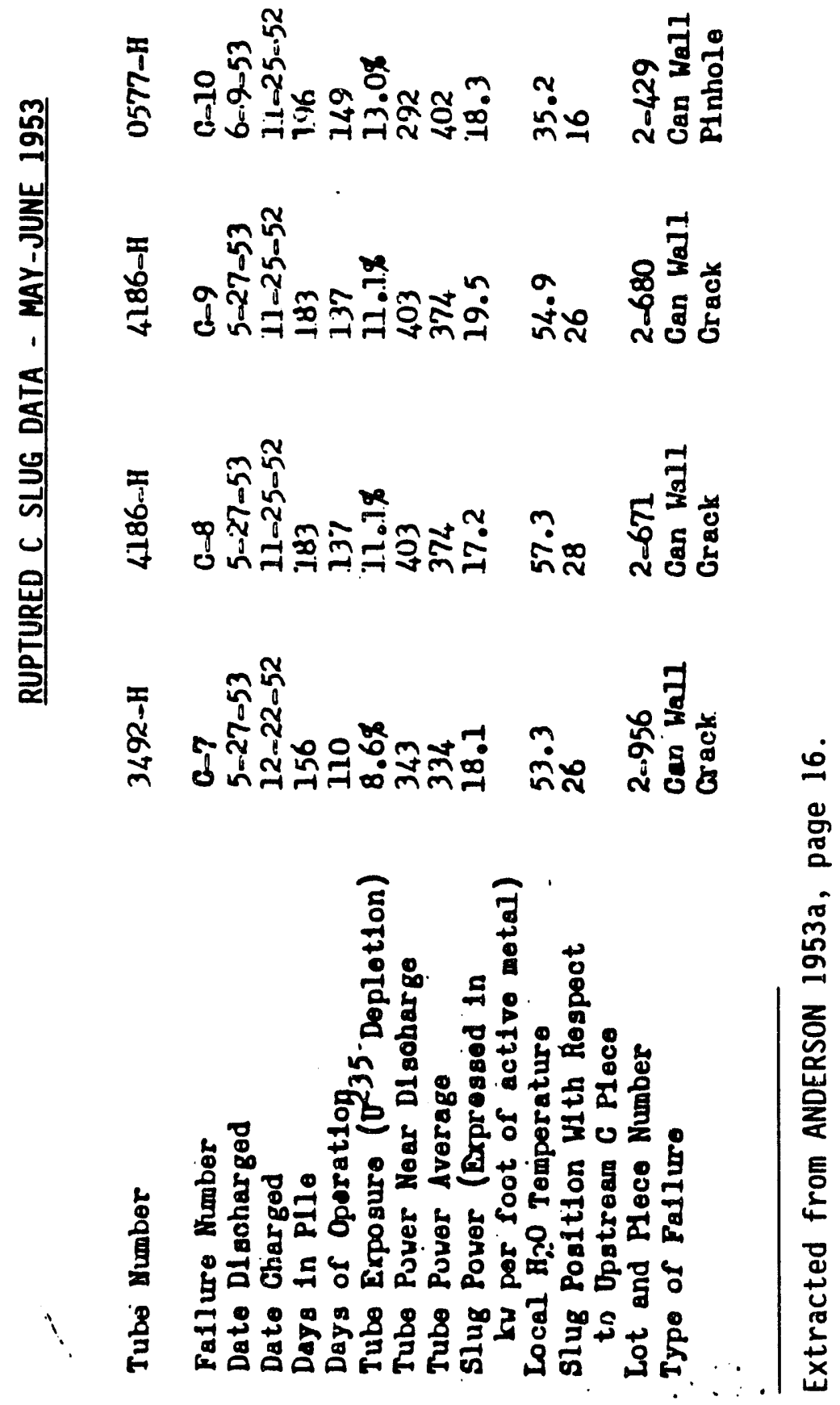

C. 8 


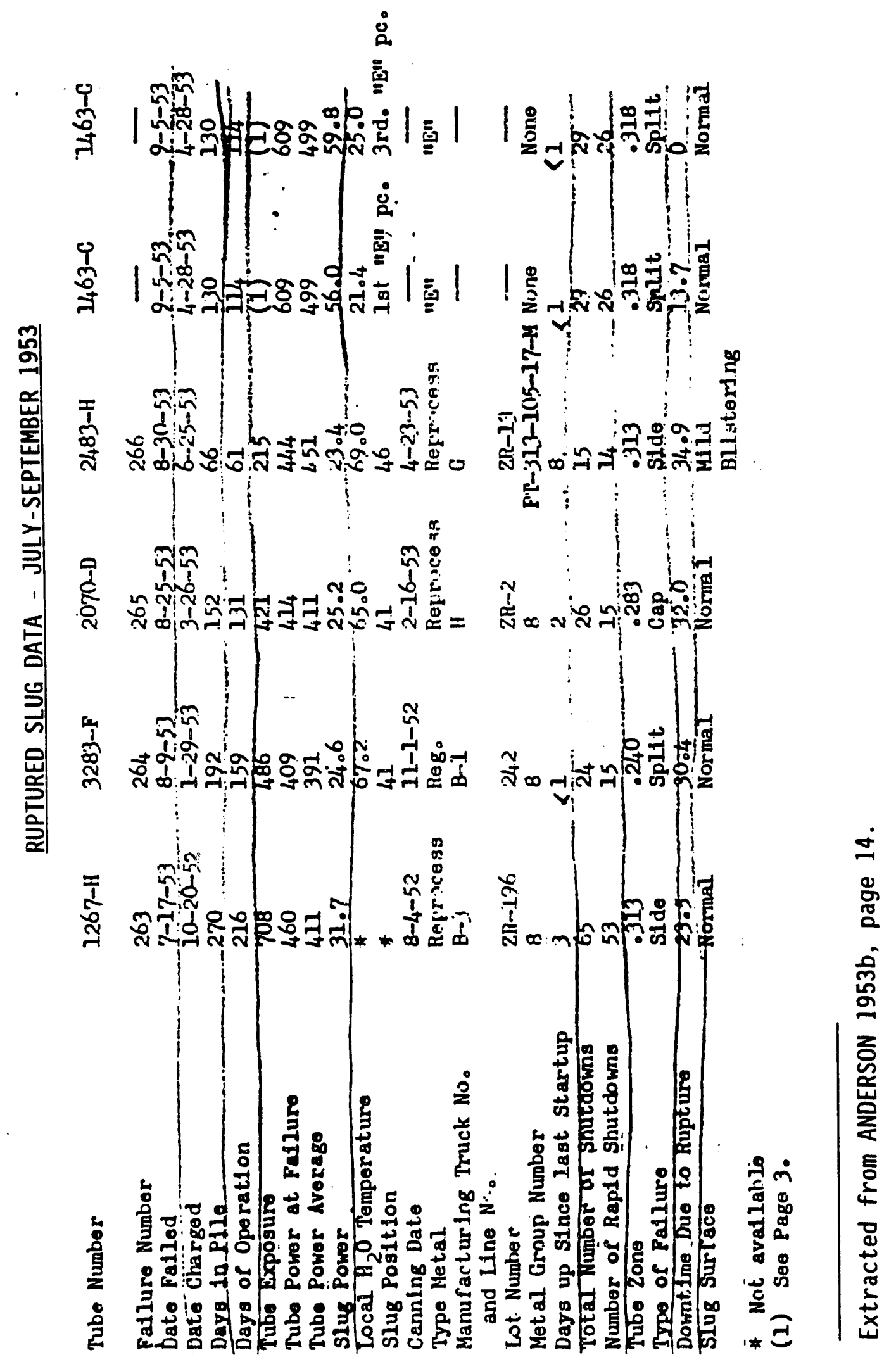




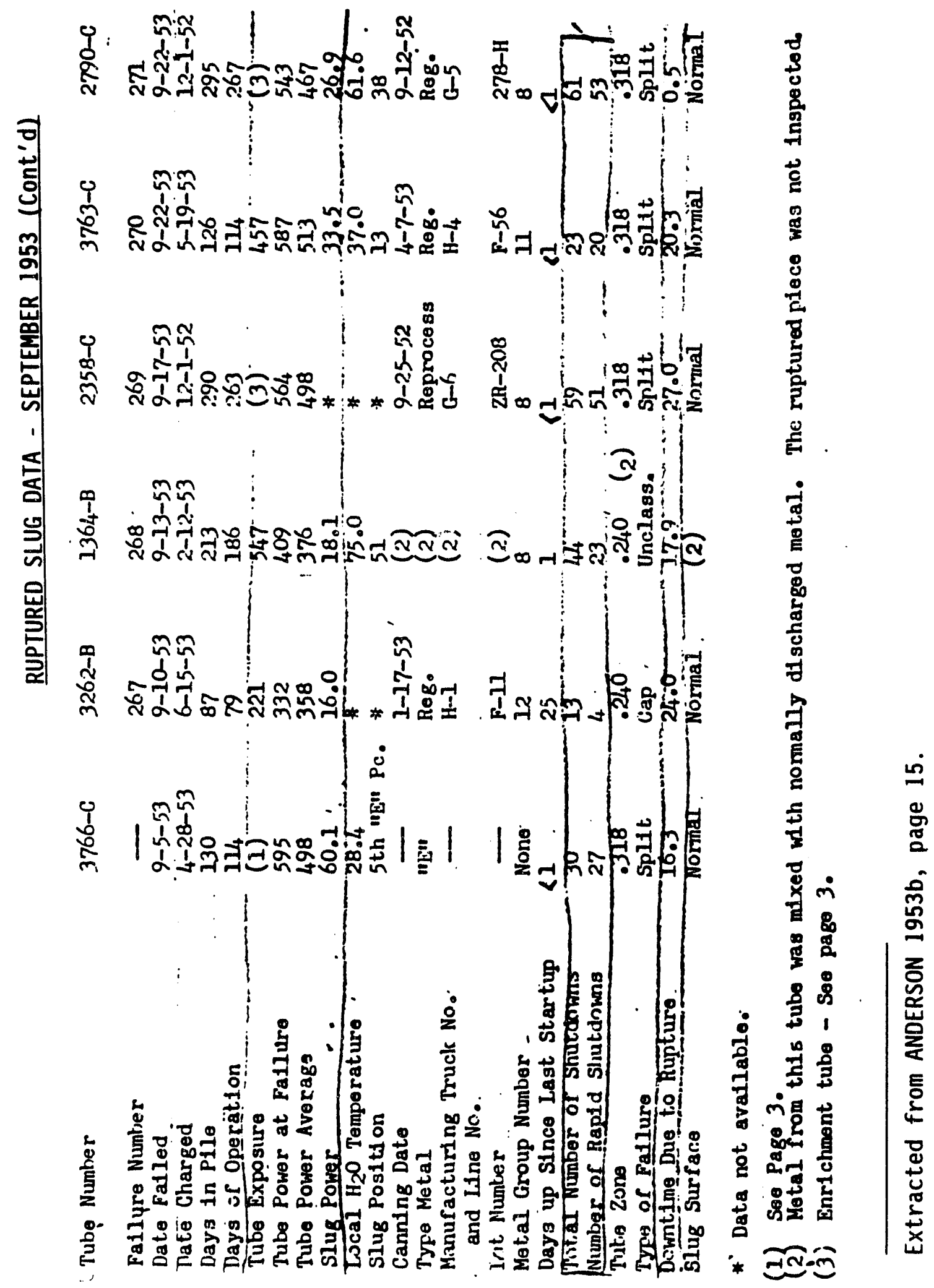




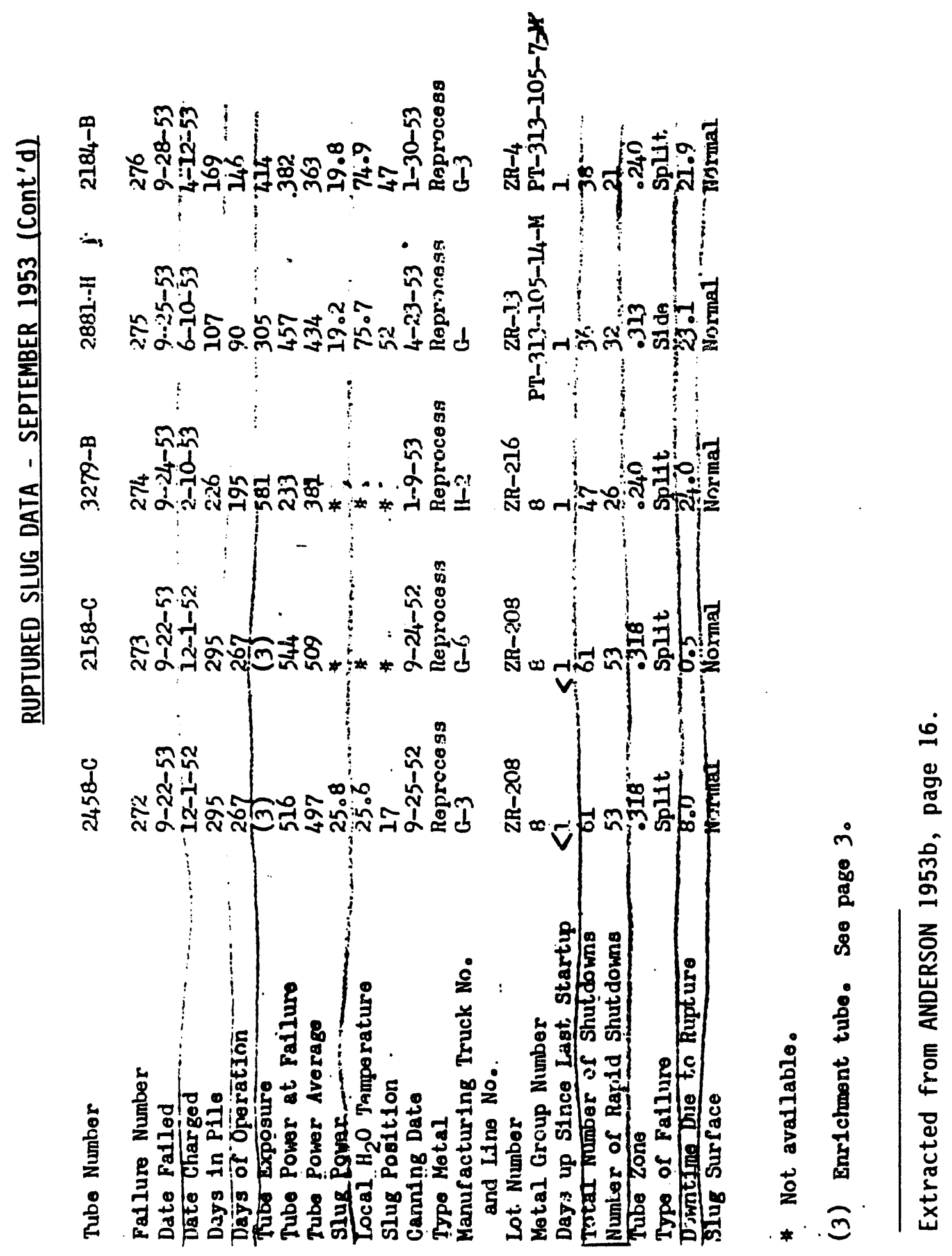




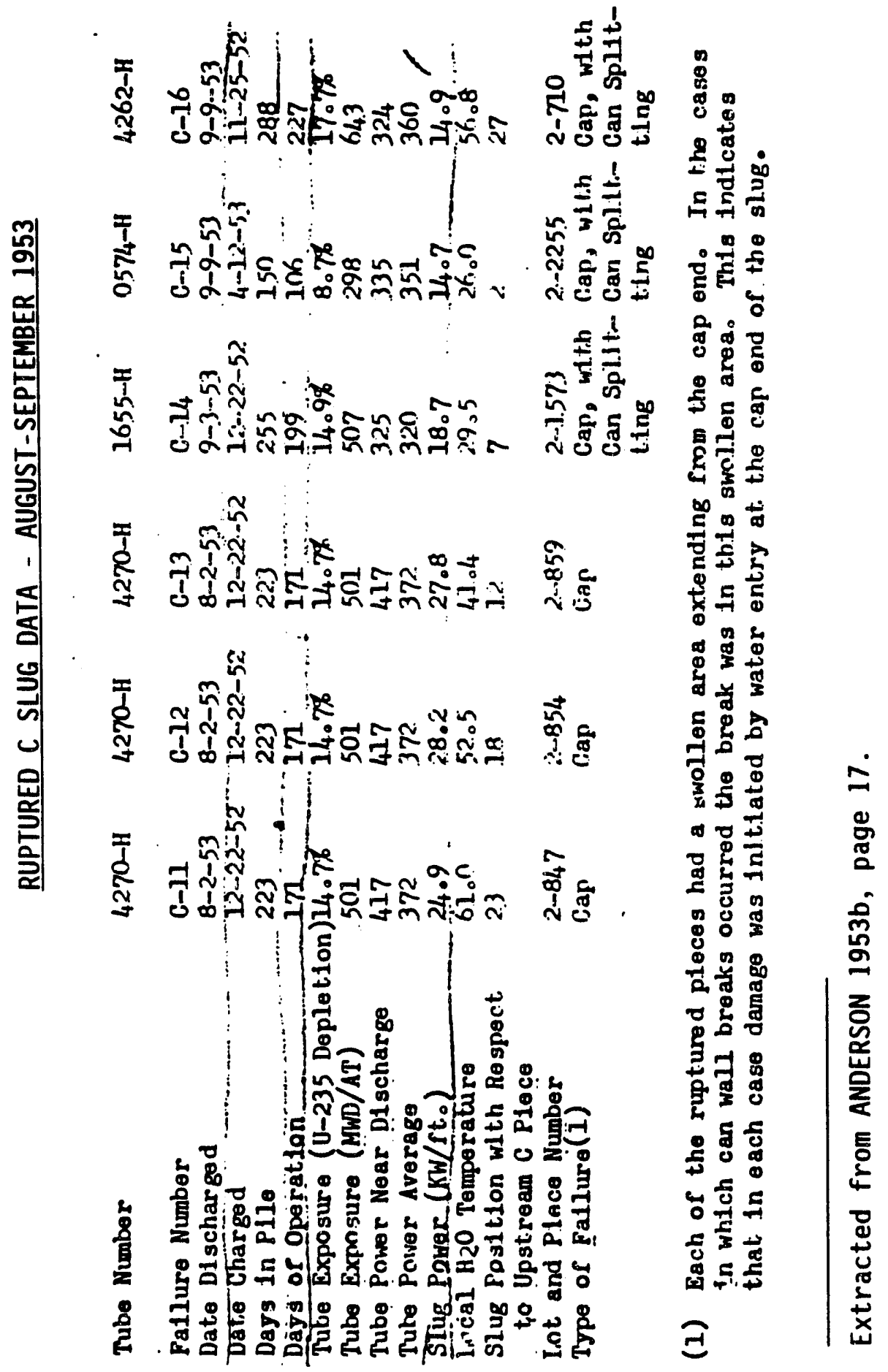

C. 12 


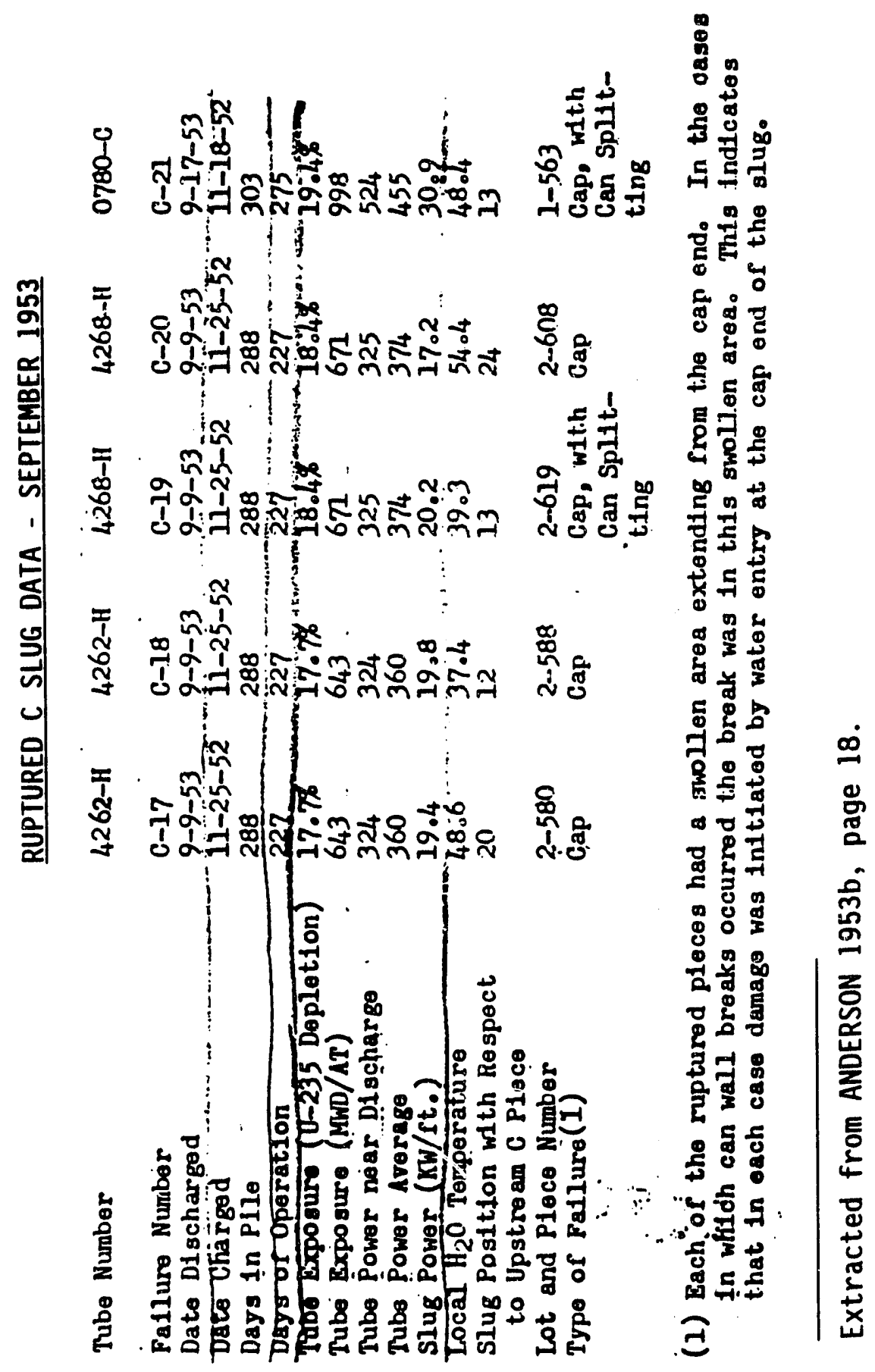




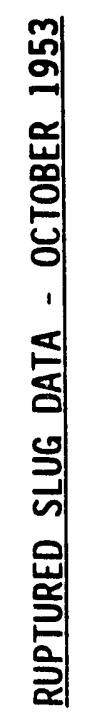

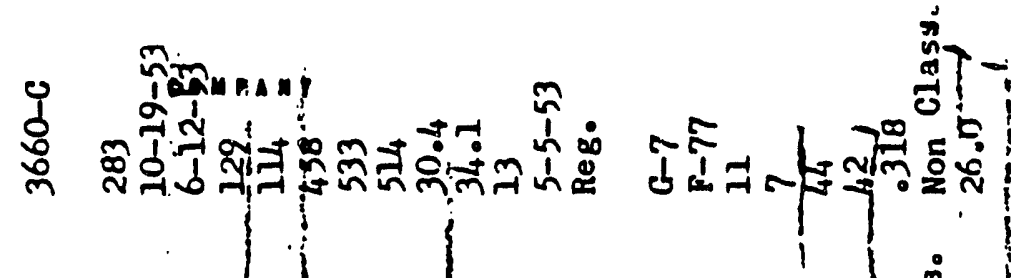

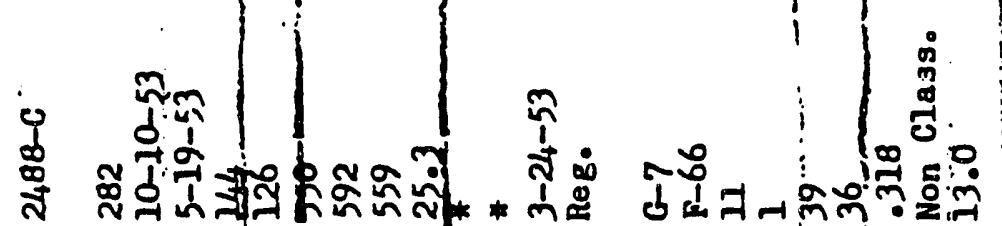

!

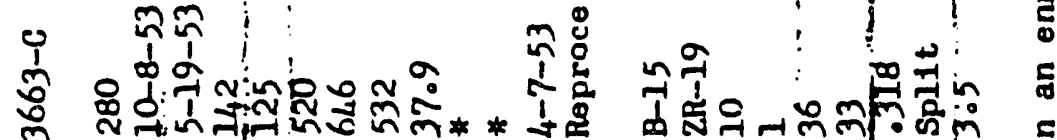

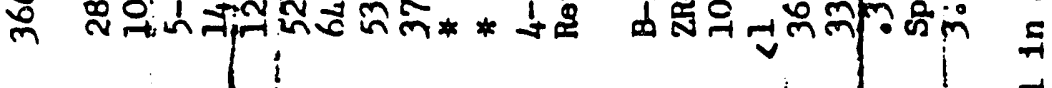

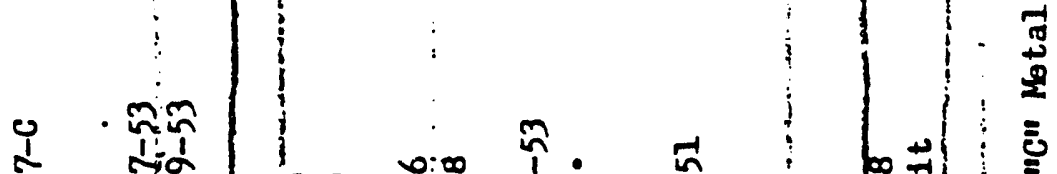

ส

i

$=$ กิ๊

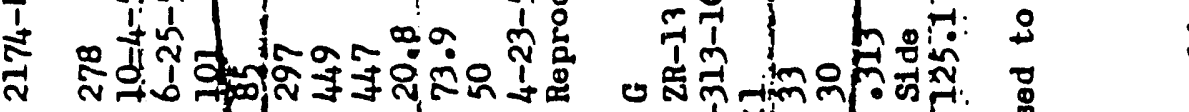

i

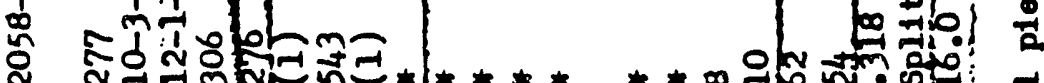

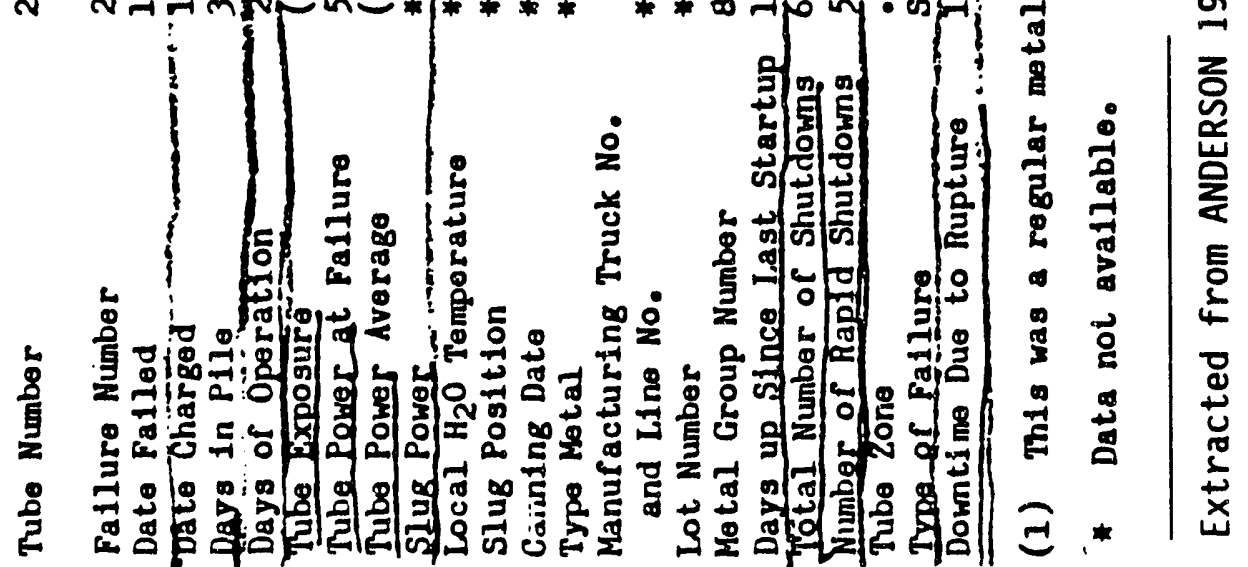




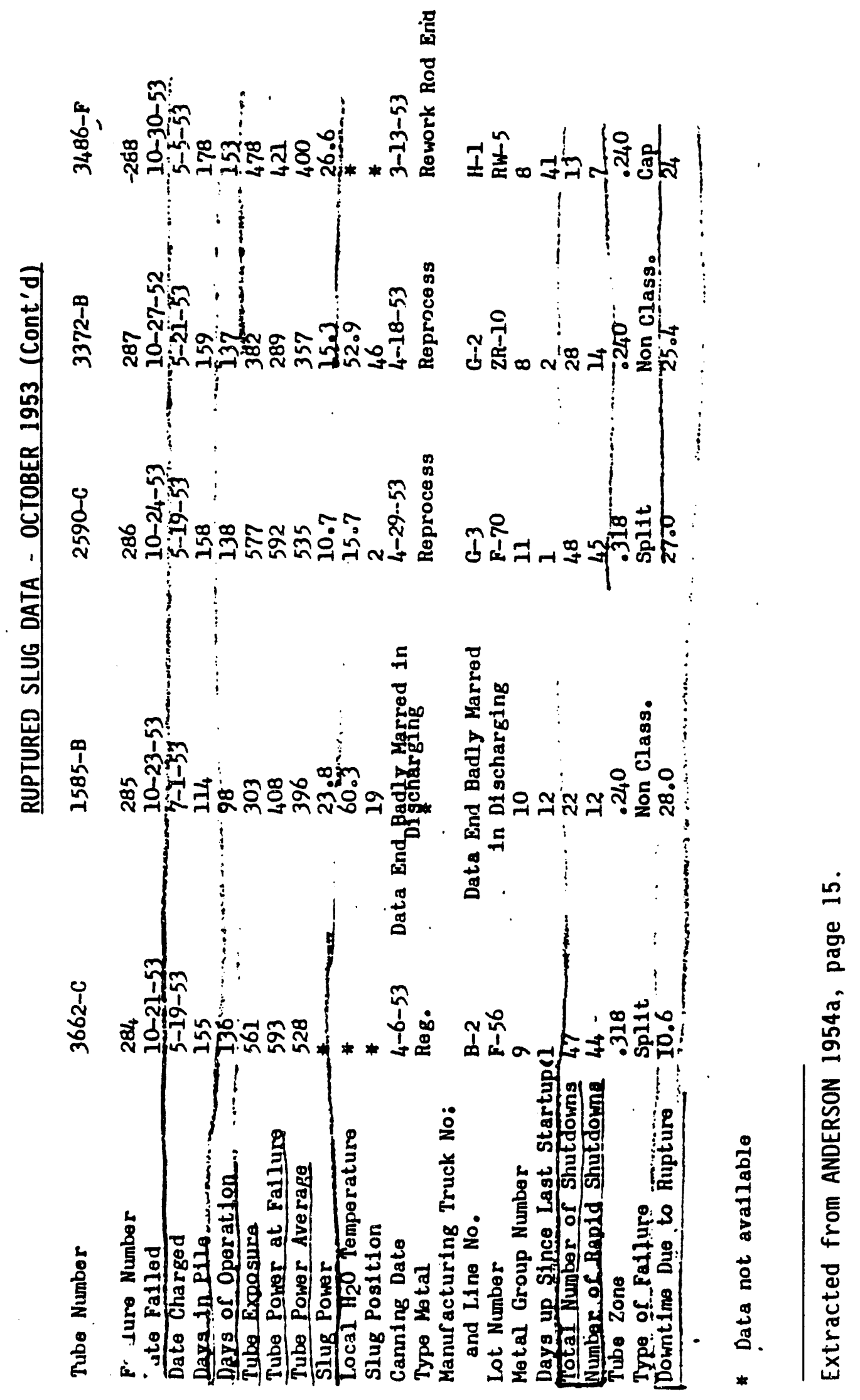

C. 15 


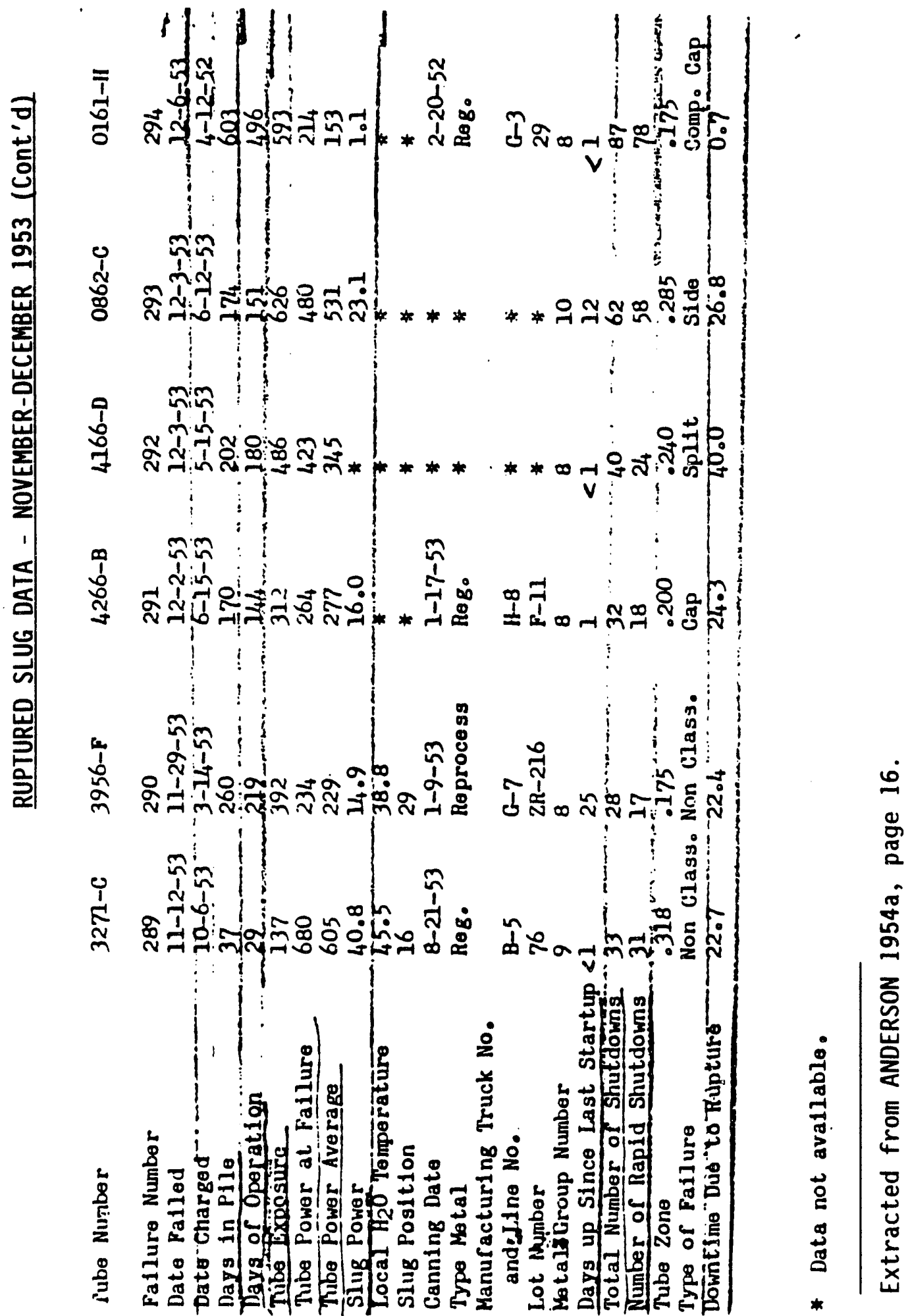

C. 16 


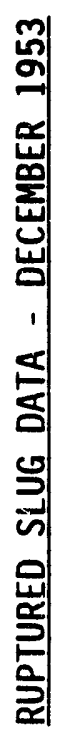

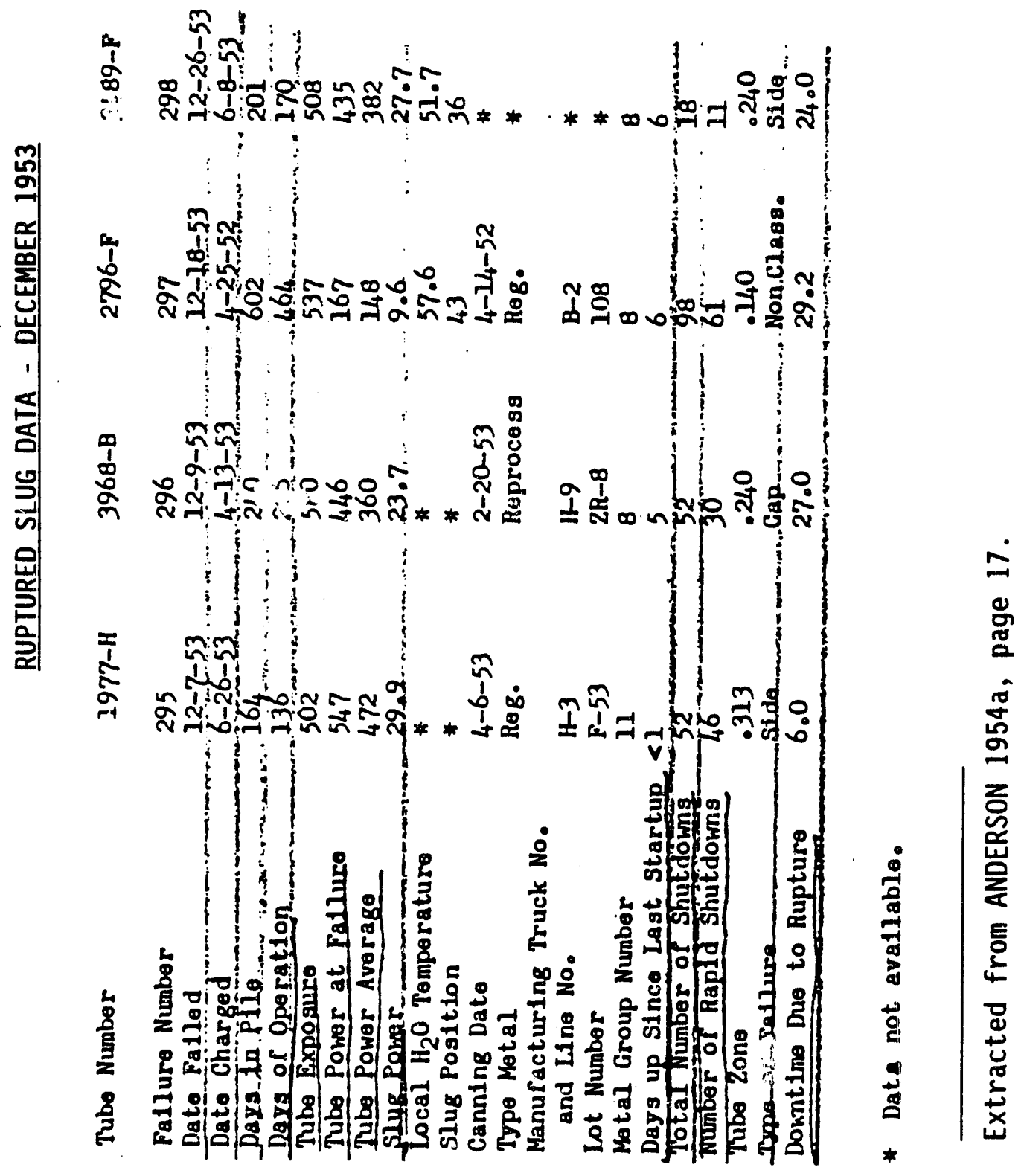




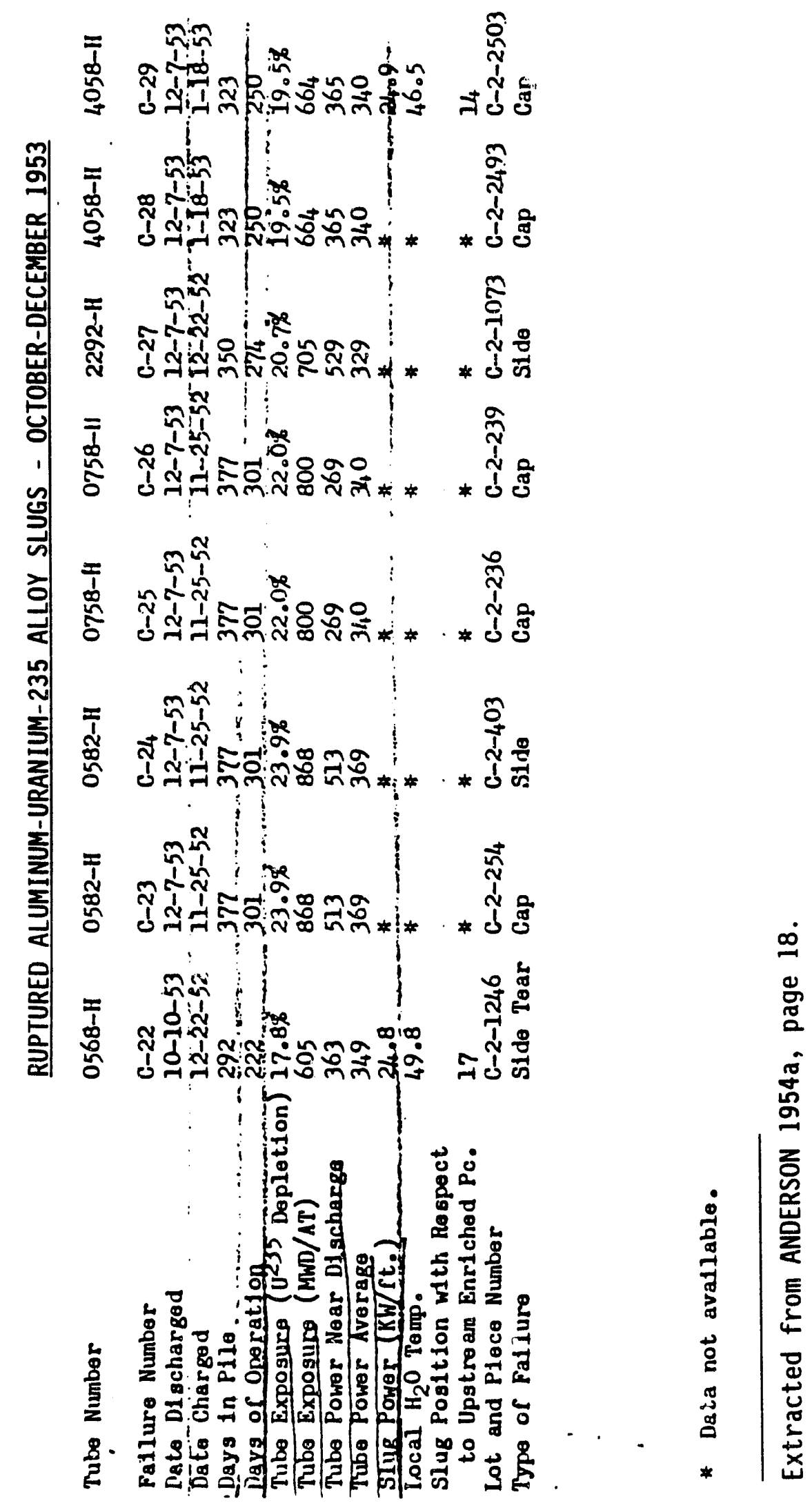

C. 18 
염

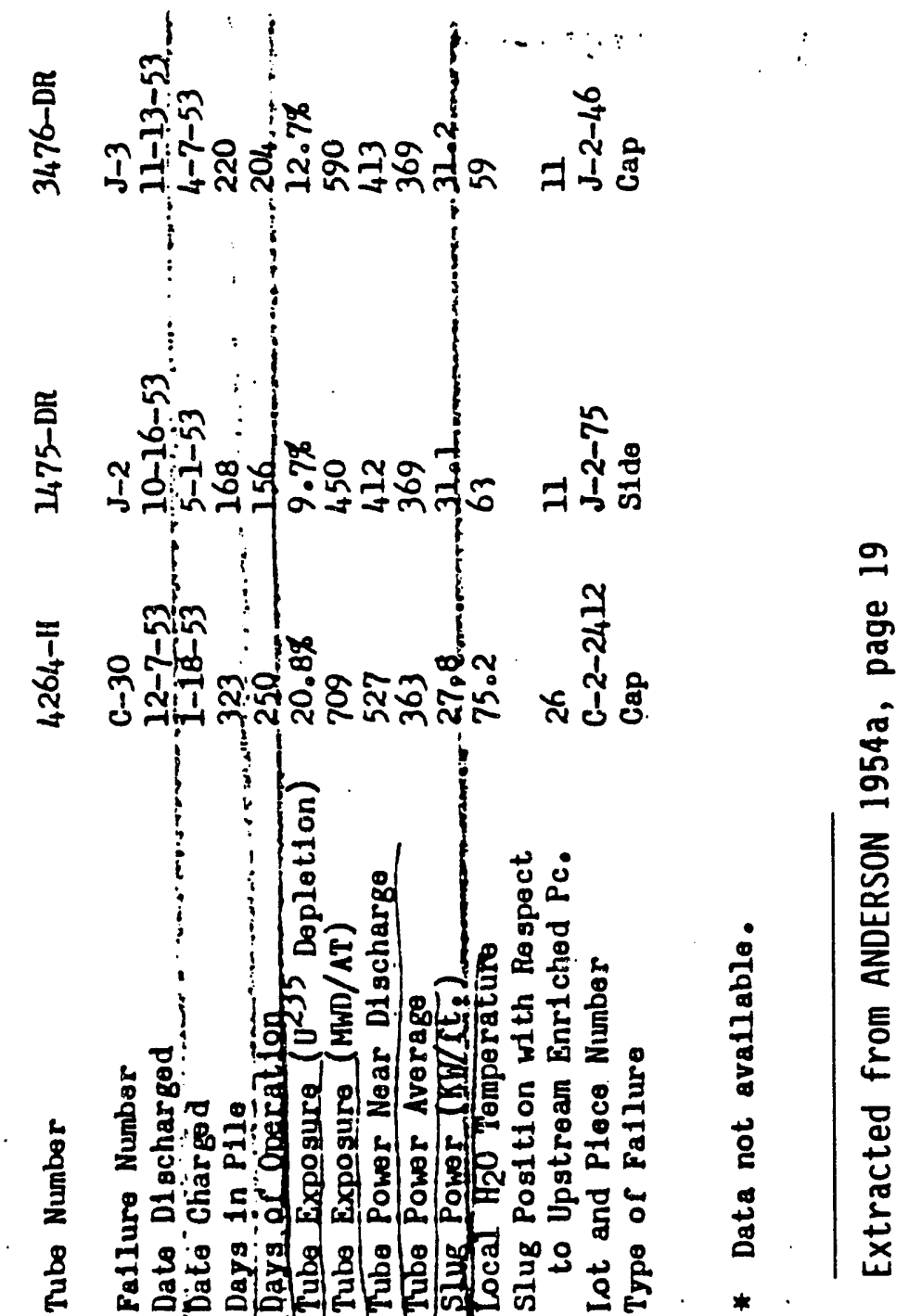


APPENDIX D

FUEL-ELEMENT FAILURE DATA FOR 1954 


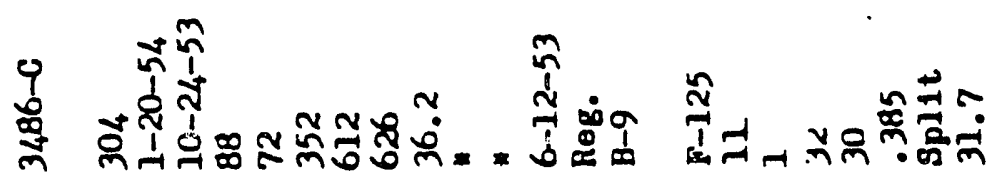

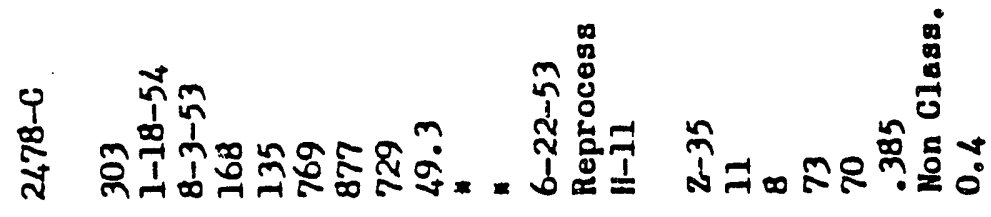

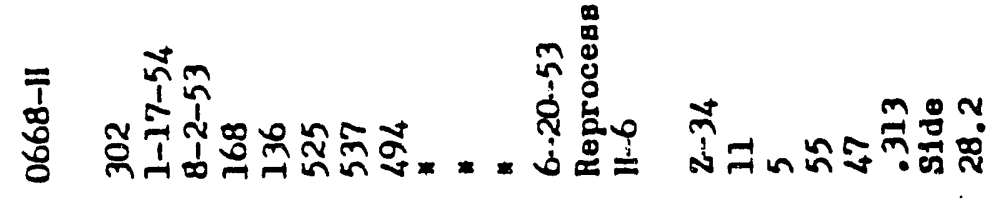

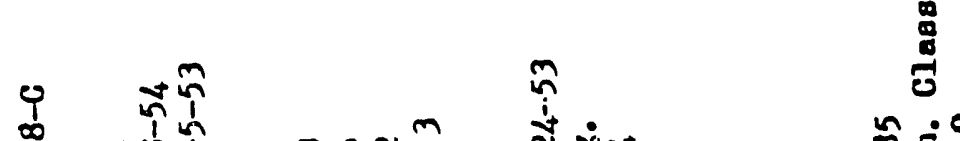

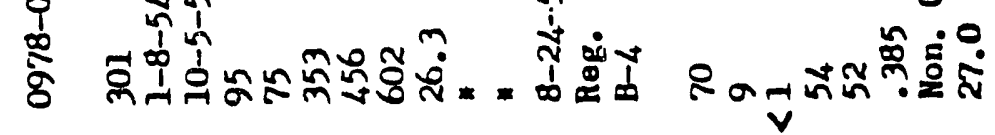

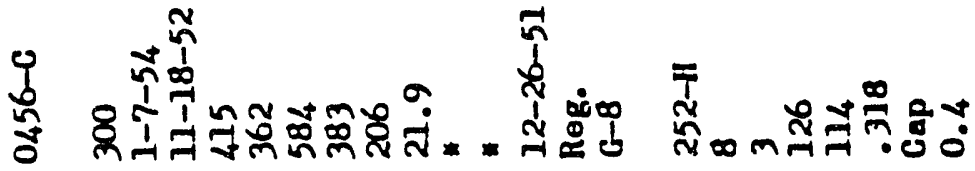

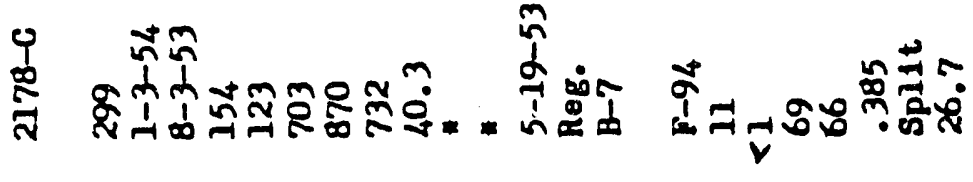

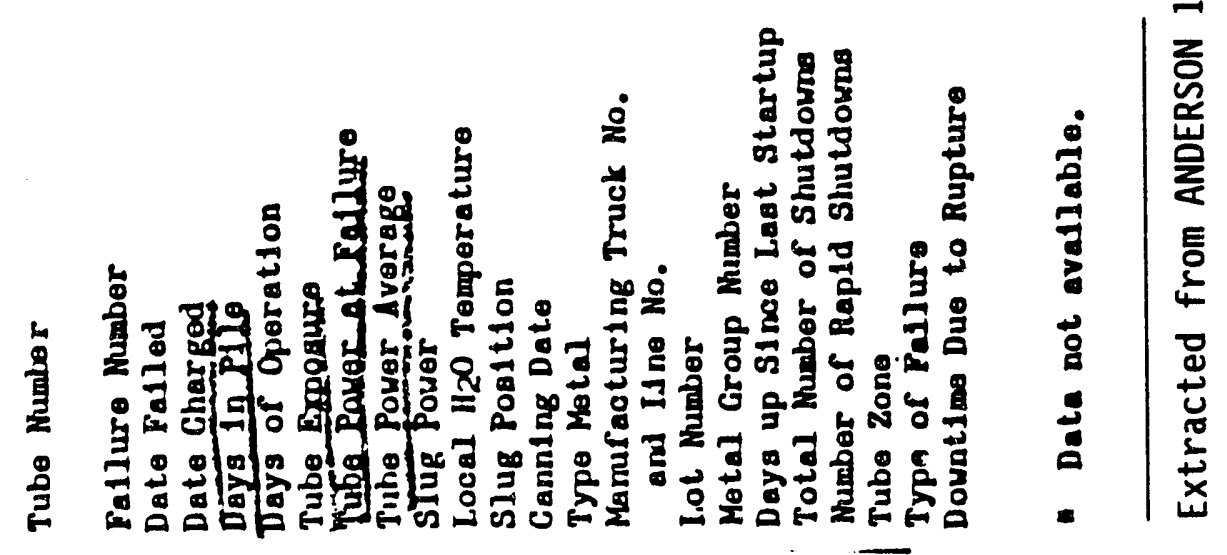




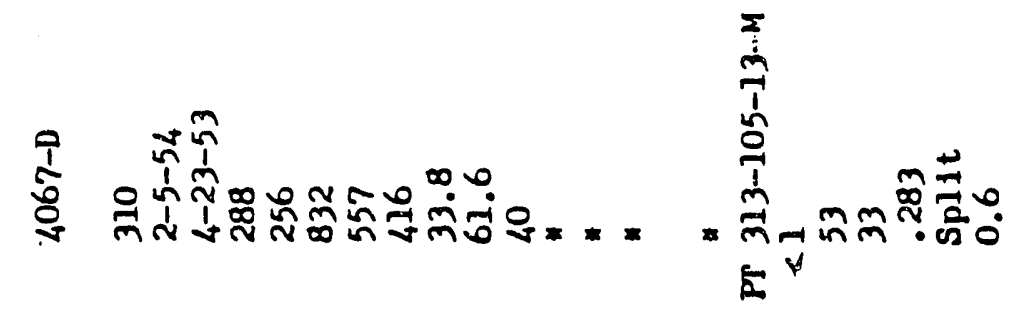

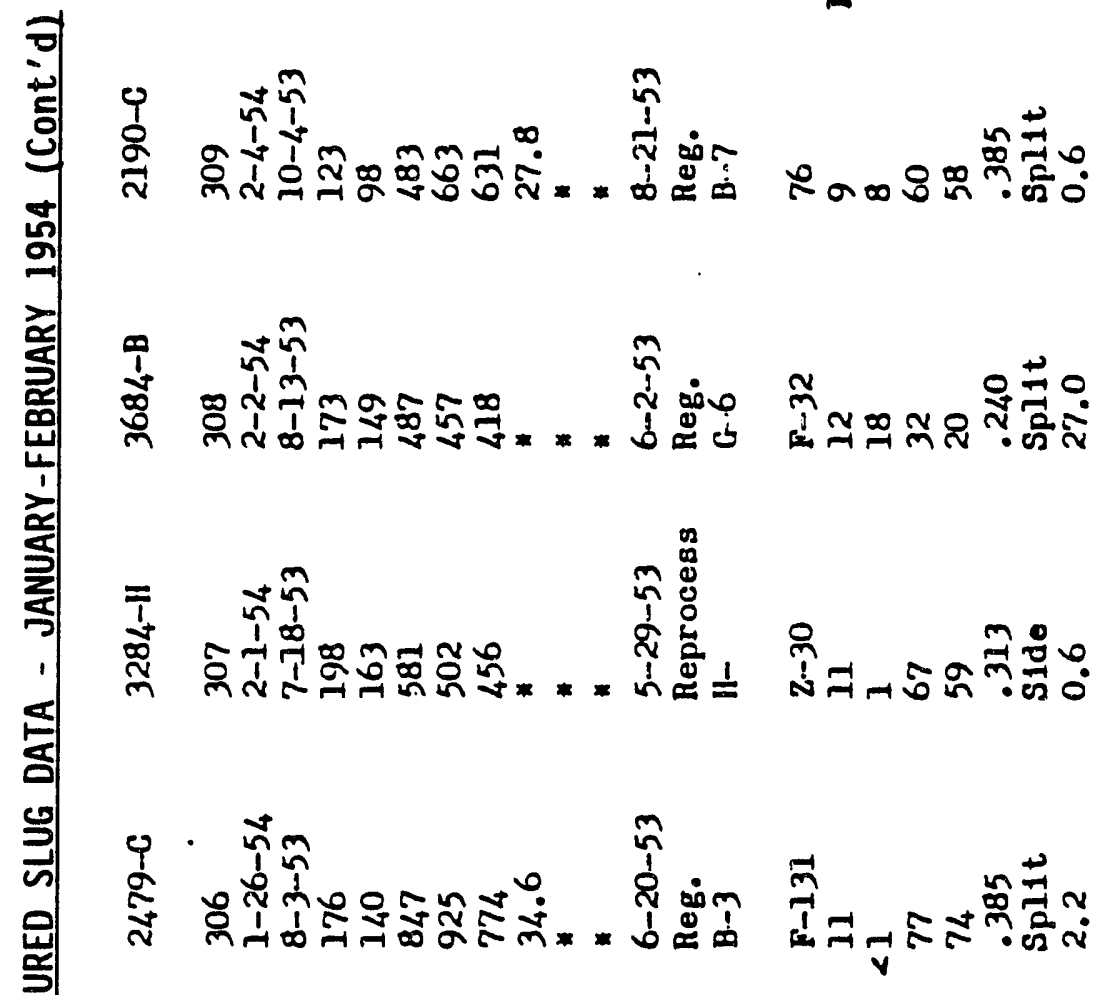

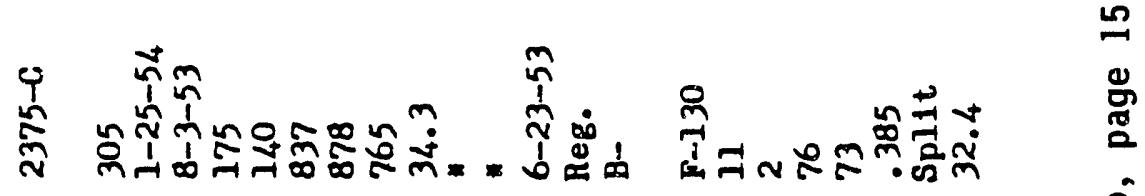

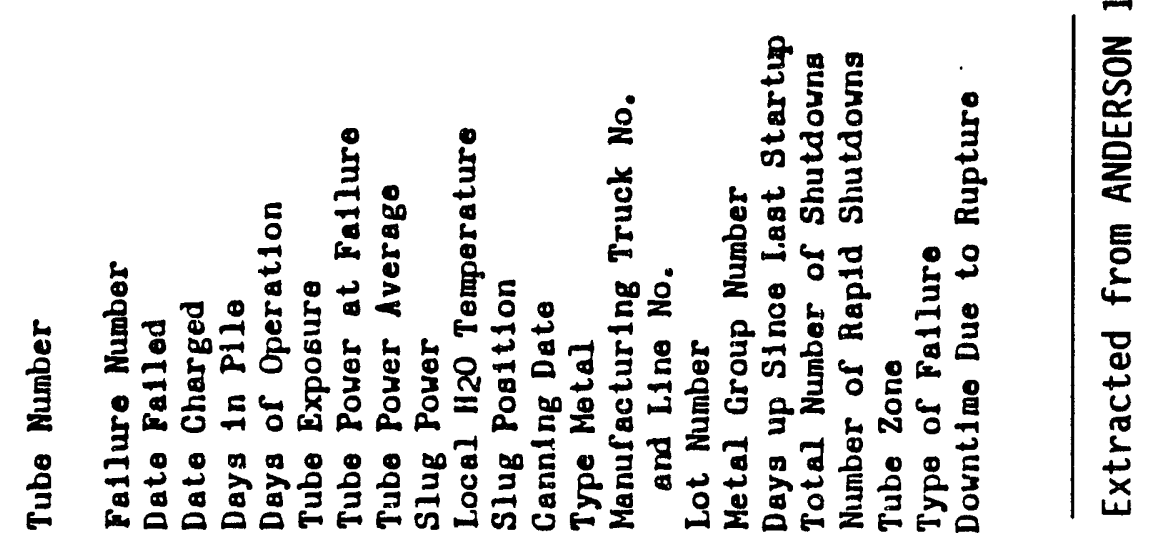

D. 2 


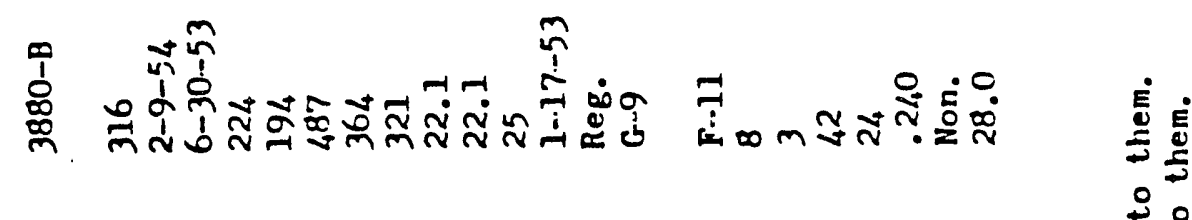

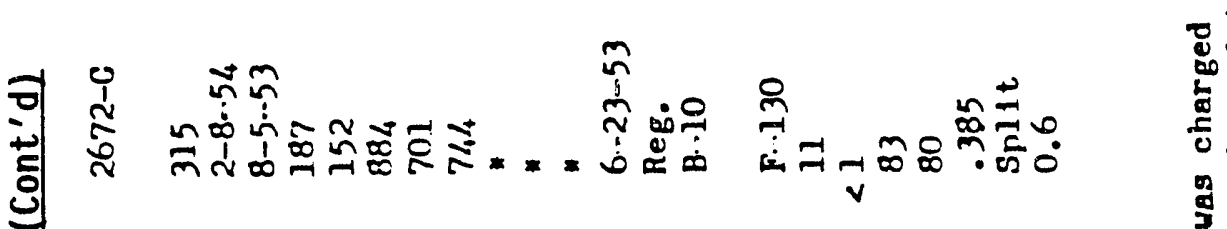

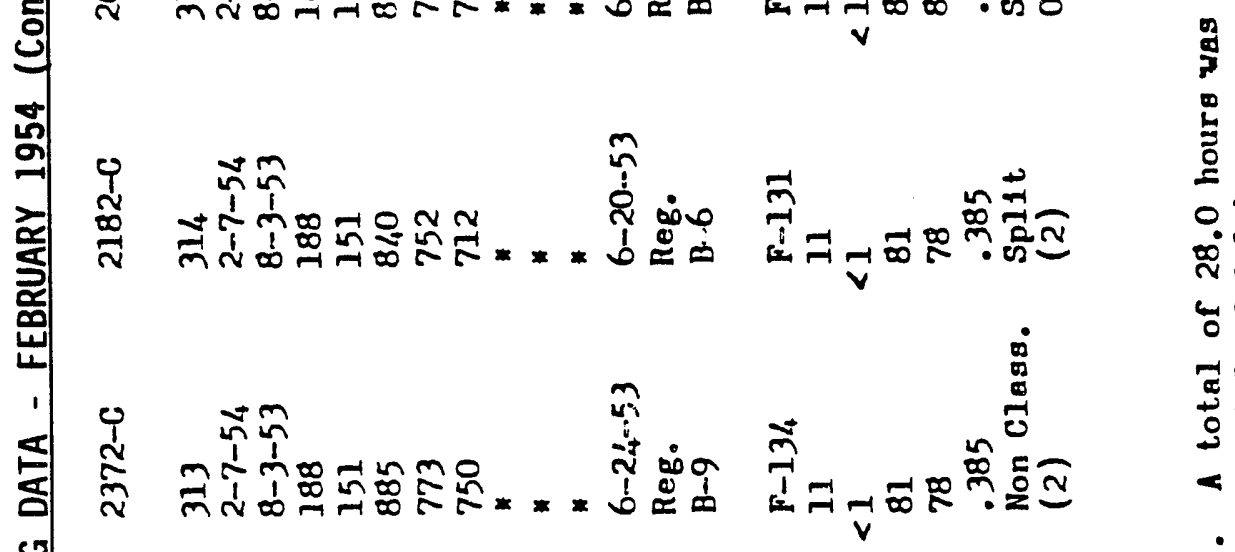

ज्ञ

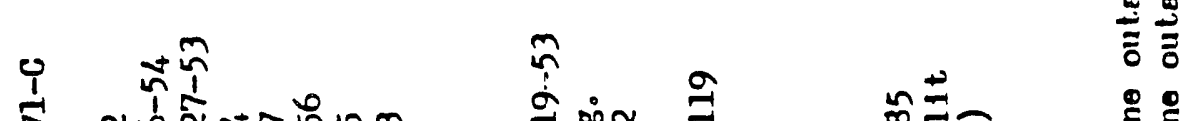

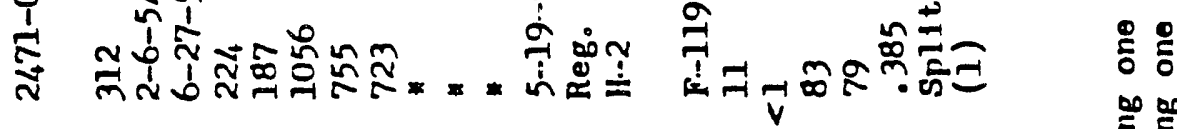
m

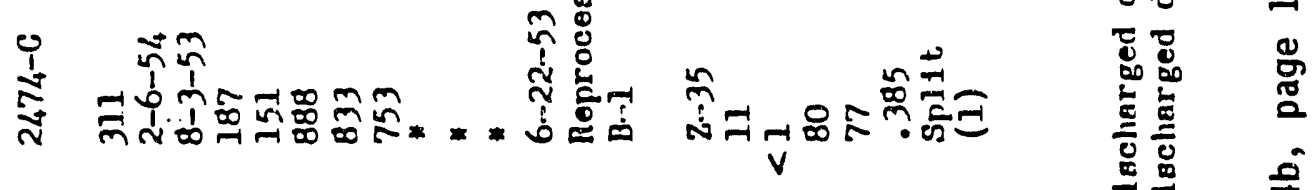

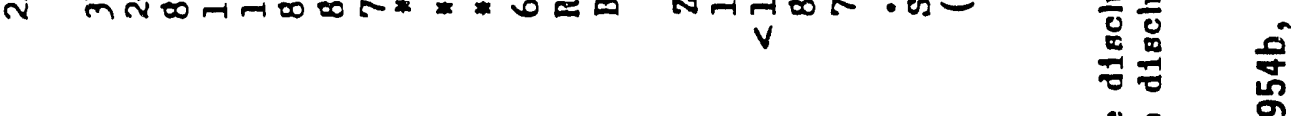

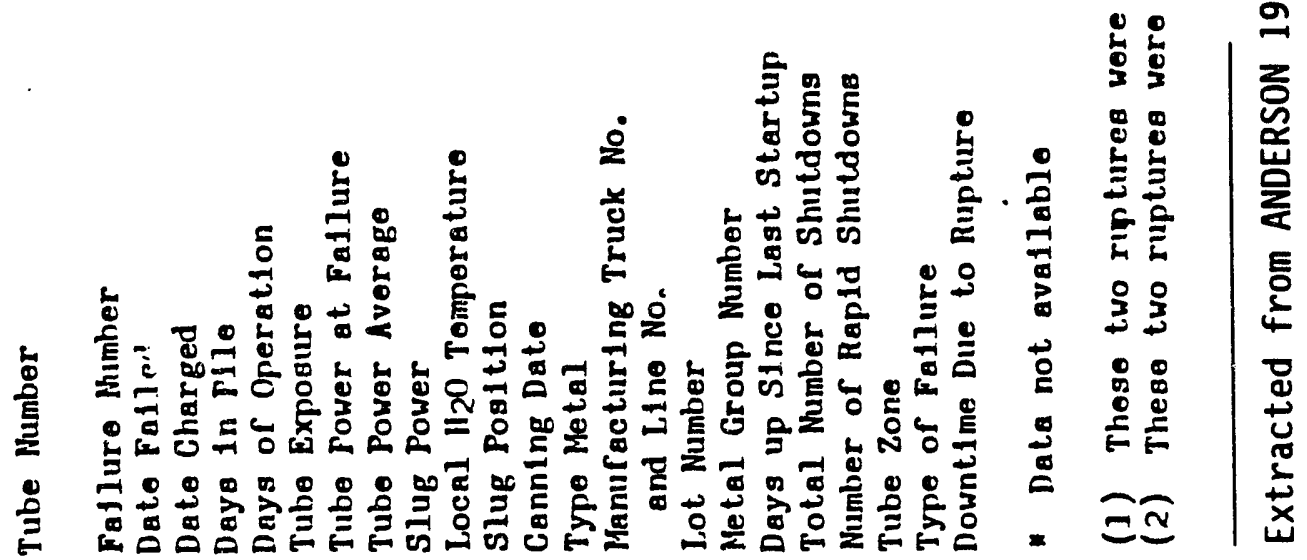


$\Xi$

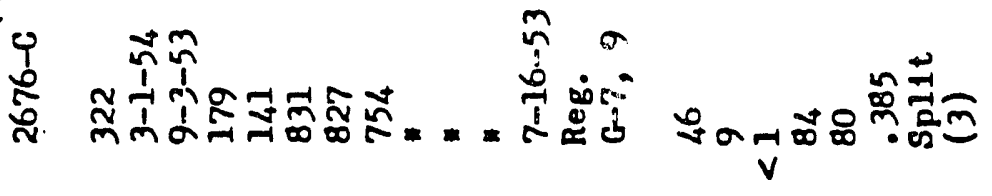

莡

$\exists$

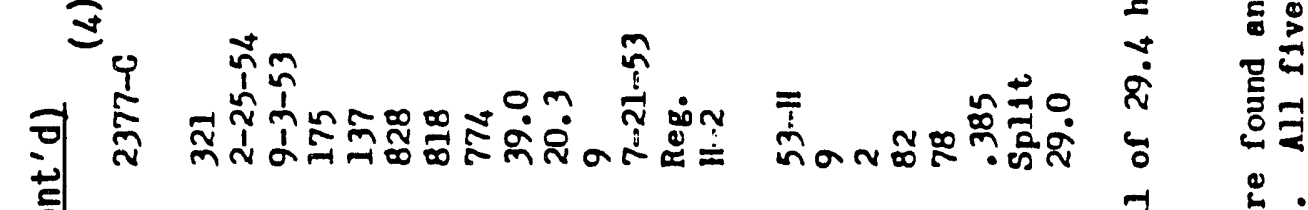

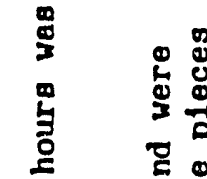

马्ष

\&े

휭

ถั้

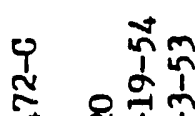

n.

영

离

ํํำ

运

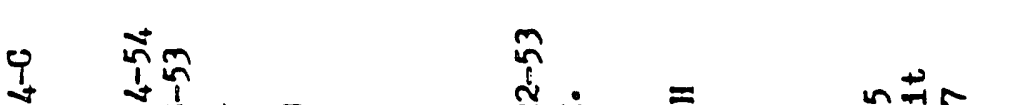

$\stackrel{0}{5}$

के

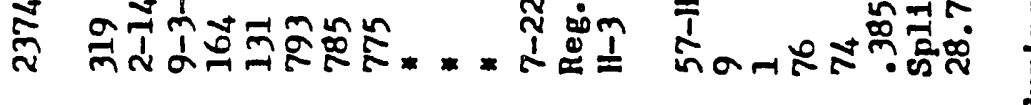

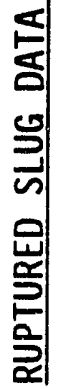

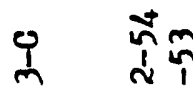

$\hat{n}$

ส

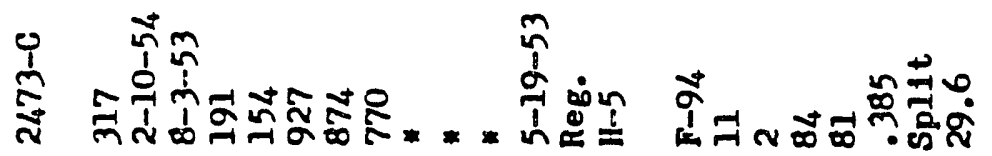

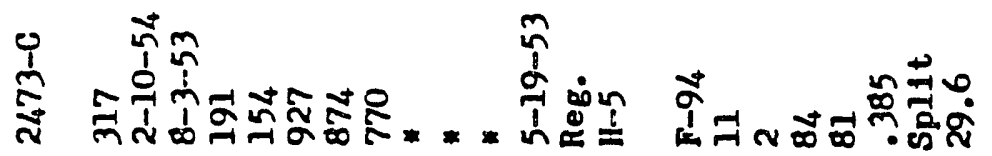

站

$\sum^{\infty}$

要

E

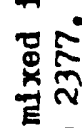

$\stackrel{5}{5}$

릉

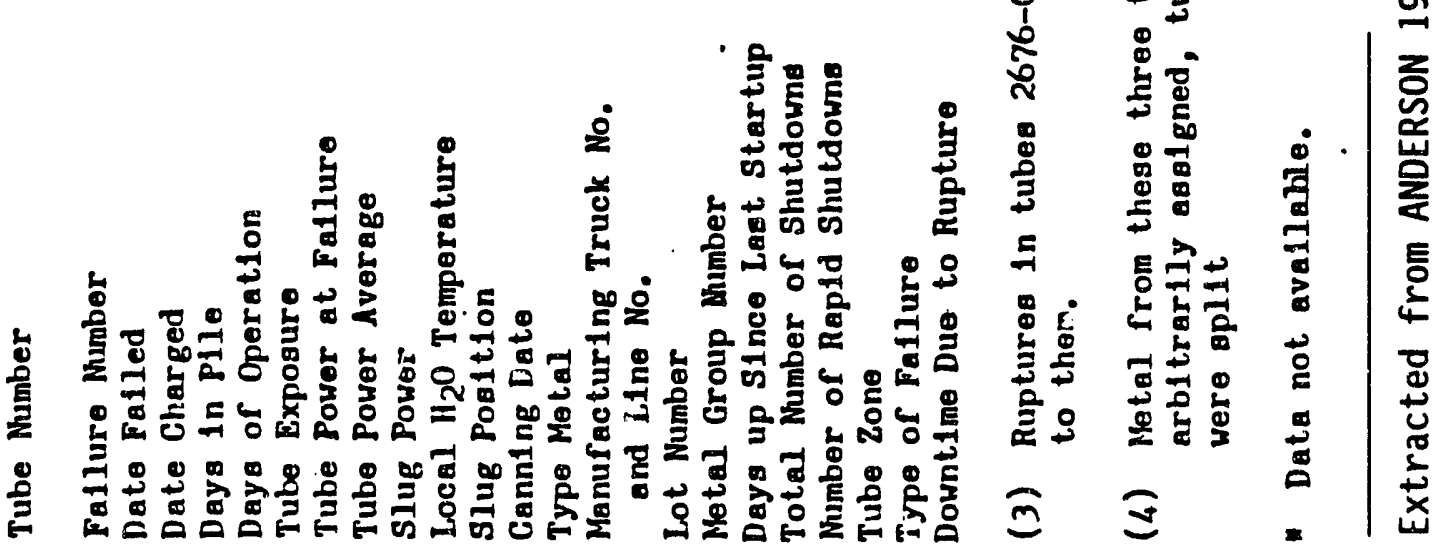




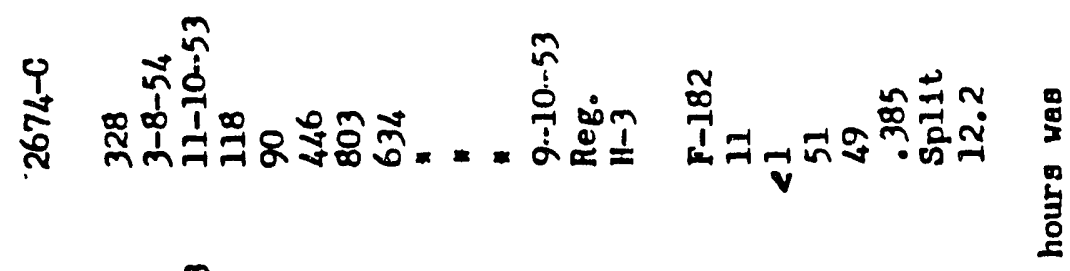

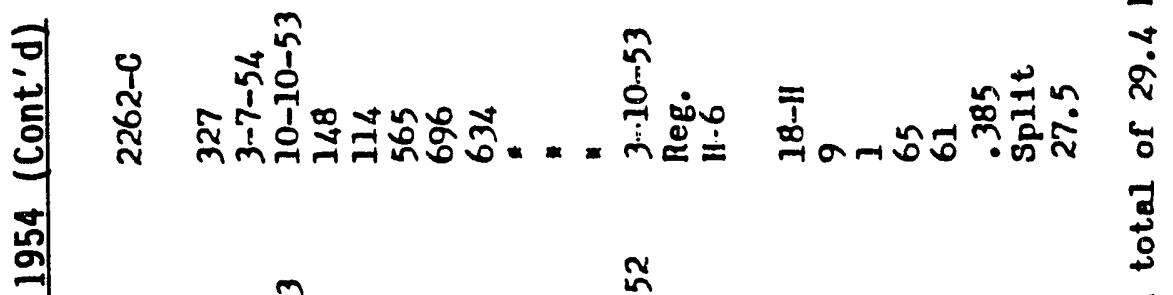

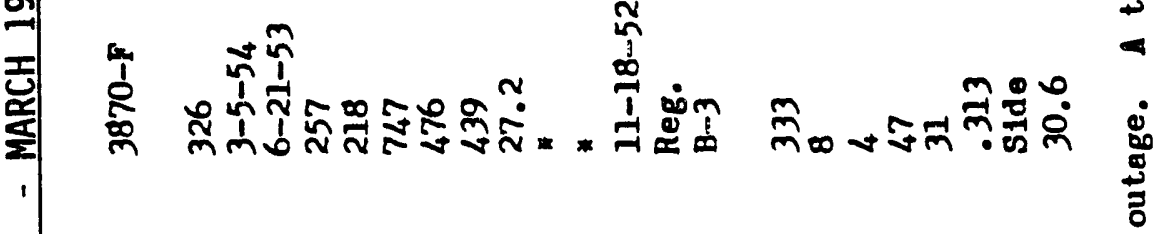

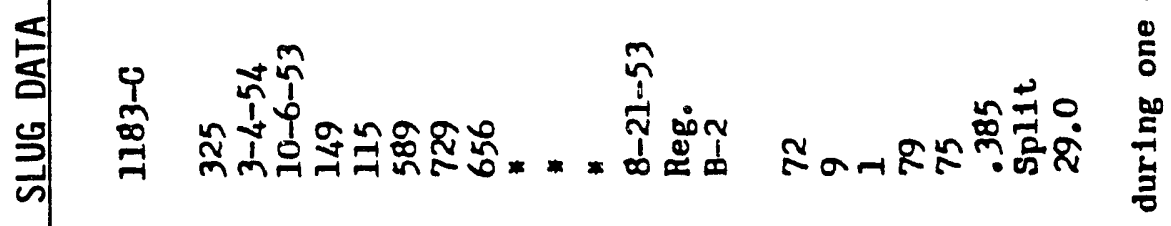

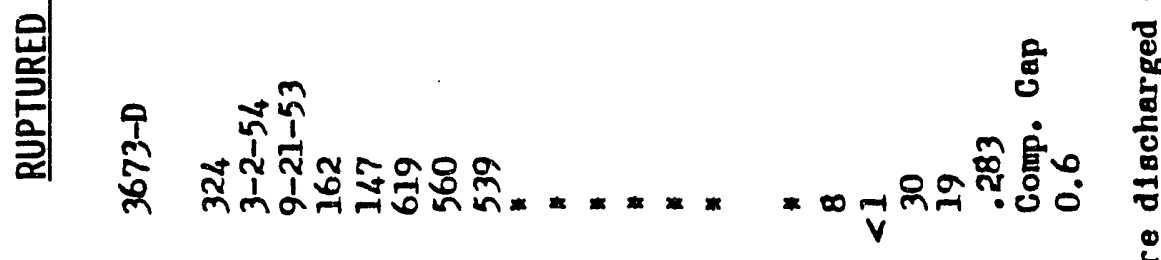

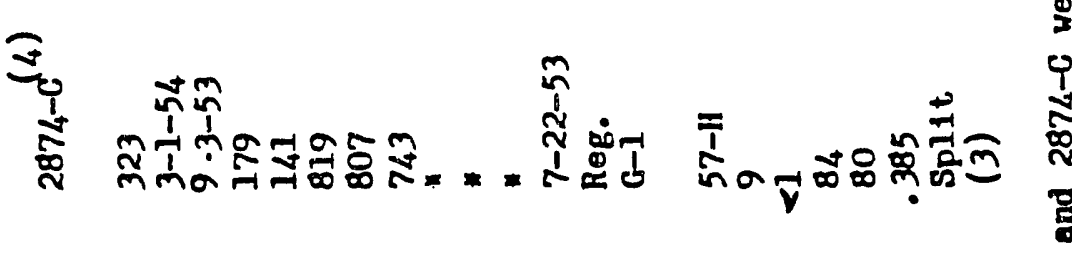

$\stackrel{\infty}{\sim}$

藏

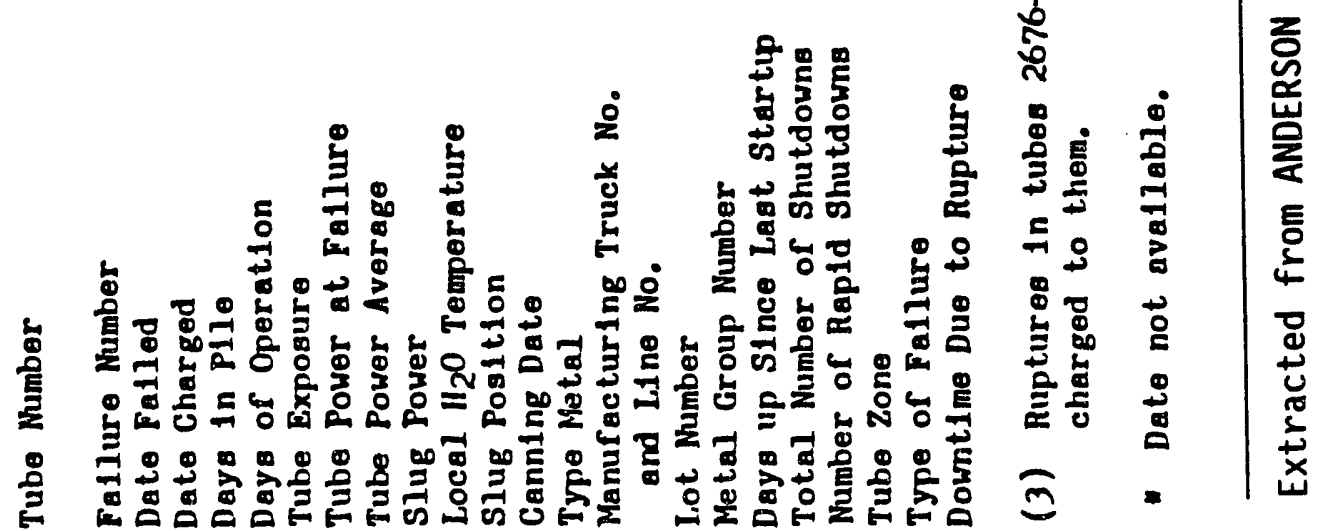




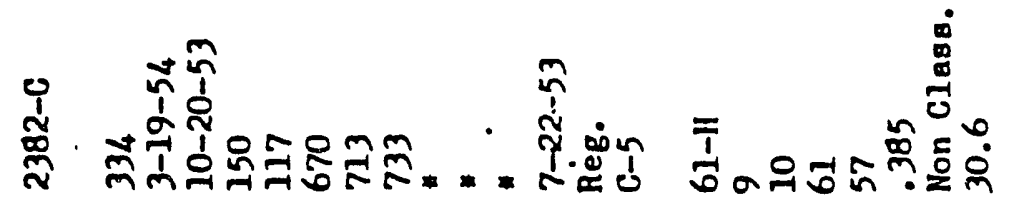

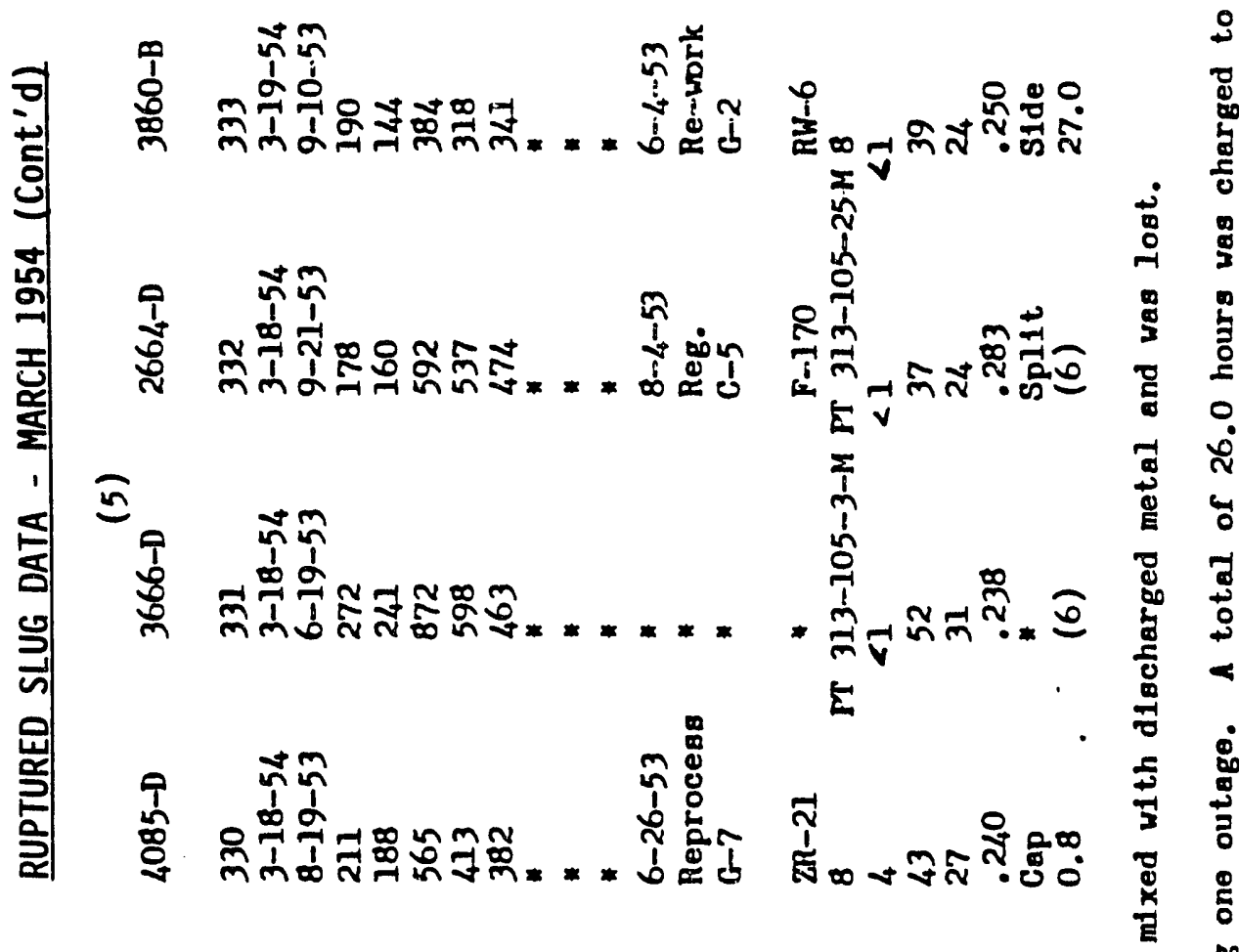

索

这

望

这

g்

$\stackrel{\square}{\circ}$ 


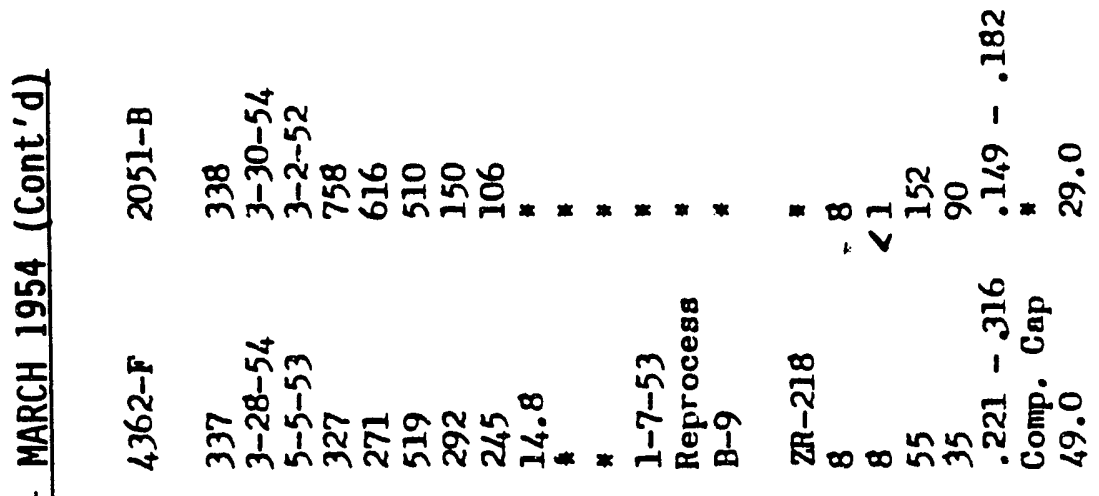

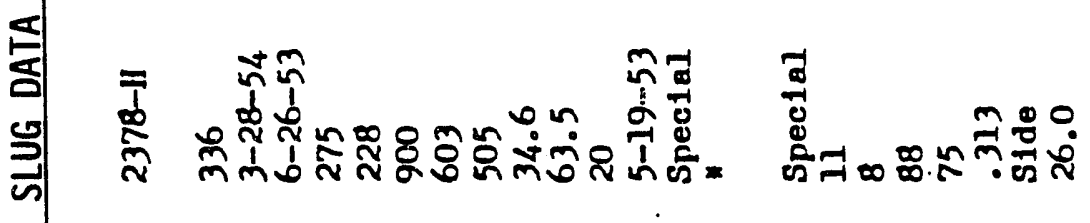

蒙 $a$ o

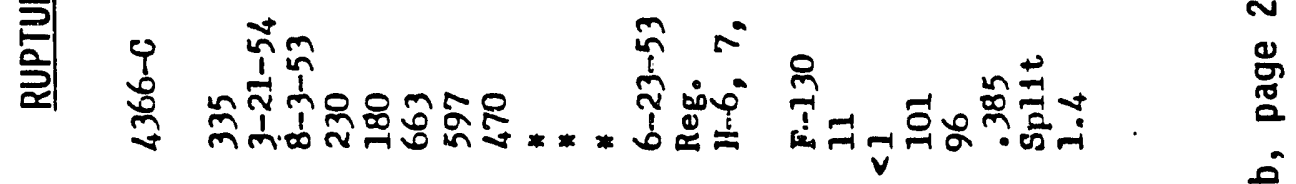

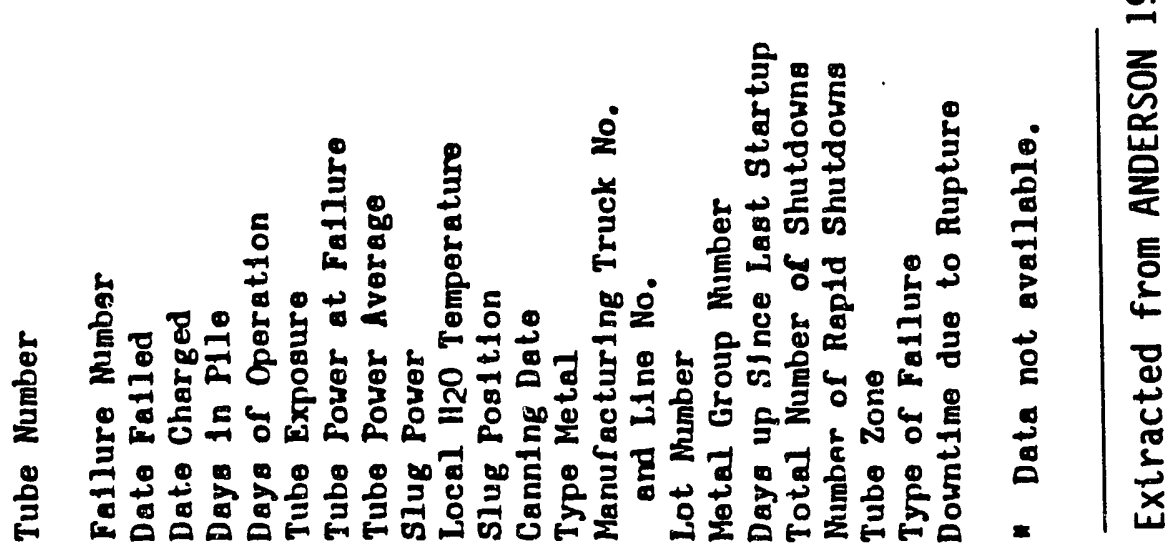

D.7 


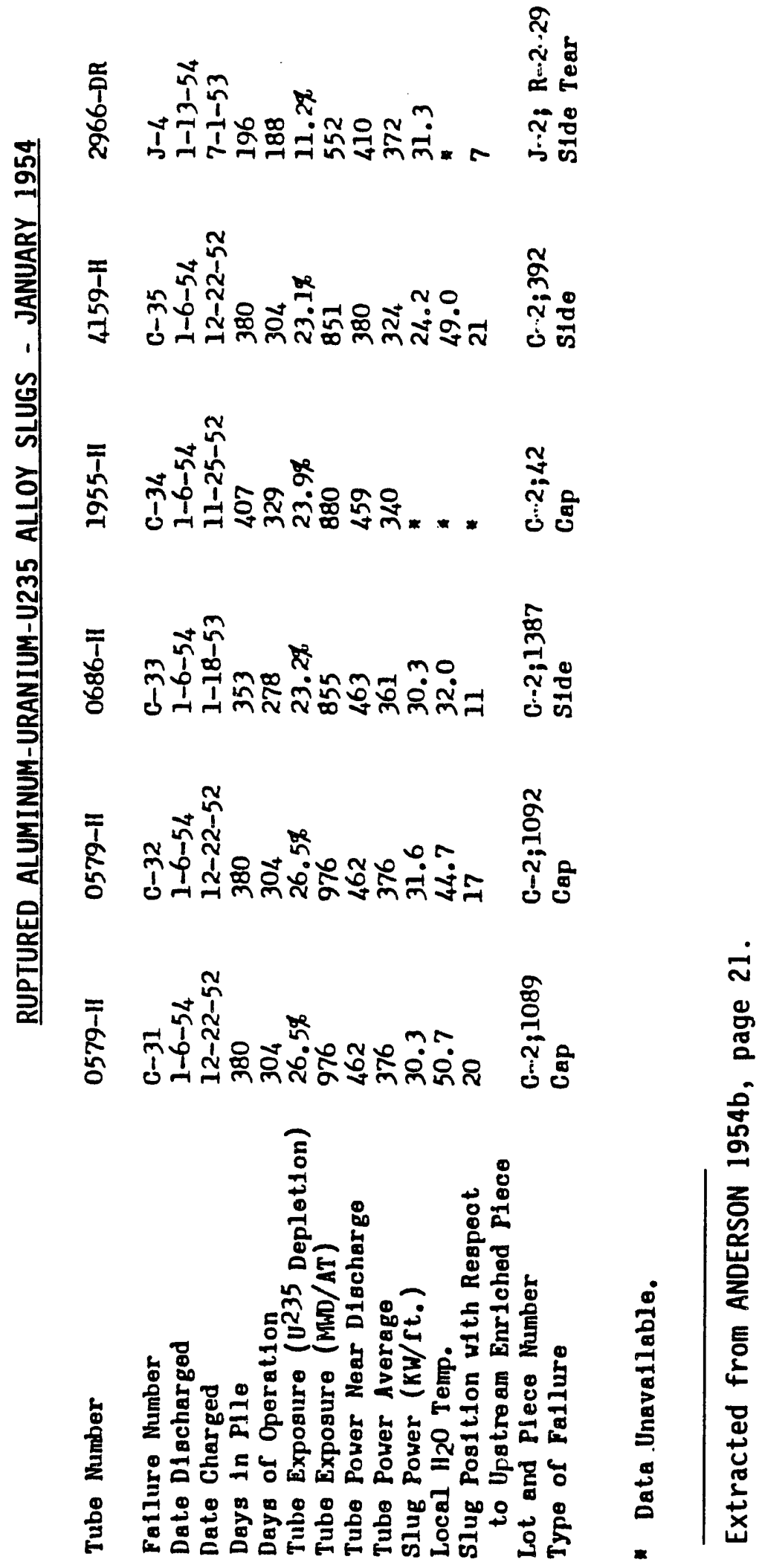

D. 8 


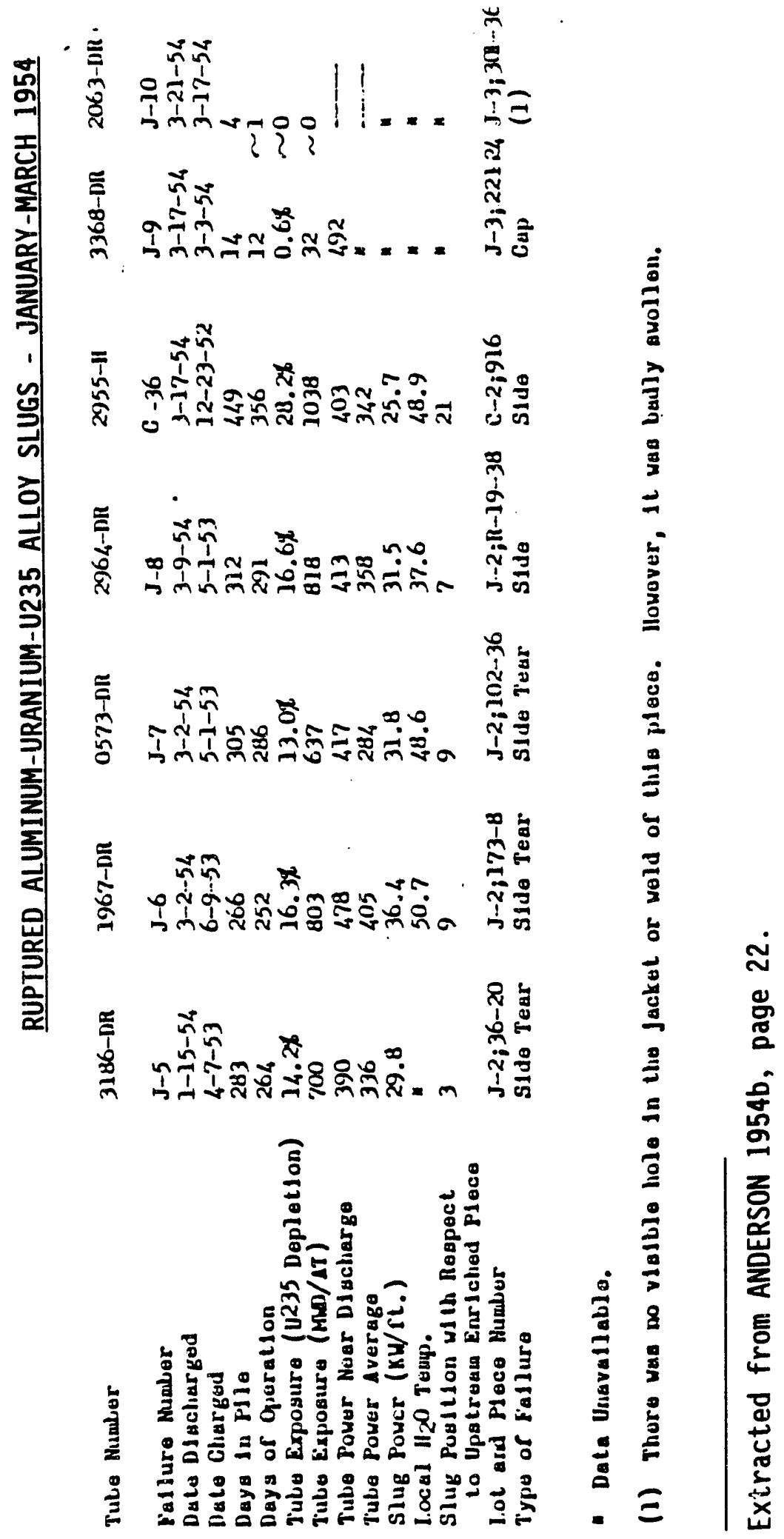

D. 9 


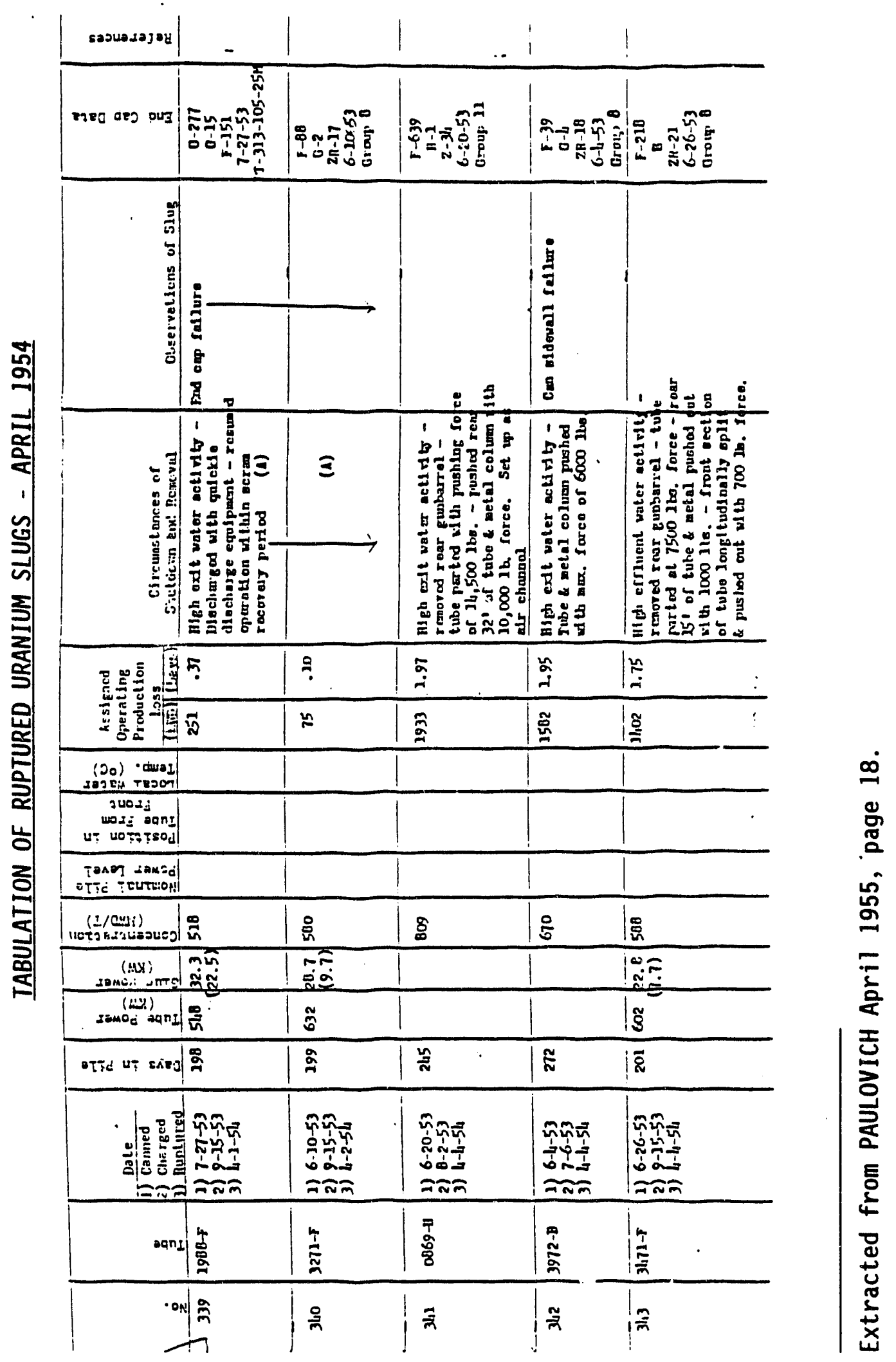

D. 10 


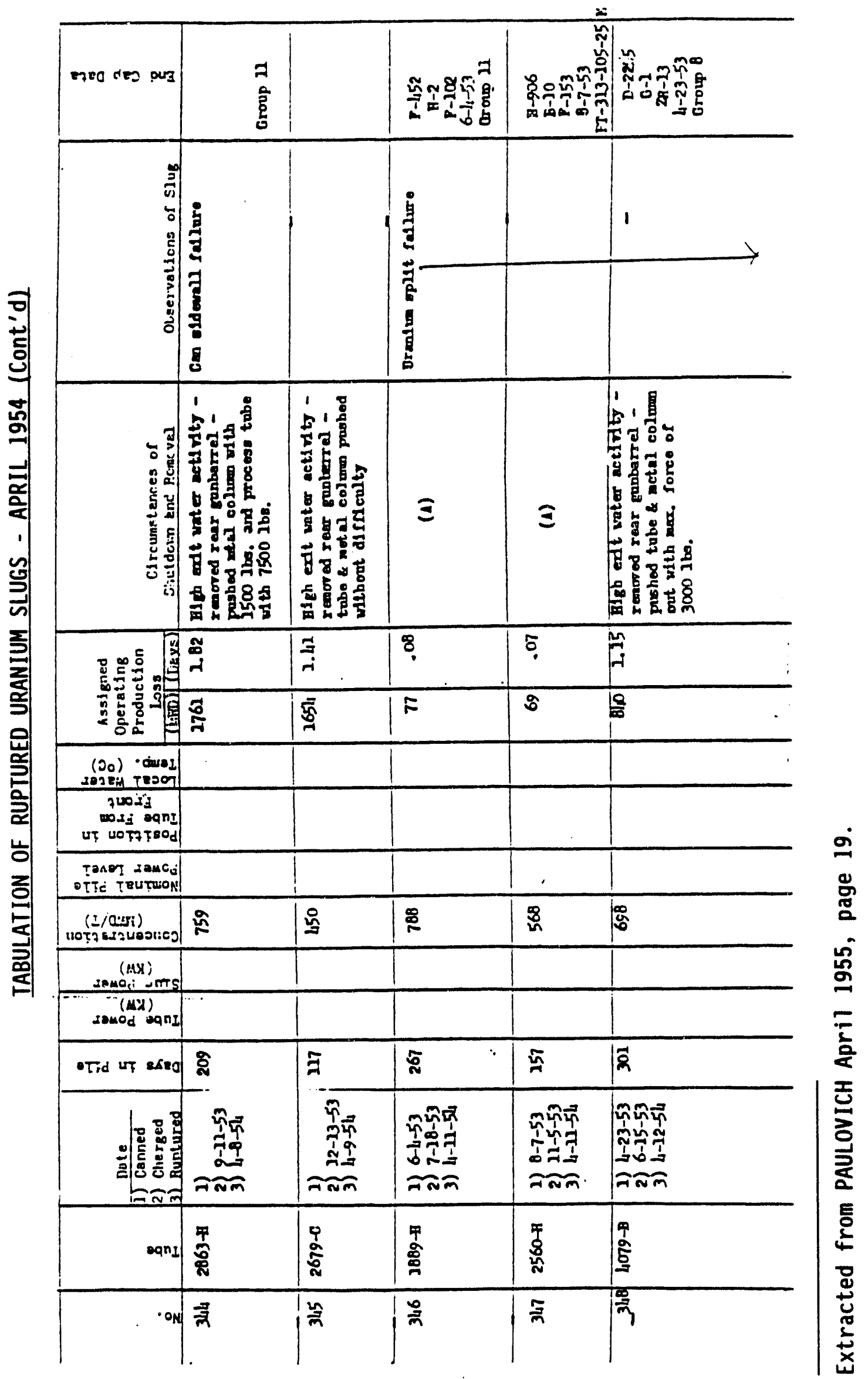

D.11 


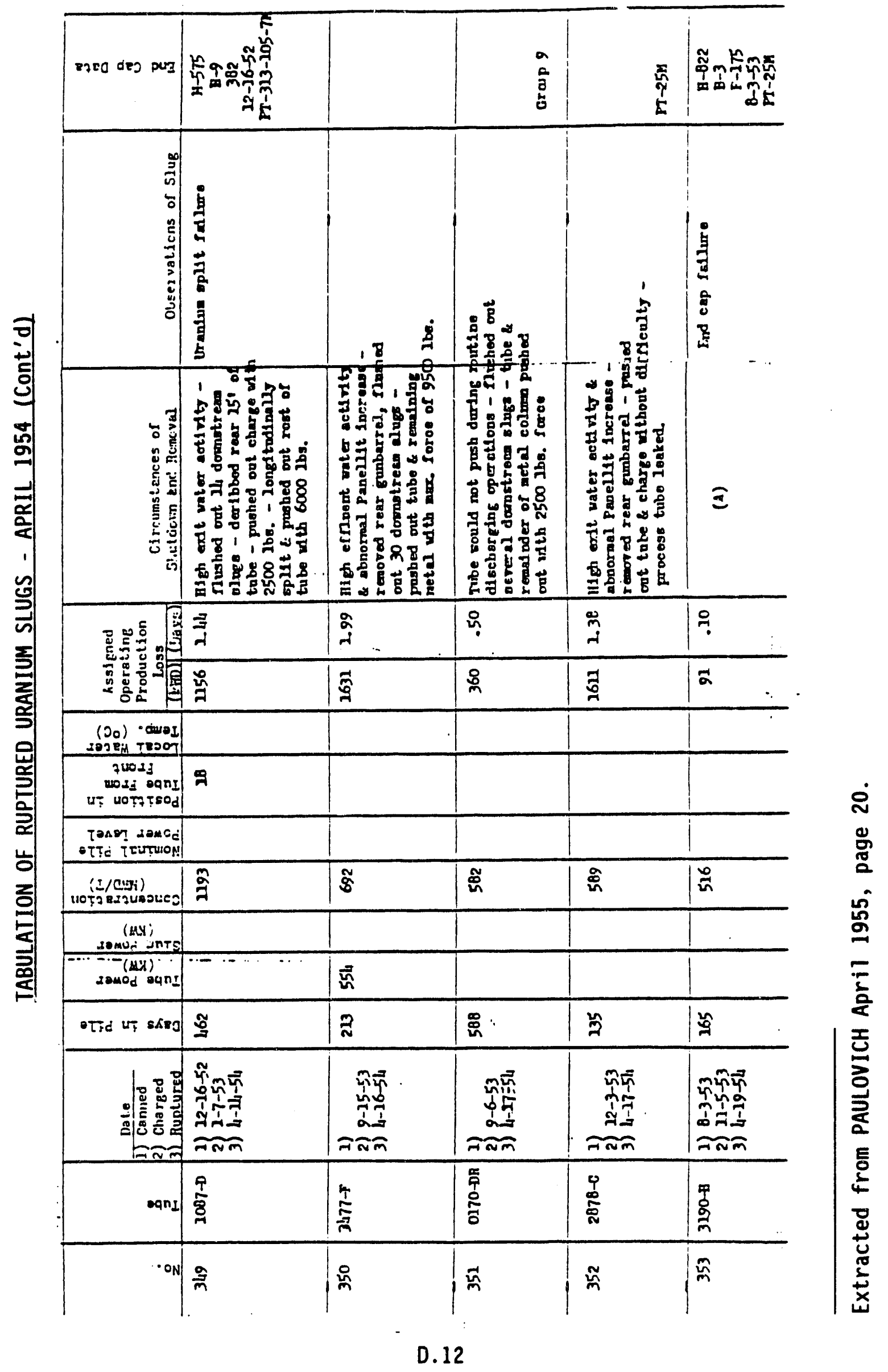




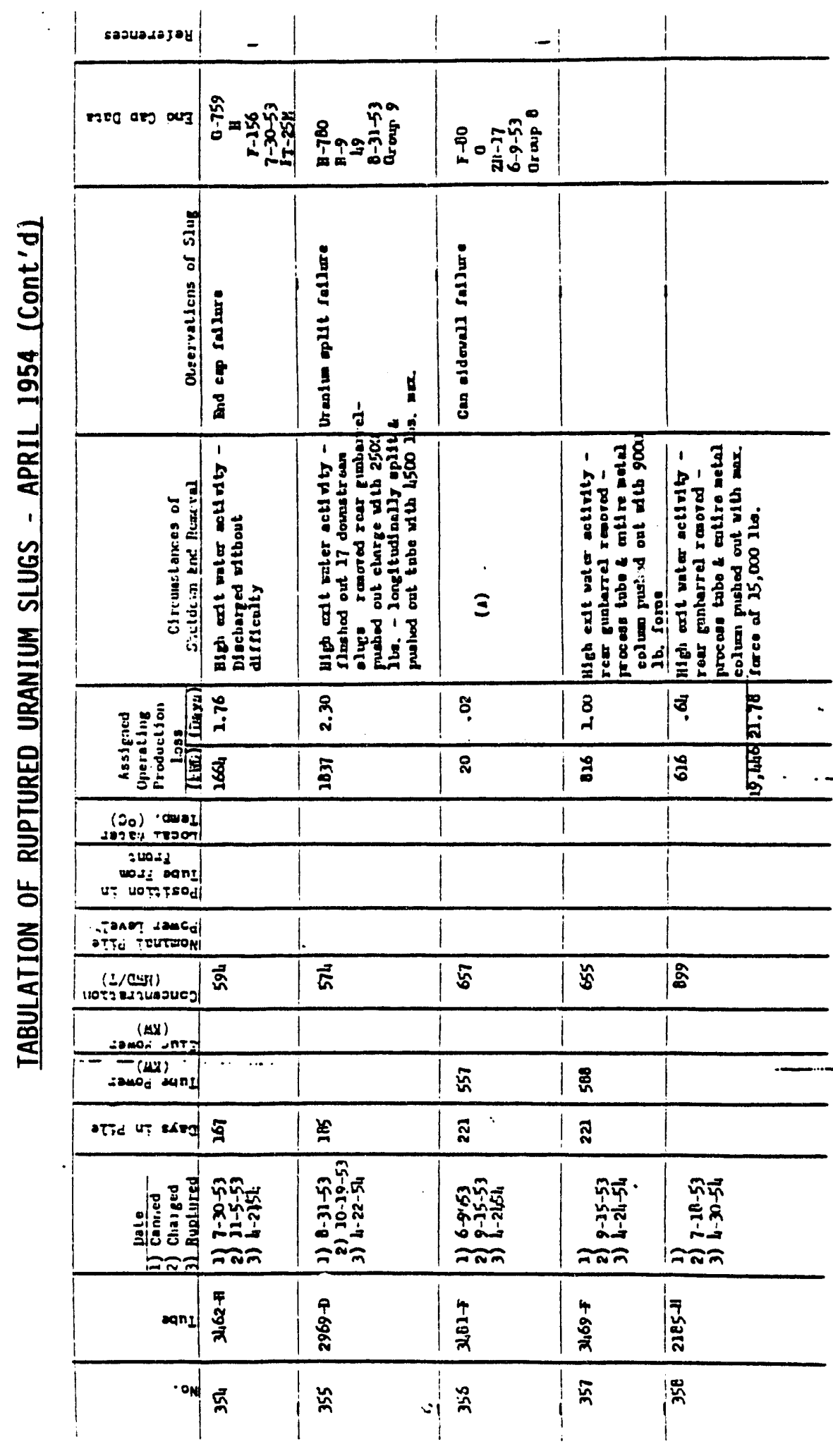

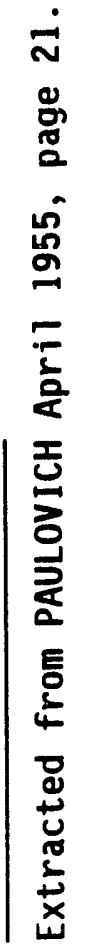

D. 13 


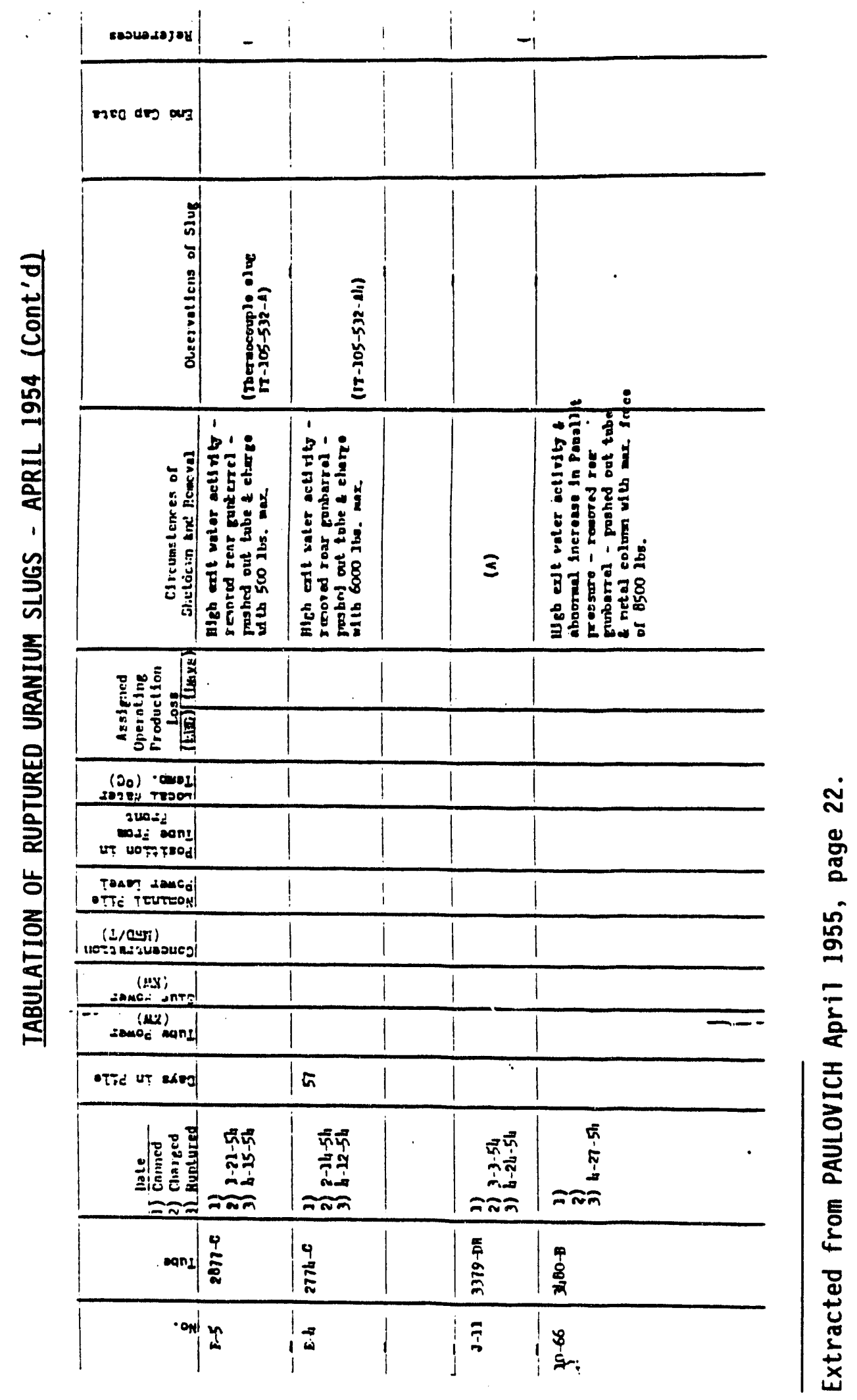




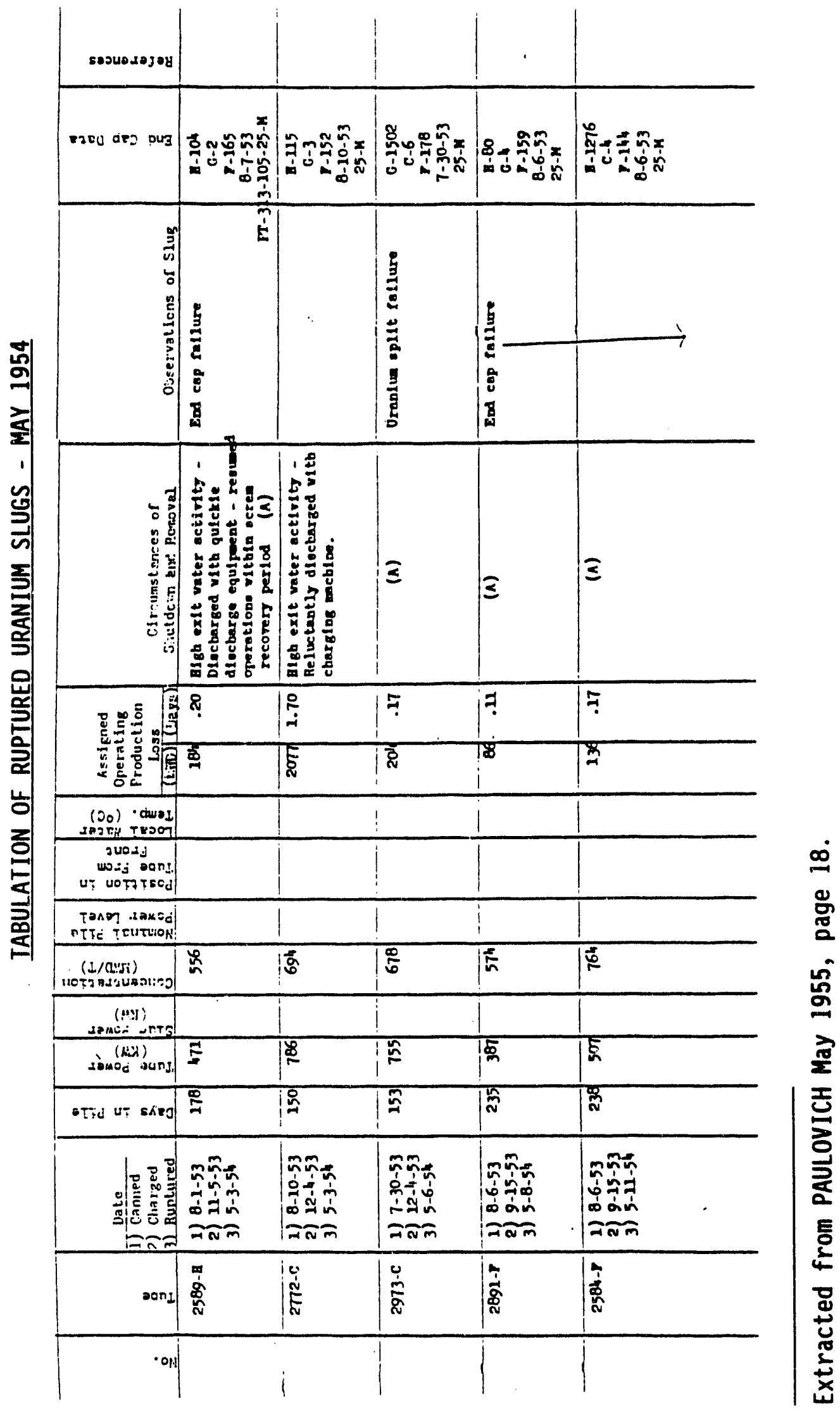

D. 15 


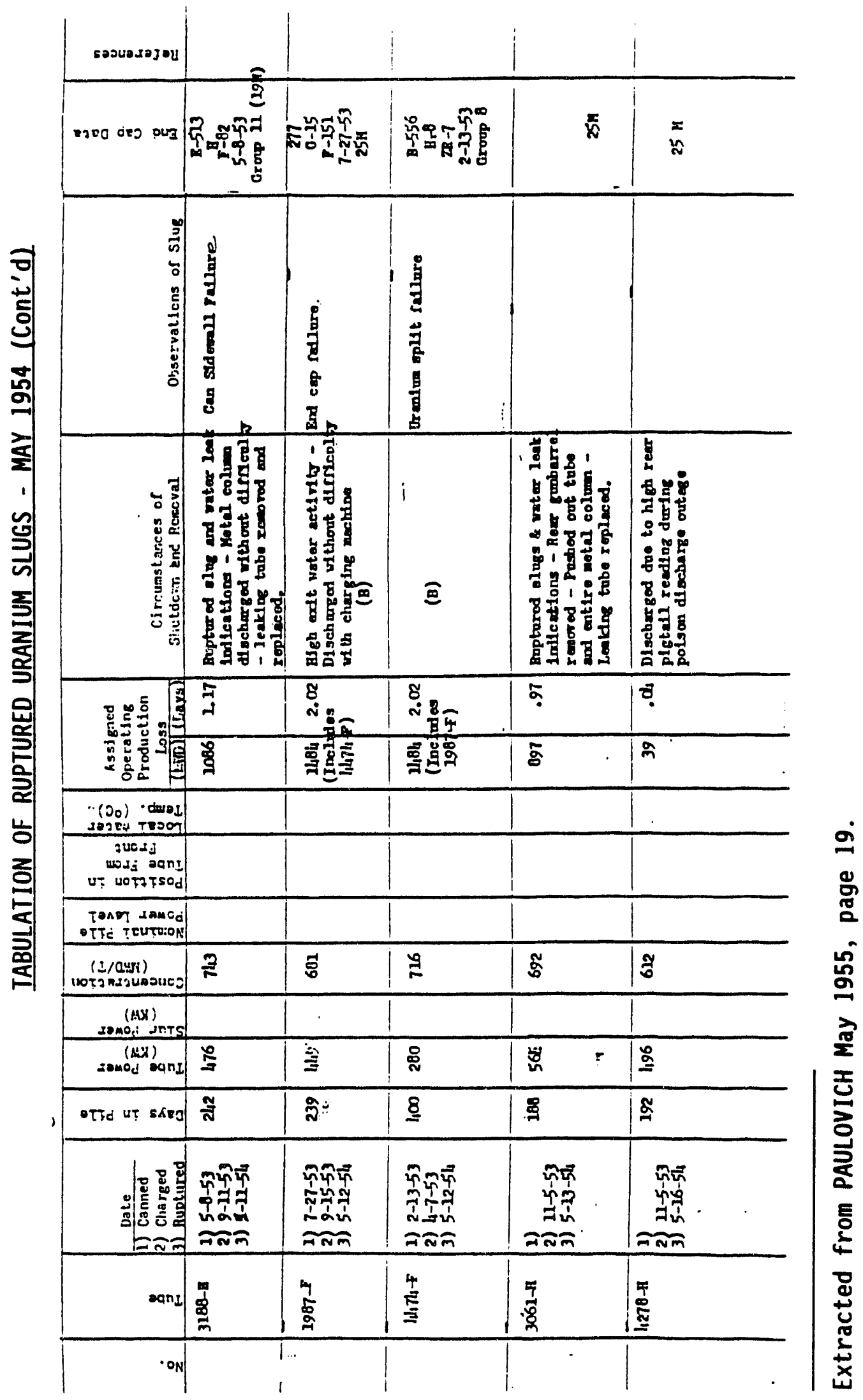

D. 16 


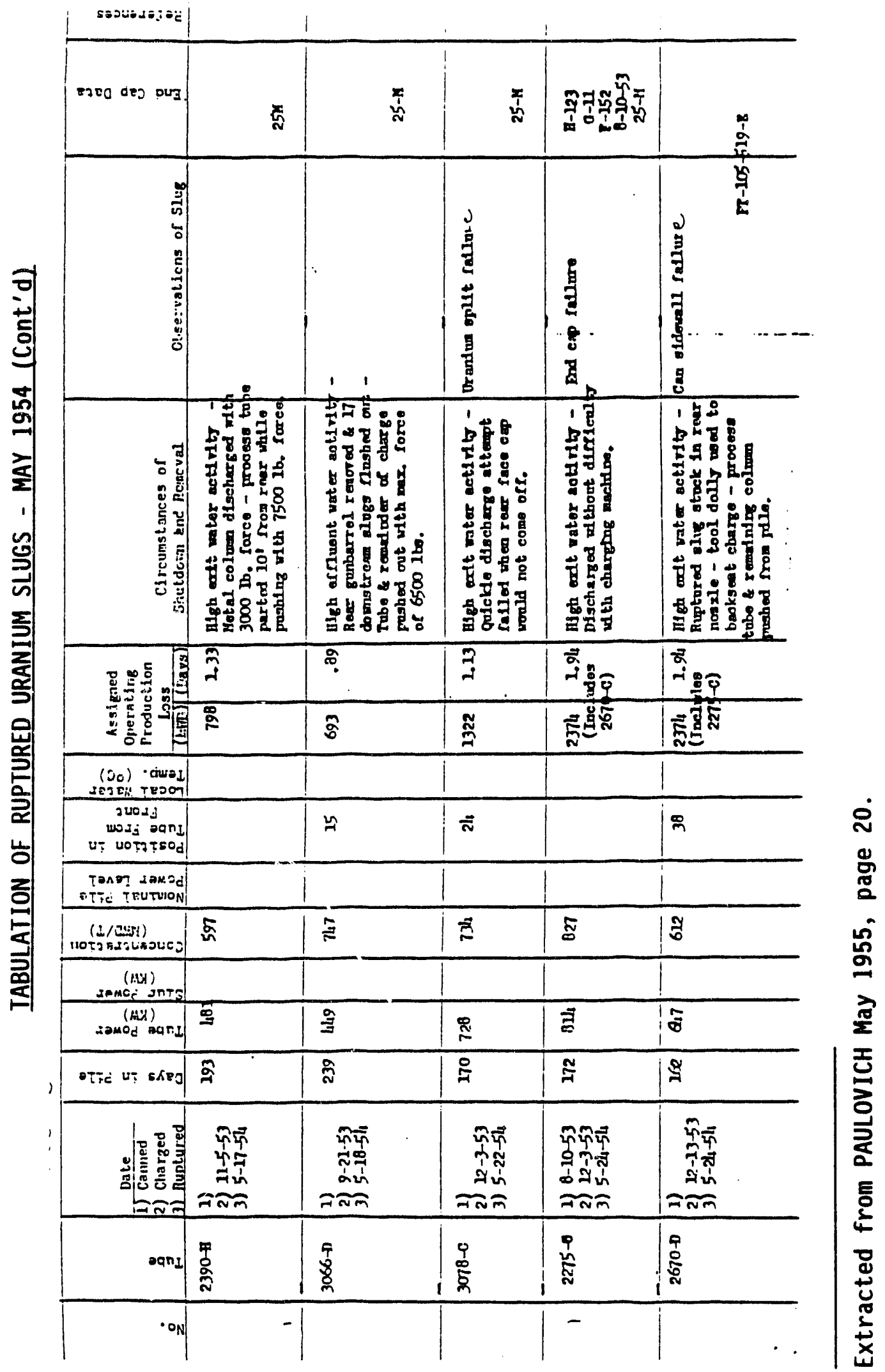

D. 17 


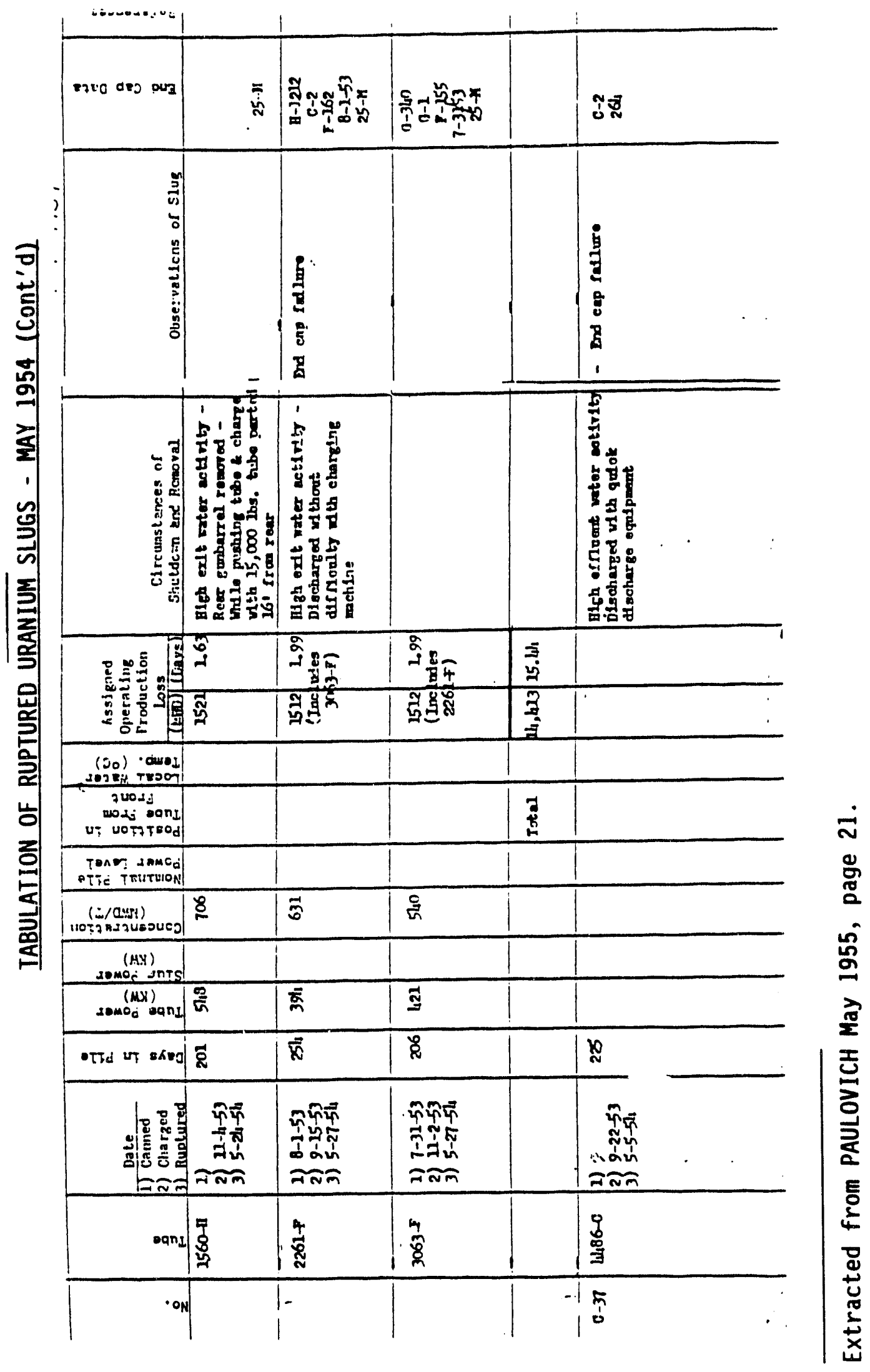

D. 18 


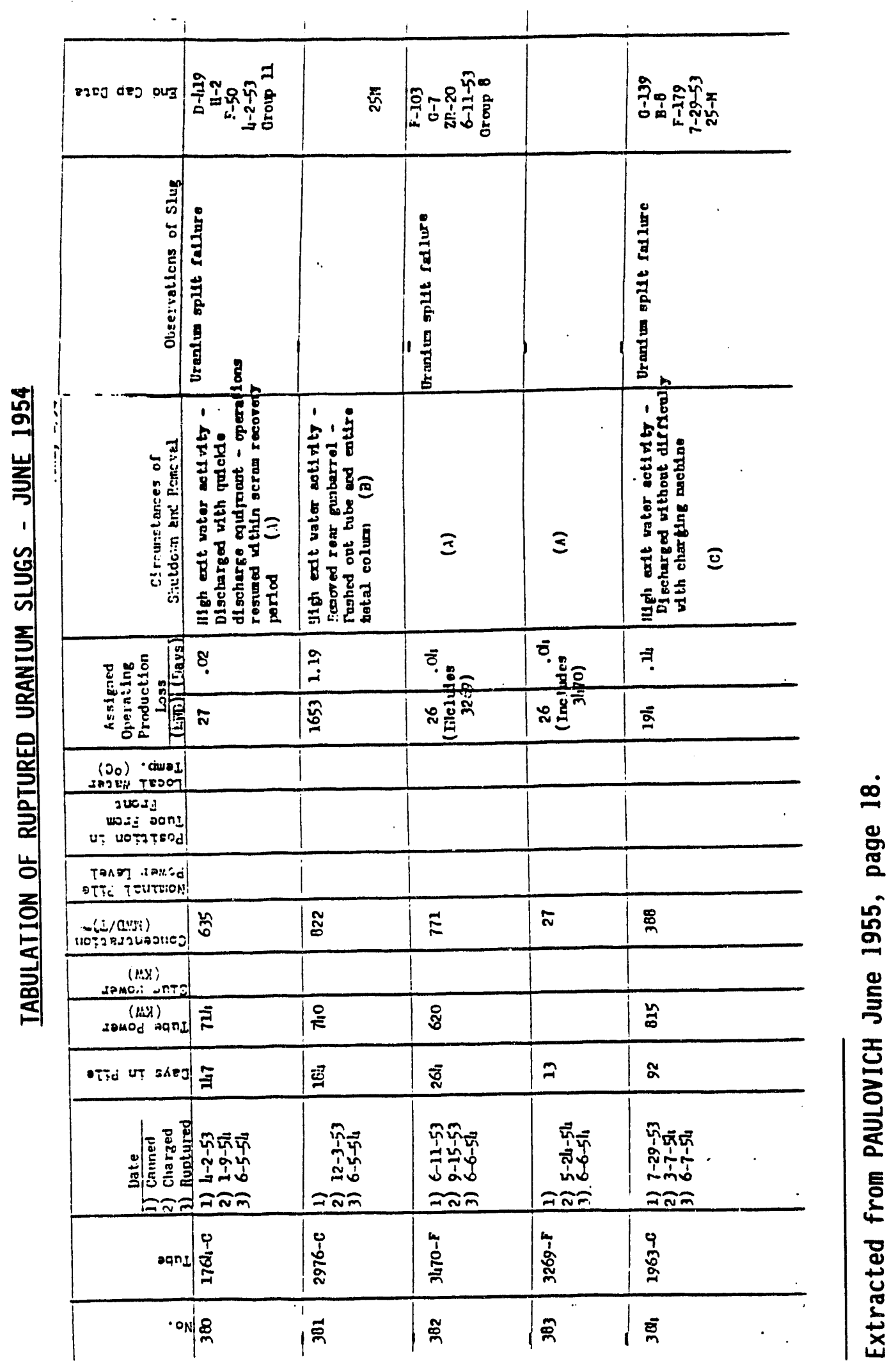




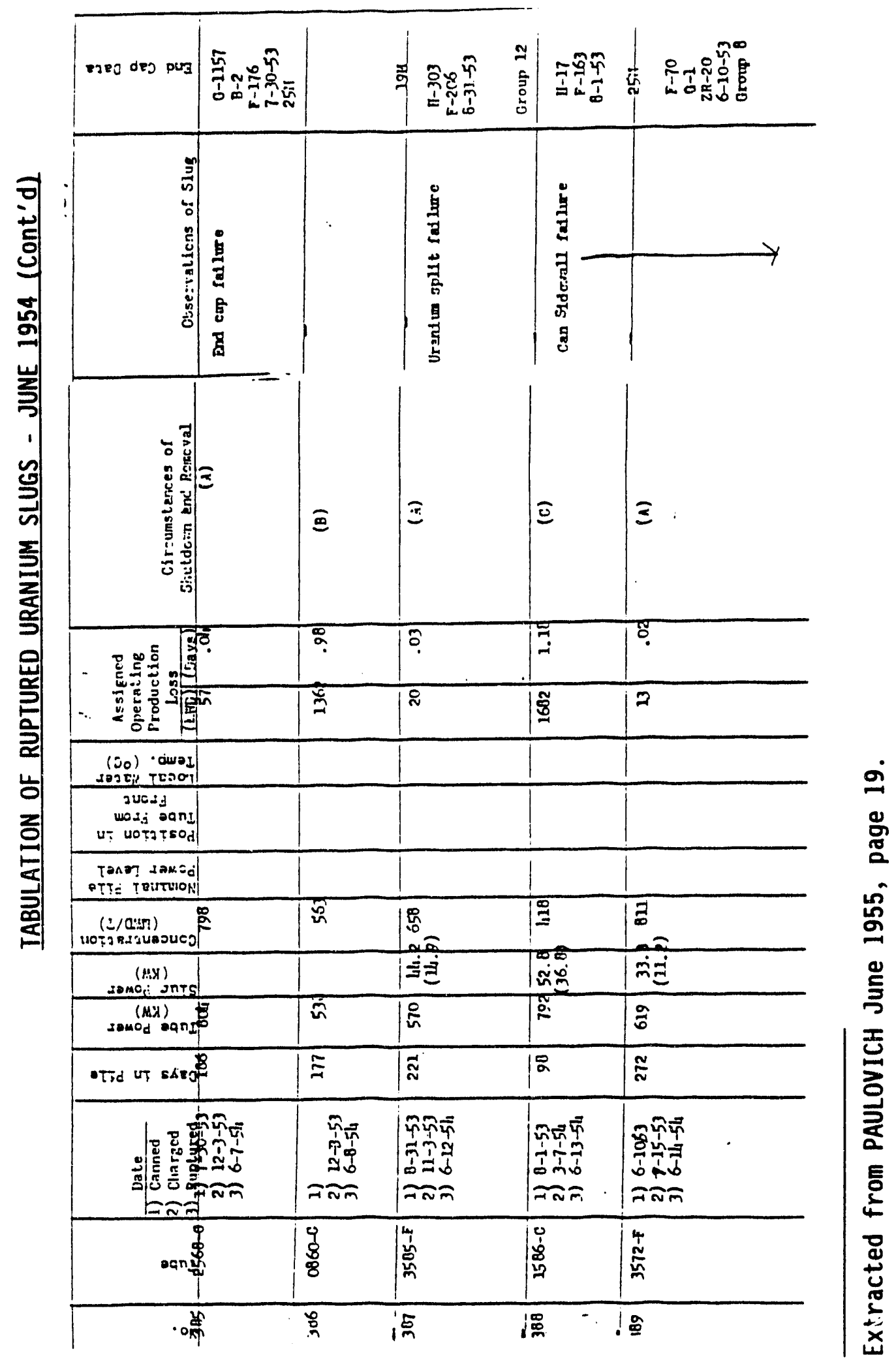




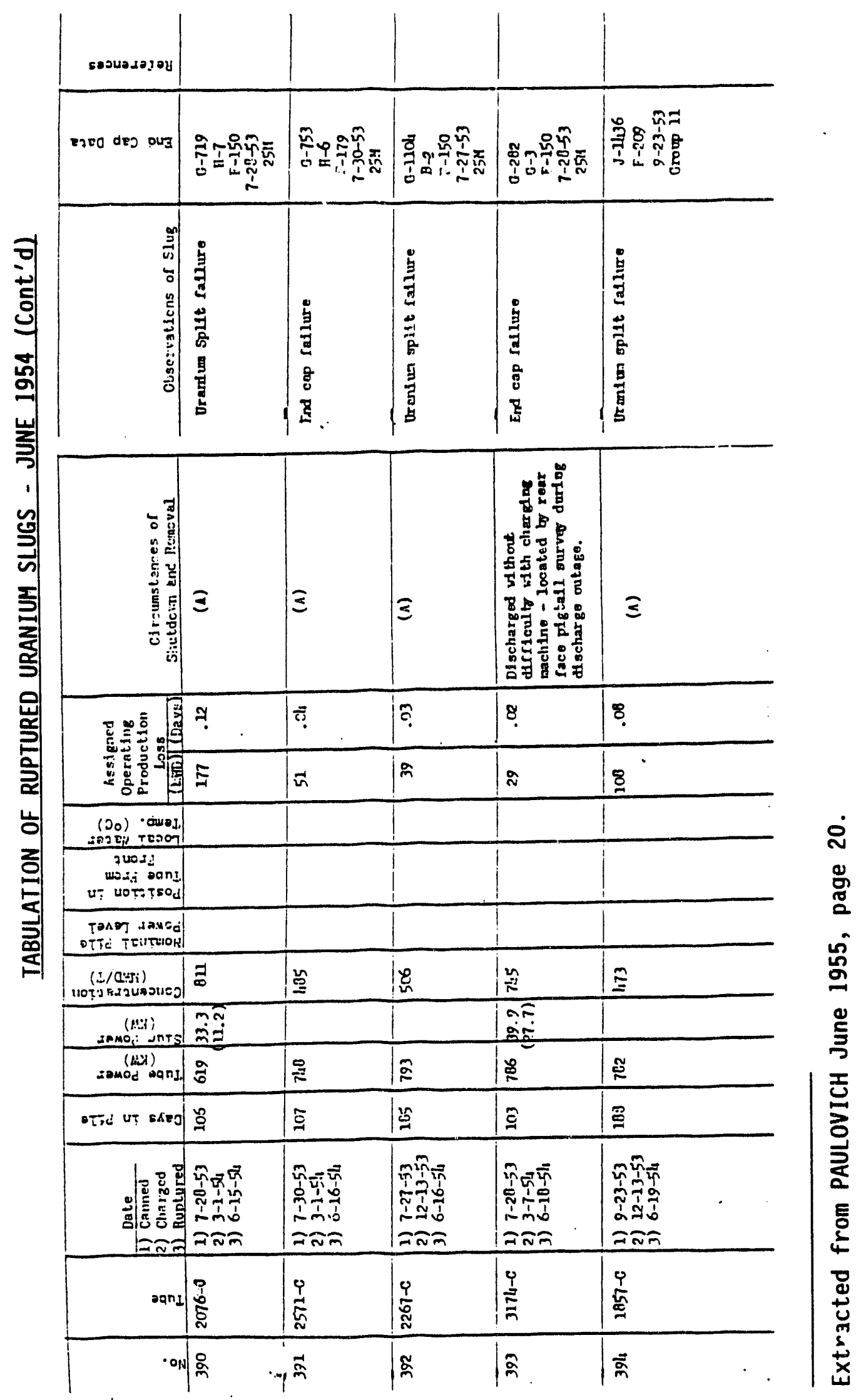




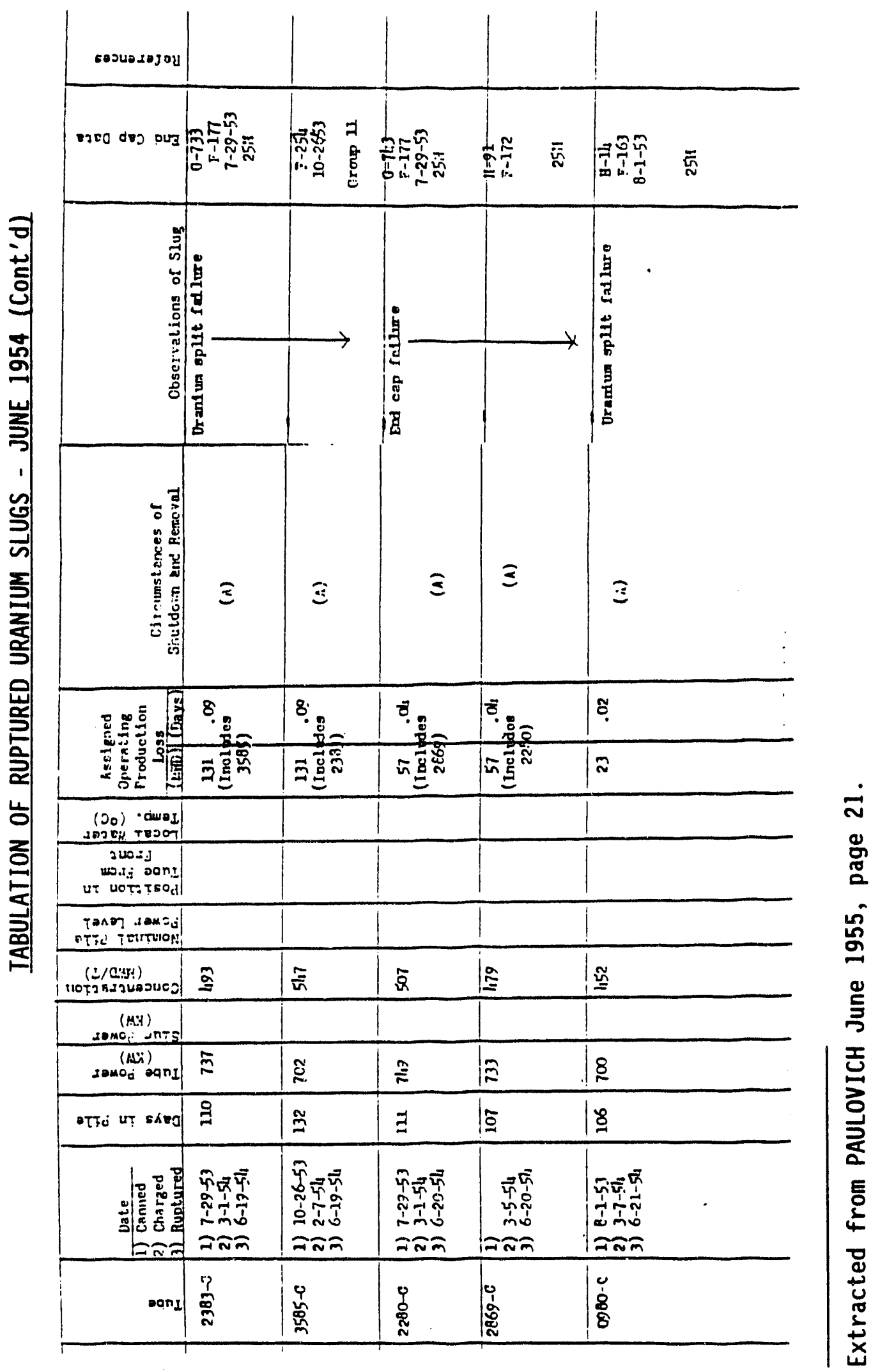




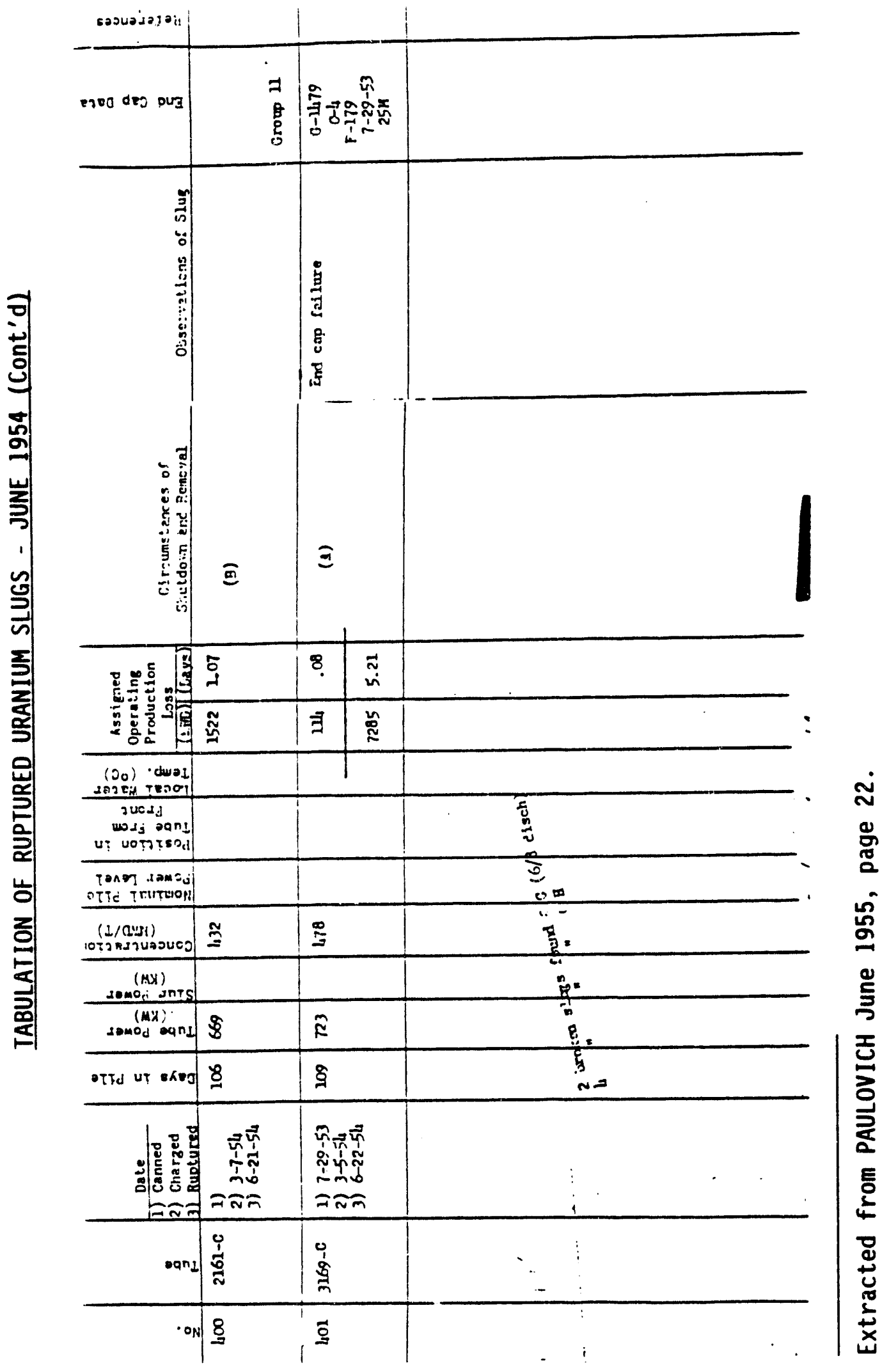

D. 23 


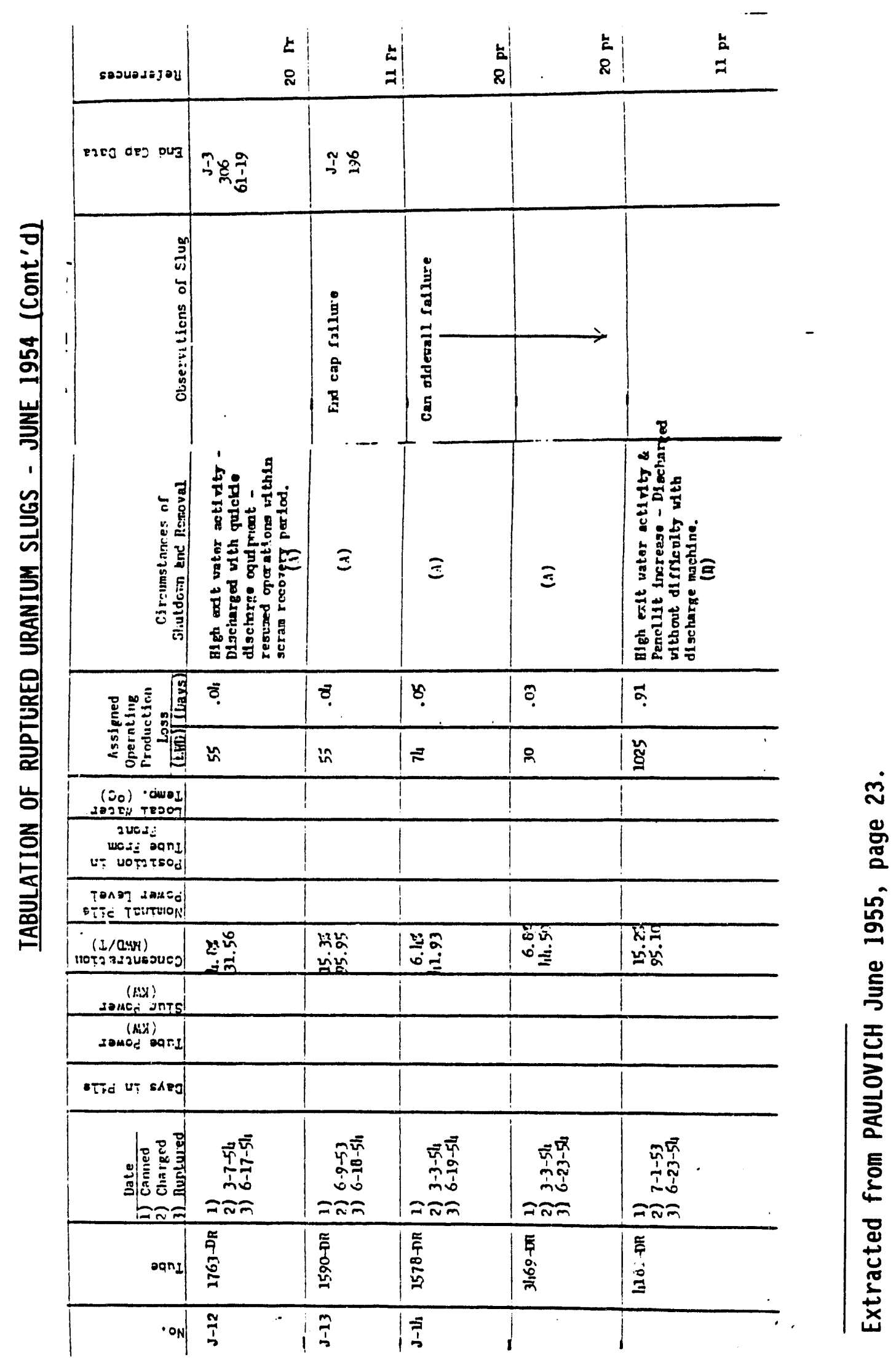




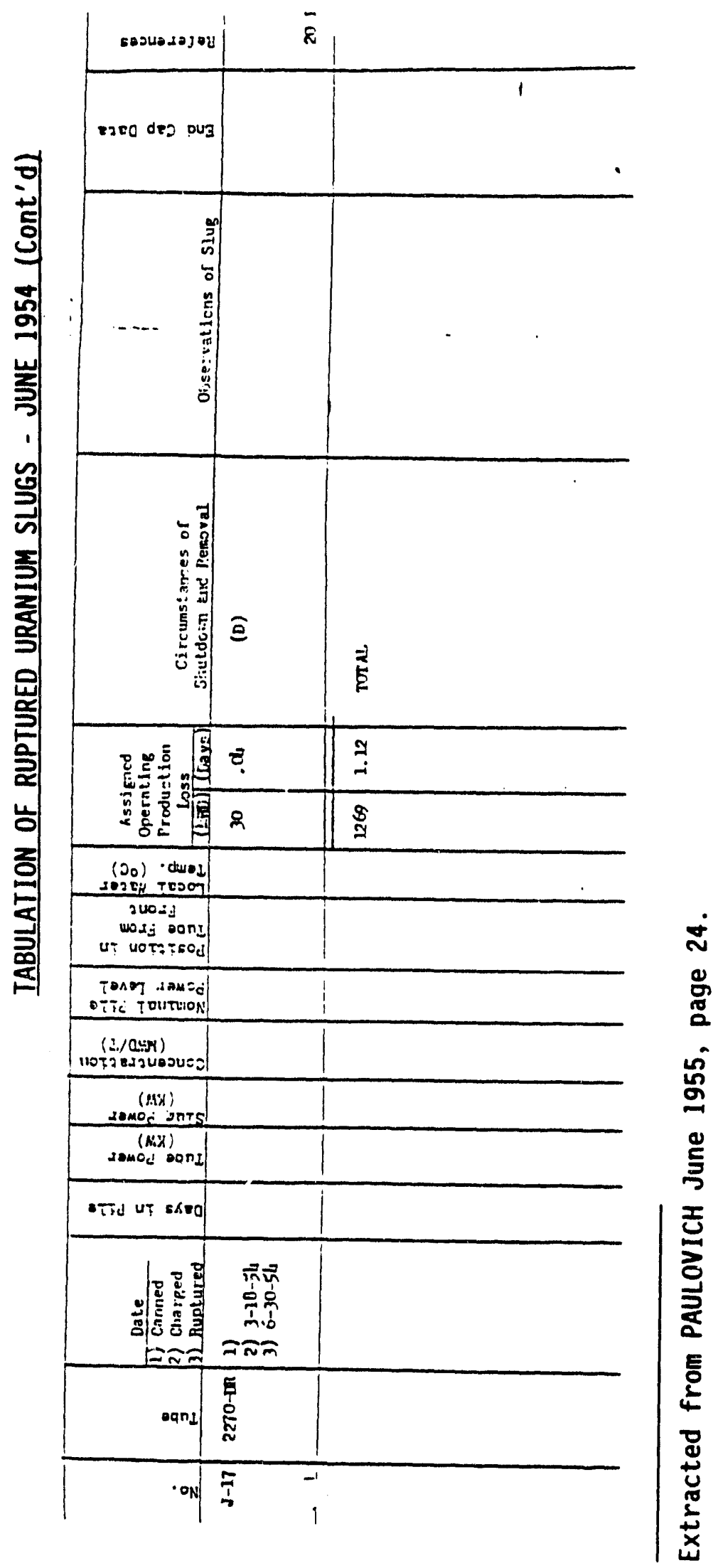

\section{25}




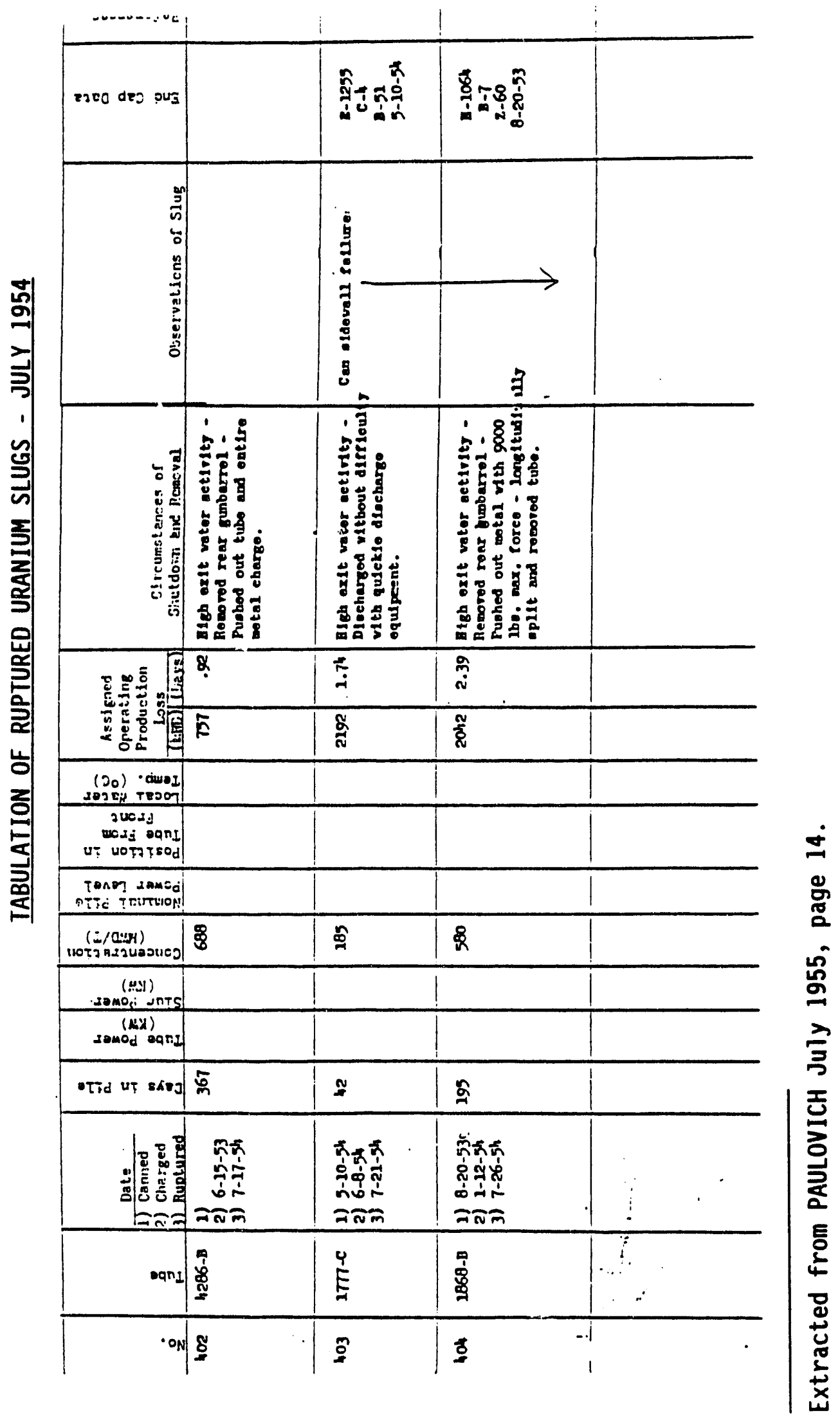




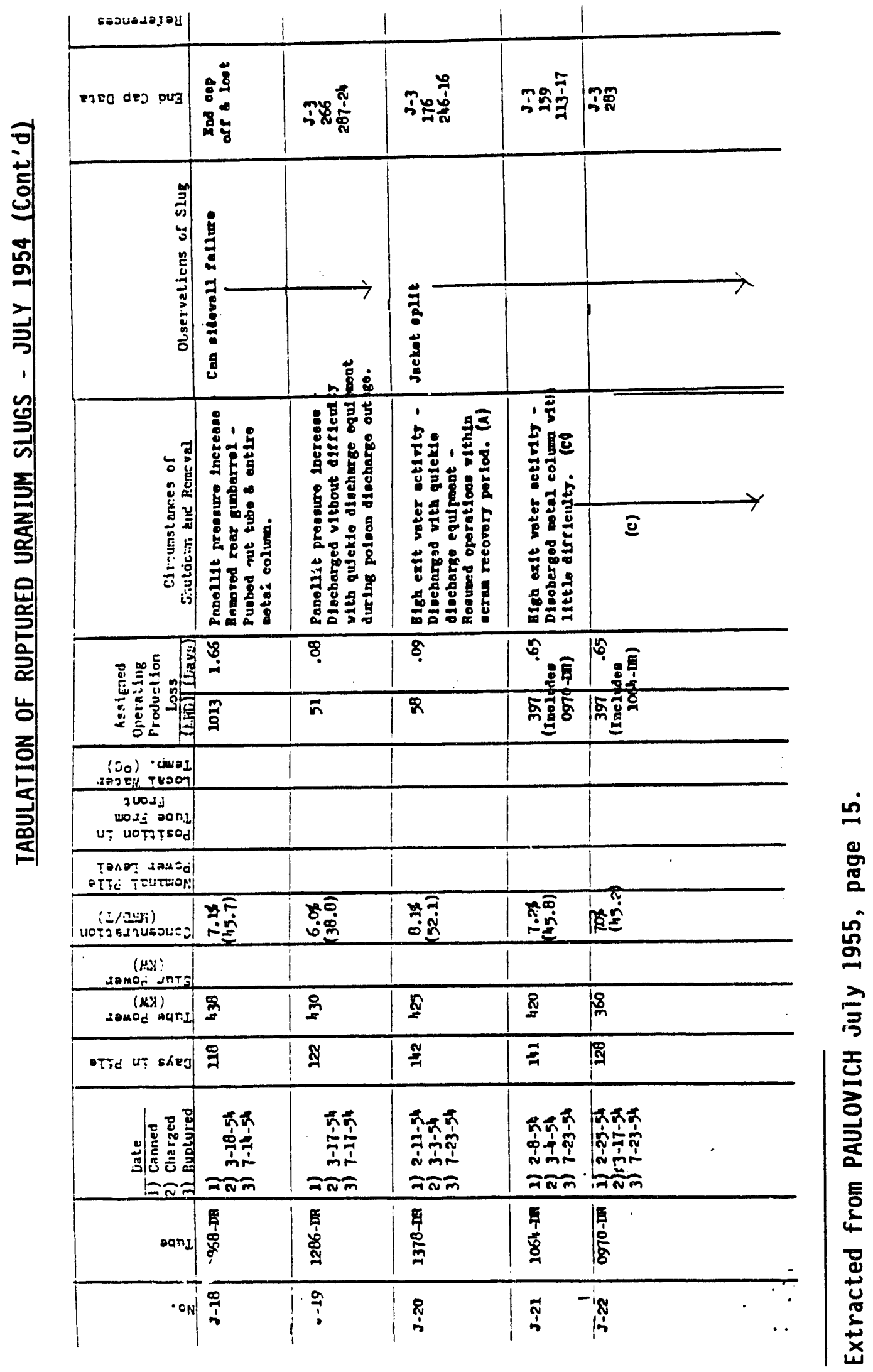

D. 27 


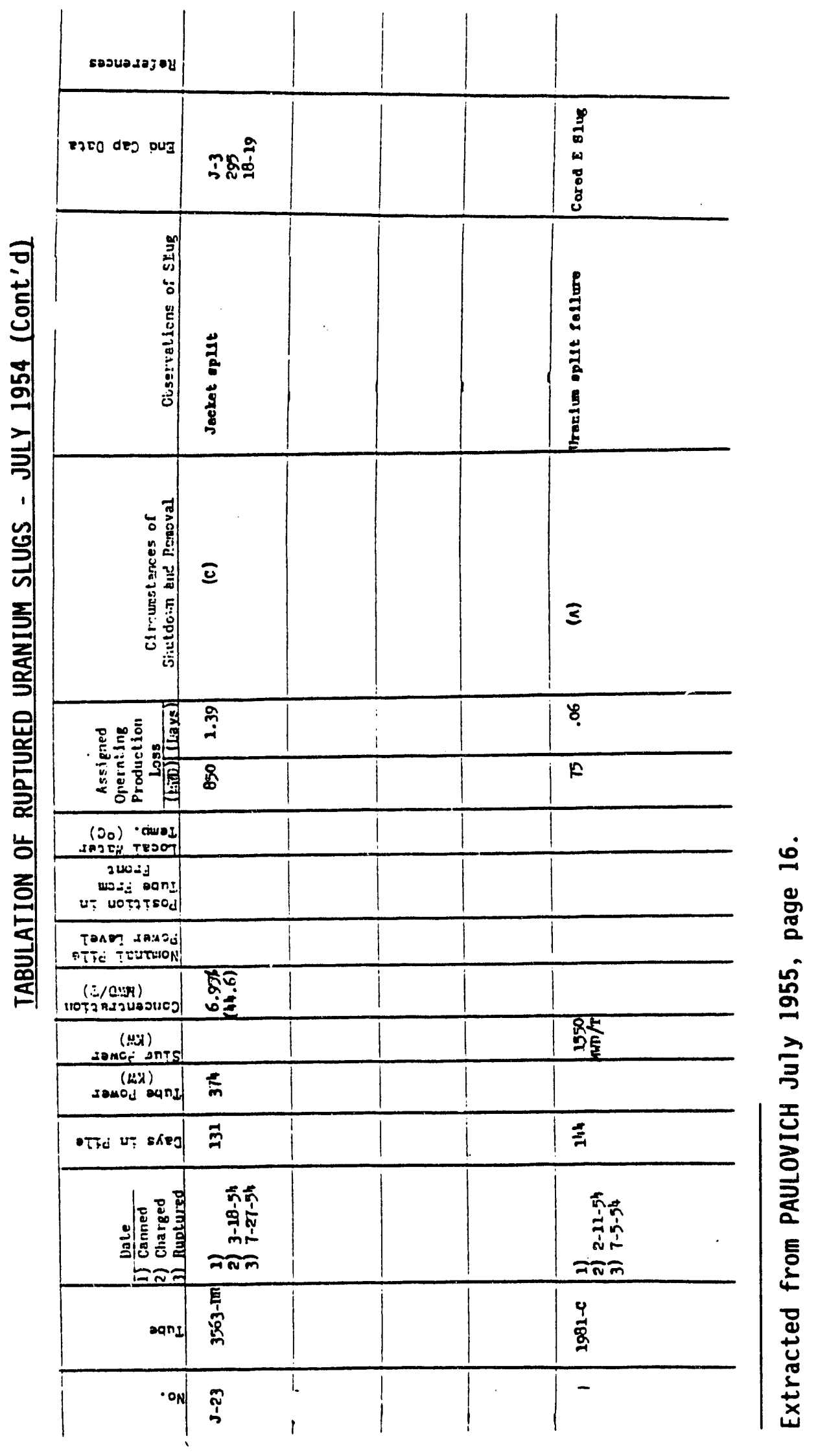

D. 28 


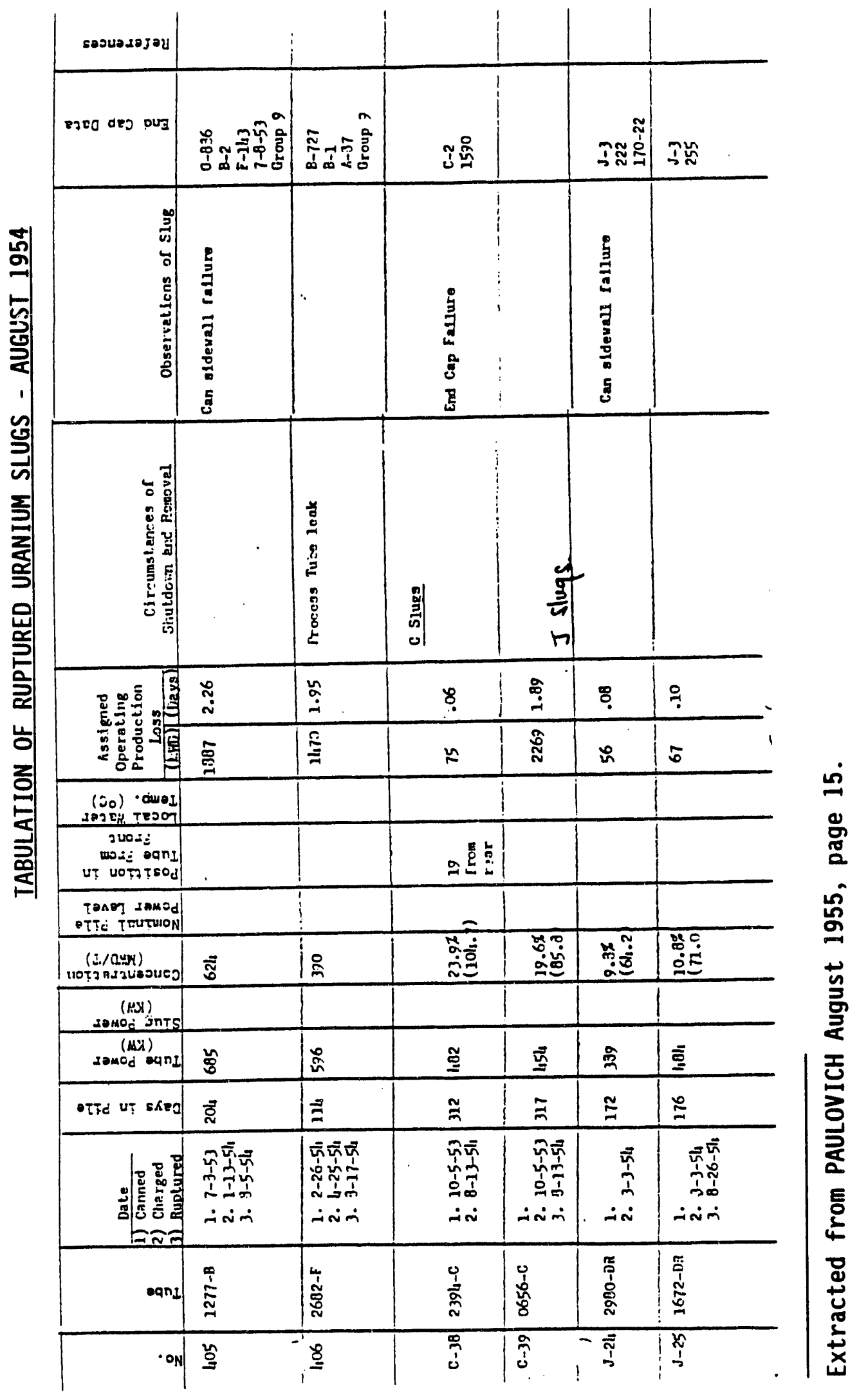




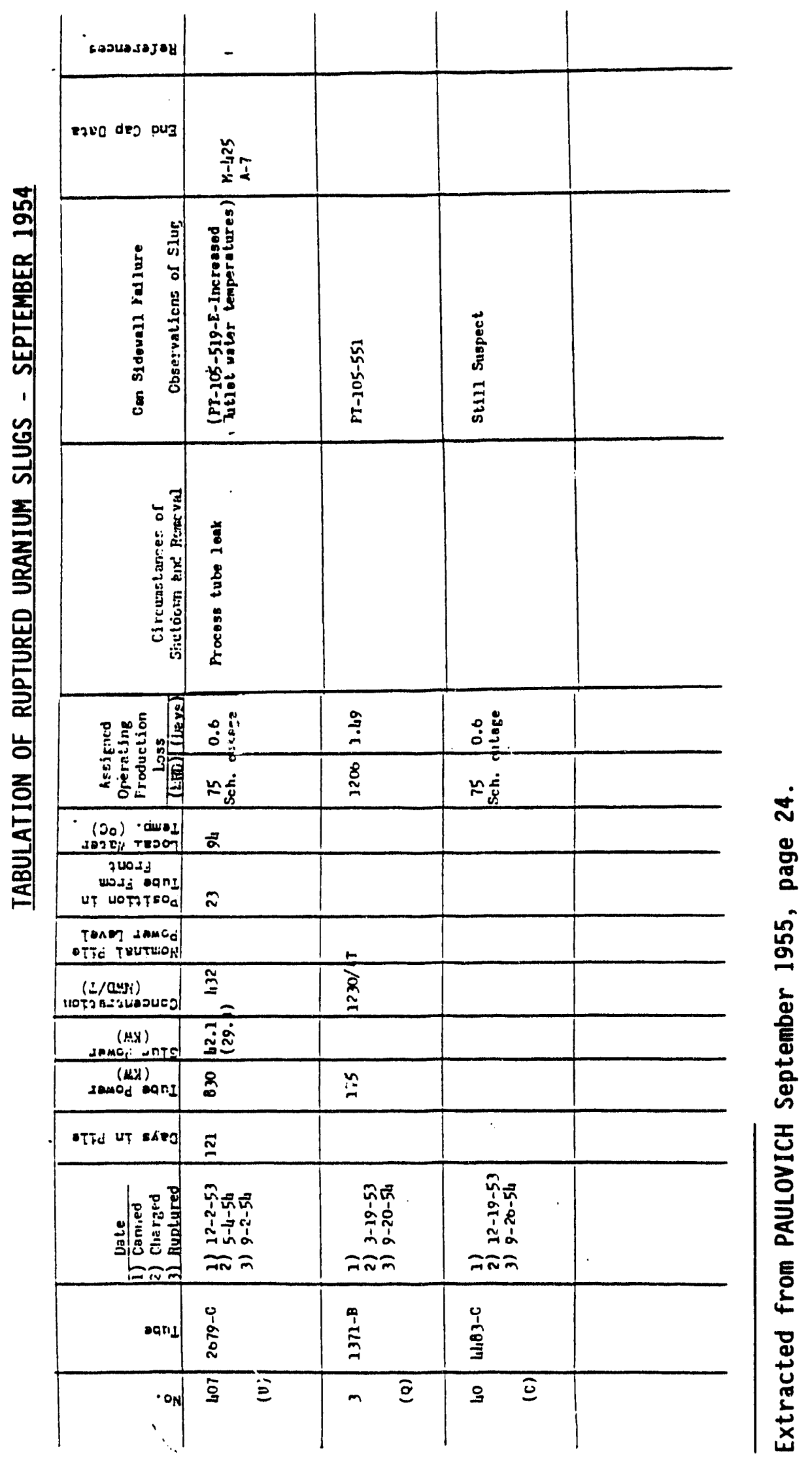




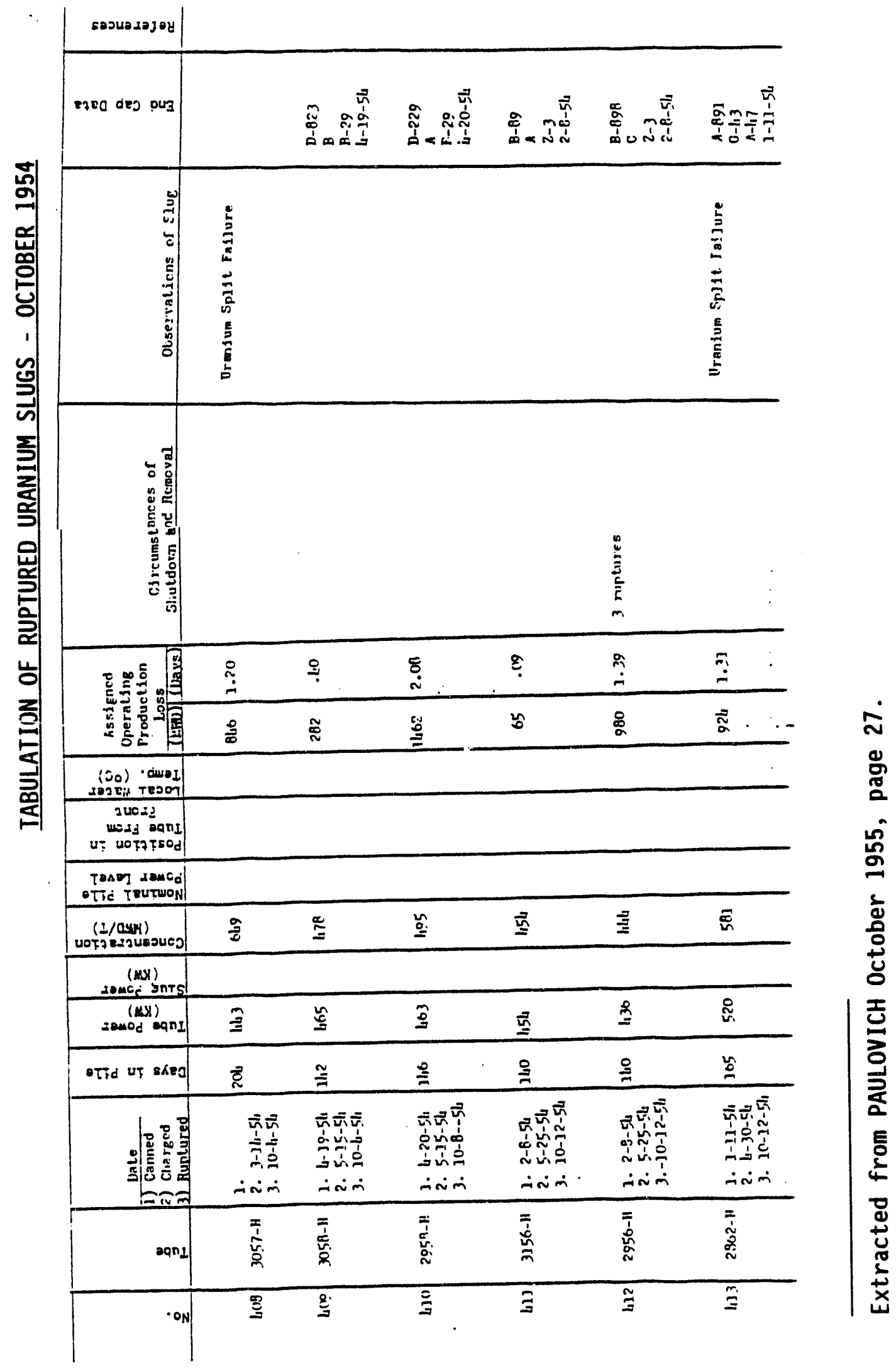

D. 31 


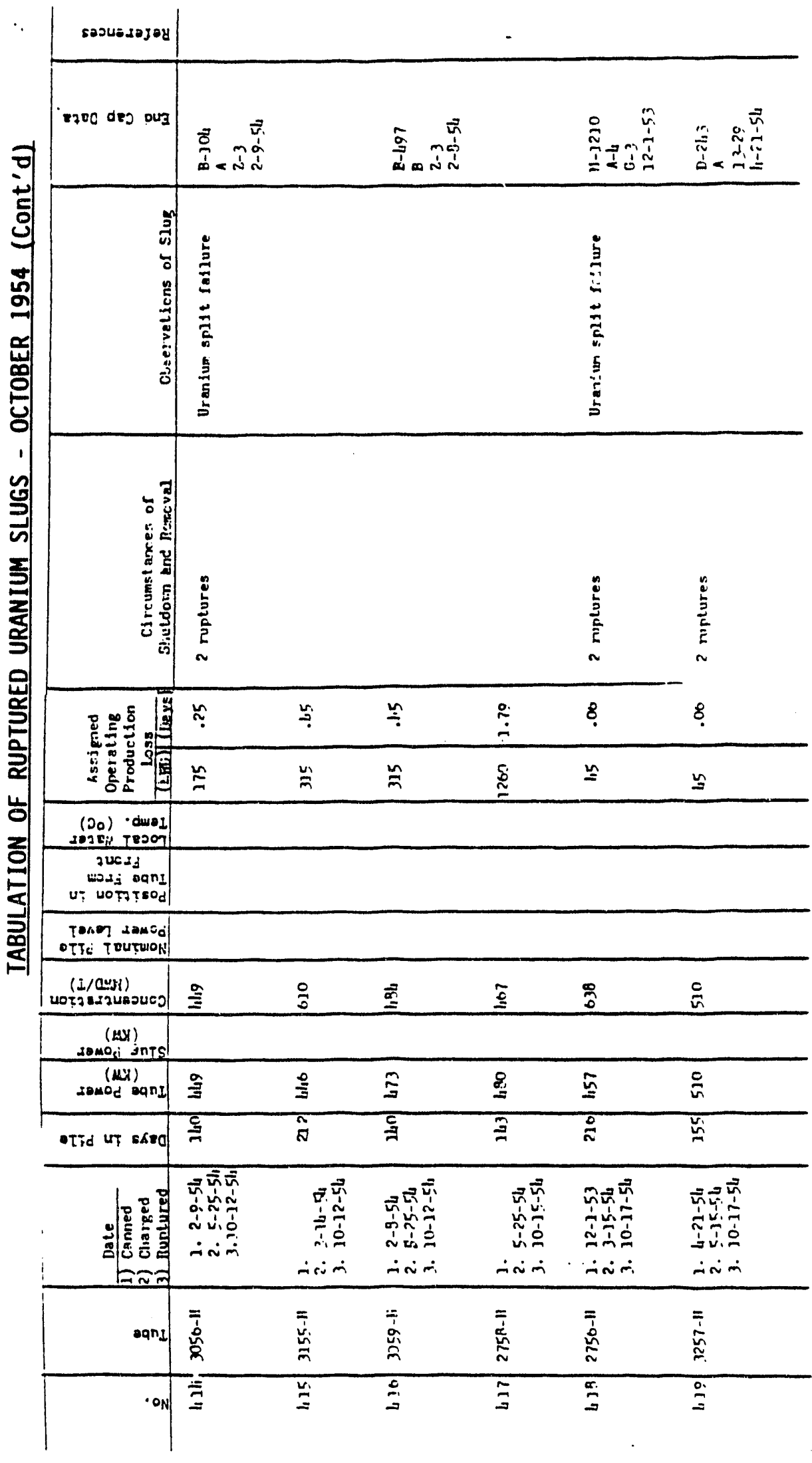

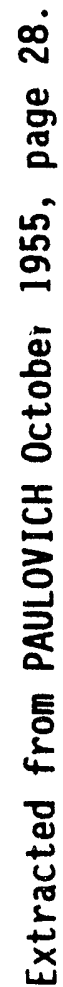




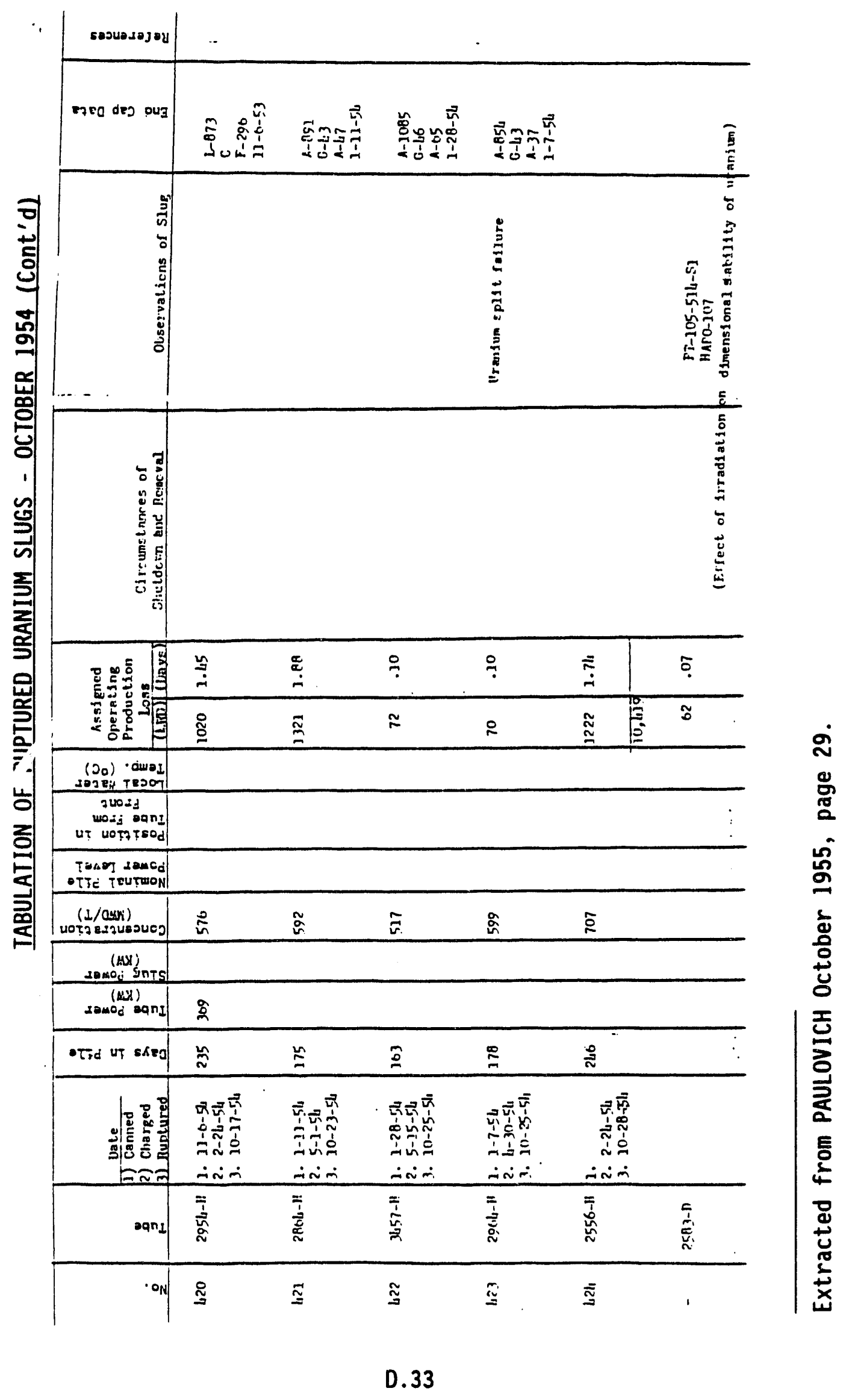




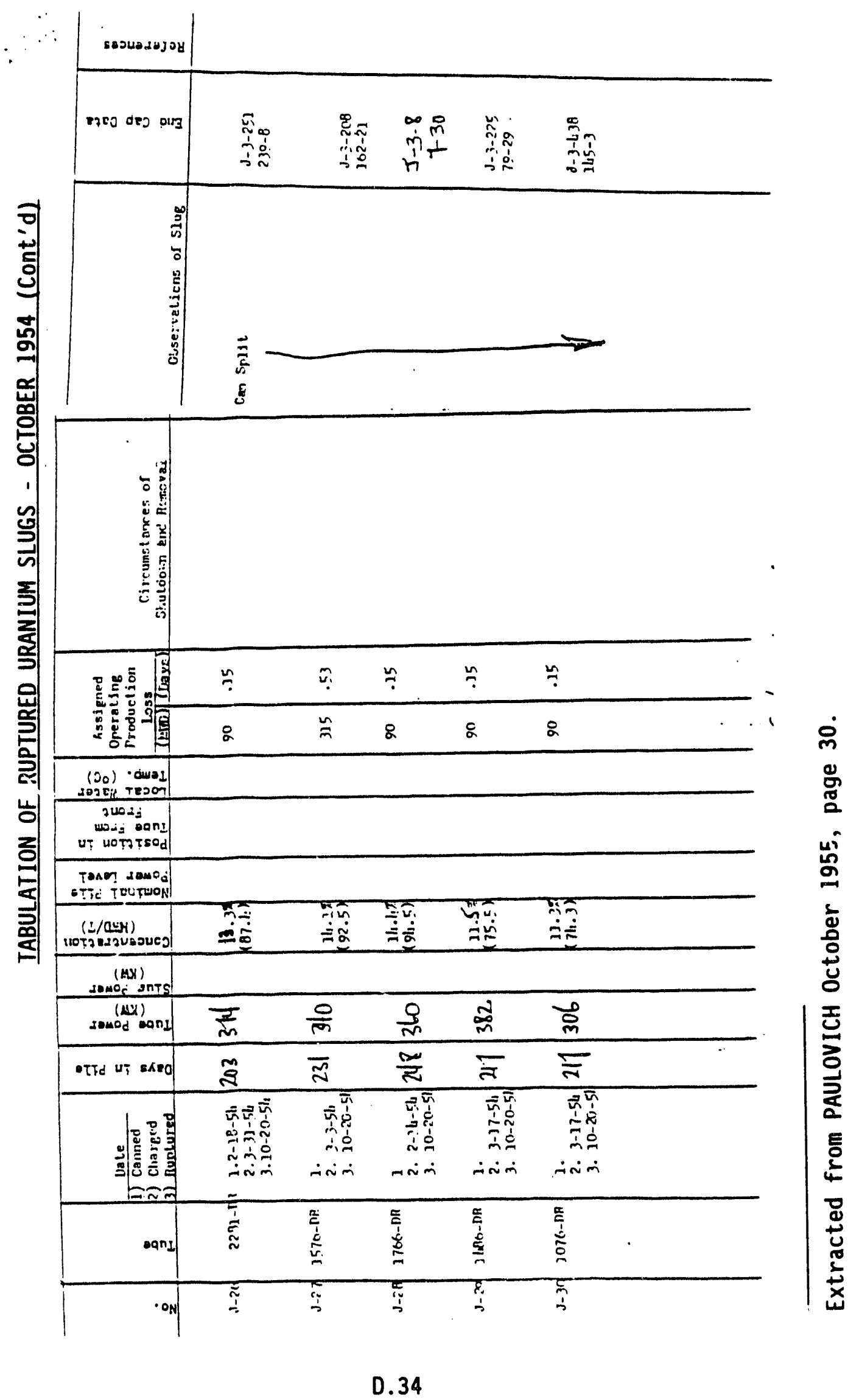




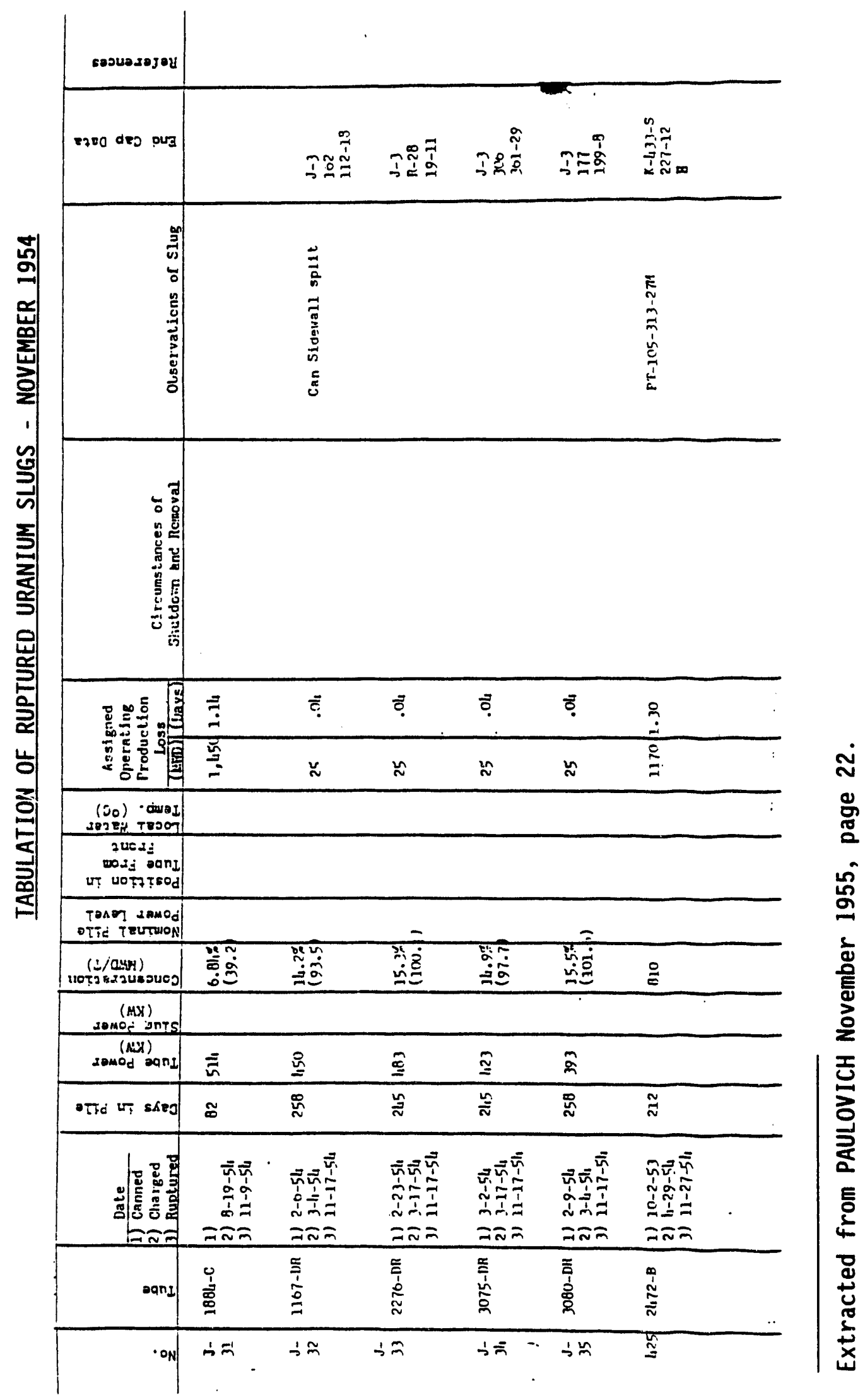




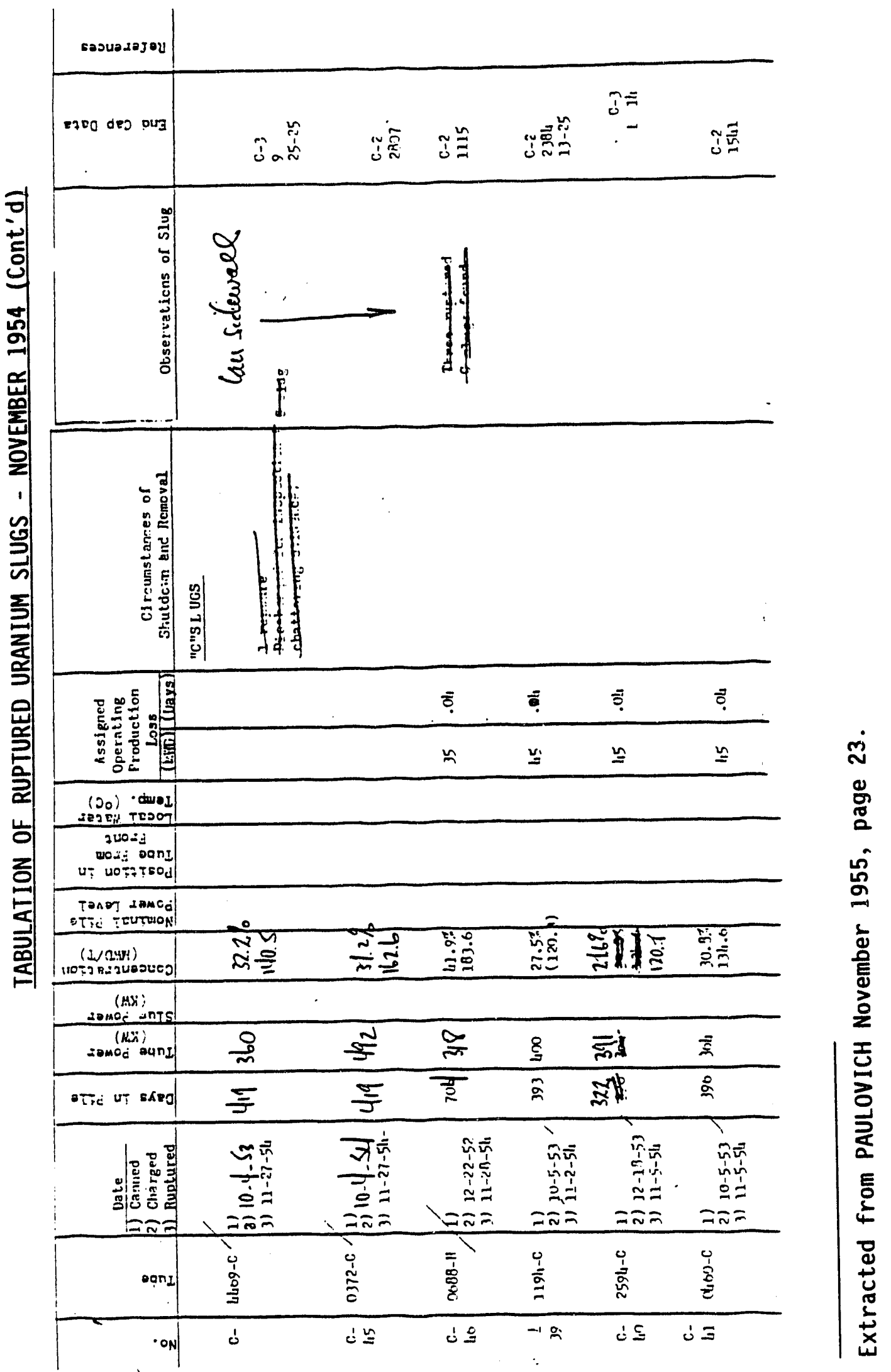




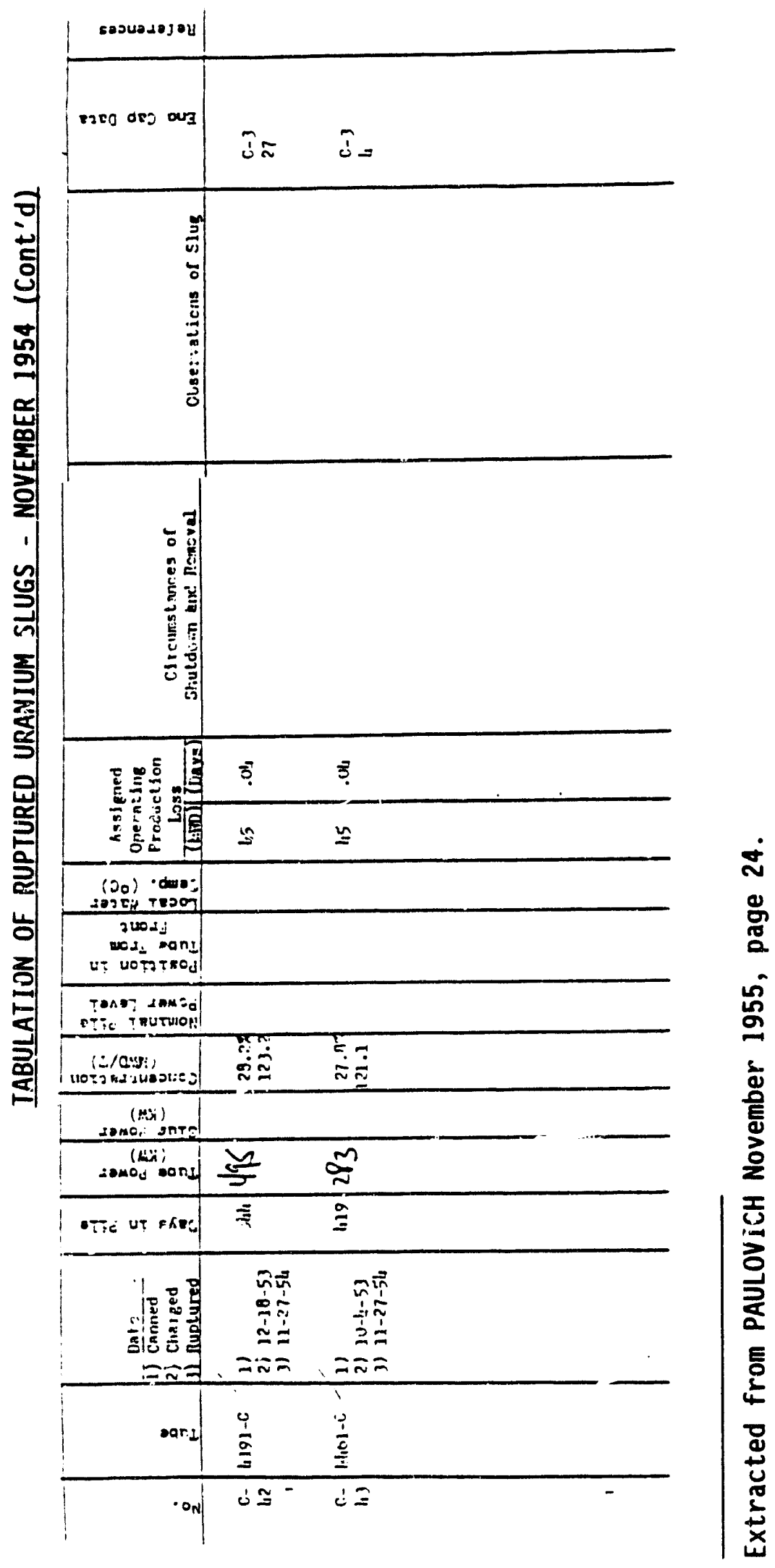




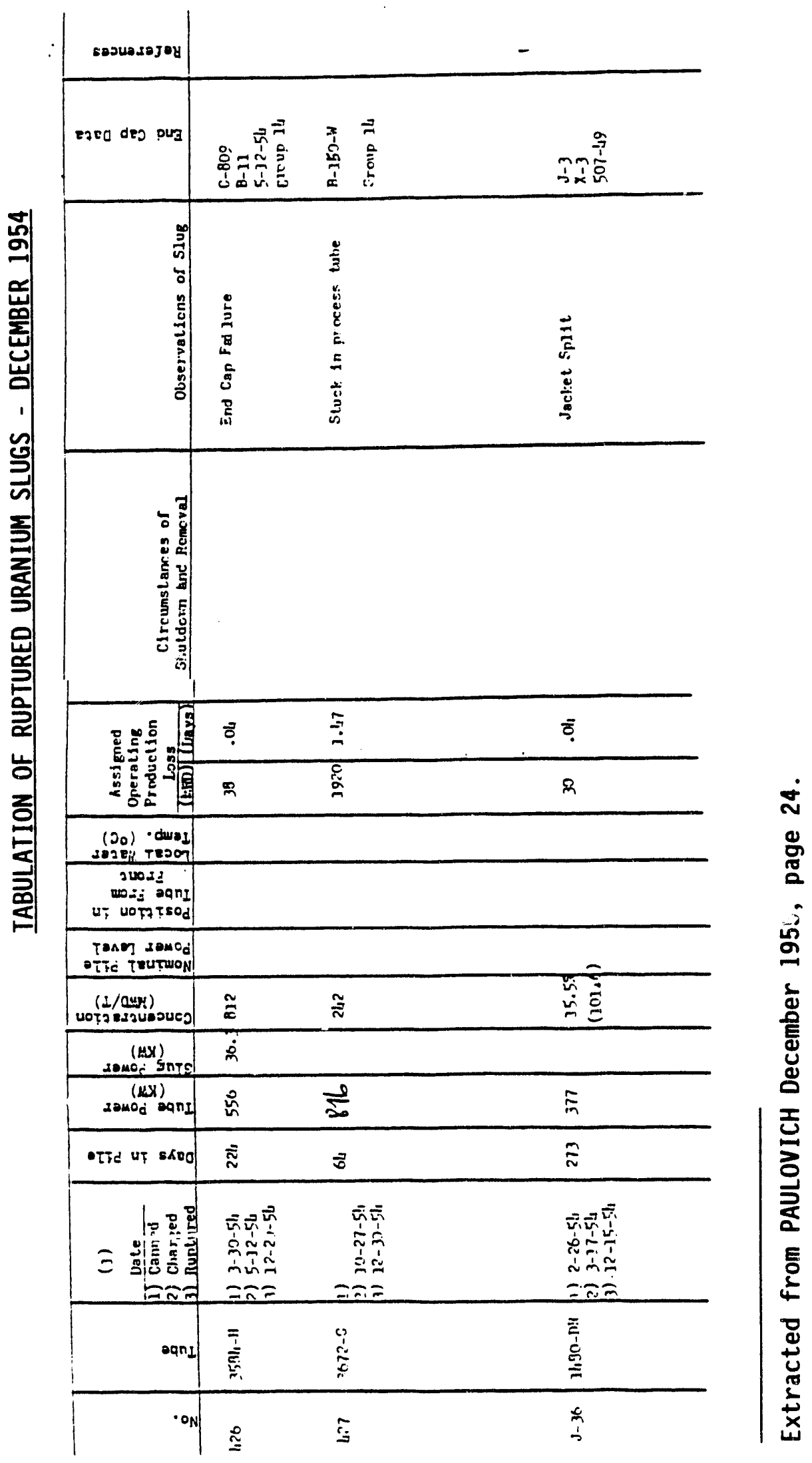


APPENDIX E

FUEL-ELEMENT FAILURE DATA FOR 1955 


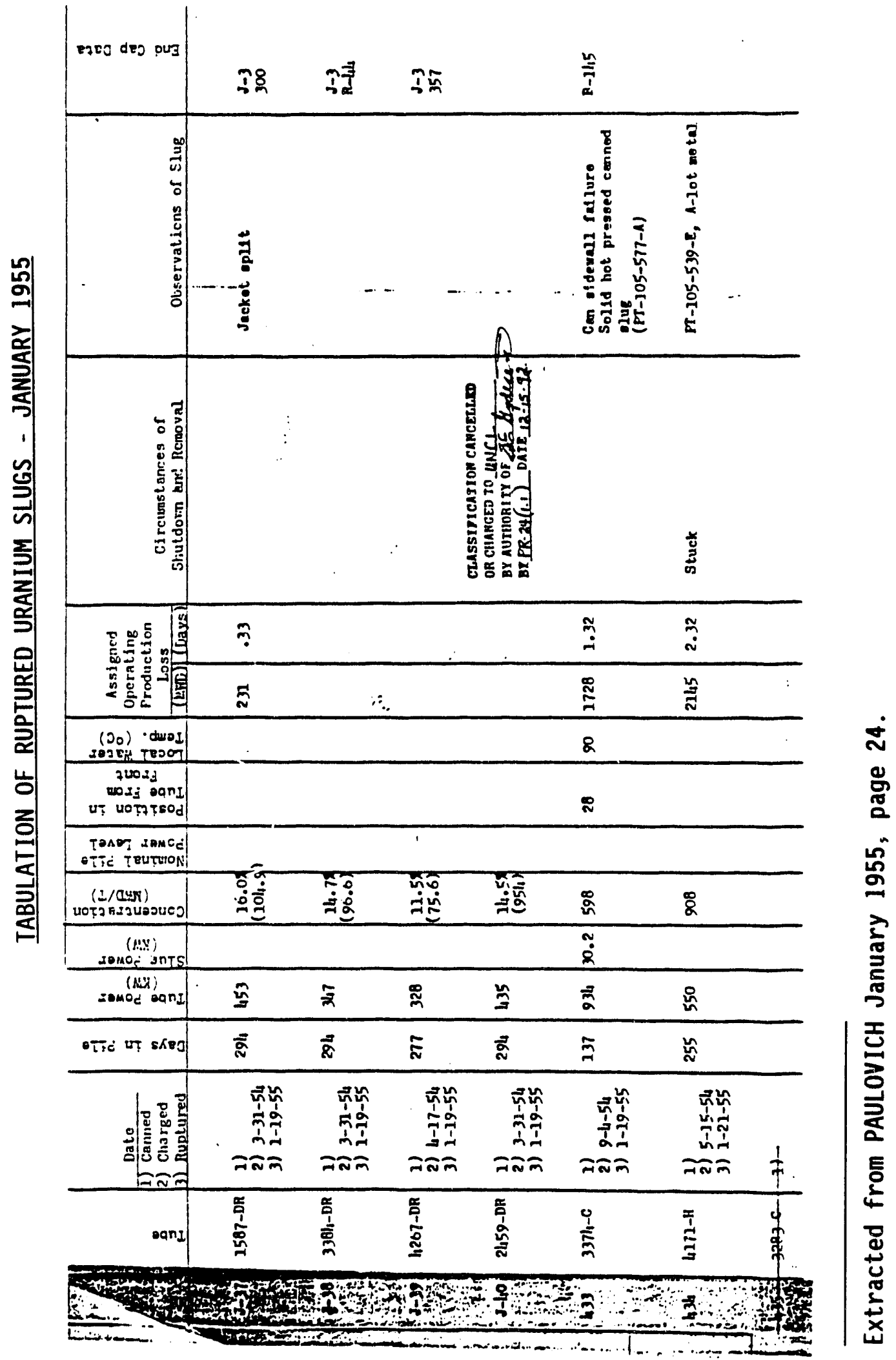

E.1 


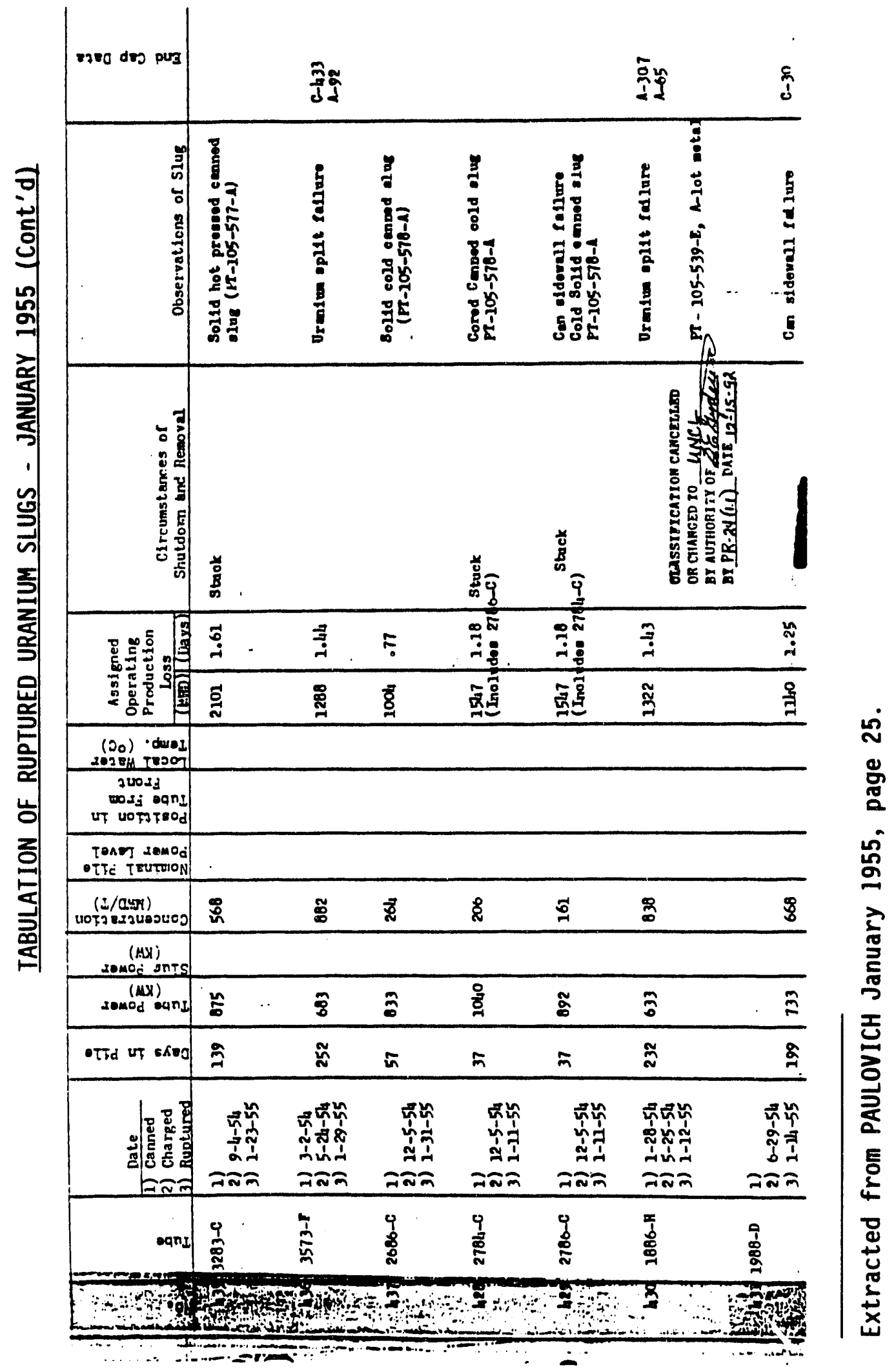

E. 2 


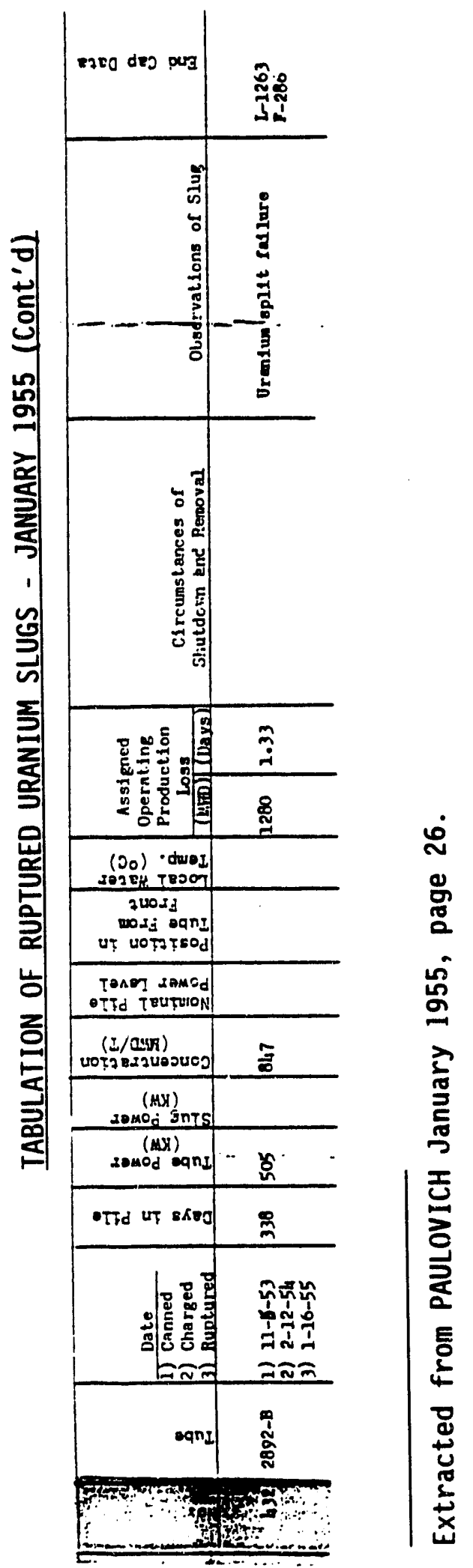

E. 3 


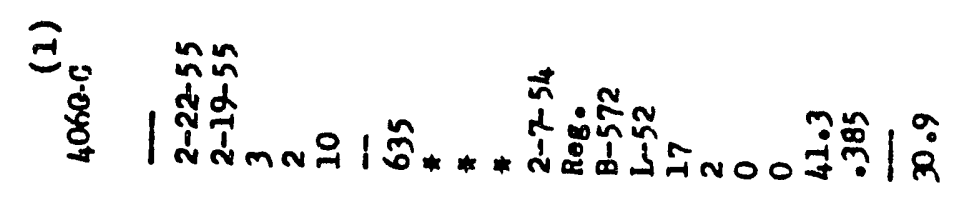

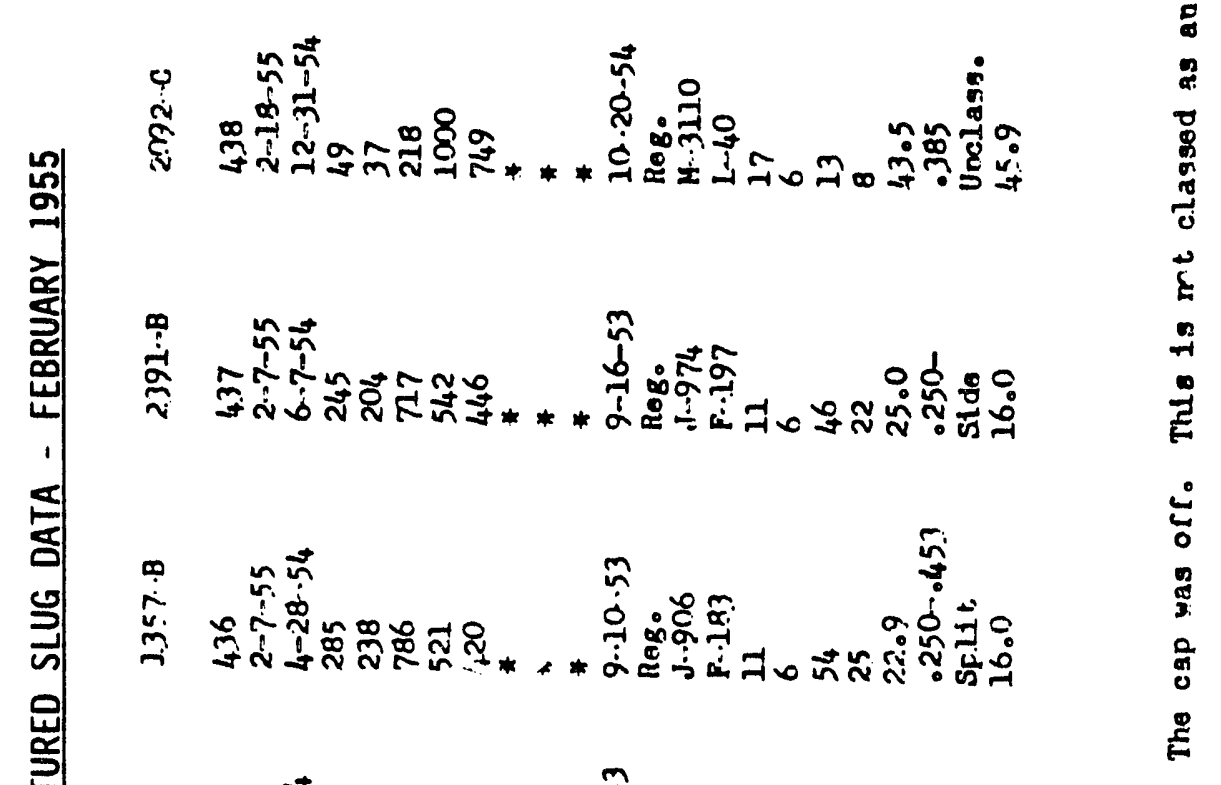

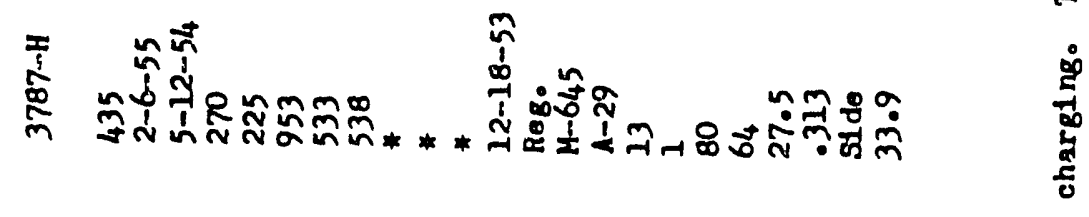

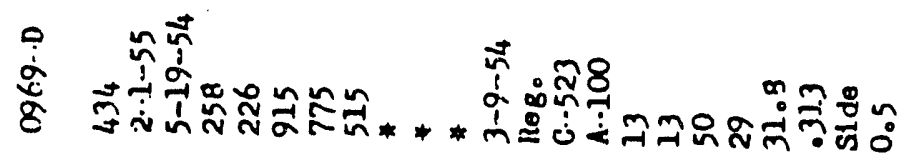

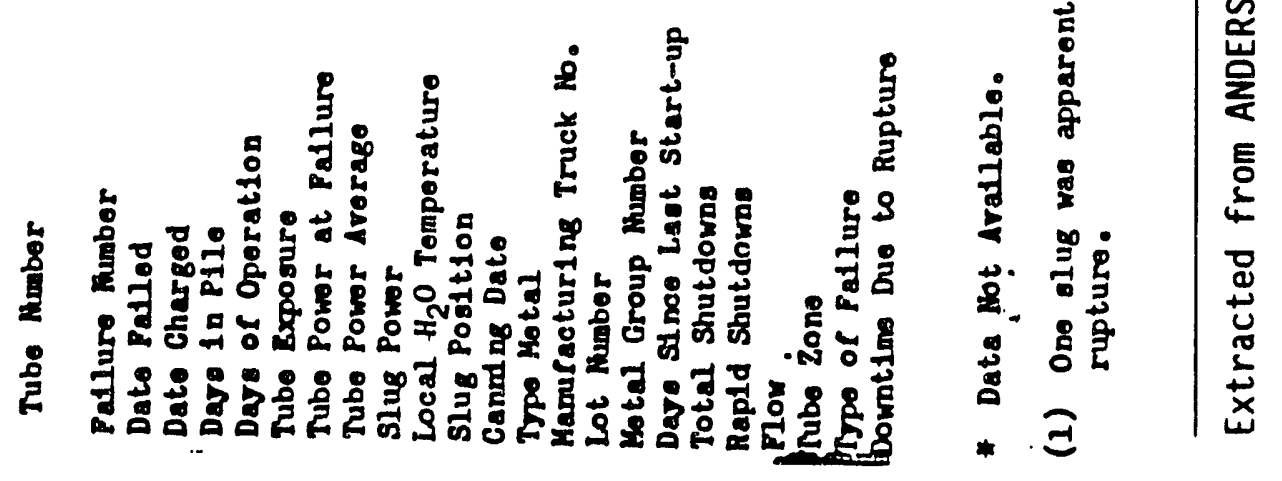




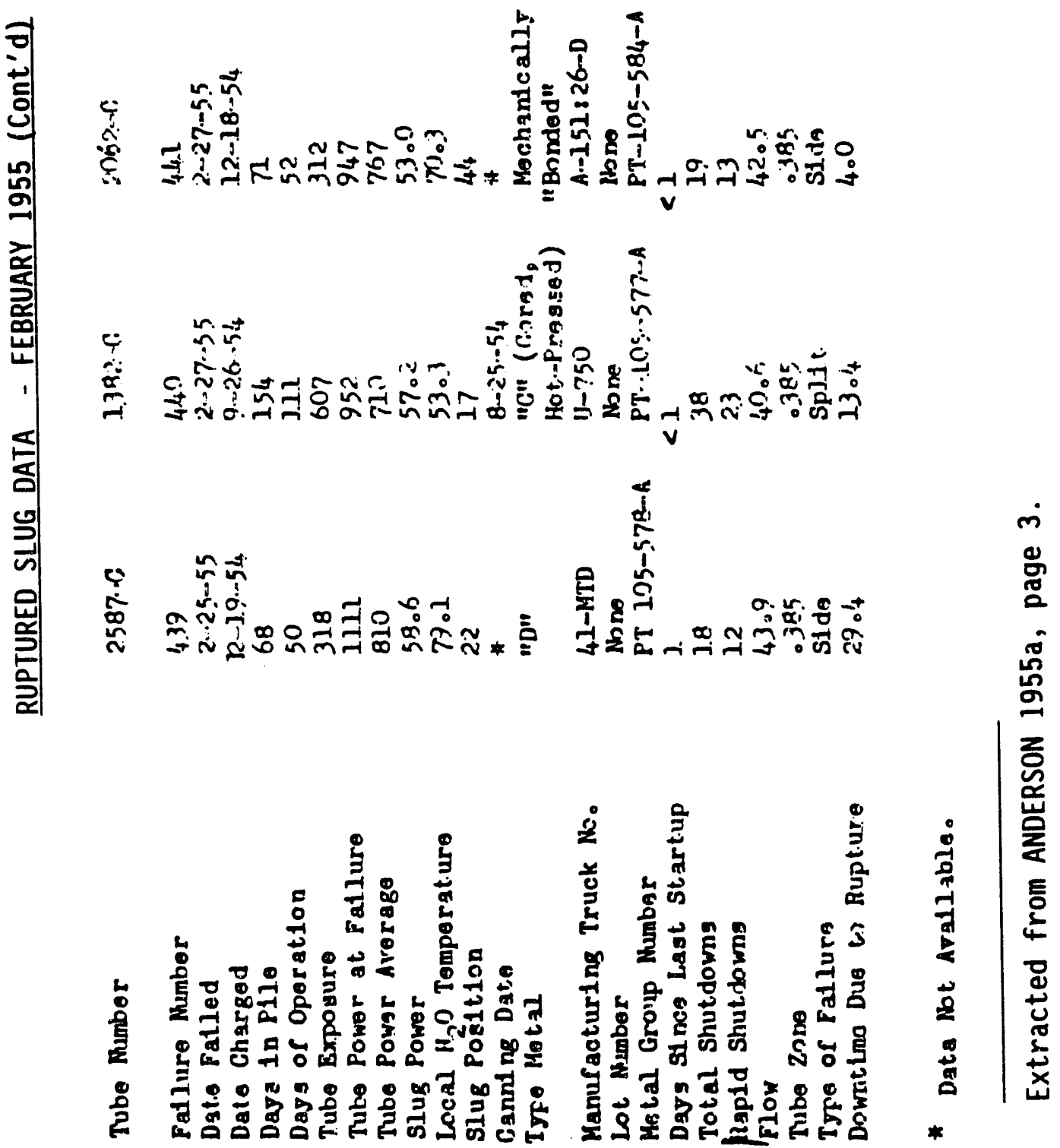


념

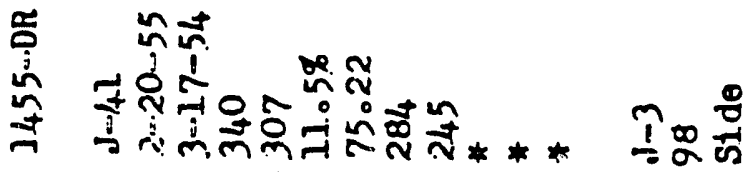

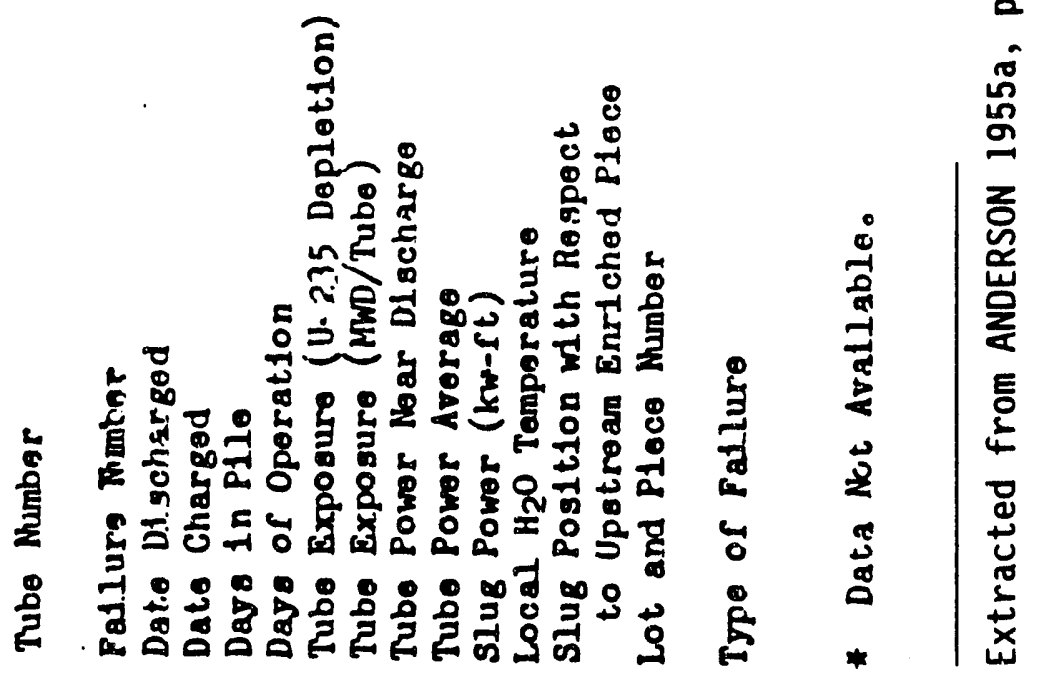




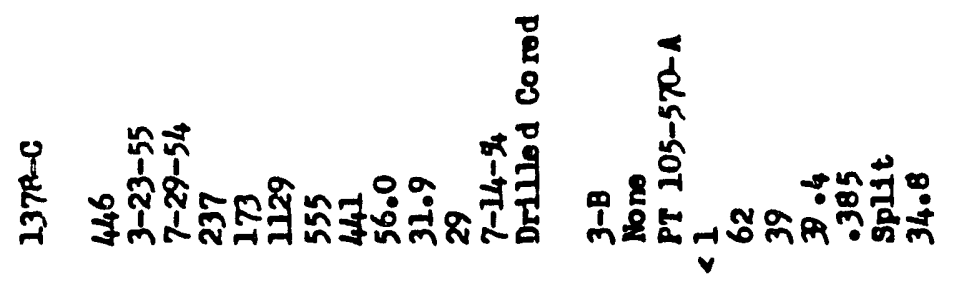

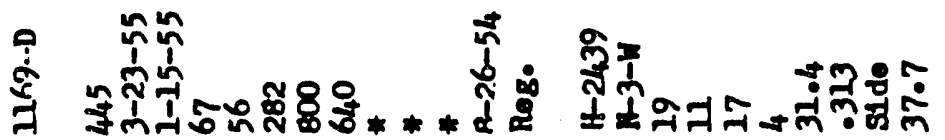

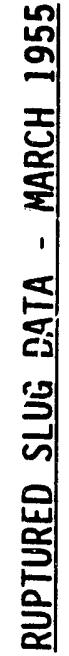

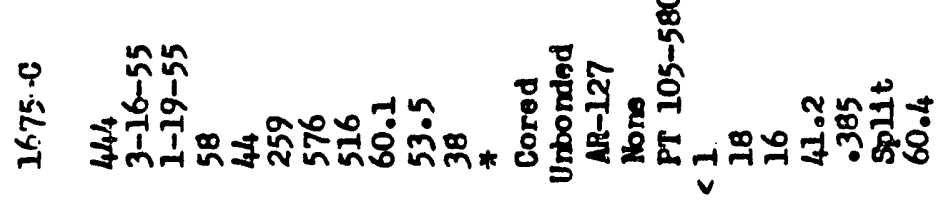

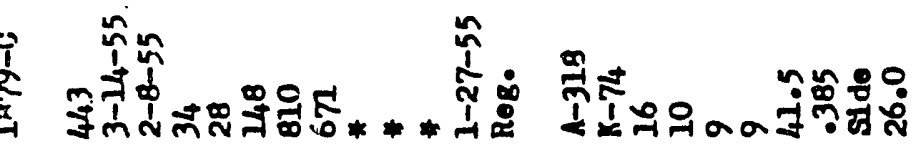

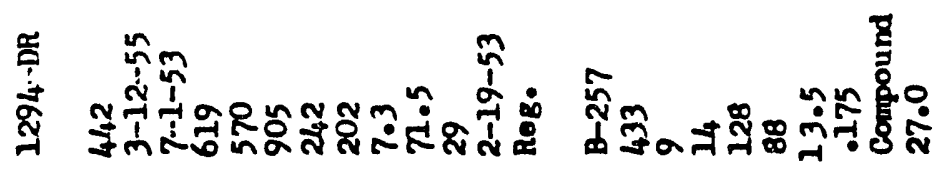
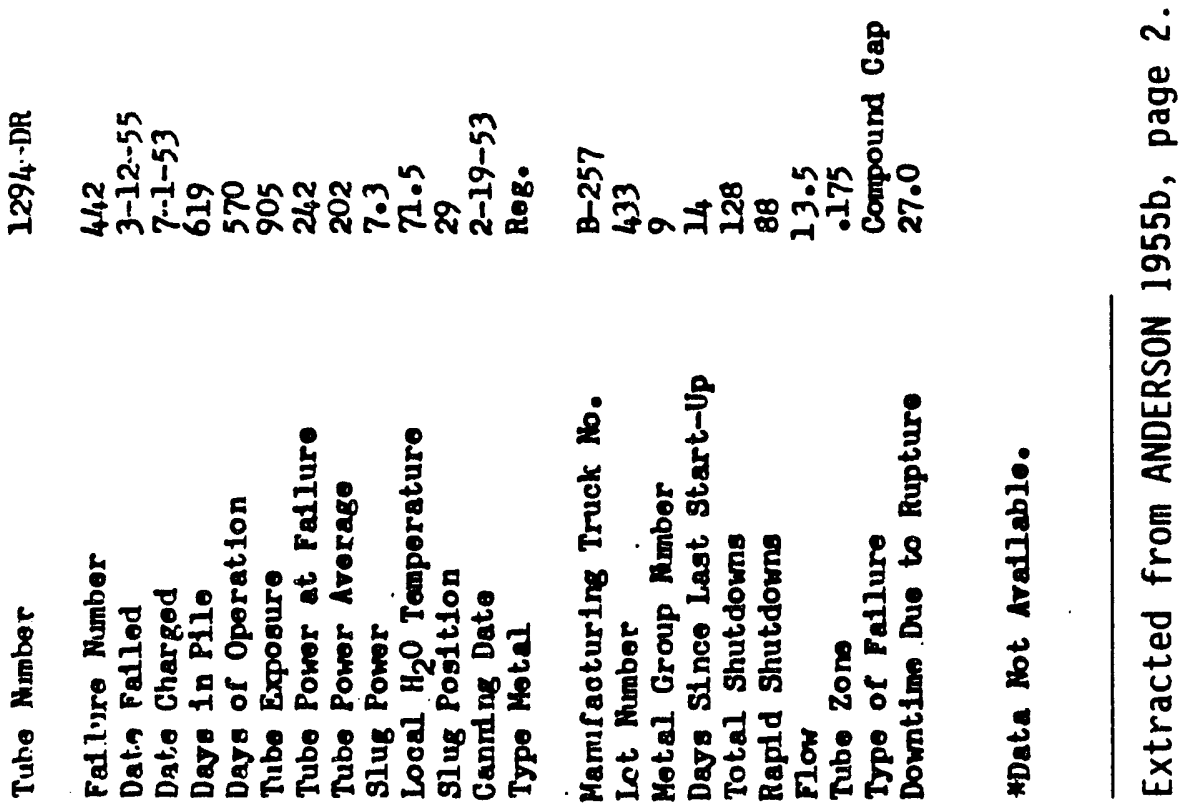

E. 7 


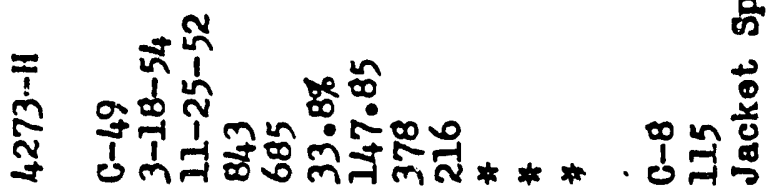

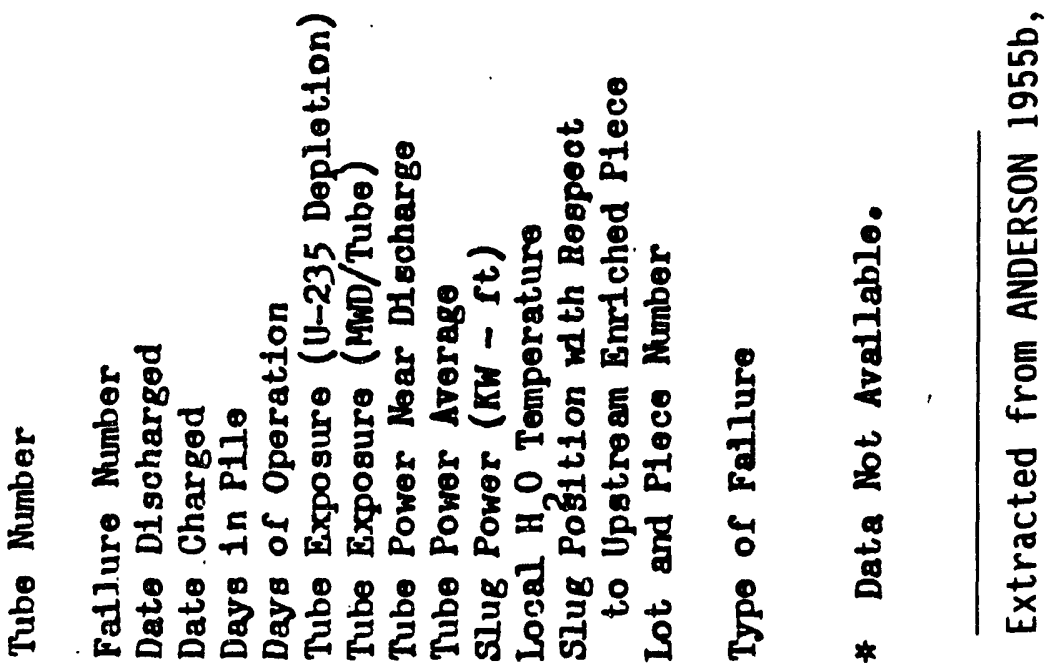




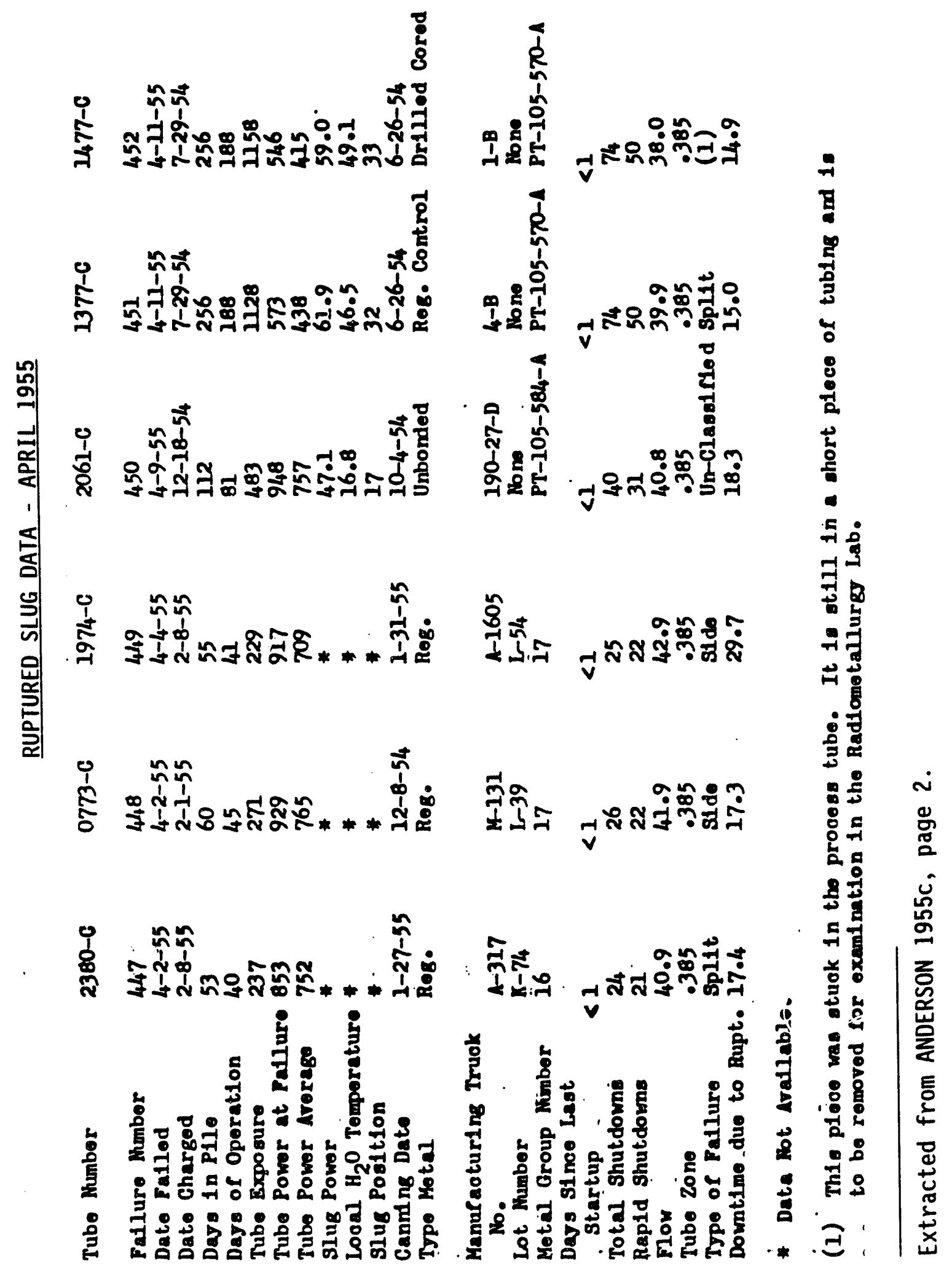




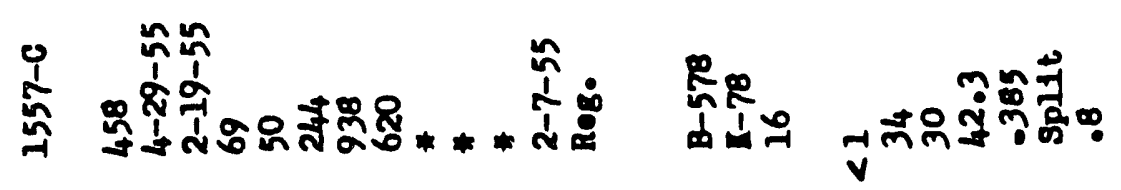

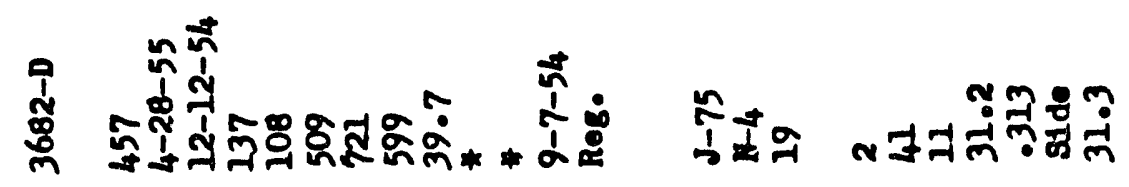

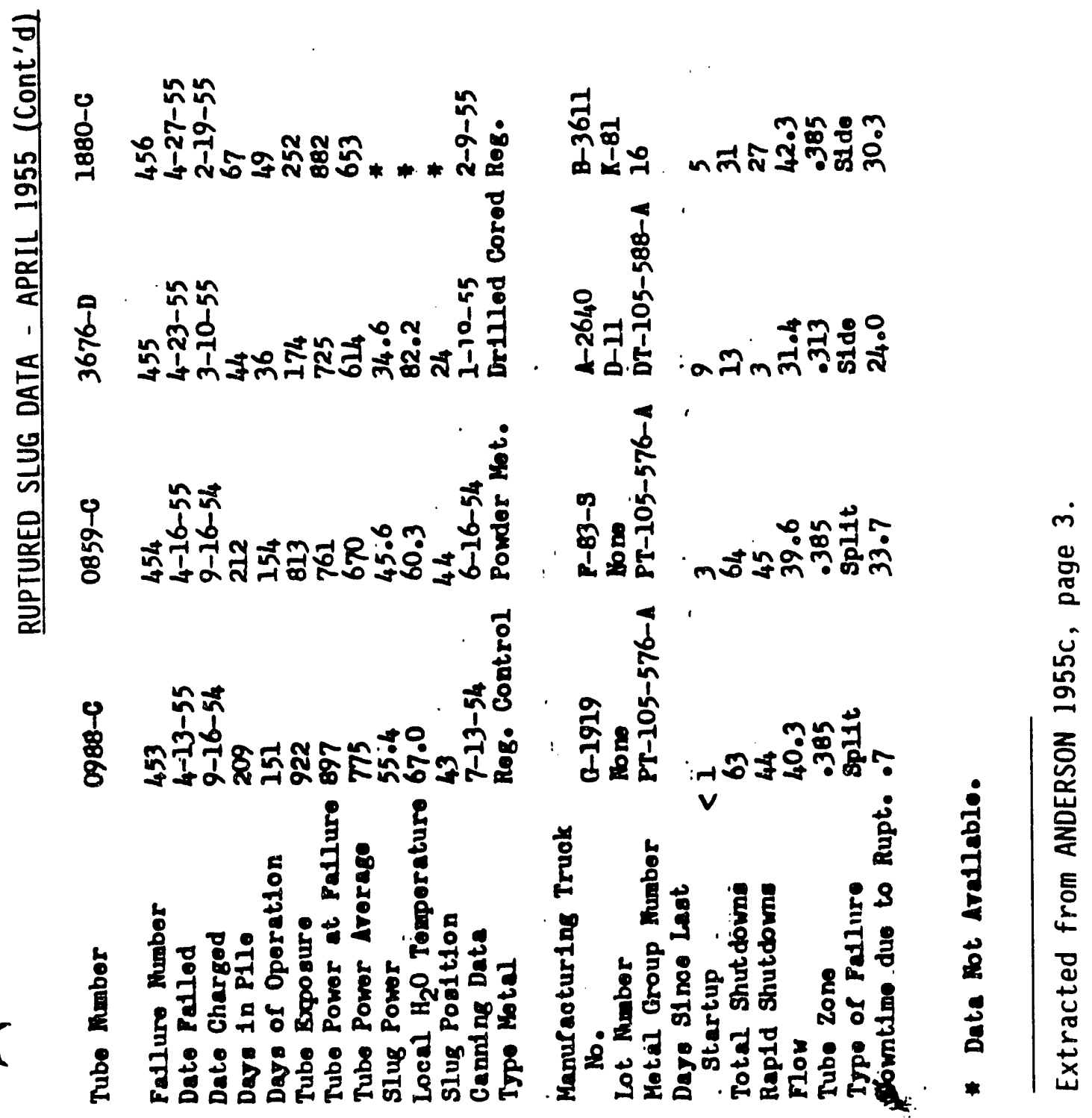




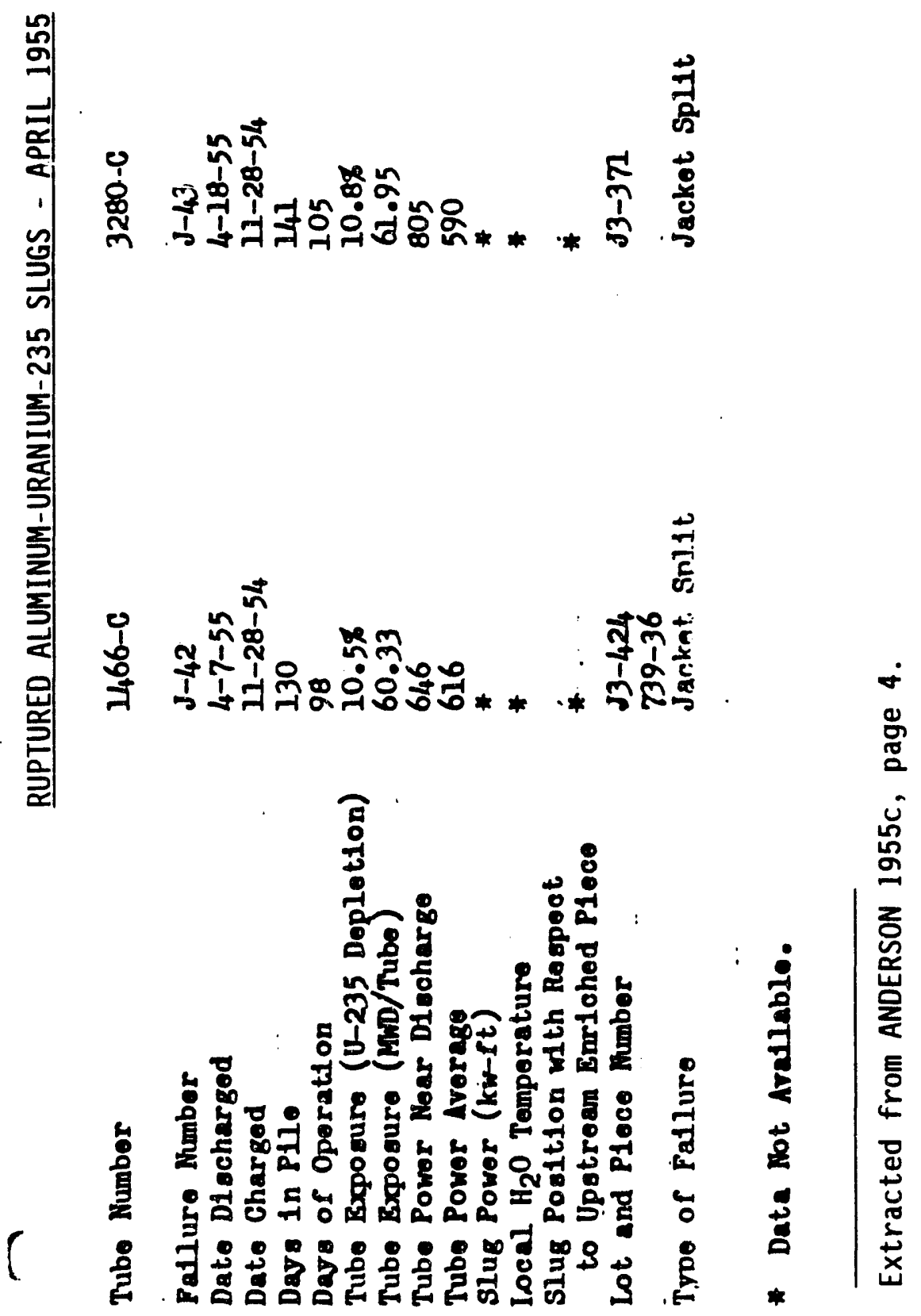


URANIUM SLUG RUPTURE EXPERIENCE - MAY 1955

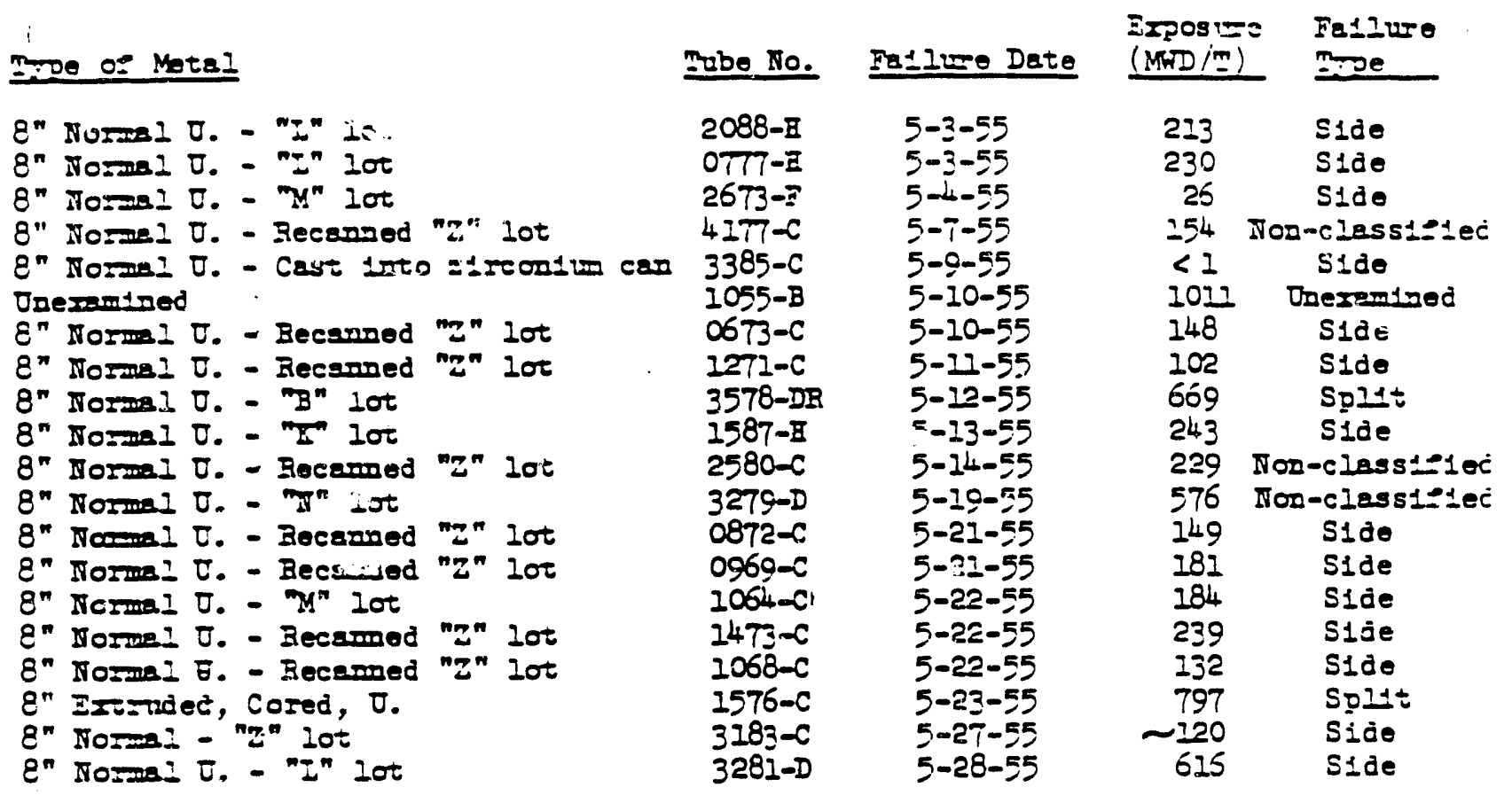

\section{ALUMINUM-URANIUM-235 ALLOY SLUG RUPTURE EXPERIENCE}

\begin{tabular}{|c|c|c|c|c|c|}
\hline$m p e$ & a Metal & Tribe II. & Fa1Iwo Dato & $\begin{array}{l}\text { Ixpostre } \\
\text { (MND/tuipe) }\end{array}$ & $\begin{array}{l}\text { Fedרare } \\
\text { mase }\end{array}$ \\
\hline & $\begin{array}{l}\text { MetaI } \\
\text { MetaI } \\
\text { MotaI } \\
\text { MetaI } \\
\text { MetaI } \\
\text { MetaI } \\
\text { MotaI } \\
\text { MetaI }\end{array}$ & $\begin{array}{l}2670-c \\
1884-c \\
2504-c \\
2092-c \\
4381-i \\
3392-c \\
2712-i \\
3676-C\end{array}$ & $\begin{array}{l}5-14-55 \\
5-15-55 \\
5-15-55 \\
5-15-55 \\
5-15-55 \\
5-10-55 \\
5-20-55 \\
5-25-55\end{array}$ & $\begin{array}{r}81.5 \\
71.5 \\
224.5 \\
206.6 \\
93.5 \\
76.9 \\
163.9 \\
104.1\end{array}$ & 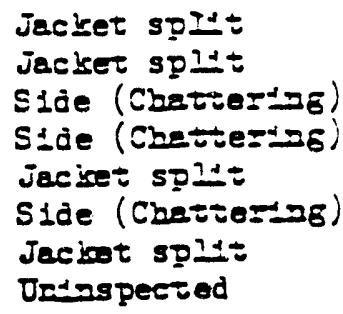 \\
\hline
\end{tabular}

It may be noted I=om Iable I thet the wojordty of the C Plle fallares thets month oc-

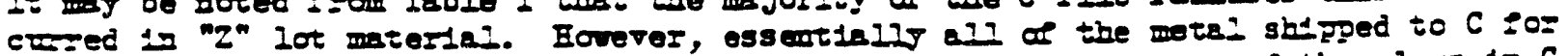
the past fer months bas bees of thes type, and the great majoraty of the sliss is $C$ Pilo at the presert time is probeibly Ieprocessed.

Extracted from HAPO 1955a, page Fb-6. 


\section{URANIUM SLUG RUPTURE EXPERIENCE - JUNE 1955}

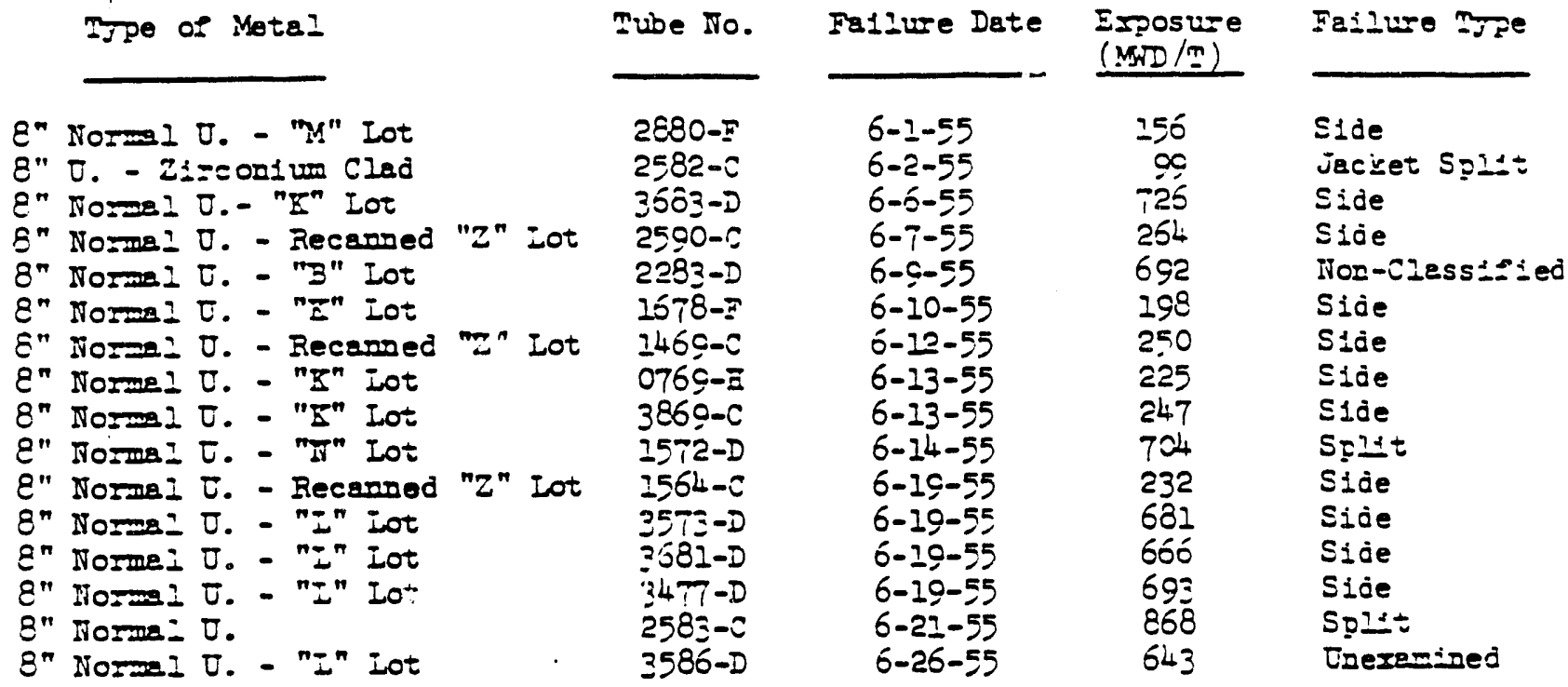

ALUMINUM-URANIUM-235 ALLOY SLUG RUPTURE EXPERIENCE

\begin{tabular}{|c|c|c|c|c|}
\hline The of Metel & mabe ro. & Faslure Dete & Exposure (MWD/tuje) & $\begin{array}{l}\text { Feinure } \\
\text { mpe }\end{array}$ \\
\hline $\begin{array}{l}\text { "O" MetaI } \\
\text { "J" Mete? } \\
\text { "J" MetaI }\end{array}$ & $\begin{array}{l}\frac{1293-C}{257 \overline{-}-\bar{E}} \\
3600-\mathrm{C}\end{array}$ & $\begin{array}{l}6-3-55 \\
6-1-55 \\
6-i 3-55\end{array}$ & $\begin{array}{r}113.2 \\
167.5 \\
03.2\end{array}$ & $\begin{array}{c}\text { jecket } \\
m \\
n\end{array}$ \\
\hline
\end{tabular}

Extracted from HAPO 1955b, page $\mathrm{Fb}-6$. 


\section{SLUG RUPTURE EXPERIENCE - JUNE-JULY 1955}

\begin{tabular}{|c|c|c|c|c|}
\hline Mope of Yetal & mebe No. & Faplare Date & $\begin{array}{l}\text { Exposire, } \\
\text { MND im }\end{array}$ & $\begin{array}{l}\text { Faslure } \\
\text { Mree }\end{array}$ \\
\hline 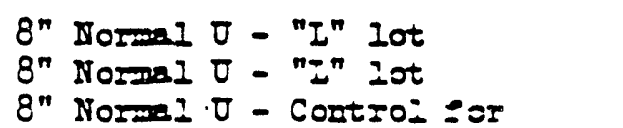 & $\begin{array}{l}24 \pi-D \\
3331-D\end{array}$ & $\begin{array}{l}6-26-55 \\
6-25-555\end{array}$ & $\begin{array}{l}709 \\
725\end{array}$ & $\begin{array}{l}\text { S1de } \\
\text { Stàe }\end{array}$ \\
\hline 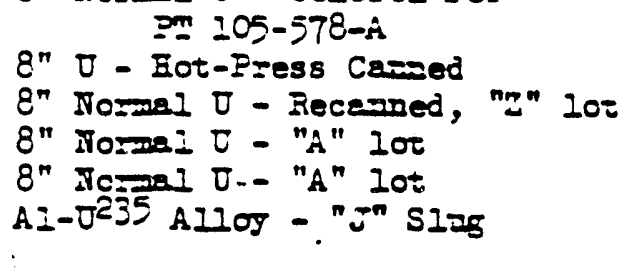 & $\begin{array}{l}2579-C \\
2276-6 \\
3880-C \\
2868-5 \\
3+2 C--29 \\
+222-2 R\end{array}$ & $\begin{array}{l}6-27-55 \\
7- \pm-55 \\
T-T-55 \\
7- \pm 7-55 \\
T= \pm j=55 \\
T=-3-55\end{array}$ & $\begin{array}{l}910 \\
407 \\
284 \\
617 \\
819 \\
135 \mathrm{MD} / \\
-0.00\end{array}$ & $\begin{array}{l}\text { Spift } \\
\text { Side } \\
\text { Side } \\
\text { Unciessi=1ed } \\
\text { Unciessi=1ed } \\
\text { Side }\end{array}$ \\
\hline 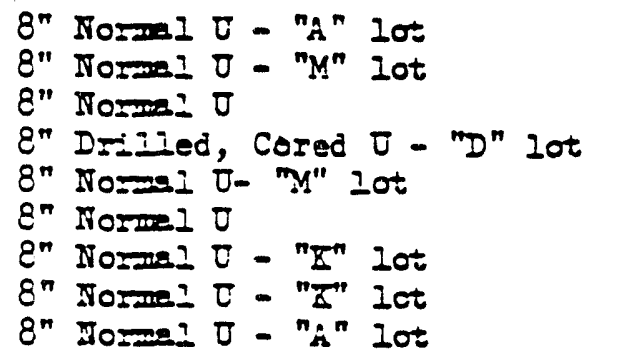 & 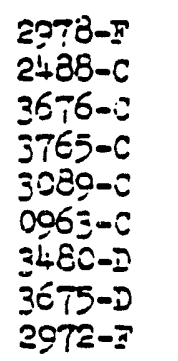 & $\begin{array}{l}T-25-55 \\
T-15-55 \\
T-18-55 \\
T-10-55 \\
T-10-55 \\
T-2 !+=5 \\
T-25-55 \\
T-25-55 \\
T-2 T-55\end{array}$ & $\begin{array}{l}678 \\
-78 \\
262 \\
505 \\
-71 \\
278 \\
559 \\
824 \\
673\end{array}$ & 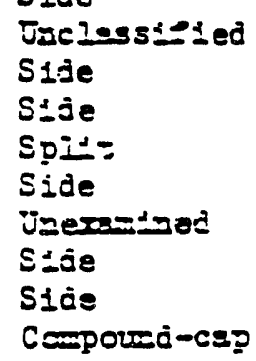 \\
\hline
\end{tabular}

Extracted from HAPO 1955c, page Fb-5. 


\section{SLUG RUPTURE EXPERIENCE - JULY-AUGUST 1955}

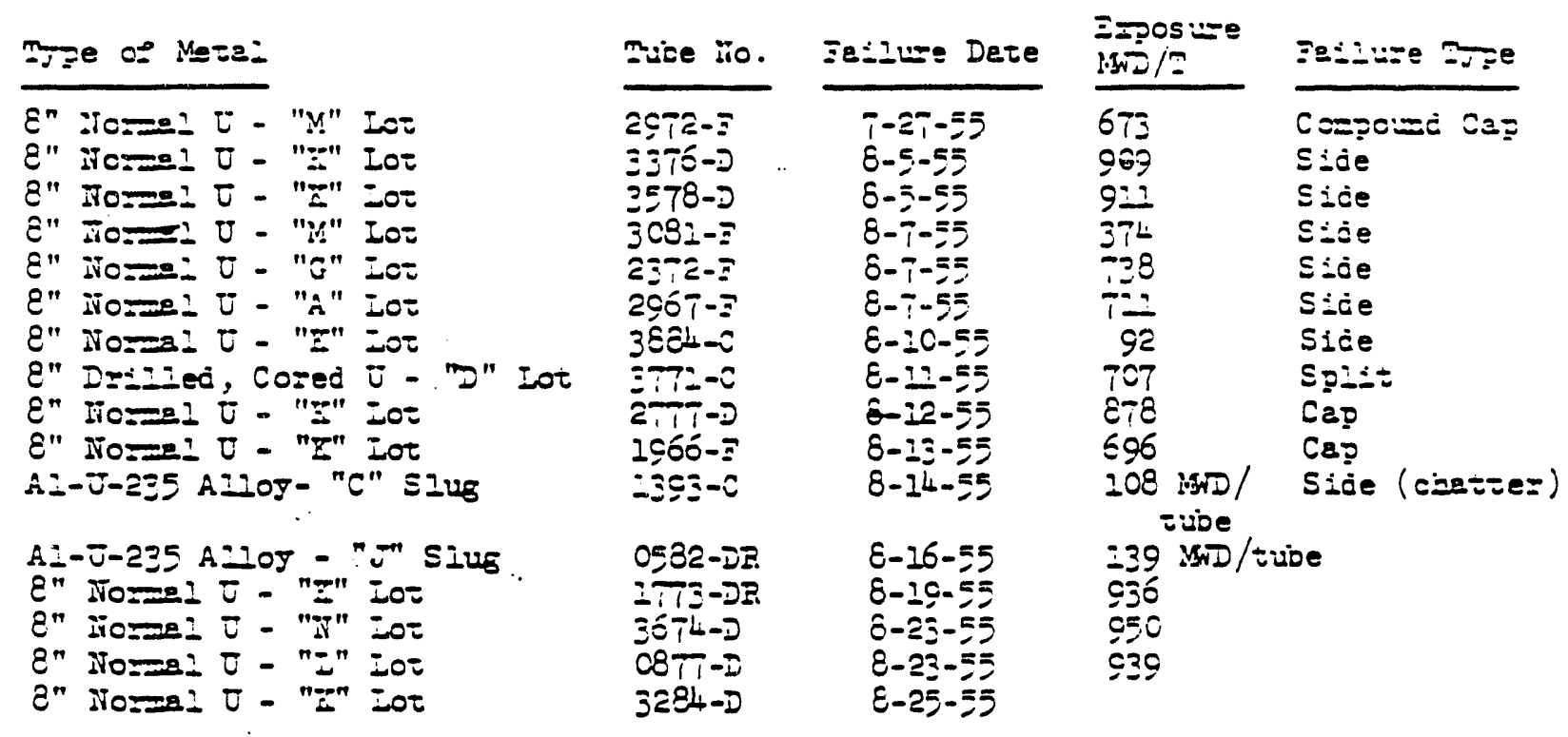

Extracted from HAPO 1955d, page Fb-11. 


\section{SLUG RUPTURE EXPERIENCE - AUGUST -SEPTEMBER 1955}

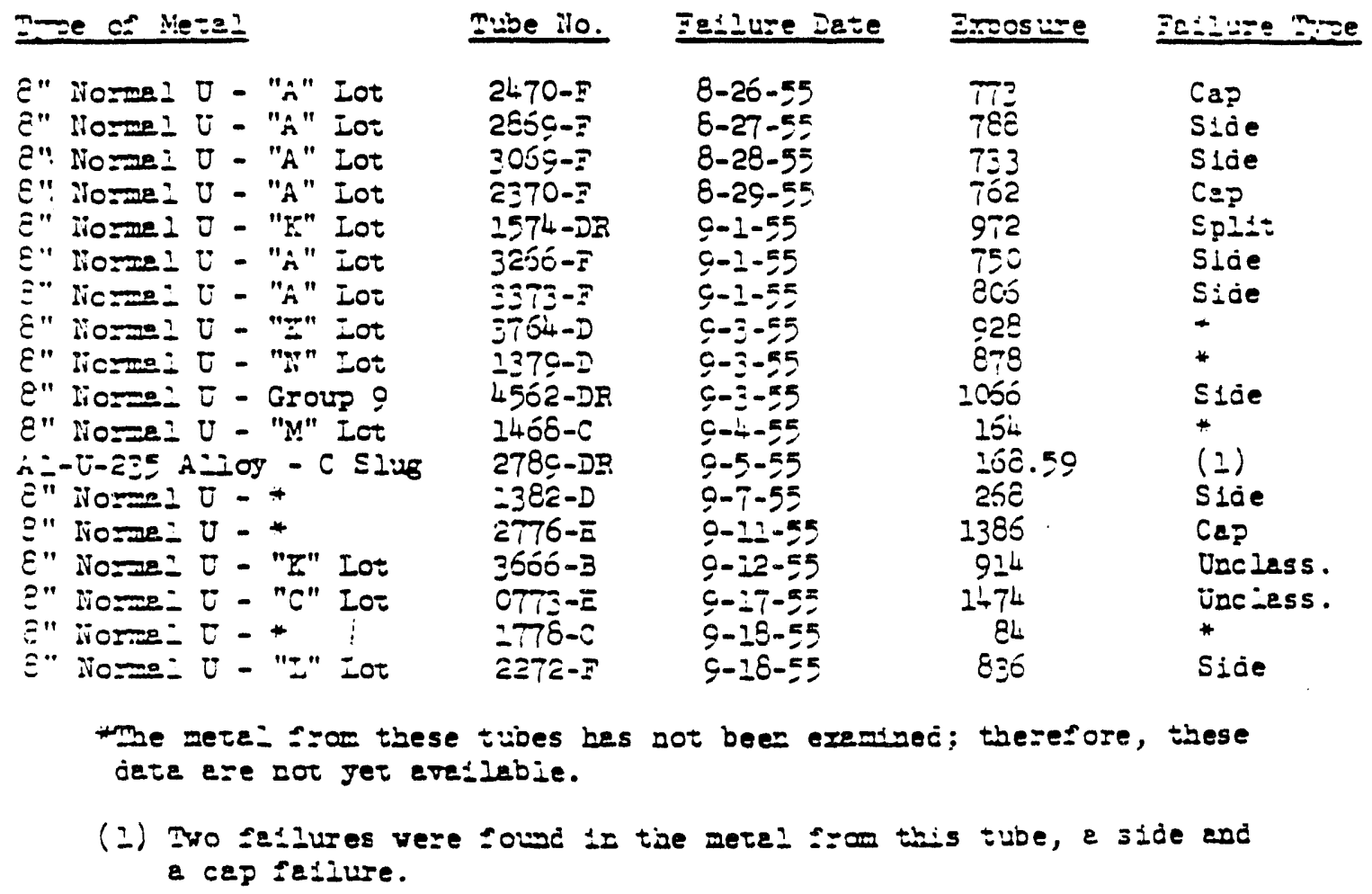

Extracted from HAPO 1955e, page Fb-7. 


\section{SLUG RUPTURE EXPERIENCE - SEPTEMBER-OCTOBER 1955}

ripe of Metal

> A $-0-235$ A $10 y$ - "J" Meta?

8n Norma 0 - "I" Lot

A $-0-235$ AIIOJ - "C" Metal

Q" Hormel U - "II" Lot

Q" Tora 1 - " "2" Lot

8" Tormeר 0 - "Y" Lot

8" NormeI 0 - "An Lot

Qn IJoreaI U - "I" Lot

Q" NozmeI U - "I" Lot*

Q" Hozene 0 - "K" Lot

8n Iormal 0 - " $\mathrm{n}^{n}$ Lot

8" No:20ii O - "K" Lot

4" Trosme? 0 - Cortsol =02 PI 105-570-A

4" NormeI 0 - Correl Ios P. 105-570-A

Q" $\mathrm{Ho=39} 0$

A-0-235 A רoy - "C" MetaI

Qn Hores O - "I" Lot*

8n Cored $U$

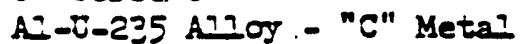

$M=0 t$

8" IoImaI D - "M" Lot
Tube No. Fatlure Date Proosure

$2462-c$

3170-F

O579-E

1081-D

$0969-C$

$0873-c$

1872-5

3773-F

107C-D

1268-D

$2271-5$

2768-D

207c-

$2780-7$

2886-

0470-C

1076-D

2368-D

$0651-$

337 -D

I 4 R-
$0-26-55$

9-28-55

$9-30-55$

10-3-55

$204-55$

$10-7-55$

10-7-55

$10-7-55$

$10-10-55$

10-10-55

10-12-55

$10-12-55$

$10-13-55$

20-14-55

10-14-55

$20-14-55$

$$
\begin{aligned}
& 10-17-55 \\
& 10-21-55 \\
& 10-22-55 \\
& 10-21-55 \\
& 10-22-55
\end{aligned}
$$

$152 \mathrm{MND} /$ tube

$829 \mathrm{MND} / \mathrm{m}$

$173 \mathrm{MWD} /$ tube

$393 \mathrm{MND} / \mathrm{m}$

$27 \mathrm{MND} / \mathrm{m}$

$200 \mathrm{MND} / \mathrm{m}$

$865 \mathrm{MHD} / \mathrm{m}$

$886 \mathrm{MHD} / \mathrm{m}$

$4 I 9 \mathrm{MND} / \mathrm{m}$

$478 \mathrm{MHD} / \mathrm{m}$

888 MWD/m

$5 T 4 \mathrm{MD} / \mathrm{T}$

$2547 \mathrm{MND} / \mathrm{m}$

$2484 \mathrm{MTD} / \mathrm{I}$

$255 \mathrm{MWD} / \mathrm{m}$

$24 \mathrm{MND} / \mathrm{tabe}$

$435 \mathrm{MWD} / \mathrm{m}$

I5J MWD/tade

$253 \mathrm{MWD} / \mathrm{m}$
Padlure mpe

Jacicet spl:t

cap

Cap

Side

Sice

Cap

Unciasst_ted

Siòe

Sice

Sice

SpIt

SElat

S돈

Uaerantned

Side

$S \pm d e$

(Chatertas)

Stce

Unera-taed

Tneraminec

Crexamined

Side

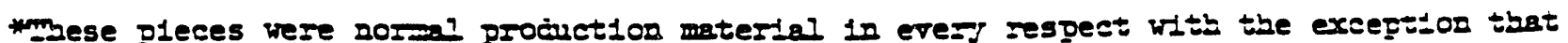
the uranium bad been cooled in air betreen the beta-transeorning and water quezai steps sor a period longer than normal.

Extracted from HAPO 1955f, page Fb-8. 


\section{SLUG RUPTURE EXPERIENCE - OCTOBER-NOVEMBER 1955}

\section{Dre of Metal}

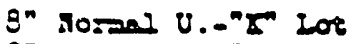

gn sosan U.."

En xceal 0.- $x^{n}$ Lot

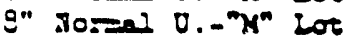

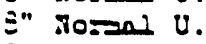

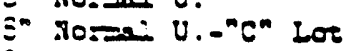

G" So: U.

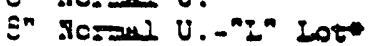

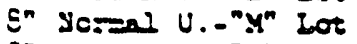

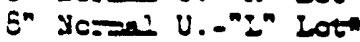

E" Tcr=a U.

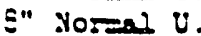

Sn Cored wist cripped U. ead pluss

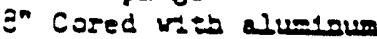
ead plides

E" VIo: $=2$ U. U.

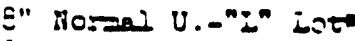

E" ilorai U. sube 10.

$0902-8$

$1069-c$

$0677-8$

$0982-8$

2779-5

$0462-8$

$3562-c$

0983-D

3985-B

$0782-D$

$3283-C$

$1364-6$

2879-B

2179-C

3291-E

1083-D

0682-8
Peine Date

20-32-55

21-5-55

21-6-55.

$212-55$

21-9-55

21-9-55

$21-20-55$

12-22-55

$21-27-55$

21-29-55

21-29-55

$12-24-55$

$21-25-55$.

22-26-55

12-26-55

21-28-55

12-28-55 aposure raslute spe

$362 \mathrm{NO} / 2 \mathrm{~g} / \mathrm{de}$

219 31da

320 s1da

343 S1de

960 Cap

189 S1de

21 Cep

376 s1de

332 S1de

394. Cap

265 Uneramlaed

282 Unernased

$398 \quad 9140$

128 Uneramined

337 Uneramined

436 Uaeramined

423 Uneromined

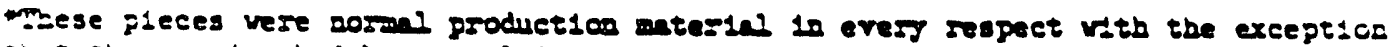

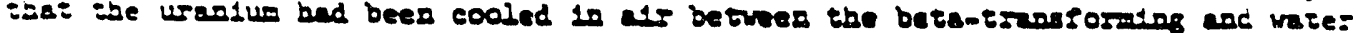
queaci steps :or a period looger than normal.

Extracted from HAPO $1955 \mathrm{~g}$, page $\mathrm{Fb}-7$. 


\section{SLUG RUPTURE EXPERIENCE - NOVEMBER-DECEMBER 1955}

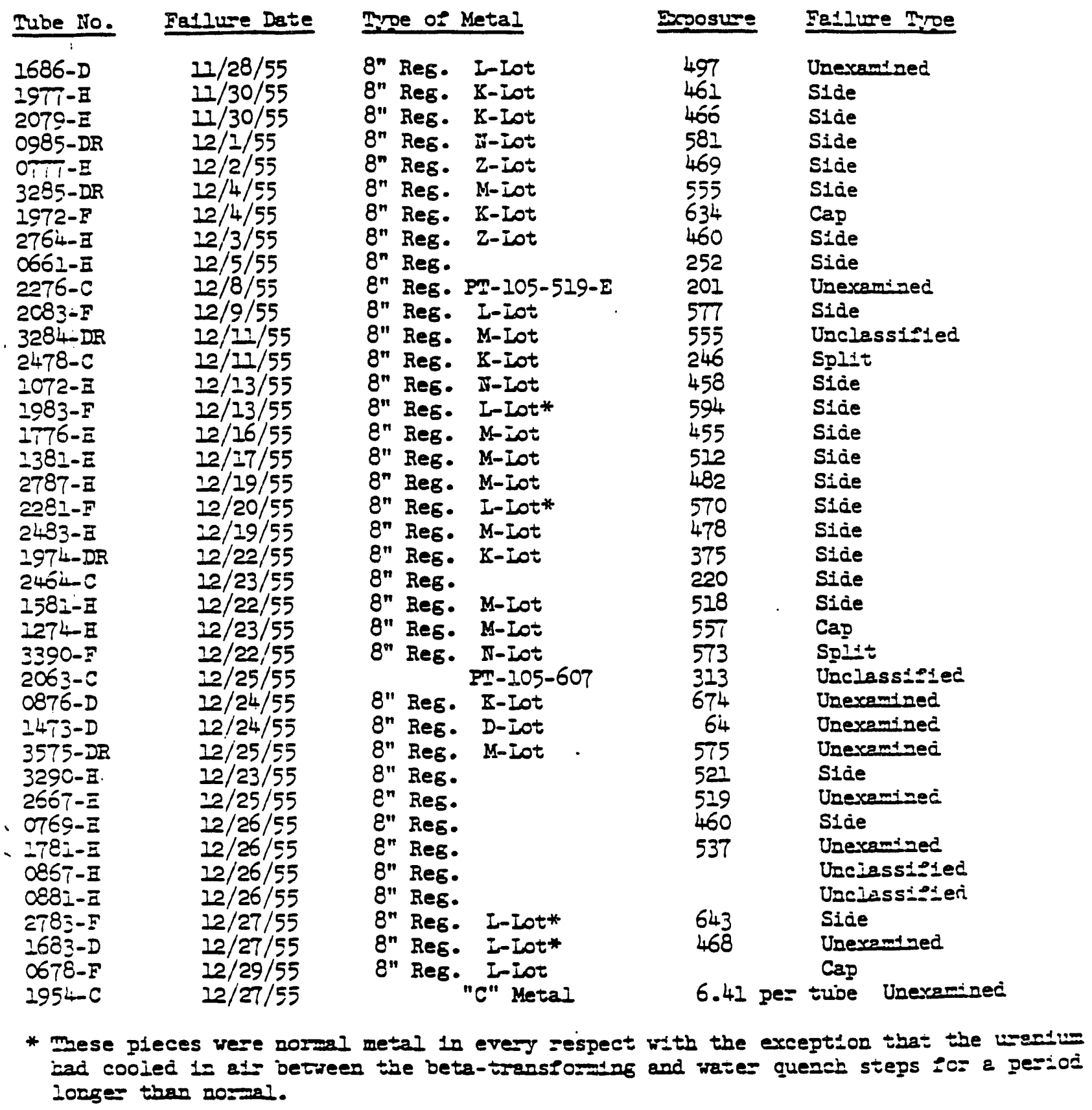

Extracted from HAPO 1956a, page Fb-8. 
APPENDIX $F$

FUEL-ELEMENT FAILURE DATA FOR 1956 


\section{RUPTURED SLUG TABULATION - JANUARY 1956}

Date Tube No. Iot No. Type Metal Bxposure Mype Failure

Begular Production Oraniom Ruptures

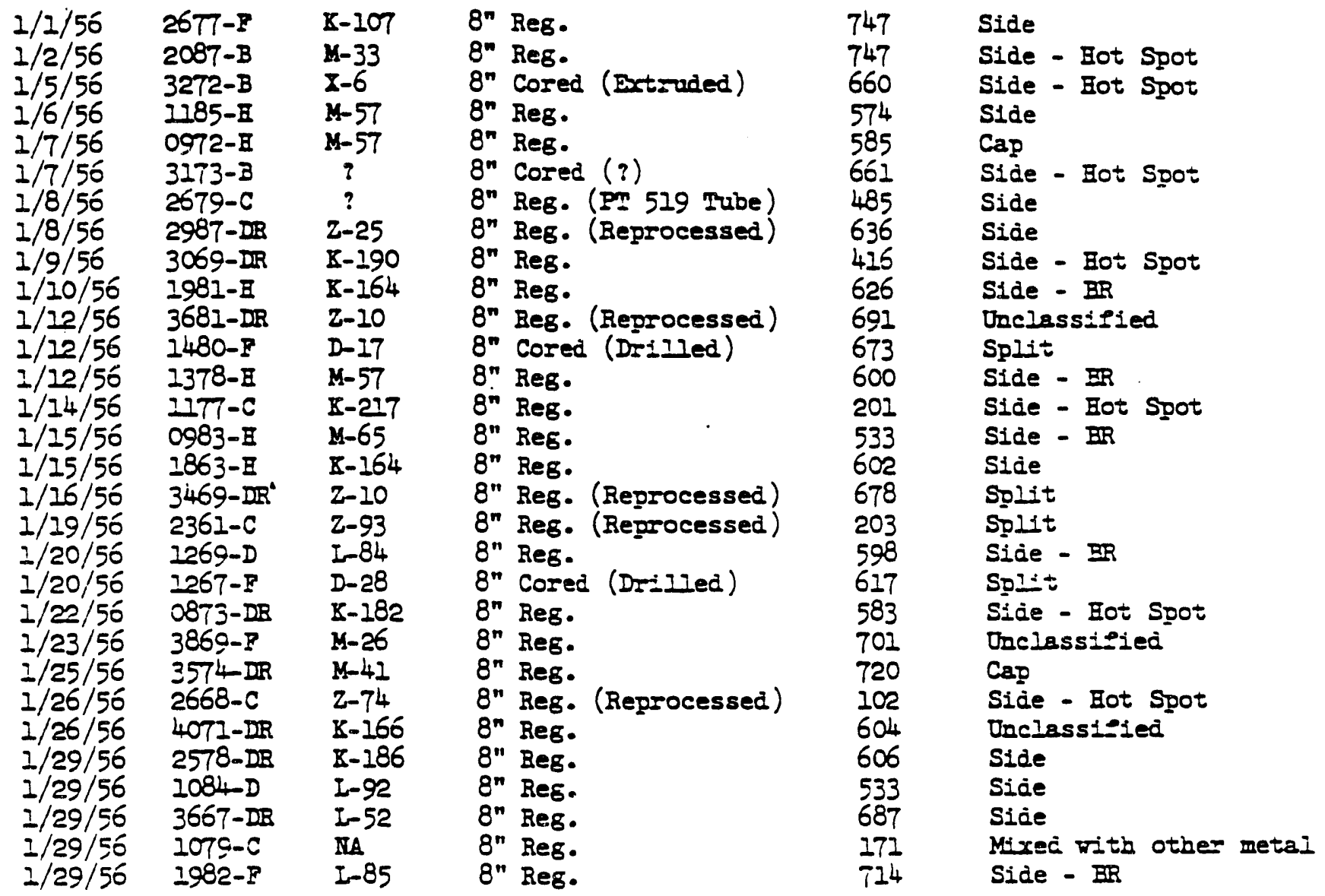

Prodaction Test and C Metal Ruptures
$1 / 3 / 56$ 2I64-C 4" Ire
4" Dre
PI 607-A
558
$1 / 4 / 56 \quad 2065-c$
PI 607-A
4 4is
$1 / 10 / 56 \quad 2073-c$
D-94
PI 603-A, Cored drialed 577
$1 / 16 / 56 \quad 2082-F \quad 8^{n}$ with crinped $U$. end pluss.
$1 / 18 / 56 \quad 4380-c$
PI 596 (Dingot)
C Metal
8445 Pieces**
54/tube J.F.
Completely Disintegratec Completely Disiatestazec Solit

Iegend and notes: BR - between Jibs

IA - not yet aveilable but should be later

? - unionorr

$\sqrt{F}$ - jeciret failure

* More examination necessary. Could be 8" regular.

Slde lailures not classifled as "hot spot" or "BR" could bave failed by the following mechanisms: pinhole penetration, "Iot Spot", "BR", unicoown mechanism.

Extracted from HAPO 1956b, page Fb-17. Also reported in ANDERSON 1956a. 


\section{RUPTURED SLUG TABULATION - FEBRUARY 1956}

\begin{tabular}{|c|c|c|c|c|c|}
\hline te & abe Ilo. & ot No. & Pe Metal & Esposure & woe Feslure \\
\hline $\begin{array}{l}? / 3 / 56 \\
? / 4 / 56 \\
? / 4 / 56 \\
? / 4 / 56 \\
? / 4 / 56 \\
? / 4 / 56 \\
? / 4 / 56 \\
? / 5 / 56 \\
? / 6 / 56 \\
? / 8 / 56 \\
? / 9 / 56 \\
? / 10 / 56 \\
? / 21 / 56 \\
? / 79 / 56 \\
? / 21 / 56\end{array}$ & $\begin{array}{l}3581-D \\
3666-D R \\
0967-B \\
1482-E \\
2184-E \\
2790-E \\
4164-E \\
3763-D \\
1063-E \\
3665-D R \\
1965-C \\
1966-E \\
4078-B \\
3282-D R \\
3573-D R\end{array}$ & 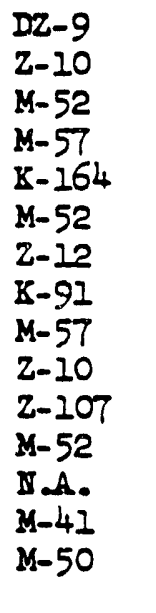 & 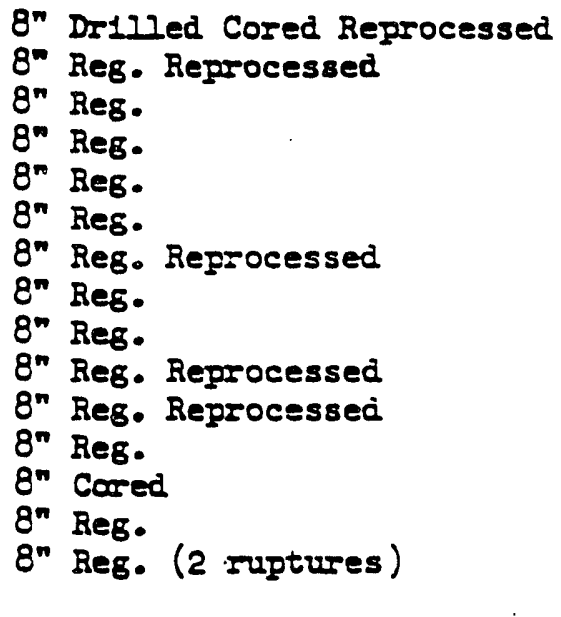 & $\begin{array}{l}765 \\
613 \\
694 \\
255 \\
651 \\
737 \\
752 \\
655\end{array}$ & $\begin{array}{l}\text { Side - Eot Spot } \\
\text { Side } \\
\text { Cap } \\
\text { Cap } \\
\text { Spift } \\
\text { Cap } \\
\text { Cap } \\
\text { li.A. } \\
\text { Jaciet Cracied } \\
\text { Side - Eot Spot } \\
\text { Side - Eot Spot } \\
\text { Side } \\
\text { Sice } \\
\text { Cap } \\
\text { I Spiit } \\
\text { I unciessi-1ed }\end{array}$ \\
\hline $\begin{array}{l}/ 12 / 56 \\
/ 22 / 56 \\
/ 13 / 56 \\
/ 13 / 56 \\
124 / 56 \\
116 \% / 56 \\
/ 16 / 56 \\
/ 21 / 56 \\
/ 21 / 56 \\
124 / 56 \\
124 / 56 \\
125 / 56 \\
121 / 56 \\
121 / 56\end{array}$ & $\begin{array}{l}2680-E \\
2363-E \\
2584-F \\
3186-F \\
4274-E \\
1779-D B \\
2890-E \\
2489-E \\
3961-E \\
3467-D R \\
3167-D \\
1769-E \\
1475-D \\
3758-D\end{array}$ & $\begin{array}{l}Z-22 \\
M-52 \\
I-89 \\
I-90 \\
M-41 \\
K-182 \\
I-A . \\
Z-60 \\
Z-40 \\
I-A . \\
I-A . \\
M-72 \\
I-84 \\
M-24\end{array}$ & $\begin{array}{l}8^{n} \text { Reg. Reprocessed } \\
8^{n} \text { Reg. } \\
8^{n} \text { Reg. } \\
8^{n} \text { Reg. } \\
8^{n} \text { Reg. } \\
8^{n} \text { Reg. } \\
8^{n} \text { Reg. } \\
8^{n} \text { Reg. Reprocessed } \\
8^{n} \text { Reg. } \\
8^{n} \text { II.A. } \\
8^{n} \text { Reg. } \\
8^{n} \text { Reg. } \\
8^{n} \text { Res. } \\
8^{n} \text { Reg. }\end{array}$ & $\begin{array}{l}705 \\
683 \\
744 \\
723 \\
622 \\
638 \\
709 \\
550 \\
597 \\
11 . A . \\
11.4 . \\
559 \\
661 \\
579\end{array}$ & 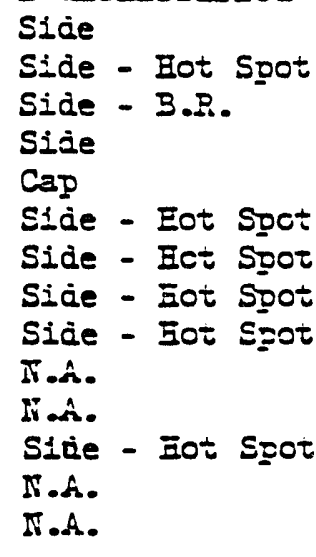 \\
\hline
\end{tabular}

P=oduction Test and C Metal Rupteres
$2 / 2 / 56 \quad 0891-c$
$2 / 2 / 56 \quad 2792-E$
$2 / 3 / 56 \quad 0990-\bar{B}$
$2,1=156 \quad 2986-C$
$2 / 27 / 56 \quad 3395-C$
$2 / 23 / 56 \quad 2163-c$
C Metal
C Metel
C Metal.
II 593, 8" Reg. Control C Metal
PE 607, 8" Reg.
(Specer for Ire)
$23 /$ - ivide
$88 /$ tribe
$178 /$ tive
568
$118 /$ iube
596
Eese and
Jackez C-acived
Jacire: Czacired
Side
liof.
SDlut
Legead and Notes:
B.R. - between zibs
II A. - not yet aratiable but should be later.

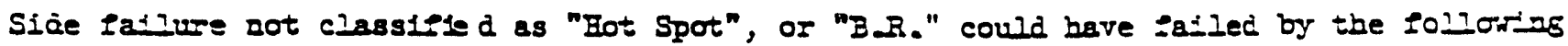

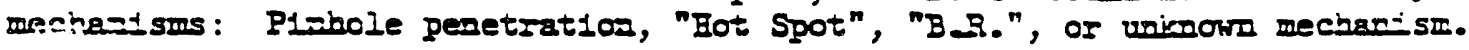

Extracted from HAPO 1956c, page Fb-18. Also reported in SOWARDS 1956a. 


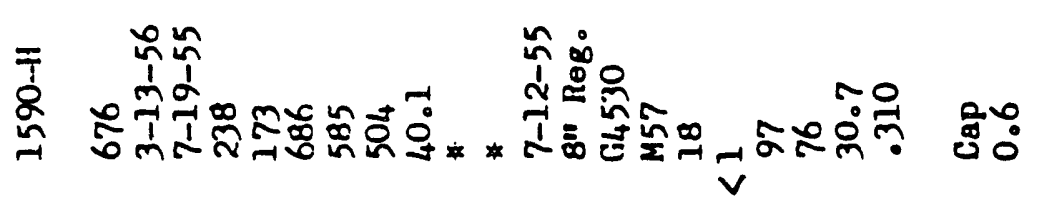

离离

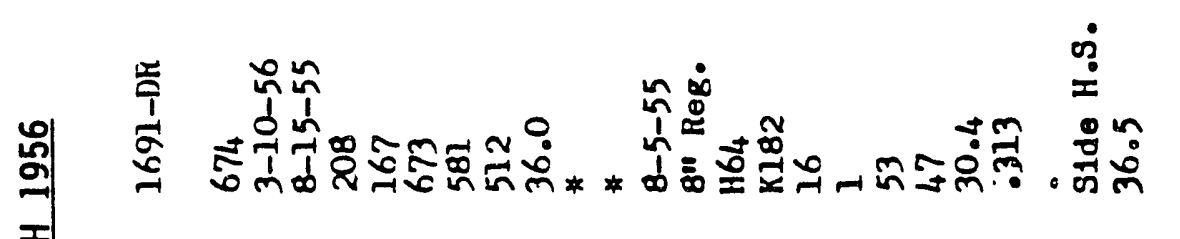

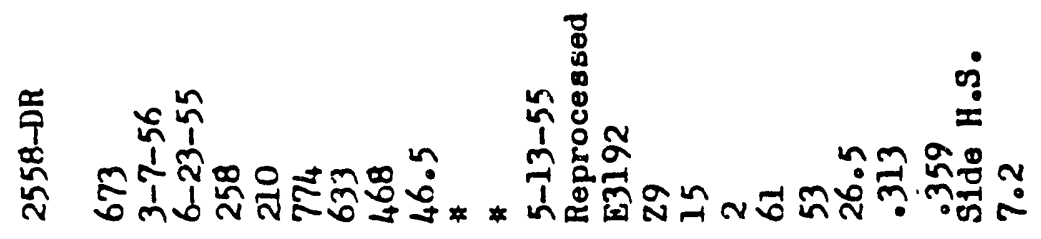

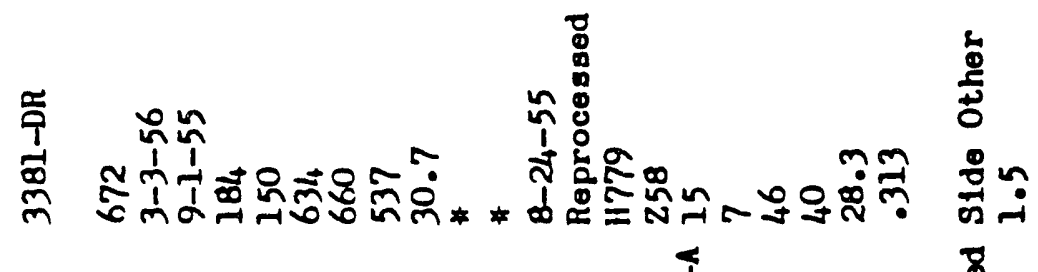

结

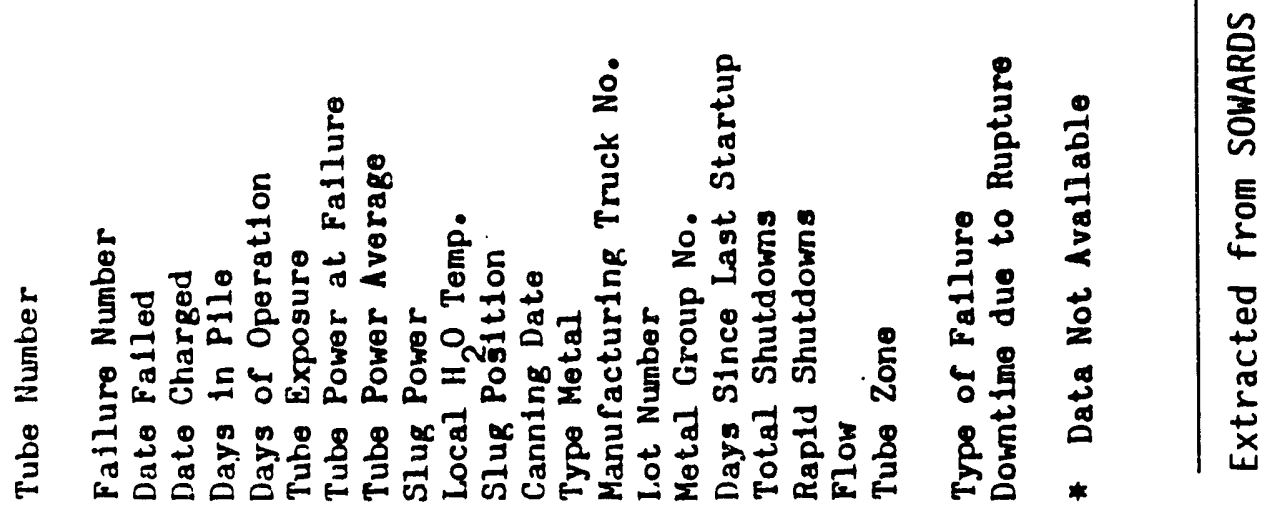




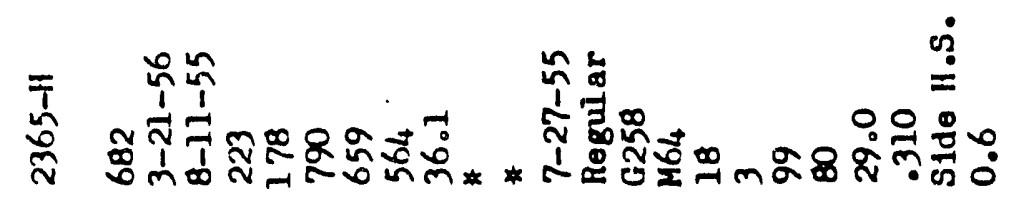

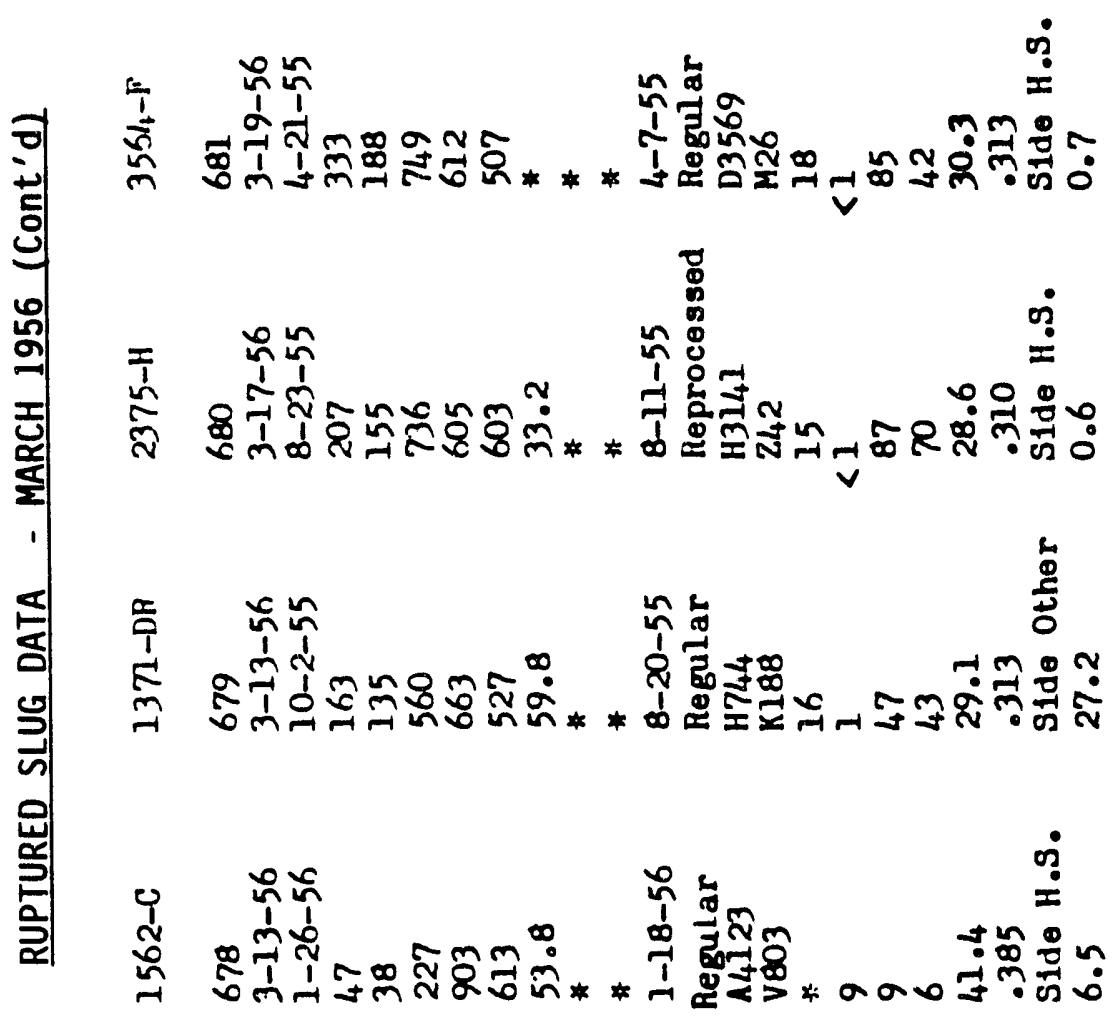

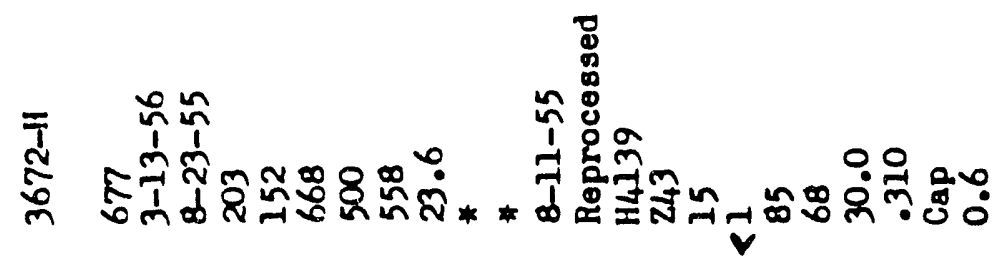

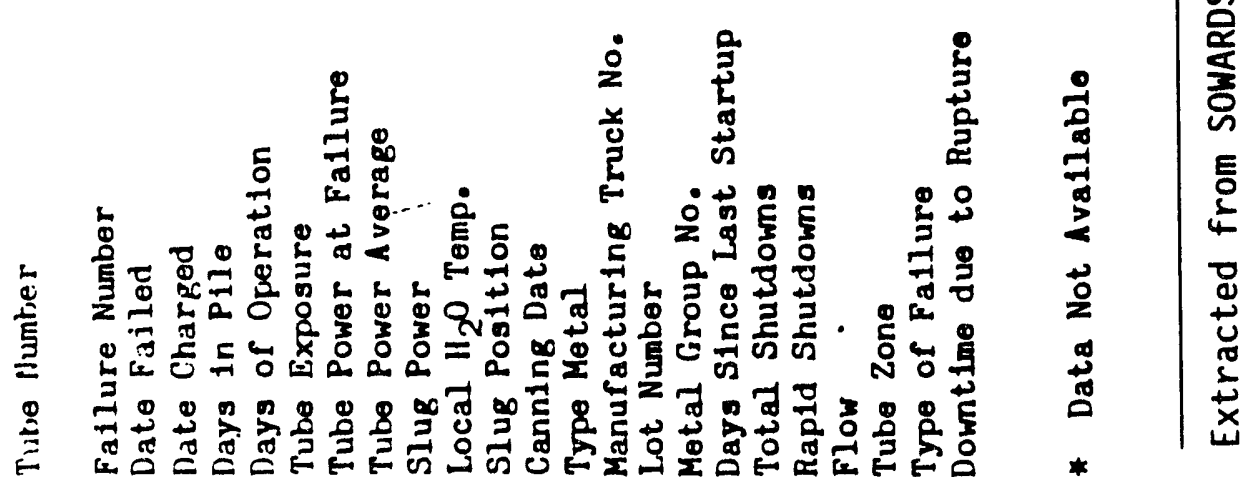




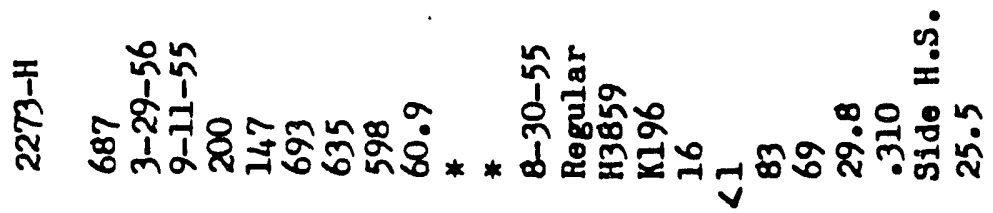

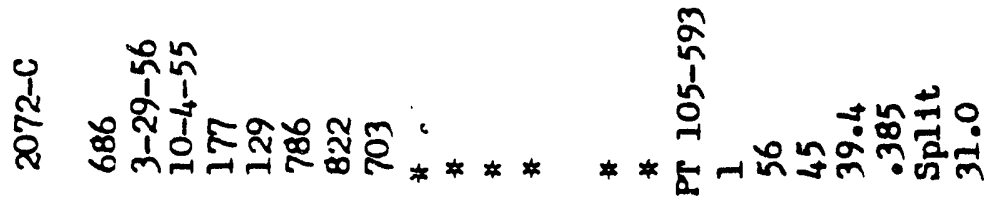

₹

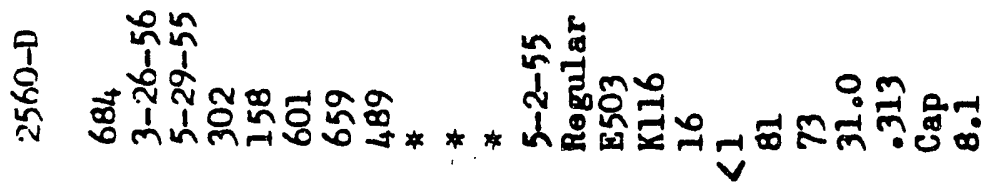

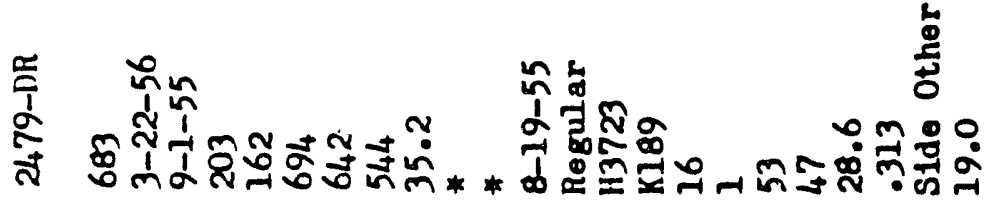




\section{RUPTURED SLUG TABULATION - MARCH-APRIL 1956}

Fatiure

Date

Tube No. Iot No. Iype Metal

$3 / 25 / 56$

ij/27/56

\section{0-D}

I- 126

8n Beg.

3884-E

$\mathrm{B}-194$

8n Beg. (2

$3 / 29 / 56$

$4 / 6 / 56$

22?3-E

$336 T-D$

2IT2-II

$\mathrm{B}-196$

D-14-A

ruptured pleces)

$4 / 7 / 56$

$2003-$ I

2970- DR

4/14/50

I0,2-C

$4 / 23 / 50$

3288- I

$1360-D R$

$4 / 24 / 56$

$4 / 24 / 56$

0975-C

$4 / 24 / 56$

$1387-B$

B- 290

8n Bes.

8n Sored

X -139

X-187

M- 205

M-69

Z- 148

8" Reg.

8" Reg.

8" Reg.

8" Res.

8" Reg.

8" Res., Reprocessed

II.A. 8" Reg.

8" Cored

Dxposure mpe Eatiure

Production Test Ruprures

3/29/56 2072-C N.A.

8" Reg. (PT 503-A conzrol pieces)

$\begin{array}{ll}601 & \text { Cap } \\ 682 & \text { I Split-t=ansterse } \\ & 1 \text { side-ES } \\ 693 & \text { Side-ES } \\ 729 & \text { I.A. } \\ 736 & \text { Side-ES } \\ 780 & \text { Cap } \\ 731 & \text { Side-ES } \\ 276 & \text { Side-ZS } \\ 795 & \text { Side-ES } \\ 786 & \text { Split-I-ansvezse } \\ 158 & \text { Side-ES } \\ 842 & \text { Side-ES }\end{array}$

Legend:

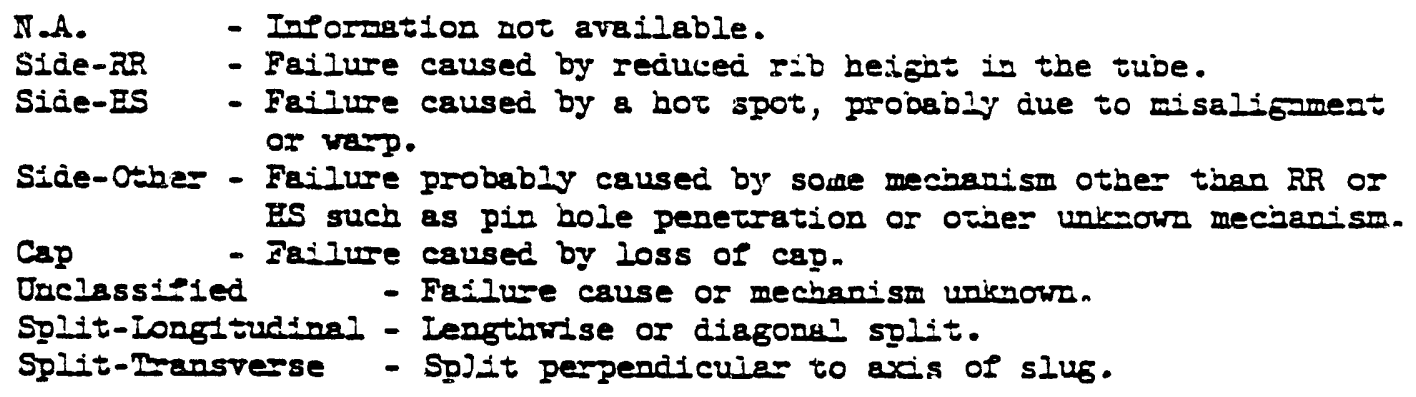

Extracted from HAPO 195Ed, page Fb-6. Also reported in SOWARDS $1956 \mathrm{c}$. 


\section{RUPTURED SLUG TABULATION - APRIL-MAY 1956}

Paslore Date

Dase

$4 / 28 / 56$

i $4 / 29 / 56$

$5 / 5 / 56$

$5 / 5 / 56$

$5 / 5 / 56$

$5 / 6 / 56$

$5 / 7 / 56$

$5 / 21 / 56$

$5 / 12 / 56$

$5 / 19 / 56$

$5 / 22 / 56$
Trube Io. Iot Io. Mroe Netal

1973-Dis

3585-

$1767-C$

1087-D

3T7-3

$0986-200$

2168-c

0,90- 201

$1877-$ I

$0986-B$

$2587-\pi$
K-190

I-226

2-120

I-84

D-78

I-279

K-228

$x-55$

u-78

D- 15

K-289 8n Rer.

8" Res.

8" Bes., Reprocessed

8" Reg. (5j-in)

8" Cored

8" Res.

8n Reg.

8" Res.

8" Res.

8n Cored

8" Reg.
Doosure mipe Patlice

795 Splet-transterse

595 Splut-1005-tudenal

196 Spl1t-10n5-tudena?

792 Side-othe:

840 Sple- Lonf-tudtar

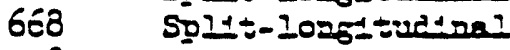

198 SIOE-ES

753 Spltt-10ng士tudton

700 Cap

604 S1de-other

22 side-othe=

Psodnct1on mest Ruptures:

5/23/56 1372-D I.A. B" SOL1d (FE 601-A- $564 \quad$ I.A.

Iegend: 53-35)

I.A. - Ineorzation not evetiable.

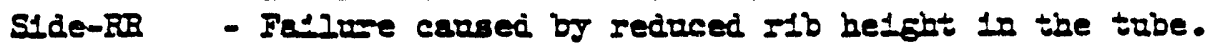

Stde-ES - Feslure caused by a hot spot.

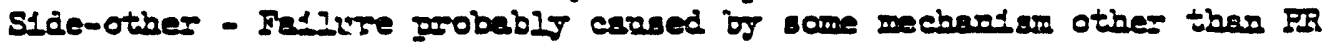
or Es such as plo hole penetration or other unicnown mechastem.

Cap - Fallare caused by 1088 of cap.

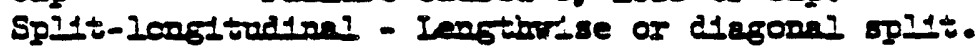

Split-transterre - Split normal to axds of alus.

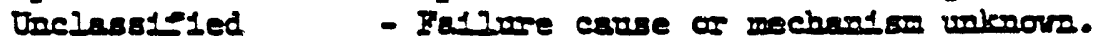

Extracted from HAPO 1956e, page Fb-6. Also reported in SOWARDS 1956d. 


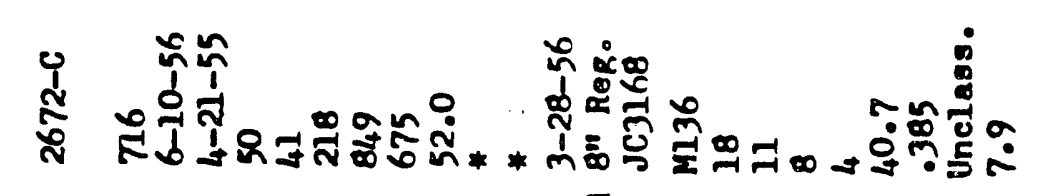

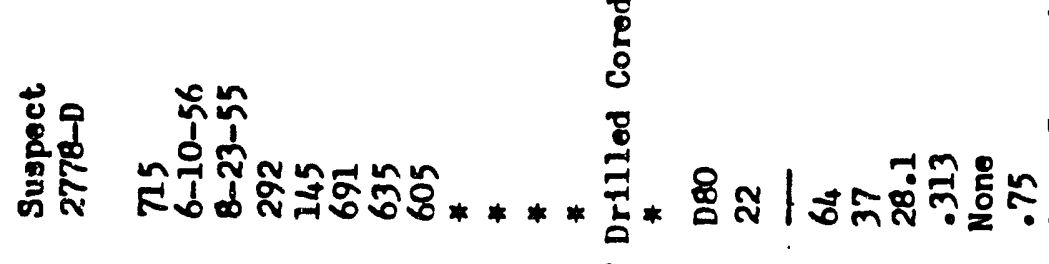

峉

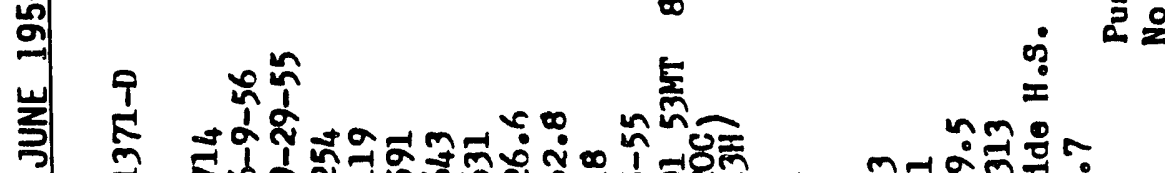

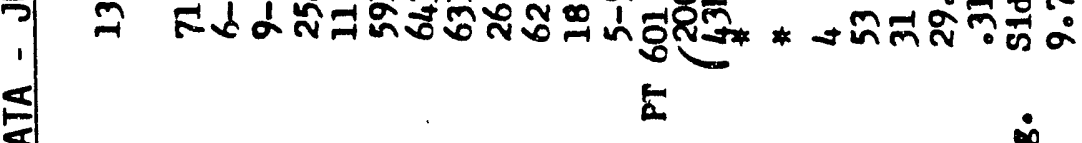

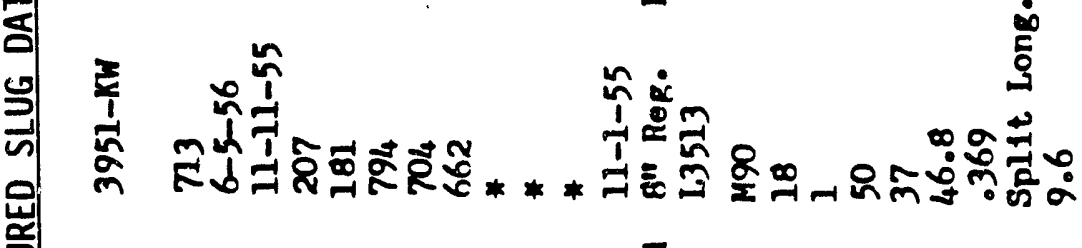

空)

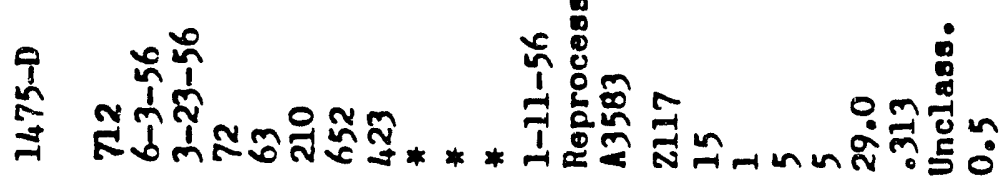

(⿻)

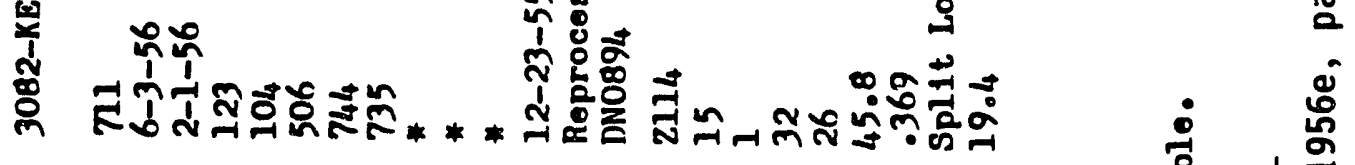

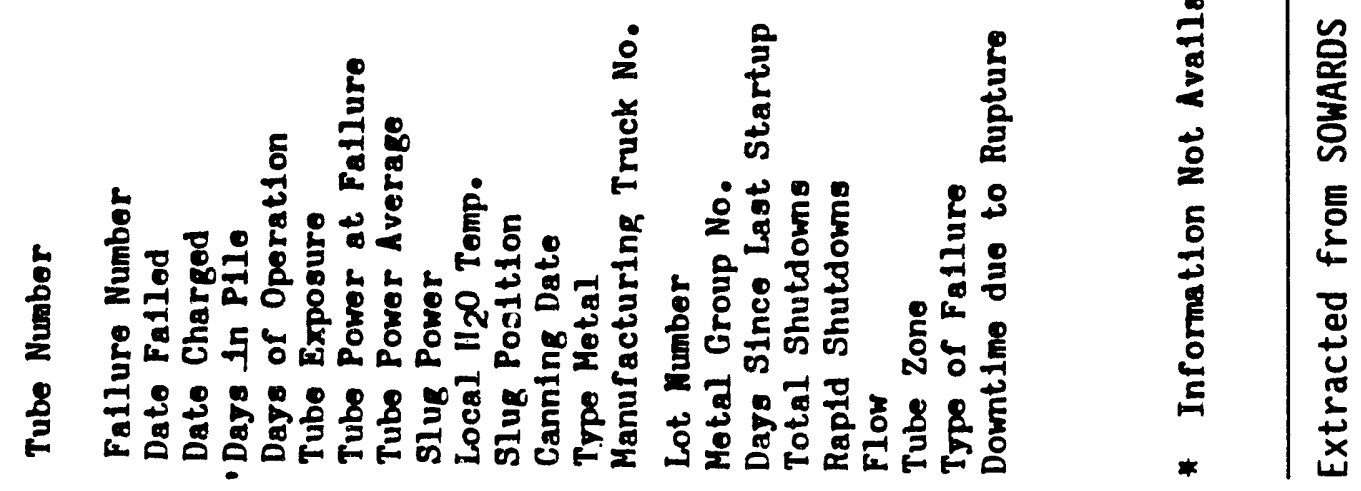




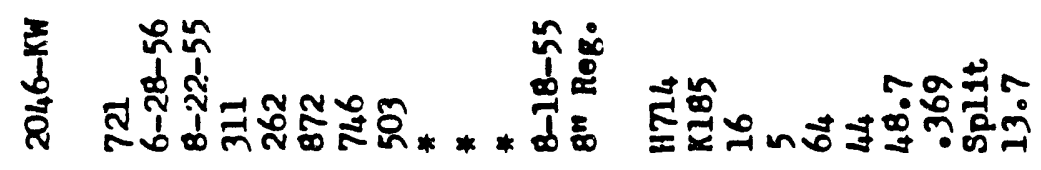

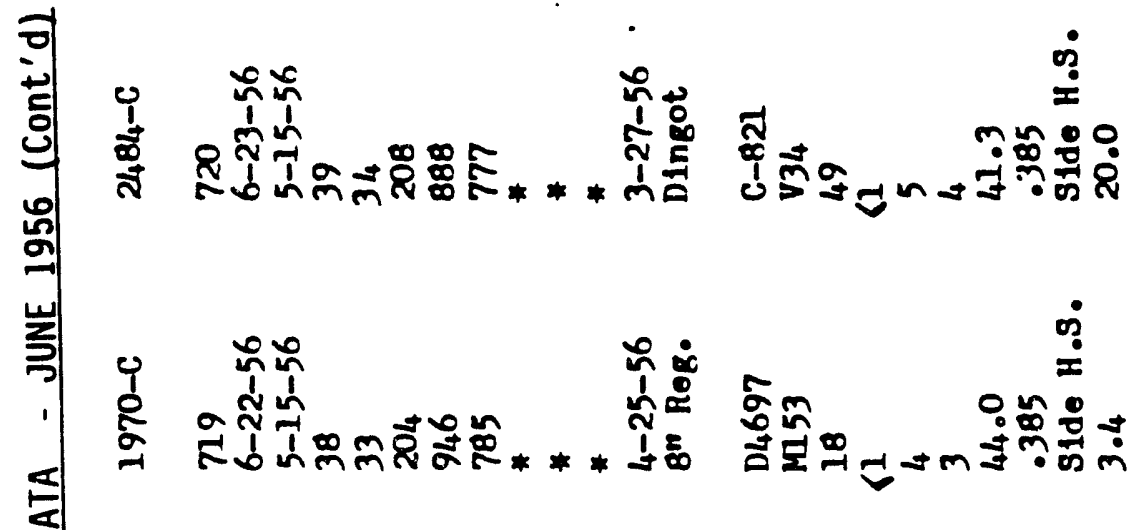

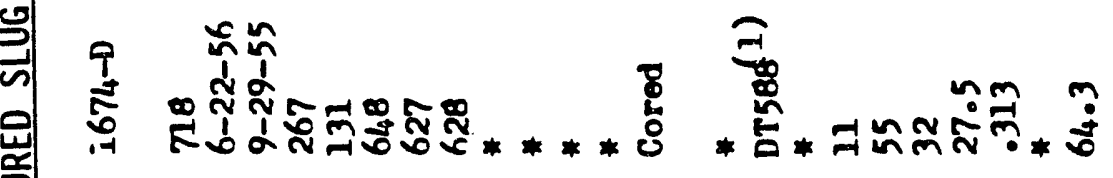

言

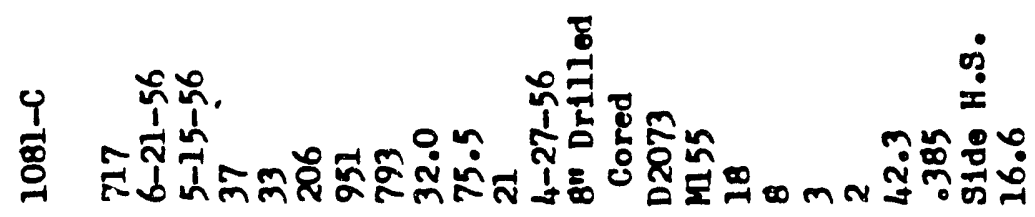

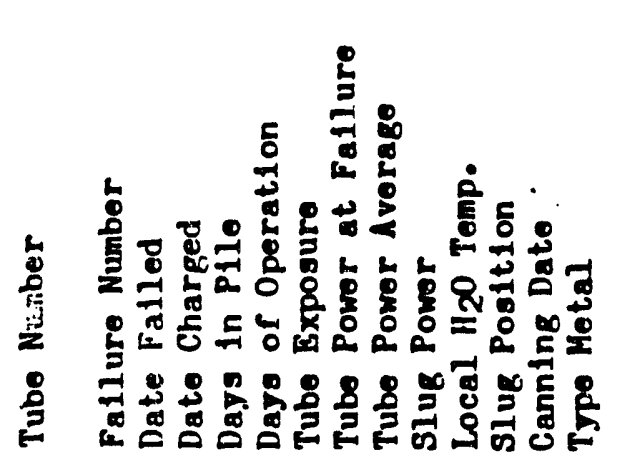

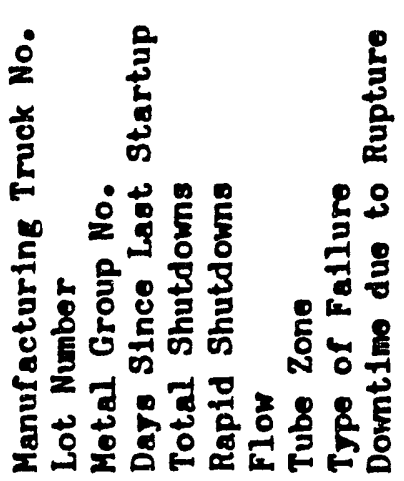

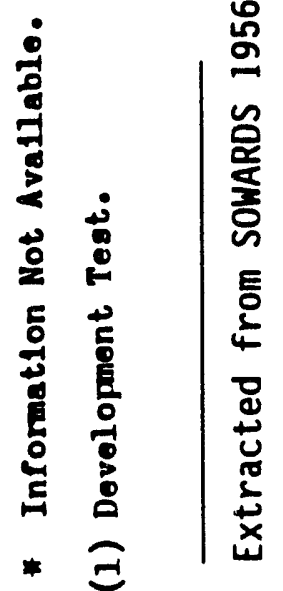


RUPTURED ALUMINUM-URANIUM-235 SLUG - JUNE 1956

Tube Number

Fatlure Nuber

Date Discharged

Date Charged

Days in Plile

Dars of Operation

Tube Exposure U-235 Depletion

Tube Expoaure MWD/Tube/

Tube Power Near Discharge

Tube Power Average

Siug Power

Iocal $\mathrm{H}_{2} \mathrm{O}$ Temp.

Slug Position with Reapect to Upstream Pleces

Lot \& Plece Number

Type of Faflure

Downtime due to Failure

* Data Not Available.

Extracted from SOWARDS 1956e, page 4.
2155-C

$0-65$

6-22-56

$5-15-56$

38

34

23.26

1012.61

656

681

$*$

*

13

Side H.S.

.5 


\section{RUPTURED SLUG TABULATION - JUNE-JULY 1956}

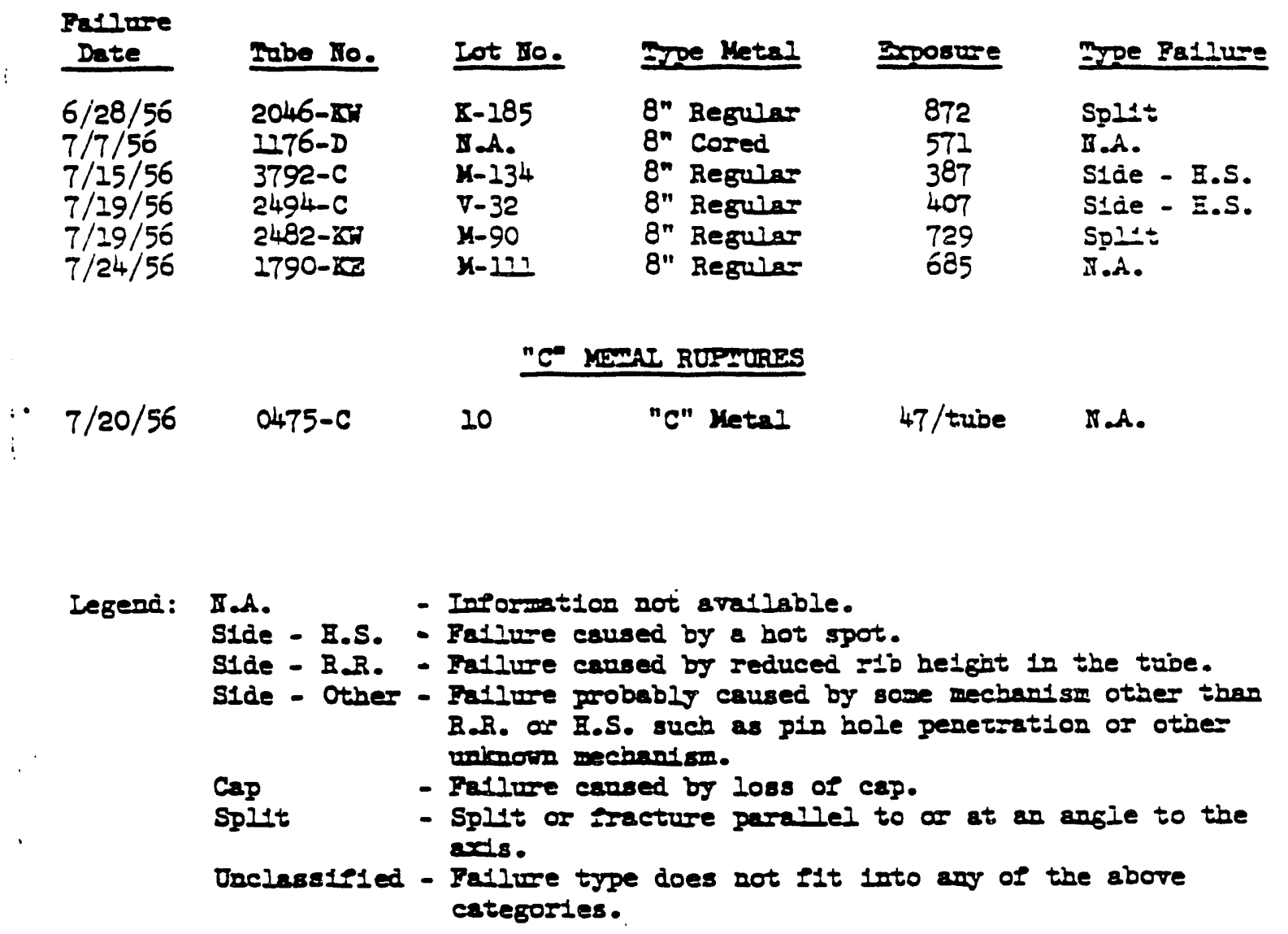

Extracted from HAPO 1956f, page Fb-7. Also reported in BLOOMSTRAND 1956a. 


\section{RUPTURED SLUG TABULATION - JULY-AUGUST 1956}

\begin{tabular}{|c|c|c|c|c|c|c|}
\hline Fo1 Iure Date & Tube & No. & Iot INo. & Mype Metal & Exposure & Mroe Fat luse \\
\hline $\begin{array}{l}7 / 27 / 56 \\
7 / 29 / 56 \\
8 / 4 / 56\end{array}$ & $\begin{array}{l}26 \pi \\
3457 \\
0370\end{array}$ & & $\begin{array}{l}z-289 \\
I-40 \\
z-36\end{array}$ & $\begin{array}{l}\text { 8n Regular } \\
\text { 8n Regular } \\
\text { 8n Reguiar - }\end{array}$ & $\begin{array}{l}334 \\
813 \\
839\end{array}$ & $\begin{array}{l}\text { Side - Other } \\
\text { S1de - IS } \\
\text { S1de - IS }\end{array}$ \\
\hline $8 / 4 / 56$ & 2784 & $-\infty$ & $\mathrm{K}-259$ & 8" Begalar & 728 & $\begin{array}{l}\text { Splat - } \\
\text { Lons-tudtral }\end{array}$ \\
\hline $\begin{array}{l}8 / 6 / 56 \\
3 / 17 / 56 \\
3 / 18 / 56\end{array}$ & $\begin{array}{l}0568 \\
3556 \\
2855\end{array}$ & & $\begin{array}{l}M-155 \\
z-110 \\
z-25\end{array}$ & $\begin{array}{l}\text { 8" Regular } \\
\text { 8" Regilar } \\
\text { 8" Regular - } \\
\text { Reprocessed }\end{array}$ & $\begin{array}{l}418 \\
767 \\
877\end{array}$ & $\begin{array}{l}\text { Onclassiz1ed } \\
\text { Side - as } \\
\text { S1de - IS }\end{array}$ \\
\hline $\begin{array}{l}8 / 20 / 56 \\
8 / 22 / 56\end{array}$ & $\begin{array}{l}4783 \\
0683\end{array}$ & $\begin{array}{l}-x \\
-C\end{array}$ & $\begin{array}{l}\text { R-247 } \\
\text { I.A. }\end{array}$ & $\begin{array}{l}g^{n} \text { Regular } \\
\text { g" Regular }^{\prime \prime} \text { Res }\end{array}$ & $\begin{array}{l}730 \\
437\end{array}$ & $\begin{array}{l}\text { TI.A. } \\
\text { Side - E.S. }\end{array}$ \\
\hline Legend: & & & & & & \\
\hline 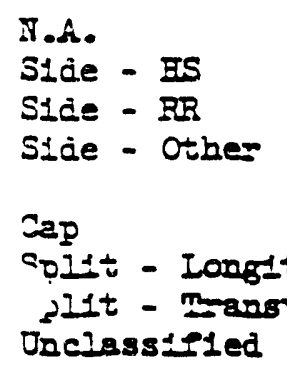 & \multicolumn{6}{|c|}{ 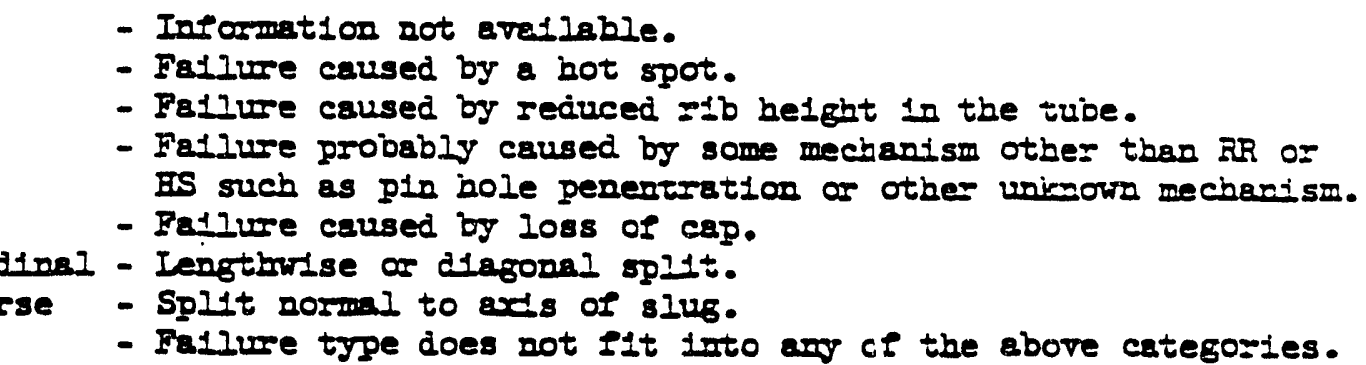 } \\
\hline
\end{tabular}

Extracted from HAPO $1956 \mathrm{~g}$, page Fb-6. Also reported in BLOOMSTRAND 1956a. 
RUPTURE EXPERIENCE - AUGUST-SEPTEMBER 1956

Production Ruptured Slug Tabulation

\begin{tabular}{|c|c|c|c|c|c|}
\hline$\frac{\text { Falluro Dato }}{1}$ & rube No, & Lot $\mathrm{NO}_{3}$ & Dep inen & Trporar & $\begin{array}{l}\text { Irpo } \\
\text { Pedine }\end{array}$ \\
\hline $6-30-56$ & $0688-c$ & $x-155$ & 80. & 490 & Onoleas. \\
\hline $9-1-56$ & $4872-16$ & 20127 & on Rog. & 657 & R.A. \\
\hline $7-2-56$ & $45790-100$ & $2-23$ & $\begin{array}{l}\text { En Rog. } \\
\text { Roprocesead }\end{array}$ & 605 & $\begin{array}{l}\text { Spltt } \\
\text { londtudinal }\end{array}$ \\
\hline $3-2-56$ & $4573-0.6$ & $2-152$ & $\begin{array}{l}\text { bu Rog. } \\
\text { Roprocieand }\end{array}$ & 560 & $\begin{array}{l}\text { Splet } \\
\text { Longterdinal }\end{array}$ \\
\hline$\frac{7-2-56}{i}$ & $47 \pi-\infty$ & $x-230$ & on Rag. & 664 & $\begin{array}{l}\text { Sputt- } \\
\text { longltrdinal }\end{array}$ \\
\hline $9-4-56$ & $4372-15$ & $2-219$ & $\begin{array}{l}\text { En Reg. } \\
\text { Raprocesend }\end{array}$ & $6 \%$ & $\begin{array}{l}\text { Spldt- } \\
\text { Longltudinal }\end{array}$ \\
\hline $3-6-56$ & $1587-H$ & $2-137$ & on Reg. & 376 & siderts \\
\hline 56 & $2788-\sqrt{8}$ & $x-24,9$ & on Be. & 743 & 31do-fis \\
\hline $9-24-56$ & $4870-103$ & $2-127$ & $\begin{array}{l}\text { 8n Rag. } \\
\text { Repiocesead }\end{array}$ & 67 & Unclase. \\
\hline -16-56 & $2287-c$ & $\nabla-816$ & 8n Rog. & 248 & na. \\
\hline $9-27-56$ & $2486-c$ & $x-335$ & 8n Rog. & 363 & Stdm-s \\
\hline \multicolumn{6}{|c|}{ Froduction Tesic Ruotures } \\
\hline $3-x-56$ & $-4863-10 B$ & $2-144$ & $\begin{array}{l}\text { 8n Rese } \\
\text { Beprocosad }\end{array}$ & 479 & Splt \\
\hline $2-23-56$ & $-1080-c$ & $\Sigma-276$ & 8n Ras. & 836 & na. \\
\hline
\end{tabular}

- 1706 Corroaion study Tube, PI 105-550-ir

$\rightarrow$ T $105-6154-63 \mathrm{MT}$ Control Tubo for IEE Production Tost.

Logond:

N.A.

Sllo - HS

jlue - RR

3 - Oener

idtl-Longltu-

innal

Spl1t-Tranavere Unclassifled
- Information not avallable.

- Fallure caused by hot apot.

- Fallure caused by roduced rib holght in the tube.

- Fallure, probably caused by gow mechandan other than RR or IS such as pin hole penetration or other unknom mechanlan.

- Falluro caused by loas of cap.

- Lonfithulso or dingonal split.

- Spirir nornal to ado of elug.

- Falluro tjpo does nor est into an of the above eategordes.

Extracted from HAPO $1956 \mathrm{~h}$, page B-20 


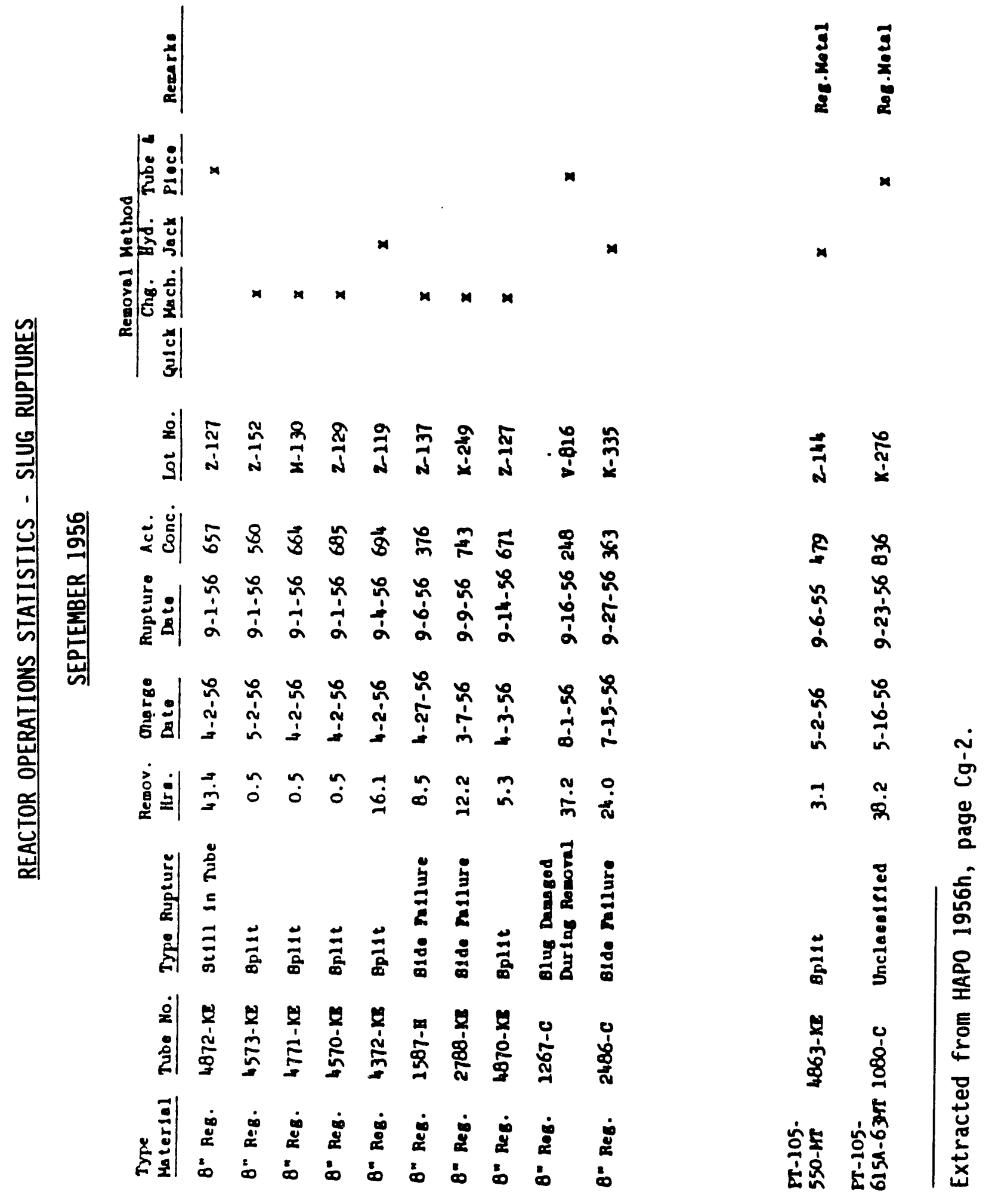


RUPTURE EXPERIENCE - OCTOBER 1956

Production Ruptured Slug Tabulation

\begin{tabular}{|c|c|c|c|c|c|}
\hline Ea17uro Dato & rube No. & Lot No, & Dap Yate? & $\begin{array}{l}\text { Eporan } \\
\text { 1.0.0. }\end{array}$ & Pas Punge \\
\hline $20-2-56$ & $2077-\mathrm{a}$ & E. & ons. & 699 & S1da-othar \\
\hline $10-8-56$ & $2067-08$ & $2-257$ & $\begin{array}{l}\text { 8m Baf. } \\
\text { Baprocesend }\end{array}$ & 628 & S1d-A. \\
\hline $20-29-56$ & $5067-65$ & $2-172$ & $\begin{array}{l}\text { or Rog. } \\
\text { Boproesecend }\end{array}$ & 598 & $\begin{array}{l}\text { Splet } \\
\text { Iandtedenal }\end{array}$ \\
\hline $20-26-56 *$ & $3373-0$ & $x-20$ & on Bace & 947 & S1da-otbar \\
\hline \multicolumn{6}{|c|}{ 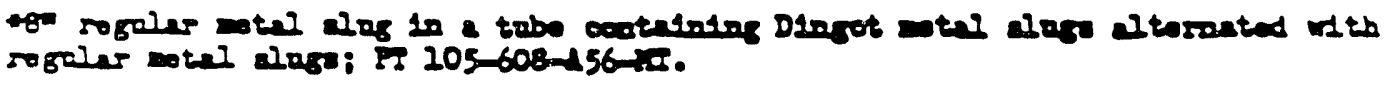 } \\
\hline \multicolumn{6}{|l|}{ Legend: } \\
\hline $\begin{array}{l}\text { Rd. } \\
\text { SIdo-r.s. } \\
\text { S100-otbor }\end{array}$ & $-\frac{\text { Inf }}{\text { - Ind }}$ & $\infty$ & $\begin{array}{l}\text { 12able. } \\
\text { aned by } 18 \\
\text { anding } \\
\text { and by }\end{array}$ & the & 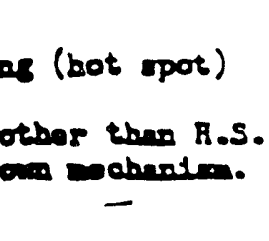 \\
\hline
\end{tabular}

Extracted from HAPO 1956i, page B-17. Also reported in BLOOMSTRAND $1956 \mathrm{~b}$. 


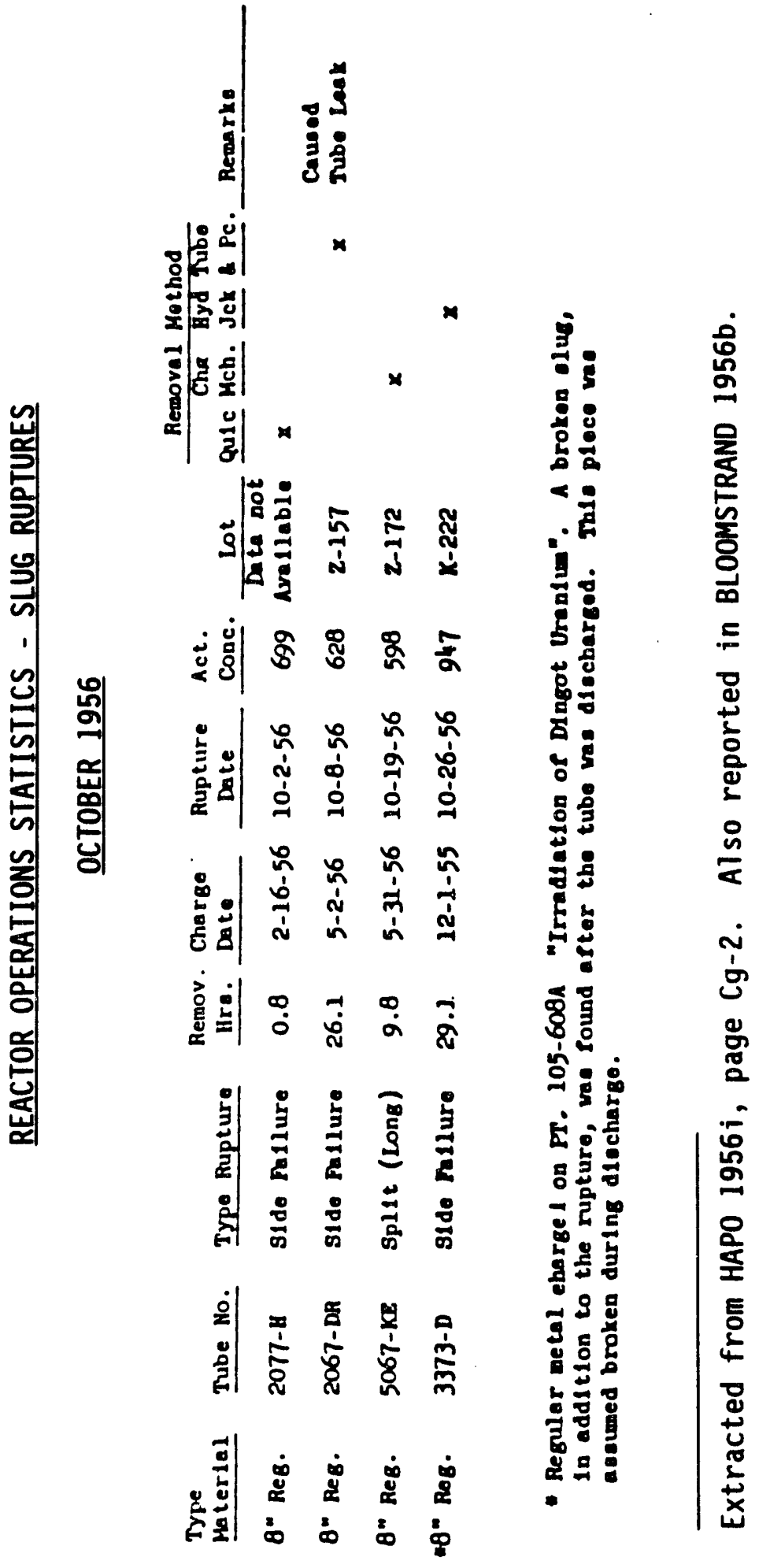


RUPTURE EXPERIENCE - NOVEMBER 1956

\section{Ruptured Slug Tabulation}

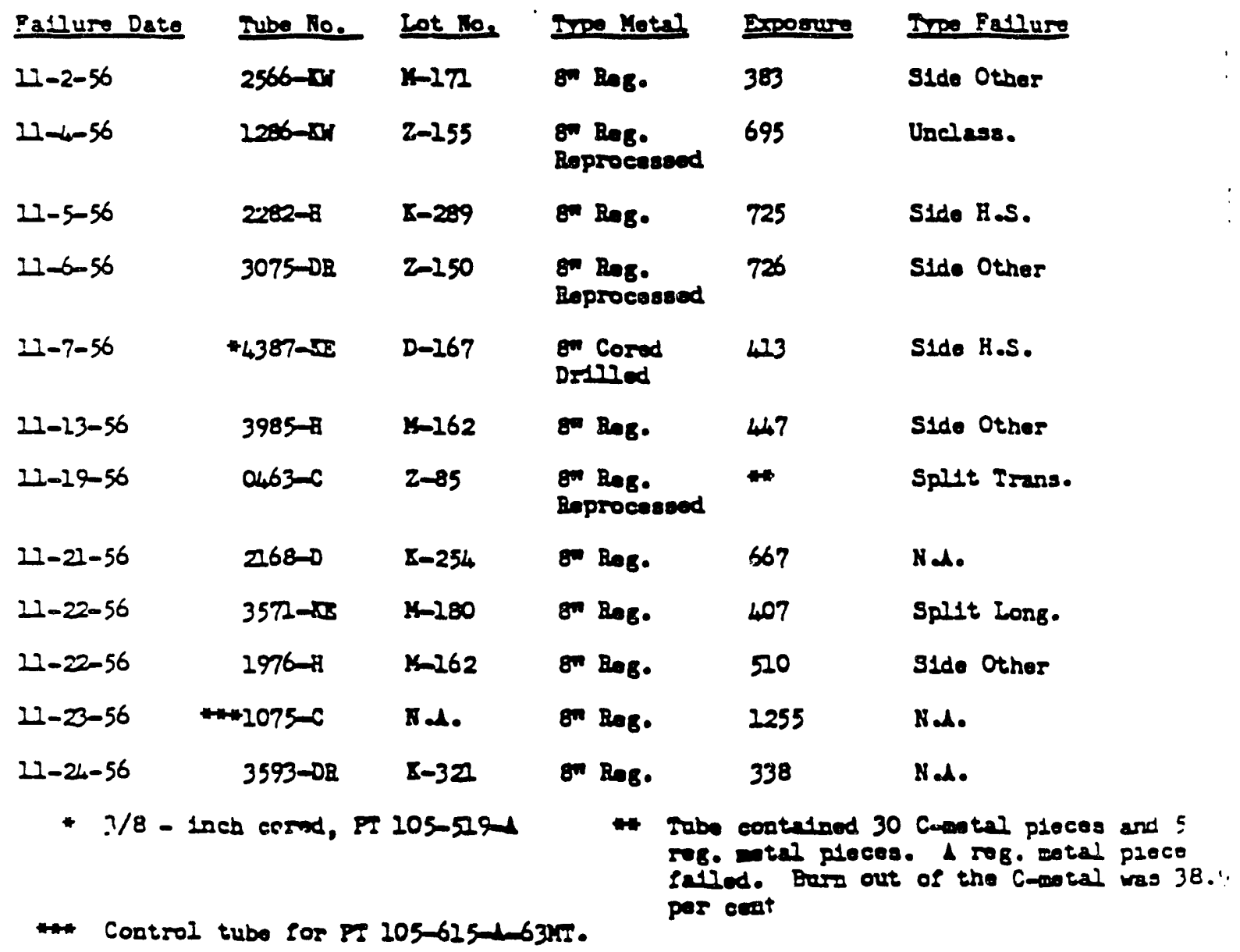

Extracted from HAPO $1956 \mathrm{j}$, page B-14. Also reported in BLOOMSTRAND $1956 \mathrm{c}$. 


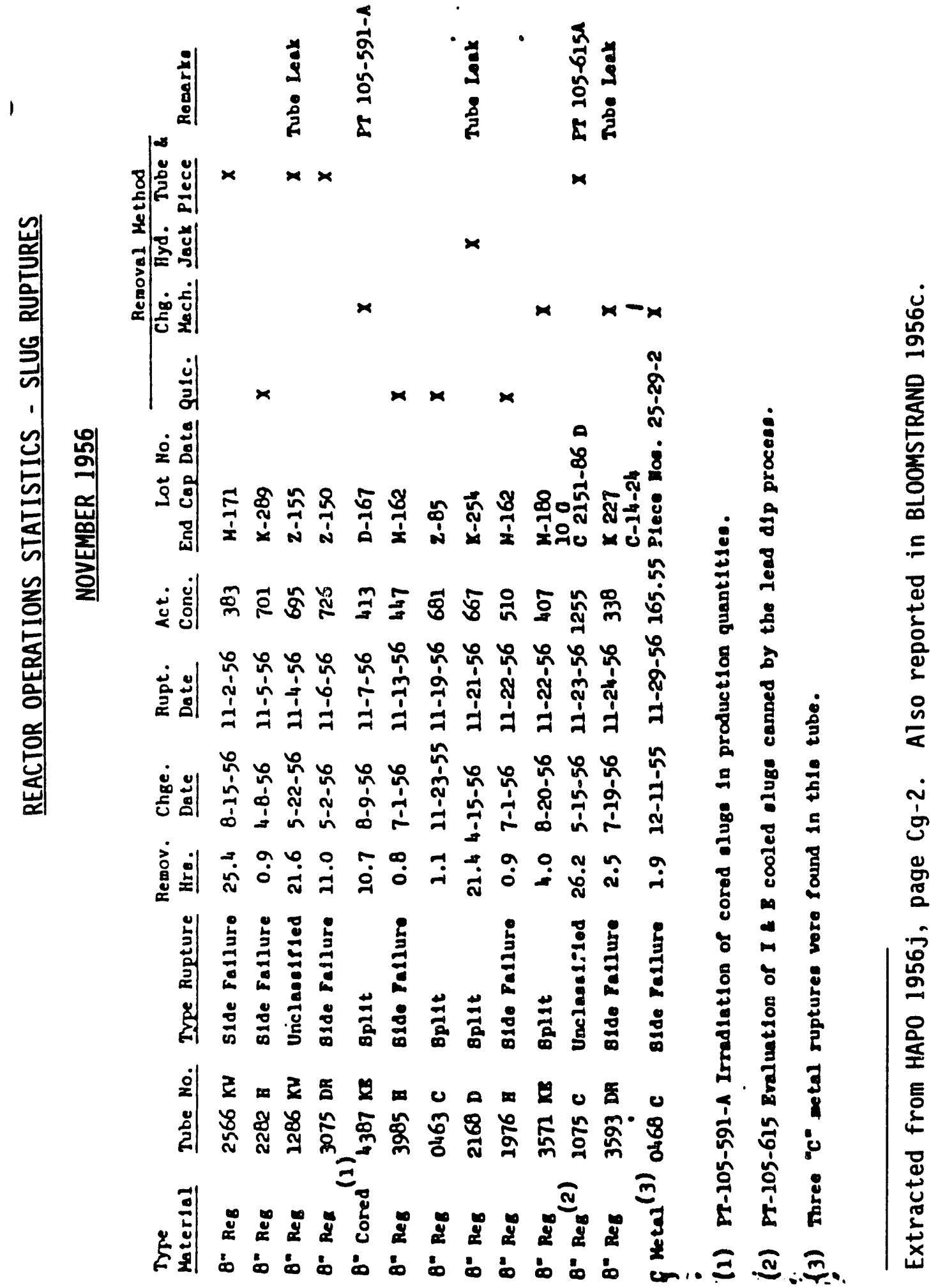




\section{RUPTURE EXPERIENCE - NOVEMBER-DECEMBER 1956}

\section{Ruptured Slug Tabulation}

Ruptures uceurrod in f1rteen cherges durang Docember. Fourteen of the ruptures

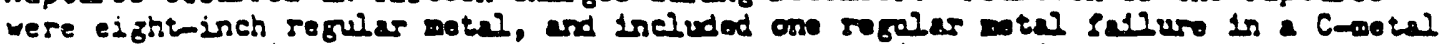

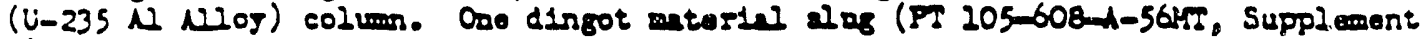

A) fallod at C Reactor. Portinest date ar tabolated belor:

\begin{tabular}{|c|c|c|c|c|c|c|}
\hline Sailure Date & Iube No. & Lot No. & No & - Motal & 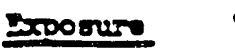 & sno Fallure \\
\hline $27-27-56$ & $0468 \sim$ & $c-2$ & $c-2$ & $(0-$ & $\begin{array}{l}37.95 \\
-235 \text { Depletson }\end{array}$ & Side Chatter \\
\hline $22-2-50$ & $2078-1$ & $8-290$ & $8 n$ & Bog. & 761 & Side K.S. \\
\hline $22-2-56$ & $299:-H$ & $I-22$ & $8 n$ & Mog. & 239 & Cap \\
\hline $12-5-56$ & $3654-2 E$ & $k-384$ & $8 n$ & Reg. & 422 & $N A_{0} \rightarrow$ \\
\hline $22-7-56$ & $2576-2-4$ & $7-64$ & $8 n$ & Dingot & 356 & Splet I. \\
\hline$=2-9-56$ & $2878-i$ & $I-35$ & 8 & Reg. & 373 & S1de R.S. \\
\hline $22-13-56$ & 32790 & $x-325$ & en & Beg. & 705 & Side - Other \\
\hline $22-17-56$ & $2584-C$ & $2-223$ & $8 n$ & Reprocesaed & 246 & SIde H.S. \\
\hline $12-18-56$ & $0777-x H$ & $\mathrm{~K}-20 \mathrm{I}$ & 8w & Log. & $\tan$ & Oneless. \\
\hline $12-20-56$ & $3281-n \pi$ & $2-196$ & $8 n$ & Reproceased & 408 & Unclass. \\
\hline $22-20-56$ & $2482 \alpha$ & $x-223$ & $8 n$ & Rog. & 349 & Unclass. \\
\hline $22-21-56$ & $276-c$ & $2-232$ & $8 n$ & Roproceseod & 250 & $N \alpha$ \\
\hline $22-23-56$ & 2469 & $\Delta-342$ & $8 n$ & Beg. & 587 & side - Other \\
\hline $12-25-56$ & บ48-H & $x-267$ & gn & Rog. & 565 & Uncelass. \\
\hline $12-25-56$ & 3085 A & $x-297$ & 8n & Beg. & Sad. & S1de - Other \\
\hline $22-32-56$ & $4263-7$ & $8-356$ & 8n & Rog. & Nג. & Nal. \\
\hline
\end{tabular}

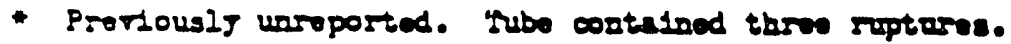

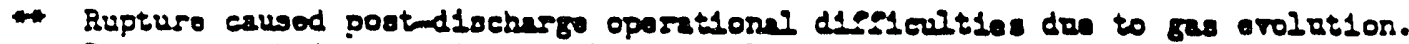

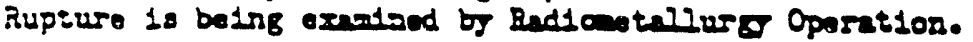

Irradiated under PT 205-608-1-56r5, Supploment 1.

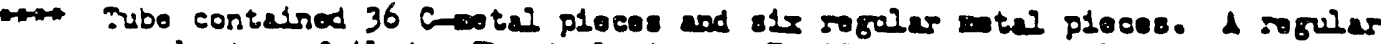
motal ploce eatied. The dopletion of 0 -235 10 the Catel we 45 par cont.

Extracted from HAPO 1957a, page B-17. Also reported in BLOOMSTRAND 1957a. 


\section{RUPTURE EXPERIENCE - NOVEMBER-DECEMBER 1956}

Ruptured Sluq Tabulation (Cont'd)

isend:

N. A.
Sise H.S.

Extracted from HAPO 1957a, page B-18. 


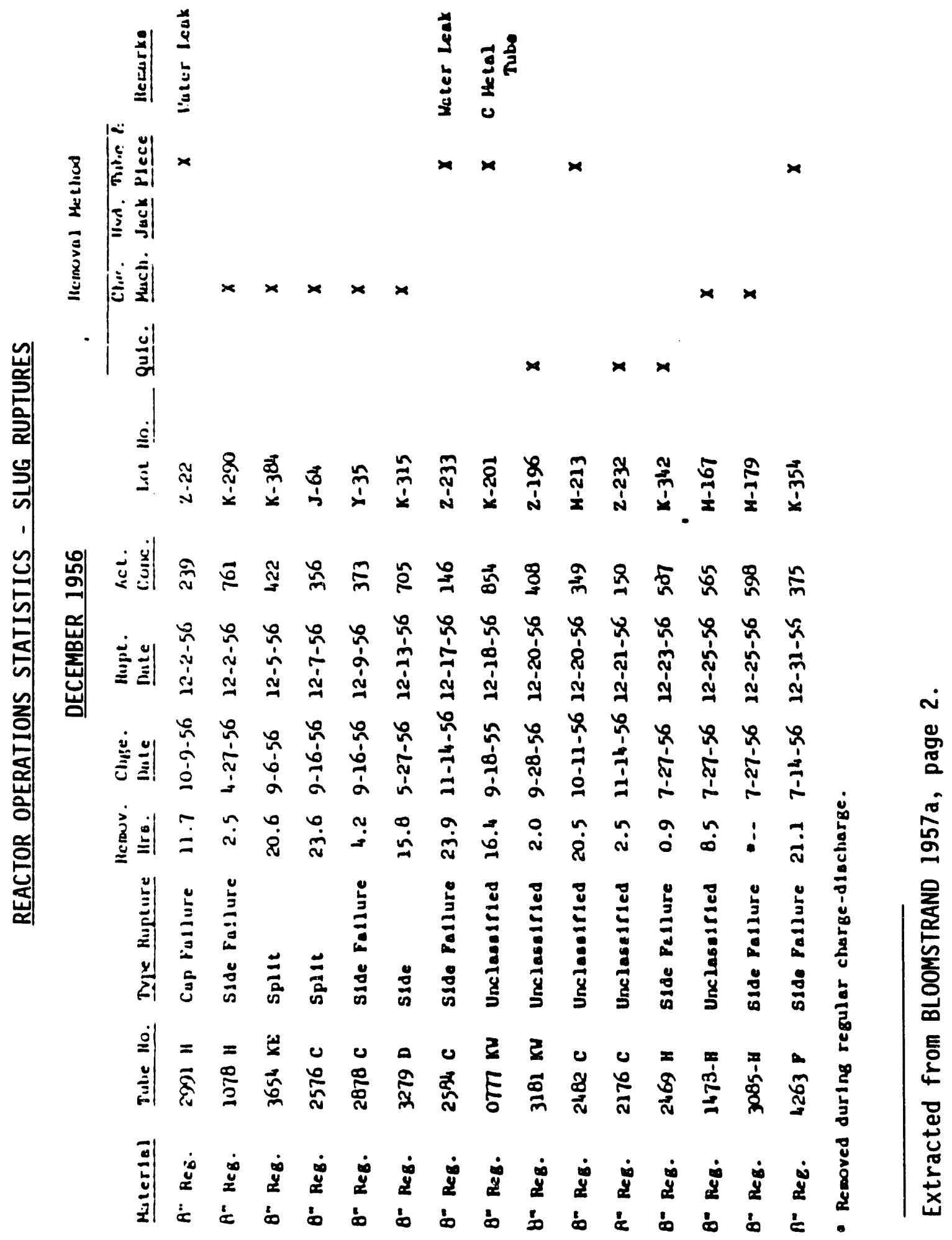


APPENDIX G

FUEL-ELEMENT FAILURE DATA FOR 1957 


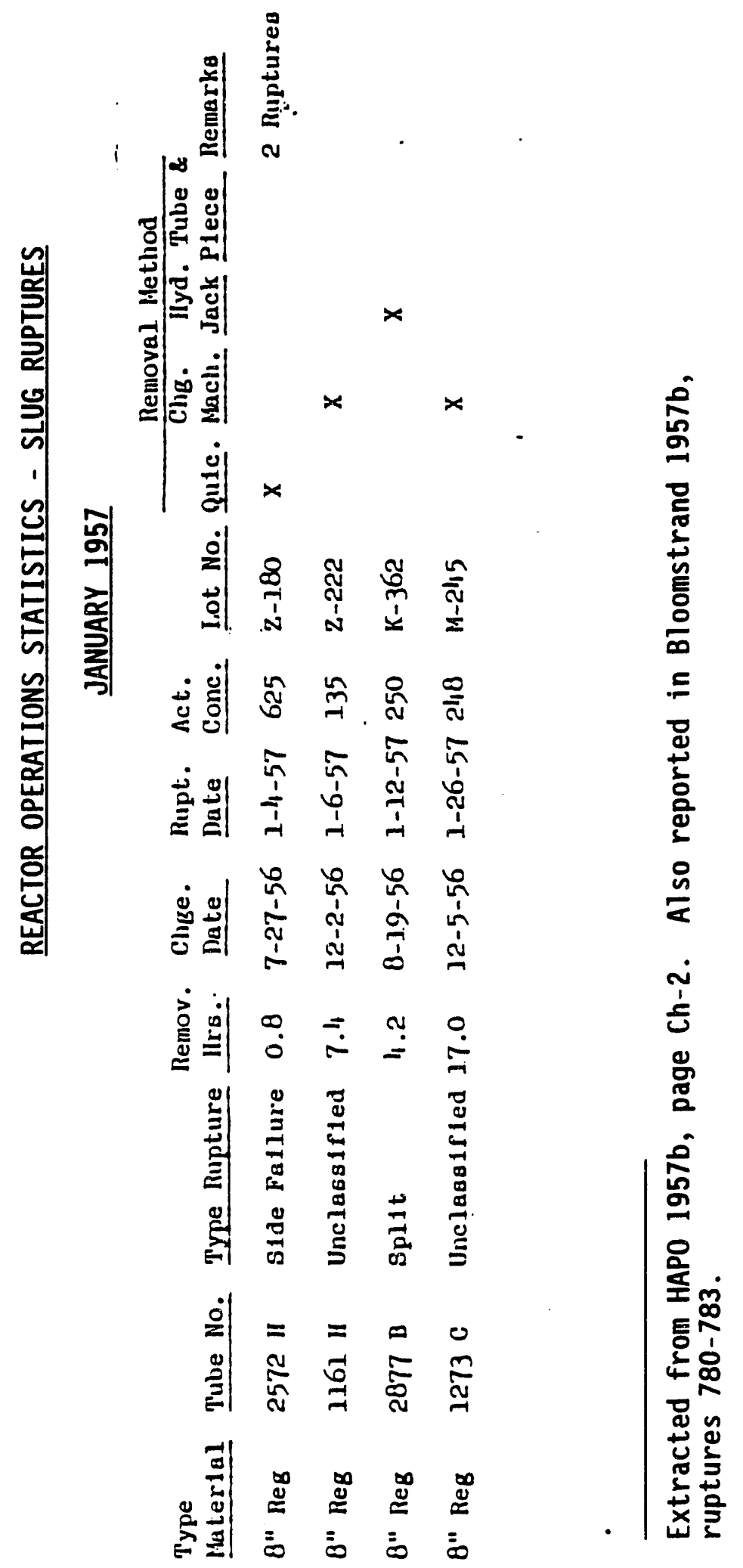

G.1 


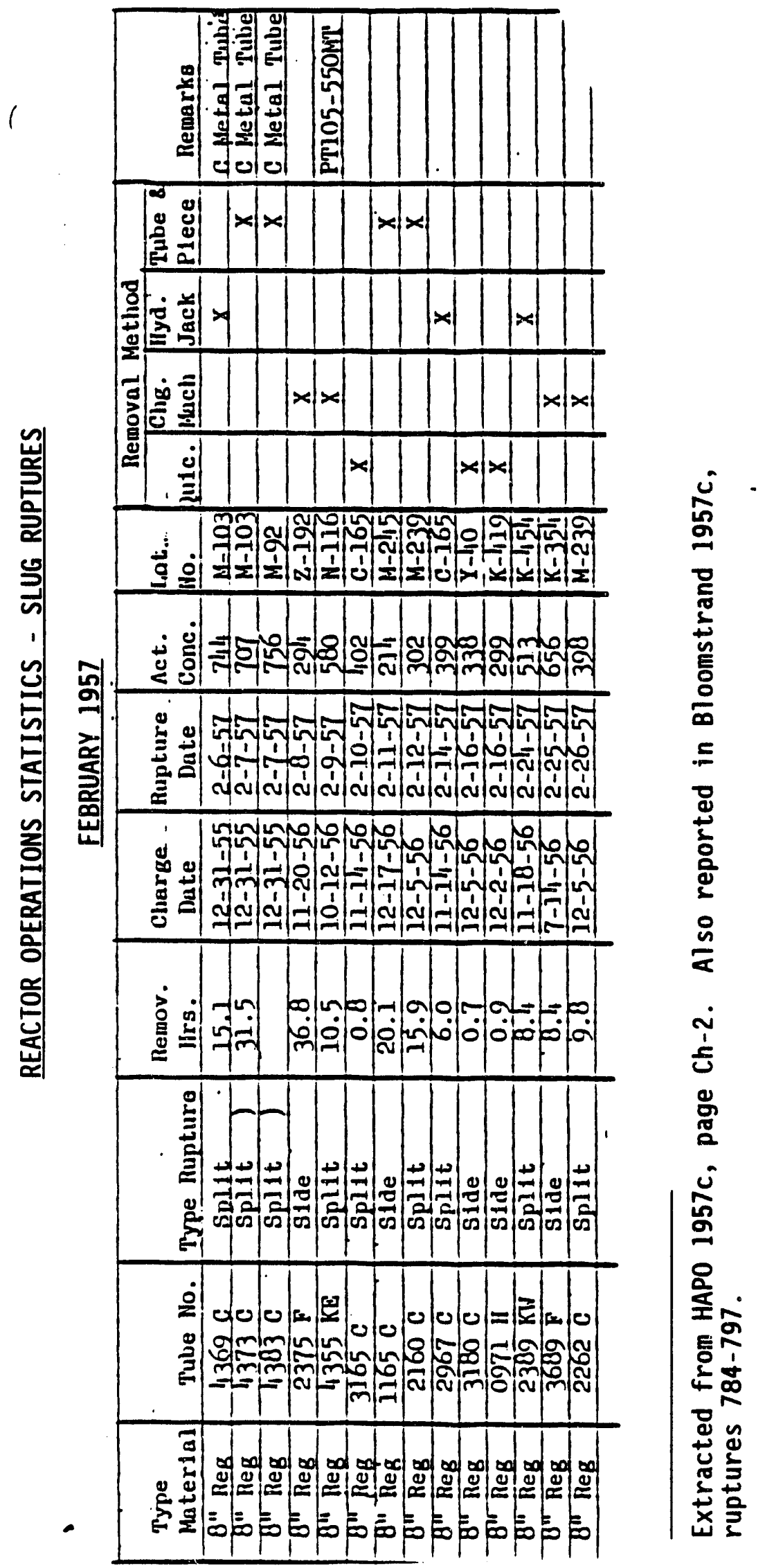

G.2 


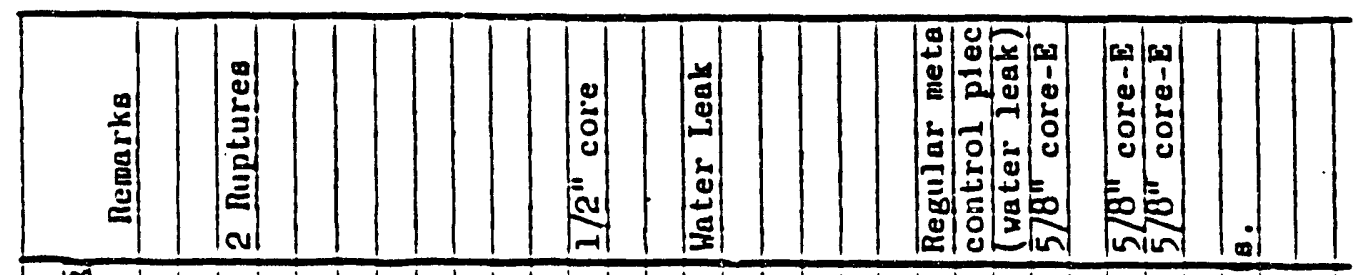

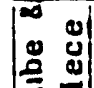

E:

물

부를

J]

$\times$

$\times$

章

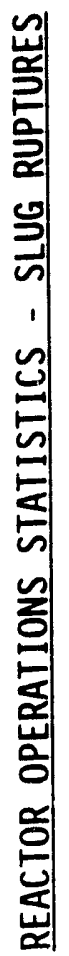

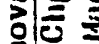

气

비 $|x|$

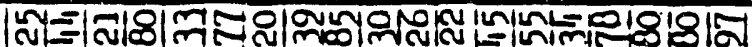

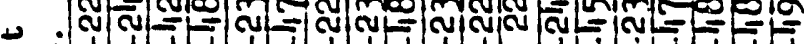

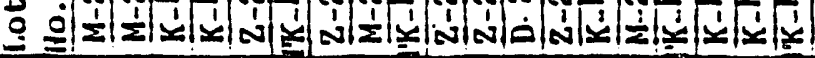

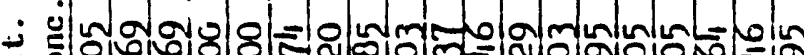

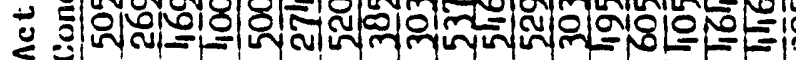

웜

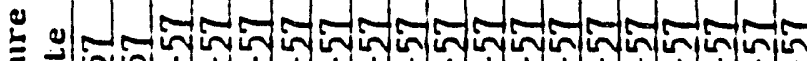

$=$ Noñ 1 ń

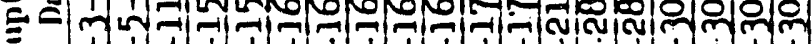

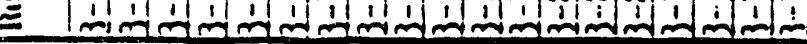

$=\frac{\pi}{\pi}$ जี

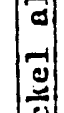

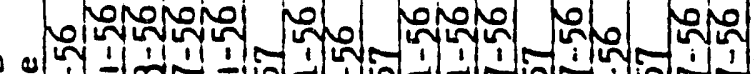

$\because$ \&

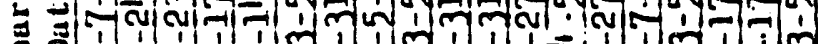

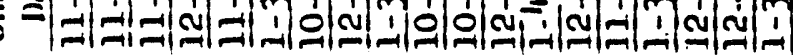

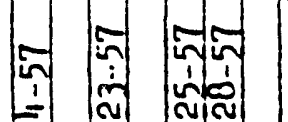

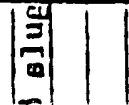

¿ :

总

\section{$=$}

- ro culoo mo mo

: $:$ :

$\exists=$

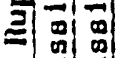

नंनें

जิ

min of

$=1$ |

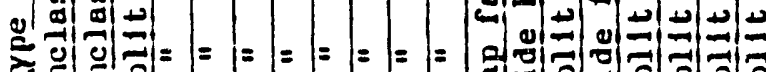

|

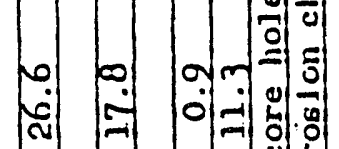

곤 $\frac{0}{5}$

$\rightarrow=-19$

7. 7 교의

-1 三ड़ि

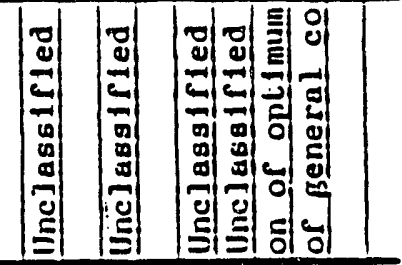

$\div$

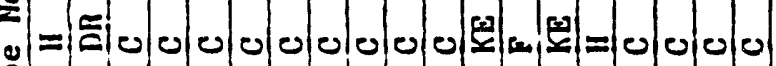

ت. Wiñ

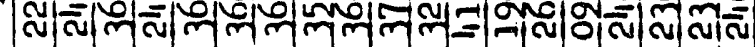

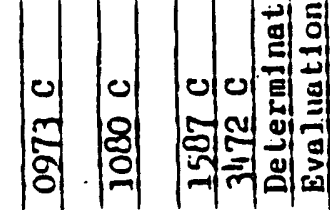

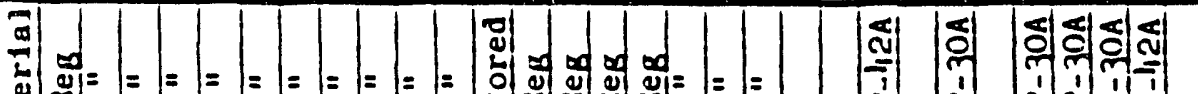

\&

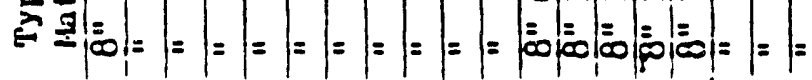


RUPTURES EXPERIENCE - APRIL 1957

\begin{tabular}{|c|c|c|c|c|c|}
\hline Eailune Date & Dube Ne. & Let No. & Toer Metal & Exposurs & Troe F3iTure \\
\hline $\begin{array}{l}4-1-57 \\
4-9-57 \\
4-13-57 \\
4-17-57 \\
4-18-57 \\
4-18-57 \\
4-19-57 \\
4-2 C-57 \\
4-28-57 \\
4-29-57 \\
4-3 C-57 \\
4-30-57 \\
4-3 C-57\end{array}$ & 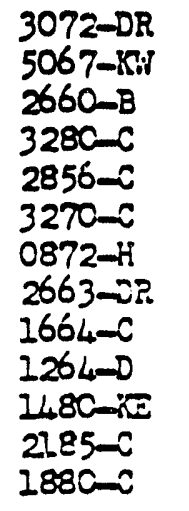 & $\begin{array}{l}K-220 \\
K-536 \\
I-34 \\
T K-503 * \\
Z-243 \\
T K-503 \\
Y-5 I \\
C-220 \\
M-265 \\
C-173 \\
D-232 \\
T K-48 \% \\
T K-539\end{array}$ & 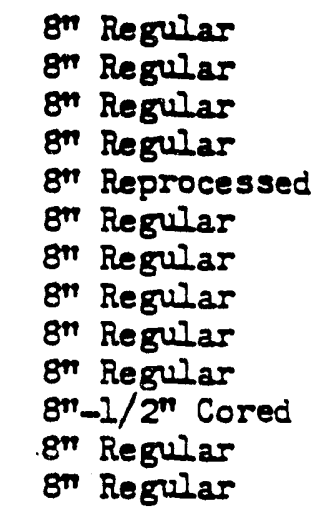 & $\begin{array}{l}455 \\
219 \\
477 \\
331 \\
490 \\
298 \\
415 \\
247 \\
370 \\
518 \\
532 \\
357 \\
269 .\end{array}$ & 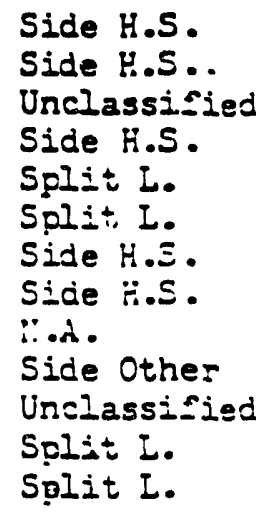 \\
\hline \multicolumn{6}{|c|}{ *' Truline - small button design. } \\
\hline \multicolumn{6}{|l|}{ Legend: } \\
\hline Side - Hot Spot & \multicolumn{5}{|c|}{$\begin{array}{l}\text { - Failure probably caused by local overheating. } \\
\text { (hot Spot) and possibly intergranular corrosion. }\end{array}$} \\
\hline SpIit & \multicolumn{5}{|c|}{$\begin{array}{l}\text { Failure probably caused by cleavage of the urarium core. } \\
\text { Slug split longitudinally (I), diagonally (D), or trans- } \\
\text { versely (T). }\end{array}$} \\
\hline Side Other & \multicolumn{5}{|c|}{$\begin{array}{l}\text { Failure probably caused by a localized corrosion penetrat } \\
\text { ion, e.g. pin hole penetration or uninown mecharisre. }\end{array}$} \\
\hline Unclassiried & \multicolumn{5}{|c|}{ - Failure types does not fit into any particular category. } \\
\hline 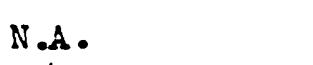 & \multicolumn{5}{|c|}{ - Information not a ailable. } \\
\hline
\end{tabular}

Extracted from HAPO 1957e, pag\%: B-21. Also reported in Bloomstrand 1957d, failures 821-833. 


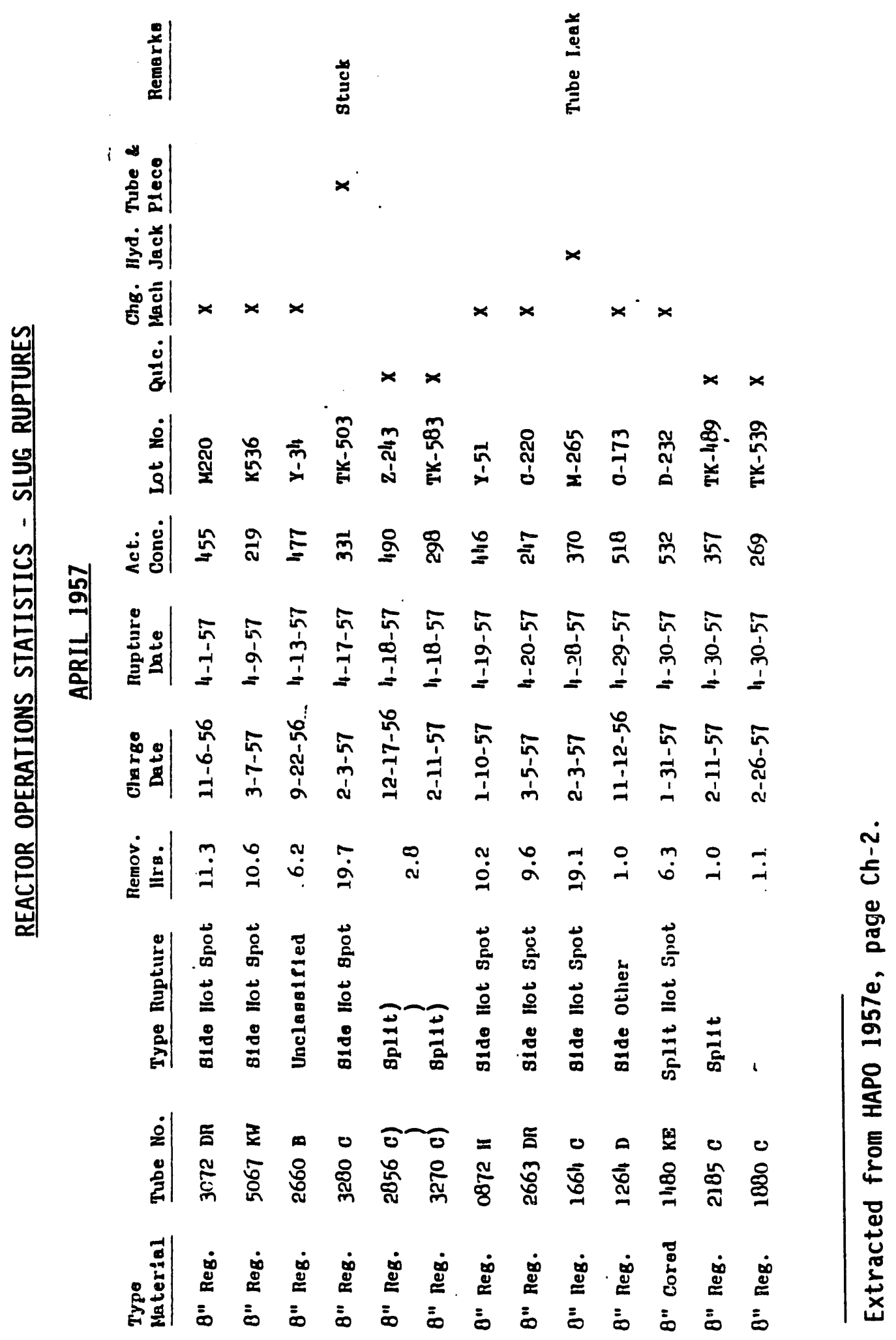




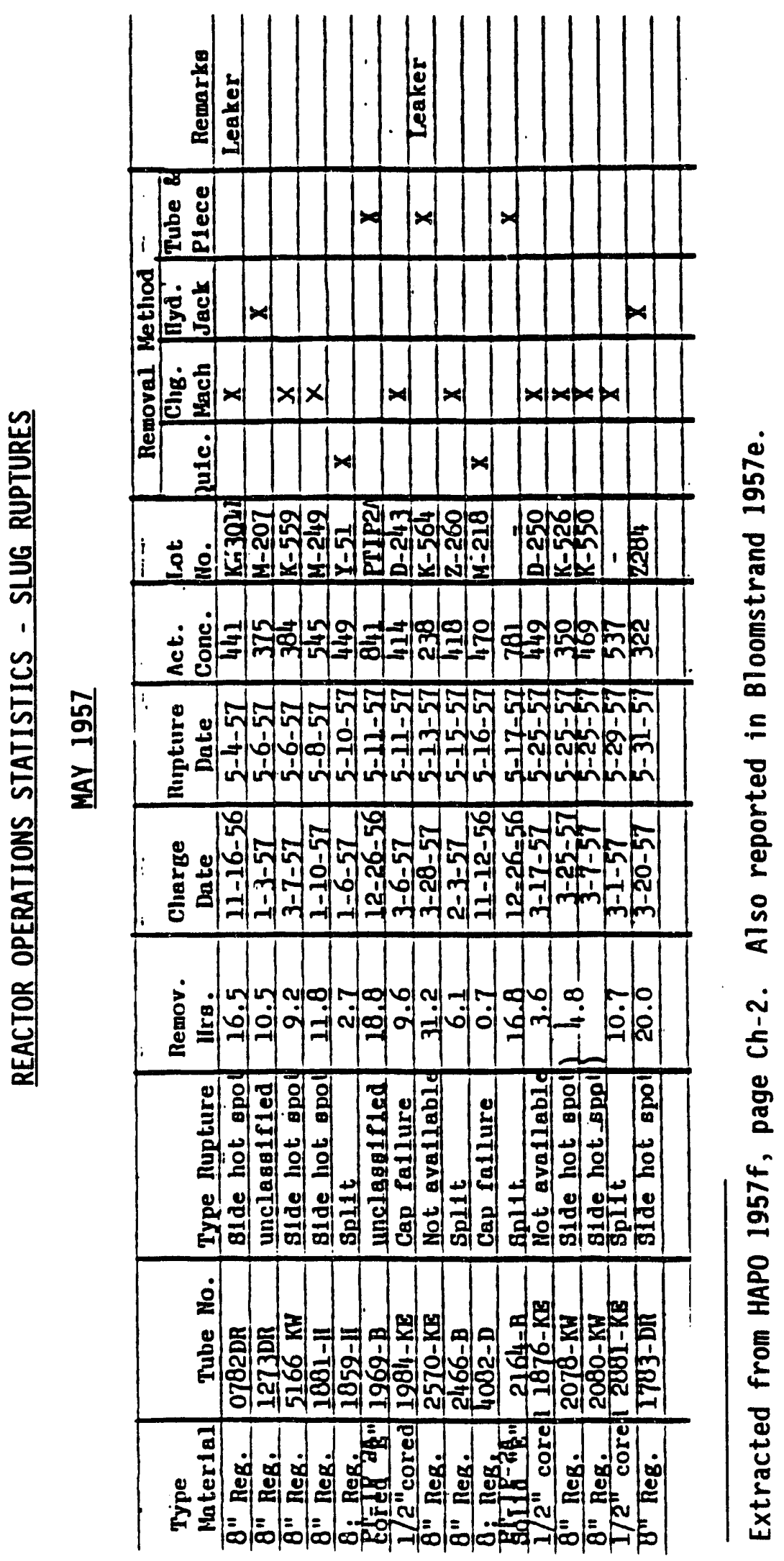

G. 6 


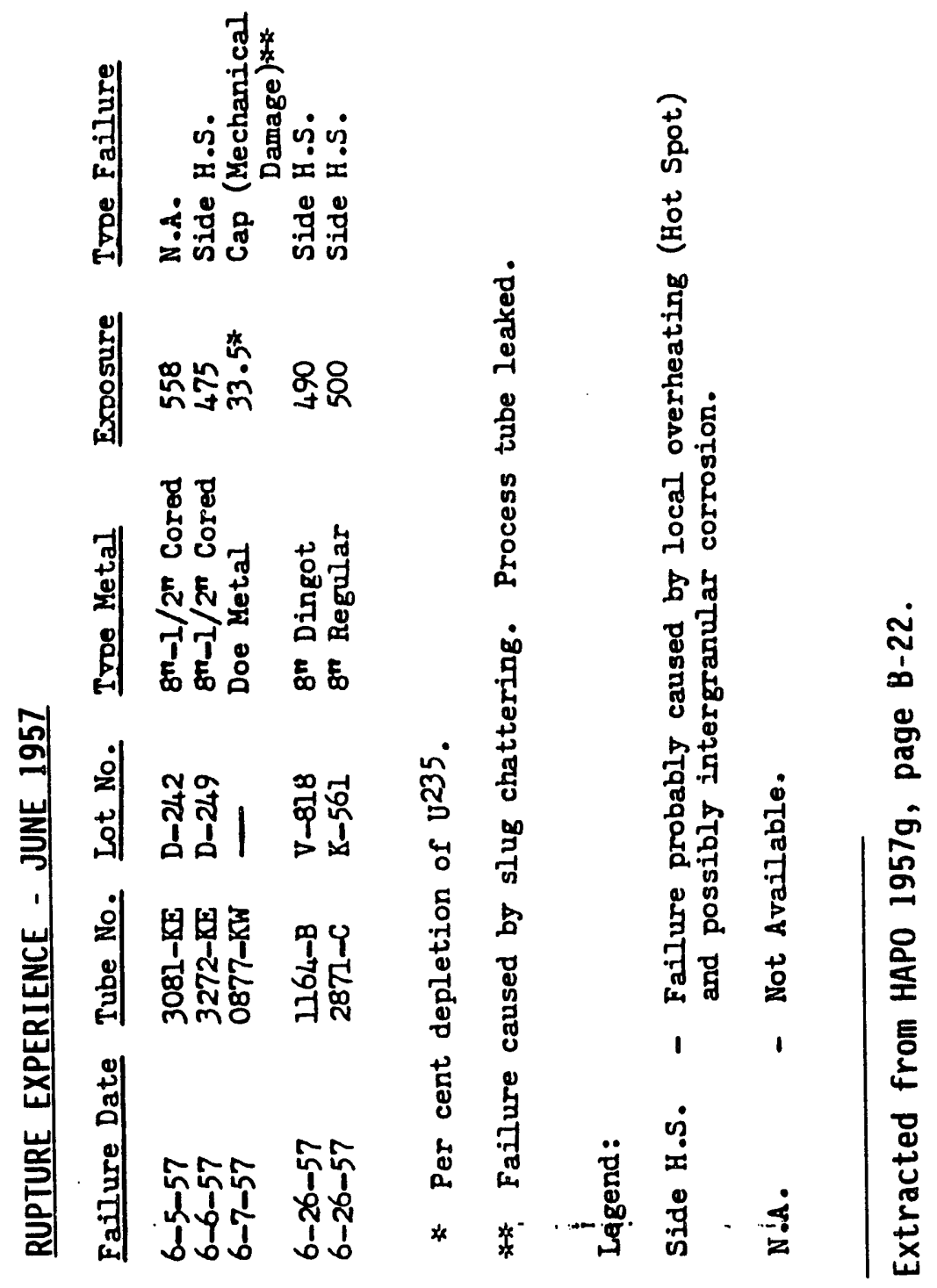

G. 7 


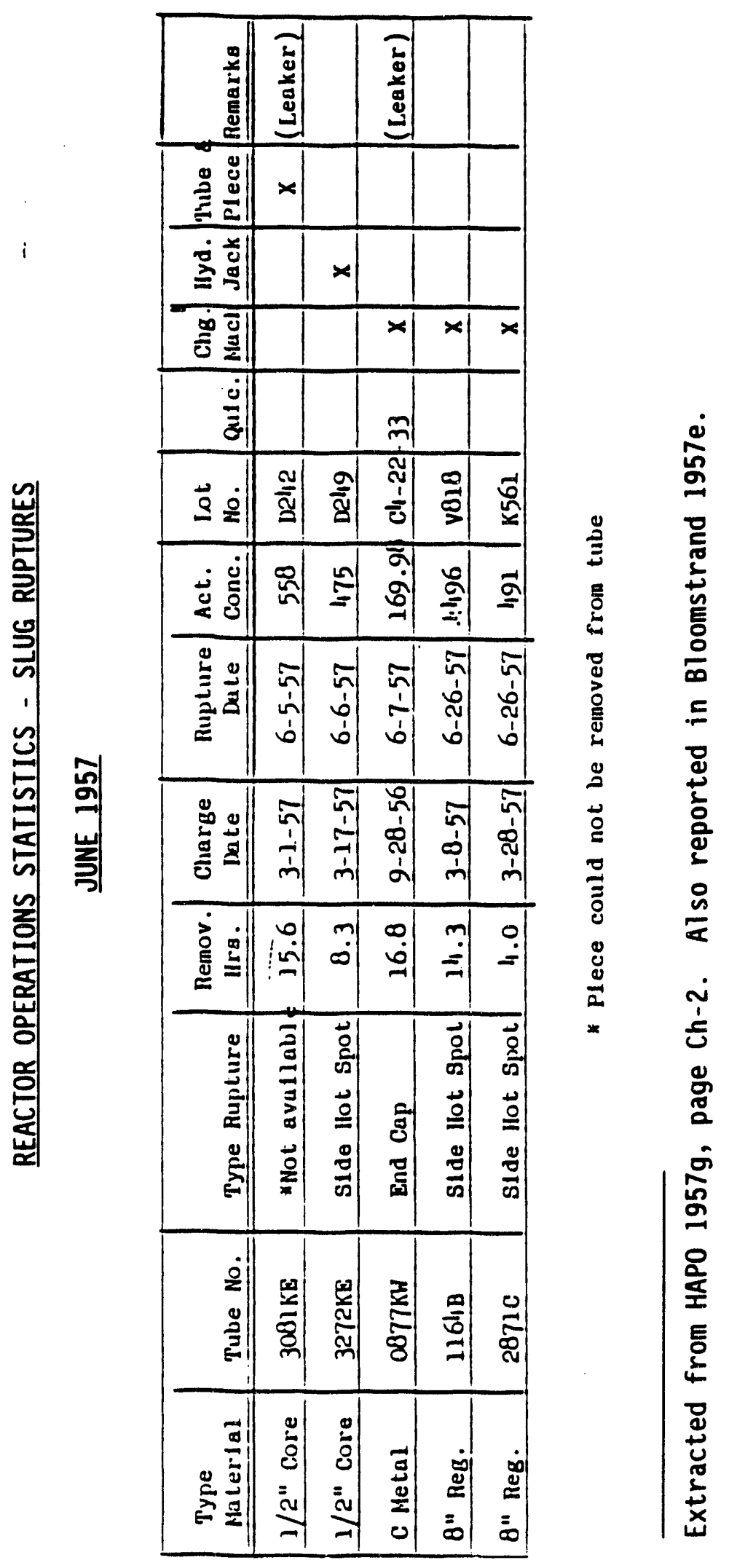




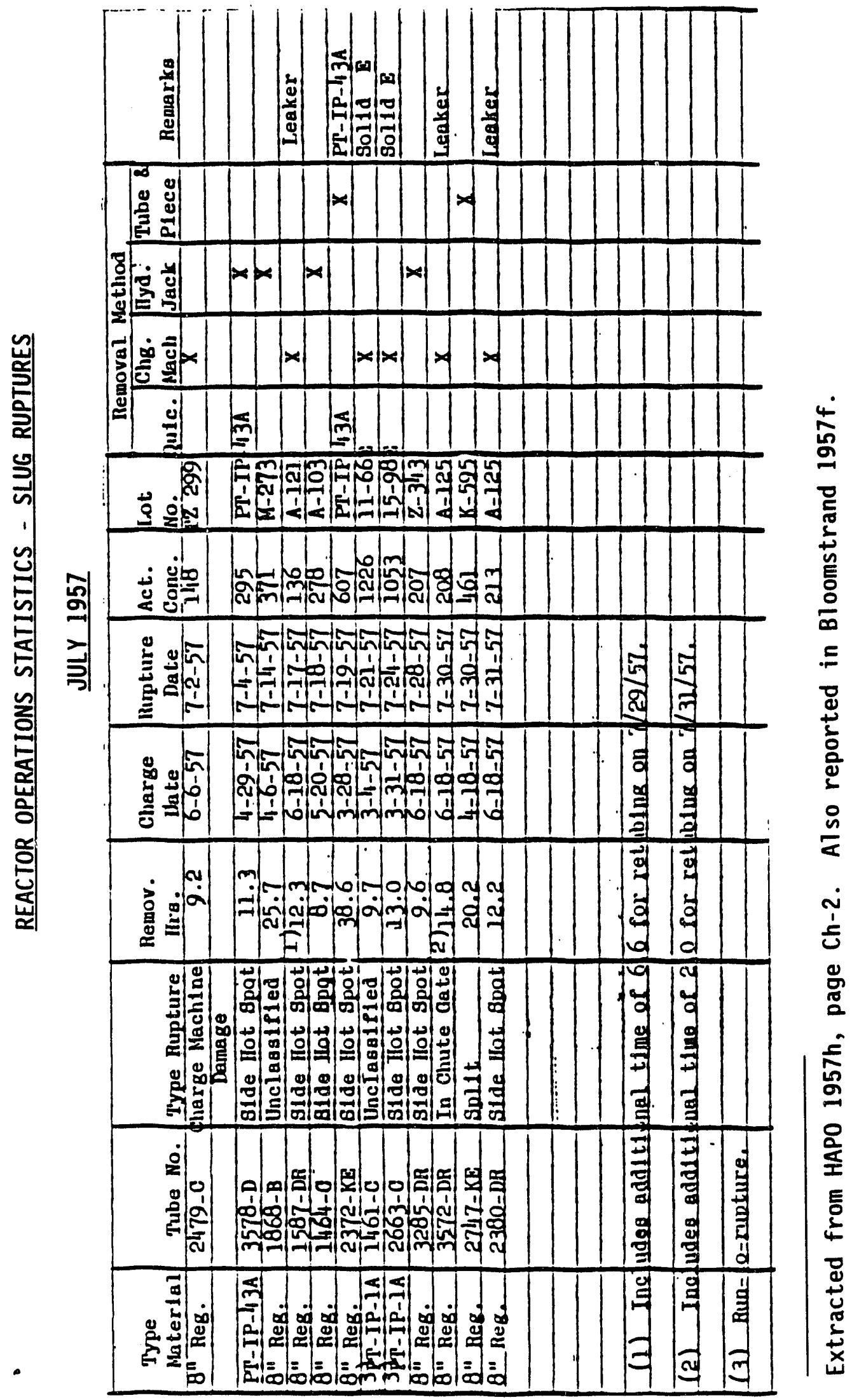




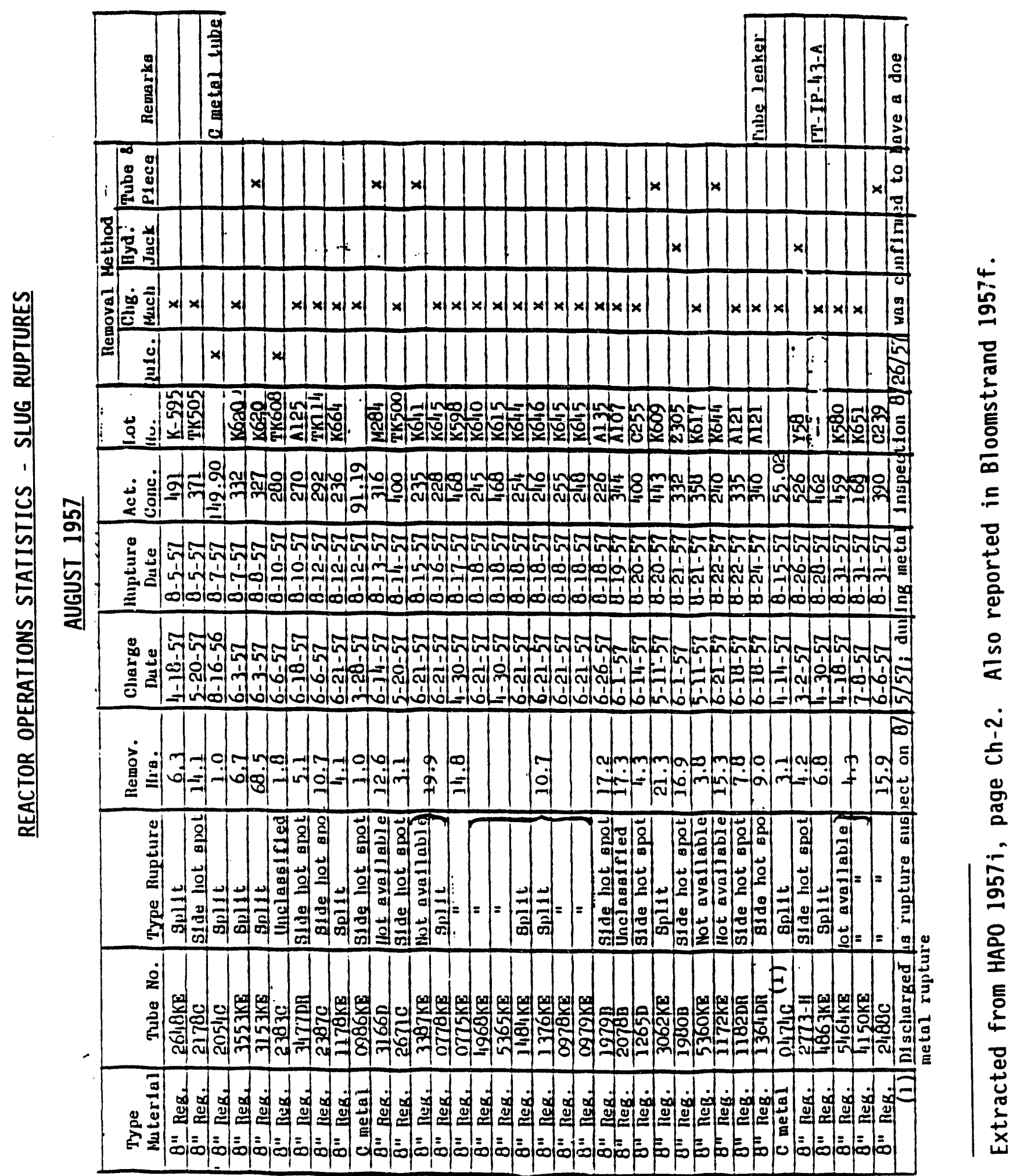


总总 = =

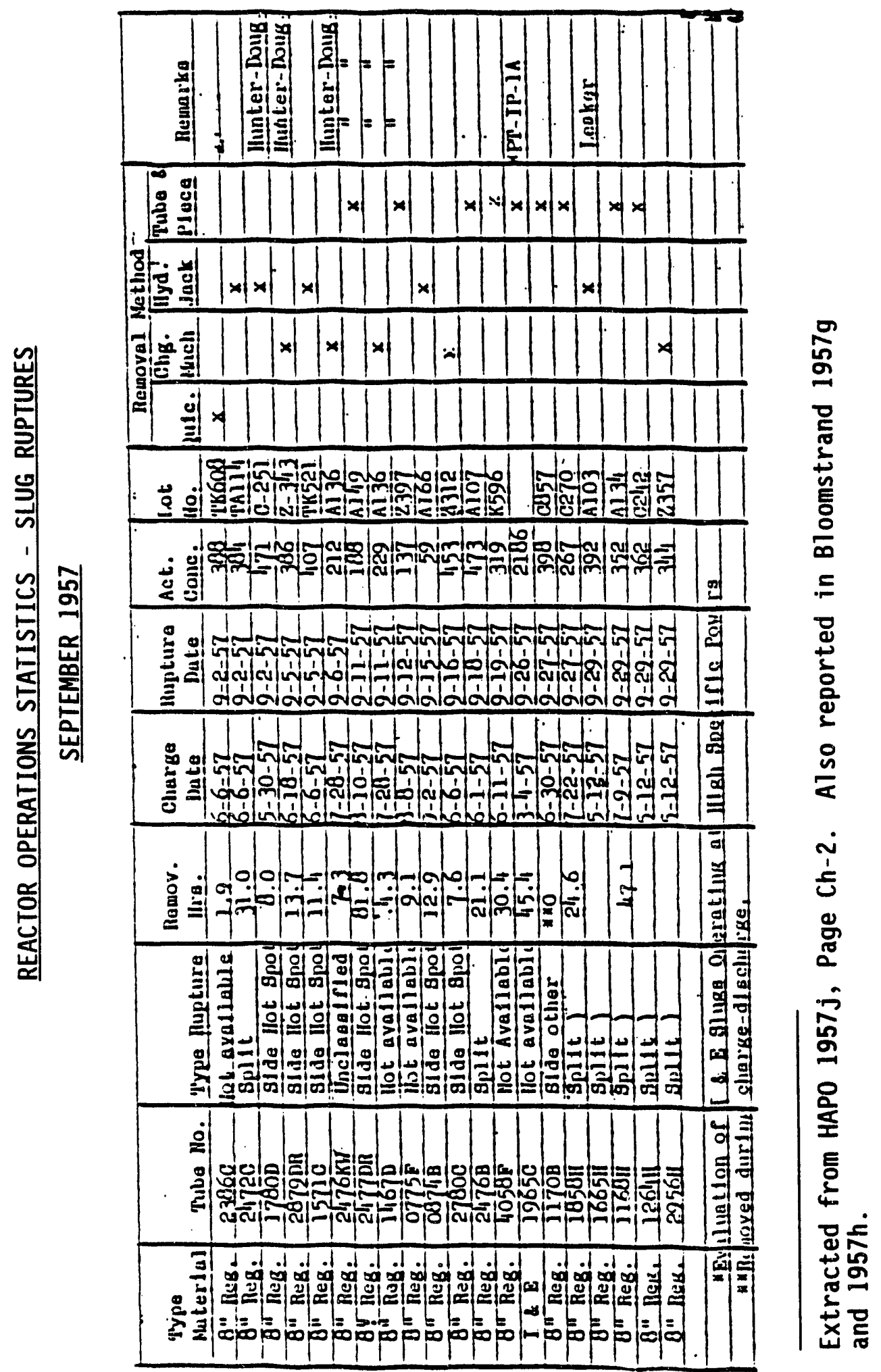




\section{RUPTURE EXPERL NCE - OCTOBER 1957}

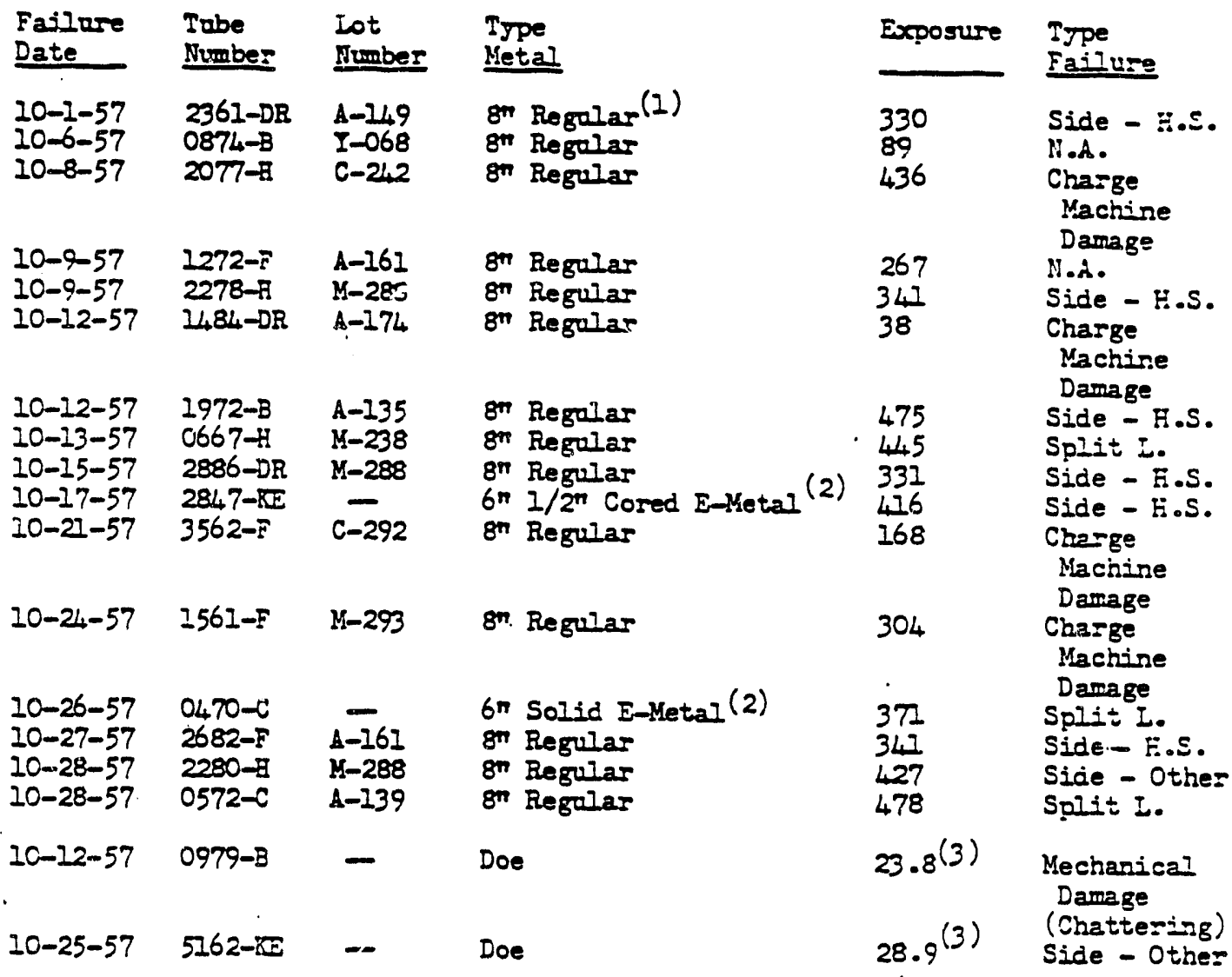

(I) Kunter Douglas Can

(2) 0.94 per cent $0-235$ enriched ararium

(3) Per cent D-235 depletion of Doe metal in the colum

Legend :

Side - R.S. - Failure probably caused by local overheasing (hot spot) and

possibly intergranular corsosion.

Split - Slug split longitadinally (I), diagonaIly (D), or transversely $(\mathrm{T})$.

Dnclassified - Failure does not elt into any particular ciscegors.

Side - Other - Failare probably caused by locainized corrosion peretratior,

N.A. $\quad$ - Information not available.

Extracted from HAPD 1957k, page B-18. Also reported in Bloomstrand 1957h and 1958a. 


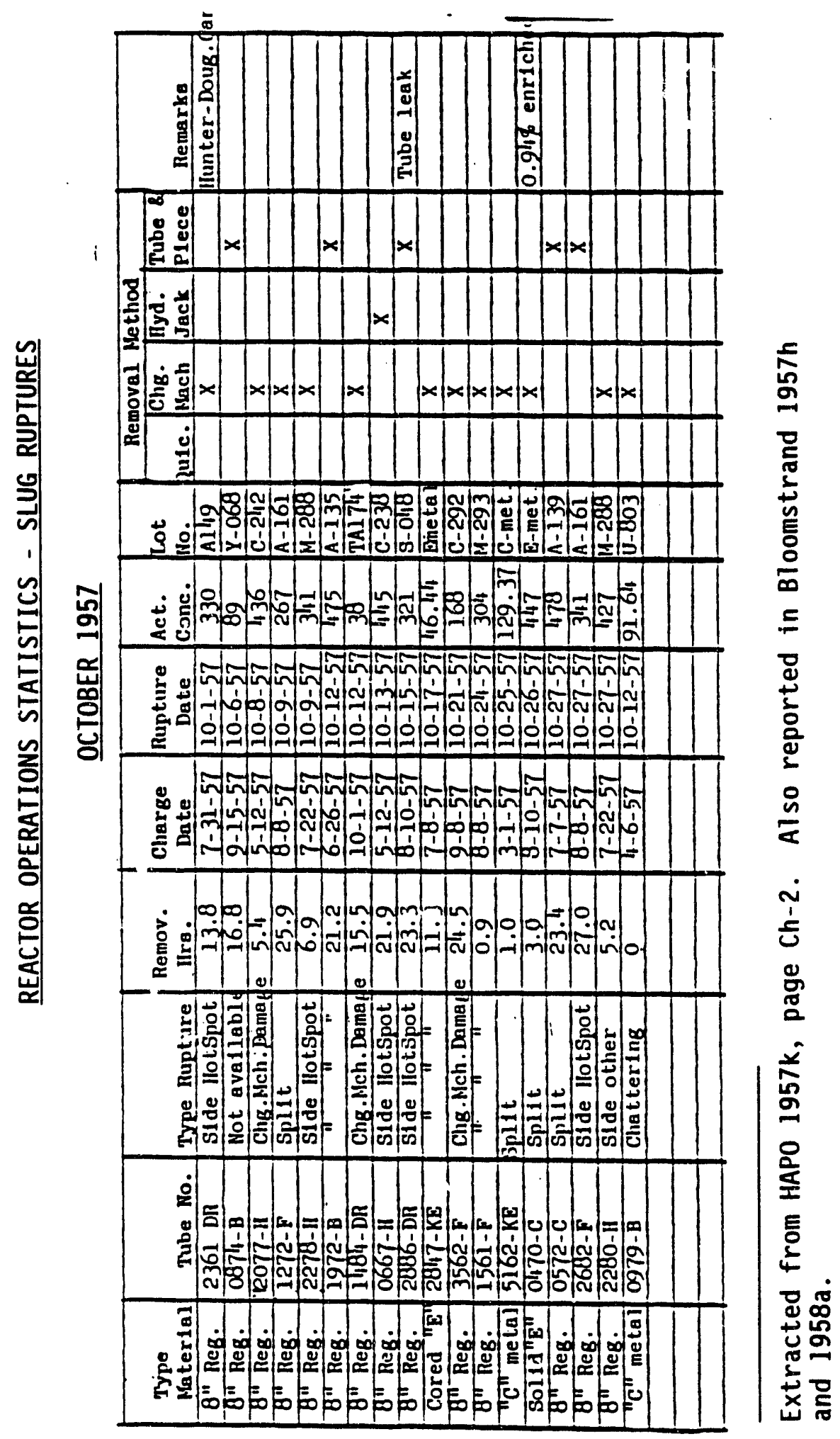

G. 13 


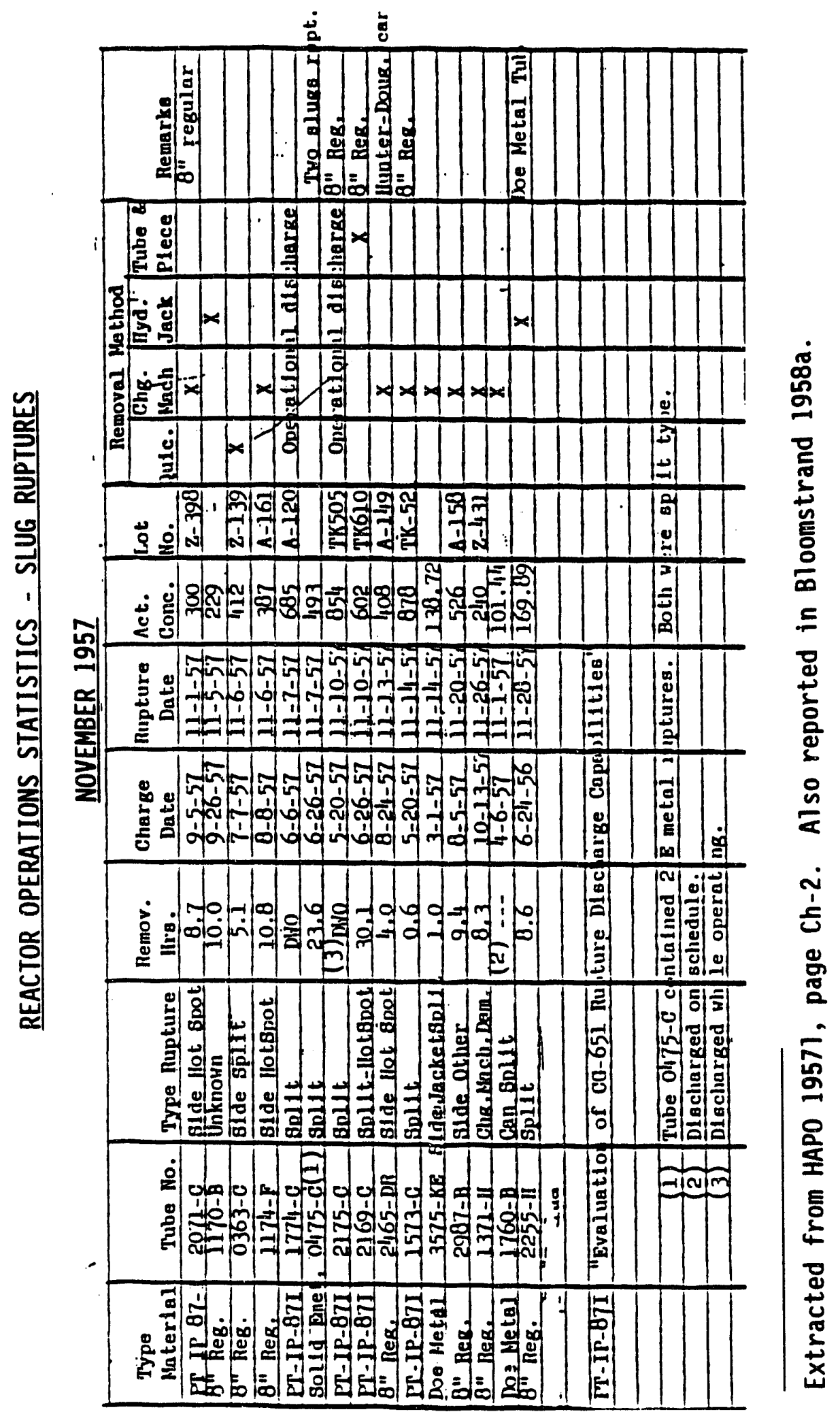




\section{RUPTURE EXPERIENCE - DECEMBER 1957}

\begin{tabular}{|c|c|c|c|c|c|}
\hline $\begin{array}{l}\text { Fal lure } \\
\text { Date }\end{array}$ & $\begin{array}{l}\text { Tube } \\
\text { Number }\end{array}$ & $\begin{array}{l}\text { Iot } \\
\text { Numbe: }\end{array}$ & $\begin{array}{l}T_{\text {Jpe }} \\
\text { Metel } \\
\end{array}$ & "xyosuג'c & $\begin{array}{l}2 y, 16 \\
\text { Fe: lure }\end{array}$ \\
\hline $\begin{array}{l}12-2-57 \\
12-3-57 \\
12-5-57 \\
12-7-57 \\
12-12-57 \\
12-13-57 \\
12-18-57 \\
12-18-57 \\
12-18-57 \\
12-18-57 \\
12-18-57 \\
12-18-57 \\
12-18-57 \\
12-18-57 \\
12-10-=7 \\
12-20-57 \\
12-20-57 \\
12-24-57 \\
12-25-57 \\
12-29-57 \\
12-30-57\end{array}$ & 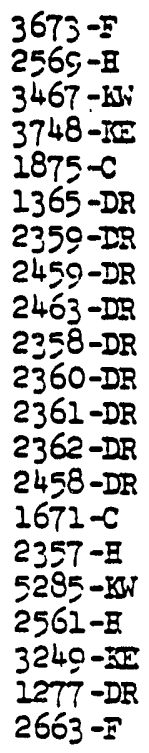 & $\begin{array}{l}M-293 \\
Y-064 \\
K-706 \\
K E-C 0=-A \\
T K-614 \\
M-290 \\
A-176 \\
Z-426 \\
K-709 \\
C-294 \\
K A-002-A \\
C-294 \\
K A-002-A \\
Z-709 \\
M-297 \\
A-171 \\
K E-002-A \\
D-268 \\
K G-014-A \\
M-290 \\
Z-421\end{array}$ & 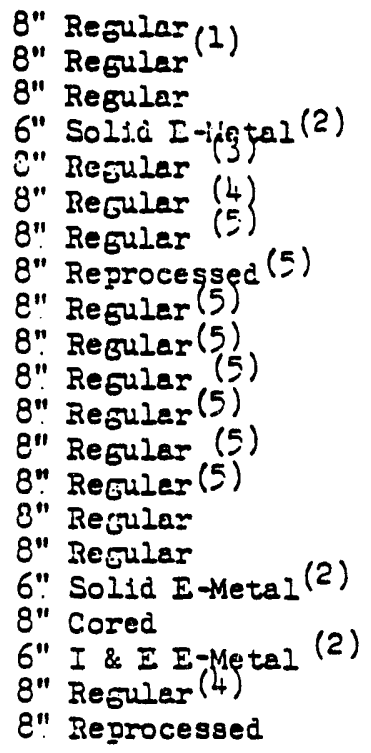 & $\begin{array}{l}514 \\
436 \\
339 \\
297 \\
174 \\
411 \\
421 \\
410 \\
357 \\
310 \\
310 \\
349 \\
322 \\
305 \\
223 \\
478 \\
232 \\
471 \\
184 \\
414 \\
521\end{array}$ & 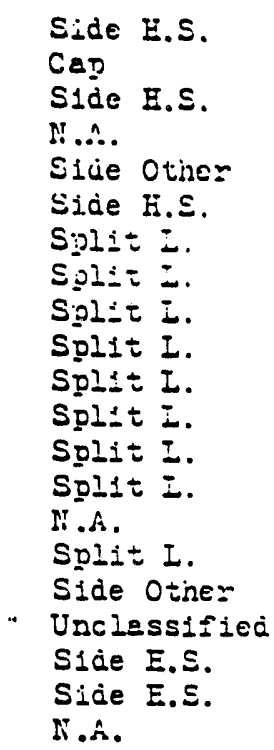 \\
\hline
\end{tabular}

(1) $M-386.1110 y$ Can

(2) 0.94 Per Cent Inriched

(3): Smell-Button Ies10n Truline

(4) Eunter-Douglas Can

(5). Associated With Crossheader Velving Difficulty (During Shutiown)

\section{Iegend:}

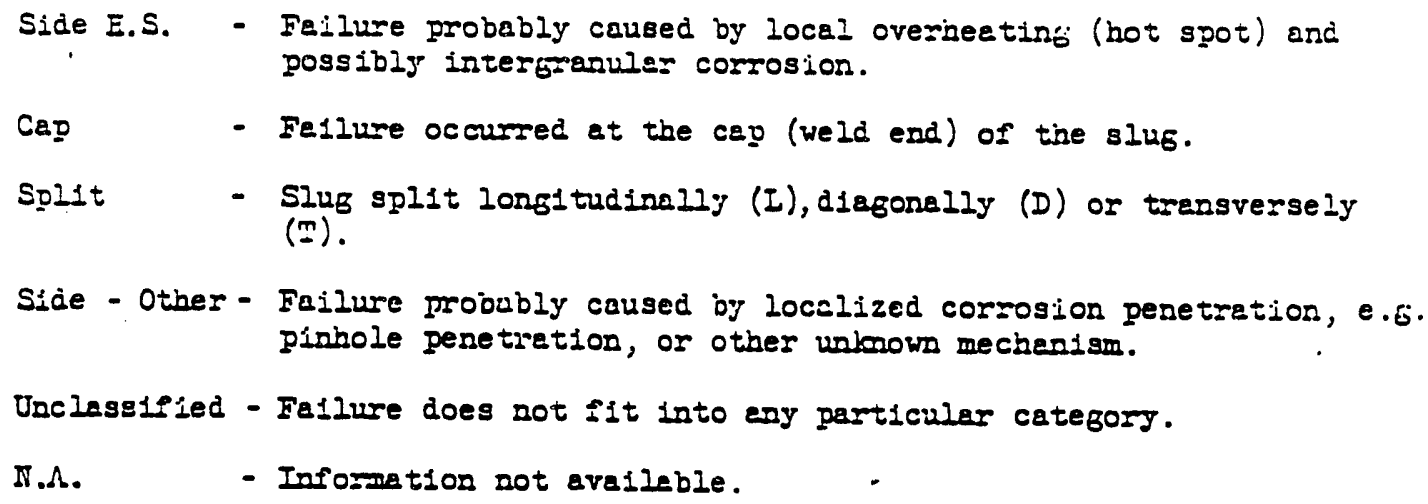

Extracted from HAPO 1958a, page B-22. Also reported in Bloomstrand 1958a. and 1958b. 


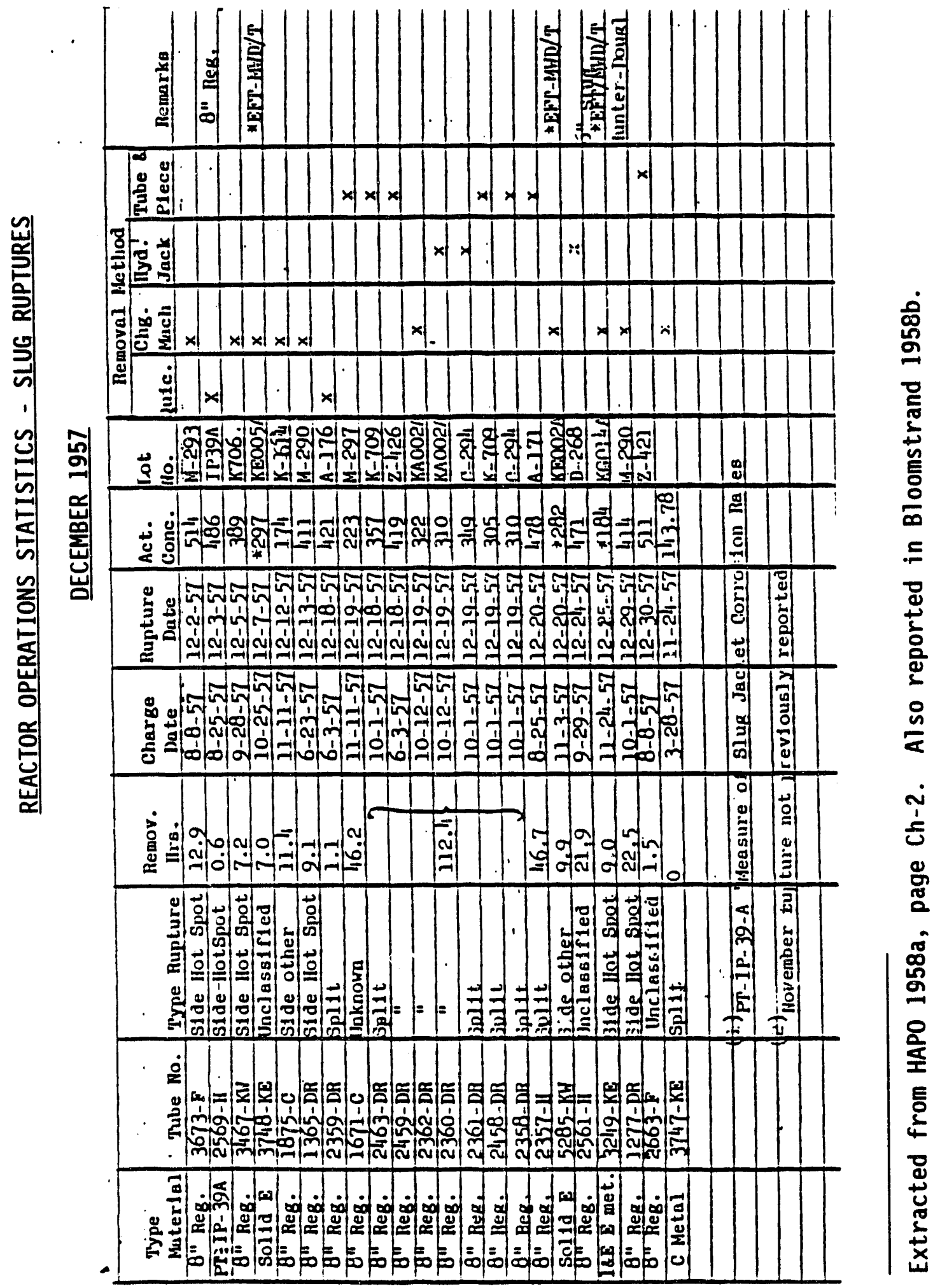




\section{APPENDIX $\mathrm{H}$}

FUEL-ELEMENT FAILURE DATA FOR 1958 


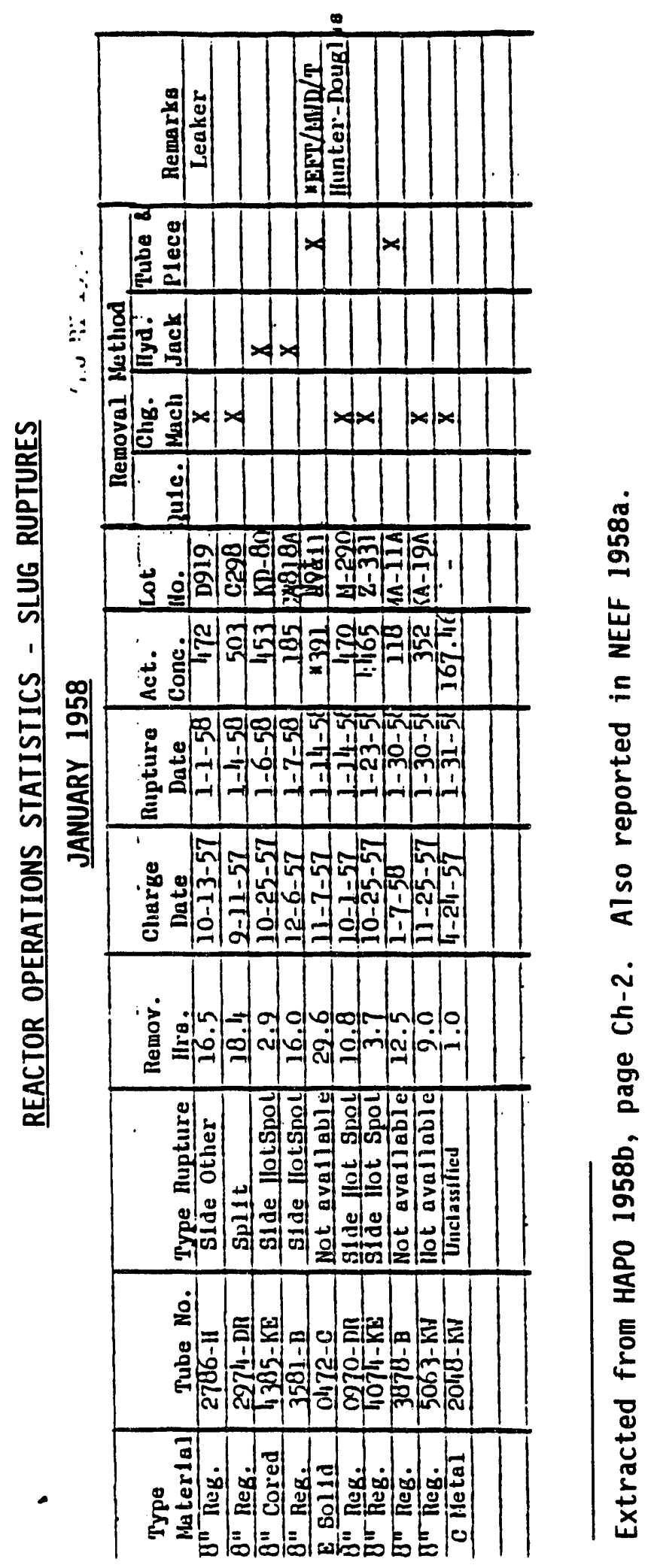

H. 1 
RUPTURE EXPERIENCE - FEBRUARY 1958

\begin{tabular}{|c|c|c|c|c|c|}
\hline Fa1lure Dete & Tube Number & Lot Number & Troe $N=t a l$ & Exposure & Troe Fa1lure \\
\hline $2-7-58$ & 2791-E & $B-300-B$ & 6" Coreq $E$ & 505 & Uncless. \\
\hline $\begin{array}{l}2-8-58 \\
2-14-58 \\
2-22-58 \\
2-22-58 \\
2-25-58\end{array}$ & $\begin{array}{l}2766-D B \\
3583-K E \\
4058-E \\
5359-K W \\
3748-1 C\end{array}$ & $\begin{array}{l}C A-051-A \\
B E-015-D \\
B E-023-A \\
B A-036-A \\
K G-014-A\end{array}$ & $\begin{array}{l}\text { 8" Regular } \\
\text { 8" Cored(5) } \\
\text { 6" E Metal (1) } \\
\text { 8" Regular } \\
\text { 6" I \& E E (2) } \\
\text { Metal (I) (2) }\end{array}$ & $\begin{array}{l}168 \\
425 \\
187 \\
352 \\
528\end{array}$ & $\begin{array}{l}\text { Side E.S. } \\
\text { Side E.S. } \\
\text { S1de E.S. } \\
\text { Uncless. } \\
\text { N.A. }\end{array}$ \\
\hline $2-27-58$ & $4858-\pi N$ & CA-044-A & 8" Regular & 317 & Unclass. \\
\hline $1-31-58$ & $1249-K \pi$ & N_A. & Doe Metal (3) & $27 \cdot 7^{(4)}$ & $\begin{array}{l}\text { Side (Jacket } \\
\text { Split) }\end{array}$ \\
\hline $\begin{array}{l}1-3 i-58 \\
2-12-58\end{array}$ & $\begin{array}{l}2048-\pi N \\
0462-C\end{array}$ & D.A. & $\begin{array}{l}\text { Doe Metal (3) } \\
\text { Doe Metal }\end{array}$ & $\begin{array}{l}30.8(4) \\
34.4(4)\end{array}$ & $\begin{array}{l}\text { Side E.S. } \\
\text { Side (Jaciset } \\
\text { SpIÉ) }\end{array}$ \\
\hline $2-27-58$ & $3054-c$ & $0-7$ & Doe & $36.0(4)$ & $\begin{array}{l}\text { Side (Jacket } \\
\text { Split) }\end{array}$ \\
\hline
\end{tabular}

(1) $0.94 \%$ Earichment

(2) Second Failure in this Lot -- Prev10us Charge in this Mube Failed 12-25-5T.

(3) Prevlously Unreported

(4) Fer cent $0^{235}$ Depletion of the Doe Metal in this Colum

(5): M-388 alloy Jaciset

Legend:

Side E.S. - Failure probably caused by local overbeating (Bot Spot) and possibly intergranular corrosion.

Jnclassified - Fallure does not fall tnto any particular category.

II.A. - Hot Arailable.

Extracted from HAPO 1958c, page B-21. Also reported in NEEF 1958a.

H.2 


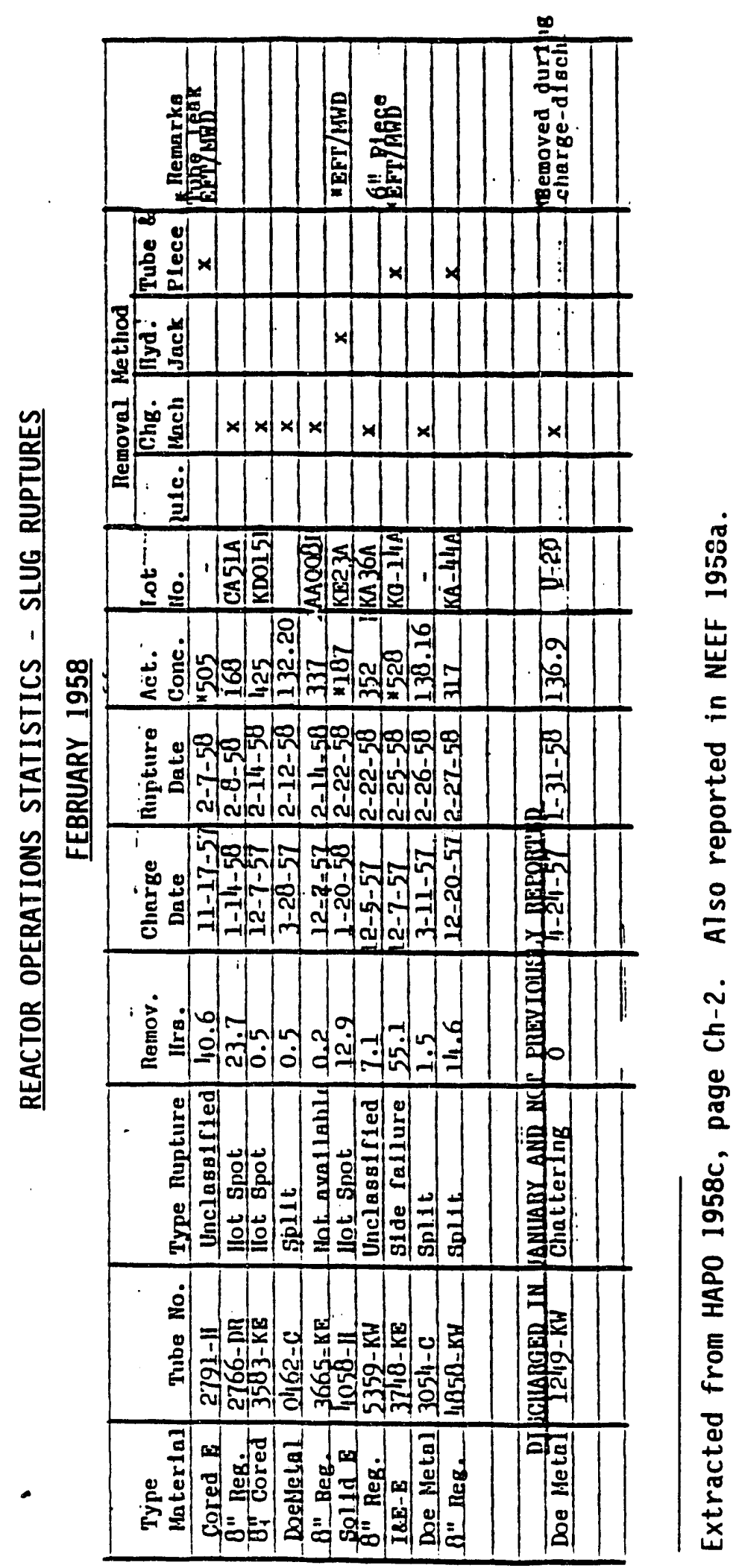

H. 3 


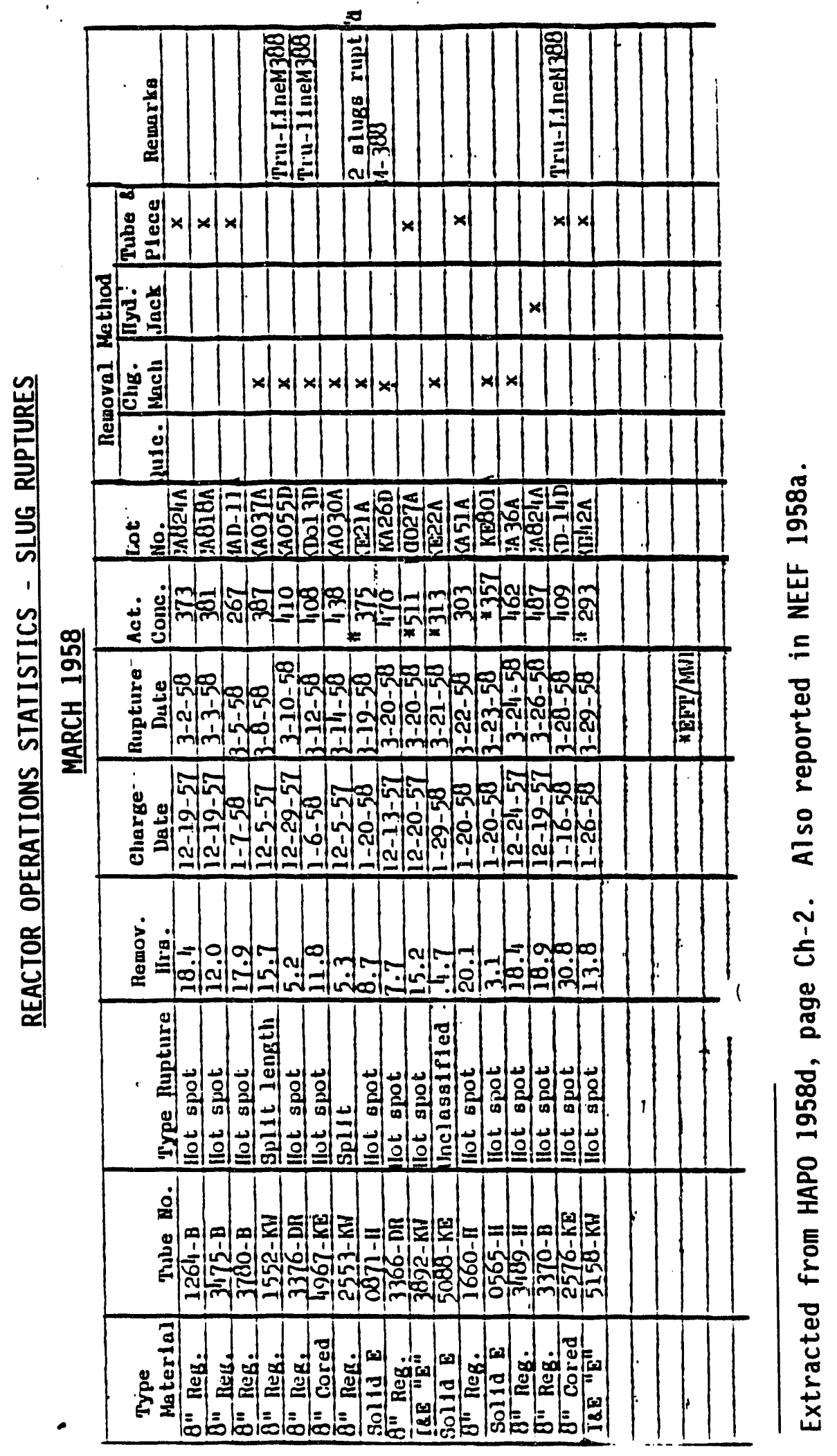




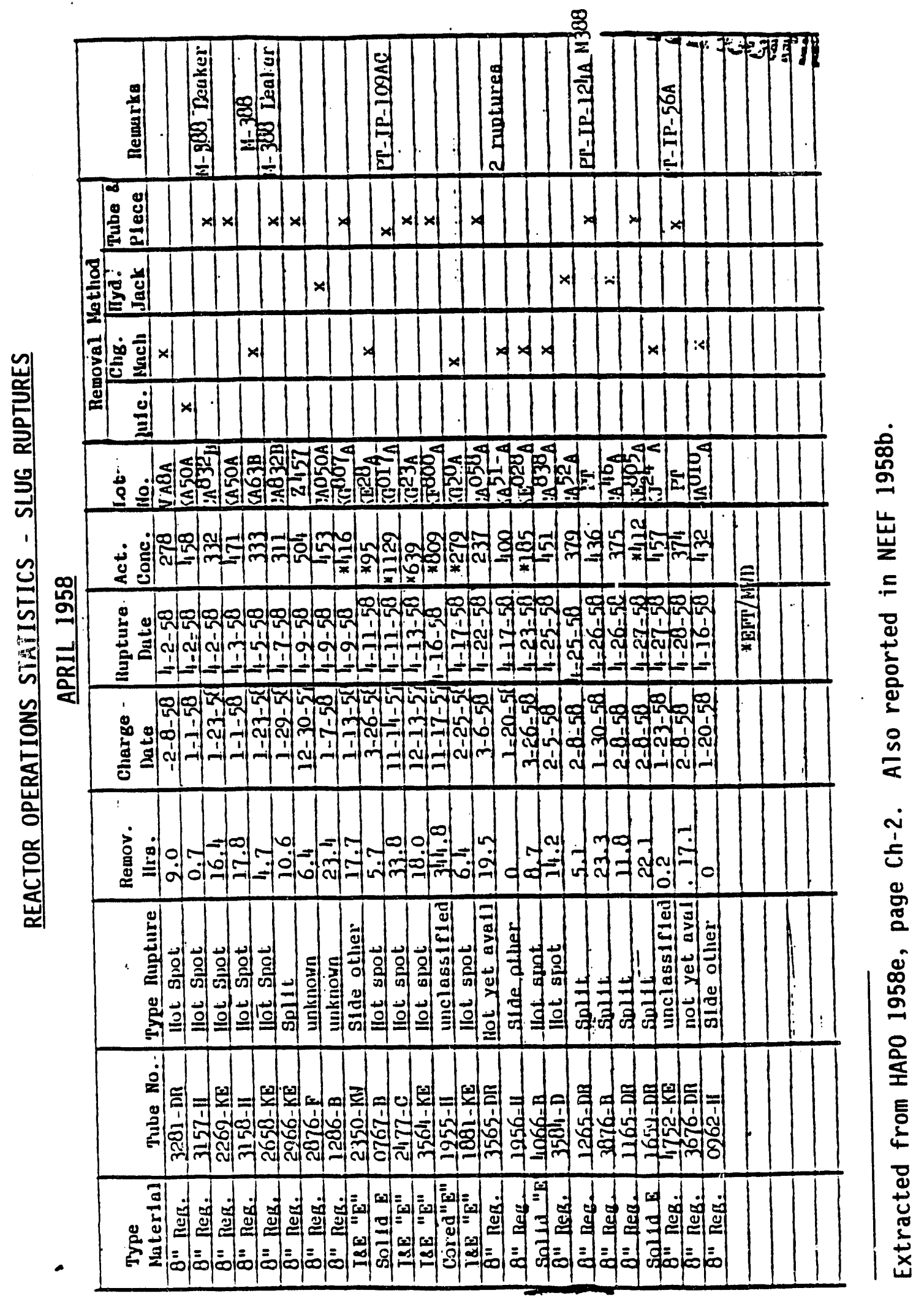


RUPTURE EXPERIENCE - MAY 1958

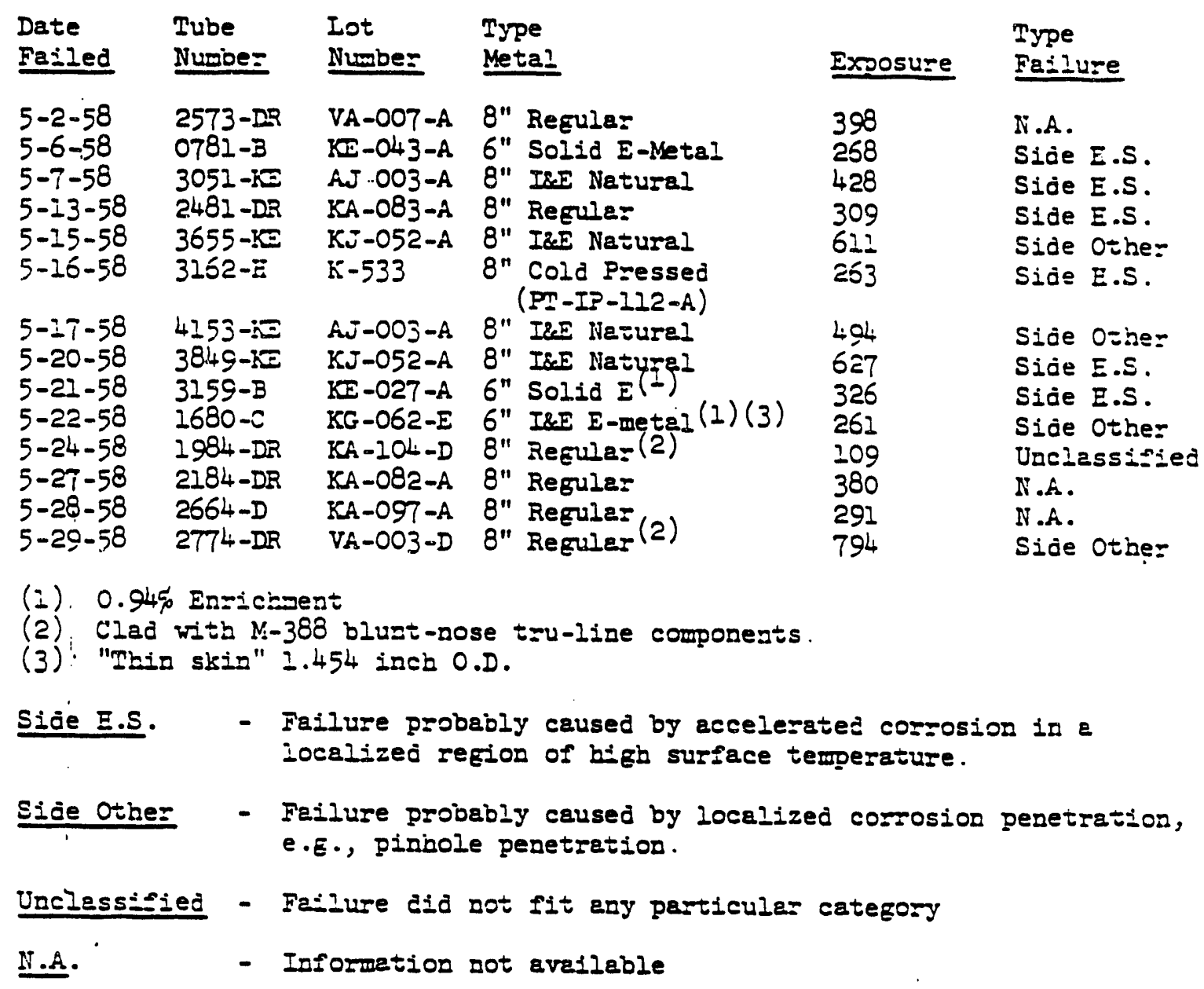

Extracted from HAPO 1958f, page B-23. Also reported in NEEF 1958c. 


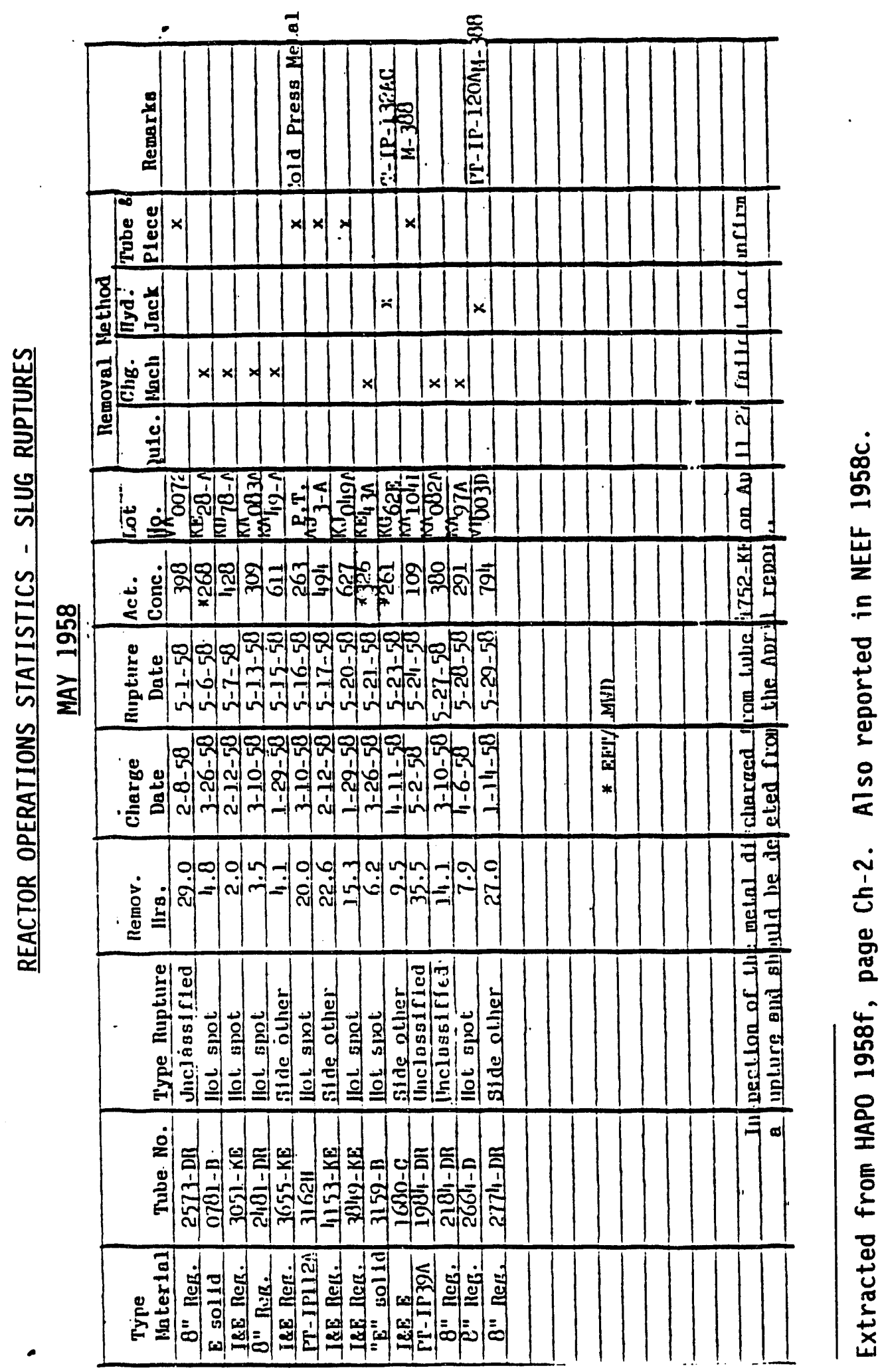

H. 7 


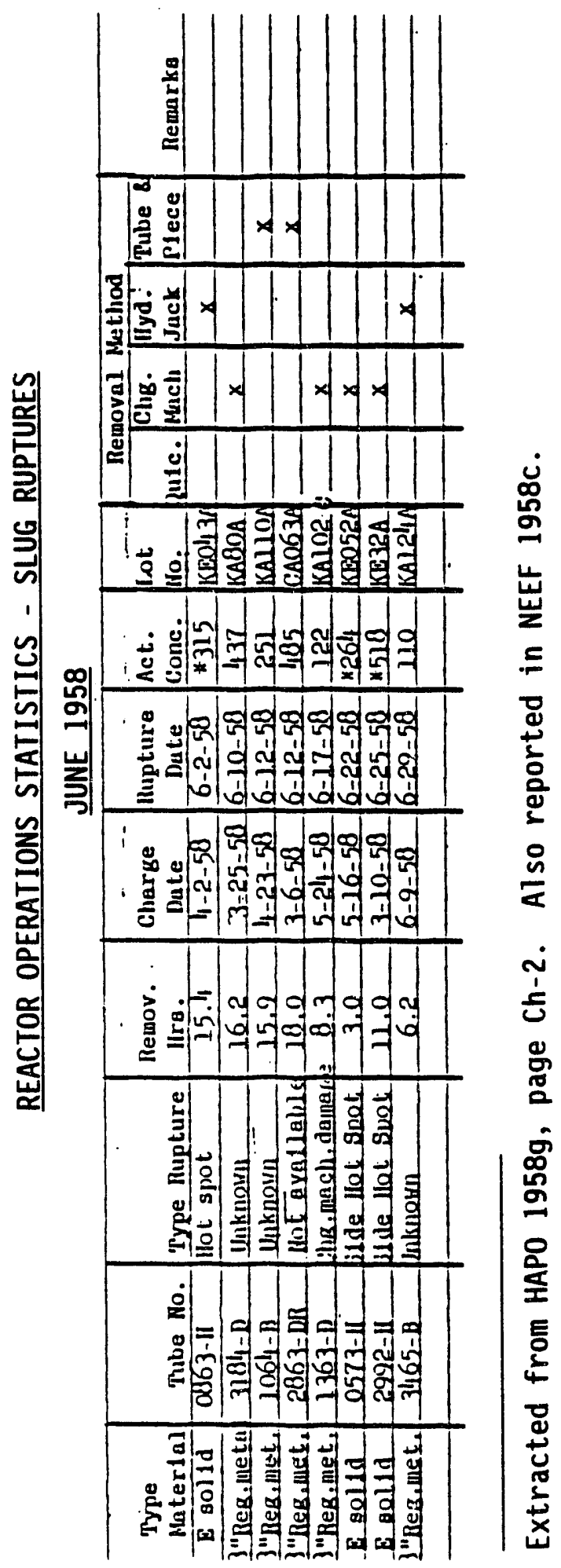

H. 8 


\section{RUPTURE EXPERIENCE - JULY 1958}

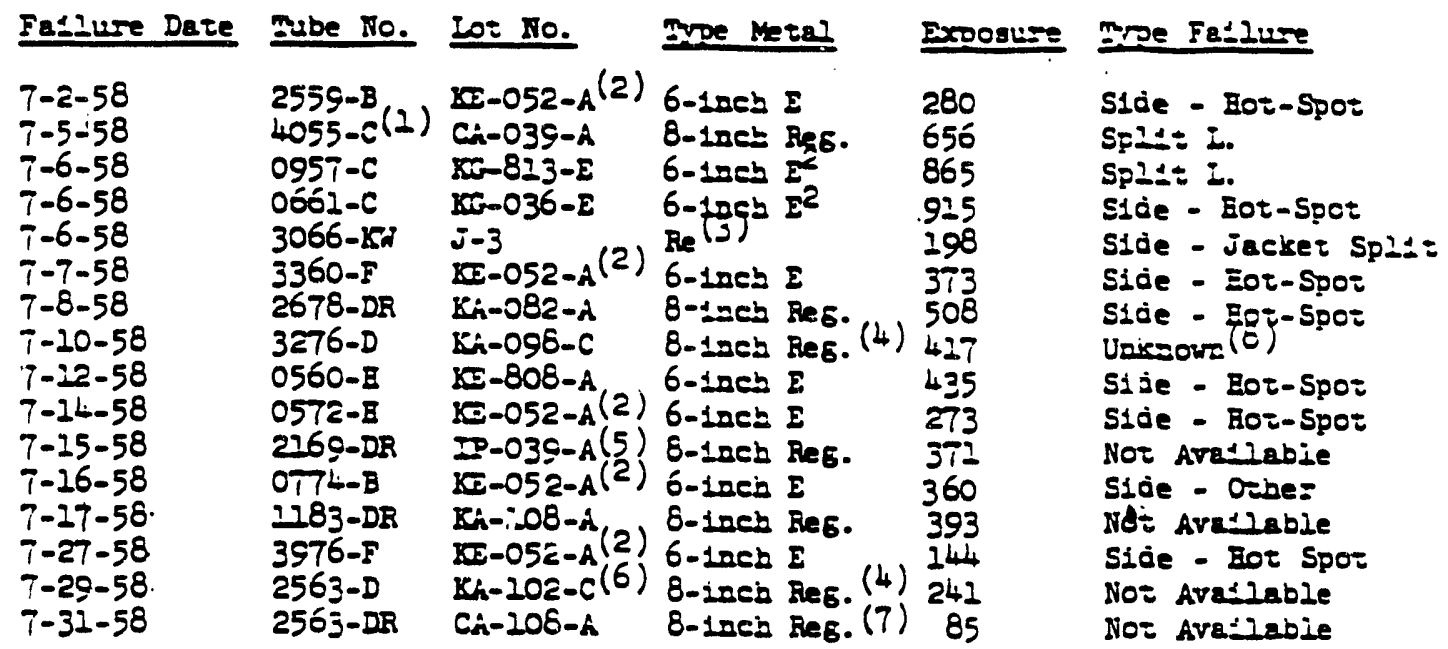

(1): Two ruptured slugs is thss tube

(2) Mitiple iailure lot

(3). PI IP-210-A (18-5, 12-Fegular and 18-N Londing)

(4)i Blusesose maldae

(5). Contadred 2245 agd M-388 jeckered slugs. Ruprure fiece stuciv anc zemoved wath tube.

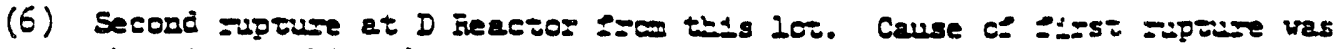
chargits macicine demage.

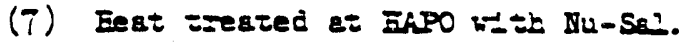

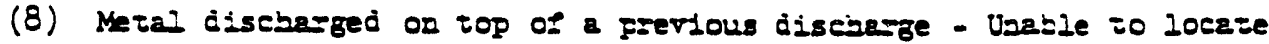
zuprexec piece.

Legend:

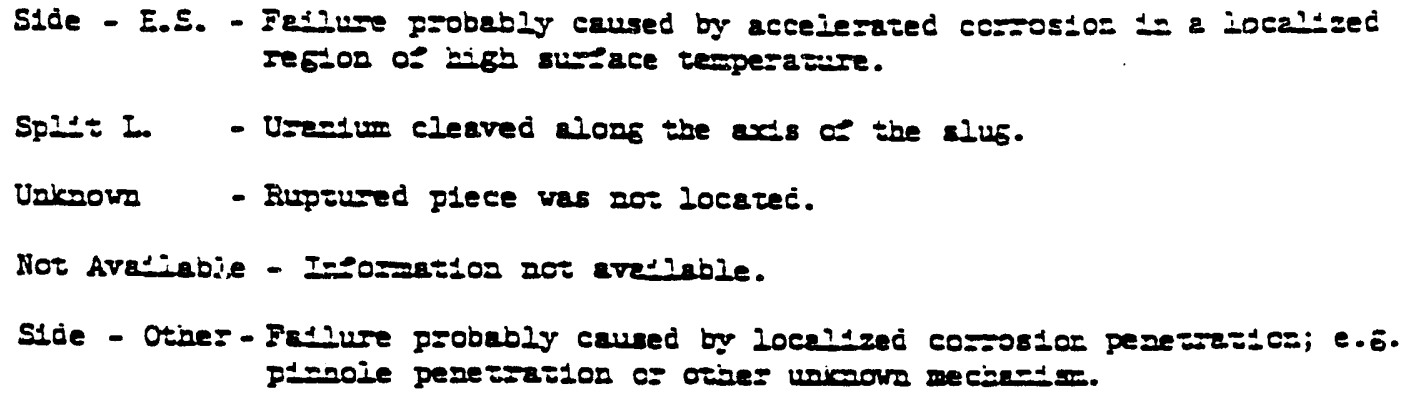

Extracted from HAPO 1958h, page B-24. Also reported in NEEF 1958d. 


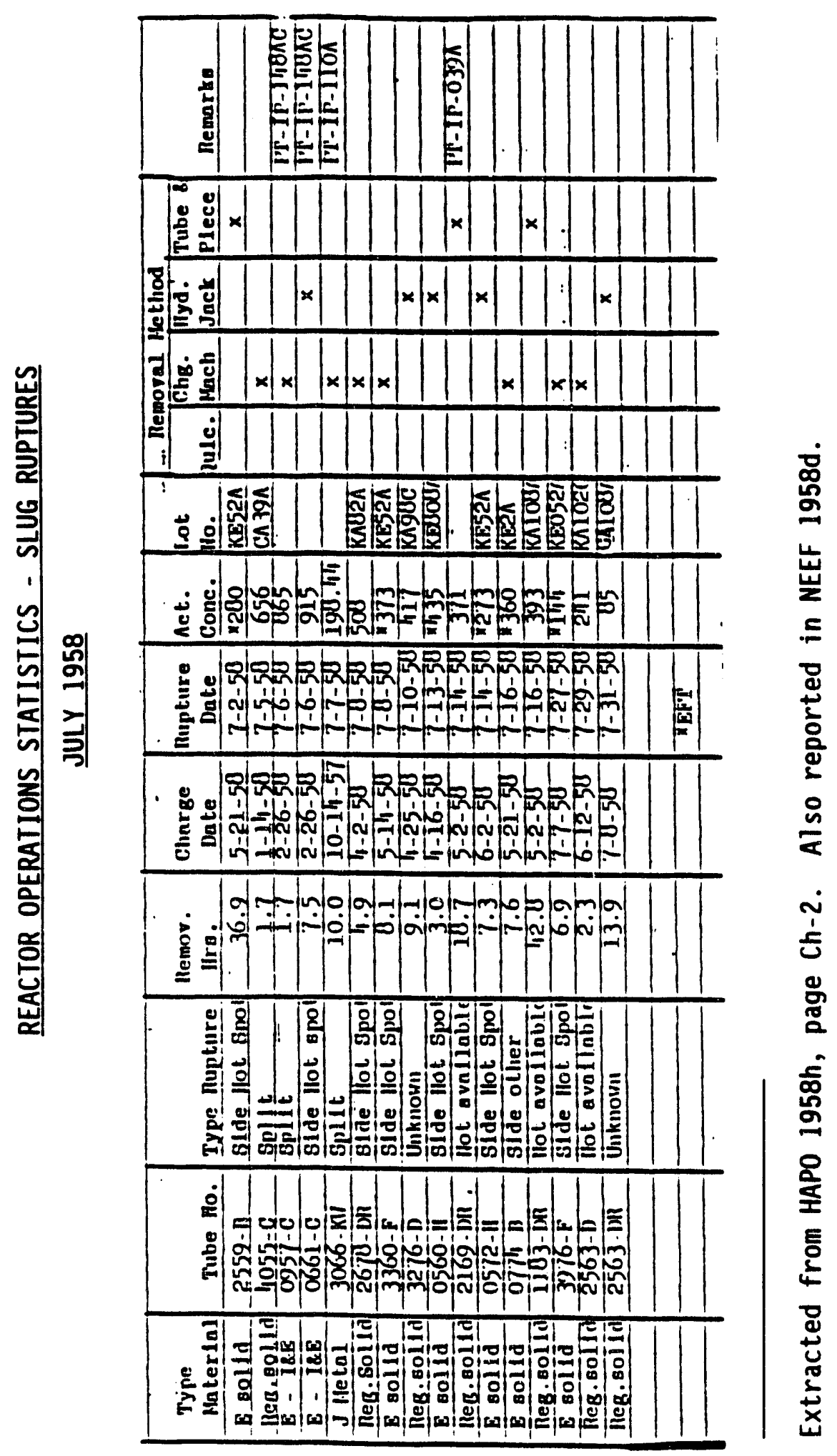




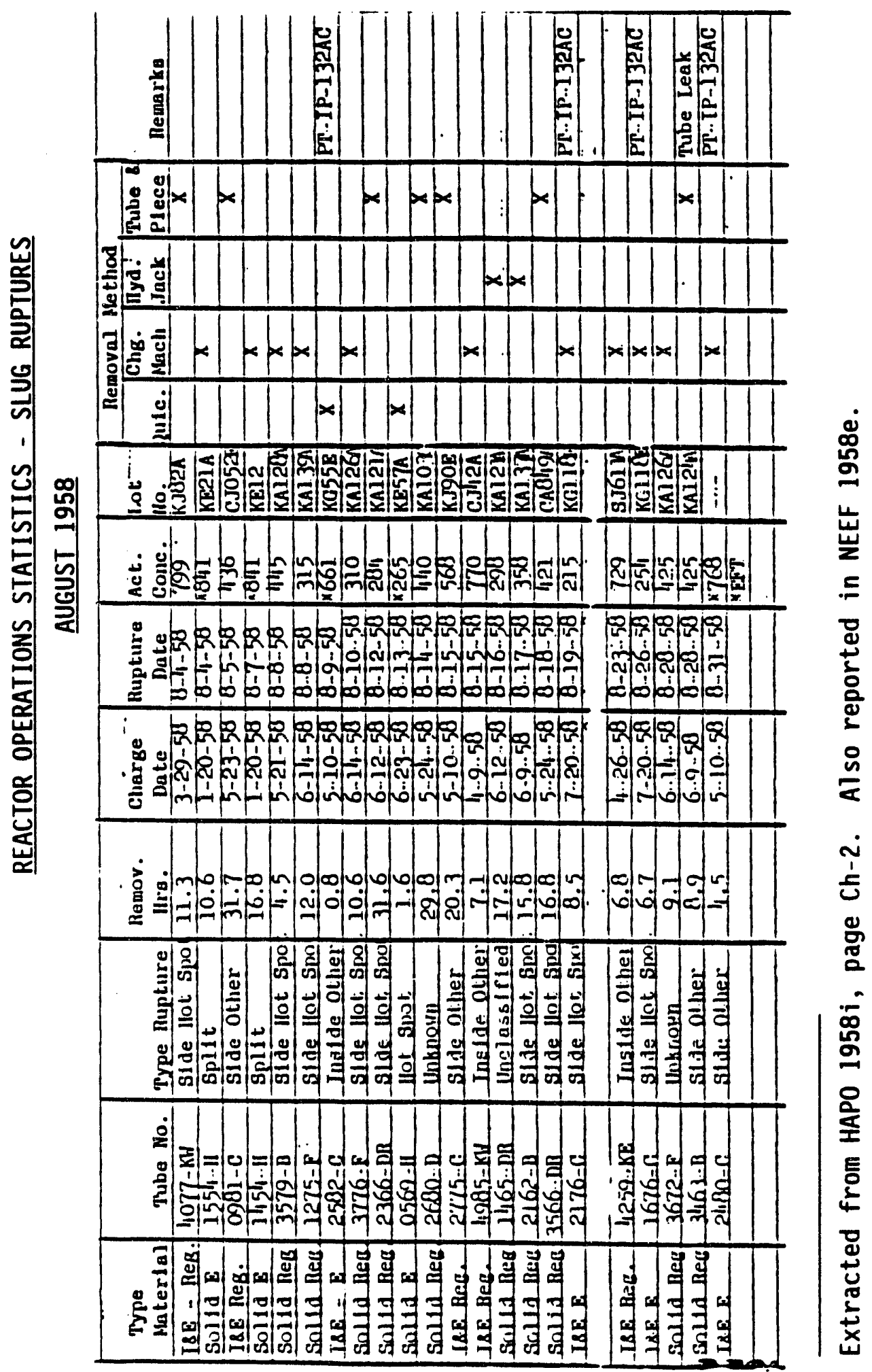




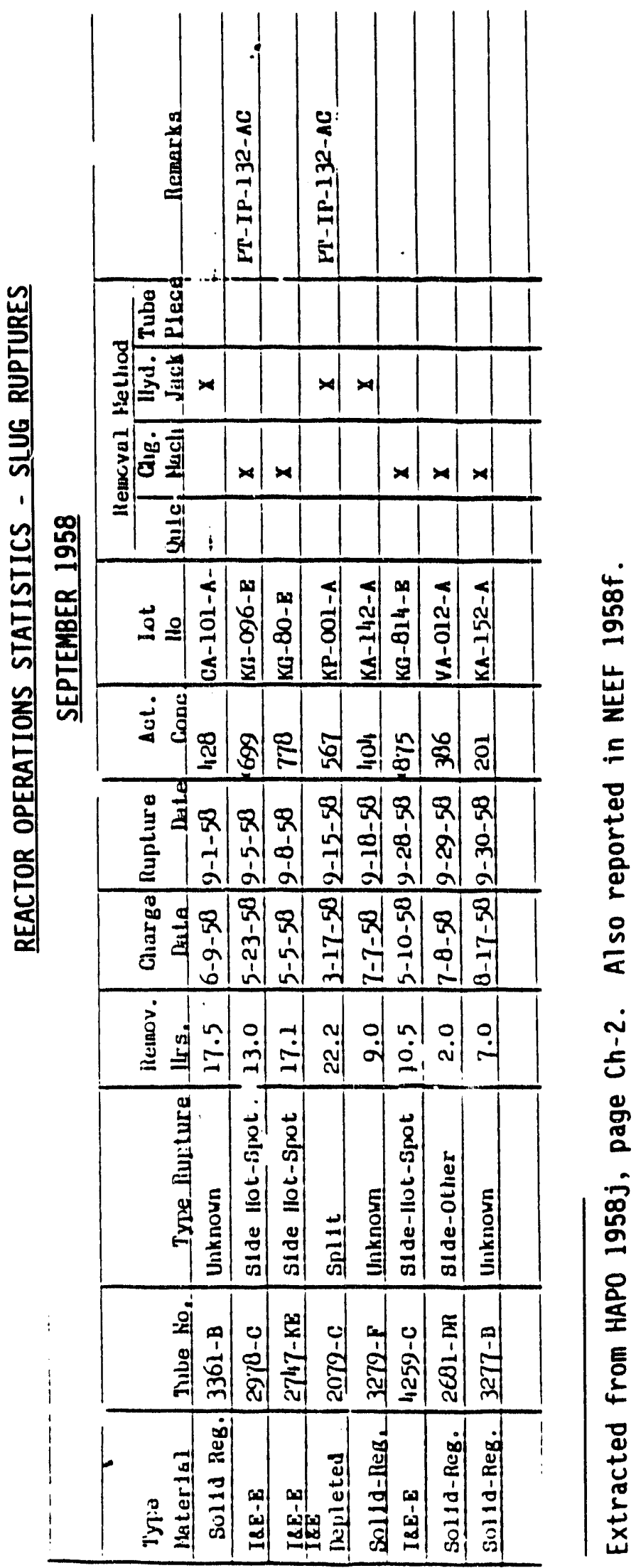

H. 12 
RUPTURE EXPERIENCE - OCTOBER 1958

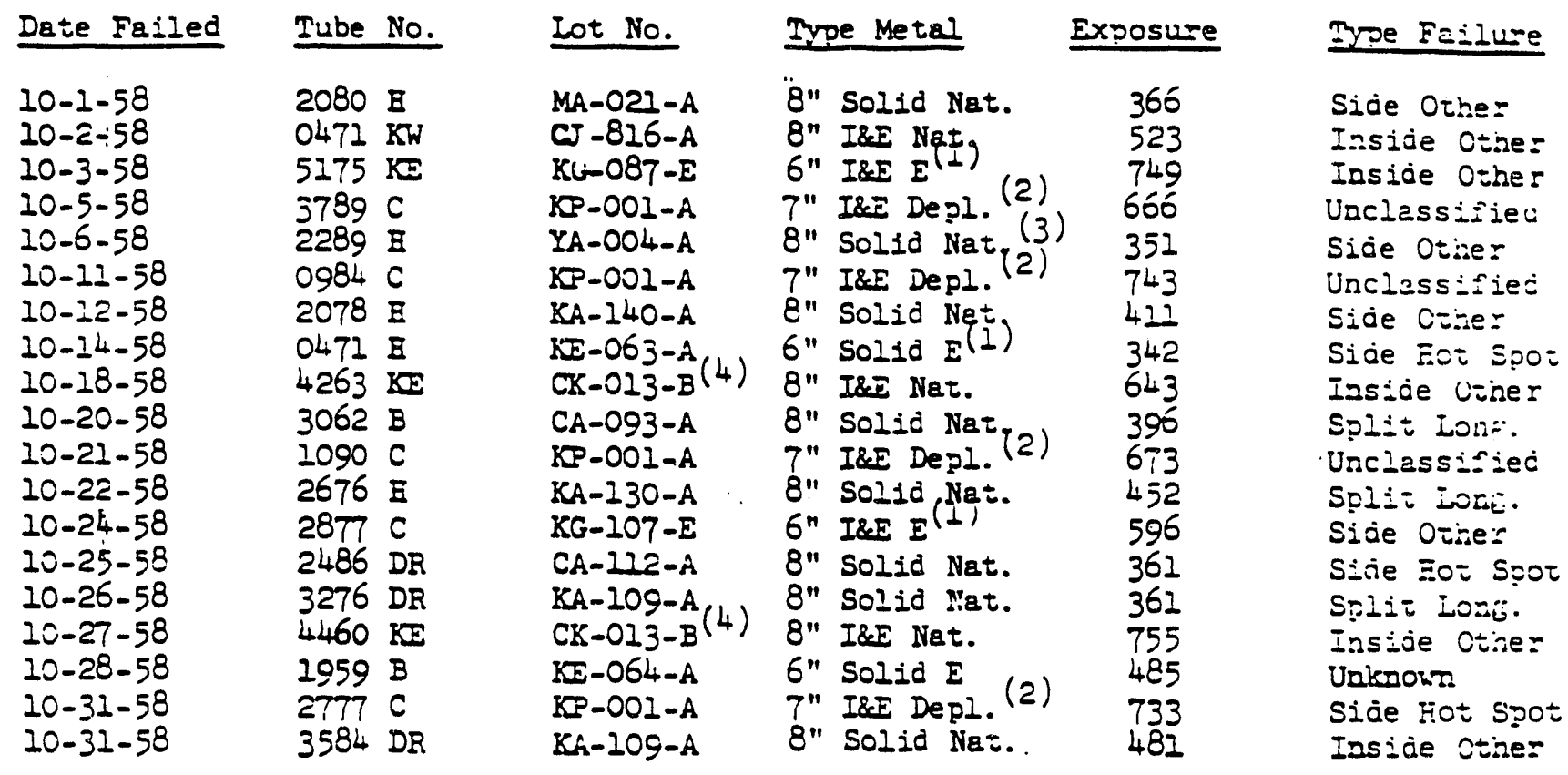

\footnotetext{
(1) $0.94 \%$ U-235

(2) I \& E depleted beize 1radiated under IF-132-AC

(3) Extruded ingot material

(4) iMaterial from reprocessed lot sK $-600-B$
}

Iegend :
Side Elot Spot - Failure probably caused by acceierated corrosion is a localizec region of high temperature.
Sice Other - Failure probably caused by corrosion or water penetration of tine can. wail or other unikgown mechanism.
Inside Other - Similar to side other except occurfing on the Inside surface of an I \& $\mathrm{E}$ slug.

Split L. - Fetlure caused by uranium cleavage along the axis of the core.

Unclassteied - Failure did not logicanly fall 1nto any of the above types.

Unicnows - Ruptured piece was lost asong other metal.

Extracted from HAPO 1958k, page B-21. Also reported in NEEF 1959a. 


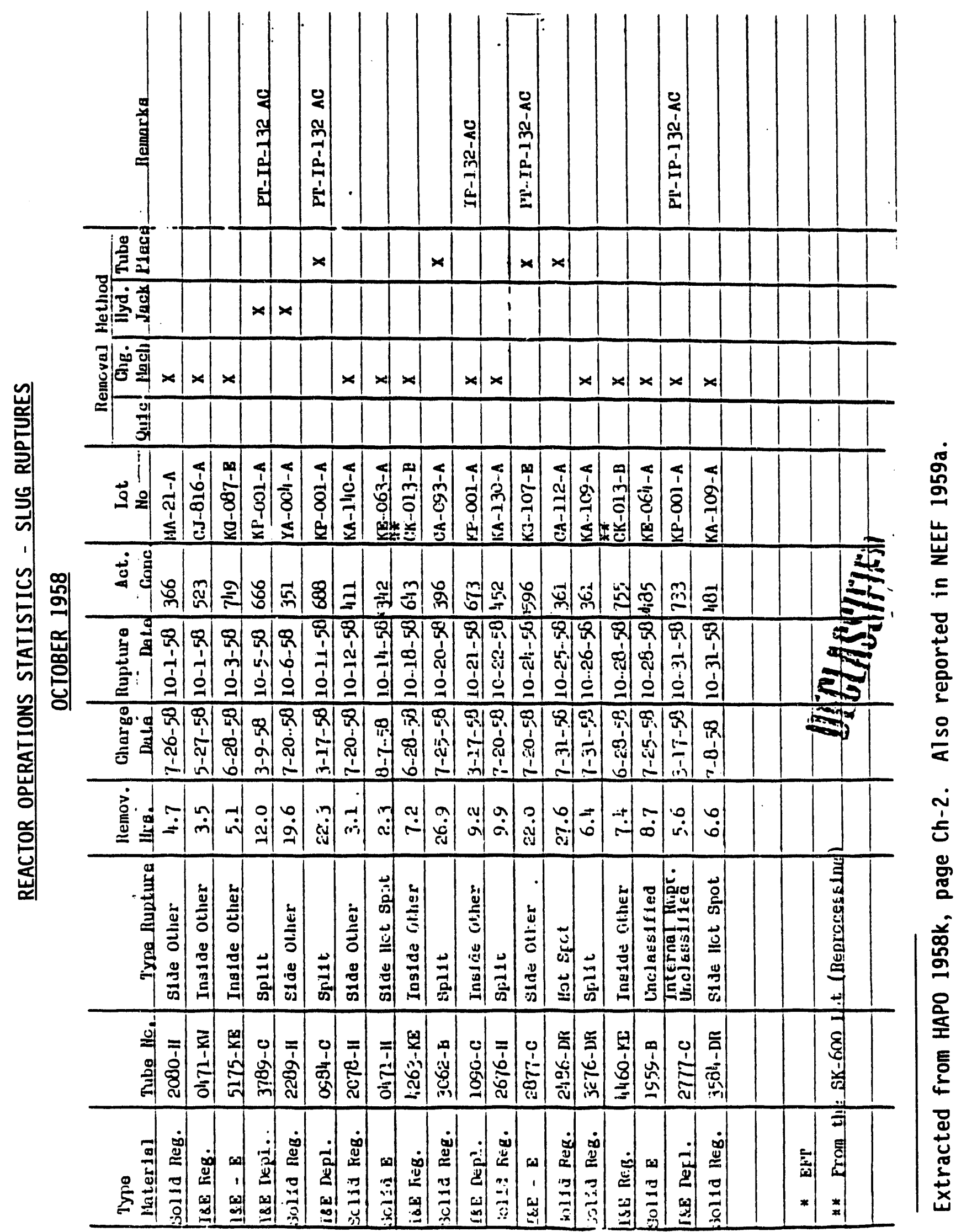


RUPTURE EXPERIENCE - NOVEMBER 1958

\begin{tabular}{|c|c|c|c|c|c|}
\hline ilure Date & dibe No. & ot No. & ype Metal & sxposure & Type Failure \\
\hline $\begin{array}{l}1 / 3 / 58 \\
1 / 3 / 58 \\
1 / 6 / 58 \\
1 / 8 / 58 \\
1 / 9 / 58 \\
1 / 15 / 58 \\
1 / 17 / 58\end{array}$ & $\begin{array}{l}0988-C \\
3093-K W \\
1453-C \\
1547-K E \\
1490-C \\
3590-C \\
4268-K E\end{array}$ & $\begin{array}{l}\text { KP-001-A } \\
\text { SK-601-B }(2) \\
K A-123-B \\
\text { SG-020-E }(3) \\
K P-001-A \\
K P-001-\ddot{B} \\
\text { UnknOWn }\end{array}$ & $\begin{array}{l}\text { 7" I\&E Depl. (I) } \\
\text { 8" I\&E Nat. } \\
\text { 8" Solid Net. } \\
\text { 6" I\&E E (4) (I) } \\
\text { 7" I\&E Depl. (I) } \\
\text { 7" I\&E Depl. (I) } \\
\text { Doe }\end{array}$ & $\begin{array}{l}778 \\
671 \\
483 \\
925 \\
762 \\
826 \\
32.51\end{array}$ & $\begin{array}{l}\text { Inside-Other } \\
\text { Inside-Otier } \\
\text { Cap } \\
\text { Inside-Other } \\
\text { Unclassified } \\
\text { Unclassifieo }\end{array}$ \\
\hline $\begin{array}{l}11 / 17 / 58 \\
11 / 17 / 58 \\
11 / 17 / 58 \\
11 / 18 / 58 \\
11 / 24 / 58 \\
11 / 30 / 58\end{array}$ & $\begin{array}{l}1269-D R \\
3057-C \\
2954-B \\
1358-B \\
2579-C \\
1276-C\end{array}$ & $\begin{array}{l}K A-155-A \\
S G-027-E \\
C A-120-A \\
K A-084-A \\
K P \text { TO1-A } \\
K P-001-A .\end{array}$ & $\begin{array}{l}\text { 8" Solid (Nat. } \\
\text { 6" I\&E E } \\
\text { 8" Solid Nat. } \\
\text { 8" Solio Nat (I) } \\
\text { 7" I\&E Depl. (I) } \\
\text { 7" I\&E Depl. }\end{array}$ & $\begin{array}{l}448 \\
589 \\
404 \\
533 \\
682 \\
748\end{array}$ & $\begin{array}{l}\text { Unclassilico } \\
\text { Unclassified } \\
\text { Side-Hot Spot } \\
\text { Side-Other } \\
\text { Inside-Other } \\
\text { Inside-Other }\end{array}$ \\
\hline
\end{tabular}

(1) Ióce depleted being irradiated under IP-13â-AC

(2) Failure piece reprocessed from lot $\mathrm{MK}-003-\mathrm{B}$

(3) Failure piece reprocessed from lot KG-O7O-E

(4) $0.94 \%$ U-235

(5) Doe metal being irradiated in KER loop \#4 under IP-190-A

(6) U-235 per cent burnout

Iegend:

Side Elot Spot - Failure probably caused by accelerated corrosion in a localized region of higin temprature.

Side Otber - Failure probably caubed by corrosion or water penetration of tine can wall or otber unknown mechanism.

Inside Other - Similar to side other except occurring on the inside surface of an I \& E slug.

Unclassified - Faflure did not logically fall 1nto any of the above types.

Cap - Rupture probably cuased by water penetration through the veld.

Extracted from HAPO 19581, page B-21. Also reported in NEEF 1959a. 


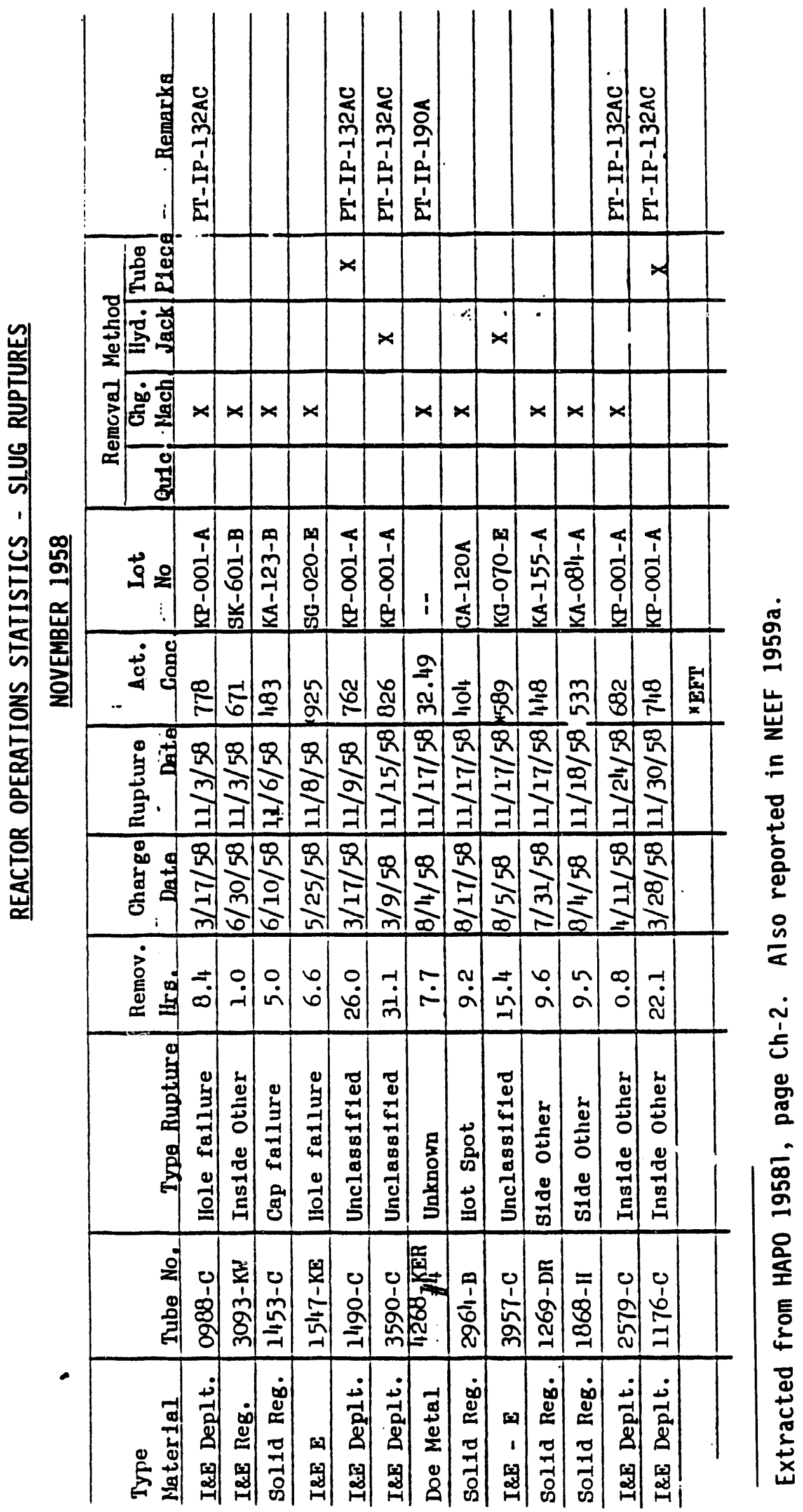

H. 16 


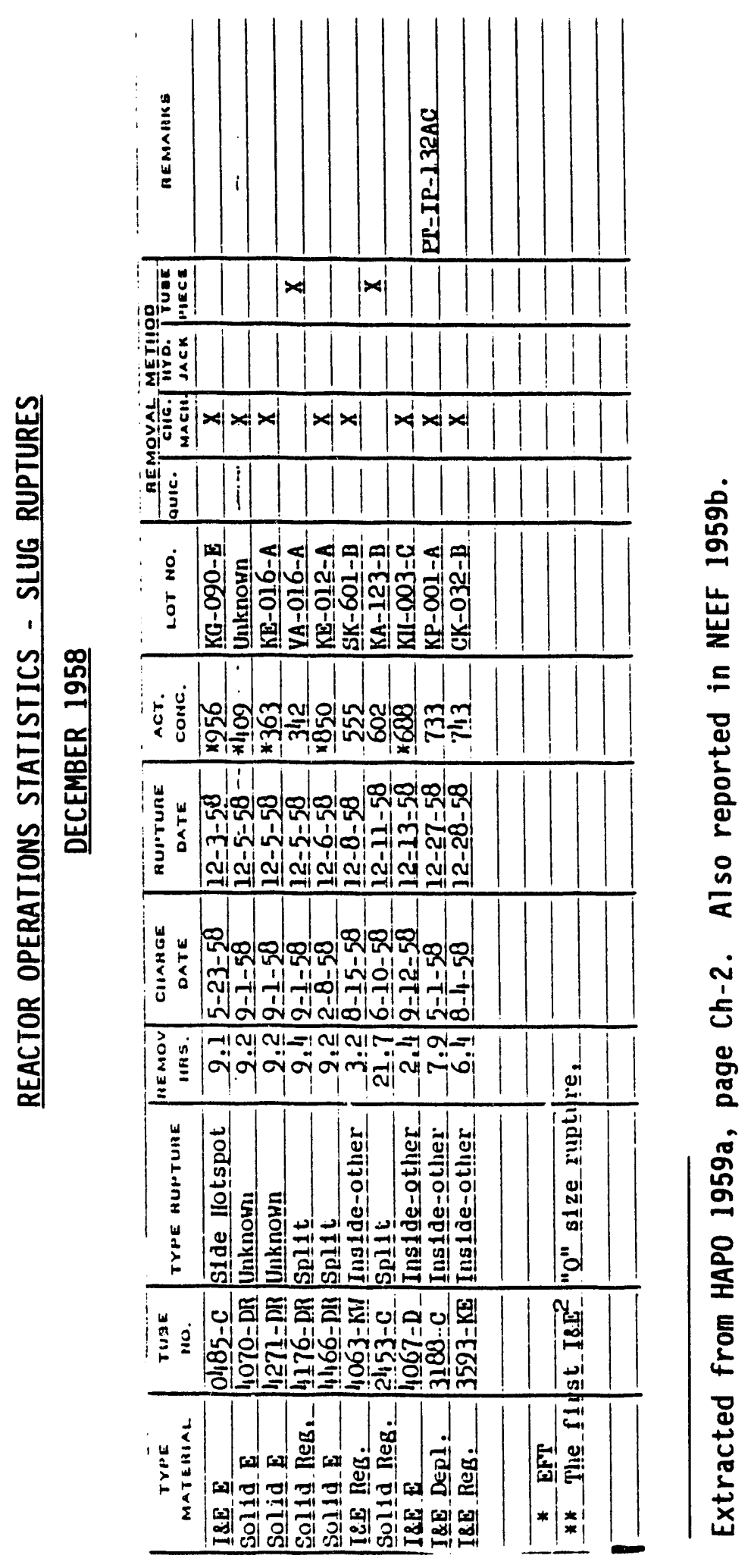


APPENDIX I

FUEL-ELEMENT FAILURE DATA FOR 1959 


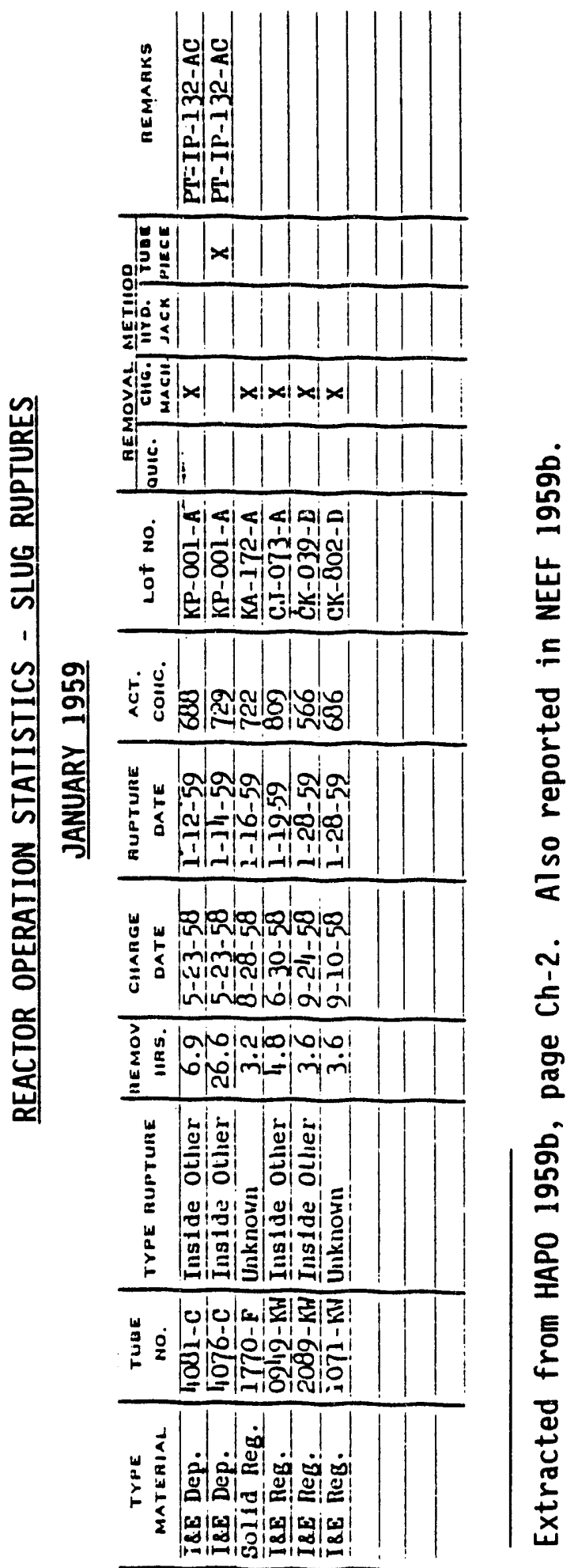

I. 1 


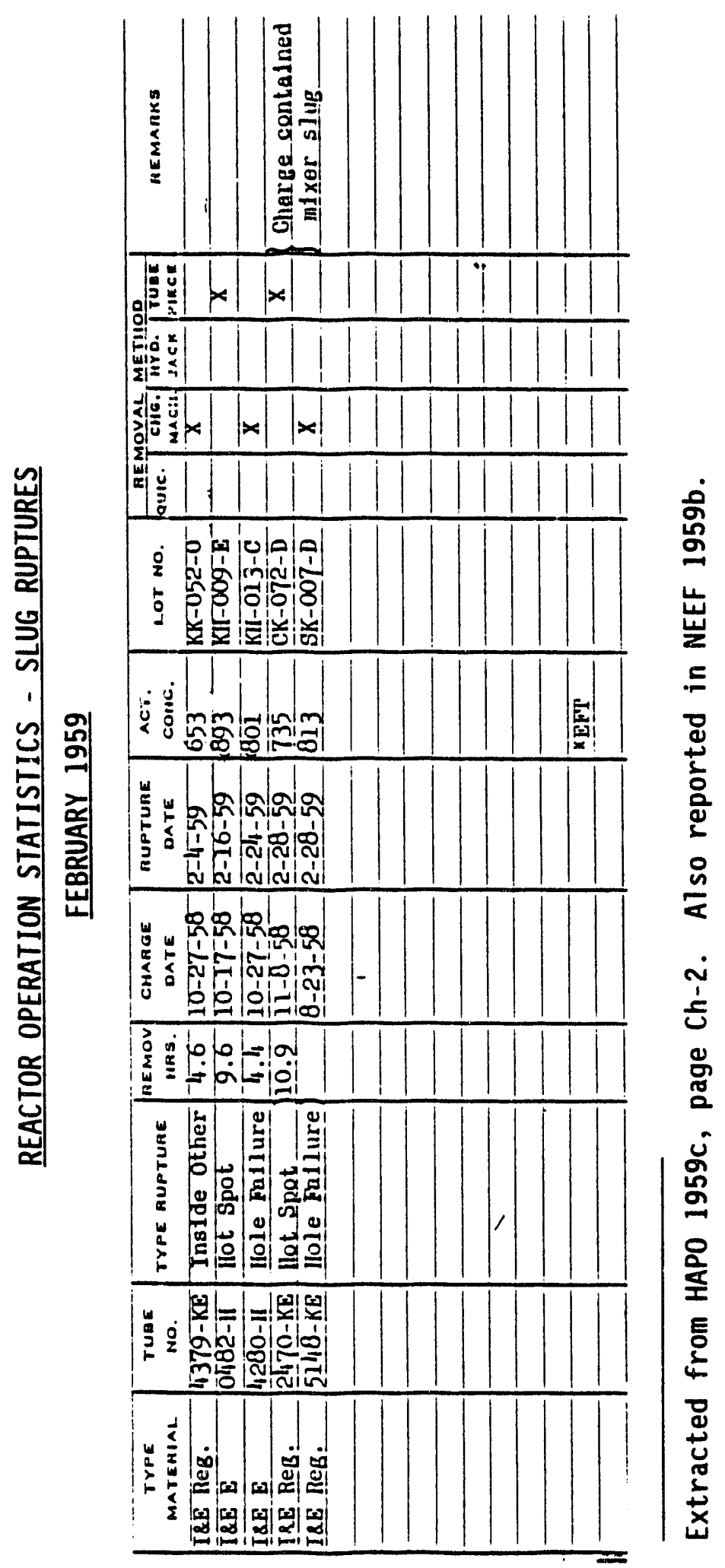




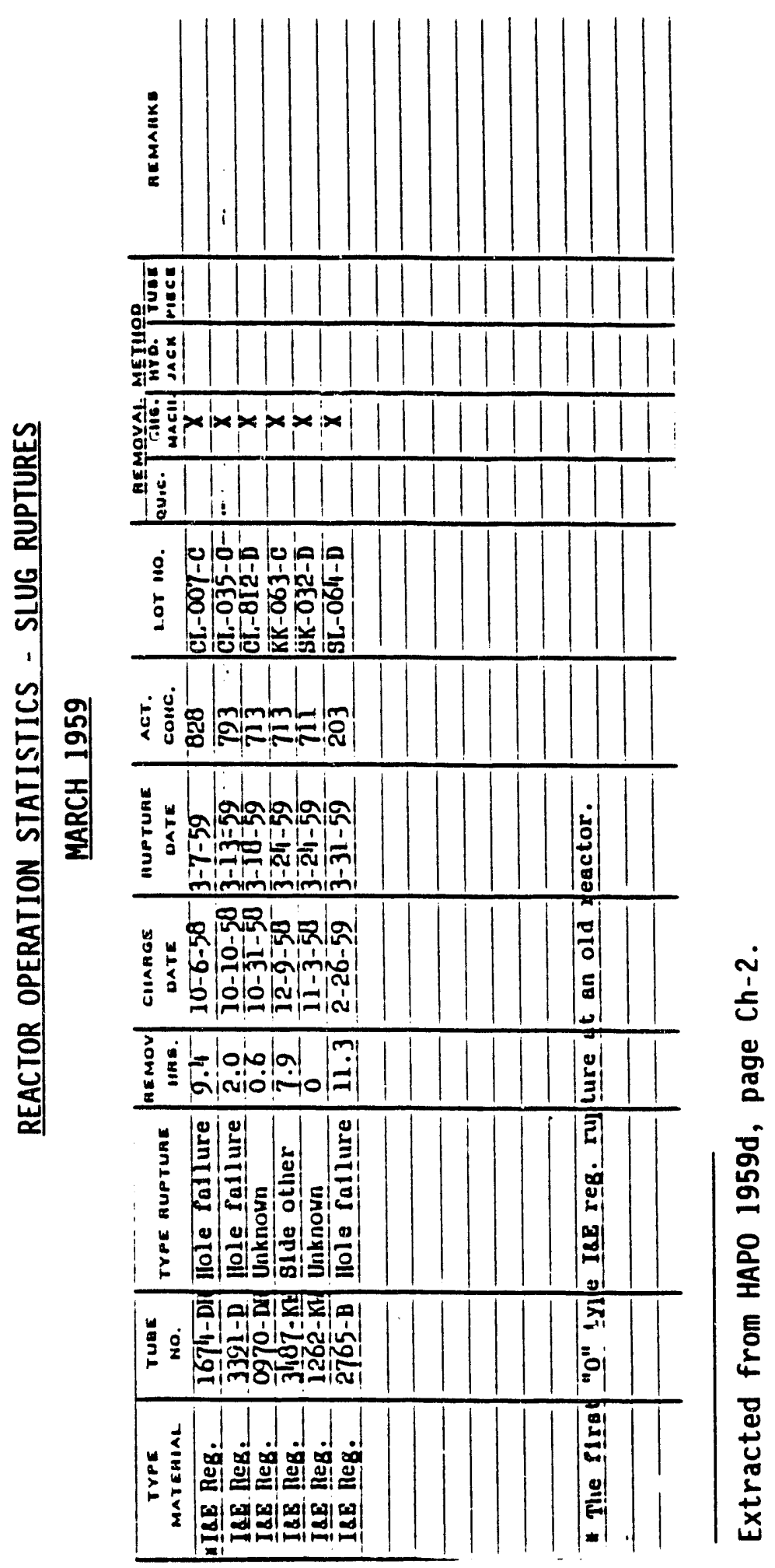




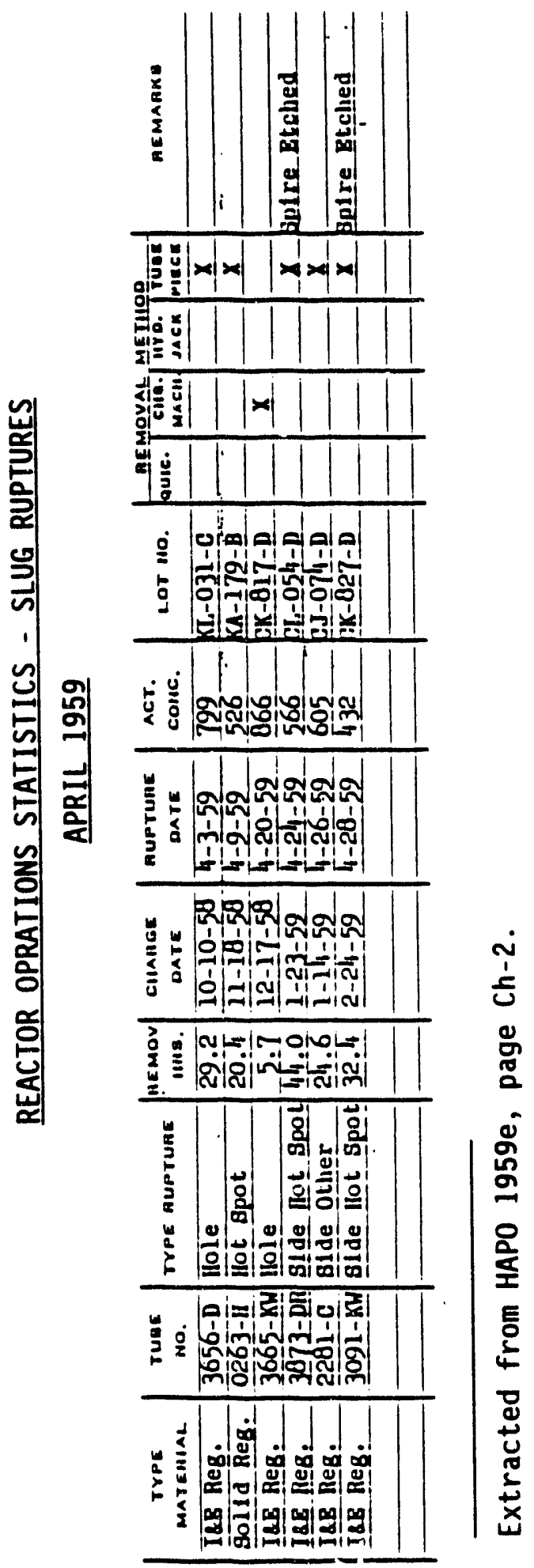


RUPTURE EXPERIENCE - MAY 1959

Fa1qure Date Pube No. Lot jo.

i $5 / 9 / 59$

$5 / 10 / 59$

$5 / 12 / 59$

$5 / 19 / 59$

$5 / 26 / 59$

$5 / 27 / 59$ $377^{8}-\mathrm{z}$

$1279-C$

$1965-2$

35T2-:Th

$3757-2$

$1587-\overline{2}$

$c 2-\infty 69-j($

c5 $081-C$

CJ $-097-6$

x. $-082-D$

Ci- $-069-D(1)$

c. $830-1$
Type Metal

8" IRE Natural

8" Ine Matural

8" The Iatural

8" IRS Thatural

$8^{n}$ res tracurel

$8^{\prime \prime}$ Th5 Jatural
Exposure Moe To11ure

412 S1de-3ot Spot

560 S1de-Eot spot

568 Eole

527 Eole

607 Bole

657 Unclassilied

(2) Multsple sacluse lot.

Legend:

Side-Eot Spot - Fatlure probably caused by eccelerated coscosion 10 a localtaed regton of be tem temerature.

Jole - Easlure or the interan ourface of a I I a plece probably caused by wate:

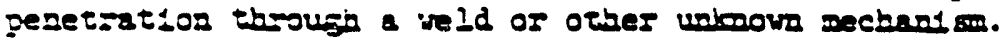

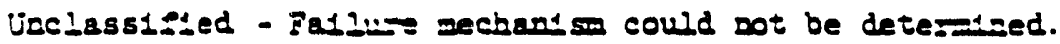

Extracted from HAPO 1959f, page B-20. 
RUPTURE EXPERIENCE - JUNE 1959

Failue Date Mube No. Lot No.

$6 / 1 / 59$

$6 / 1 / 59$

$6 / 6 / 59$

$6 / 13 / 59$

$6 / 25 / 59$

$6 / 20 / 59$

$6 / 22 / 59$

$6 / 29 / 50$

\section{5}

$2778 \mathrm{C}$

37818

32520

22765

40620

iาTE

27038
Mroe Meza?

8" Solid

8" Ire Interal

8n. Ise. Netwral

8n Ise Neterel

8" Ine Thever

8" IRE Netist

8" Ife Naseral

8" I E Natura
Excosute Mope Iedlure

83

Ubelassteled (4)

ถ⿻-19T-D

ST-070-D (2)

B-107-D(3)

CD-OFT-D

उ-06I-0
792

725

642

497

785

754

851
Bole

Side Other

inle

Bole

Eole

Eole
Unelessieled

(1) Ci-097-c was the pareze lot.

(2) J-150-D vas the parest lot.

(3) 2 soptures is tube.

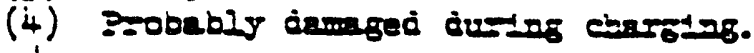

Iegero: :

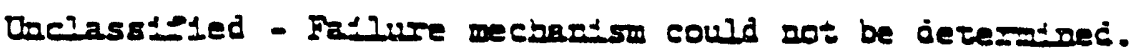

Eole - Fafluze on the lateral surace of an I \& E piece probrbiy caused by

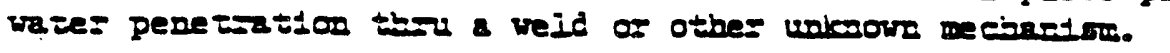

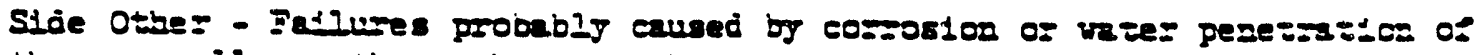

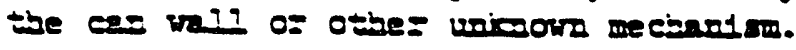

Extracted from HAPO $1959 \mathrm{~g}$, page B-18. 


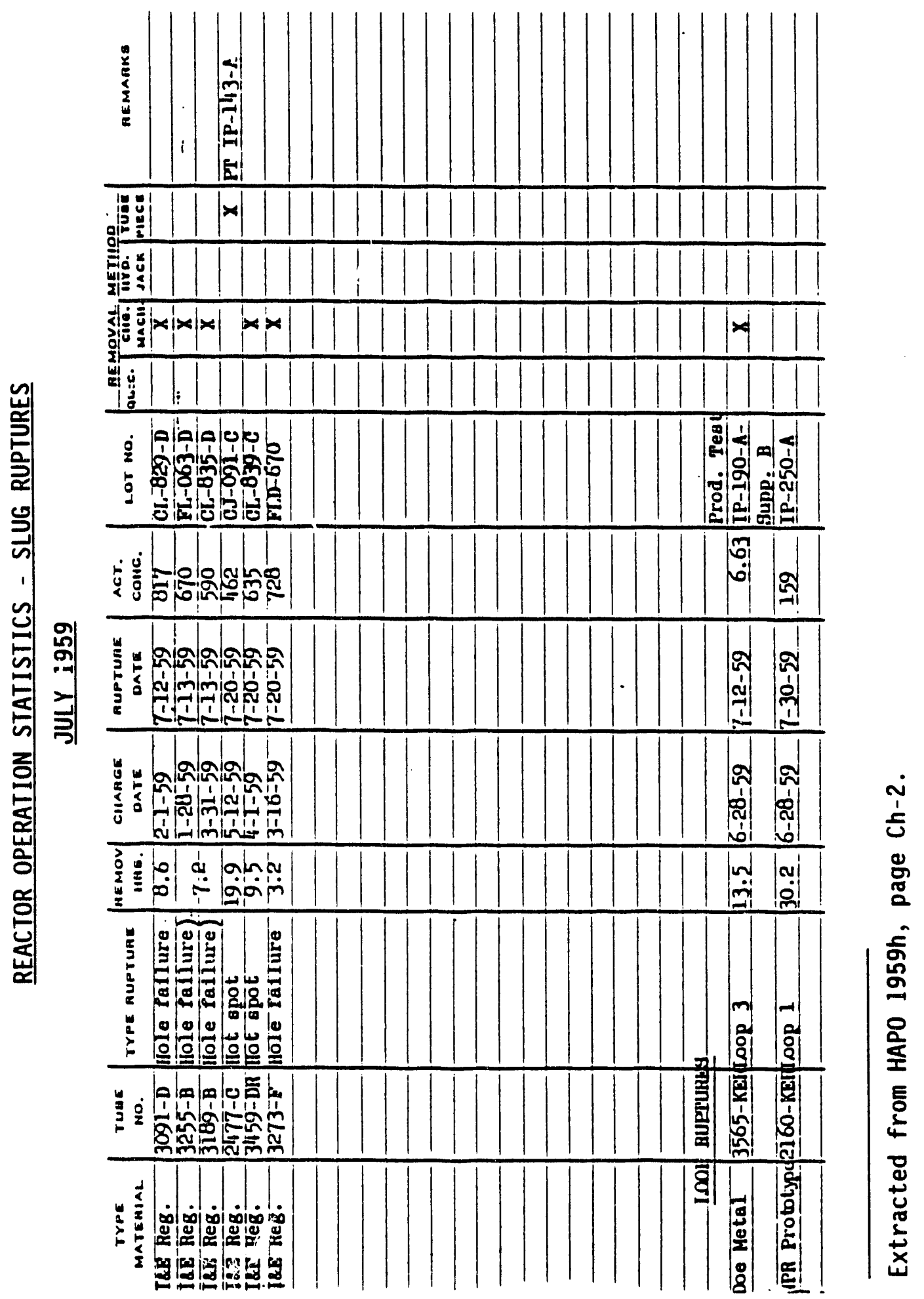




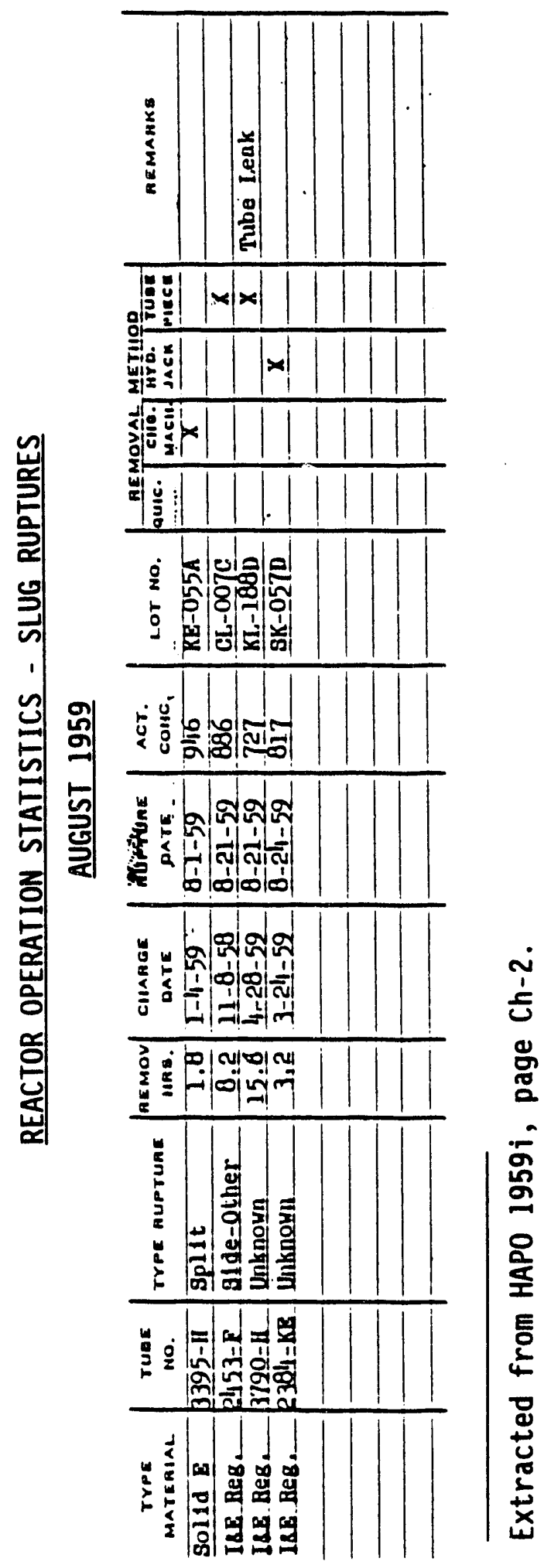




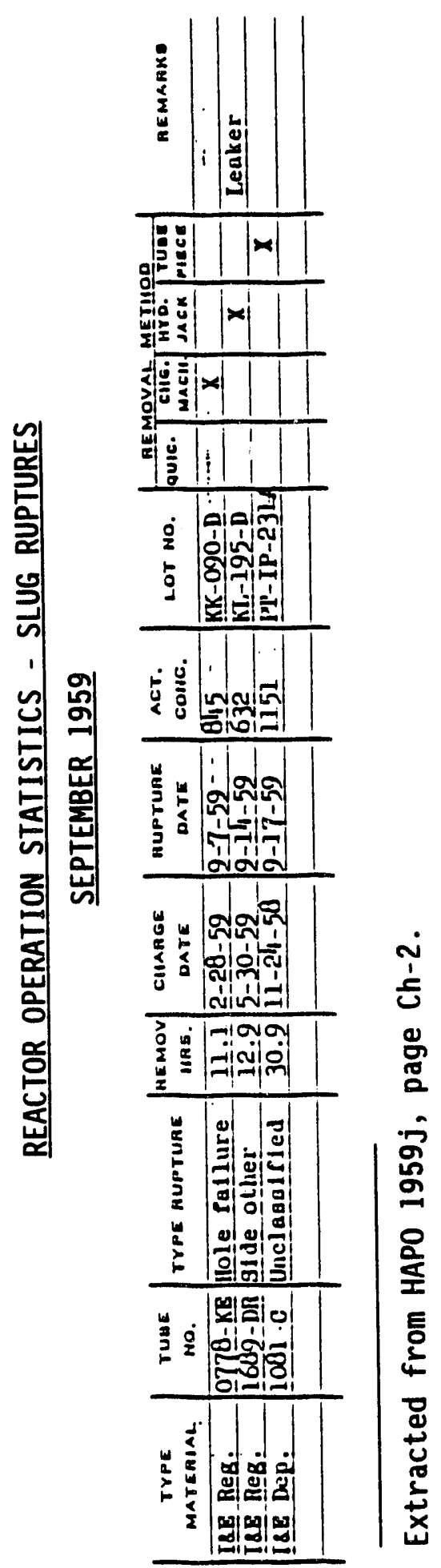

1.9 


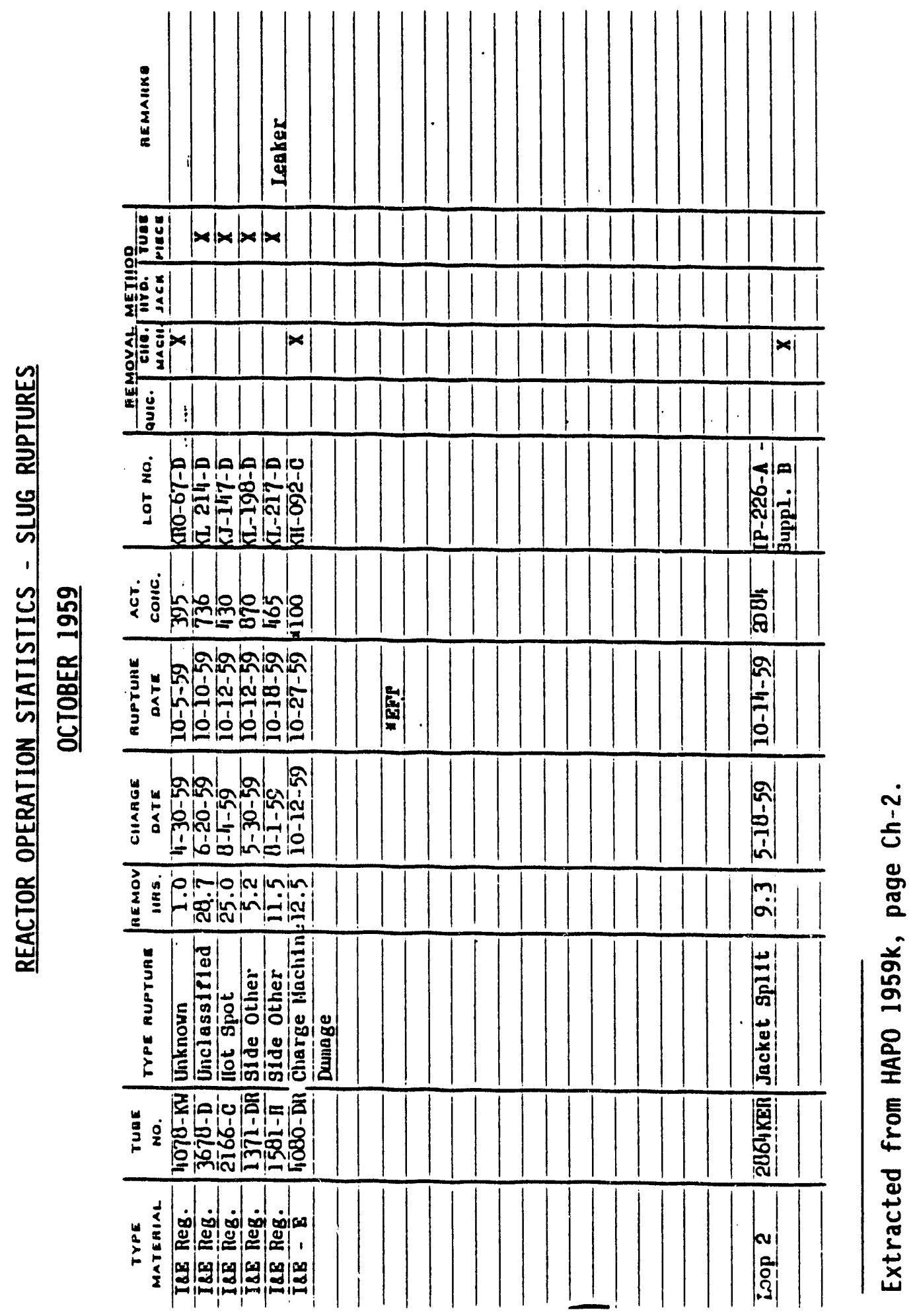




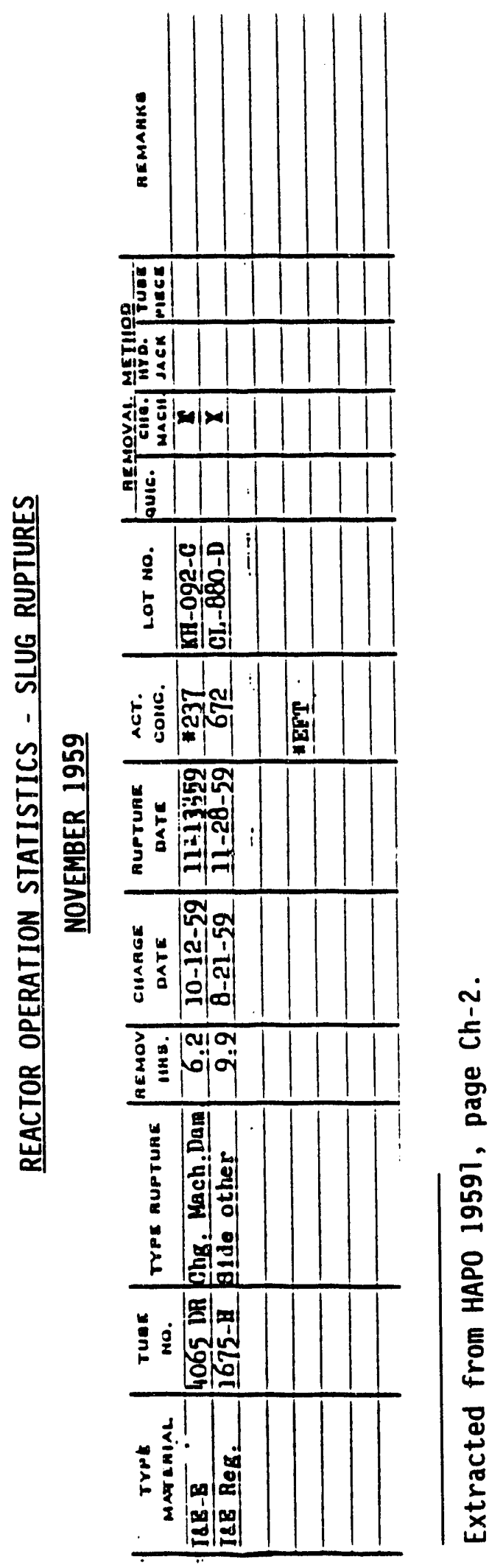

I. 11 


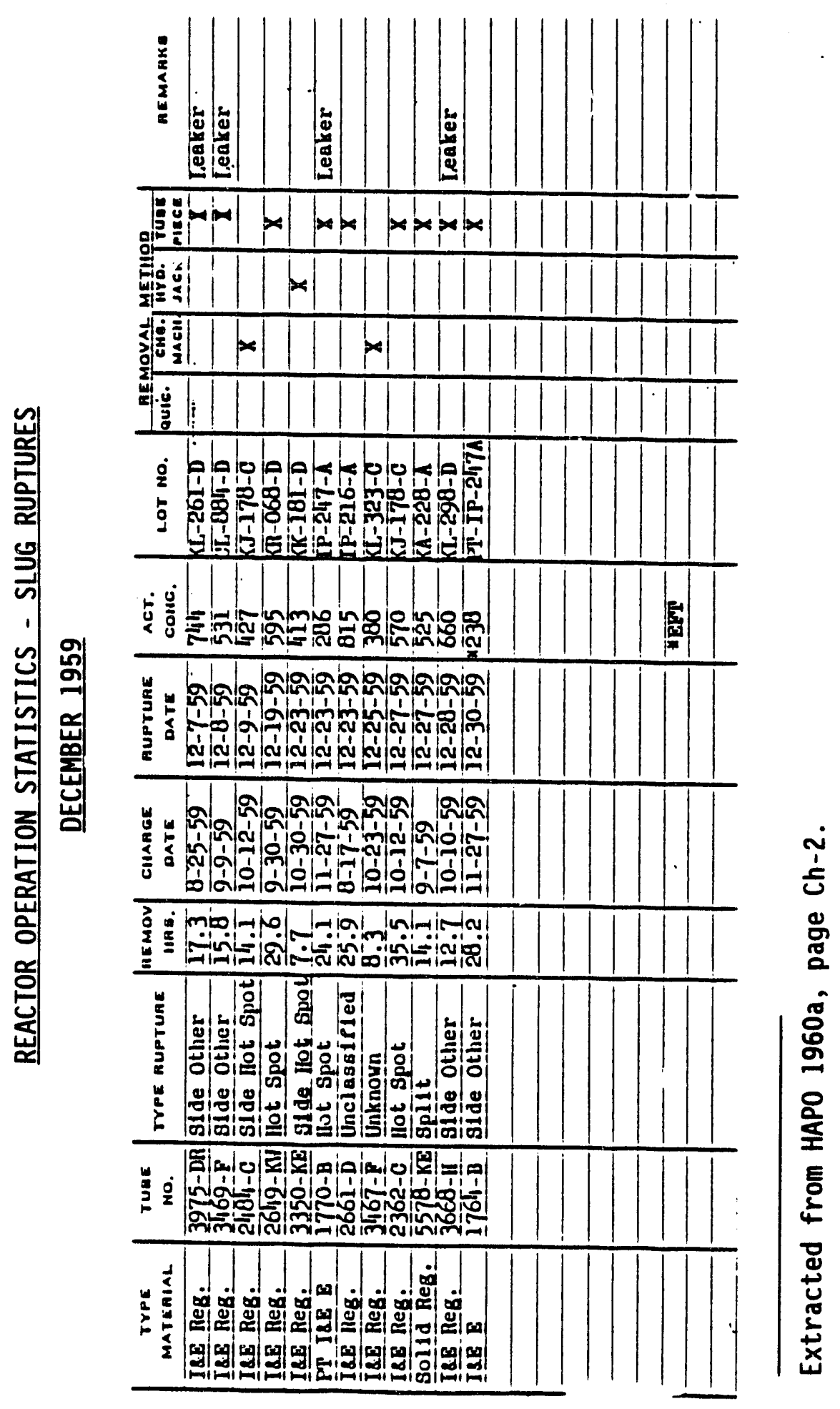


APPENDIX $\mathrm{J}$

FUEL-ELEMENT FAILURE DATA FOR 1960 


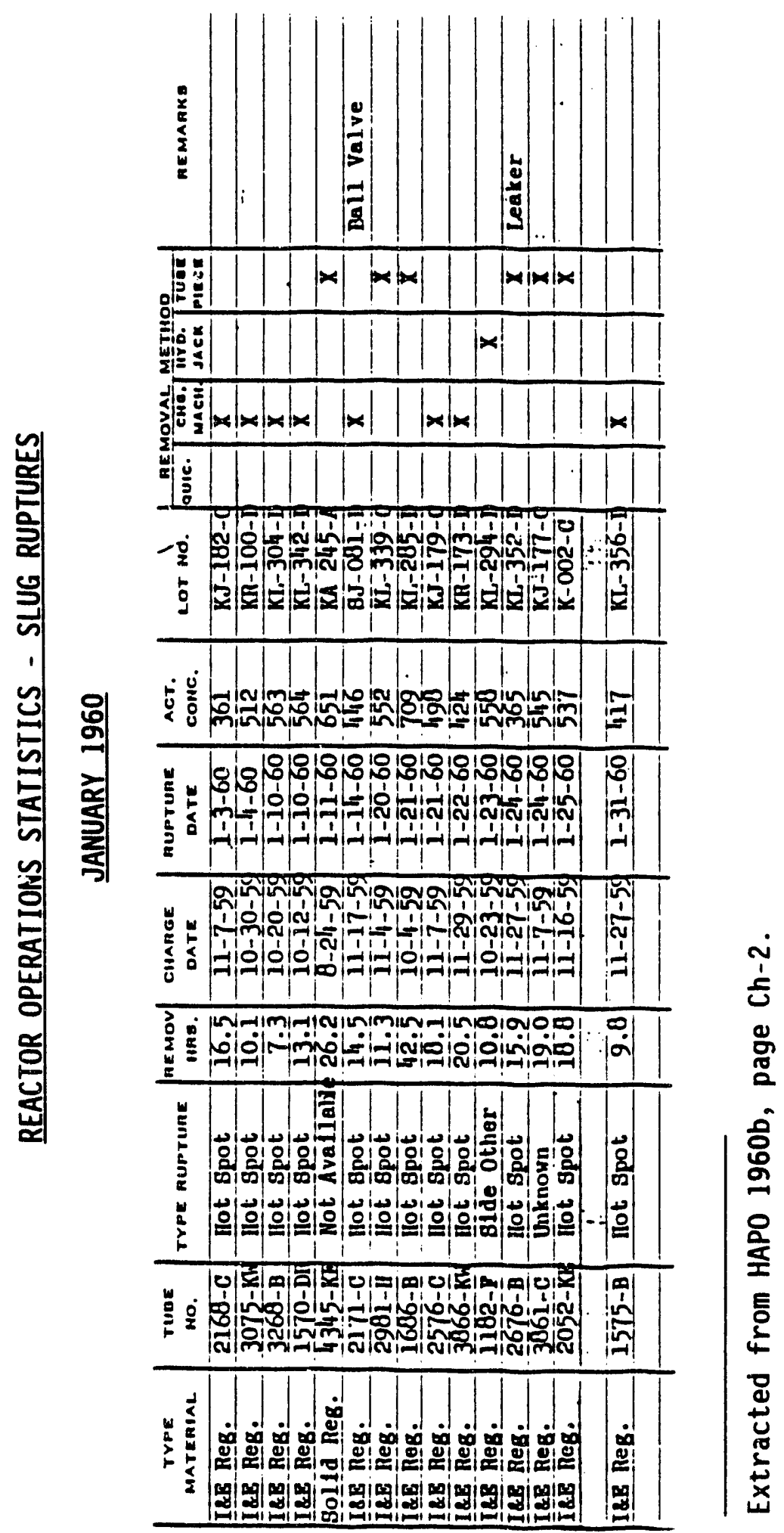




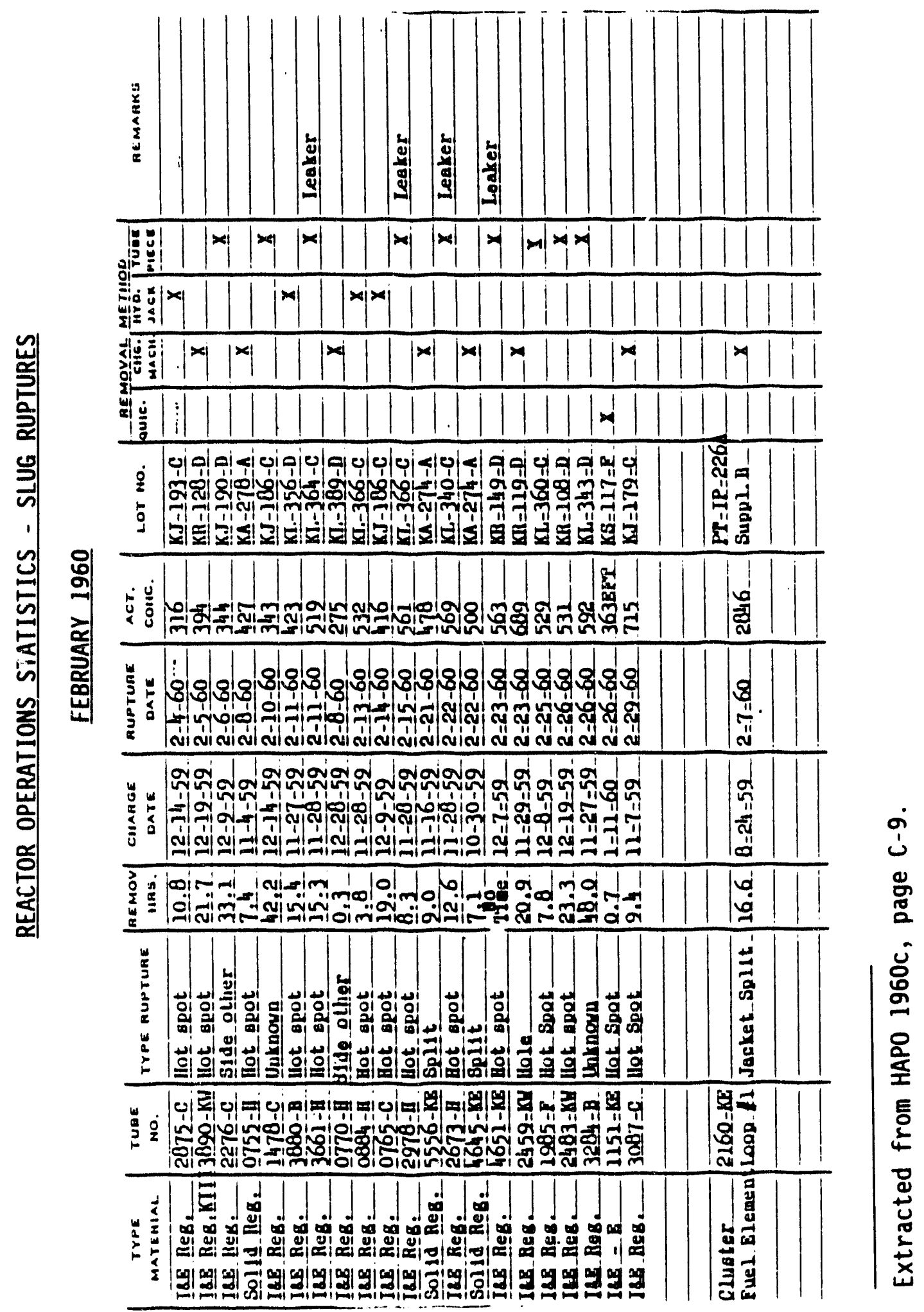


RUPTURE EXPERIENCE - MARCH 1960

\begin{tabular}{|c|c|c|c|c|c|}
\hline $\begin{array}{l}\text { Faizure } \\
\text { Daze }\end{array}$ & $\begin{array}{l}\text { Tube } \\
\text { Tumioe: }\end{array}$ & $\begin{array}{l}\text { Iot } \\
\text { Numbe: }\end{array}$ & myoe of Metal & Essosure & $\begin{array}{l}\text { Type of } \\
\text { Ea-ivere }\end{array}$ \\
\hline $3 / 1 / 60$ & $2369-7$ & $\pi-364-c^{(1)}$ & 8" I\&B Natural & 376 & $\begin{array}{l}\text { Side Hot } \\
\text { Spot }\end{array}$ \\
\hline $\begin{array}{l}3 / 19 / 60 \\
3 / 13 / 60 \\
3 / 13 / 60 \\
3 / 97 / 60\end{array}$ & $\begin{array}{l}2060-k \pi \\
2184-3 \\
2562-\pi / 4 \\
3557-507\end{array}$ & $\begin{array}{l}K R-108-D(1) \\
K R-360-D \\
K R-140-D \\
K R-141-D\end{array}$ & $\begin{array}{l}\text { 8" I\&E Natural } \\
\text { 8" I\&B Natural } \\
\text { 8" I\&B Naturel } \\
\text { 8" I\&E Natural }\end{array}$ & $\begin{array}{l}661 \\
571 \\
373 \\
387\end{array}$ & $\begin{array}{l}\text { Unjeown } \\
\text { Univown } \\
\text { Uni=own } \\
\text { Unis=own }\end{array}$ \\
\hline $3 / 27 / 60$ & $0560-6$ & $K G-244-\bar{E}$ & 6" IRs Earicied (2) & $4+2$ & $\begin{array}{c}\text { Side Eot } \\
\text { Spot }\end{array}$ \\
\hline $3 / 22 / 60$ & $3565-D$ & D-8.92-D & 8" I3e Natural & 548 & $\begin{array}{l}\text { Side } \\
\text { otbe }\end{array}$ \\
\hline $3 / 22 / 60$ & $38 T \pi-D R$ & $K-418-D$ & 8" Ises Naturei & 272 & $\begin{array}{l}\text { Side } \\
\text { other }\end{array}$ \\
\hline $\begin{array}{l}3 / 23 / 60 \\
3 / 25 / 60\end{array}$ & $\begin{array}{l}2044-i B \\
0859-D R\end{array}$ & $\begin{array}{l}M A-279-A \\
S B-017-C(3)\end{array}$ & $\begin{array}{l}\text { 8" Solid Natural } \\
\text { 6" Ias Enflabed(2) }\end{array}$ & $\begin{array}{l}402 \\
377\end{array}$ & $\begin{array}{l}\text { Spבz IODS } \\
\text { Side Eot } \\
\text { SDot }\end{array}$ \\
\hline $\begin{array}{l}3 / 25 / 60 \\
3 / 20 / 60 \\
3 / 29 / 60\end{array}$ & $\begin{array}{l}146 j-D \\
387 \bar{j}-5 \\
2567-C\end{array}$ & $\begin{array}{l}\sqrt{1}-009-D \\
K \pi-378-C \\
K J-197-C\end{array}$ & $\begin{array}{l}\text { 8" I\&B NeturaI } \\
\text { 8" I\&S NetureI } \\
\text { 8" I\&S NeturaI }\end{array}$ & $\begin{array}{l}557 \\
553 \\
429\end{array}$ & $\begin{array}{l}\text { Unci. } \\
\text { S1de Ctier } \\
\text { Side Eot } \\
\text { Spoz }\end{array}$ \\
\hline$j / \equiv \geq / 60$ & $140 \div-E$ & $C I-991-D$ & 8" I\&E Nazural & 650 & $U=0$ ons \\
\hline
\end{tabular}

(2) Multiple Eallure Iot

(2) .94 pez ceat U-235

(3) pareat lot $\mathrm{ke}-134-\mathrm{C}$

Iegend:

Side Eot Spot - Fasulure probably caused by accelerated corcosion of the exterpel cen wall in a localized region of bigin temperature.

Side Other - Failure probaily caused by cososion of water penetration cI tine exrezal can weil or otiber unienorm mechand sm.

Split Ions - Foilure caused by uracium cleavage along the axds of the core.

Unclessifieo - Feilure did pot logicelly fall tato ing of the usuel types.

Unipown - Feiled piece could not be located or bad not been examined at the time of wettiag.

Extracted from HAPO 1960d, pages B-14 \& B-15. 


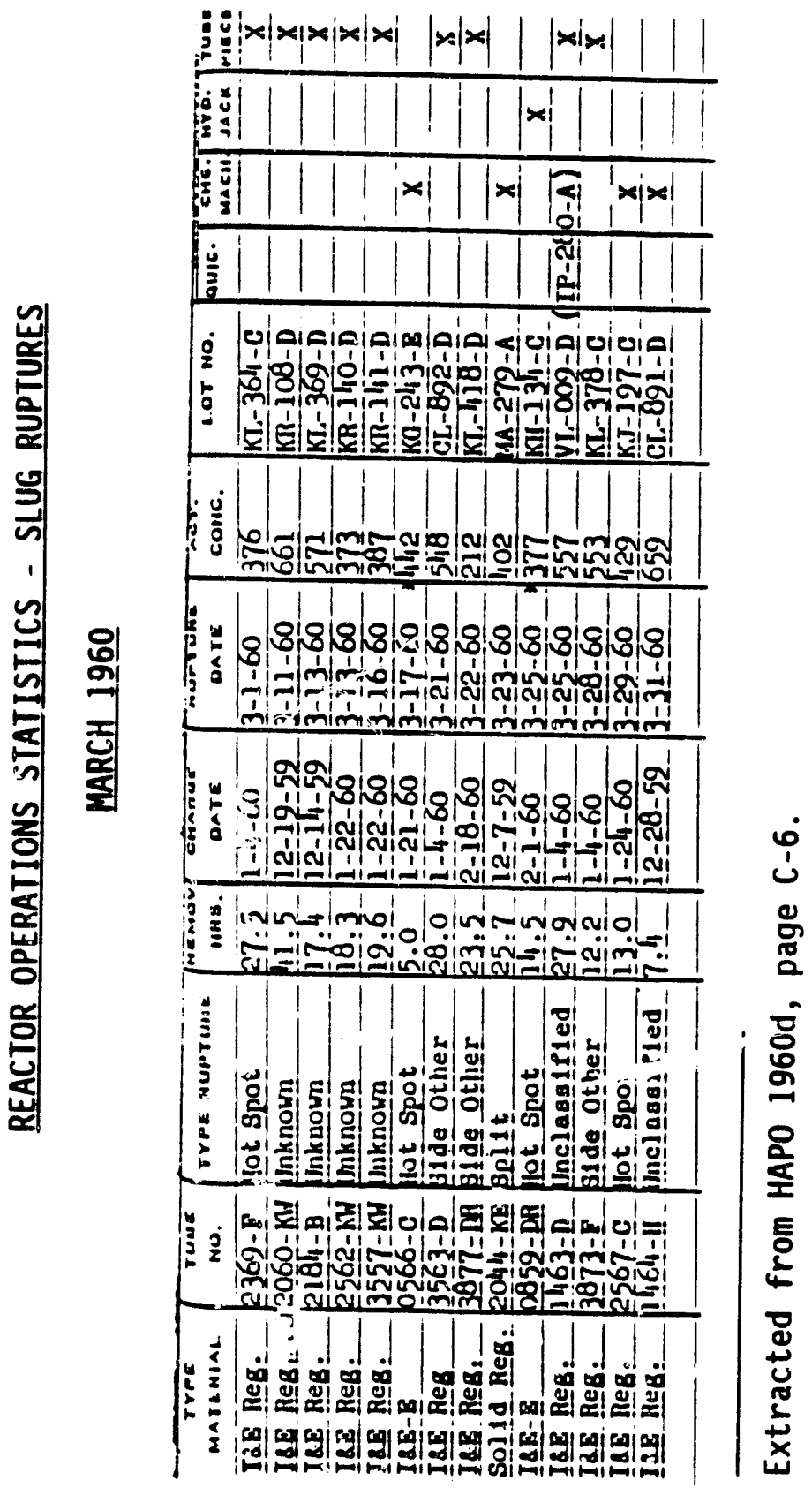




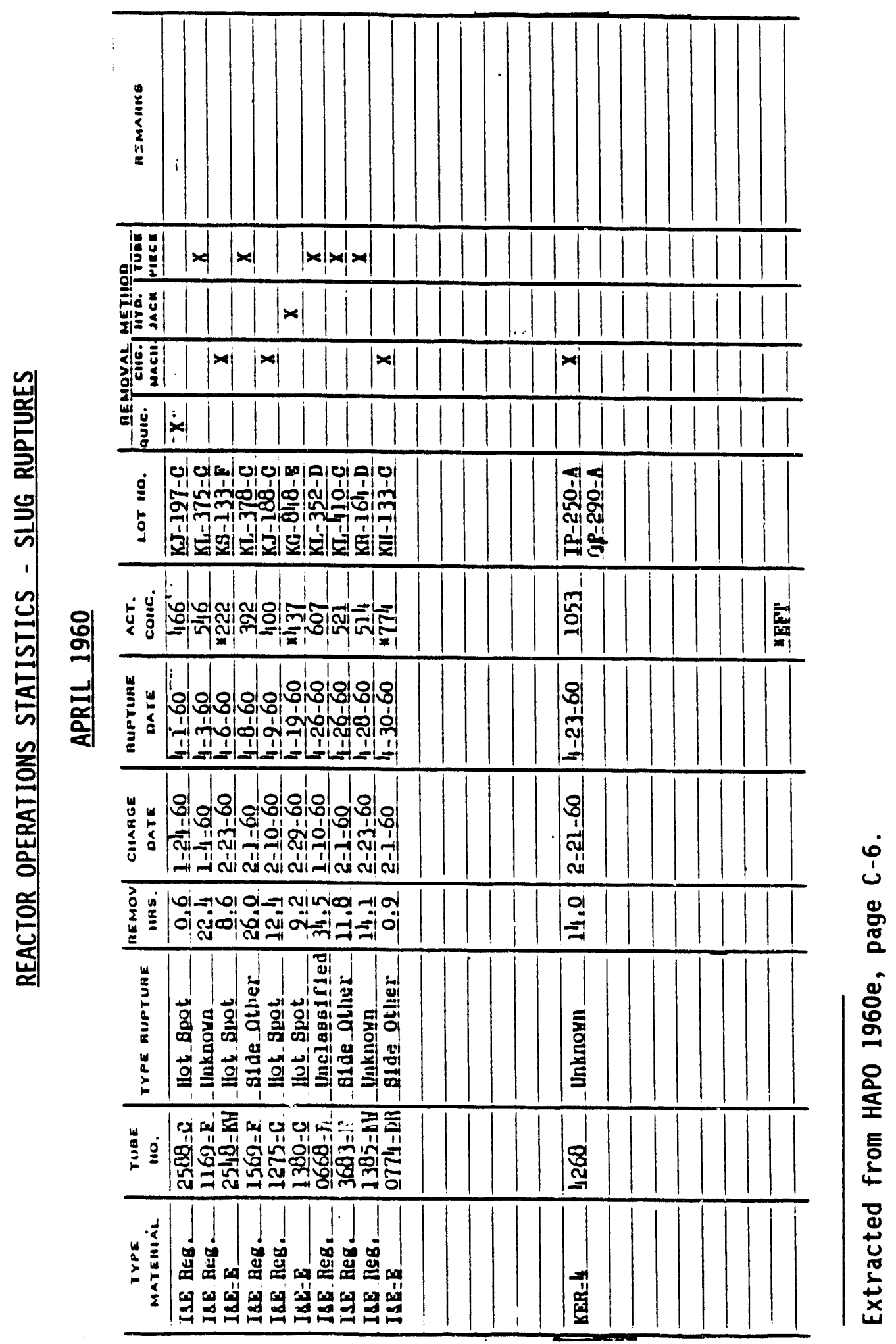




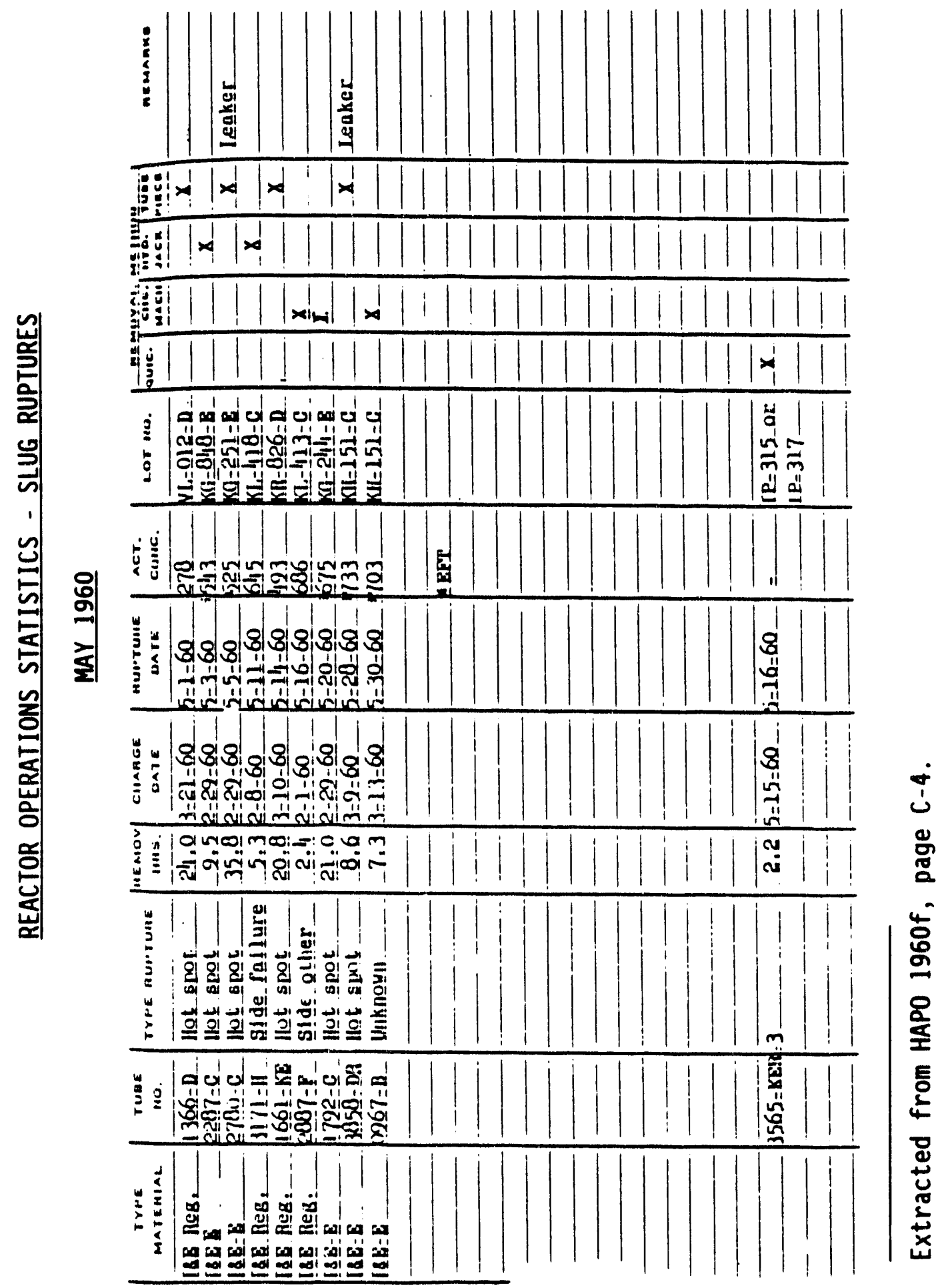


RUPTURE EXPERIENCE - JUNE 1960

\begin{tabular}{|c|c|c|c|c|c|}
\hline $\begin{array}{l}\text { Paslure } \\
\text { Date } \\
\end{array}$ & $\begin{array}{l}\text { Tube } \\
\text { Numbe= }\end{array}$ & $\begin{array}{l}\text { Lot } \\
\text { Number }\end{array}$ & $\begin{array}{l}\text { Type of } \\
\text { Netal }\end{array}$ & Exposure & $\begin{array}{l}\text { Type of } \\
\text { Failure }\end{array}$ \\
\hline $6 / 1 / 60$ & $1583-E$ & $k=-458-C$ & IrE Natural & 424 & Side-otber \\
\hline $6 / 2 / 60$ & $2472-F$ & $B C-4+4-D$ & I\&E Natural & 466 & End Hale \\
\hline $6 / 3 / 60$ & $3553-E$ & $M A-282-1$ & Solfd Netural & 588 & Splitー-Iمng. \\
\hline $6 / 9 / 60$ & 3964-D & $K E=-150-C$ & I\&B Enriched (1) & 807 & Side-Eot Spot \\
\hline $6 / 16 / 60$ & $2178-E$ & $k \pi-458-c^{(2)}$ & I\&S Natural & 468 & Side-Bot Spot \\
\hline $6 / 17 / 60$ & $2755-D R$ & $M A-285-d$ & Solida Naturel & 554 & Side-Hot Spot \\
\hline $6 / 28 / 60$ & $2456-c$ & WG $-256-\Sigma$ & IaB Enriched(1) & 697 & Side-Hot Spot \\
\hline $6 / 20 / 60$ & $1889-C$ & DJ-222-D & IRs Natural & 488 & Side-Hot Spot \\
\hline $6 / 26 / 60$ & $3277-B$ & $5 \pi-433-n$ & IRS Natural & 683 & Unkenown \\
\hline
\end{tabular}

(1) 0.94 per cent U-235

Iegerd:

Side Eot Spot - Faclure probably caused by accelerated corrosion of the external can wall in a locallzed region of blgh temperature.

Side Otber - Fallure probably caused by corrosion or water penetration of the external cas wall or other unicnown mechanism.

Split-Iong. Fallure caused by uranium eleavage along the axis of the core.

Unkowown - Failed piece could not be located or had not been examined at the time of writing.

Ind - Feilure on the end of an I \& E piece, probably caused by vater penetration through a weld.

Extracted from HAPO $1960 \mathrm{~g}$, page B-16. 


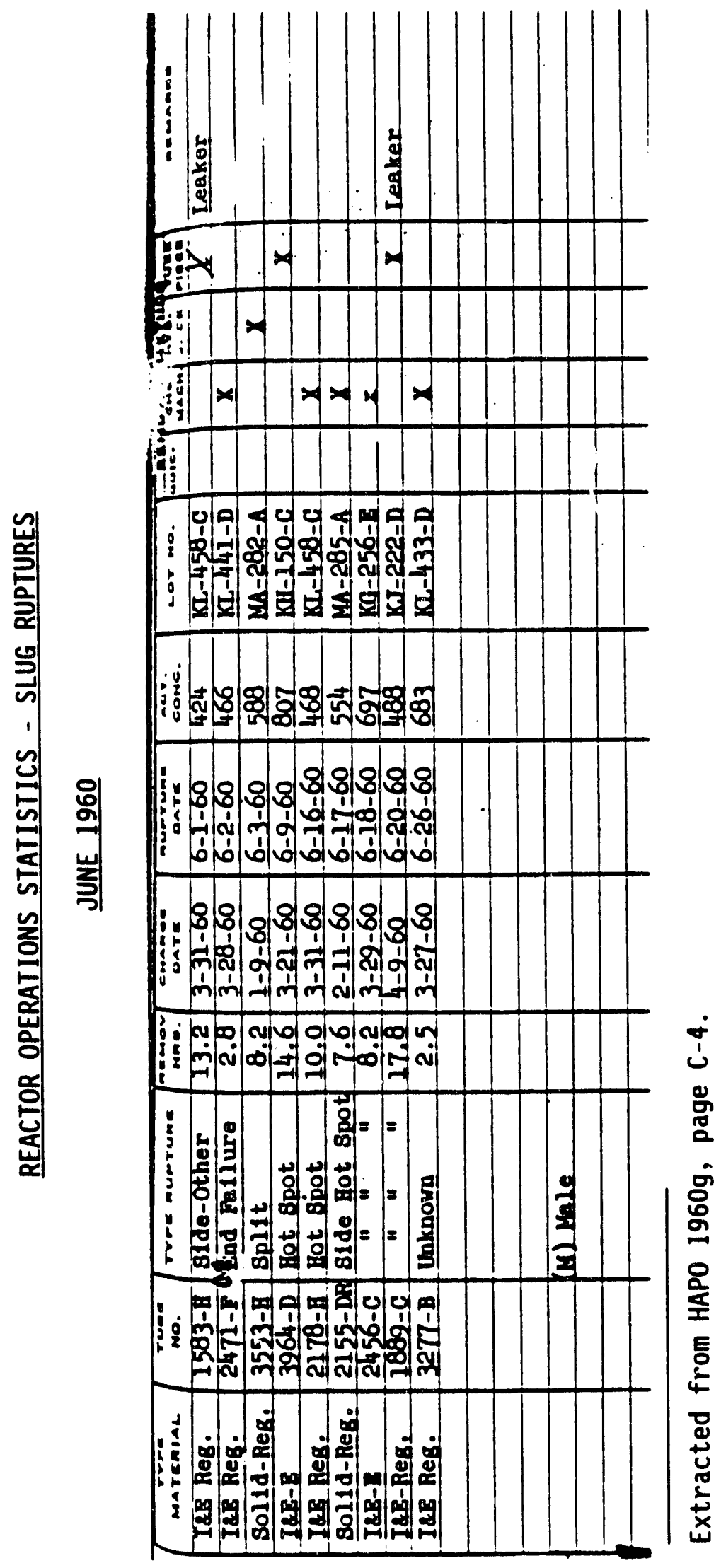




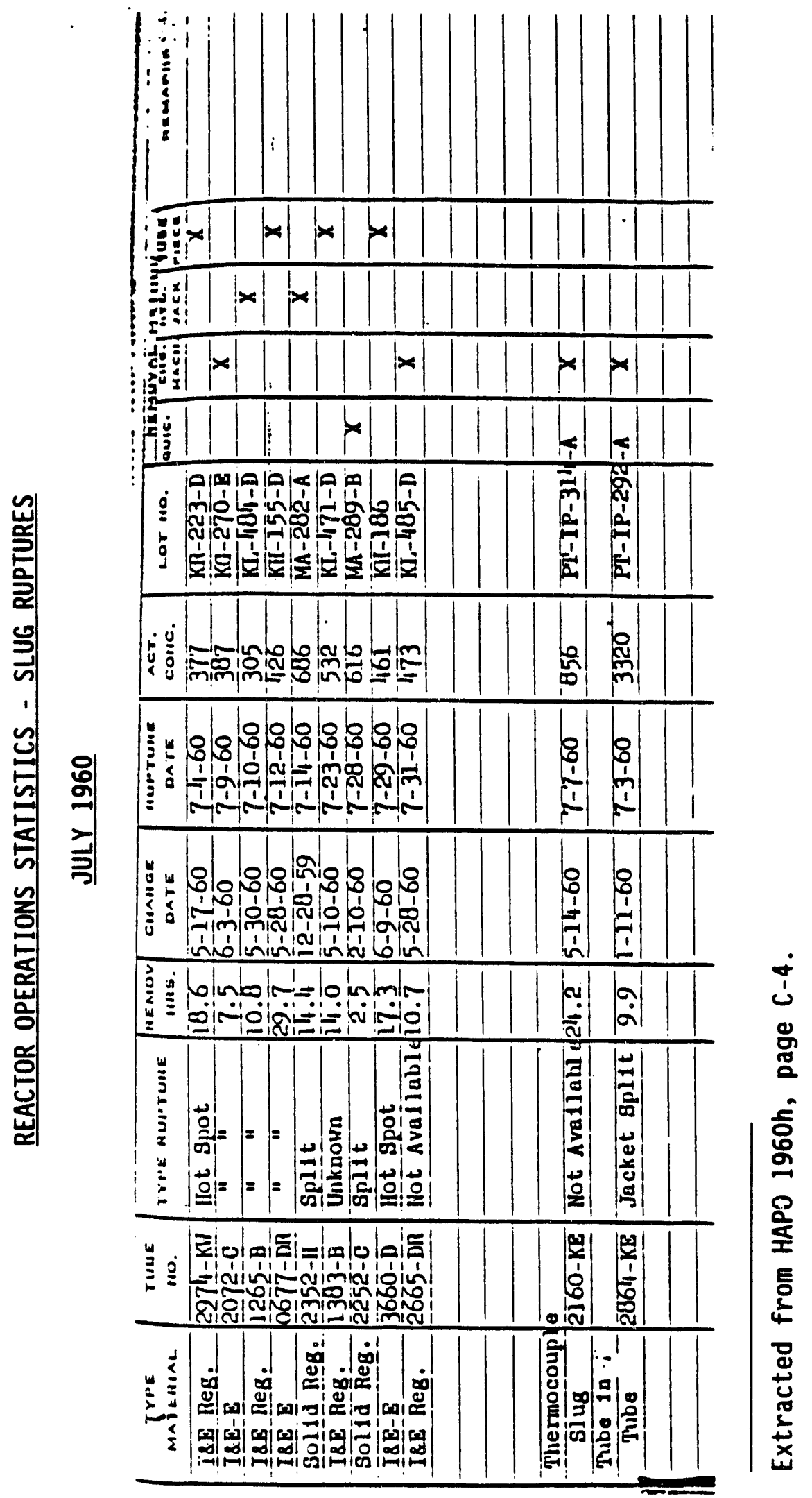




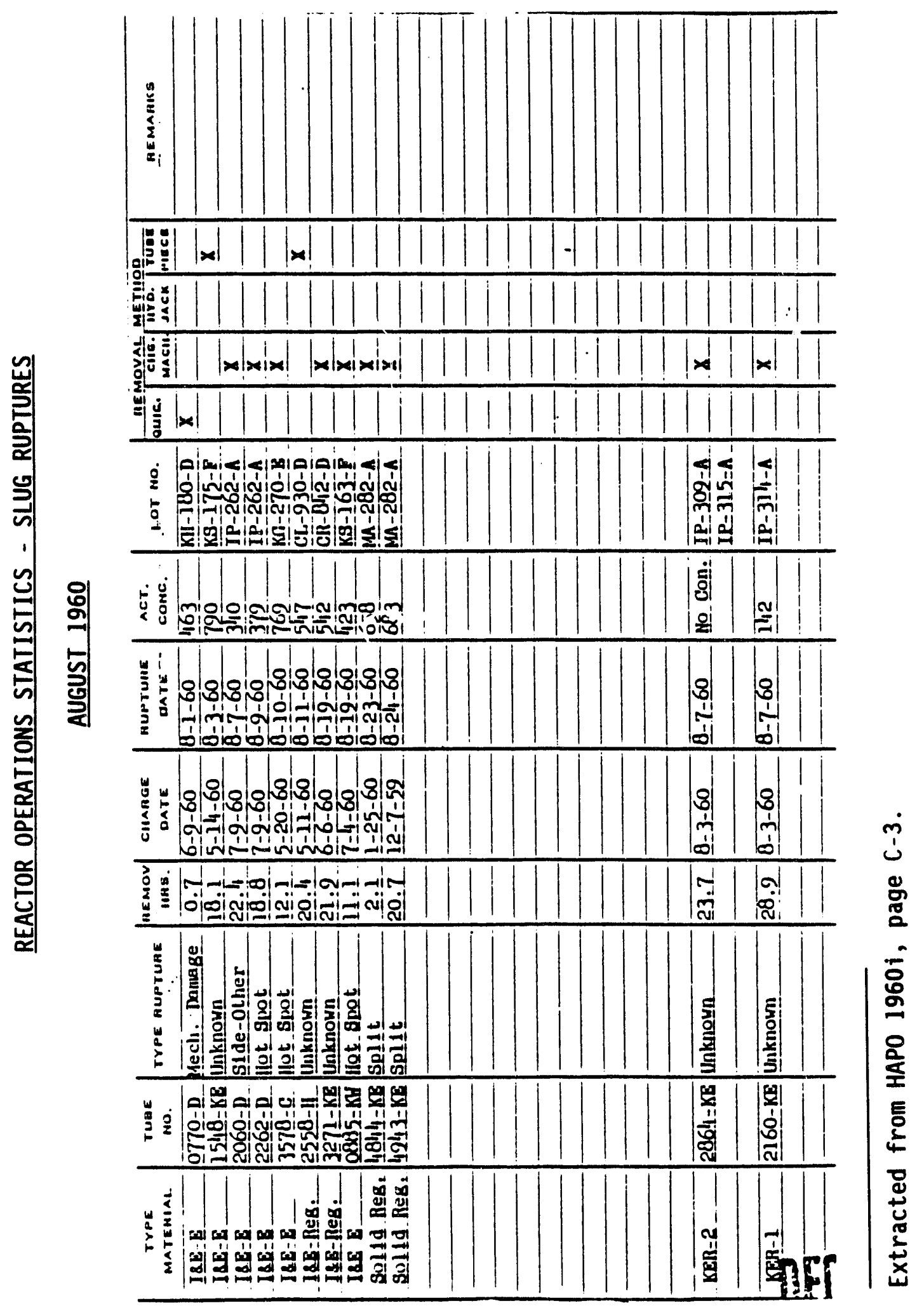




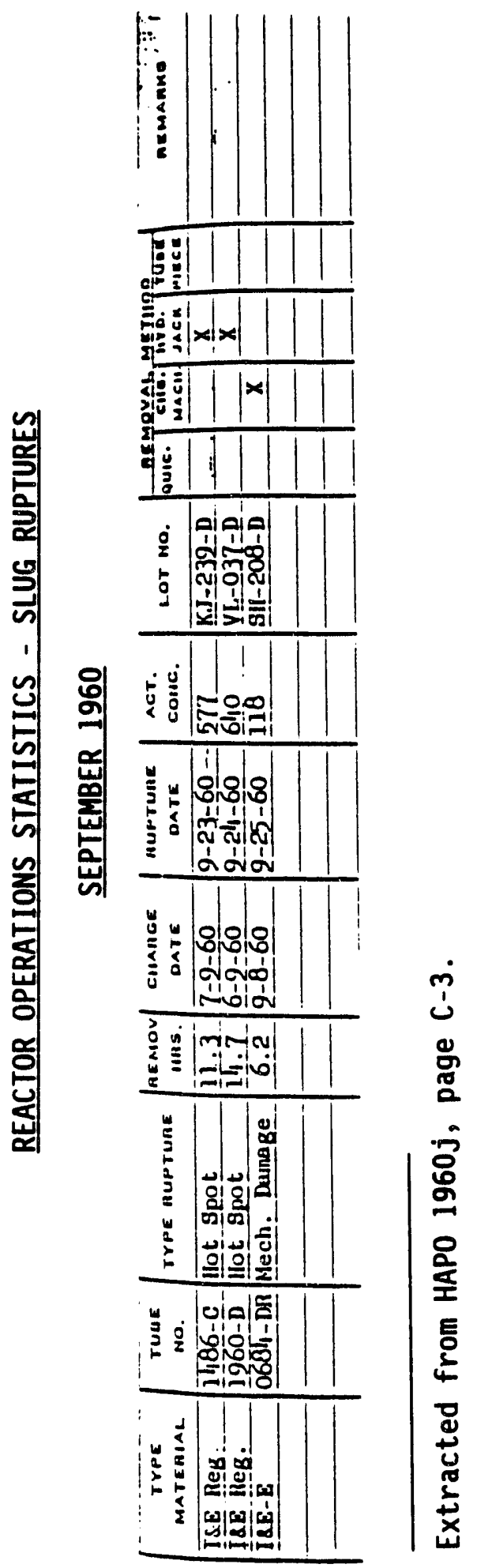

J.11 


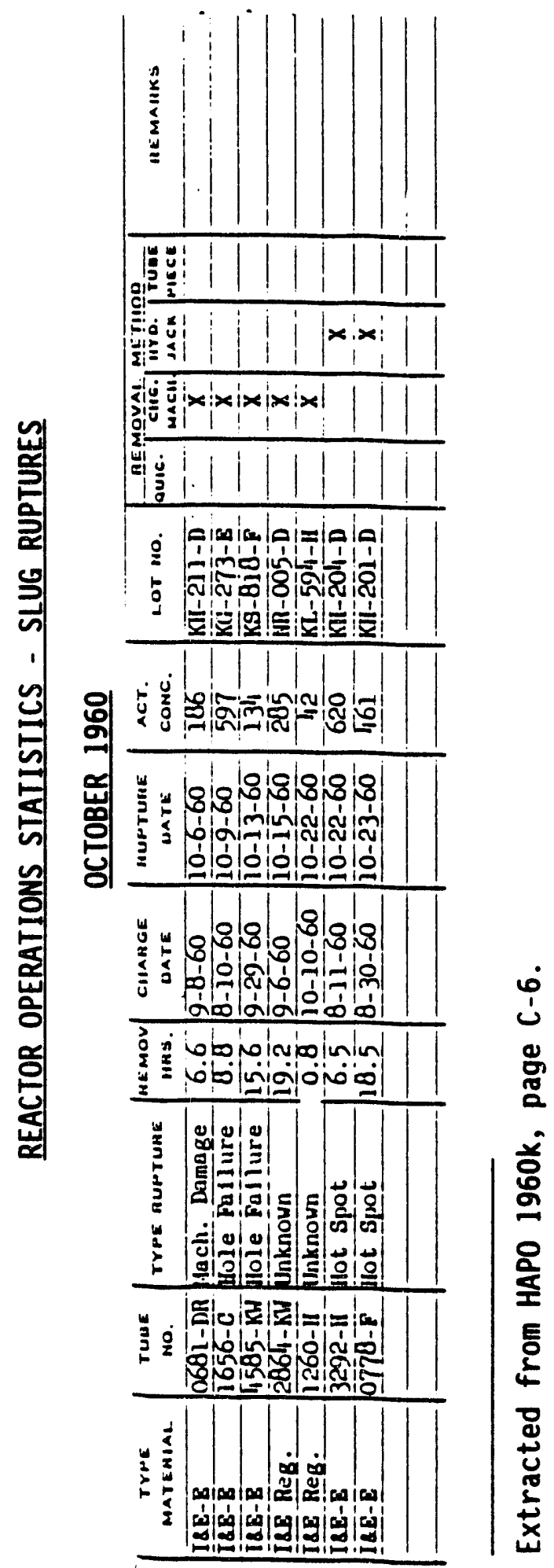

J. 12 
RUPTURE EXPERIENCE - OCTOBER 1960

\begin{tabular}{|c|c|c|c|c|c|}
\hline $\begin{array}{l}\text { Failure } \\
\text { Dere }\end{array}$ & $\begin{array}{l}\text { Tube } \\
\text { Numiber }\end{array}$ & $\begin{array}{l}\text { Lot } \\
\text { Numioe: }\end{array}$ & $\begin{array}{l}\text { Mype of } \\
\text { Mezerigl }\end{array}$ & Exposure & $\begin{array}{l}\text { Type of } \\
\text { Fa lure }\end{array}$ \\
\hline $\begin{array}{l}10 / 6 / 60 \\
10 / 9 / 60 \\
10 / i 3 / 60 \\
10 / 75 / 60 \\
10 / 22 / 60 \\
10 / 22 / 60 \\
10 / 23 / 60\end{array}$ & $\begin{array}{l}0601-D R \\
1656-C \\
4585-20 N \\
2864-K N \\
3202-\bar{I} \\
1260-\bar{E} \\
0778-\bar{F}\end{array}$ & $\begin{array}{l}K E-211-D \\
K S-273-E \\
K S-818-T \\
N R-005-D \\
K E-204-D \\
K E-594-E \\
K E-201-D\end{array}$ & 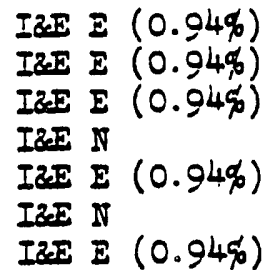 & $\begin{array}{r}186 \\
597 \\
234 \\
285 \\
620 \\
42 \\
461\end{array}$ & $\begin{array}{l}\text { Mech. Demage } \\
\text { Eoie } \\
\text { Eole } \\
\text { Unienown } \\
\text { Side Eor Spot } \\
\text { Unkeown } \\
\text { Side DoI Spot }\end{array}$ \\
\hline
\end{tabular}

\section{Iegenci}

I2E N - This is the symol for taternelly and extersaliy cooled produc:ic= Feacjo: fulel elemeass of naturai uracium.

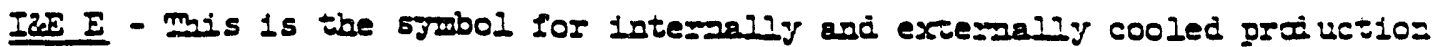
reactor fuel elements with urantive cores erricied is U-235. The weighi per ceav U-235 in the core material is stated.

Mecharical Damage - The failure vas probably caused by mesiarical damage of ine fue- ejement before irraciation.

Side EcT SDOT - The fatlure was probably caused by acselezated corfosion of ine exrerzai can wali to a localized region of high temperazure.

Eole - Failure occurzed on the interal surface of an interaling ard exrernaiy cooled fuel element. The failure was probably causec by water pezetraisce th=cuigin a weld or other unkeown meciantsm.

Uninowa - The fuel elexent fatlure could not be located or bad not bee: exarized at the tide the report was writien.

Extracted from HAPO 1960k, pages B-14 \& B-15. 


\section{RUPTURE EXPERIENCE - NOVEMBER 1960}

\begin{tabular}{|c|c|c|c|c|c|}
\hline $\begin{array}{l}\text { ailure } \\
\text { aze }\end{array}$ & $\begin{array}{l}\text { Tube } \\
\text { Numioez }\end{array}$ & $\begin{array}{l}\text { Lot } \\
\text { Number }\end{array}$ & $\begin{array}{l}\text { Type of } \\
\text { Marezial }\end{array}$ & Exposure & $\begin{array}{l}\text { Type of } \\
\text { Tailure }\end{array}$ \\
\hline $\begin{array}{l}1 / 6 / 60 \\
1 / 6 / 60 \\
1 / 11 / 60 \\
1 / 11 / 60 \\
1 / 12 / 60 \\
1 / 14 / 60 \\
1 / 20 / 60 \\
1 / 22 / 60 \\
1 / 27 / 60 \\
1 / 30 / 60 \\
1 / 30 / 60\end{array}$ & $\begin{array}{l}1361-\mathrm{B} \\
2180-\mathrm{C} \\
1559-\mathrm{KI} \\
3275-\mathrm{KN} \\
1366-\mathrm{D} \\
1166-\mathrm{B} \\
1192-\mathrm{DR} \\
2455-\mathrm{DR} \\
1471-\mathrm{K} \\
3566-\mathrm{B} \\
1677-\mathrm{F}\end{array}$ & $\begin{array}{l}K L-491-D \\
C J-855-C \\
K R-284-D \\
K S-209-\bar{I} \\
K 0-108-C(1) \\
K L-536-D \\
C A-161-\bar{D} \\
C A-902-\bar{D} \\
K R-279-D \\
K L-561-D \\
K L-512-D\end{array}$ & 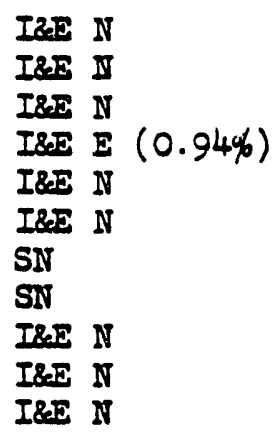 & $\begin{array}{l}6 \\
50 \\
4 \\
6 \\
6 \\
6\end{array}$ & $\begin{array}{l}\text { Side Eot Spo } \\
\text { Eole } \\
\text { Unionown } \\
\text { S1de Eot-Spo } \\
\text { Side-Otbe: } \\
\text { Side Eot-Spo } \\
\text { Split-Iong } \\
\text { Spl1t-Iong } \\
\text { Eole } \\
\text { Sidenother } \\
\text { Unionown }\end{array}$ \\
\hline
\end{tabular}

(1) Six inci Fuel element with attached water mixer spool.

\section{Iegend:}

I32 N - This is the symbol for internally and exterpally cooled production Feactor fuel elements of natural uranium.

Ibs E - Mis is the symbol for Interally and extersally cooled production reactor fuel elements with uracium cores ex-1ched in U-235. The veight per ceas U-235 in the core material is stated.

SN - Mais is the symbol for production reactor fuel elements having a solid cylindrical core of aptural uraniur.

Extracted from HAPO 19601, page B-12. 


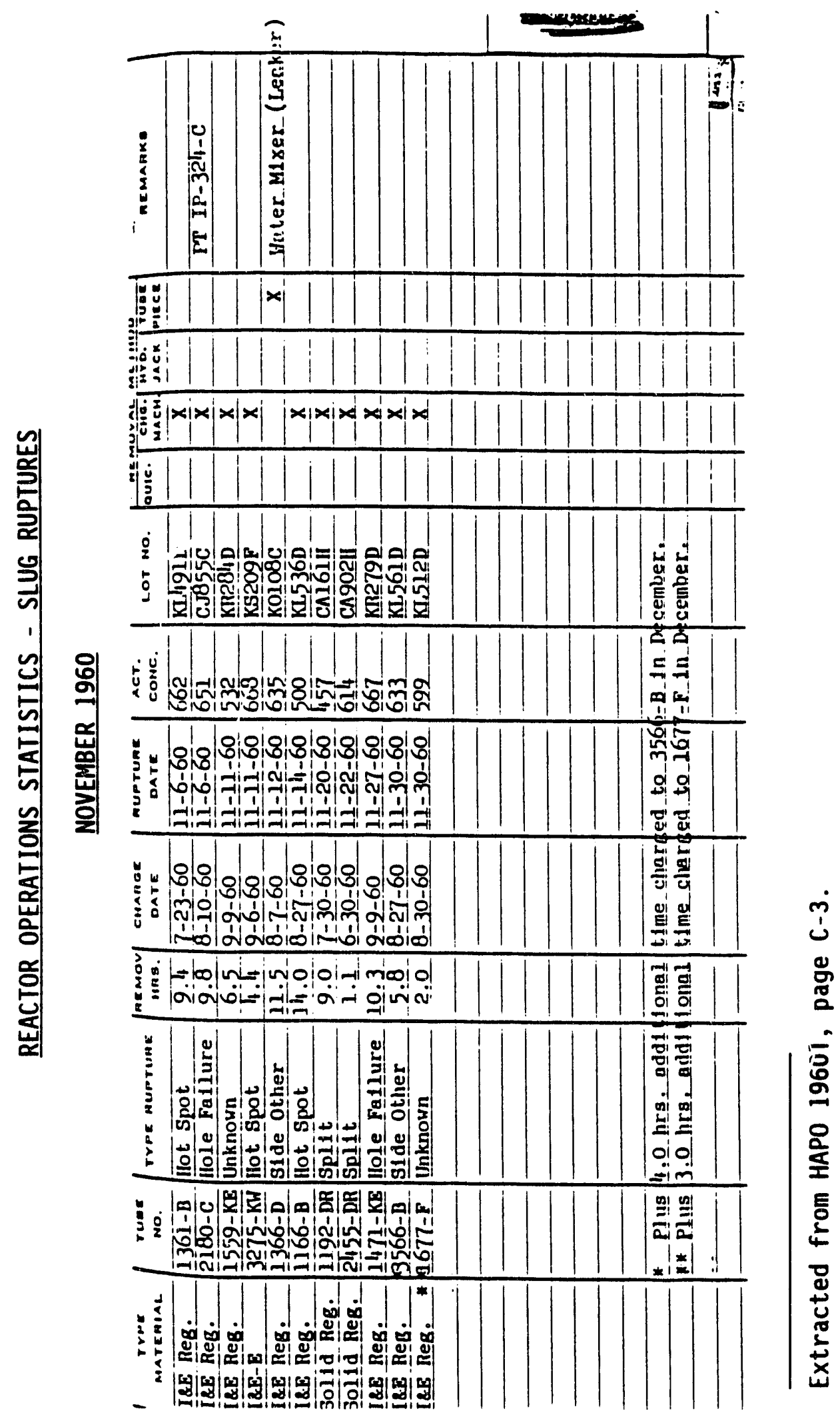




\section{RUPTURE EXPERIENCE - DECEMBER 1960}

\begin{tabular}{|c|c|c|c|c|c|}
\hline $\begin{array}{l}\text { Eailure } \\
\text { Daie }\end{array}$ & $\begin{array}{l}\text { Tube } \\
\text { Number }\end{array}$ & $\begin{array}{l}\text { Lot } \\
\text { Number }\end{array}$ & $\begin{array}{l}\text { Type of } \\
\text { Meieriel }\end{array}$ & Exposure & $\begin{array}{l}\text { Tyoe o: } \\
\text { Feilure }\end{array}$ \\
\hline $\begin{array}{l}12 / 4 / 60 \\
12 / 7 / 60 \\
12 / 9 / 60 \\
12 / 10 / 60 \\
12 / 11 / 60 \\
12 / 14 / 60 \\
12 / 15 / 60 \\
12 / 27 / 60 \\
12 / 18 / 60 \\
12 / 21 / 60 \\
12 / 21 / 60 \\
12 / 25 / 60 \\
22 / 26 / 60\end{array}$ & 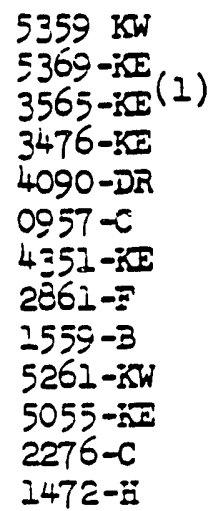 & $\begin{array}{l}K S-227-F \\
K S-022-F \\
I P-309-A \\
K R-277-D \\
C A-903-ت \\
K G-270-F \\
K R-321-D \\
K L-560-D \\
K L-555-D \\
K S-232-\bar{F} \\
K S-221-D \\
K G-856-A \\
K L-521-D\end{array}$ & 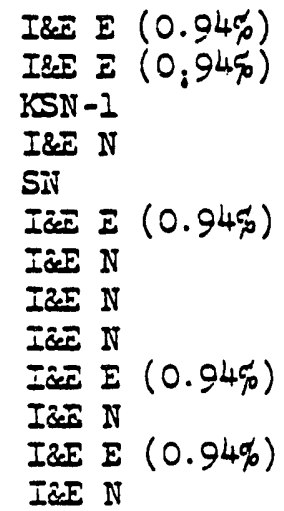 & $\begin{array}{l}619 \\
603 \\
2733 \\
495 \\
793 \\
590 \\
530 \\
499 \\
603 \\
426 \\
535 \\
5=4 \\
524\end{array}$ & 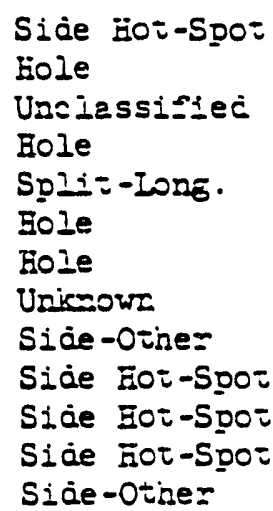 \\
\hline
\end{tabular}

(i) KER I0Op $\div 3$

Iegenc:

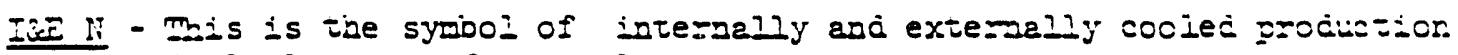
Feactor fuel elements of natural wranium.

IjeE $\equiv$ - This 15 the symbol for interally and exremally cooled prociction reactor fuel elements witb uraniun cores enricied in U-235. Tne weigi= per cent U-235 in the core material is stated.

Sii - This is the symol for production reactor fuel elemeats baving a solic cylincirical core of paiural uranium.

Extracted from HAPO 1961a, page B-15. 


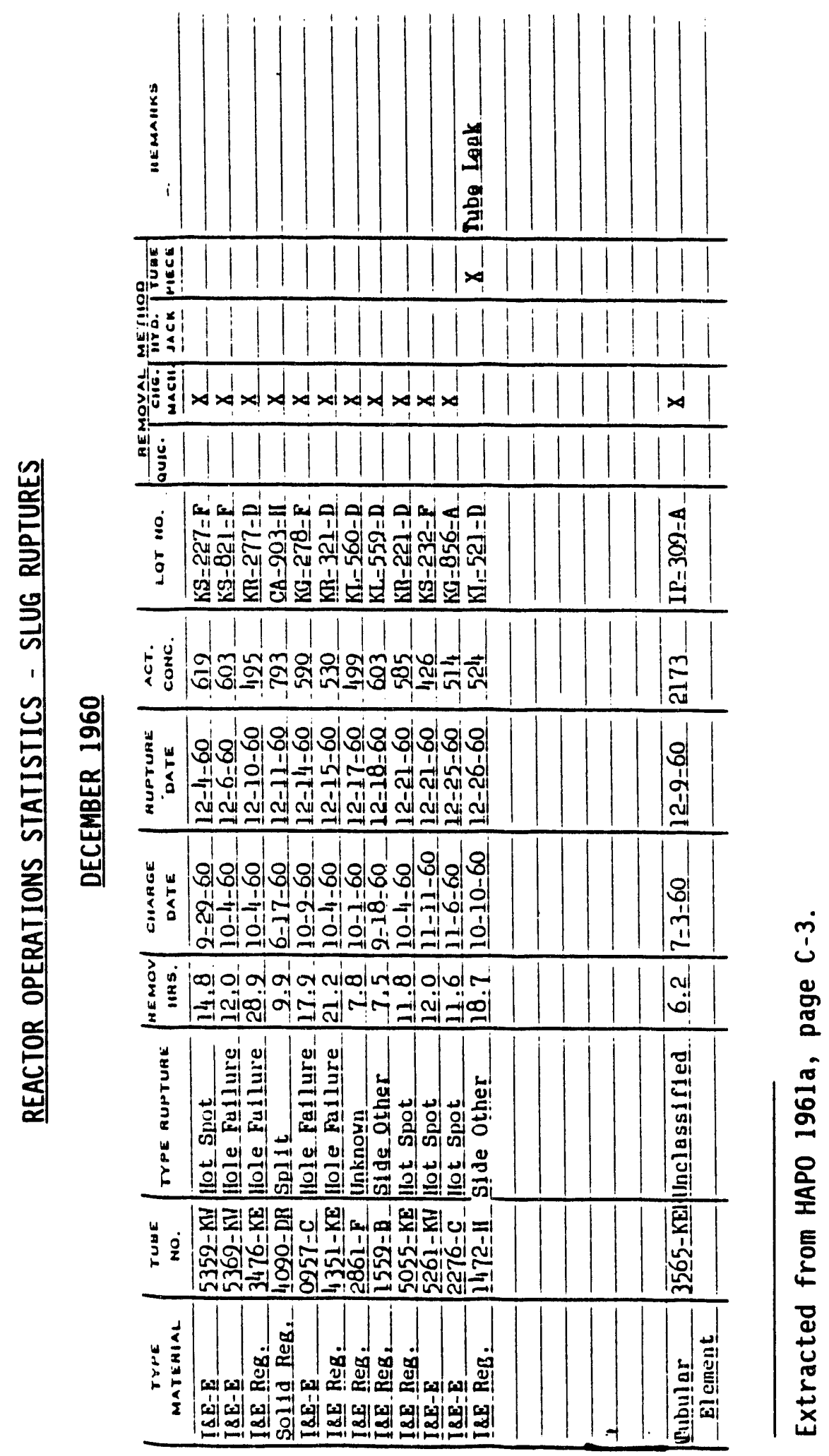


APPENDIX K

FUEL-ELEMENT FAILURE DATA FOR 1961 
RUPTURE EXPERIENCE - JANUARY 1961

\begin{tabular}{|c|c|c|c|c|c|}
\hline $\begin{array}{l}\text { Failure } \\
\text { Date }\end{array}$ & $\begin{array}{l}\text { Tube } \\
\text { Numiber }\end{array}$ & $\begin{array}{l}\text { Iot } \\
\text { Numioex }\end{array}$ & $\begin{array}{l}\text { Type of } \\
\text { Material }\end{array}$ & Exposure & $\begin{array}{l}\text { Type of } \\
\text { Failure }\end{array}$ \\
\hline $\begin{array}{l}1 / 2 / 61 \\
1 / 9 / 61 \\
1 / 13 / 61 \\
1 / 13 / 61 \\
1 / 21 / 61 \\
1 / 23 / 61 \\
1 / 23 / 61 \\
1 / 23 / 61 \\
1 / 26 / 61\end{array}$ & $\begin{array}{l}0868-F \\
3781-H \\
1366-D \\
3167-\mathrm{H} \\
2676-D \\
3090-\mathrm{KN} \\
0982-\mathrm{H} \\
5384-\mathrm{KN} \\
2878-D\end{array}$ & $\begin{array}{l}K L-534-D \\
K L-550-\mathbb{Z} \\
C I-95 I-A \\
K R-249-D \\
K I-530-D \\
K S-228-F \\
K O-108-C(I) \\
C R-865-A \\
K L-530-D\end{array}$ & 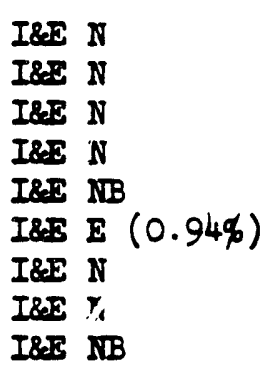 & $\begin{array}{l}614 \\
648 \\
512 \\
642 \\
957 \\
841 \\
-223 \\
621 \\
966\end{array}$ & $\begin{array}{l}\text { Unionown } \\
\text { Unclassified } \\
\text { Side Bot spot } \\
\text { Unicoown } \\
\text { Side-Other } \\
\text { Side Eot-Spot } \\
\text { Side-Other } \\
\text { Hole } \\
\text { Side-Other }\end{array}$ \\
\hline $\begin{array}{l}2 / 28 / 61 \\
i / 31 / 61\end{array}$ & $\begin{array}{l}2657-\mathrm{DR} \\
3379-\mathrm{E}\end{array}$ & $\begin{array}{l}\mathrm{KB}-219-\mathrm{D} \\
\mathrm{KI}-5,96-\mathrm{A}\end{array}$ & $\operatorname{IrE} \underset{N}{\operatorname{Igex}}(0.94 \%)$ & $\begin{array}{l}775 \\
757\end{array}$ & $\begin{array}{l}\text { S1de-Other } \\
\text { Eole }\end{array}$ \\
\hline
\end{tabular}

(i) Six Incin element with attached water mixer spool.

\section{Iegend}

IfEN - This is the symbol for internally and externally cooled production reactor fuel elements of natural uranium.

I\&E E - This is the symol for internally and externally cooled production reactor fuel elements with uranium cores enriched in U-235. The welght per cent $U-235$ in the core material is stated.

I E I IB - This is the symbol for internally and externally cooled production reactor fuel elements of naturai uranium winich have projections (oumpers) welied to tide fuel element jacket.

Unienown - Failed piece could not be located or had not been examined at the ijme of writiog.

Unclassified - The fallure did not logically fall into any of the usual types.

Side Eot-Spot - Failure probably caused by accelerated corrosion of the exuerasil can wall in a locallzed region of bigh temperature.

Sige-other - Fallure probaily caused by corrosion or water penetration of the external can wall or otiner unicoown mechanism.

Eole - Failure occursed on the internal surface of an 1nternaly and externaify fuel element. The failure was probably caused by water penetration through a weld.

Extracted from HAPO 1961B, page B-15. 


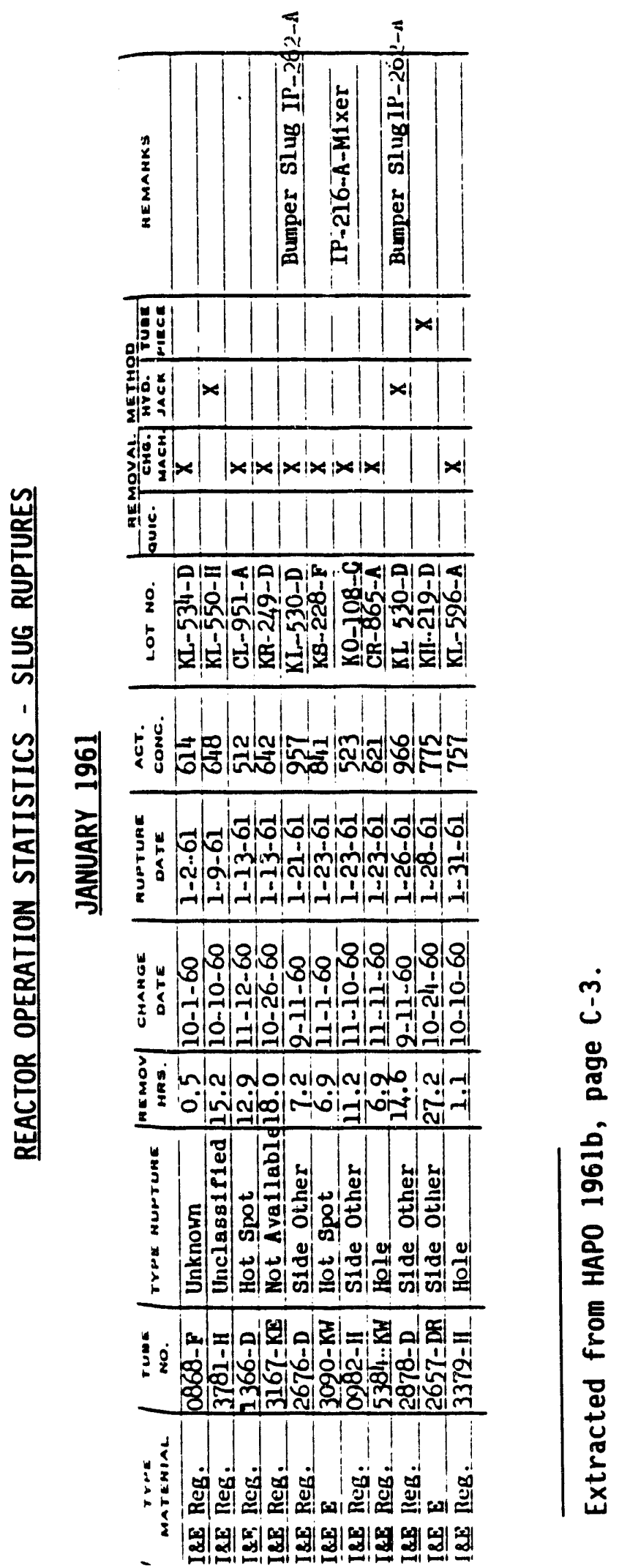




\section{RUPTURE EXPERIENCE - FEBRUARY 1961}

\begin{tabular}{|c|c|c|c|c|c|}
\hline $\begin{array}{l}\text { aslure } \\
\text { ate }\end{array}$ & $\begin{array}{l}\text { Tube } \\
\text { Numbez }\end{array}$ & $\begin{array}{l}\text { Lot } \\
\text { Number }\end{array}$ & $\begin{array}{l}\text { Type of } \\
\text { Meter18l }\end{array}$ & Exposure & $\begin{array}{l}\text { Type of } \\
\text { Foilure }\end{array}$ \\
\hline $\begin{array}{l}2-2-61 \\
2-11-61 \\
2-12-61 \\
2-12-61 \\
2-21-61 \\
2-25-61\end{array}$ & $\begin{array}{l}3275-C \\
1686-B-1 \\
4072-D \\
4173-B \\
1686-B \\
236 i-C\end{array}$ & $\begin{array}{l}D M-023-3(I) \\
B S-26 j-A \\
B E-201-D \\
W E-234-D \\
\nabla L-035-D \\
C J-865-A\end{array}$ & 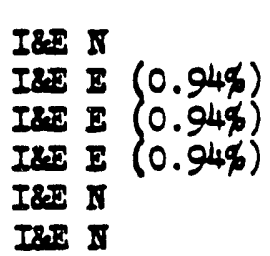 & $\begin{array}{l}706 \\
583 \\
638 \\
825 \\
774 \\
702\end{array}$ & $\begin{array}{l}\text { Eole } \\
\text { S1de B } \\
\text { Uncles } \\
\text { S1de E } \\
\text { Side B } \\
\text { S1de E }\end{array}$ \\
\hline
\end{tabular}

(1) S1x-1ach fluel eleweat with attacbed water mixer spool.

Iesend

Ira II - This is the symbol for Interas IIy and exterasily cooled product100 reactor suel elements of astural urantur.

Ires E - Tass is the symbol for 1nternelly and exterasily cooled product100 reector suel elements w1th uranium cores eariched in 0235 . The velight pe: cent 0235 in the core material 18 stated.

Bole - Fallure occurred on the 1aterwal surface of an lateraally and extercalis fuel elemeat. The faslure was probubly caused by veter penetration tbrough a rield.

S1de Bot-Spot - Fallure probably caused by accelerated corrosion of the exterasi cas rall in a locallzed region of blgh temperature.

Uaciasofeled - The fallure did aot loglcally fall lato ang of the usual Eypes.

Extracted from HAPO 1961c, page B-16. 


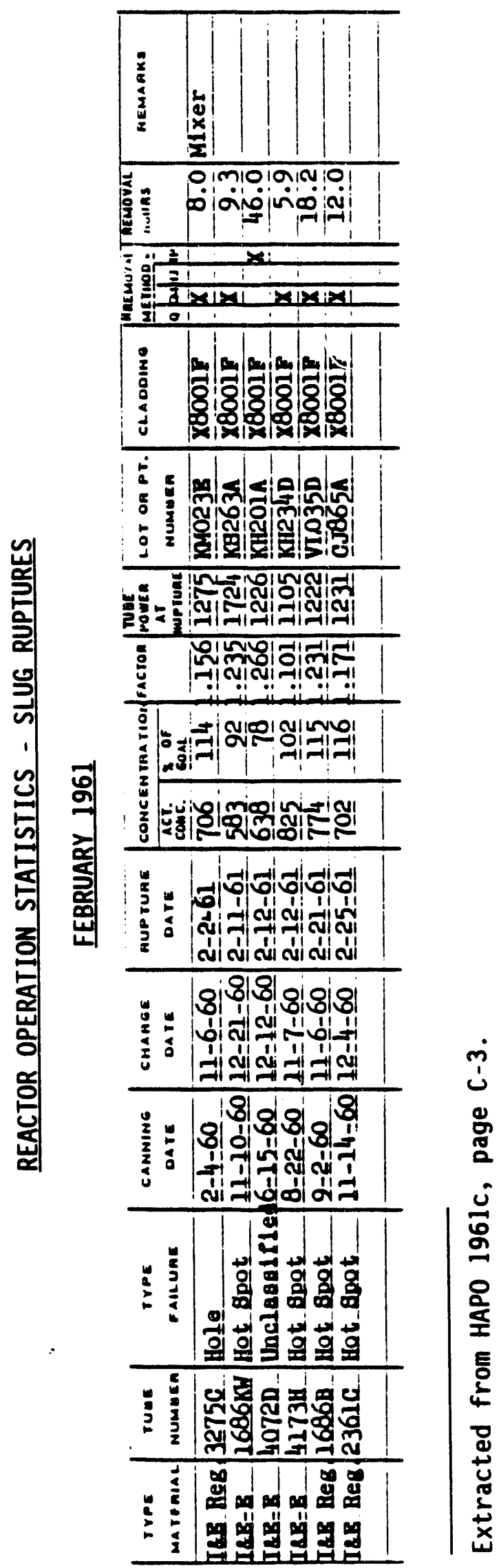

K. 4 
RUPTURE EXPERIENCE - MARCH 1961

\begin{tabular}{|c|c|c|c|c|c|}
\hline $\begin{array}{l}\text { Failure } \\
\text { Date }\end{array}$ & $\begin{array}{l}\text { Tube } \\
\text { Number }\end{array}$ & $\begin{array}{l}\text { Lot } \\
\text { Number }\end{array}$ & $\begin{array}{l}\text { Type of } \\
\text { Mate=tal }\end{array}$ & Exposure & $\begin{array}{l}\text { Type of } \\
\text { Failure }\end{array}$ \\
\hline $\begin{array}{l}3 / 5 / 61 \\
3 / 5 / 61 \\
3 / 8 / 61 \\
3 / 8 / 61 \\
3 / 8 / 61 \\
3 / 10 / 61 \\
3 / 19 / 61\end{array}$ & $\begin{array}{l}1467-D R \\
4177-K N \\
2776-K D \\
0571-H \\
2892-i \\
2178-K W \\
5684-K D\end{array}$ & $\begin{array}{l}\mathrm{KR}-234-\mathrm{D} \\
\mathrm{KR}-316-\mathrm{A} \\
\mathrm{CR}-878-\mathrm{A} \\
\mathrm{KH}-234-\mathrm{D} \\
\mathrm{KE}-211-\mathrm{D} \\
\mathrm{KS}-262-\mathrm{A} \\
\mathrm{KR}-295-\mathrm{D}\end{array}$ & $\begin{array}{l}\text { IRE E }(0.94 \%) \\
\text { I\&E N } \\
\text { I\&E N } \\
\text { IRE E }(0.94 \%) \\
\text { I\&E E }(0.94 \%) \\
\text { I\&E E }(0.94 \%) \\
\text { I\&E N }\end{array}$ & $\begin{array}{l}960 \\
672 \\
424 \\
598 \\
447 \\
499 \\
785\end{array}$ & $\begin{array}{l}\text { Side Hot-Spot } \\
\text { Unknown } \\
\text { Side Hot-Spot } \\
\text { Side Hot-Spot } \\
\text { Side Hot-Spot } \\
\text { Unknown } \\
\text { Eole }\end{array}$ \\
\hline $\begin{array}{l}3 / 20 / 61 \\
3 / 27 / 61 \\
3 / 28 / 61 \\
3 / 28 / 61 \\
3 / 28 / 61\end{array}$ & $\begin{array}{l}3764-B \\
3183-K N \\
3362-B \\
0572-B \\
2991-B\end{array}$ & $\begin{array}{l}K L-587-Z \\
K S-253-A \\
C L-964-B \\
K B-816-D \\
K B-242-A\end{array}$ & 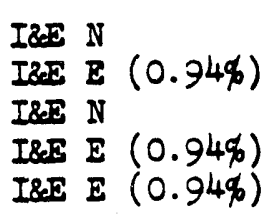 & $\begin{array}{l}517 \\
649 \\
554 \\
634 \\
507\end{array}$ & $\begin{array}{l}\text { Unclassified } \\
\text { Side \#ot-Spot } \\
\text { Side \#ot-Spot } \\
\text { Unknown } \\
\text { Side Bot-Spot }\end{array}$ \\
\hline
\end{tabular}

\section{Legend}

I\&E N - This is the symol for internally and externally cooled production reactor fuel elements of natural uranium.

I\&E E - This is the symbol for internally and externally cooled production Feactor fuel elemenis with uraoium cores enriched in U-235. The weight pe: cent $U-235$ in the core material is stated.

Side Jot-Soot - Failure probably caused by accelerated corrosion of the external can wall in a localized region of bigh temperature.

Unknown - Failed piece could not be located or had not been examined at the time of writing.

Bole - Failure ocsured on the internal surface of an internally and externaliy fuel element. The fallure was probably caused by water penetration througin a. weld.

Unclassified - The failure did not logically fall into any of the usual ijpes.

Extracted from HAPO 1961d, pages B-13 \& B-14. 


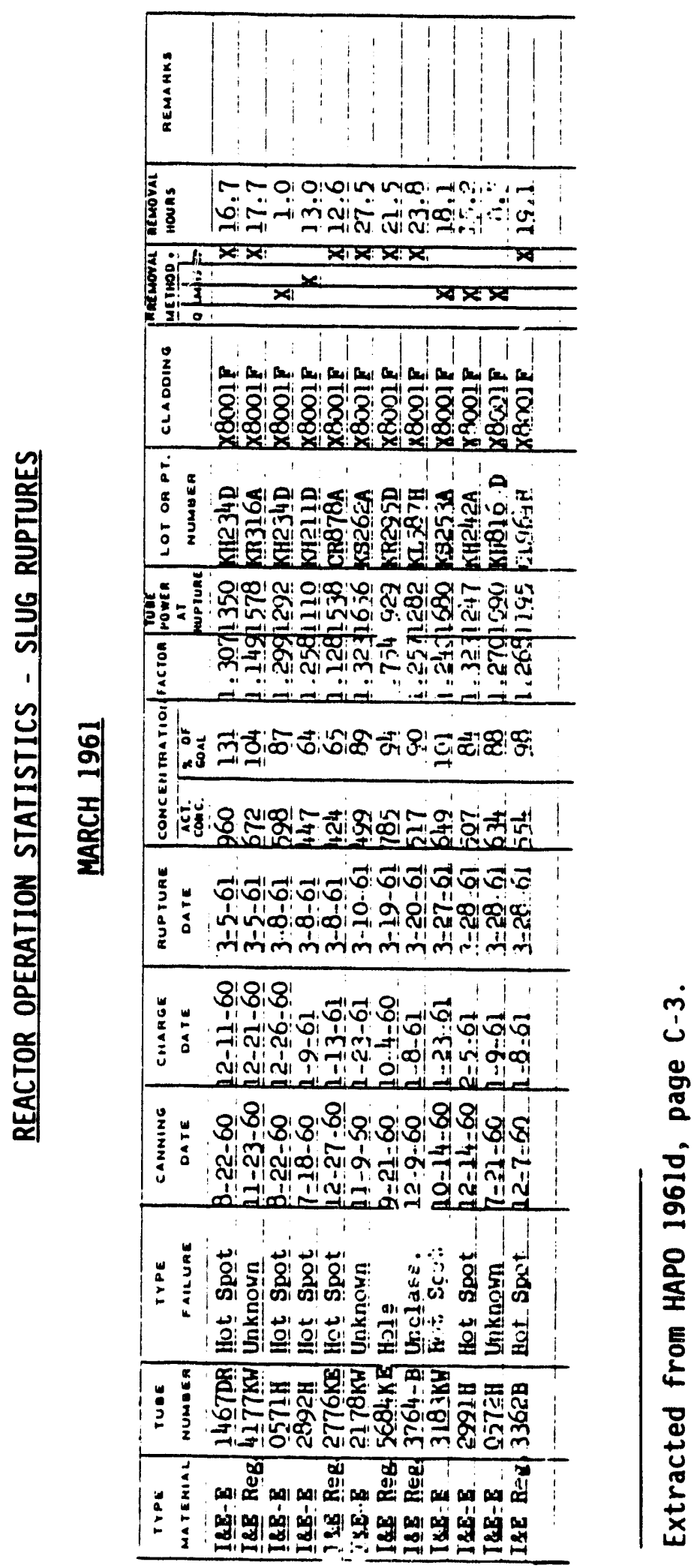

K. 6 


\section{RUPTURE EXPERIENCE - APRIL 1961}

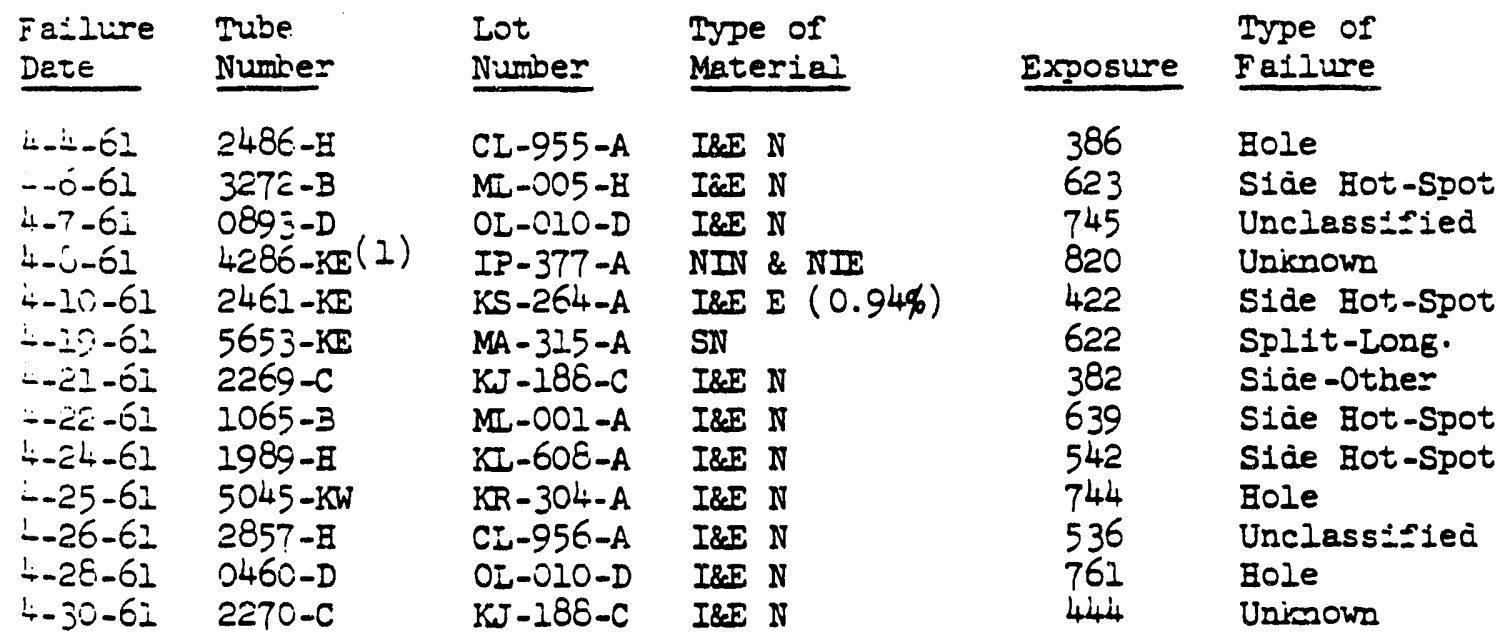

i. Kहn LOOP \#

Legend

IRE N - This is the symool for intemally and externally cooled production reactor fuel elements of natural urantum.

I\&E E - This is the symbol for internally and externally cooled production reactor fuel elements with uranium cores enriched in U-235. The weight per cent in the core material is stated.

SN - This is the symbol for production reactor fuel elements having a solid sylindricai core of netural urentum.

IVIN - This is the symbol for NPR inner tube fuel elements of natural urenium. NIE - This is the symbol for NPR 1nner tube fuel elements of enriched uraniur.

Bole - Failure on the internal surface of an I \& E plece probably caused by water penetration through a weld or other unknown mechanism.

Sice Hot-Spot - Failure probably caused by accelerated corrosion of the externei can wall in a localized region of high temperature.

Unknown - Failed piece could not be loceted or had not been examined at the iime of writing.

Unclassified - The failure did not logically fell into any of the usual types.

Split-Long.-Failure caused by uranium cleavage along the axis of the core.

Siaje-Other - Feilure probably caused by corrosion or water penetration of the exteznai car wall or other unimown mechanism.

Extracted from HAPO 196le, pages B-13 \& 14. 


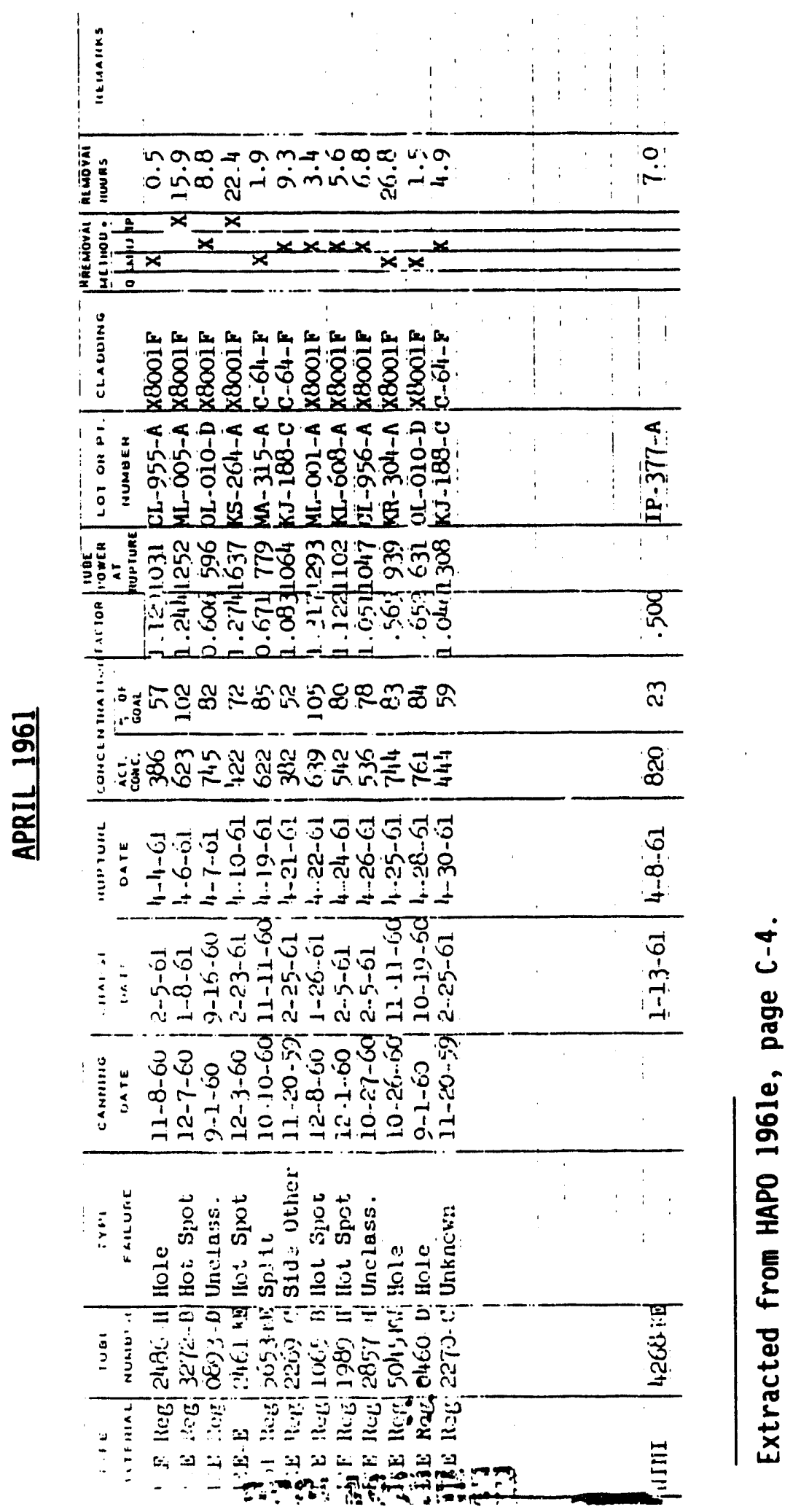

K. 8 
RUPTURE EXPERIENCE - MAY 1961

\begin{tabular}{|c|c|c|c|c|c|}
\hline $\begin{array}{l}\text { Fallure } \\
\text { Date }\end{array}$ & $\begin{array}{l}\text { Tube } \\
\text { Number }\end{array}$ & $\begin{array}{l}\text { Iot } \\
\text { Number }\end{array}$ & Type of Material & Exposure & $\begin{array}{l}\text { Type of } \\
\text { Feilure }\end{array}$ \\
\hline $\begin{array}{l}5 / 1 / 61 \\
5 / 2 / 61 \\
5 / 13 / 61 \\
5 / 17 / 61\end{array}$ & $\begin{array}{l}2370-B \\
1959-B \\
2160-K D(1) \\
2657-D R\end{array}$ & $\begin{array}{l}I Z-500-A \\
I Z-500-A \\
I P-3 I 4-A \\
K E-252-A\end{array}$ & $\begin{array}{l}\operatorname{IRE} \mathbb{N} \\
\operatorname{IRE} \mathrm{N} \\
\operatorname{LSRN}-2 \\
\operatorname{IRS} \mathrm{B}(0.94 \%)\end{array}$ & $\begin{array}{c}491 \\
450 \\
\text { Iess then } 1 \\
890\end{array}$ & $\begin{array}{l}\text { Side Hot-Spot } \\
\text { Unclessifiec } \\
\text { Unclossifiec } \\
\text { Bnd-Mele }\end{array}$ \\
\hline
\end{tabular}

\section{Iegend}

I\&E N - This is the symbol for internally and externally cooled production reactor fuel elements of natural uranium.

KSRN-2 - This is the symbol for K Reactor single-tube fuel elements of nature: uranium.

I\&E E - This is the symbol for interally and externelly cooled production reactor fuel elements with uranium cores enriched in U-235. The weight per cent U-235 in the core material is stated.

Side Hot-Spot - Failure probably caused by accelereted corrosion of the external can wall in a localized region of bigh temperature.

Unclassified - The failure did not logicaliy fall into any of the usual types.

End-Male - Failure on the end of the fuel elements probably caused by weter penetration through a weld.

\section{WATERR TREATMEAVT}

Alum feed rates of $18 \mathrm{ppm}$ have been accomplished at 100-F during the month. Although water is difficult to treat during this period, good water quality hes been maintained and no reduction in flow to the reactor has been requires.

(I) KKR IOOP

Extracted from HAPO 1961f, page B-16. 


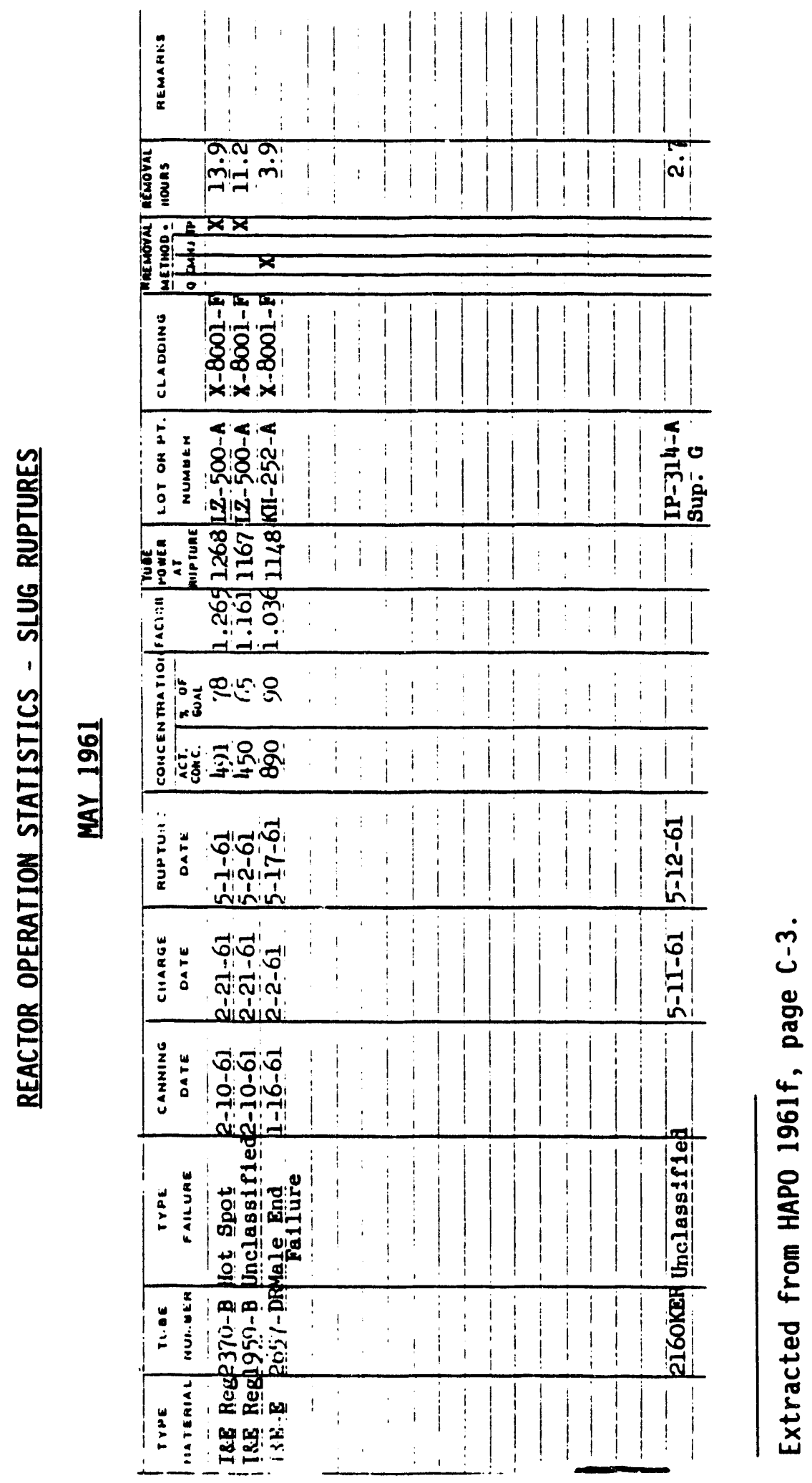




\section{RUPTURE EXPERIENCE - JUNE 1961}

\begin{tabular}{|c|c|c|c|c|c|}
\hline $\begin{array}{l}\text { Fallure } \\
\text { Dete }\end{array}$ & $\begin{array}{l}\text { Tube } \\
\text { Number }\end{array}$ & $\begin{array}{l}\text { Iot } \\
\text { Numioer }\end{array}$ & Type of Material & Exposure & $\begin{array}{l}\text { Type of } \\
\text { Feilure }\end{array}$ \\
\hline $\begin{array}{l}6 / 5 / 61 \\
6 / 9 / 61 \\
6 / 9 / 61 \\
6 / 22 / 61 \\
6 / 17 / 61 \\
6 / 18 / 61 \\
6 / 24 / 61\end{array}$ & $\begin{array}{l}3766-C \\
2986-D R \\
2352-C \\
3457-F \\
0967-B \\
2867-E \\
2264-F\end{array}$ & $\begin{array}{l}C J-863-A \\
U Z-014-A \\
C A-909-A \\
K E-8290-A \\
V Z-006-B \\
K E-289-D \\
U Z-015-E\end{array}$ & 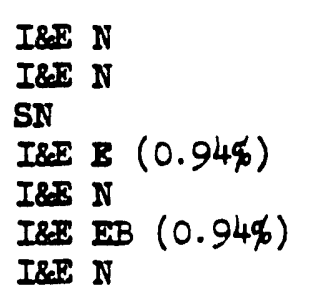 & $\begin{array}{l}727 \\
305 \\
723 \\
716 \\
252 \\
314 \\
208\end{array}$ & $\begin{array}{l}\text { Side Hot-Sp } \\
\text { Split-Trans } \\
\text { Spl1t-IOnE. } \\
\text { End-Female } \\
\text { End-Naie } \\
\text { End-Male } \\
\text { Unionown }\end{array}$ \\
\hline
\end{tabular}

Iegend:

IfE N - This is the symbol for internally and externally cooled production reactor fuel elements of natural uranium.

I EE E - This is the symbol for internally and externelly cooled production reactor fuel elements with uranium cores enriched in U-235. The weight per cent U-235 in the core material is stated.

Ife EB This is the symbol for internally and externally cooled production reactor fuel elements which have projections (bumpers) welded to fuel element jackets. The urantum cores are enriched in U-235 and the weight per cent $U-235$ in the core material is stated.

SN - This is the symbol for production reactor fuel elements bave a solic cylinaricel core of natural uranium.

Side jot-Spot - Failure probably caused by accelerated corrosion of the exrernal can wail in a locallzed region of bugh temperature.

Sol1t-Iransverse - Fallure caused by uranium cleavage across or at an angle to the axis of the core.

Solit-Iongitudinal - Failure caused by uranium cleavage along the axds of the core.

and - Failure on the end of the fuel element probaily caused by water penetration through a weld.

Unionown - Failure caused by urantum cleavage along the axts of the core.

Extracted from HAPO $1961 \mathrm{~g}$, pages B-16 \& B-17. 


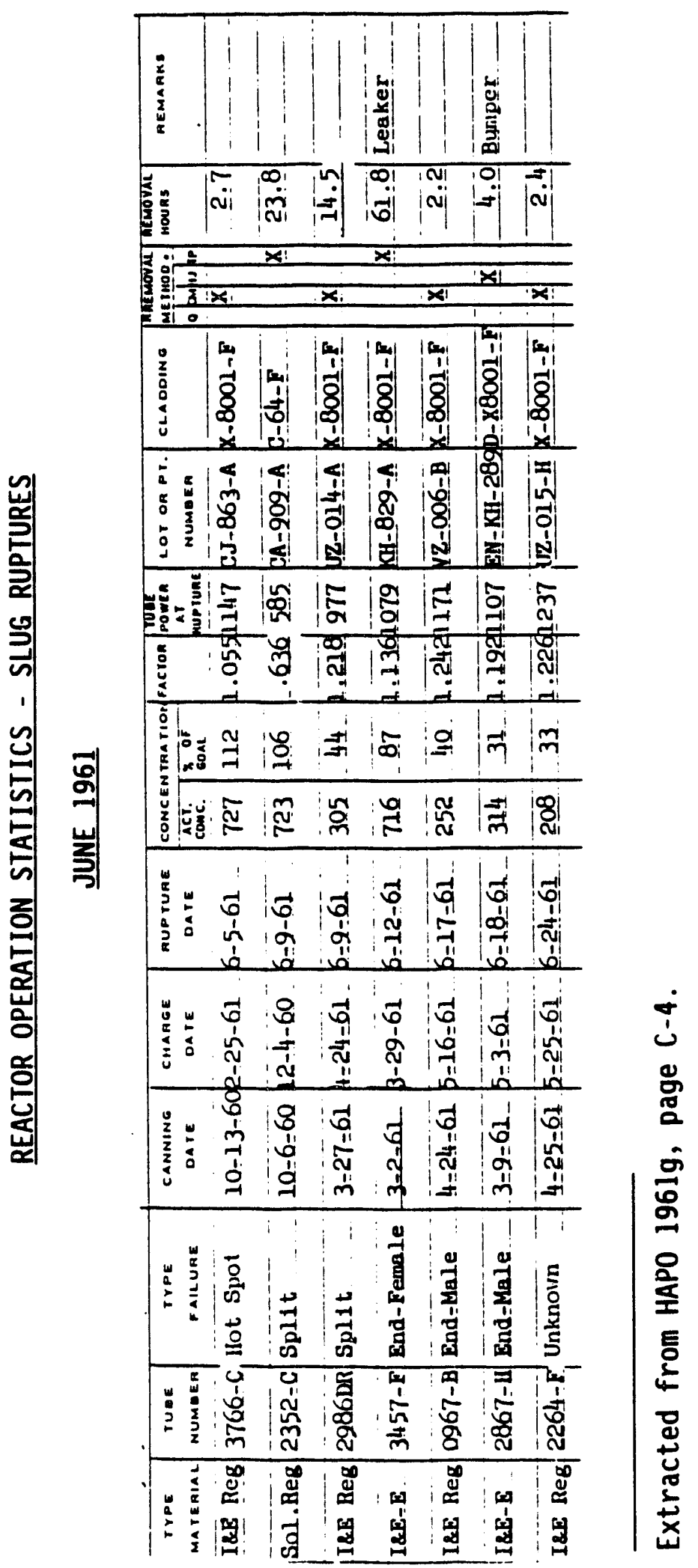


RUPTURE EXPERIENCE - JULY 1961

Failure Tube Iot Type of

Date Number Number Type of Material Exposure Failure

$7 / 1 / 61$ 2485-F UZ-015-E I8E N 419 SplitTransverse

Iegend:

IfE N - Tais is the symbol for internally and externaliy cooled production reactor fuel elements of natural uranium.

Solit-Transverse - Failure caused by uranilum cleavage across or at an angle to the axis of the core.

Extracted from HAPO 1961h, page B-13. 


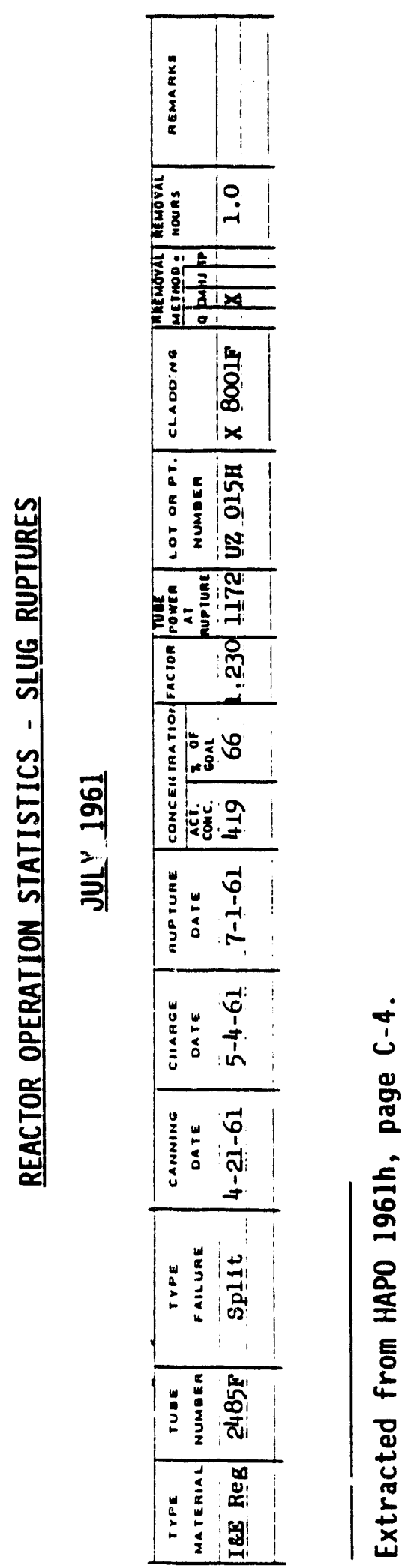

K. 14 
RUPTURE EXPERIENCE - AUGUST 1961

\begin{tabular}{|c|c|c|c|c|c|}
\hline $\begin{array}{l}\text { Failure } \\
\text { Date }\end{array}$ & $\begin{array}{l}\text { Tube } \\
\text { Number }\end{array}$ & $\begin{array}{l}\text { Lot } \\
\text { Number }\end{array}$ & Type of Material & Exposure & $\begin{array}{l}\text { Type of } \\
\text { Feilure }\end{array}$ \\
\hline $\begin{array}{l}8-8-61 \\
8-24-61 \\
8-29-61 \\
8-30-61 \\
8-31-61 \\
8-31-61\end{array}$ & $\begin{array}{l}2476-\mathrm{E} \\
1363-\mathrm{KN} \\
1163-\mathrm{D} \\
1379-\mathrm{E} \\
2470-\mathrm{DR} \\
2478-\mathrm{DR}\end{array}$ & $\begin{array}{l}K H-289-D \\
K K-081-A \\
K Z-064-E \\
K K-273-A \\
C Z-814-A \\
P Z-010-A\end{array}$ & $\begin{array}{l}\text { I\&E } \mathrm{EB}(0.94 \%) \\
\text { I\&E } \mathrm{N}(0.94 \%) \\
\text { I\&E NB } \\
\text { I\&E } \mathrm{NB}(0.94 \%) \\
\text { I\&E N } \\
\text { I\&E } \mathrm{N}\end{array}$ & $\begin{array}{l}663 \\
554 \\
795 \\
745 \\
768 \\
786\end{array}$ & $\begin{array}{l}\text { Hole } \\
\text { Sice Hot-Spot } \\
\text { Hole } \\
\text { Hoi } \\
\text { Hi-t: } \\
\text { Hoile }\end{array}$ \\
\hline
\end{tabular}

Legend:

I\&E EB - This is the symol for internally and externally coolec production reactor fuel elements which heve projections (bumpers) welded to the fuel element jacisets. The uranium cores are enriched in U-235 and the weigint per cent U-235 in the core material is stated.

I\&E E - This is the symbol for 1nternally and externally cooled production reactor fuel elements with uranium cores enriched in U-235. The weight per cent U-235 in the core material is stated.

I\&E NB - This is the symol for internally and externally cooled production. reactor fuel elements of netural uranium which have projections (bumpers) welded to the fuel element faciret.

I\&E N - This is the symbol for internally and externally cooled production reactor fuel eiements of natural uranium.

Eole - Failure on the internal surface of an IRE piece probably caused by water penerration tbrough a weld or othe: unirnown mecinanism.

Side EOT-Spot - Failure probably caused by accelerated corrosion of the externai can wail in a iocalizec region of bigh temperature.

Extracted from HAPO 1961i, page B-15. 


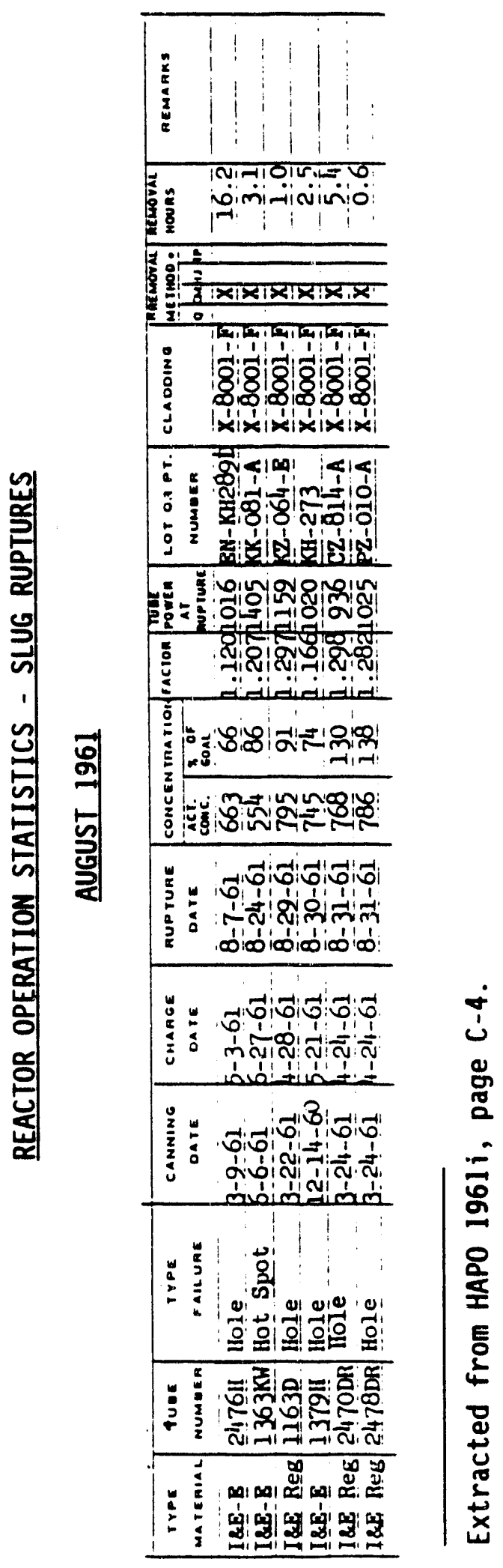

K. 16 


\section{RUPTURE EXPERIENCE - SEPTEMBER 1961}

\begin{tabular}{|c|c|c|c|c|c|}
\hline $\begin{array}{l}\text { Failiure } \\
\text { Date }\end{array}$ & $\begin{array}{l}\text { Tube } \\
\text { Numiber }\end{array}$ & $\begin{array}{l}\text { Iot } \\
\text { Number }\end{array}$ & Mype of Materigl & Exposure & $\begin{array}{l}\text { Type of } \\
\text { Feilure }\end{array}$ \\
\hline $\begin{array}{l}9 / 2 / 61 \\
9 / 3 / 61\end{array}$ & $\begin{array}{l}2667-\mathrm{I} \\
1465-\mathrm{D}\end{array}$ & $\begin{array}{l}\mathrm{KET}-274-\mathrm{A} \\
\mathrm{BZ}-067-\mathrm{E}\end{array}$ & $\begin{array}{l}I \& E \\
I \& E \\
\mathrm{IBB}\end{array}$ & $\begin{array}{r}843 \\
13\end{array}$ & $\begin{array}{l}\text { Hole } \\
\text { Mechanical } \\
\text { Demage }\end{array}$ \\
\hline $\begin{array}{l}9 / 5 / 61 \\
9 / 6 / 61 \\
9 / 15 / 61 \\
9 / 15 / 61 \\
9 / 18 / 61 \\
9 / 19 / 61 \\
9 / 26 / 61\end{array}$ & $\begin{array}{l}2178-F \\
1581-\mathrm{E} \\
4072-\mathrm{D} \\
2384-\mathrm{F} \\
2561-\mathrm{DR} \\
2795-\mathrm{F} \\
0766-\mathrm{DR}\end{array}$ & $\begin{array}{l}K Y-006-A \\
K Y-081-D \\
K Y-024-D \\
K Y-062-A \\
K Y-096-A \\
K I Y-22 I-D \\
K Y-096-A\end{array}$ & 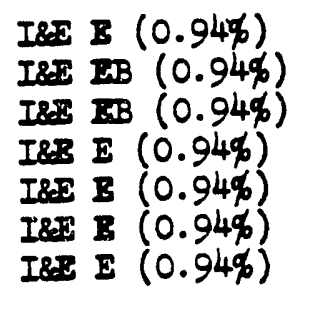 & $\begin{array}{r}338 \\
544 \\
986 \\
434 \\
93 \\
1098 \\
154\end{array}$ & $\begin{array}{l}\text { Side EOT-Spo } \\
\text { Side-Other } \\
\text { Bole } \\
\text { Side Eot-Spo } \\
\text { Side Hot-Spo } \\
\text { Side-Other } \\
\text { Mechanical } \\
\text { Damage }\end{array}$ \\
\hline
\end{tabular}

Iegend:

I\&S EB - This is the symbol for Internally and externally cooled production reactor fuel elements which bave projections (bumpers) welded to tine Puel element jacisets. The uranium cores are enriched in U-235 and the weight per cent $U-235$ in the core material is stated.

I\&B NB - This is the symbol for intermally a wernally cooled production reactor flul elements of natural uranium wich bave projections (bumpers) weloed to the fuel element jacket.

I\&E E - This is the symbol for internally and exterally cooled production reactor fuel elements with uranium cores enriched is U-235. The weight per cent U-235 in the core material is stated.

Hole - Failure on the internal surface of an I\&E piece probably causes by water penetration through a weld or other unknown mecinanism.

Mechanical Damage - Failure probably cuased by mechanical damage to the fuel element prior to irradiation.

Side Hot-Spot - Failure probably caused by accelerated corrosion of the external can wall in a localized region of high temperature.

Side-other - Failure probably caused by corrosion or water penetration of the exrernal can wall or other unknown mechanism.

Extracted from HAPO $1961 j$, page B-15. 


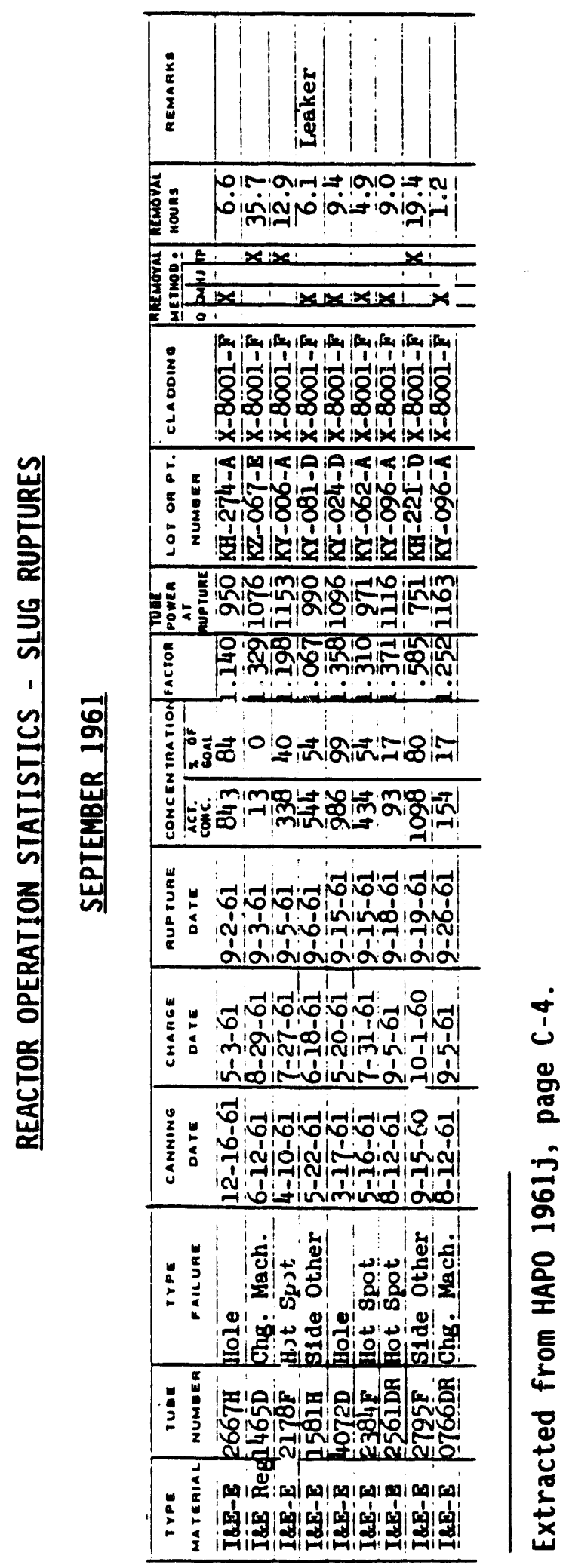



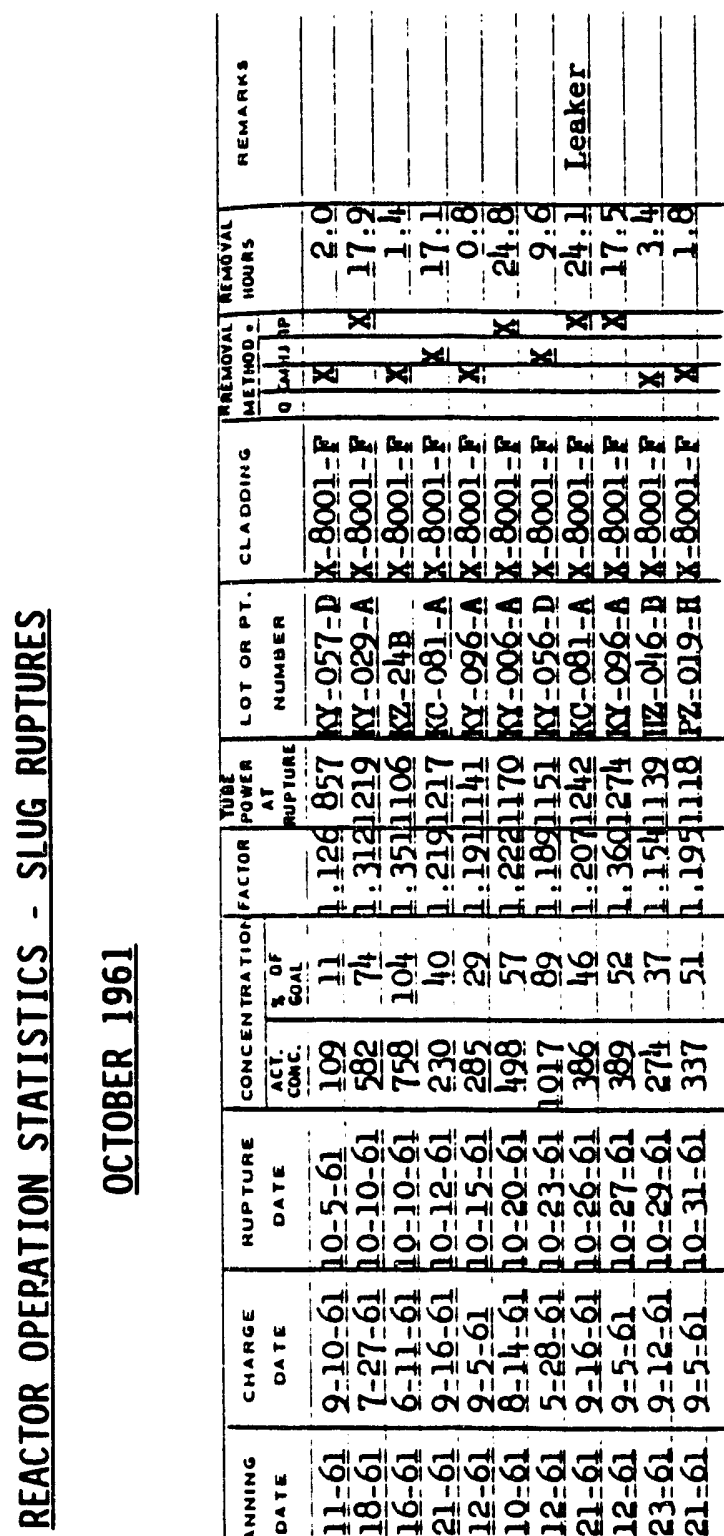

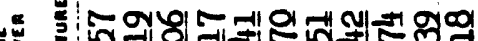

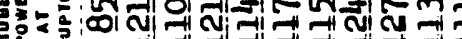

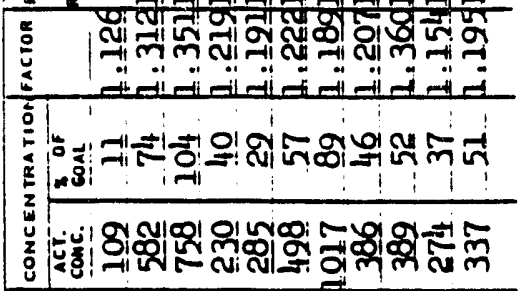

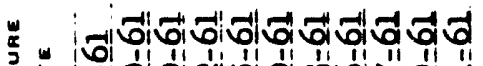

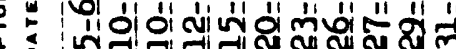
จ

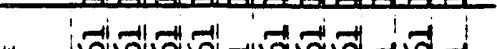

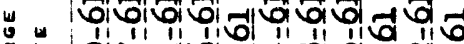

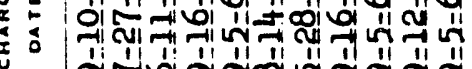

on or ovel wor or or

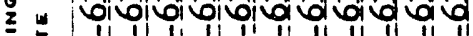

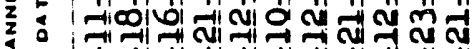

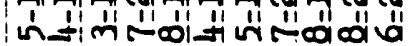

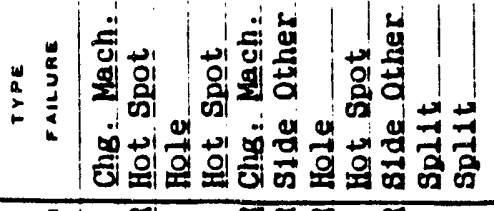

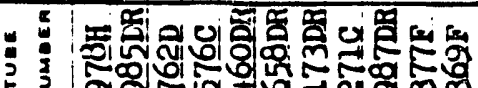

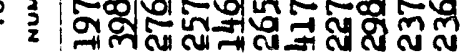

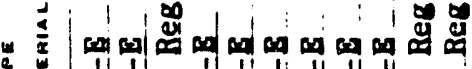

(

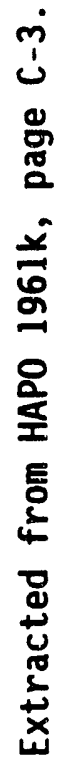


RUPTURE EXPERIENCE - NOVEMBER 1961

\begin{tabular}{|c|c|c|c|c|c|}
\hline $\begin{array}{l}\text { Failure } \\
\text { Date }\end{array}$ & $\begin{array}{l}\text { Tube } \\
\text { Number }\end{array}$ & $\begin{array}{l}\text { Iot } \\
\text { Number }\end{array}$ & Type of Materie? & $\begin{array}{l}\text { Exposure } \\
(\mathrm{MND} / \mathrm{T})\end{array}$ & Type of Feilure \\
\hline $11 / 27 / 61$ & $0774-D$ & $E Y-059-D$ & IES $(0.94 \%)$ & 499 & Fnd \\
\hline
\end{tabular}

Iegend:

I\&E EB - This is the symbol for interneily and excernelly cooled production reactcr fuel elements wich have projections (inumers) welded to the fuel element jacisets. The uranium cores are enriched in $1-235$ and the weight per cent $U-235$ in tine core materiel is stated.

Ena - Feilure on the end cf the fuel element probabily cusea by water penetreiion througin $a$ weli.

Extracted from HAPO 19611, page B-12. 


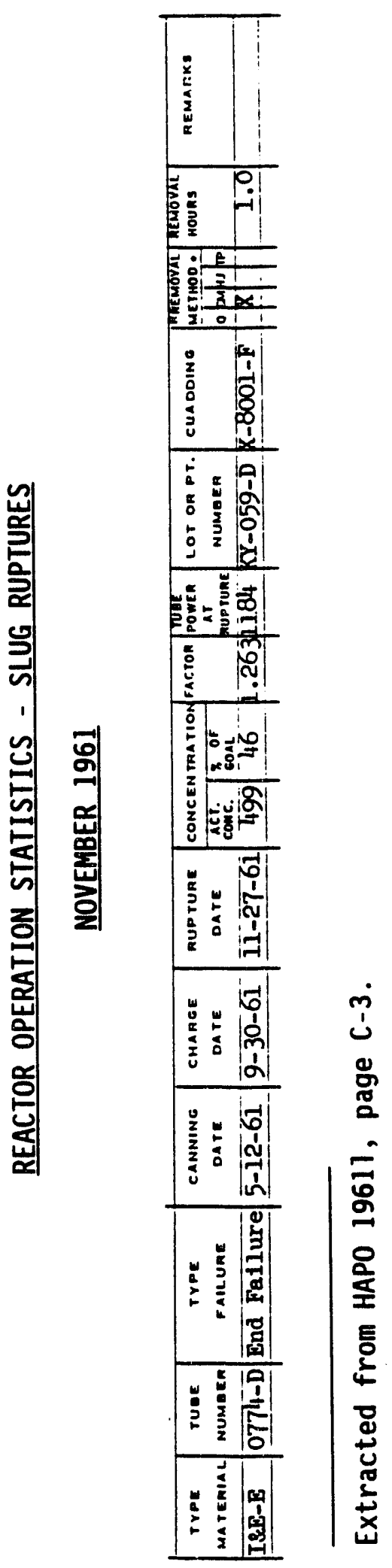

K. 21 
RUPTURE EXPERIENCE - DECEMBER 1961

\begin{tabular}{|c|c|c|c|c|c|}
\hline $\begin{array}{l}\text { Failure } \\
\text { Dete }\end{array}$ & $\begin{array}{l}\text { Tube } \\
\text { Numiber }\end{array}$ & $\begin{array}{l}\text { Iot } \\
\text { Number }\end{array}$ & Type of vaterial & $\begin{array}{l}\text { Exposure } \\
\text { (N:D/T) }\end{array}$ & $\begin{array}{l}\text { Type of } \\
\text { Feilure }\end{array}$ \\
\hline $\begin{array}{l}12 / 8 / 61 \\
12 / 13 / 61 \\
12 / 14 / 61 \\
12 / 23 / 61 \\
12 / 27 / 61 \\
12 / 28 / 61 \\
12 / 31 / 61\end{array}$ & $\begin{array}{l}3762-C \\
2864-B \\
3264-D R \\
4277-E \\
0867-D R \\
2872-E \\
2667-E\end{array}$ & 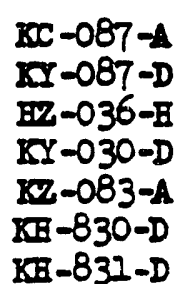 & 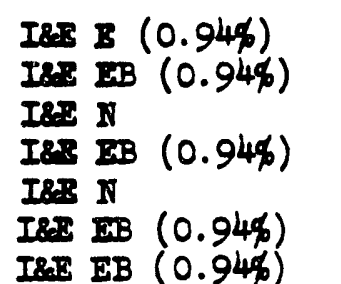 & $\begin{array}{r}595 \\
605 \\
780 \\
1050 \\
750 \\
760 \\
793\end{array}$ & $\begin{array}{l}\text { Side Bot Spot } \\
\text { Split-IOng. } \\
\text { Side Bot Spot } \\
\text { Side Other } \\
\text { Side Eot Spot } \\
\text { Hole } \\
\text { Hole }\end{array}$ \\
\hline
\end{tabular}

\section{Iegend}

Igs N - This is the symbol for internally and externally cooled production reactor fuel elements of natural uranium.

I8s B - This is the symbol for internally and externaliy cooled production reactor fuel elements with uranium cores enriched in $0-235$. The weight per cent $U-235$ in the core material 15 stated.

Ifs EB - This is the symbol for internally and externally cooled production reactor fuel elemexts which bave projections (bumpers) welded to the fuel element jackets. The uranium cores are enriched in U-235 and the welght per cent U-235 is the core material is stated.

Side Hot-Spot - Failure probably caused by accelerated corrosion of the external car wall in a localized region of high temperature.

Split-Iong. - Fallure caused by uranium cleavage along the'. axds of the core.

Side-Other - Fallure probably caused by corrosion or water penetration of the external can wall or other unicnown mechanism.

Hole - Failure on the 1nternal ourface of an IsE plece probably caused by water penetration through a weld or other unknown mechanism.

Extracted from HAPO 1962a, pages B-14 \& B-15. 


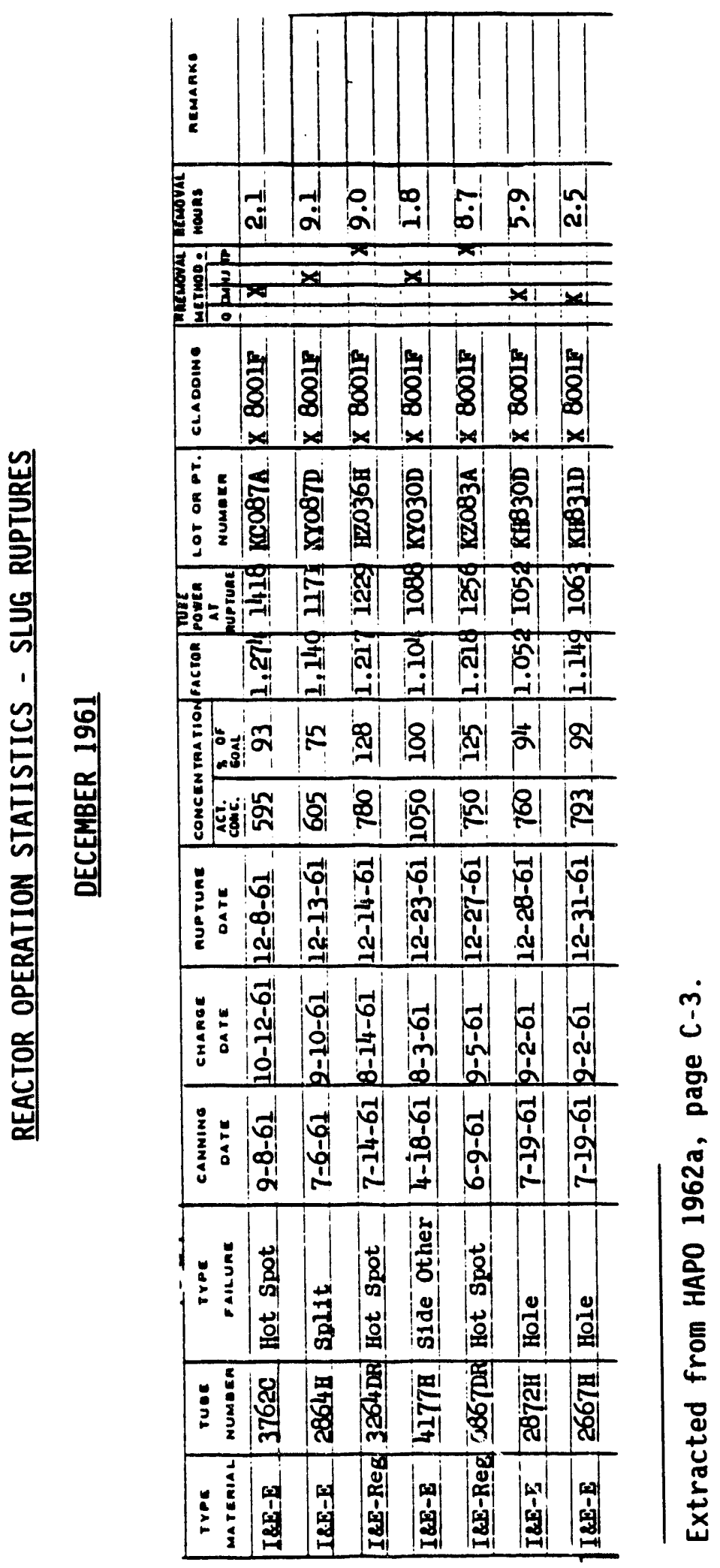


APPENDIX L

FUEL-ELEMENT FAILURE DATA FOR 1962 
RUPTURE EXPERIENCE - JANUARY 1962

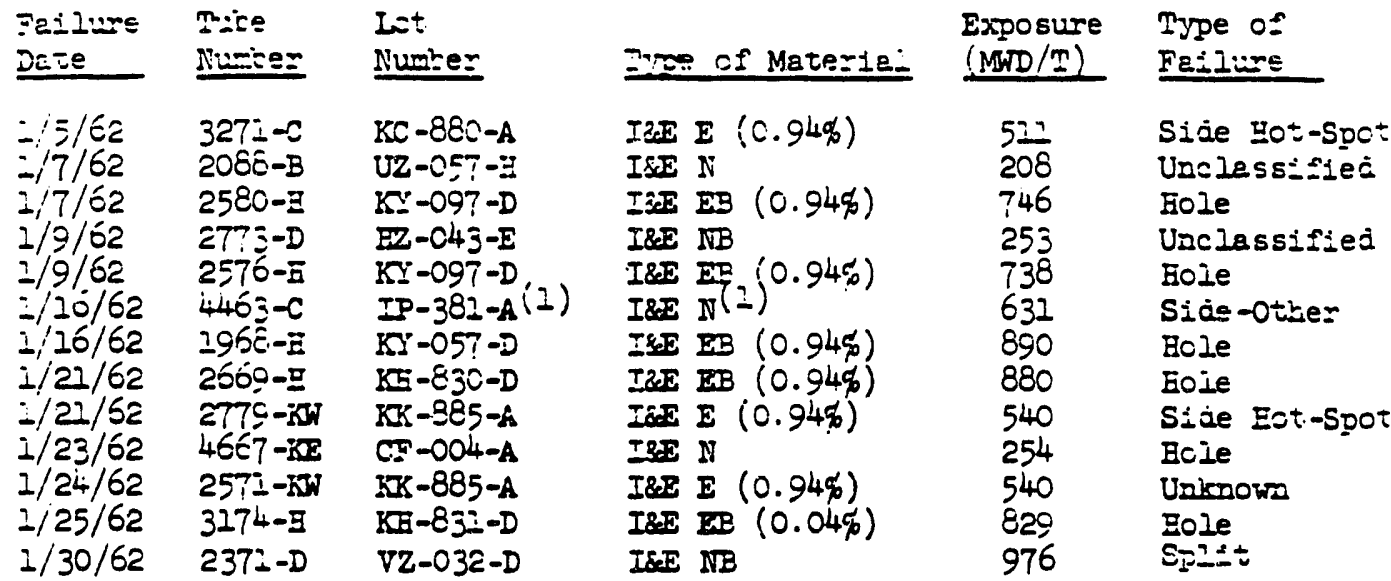

Iegend

Ire E - This is the symici for i=ternai-y and extemaily ccoied production

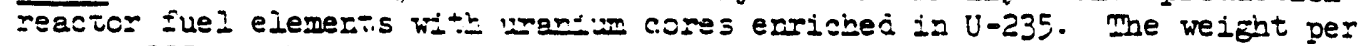
sezt li-23E in the cors materiai is ster.es.

İE ji - mais is the symbol for internaicy anc externaily coolej procuction reastor fuel elements of natural irazi-um.

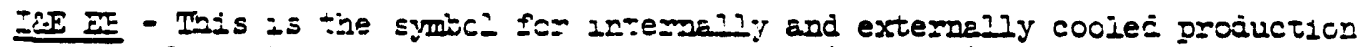
reactor Suel $\in$ iements which javs projestsons (bumpers) weljoc to the fueI ejezent jecire:s. The uraniur cores are anriched in $0-235$ and the weight pez: sent U-235 in the core mateziai is s,azej.

Ias NB - This is the symbol for internally and externally cooled production reacto: fuel elements of natural uraniur which bave projections (bumpers) welojed to tine fuel element jacket.

Side Hot-Spot - Fallure probably caused by accelerated corrosion of the externai can well in a localized region of bigh temperature.

Uaclessified - The faflure did not loglcally fall into any of the usual types

Eole - Failure on the Interval surface of an Ise piece probably caused by water penetration through a weld or other uaknown mechani am.

Side-Other - Faslure probably caused by corrosion or weter penetration of the externai can vell or otber untnown mechantam.

Unknown - Failed piece could not be located or had not been examined at the ine of walting.

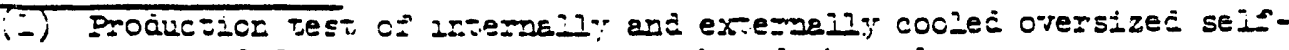
supporteo fue $i$ jemenis in an srerjored sinannei.

Extracted from HAPO 1962b, pages B-15 \& B-16. 


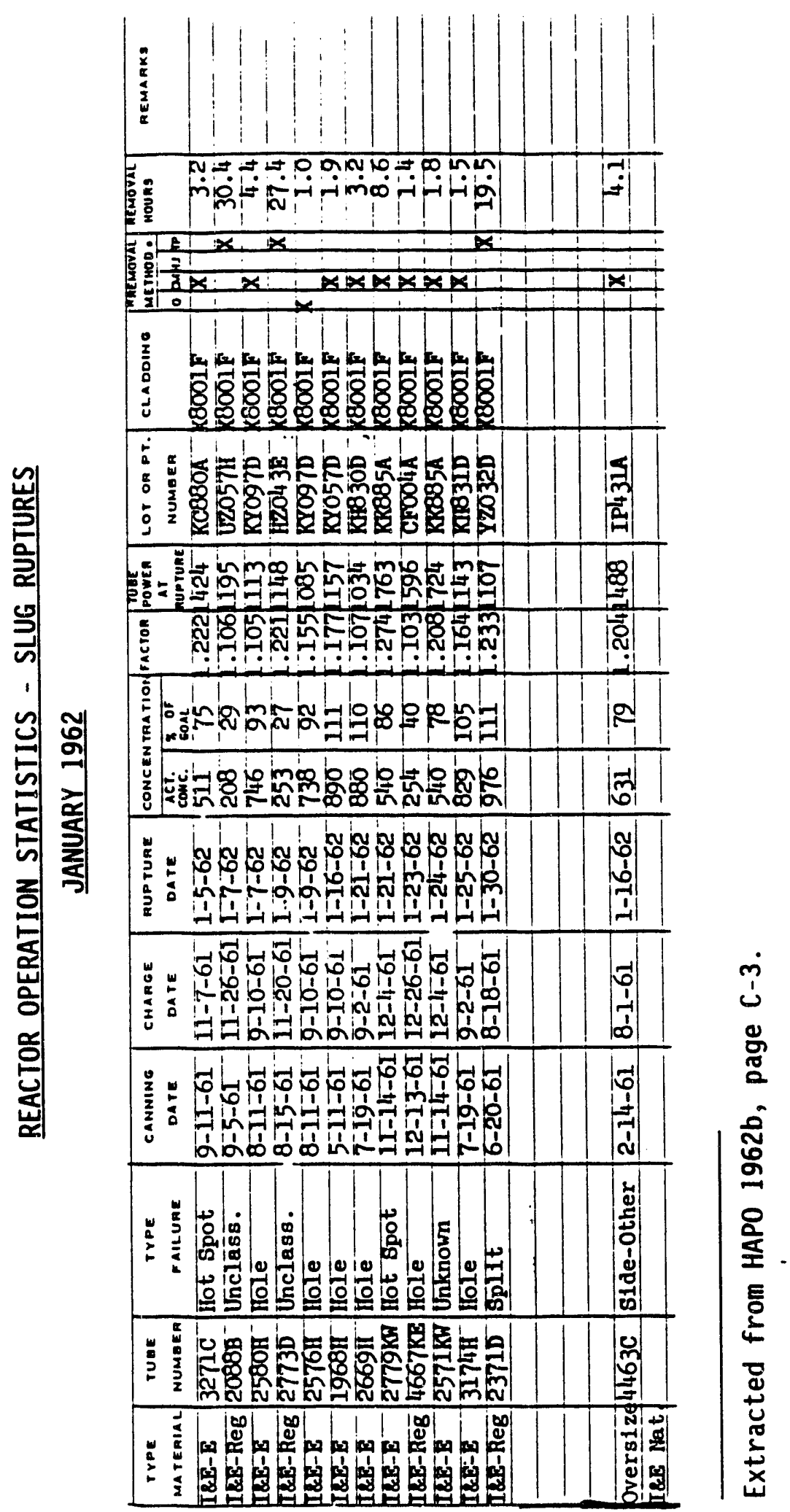

L. 2 


\section{RUPTURE EXPERIENCE - FEBRUARY 1962}

\begin{tabular}{|c|c|c|c|c|c|}
\hline $\begin{array}{l}\text { Failure } \\
\text { Dete }\end{array}$ & $\begin{array}{l}\text { Tube } \\
\text { Number }\end{array}$ & $\begin{array}{l}\text { Iot } \\
\text { Number }\end{array}$ & Type of Materiel & $\begin{array}{l}\text { Exposure } \\
\mathrm{MD} / \mathrm{T}\end{array}$ & $\begin{array}{l}\text { Type of } \\
\text { Failure }\end{array}$ \\
\hline $\begin{array}{l}2 / 3 / 62 \\
2 / 18 / 62 \\
2 / 26 / 62\end{array}$ & $\begin{array}{l}2289-C \\
3775-D \\
2453-B\end{array}$ & $\begin{array}{l}K T-019-A(I) \\
C Z-806-E \\
I Y-082-A\end{array}$ & $\begin{array}{l}\text { IrE } \mathrm{N}(1) \\
\text { IRE NB } \\
\text { IRE } \mathrm{B}(0.94 \%)\end{array}$ & $\begin{array}{r}1003 \\
1158 \\
270\end{array}$ & Split \\
\hline
\end{tabular}

\section{Iegend}

I8E N - This is the eymbol for interneliy and externally cooled production reactor fuel elementis of natural urantum.

I\&B NB - This is the symbol for internelly and externelly cooled proauction reactor fuel elements of natural urantium which bave projections (bumpers) welded to the fuel elemert jacket.

I\&B B - Ihis is the symol for interneliy and externally cooled production reactor fuel elements with urantum cores enricied in U-235. The weight per cent U-235 in the core materiai is siatec.

Siae-other - Failure probetiy calised by eorrosior or water penetration of tine external can wall or other luknown mechaniax.

Split-Trans. - Failure caused hy uraniur aleavage across or at an angle to the axis of the core.

(1) This rupture occured in a normal size natural self-supported fuel element charged in a ribless zirconium process tube. This charge was being irradiated under the auspices of Production Test IP-272-A.

Extracted from HAPO 1962C, page B-16. 


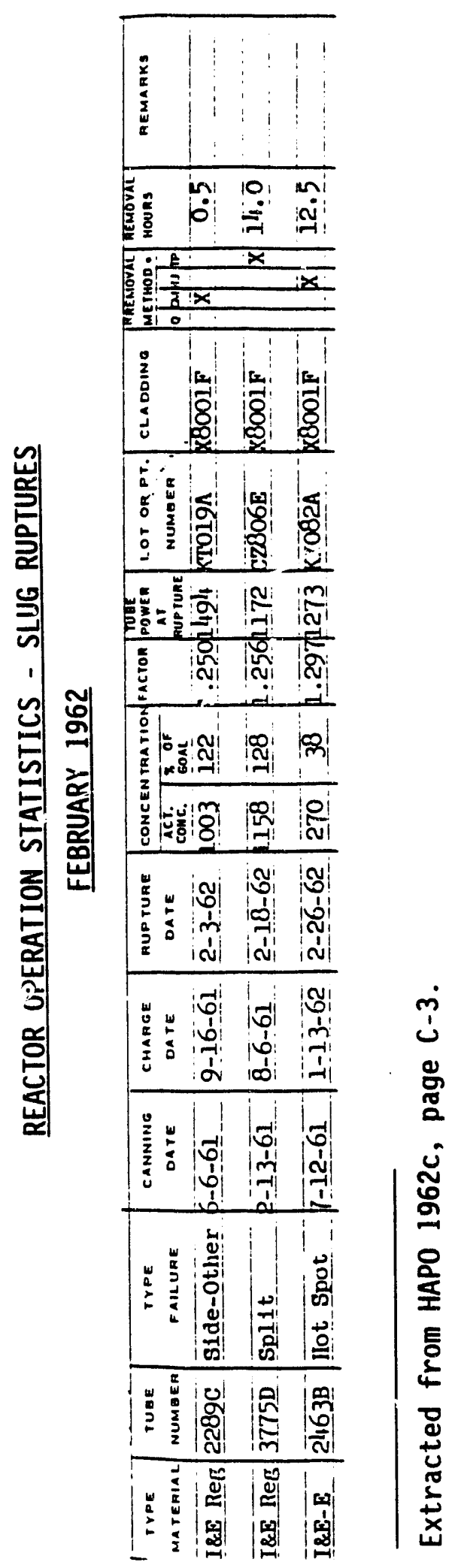




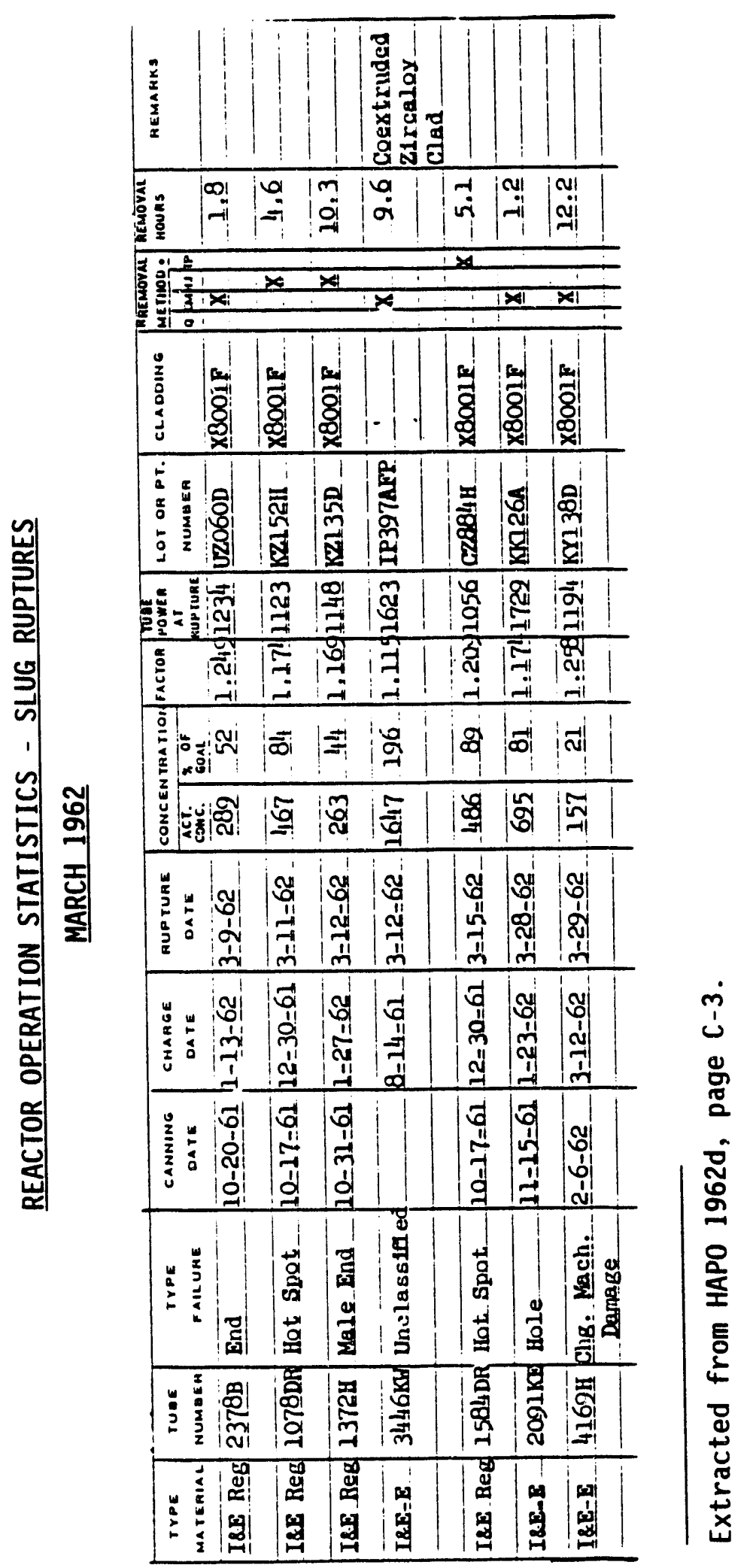




\section{RUPTURE EXPERIENCE - MARCH-APRIL 1962}

\begin{tabular}{|c|c|c|c|c|c|c|}
\hline $\begin{array}{l}\text { Pasline } \\
\text { Dete }\end{array}$ & $\begin{array}{l}\text { Tube } \\
\text { Iumioe: }\end{array}$ & $\begin{array}{c}\text { Dot } \\
\text { Irumber } \\
\end{array}$ & $\begin{array}{l}\text { Type of } \\
\text { Materiel }\end{array}$ & $\begin{array}{l}\text { Tube } \\
\text { Pores at } \\
\text { Faslune } \\
\text { (ks) }\end{array}$ & $\begin{array}{l}\text { ropomere } \\
\text { Mp/T }\end{array}$ & $\begin{array}{l}\text { Iype of } \\
\text { Fetiure }\end{array}$ \\
\hline $\begin{array}{l}3 / 28 / 62(1) \\
3 / 29 / 62\end{array}$ & $\begin{array}{l}2091-\pi \\
4169-1\end{array}$ & $\begin{array}{l}x-126-1 \\
E-136-D\end{array}$ & $\begin{array}{l}\operatorname{In} E(0.94(x) \\
\operatorname{Iex}=(0.94 x)\end{array}$ & $\begin{array}{l}1729 \\
2194\end{array}$ & $\begin{array}{l}695 \\
157\end{array}$ & $\begin{array}{l}\text { Bole } \\
\text { Moceb. }\end{array}$ \\
\hline $\begin{array}{l}\dot{1} \\
4 / 4 / 62 \\
4 / 5 / 62\end{array}$ & $\frac{2760-x^{(2)}}{2955-c^{(2)}}$ & $\underset{x-1097-A}{x-14}$ & $\begin{array}{l}\operatorname{coseg}^{(2)}(1.6 x) \\
\operatorname{Ix}=(0.94 x)\end{array}$ & 2585 & 796 & $\begin{array}{l}\text { Uaci. } \\
\text { Side }\end{array}$ \\
\hline $\begin{array}{l}4 / 24 / 62 \\
4 / 26 / 62 \\
4 / 26 / 62\end{array}$ & $\begin{array}{l}2281-D \\
1684-D \\
3062-C\end{array}$ & $\begin{array}{l}E-889-D \\
Z=-065-D \\
\square-38 I-\alpha\end{array}$ & 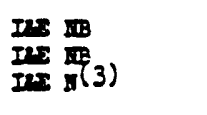 & $\begin{array}{l}1246 \\
1267 \\
1439\end{array}$ & $\begin{array}{r}77 \\
79 \\
672\end{array}$ & $\begin{array}{l}\text { Hot fp } \\
\text { Bad } \\
\text { Bole } \\
\text { 8p21=- }\end{array}$ \\
\hline $\begin{array}{l}4 / 20 / 62 \\
4 / 21 / 62 \\
4 / 26 / 62\end{array}$ & $\begin{array}{l}2064-D R \\
1962-D \\
3079-D R\end{array}$ & 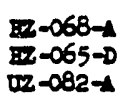 & 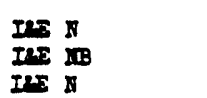 & $\begin{array}{l}2167 \\
2179 \\
2183\end{array}$ & $\frac{121}{2218}$ & $\begin{array}{l}\text { Bole } \\
\text { Boc } \\
\text { Ut-ind }\end{array}$ \\
\hline
\end{tabular}

Lenend

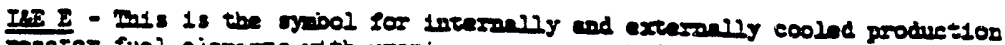

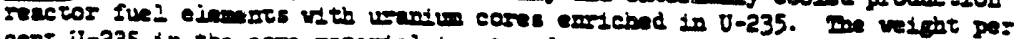
cent ij-235 in the core wererall is stated.

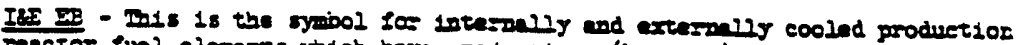

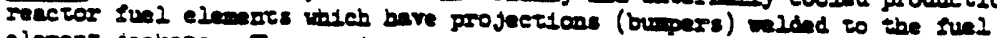
element jackets. The urantum corse are eneriebad in 0-235 and the weight per cent U-235 in the core suretel is stered.

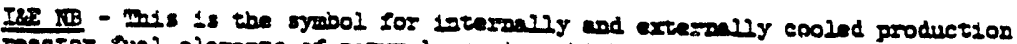

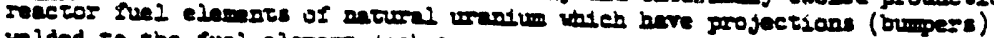
weldad to the suel elewere jecket.

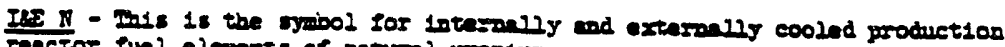

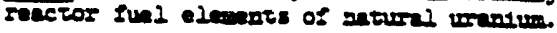

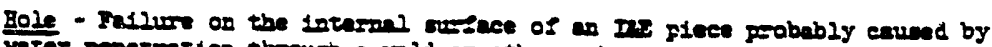

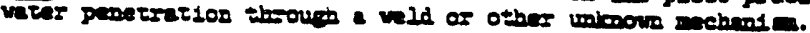

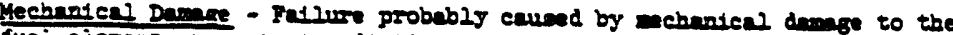

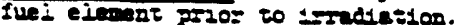

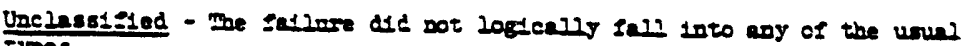
Eypes.

Side Bot-60ot - Falline probably caused by aceelereted corrosion of the

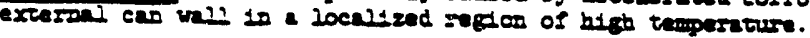

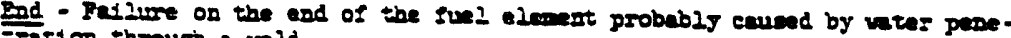
Fration througio a vid.

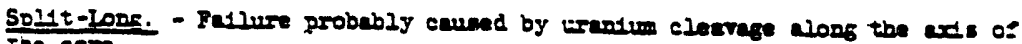
whe core.

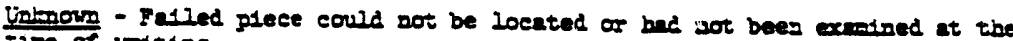
ine of vestsog.

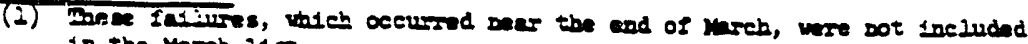
in the vareb $21 \pi$.

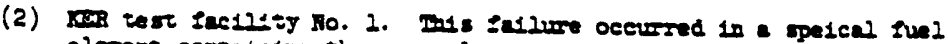
element convedating thermocouples. A complete dencription of the

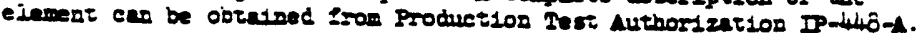

(3) Profuction tost of iresanly and exterandy cooled oversized selp upported tuel elemets in un overjored chnonel.

Extracted from HAPO 1962e, pages C-13 \& 14. 


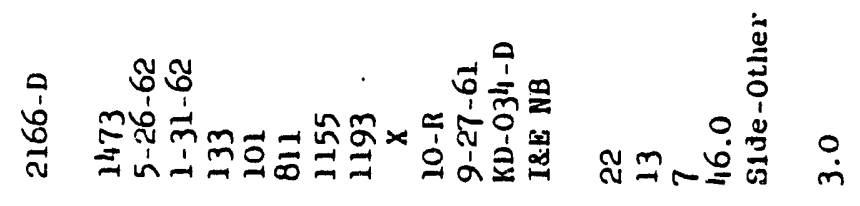

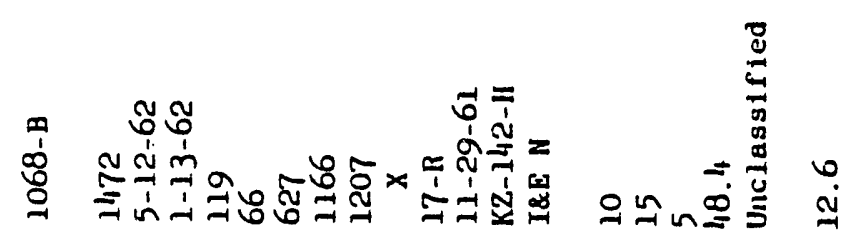

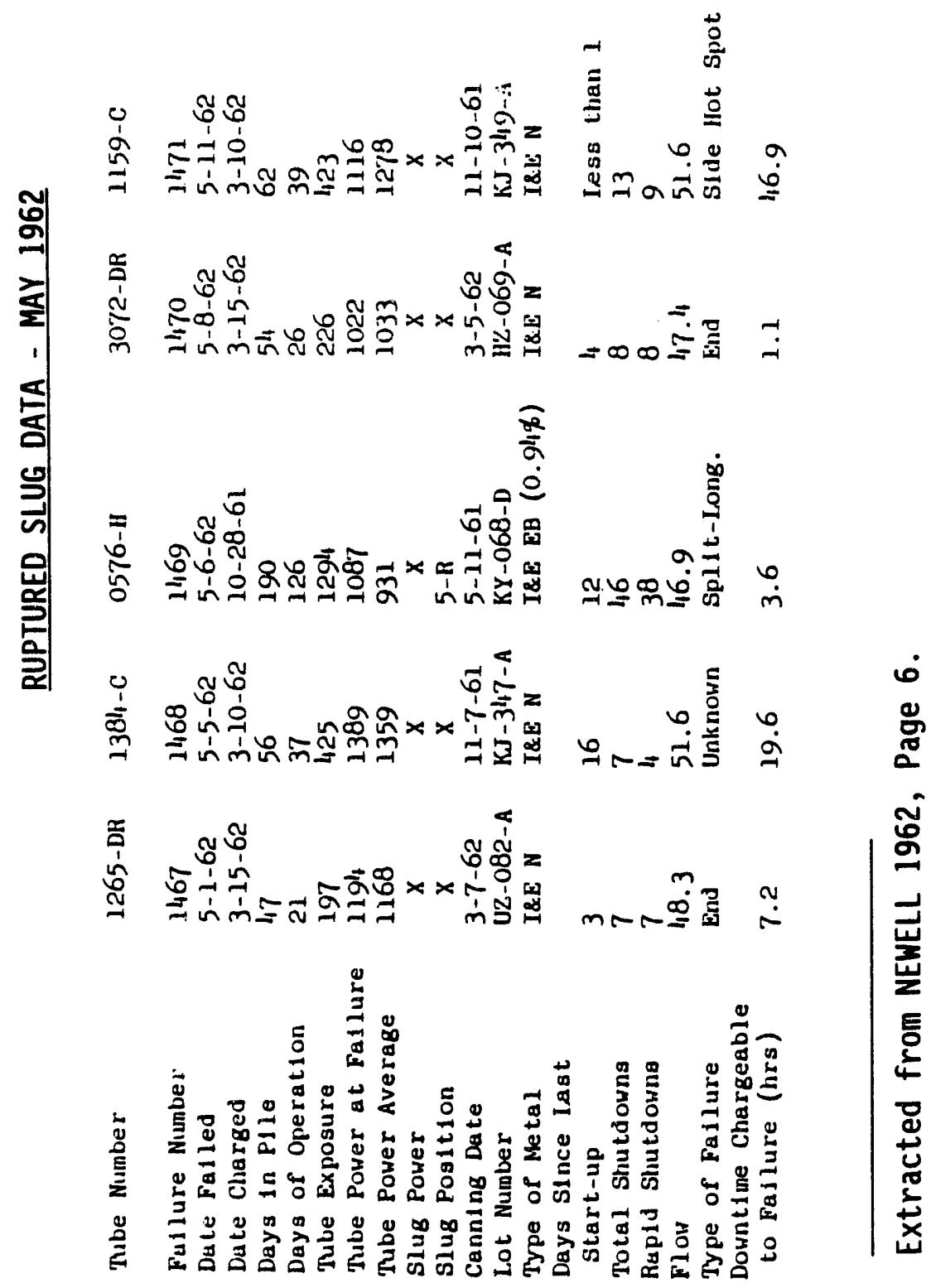




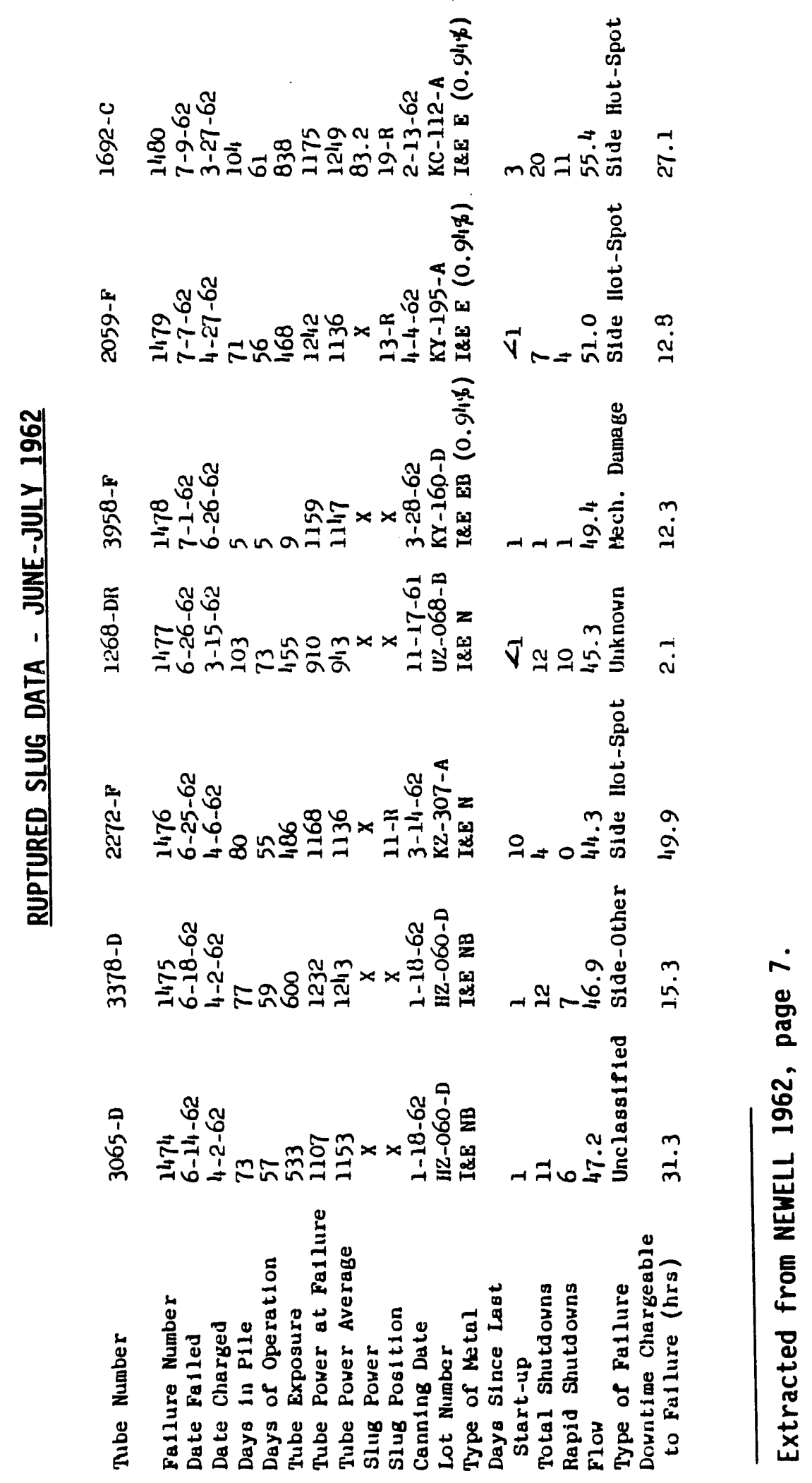




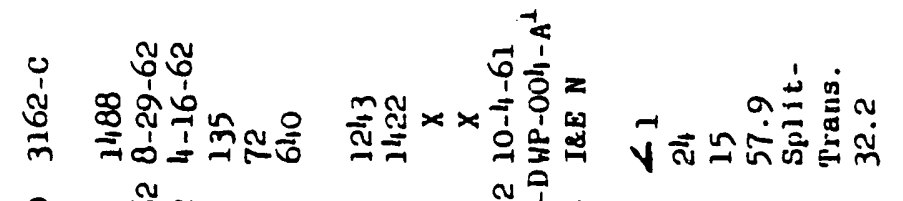

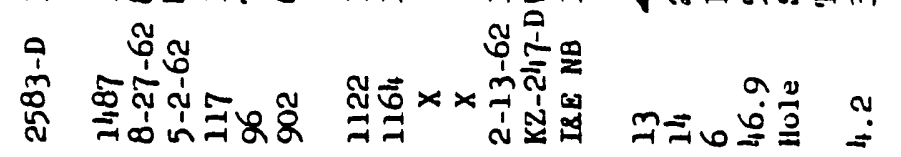

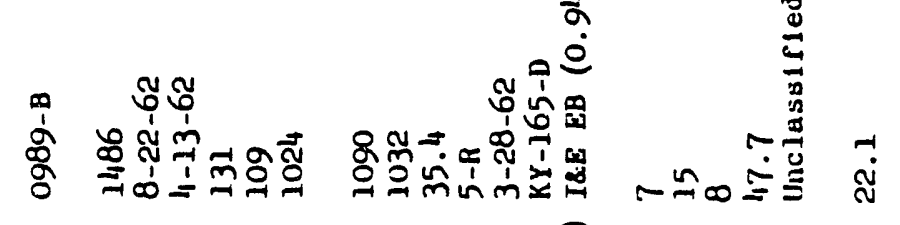

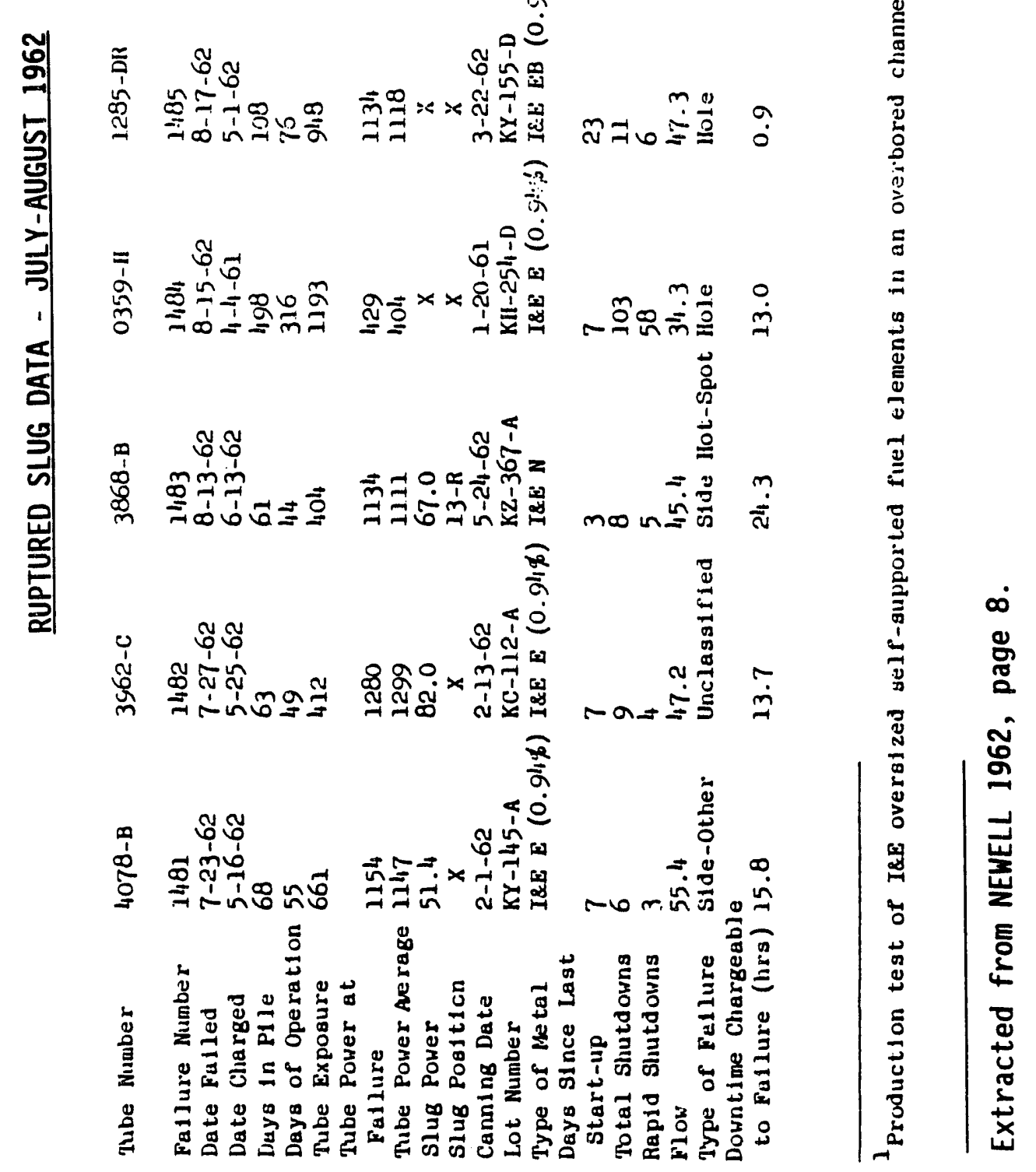




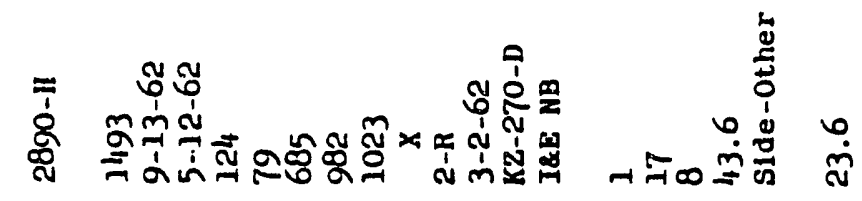

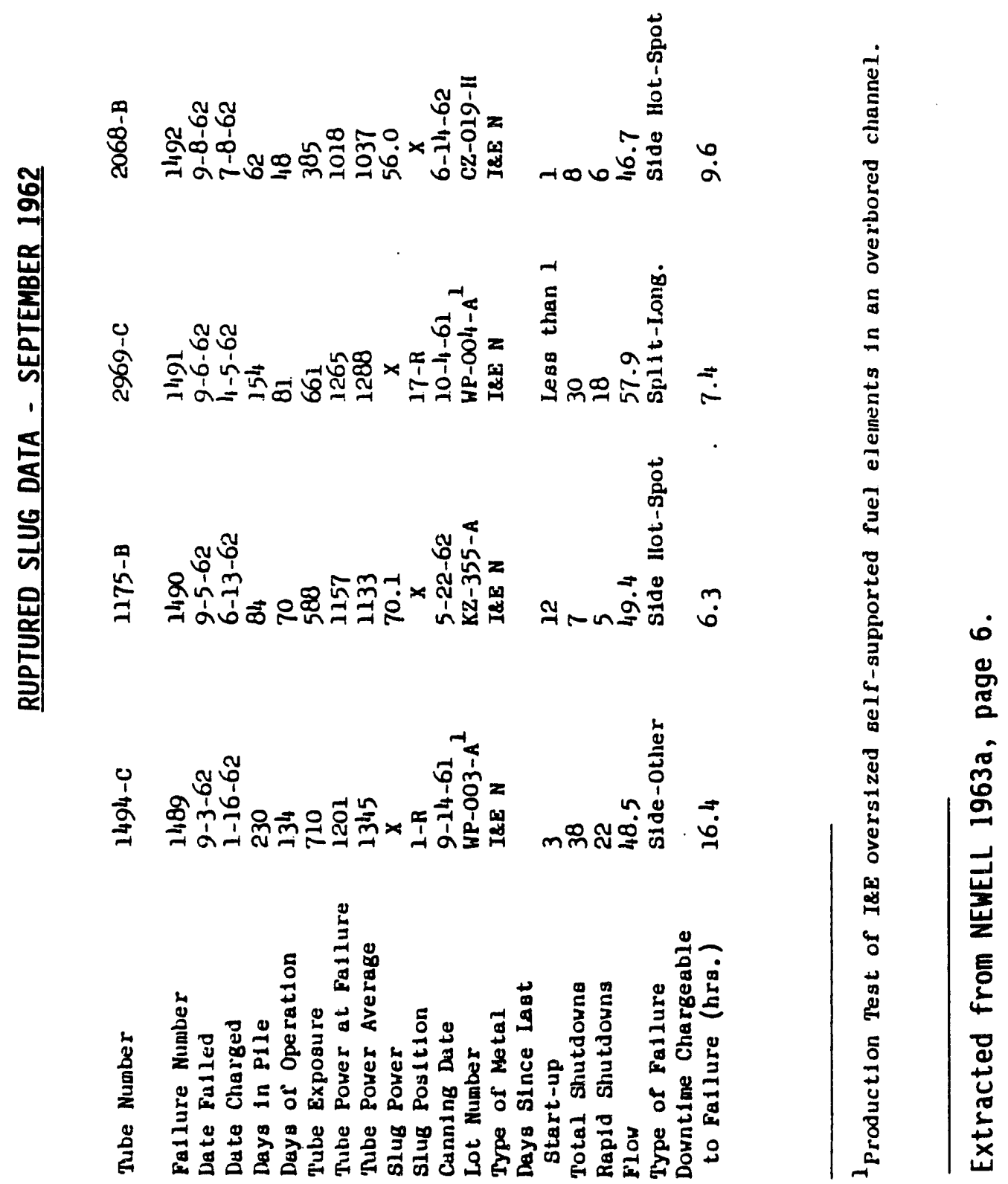


乏)

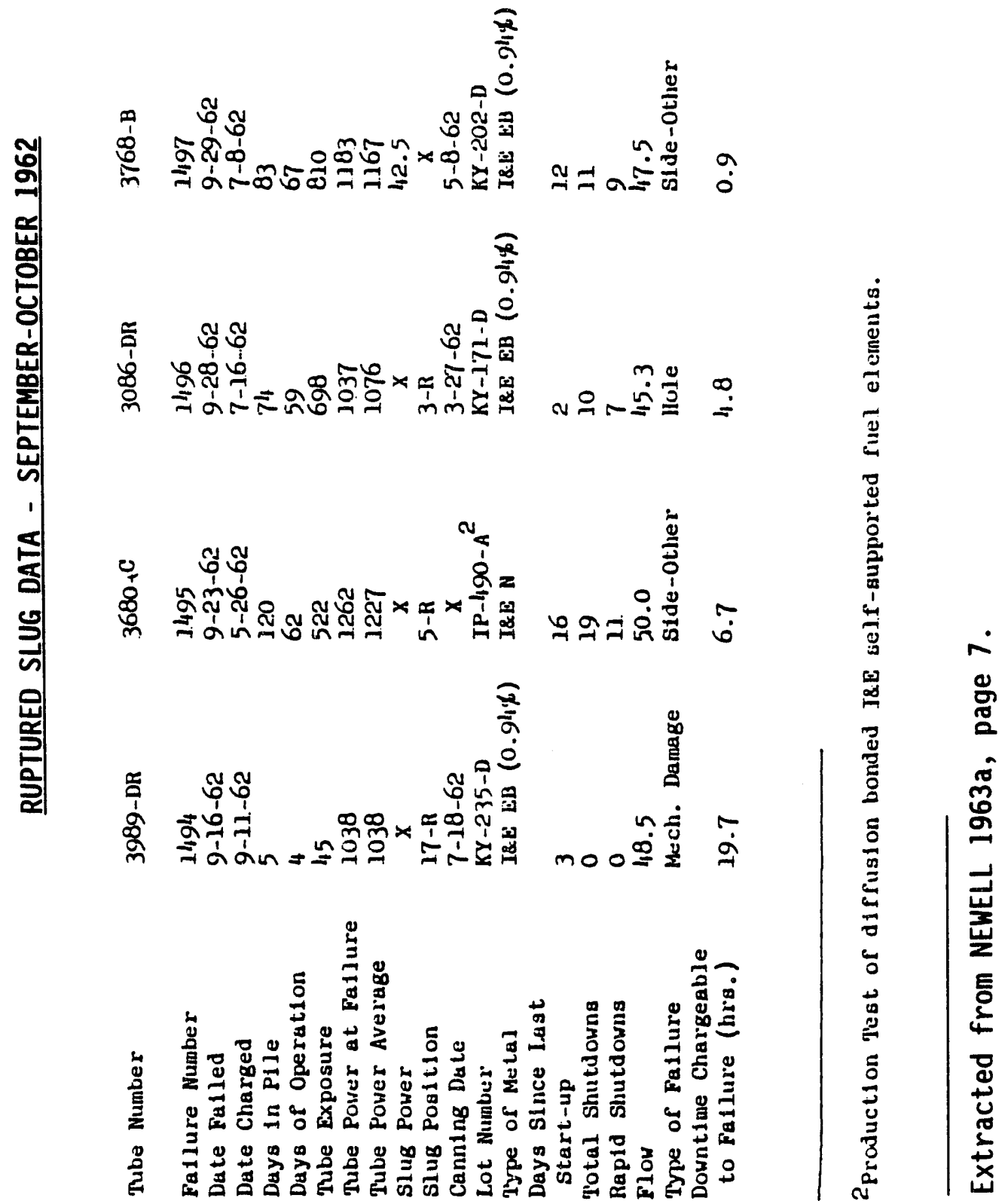




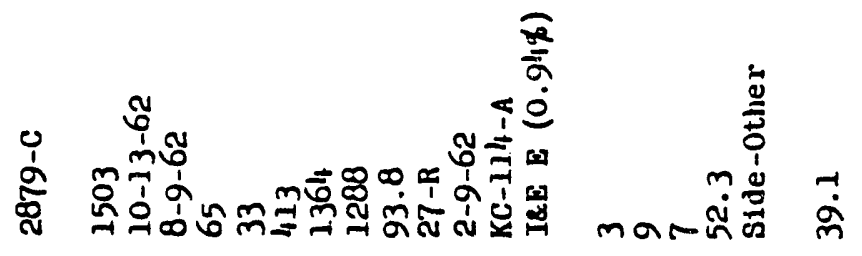

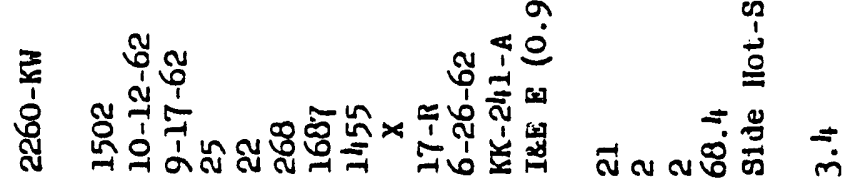

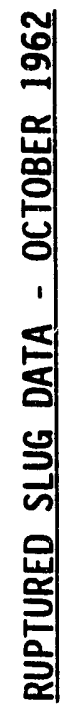
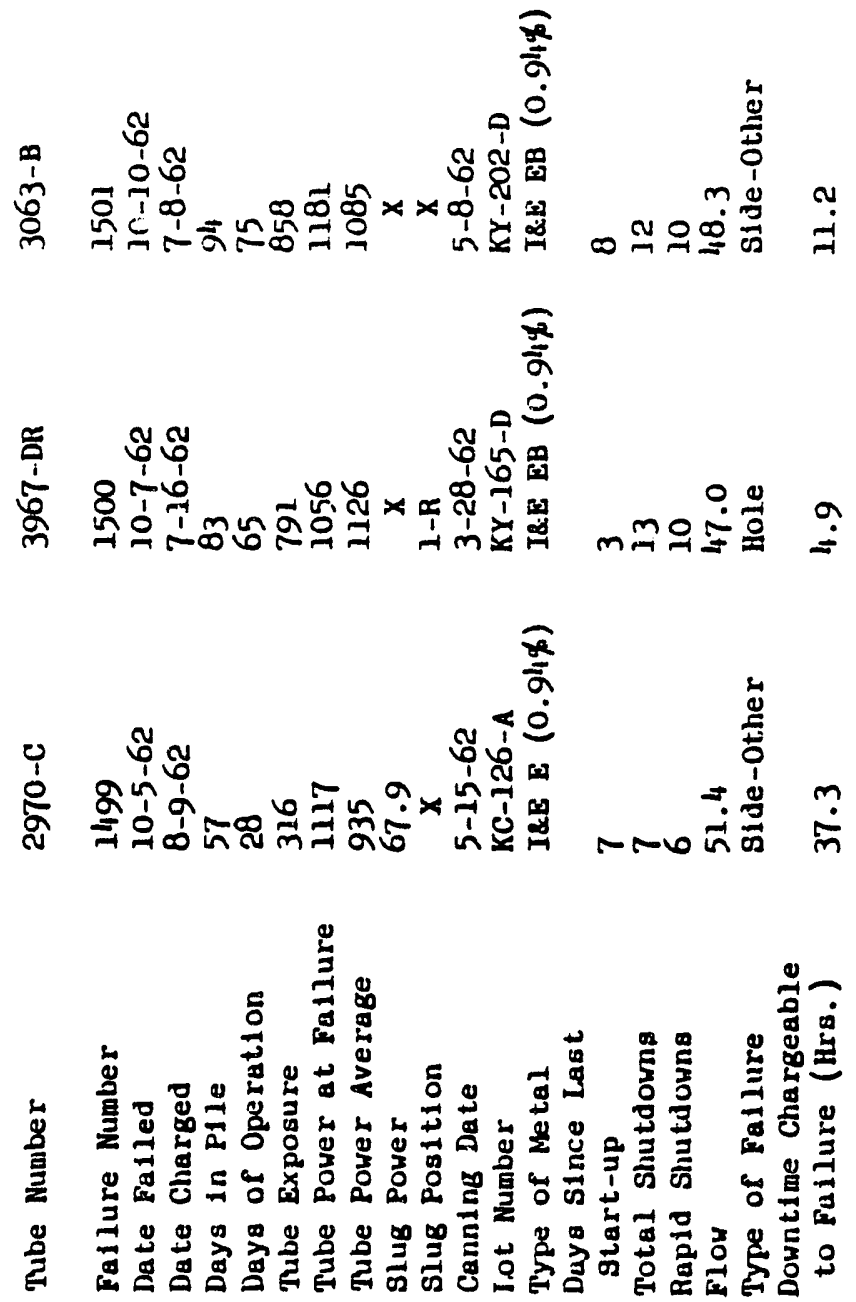

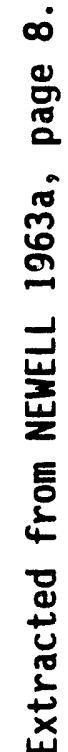




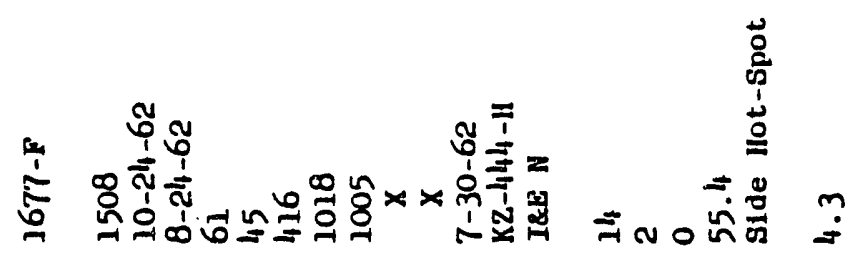

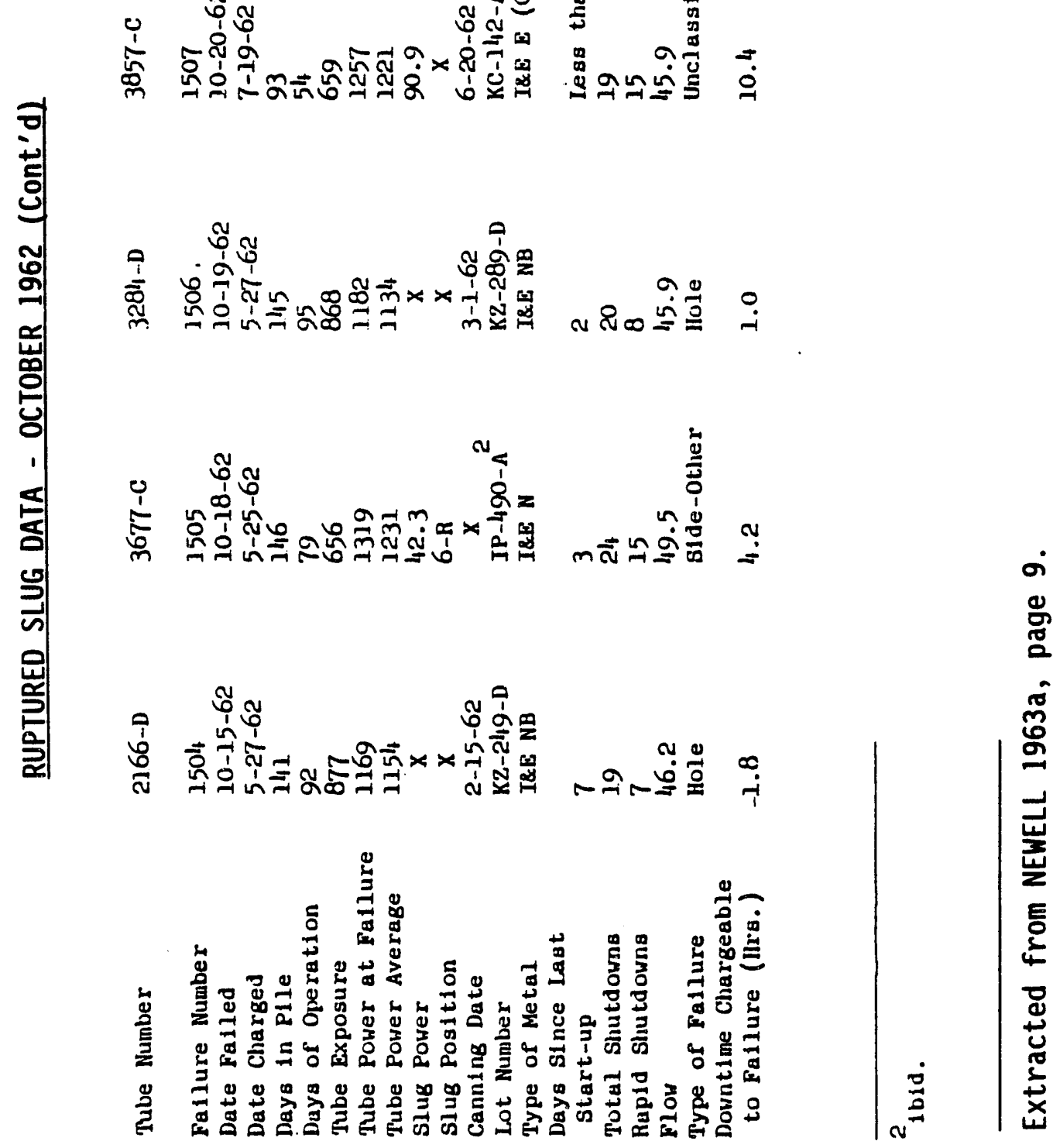




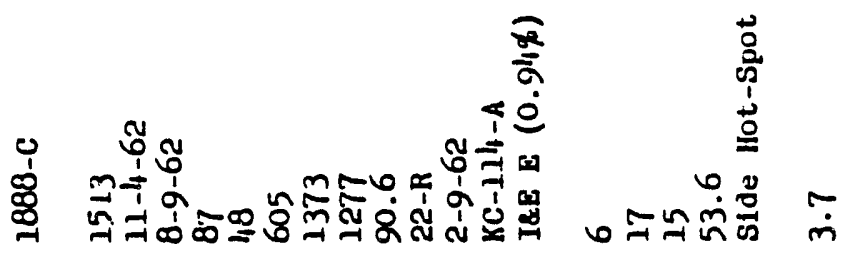

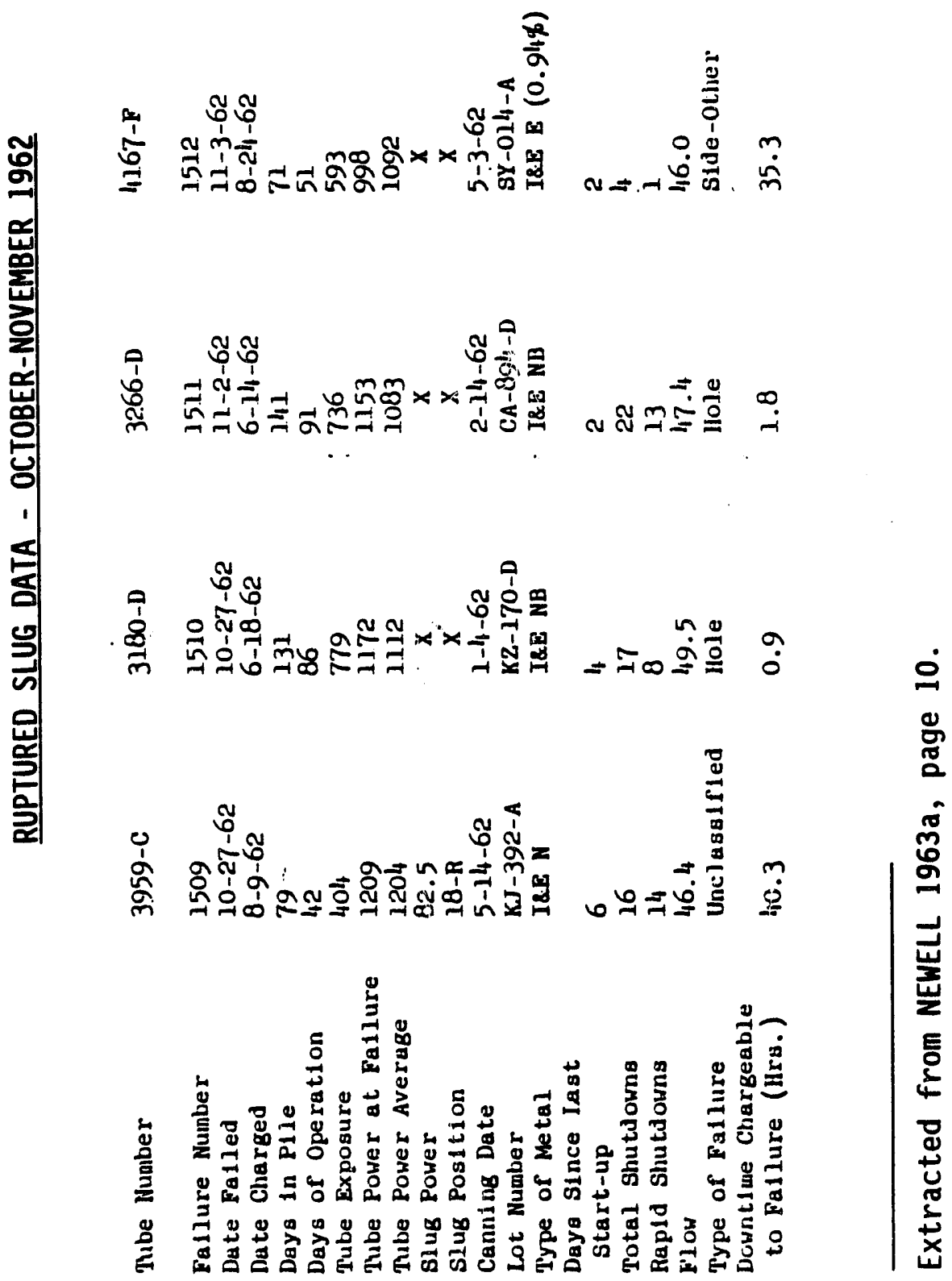




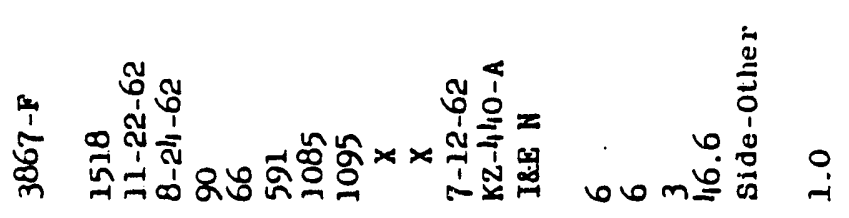

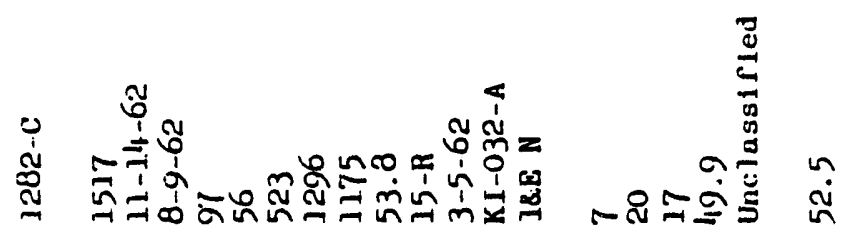

임
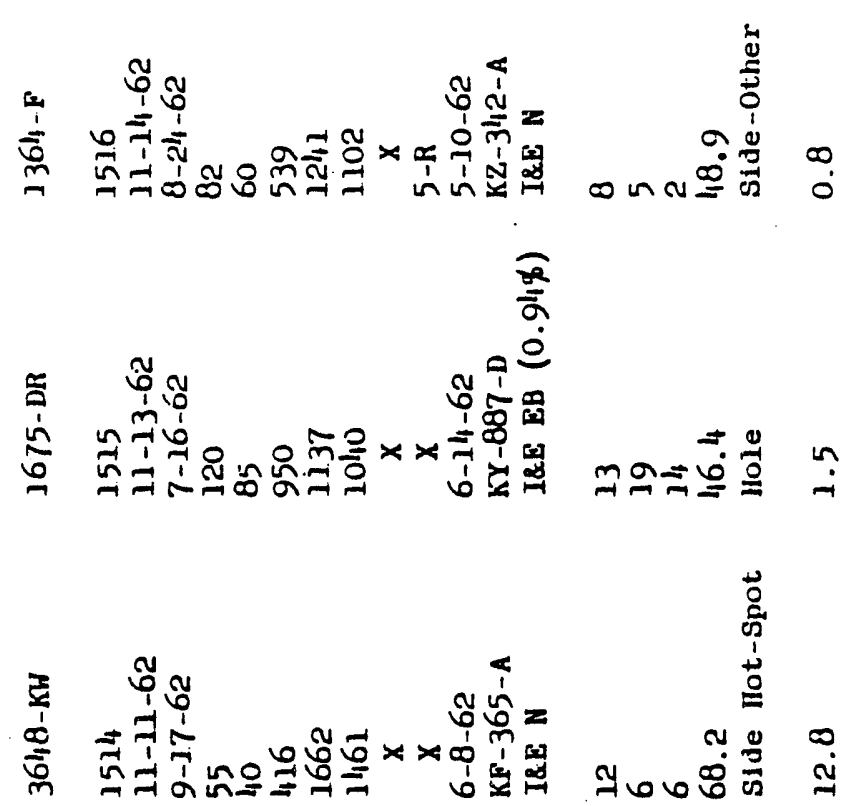

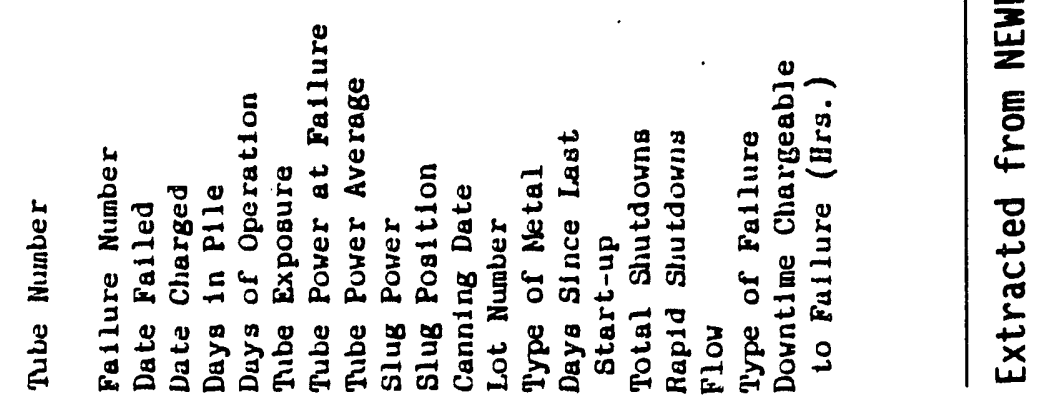


至

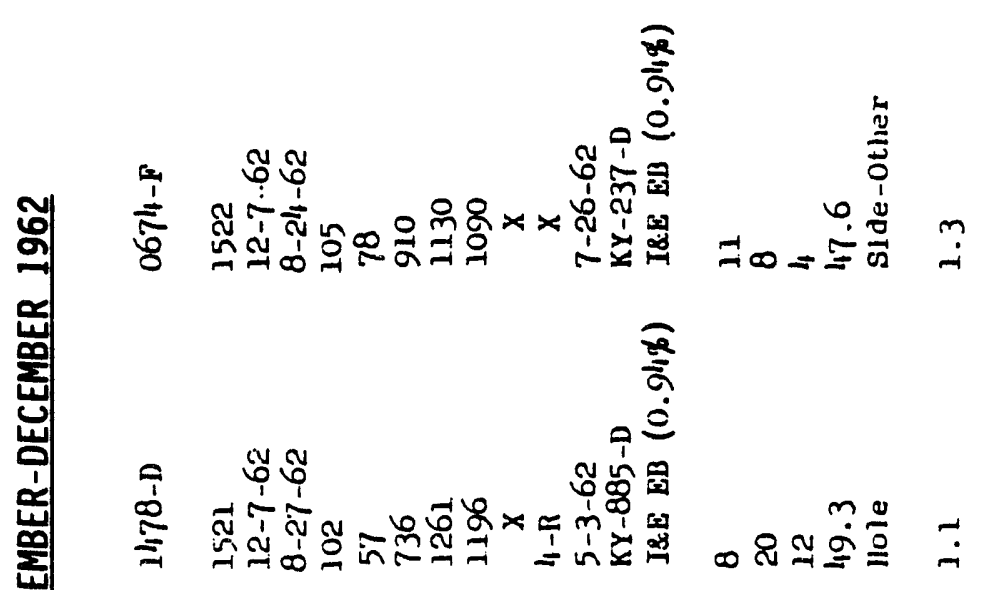

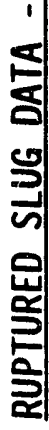

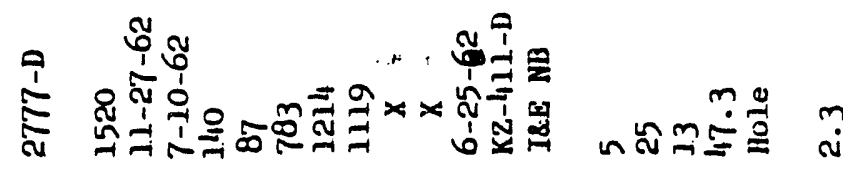

$\underset{1}{ }$

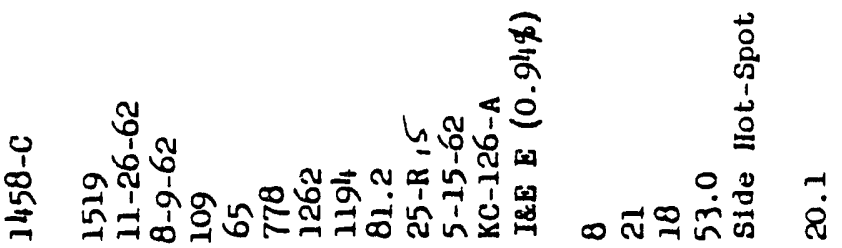

苋

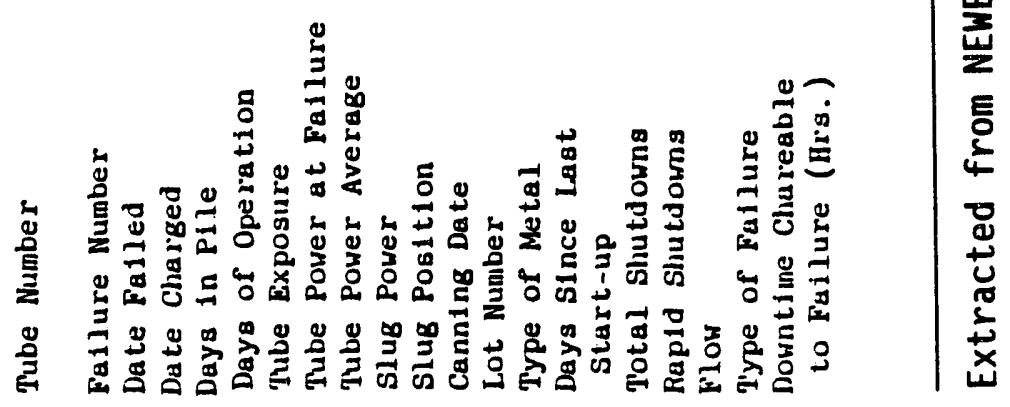




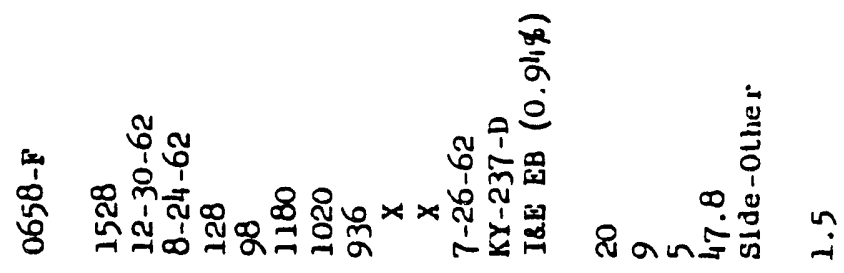

落

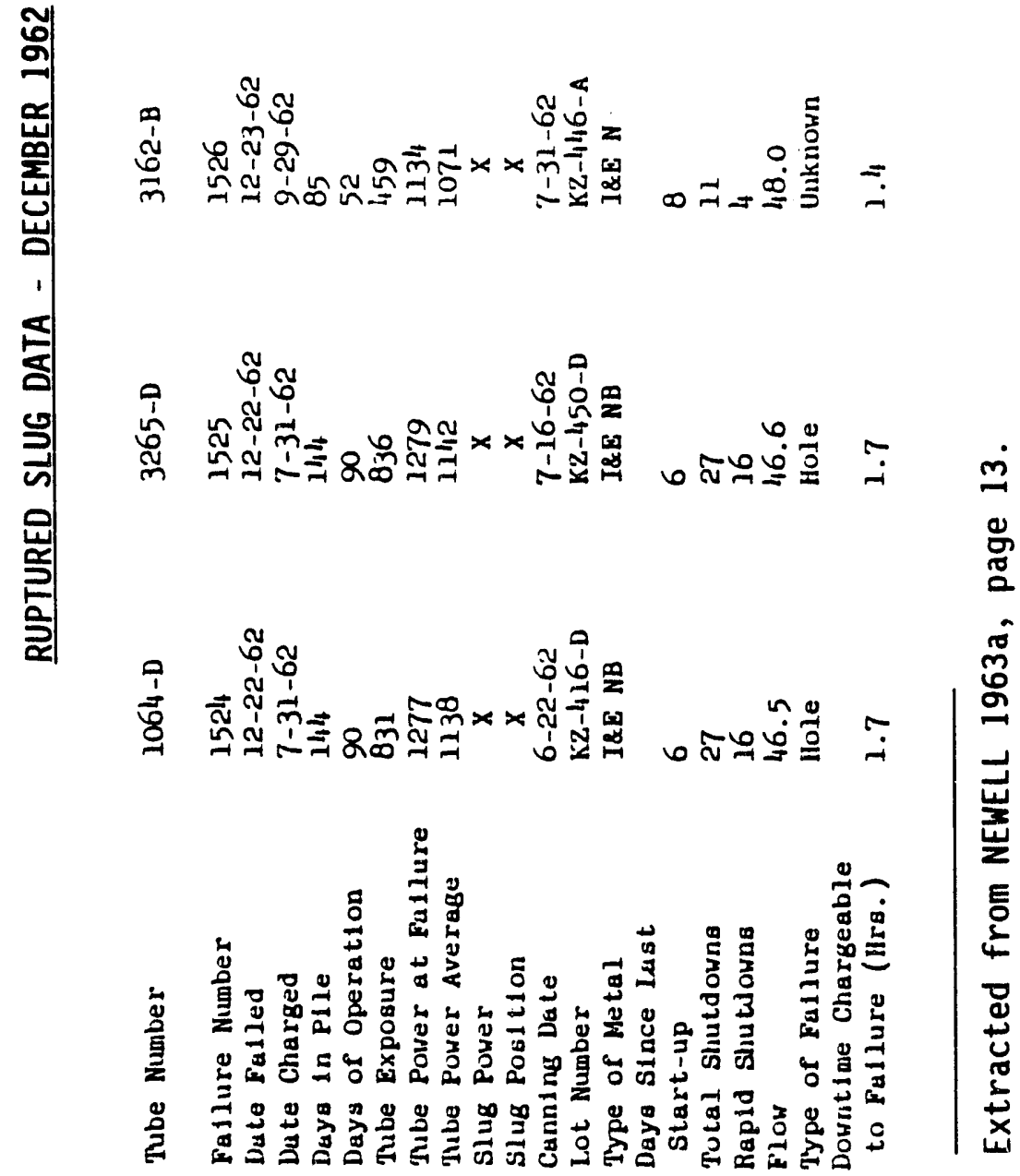




\section{APPENDIX M}

FUEL-ELEMENT FAILURE DATA FOR 1963 


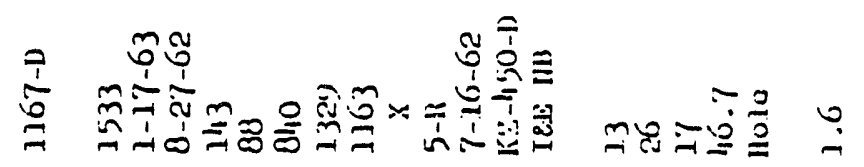

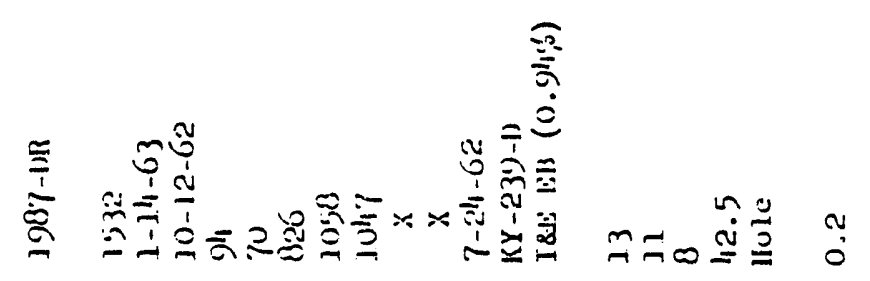

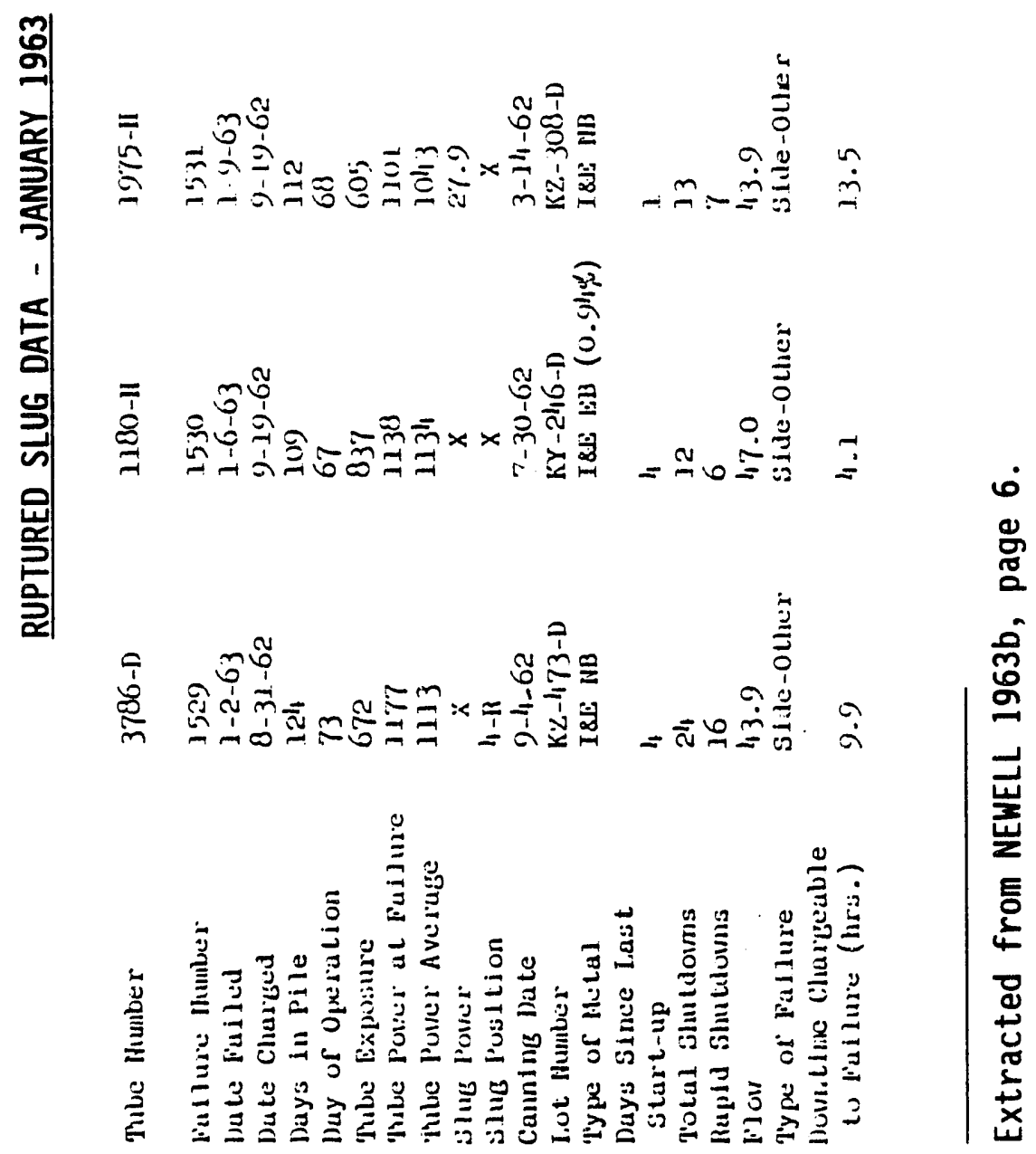

M.1 
咅

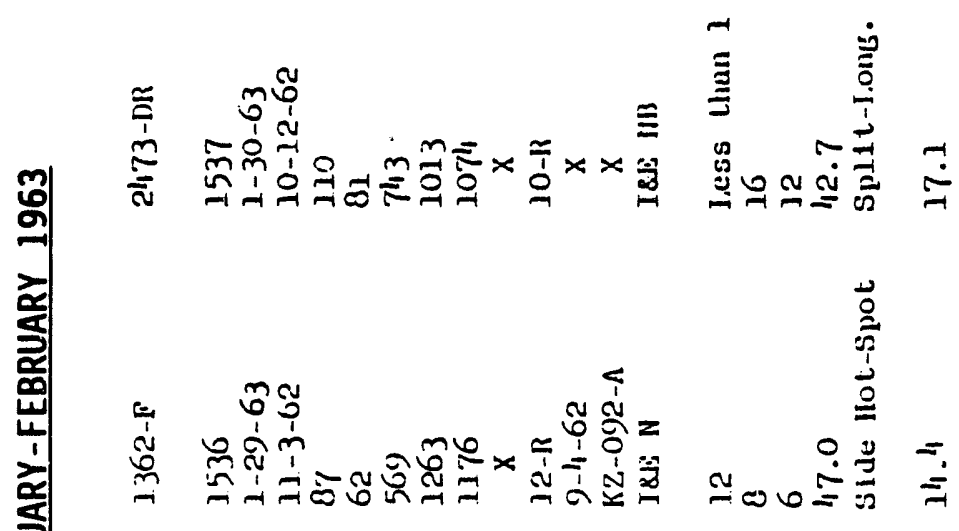

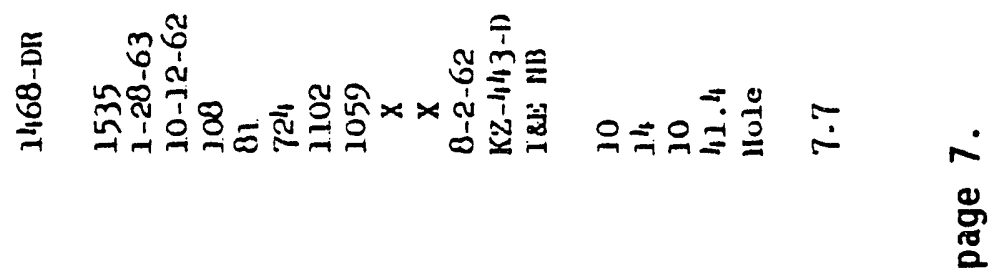

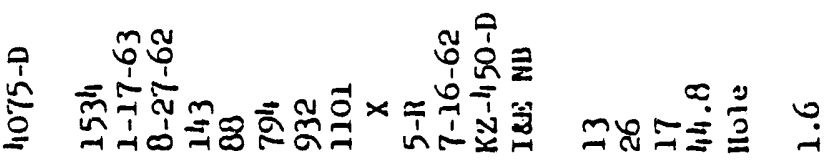

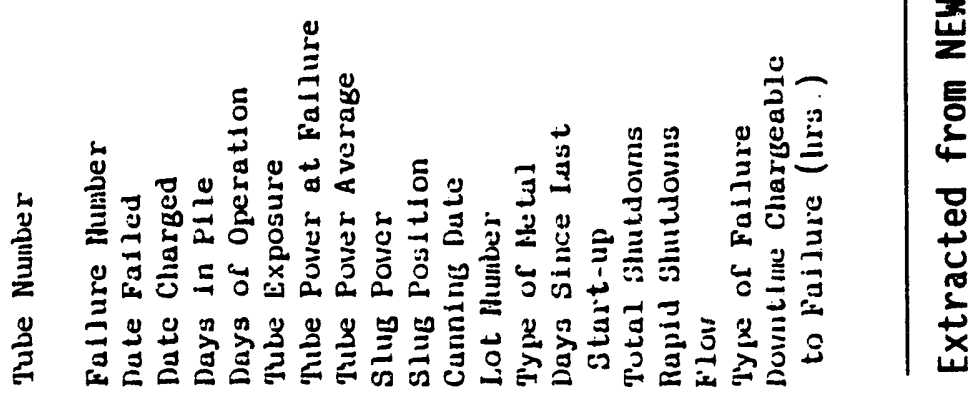

\section{2}




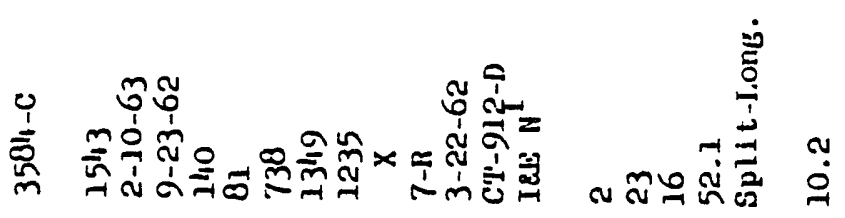

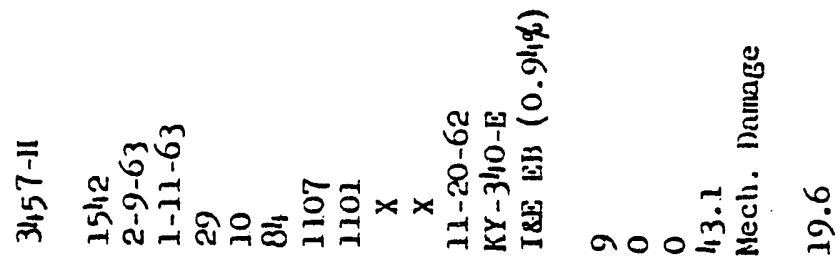

章

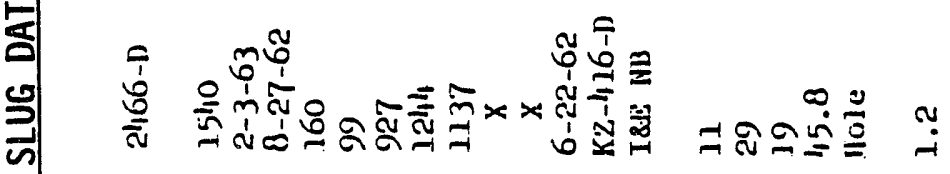

营

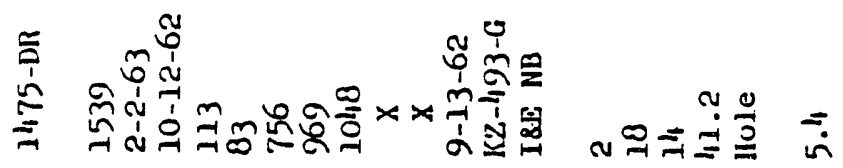

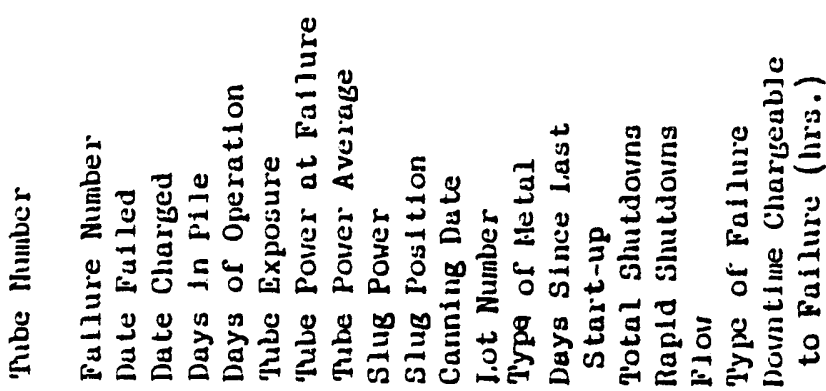

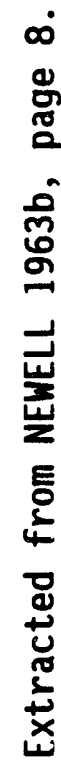

ติ. 3 


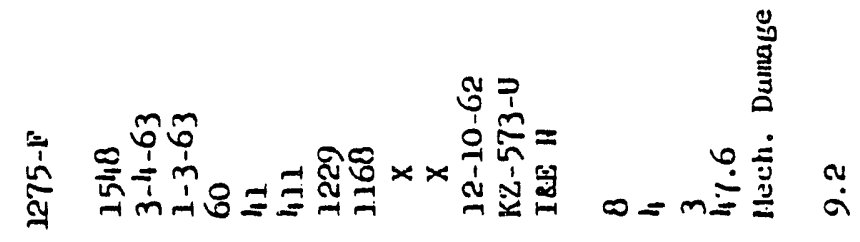

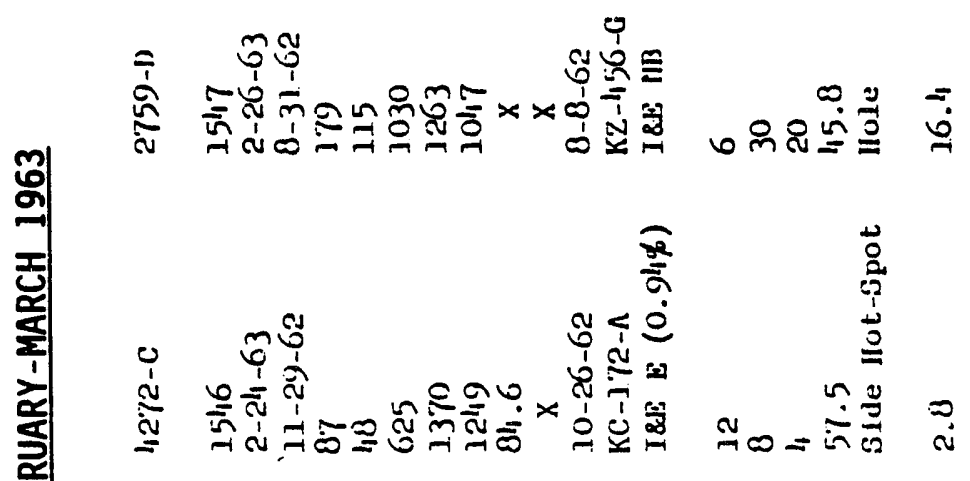

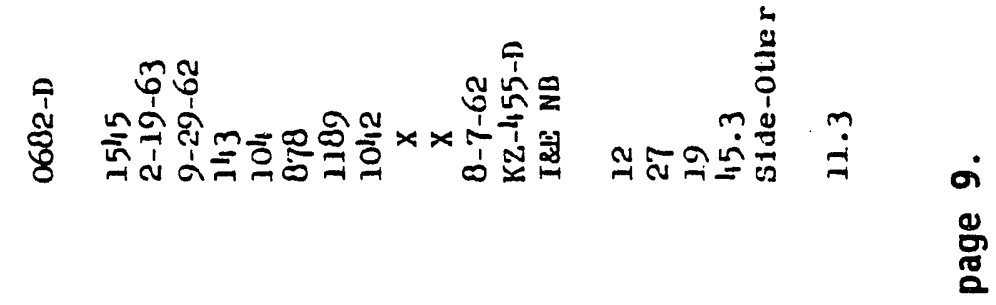

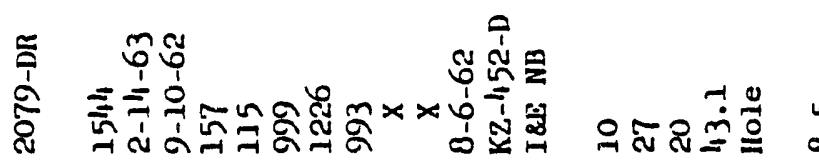

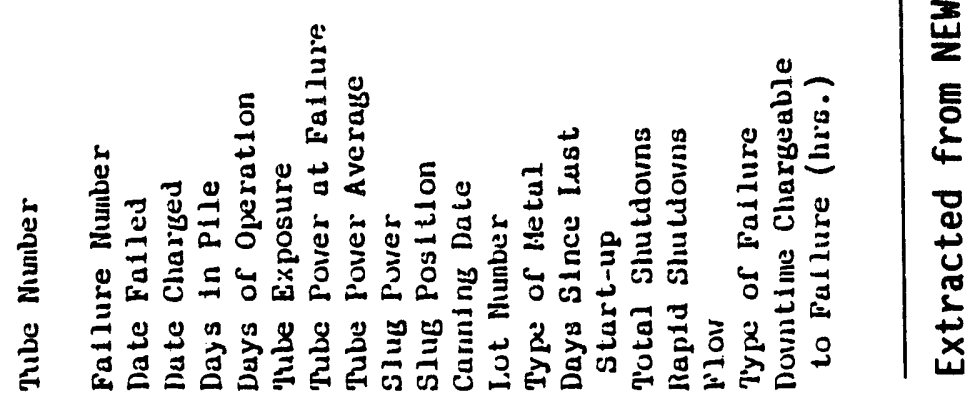




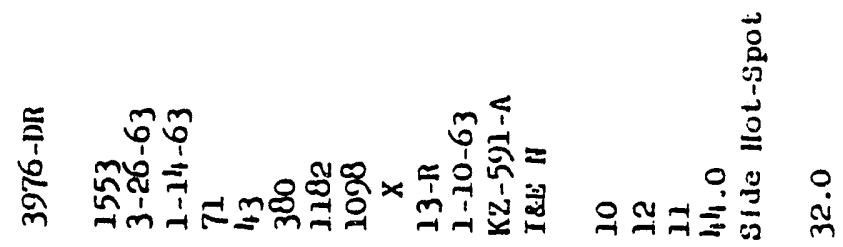

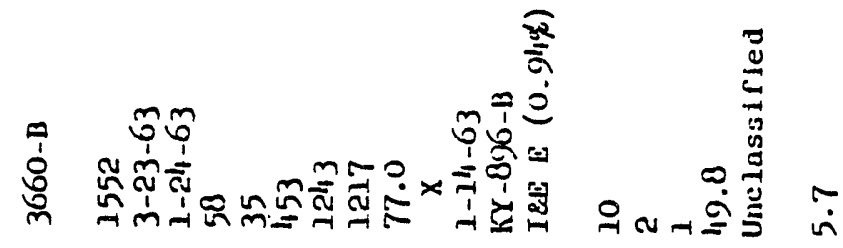

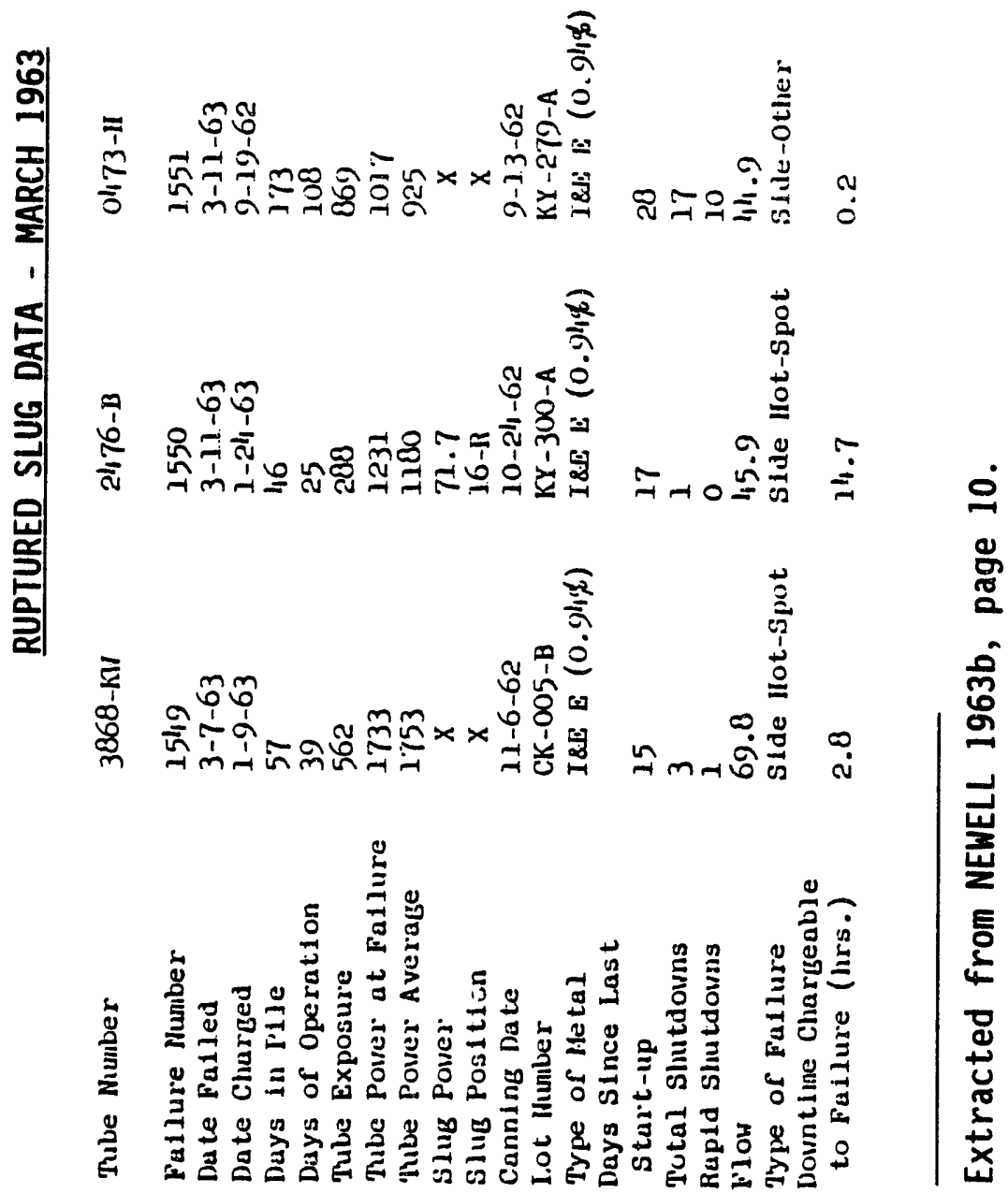




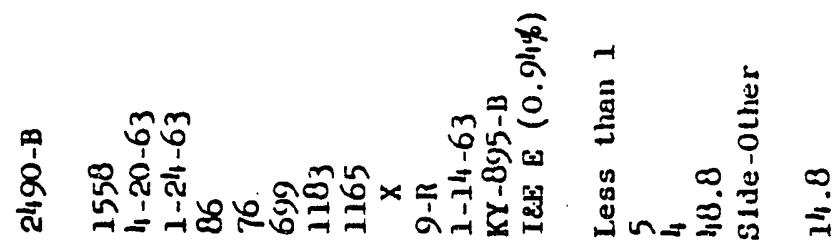

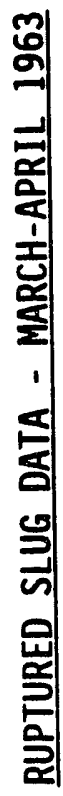

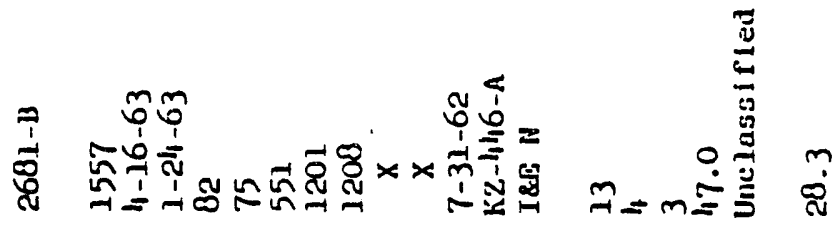

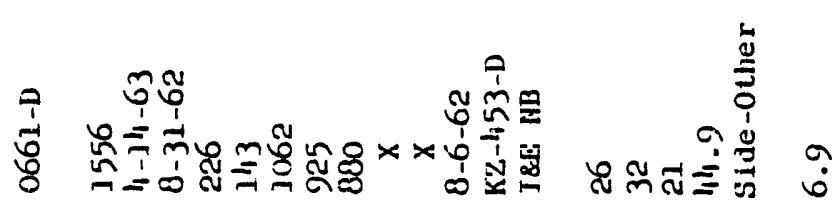

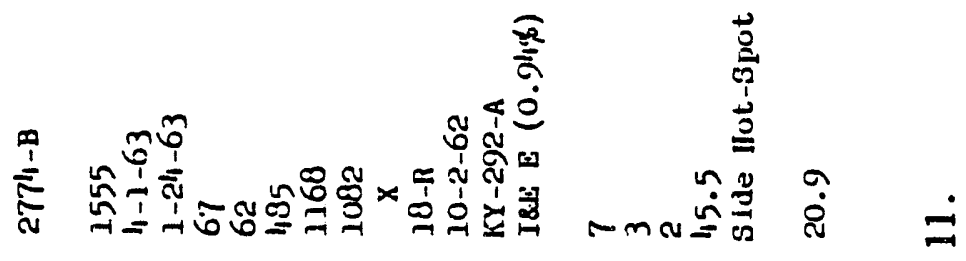

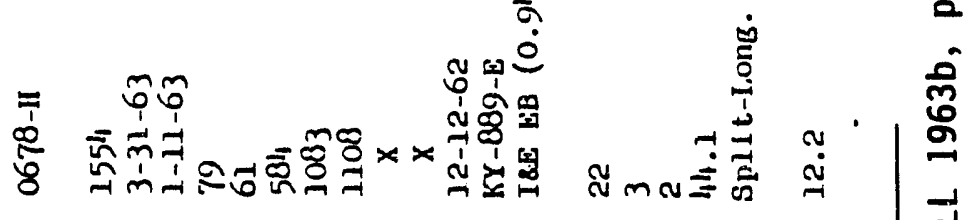

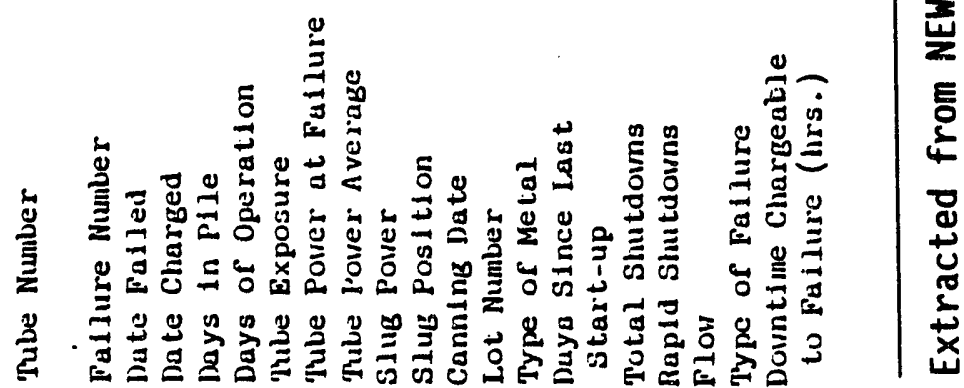

M. 6 


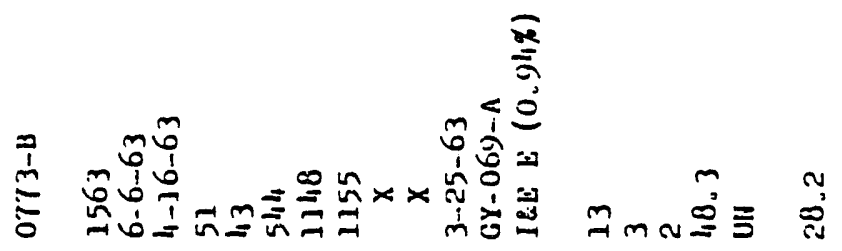

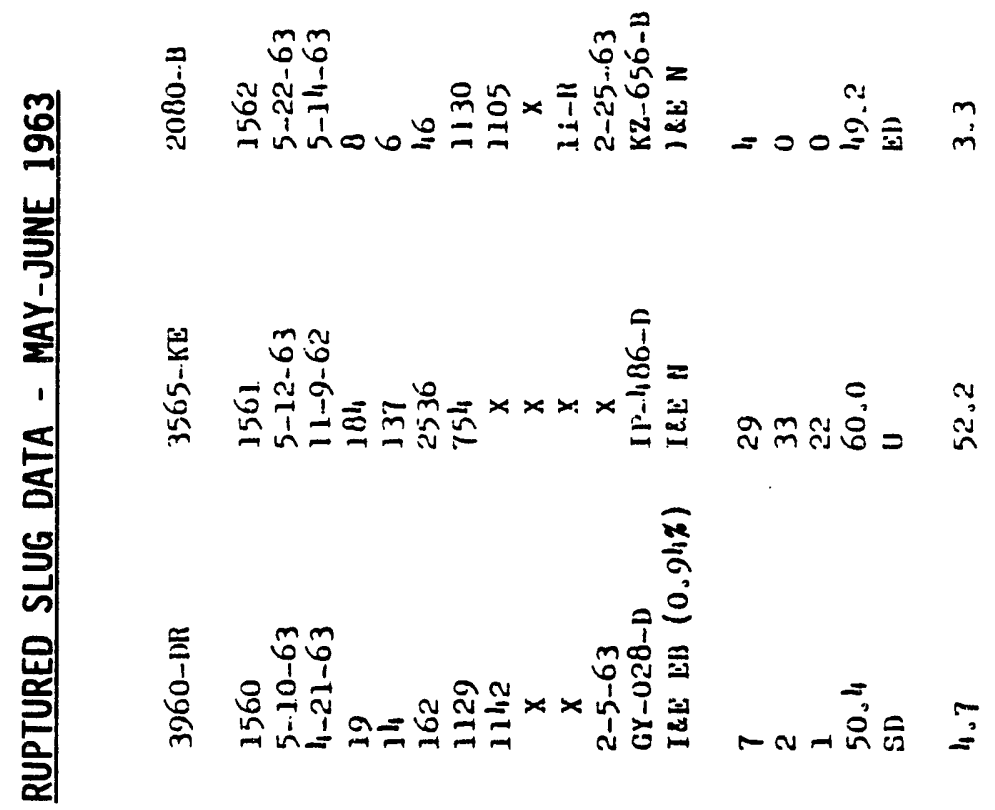

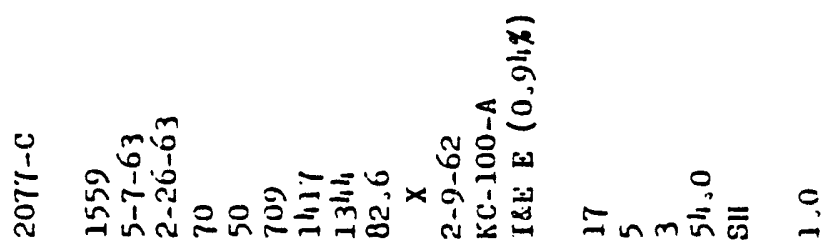

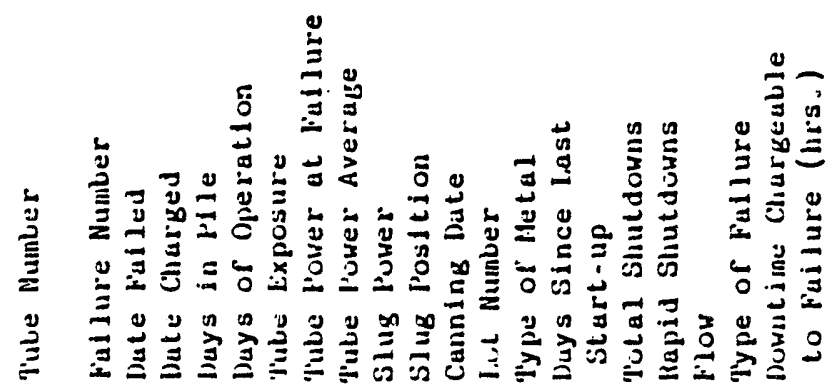




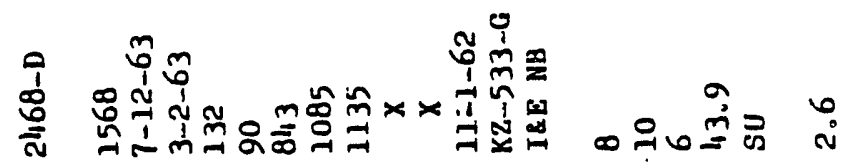

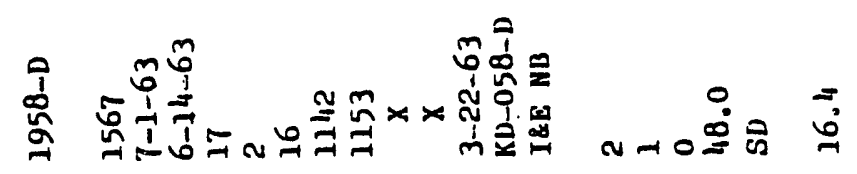

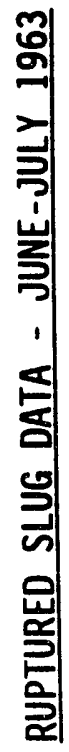

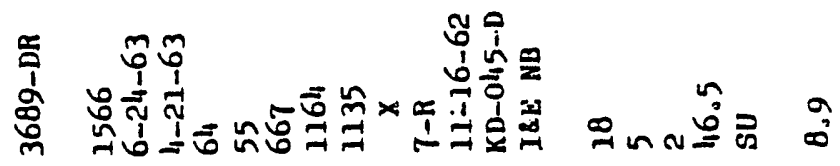

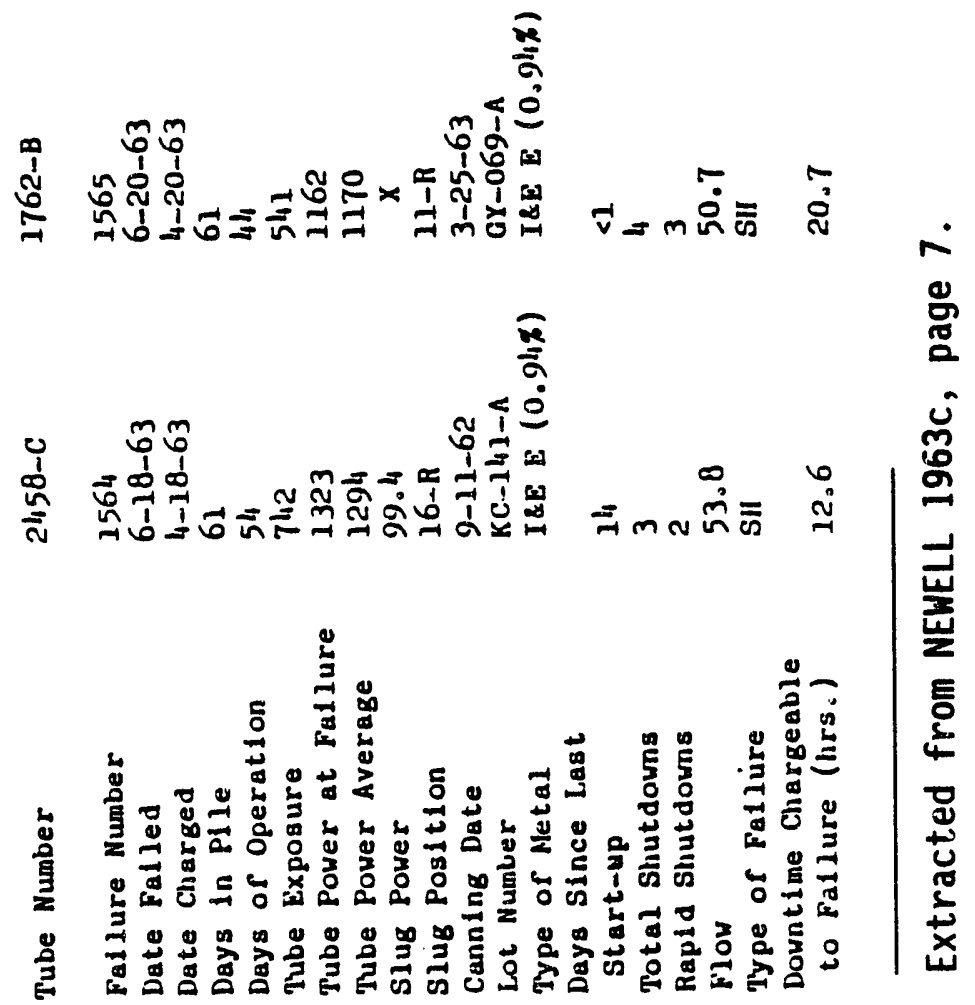




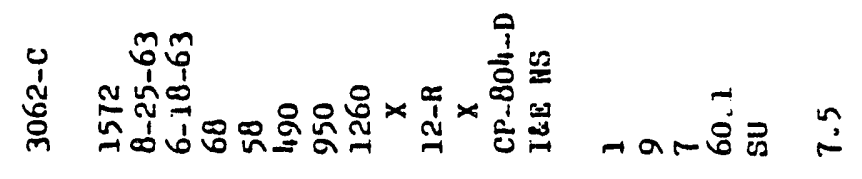

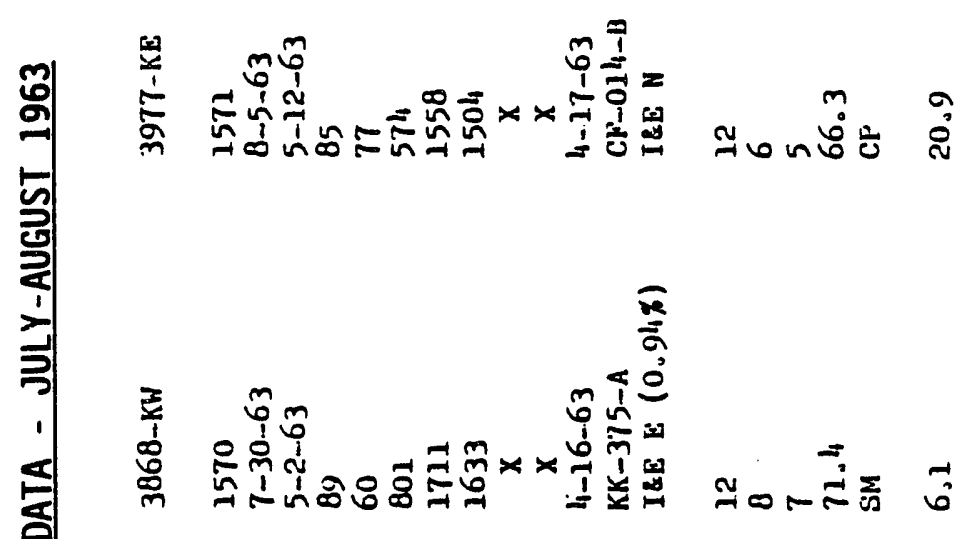

离

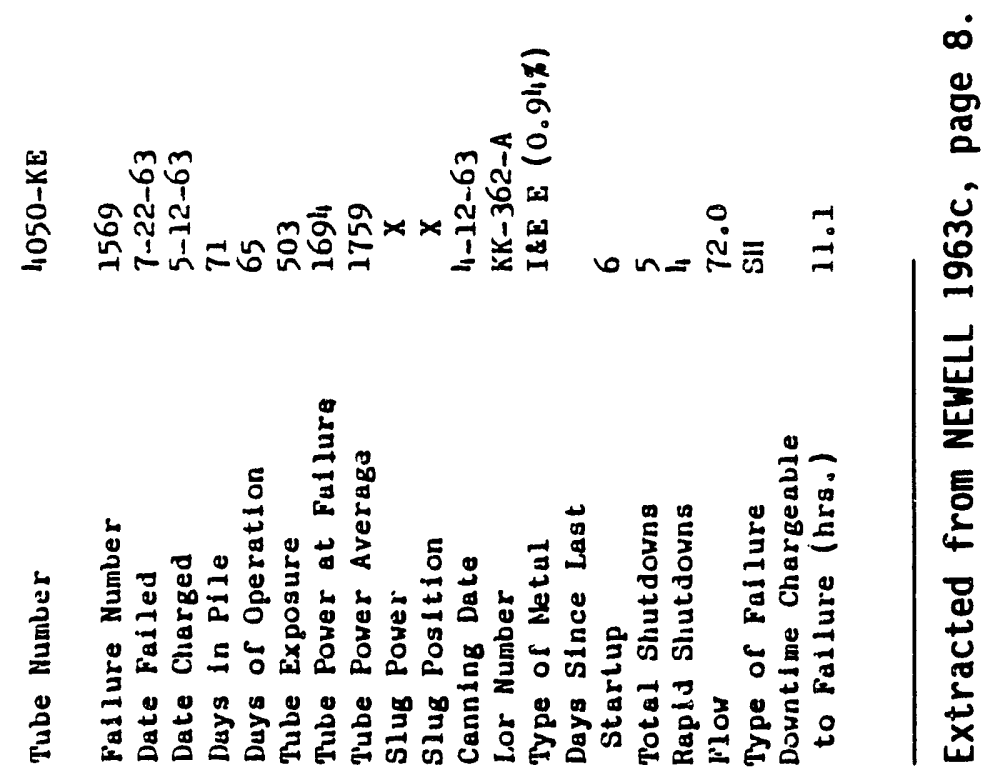

M. 9 


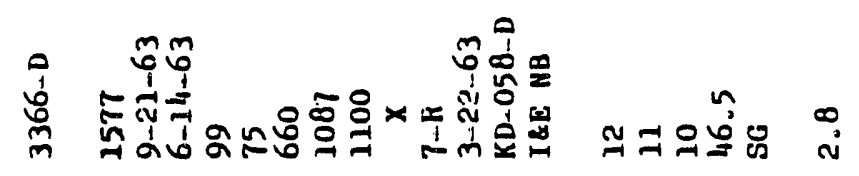

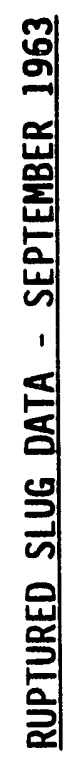

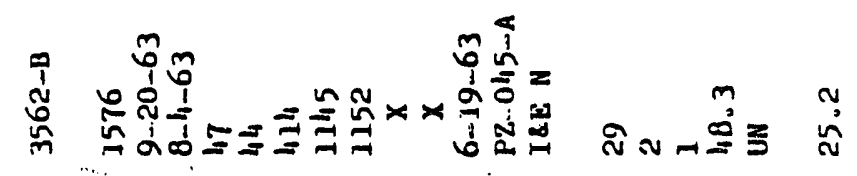

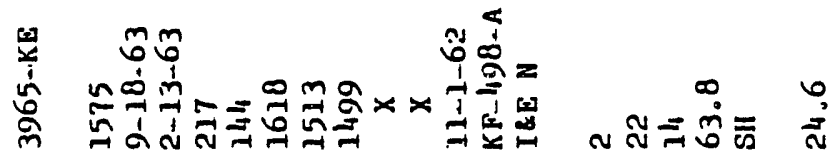

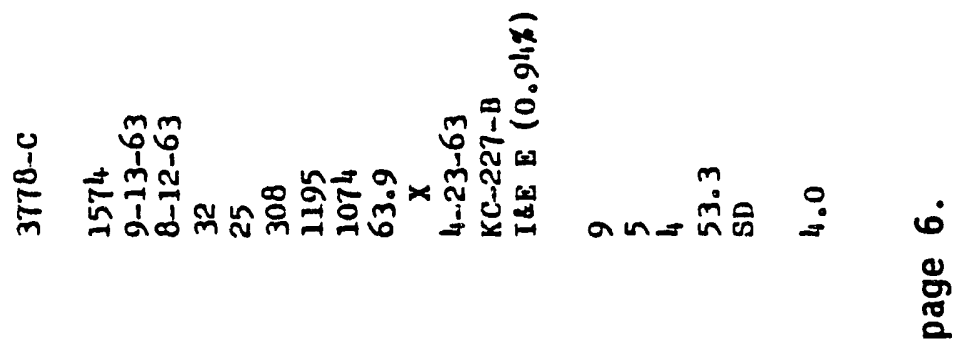

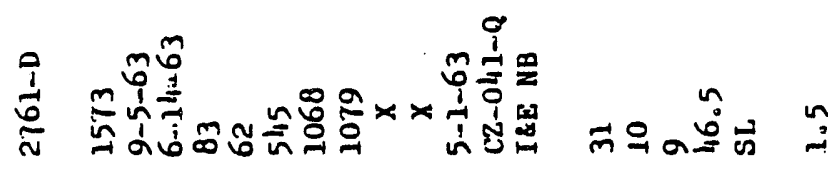

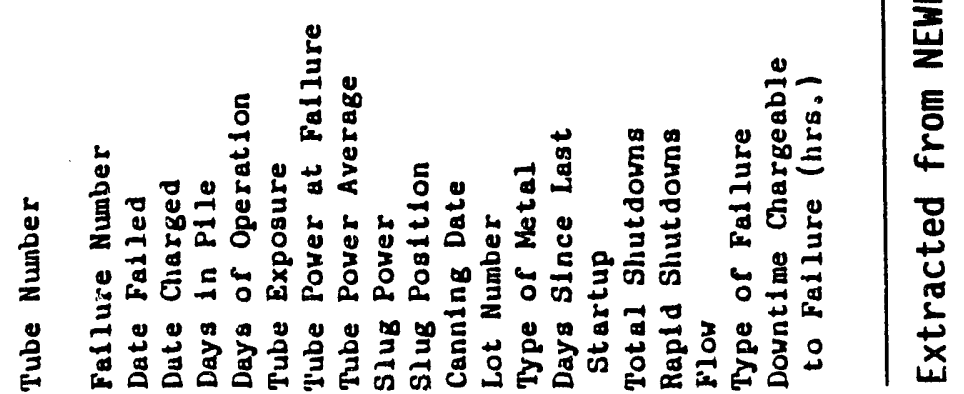

M. 10 


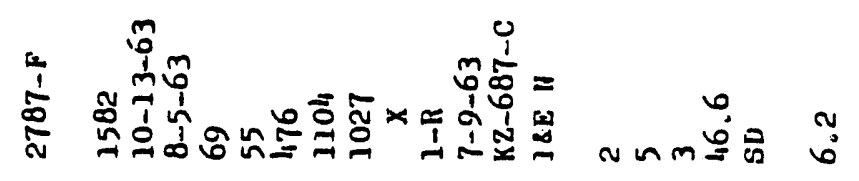

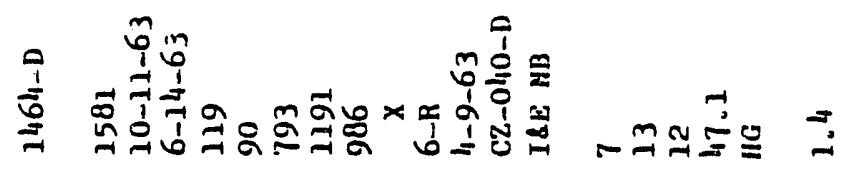

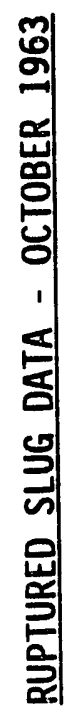

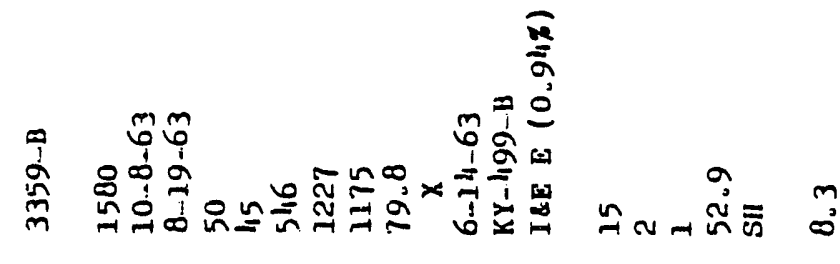

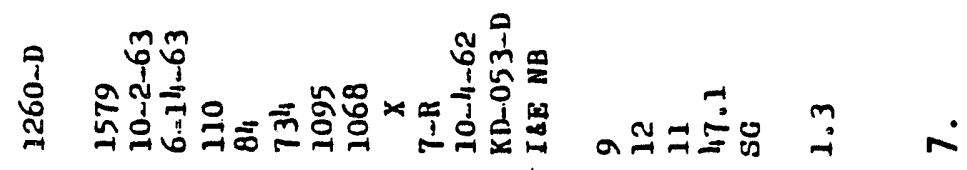

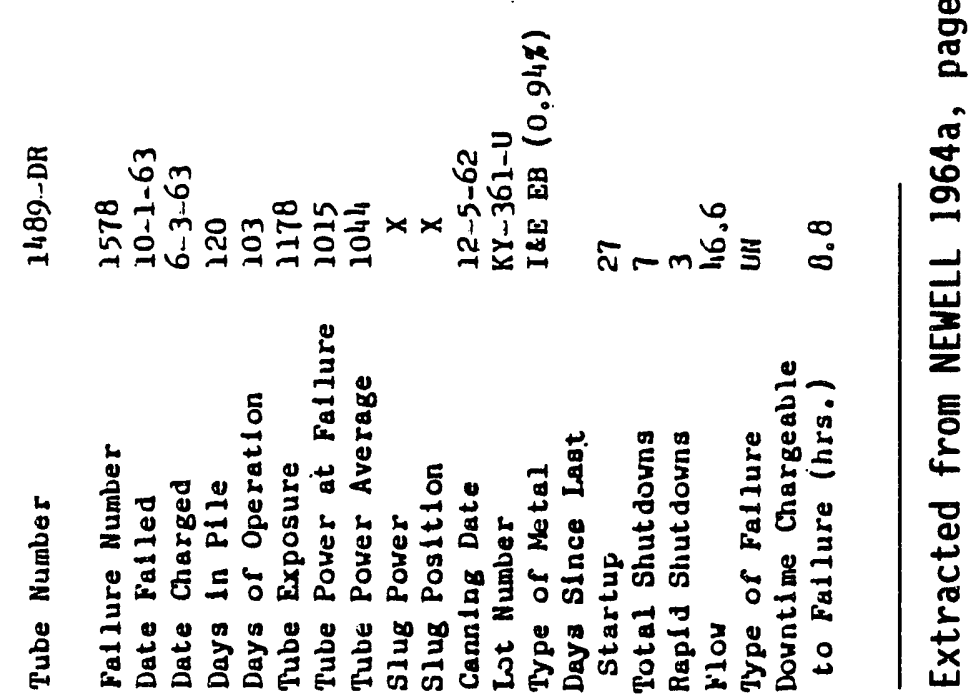

M.11 


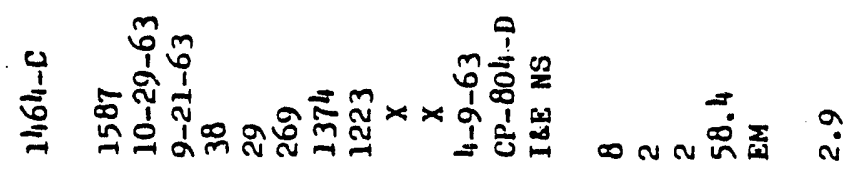

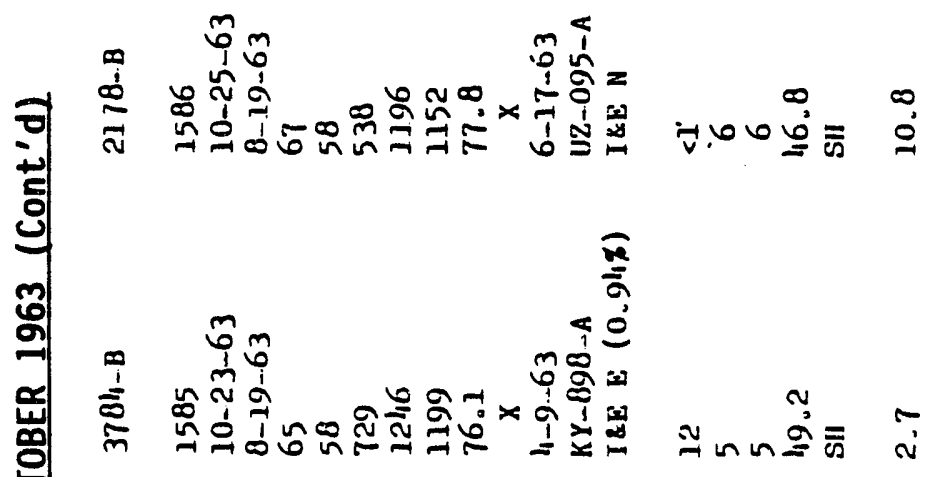

ज़

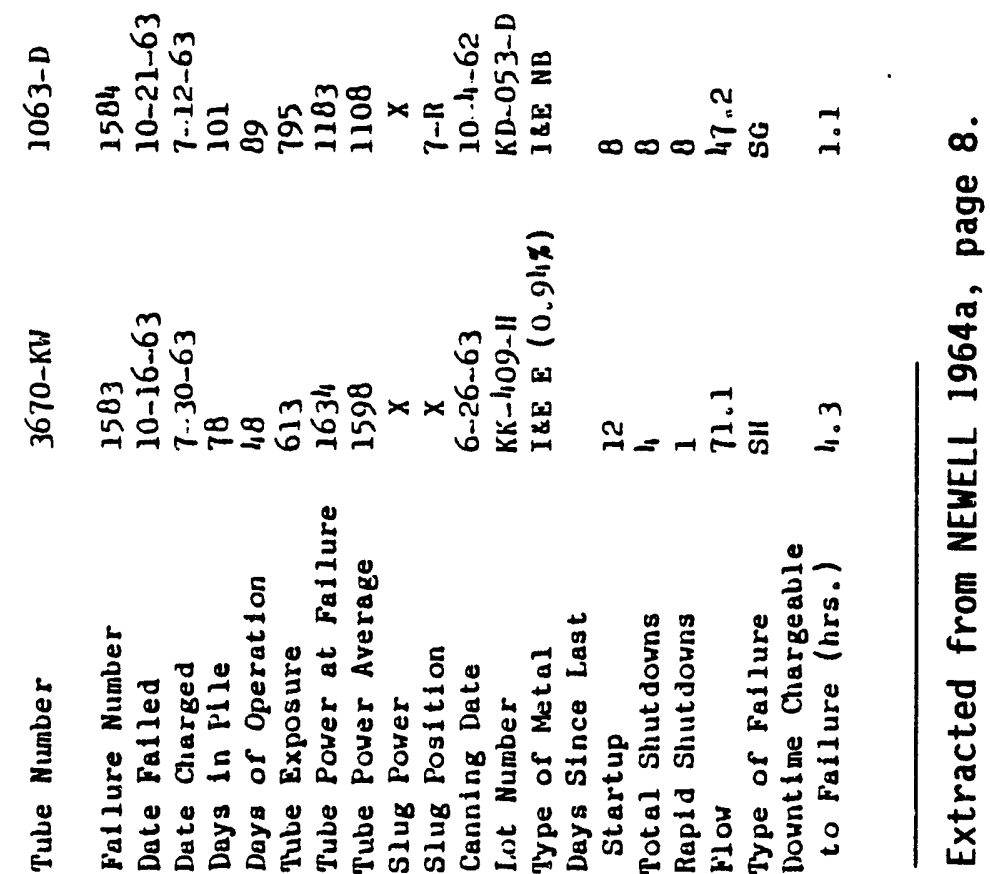


=

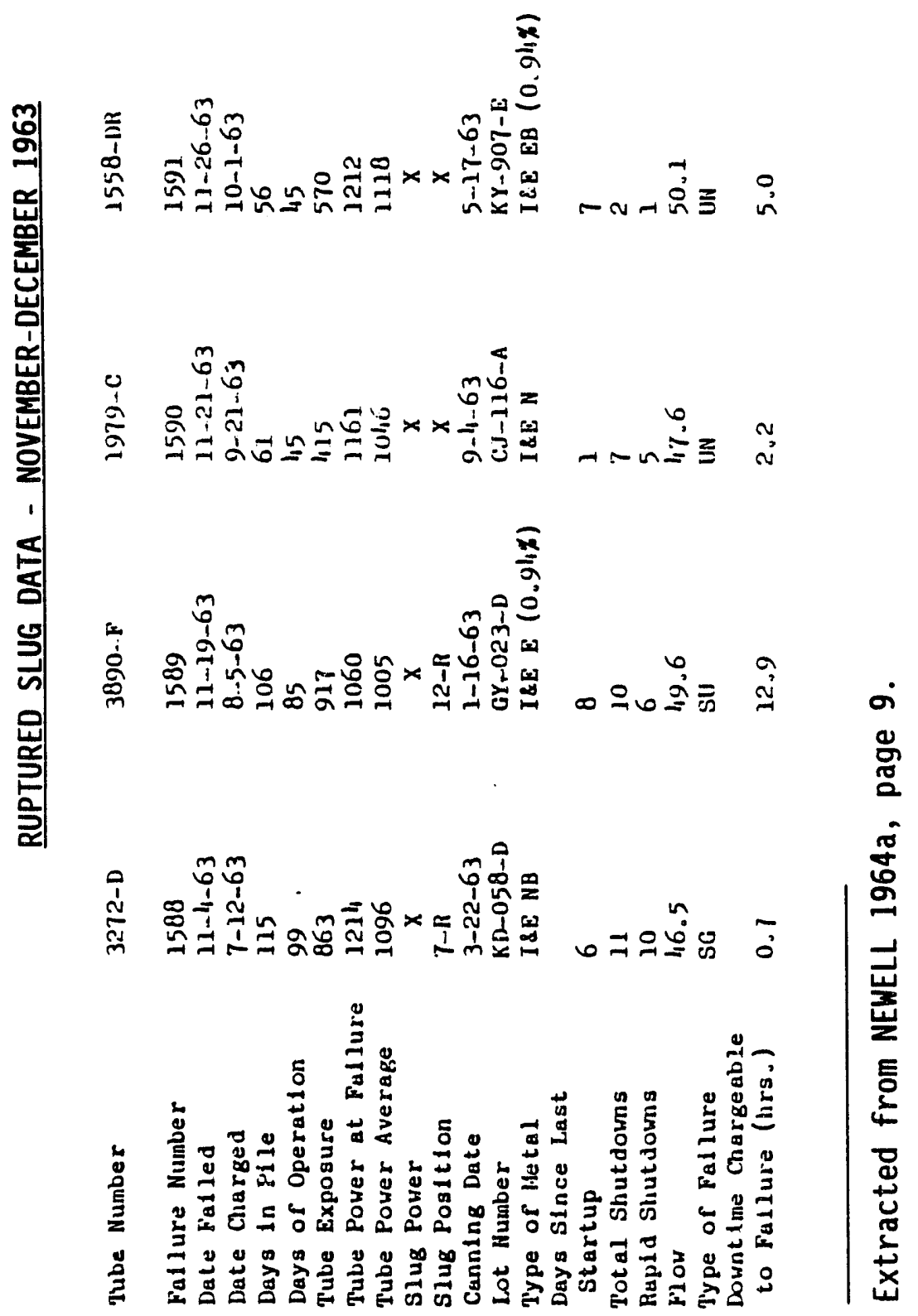




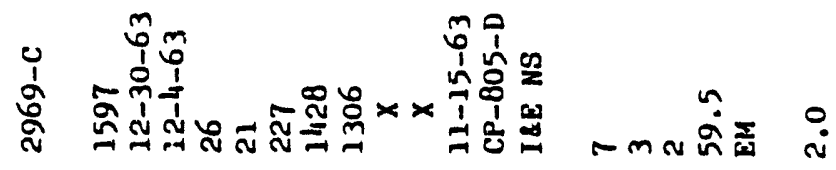

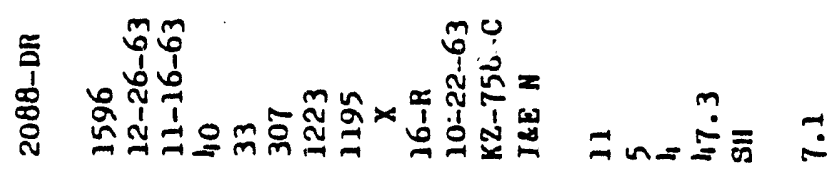

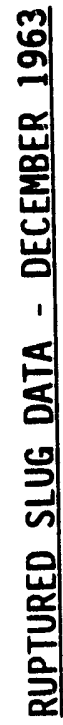

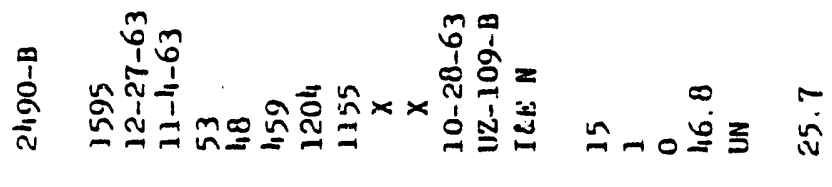

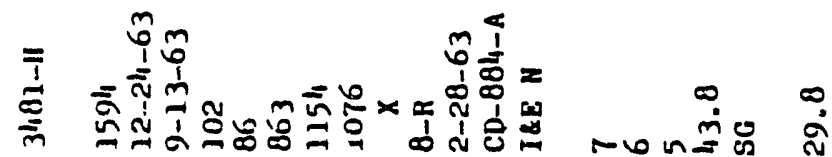

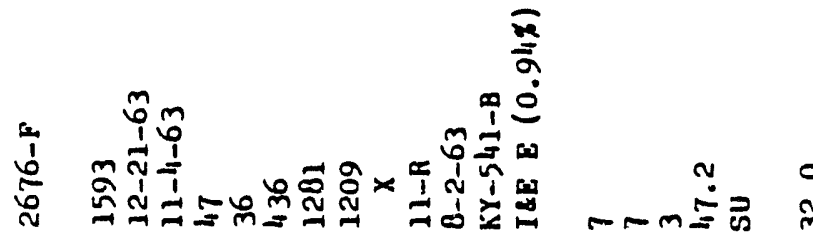

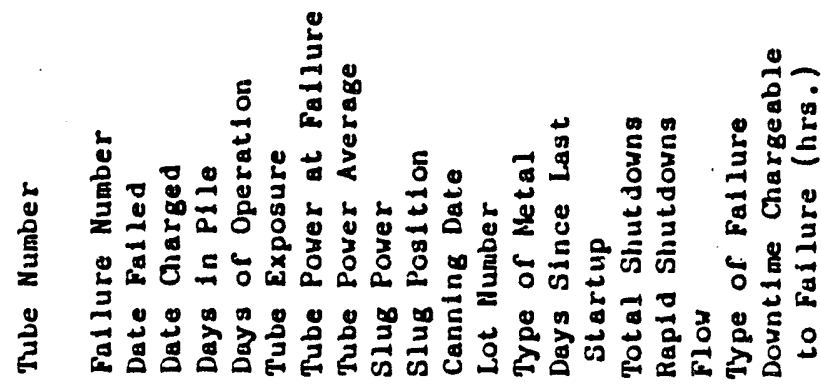

$\stackrel{\circ}{\stackrel{0}{8}}$

M. 14 
APPENDIX N

FUEL-ELEMENT FAILURE DATA FOR 1964 


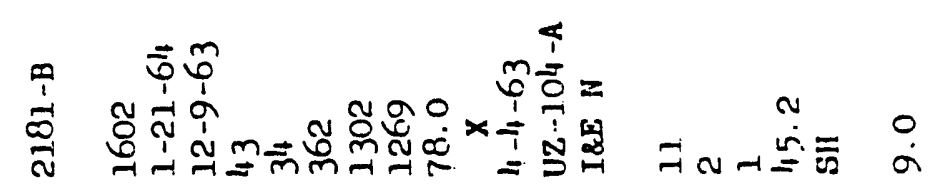

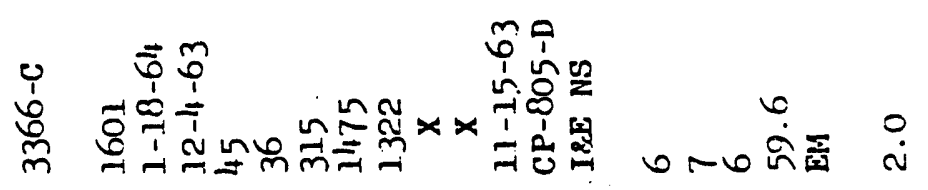

章

吕

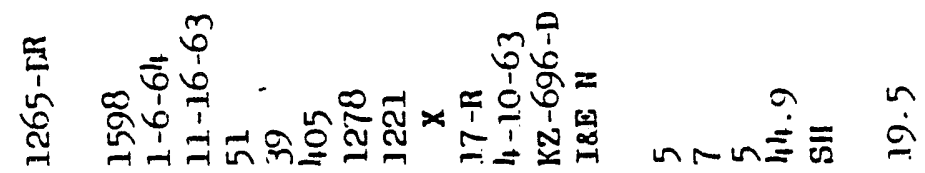

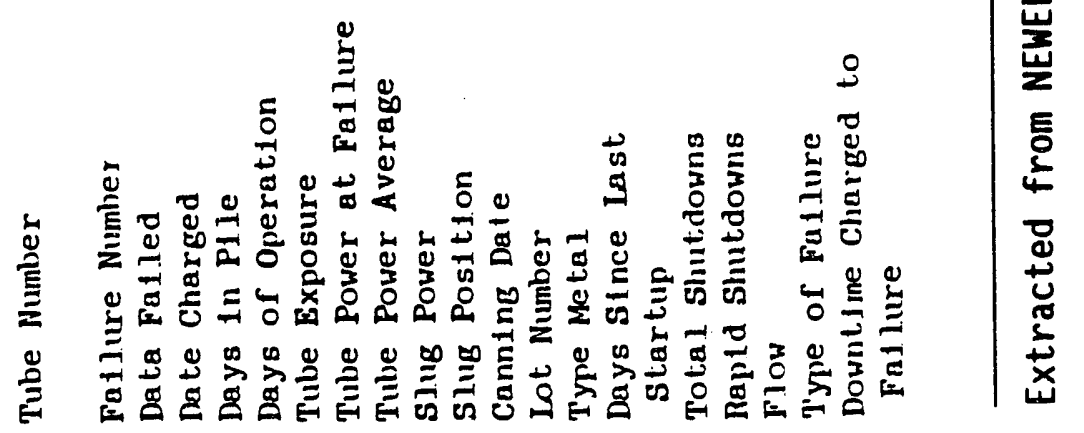

N. 1 


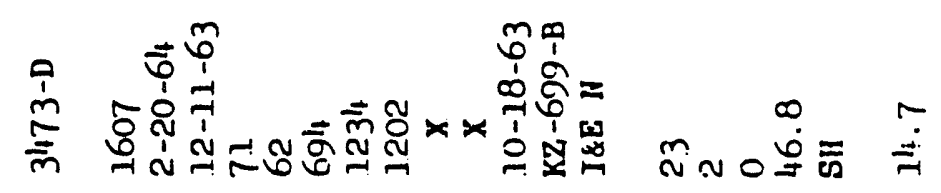

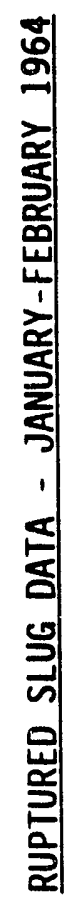

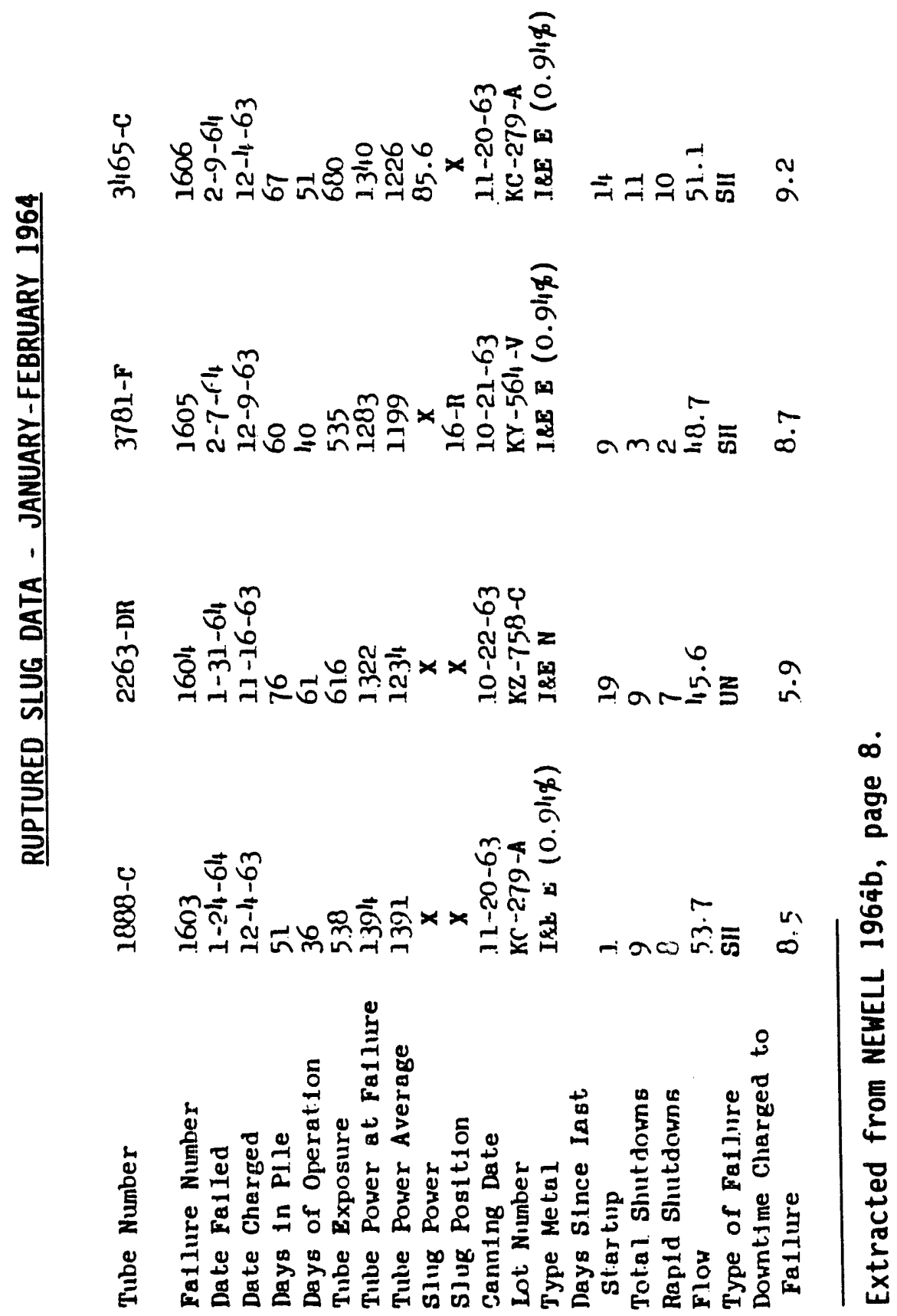


혐

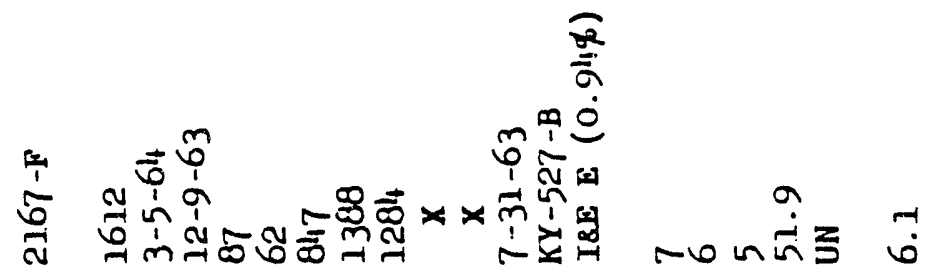
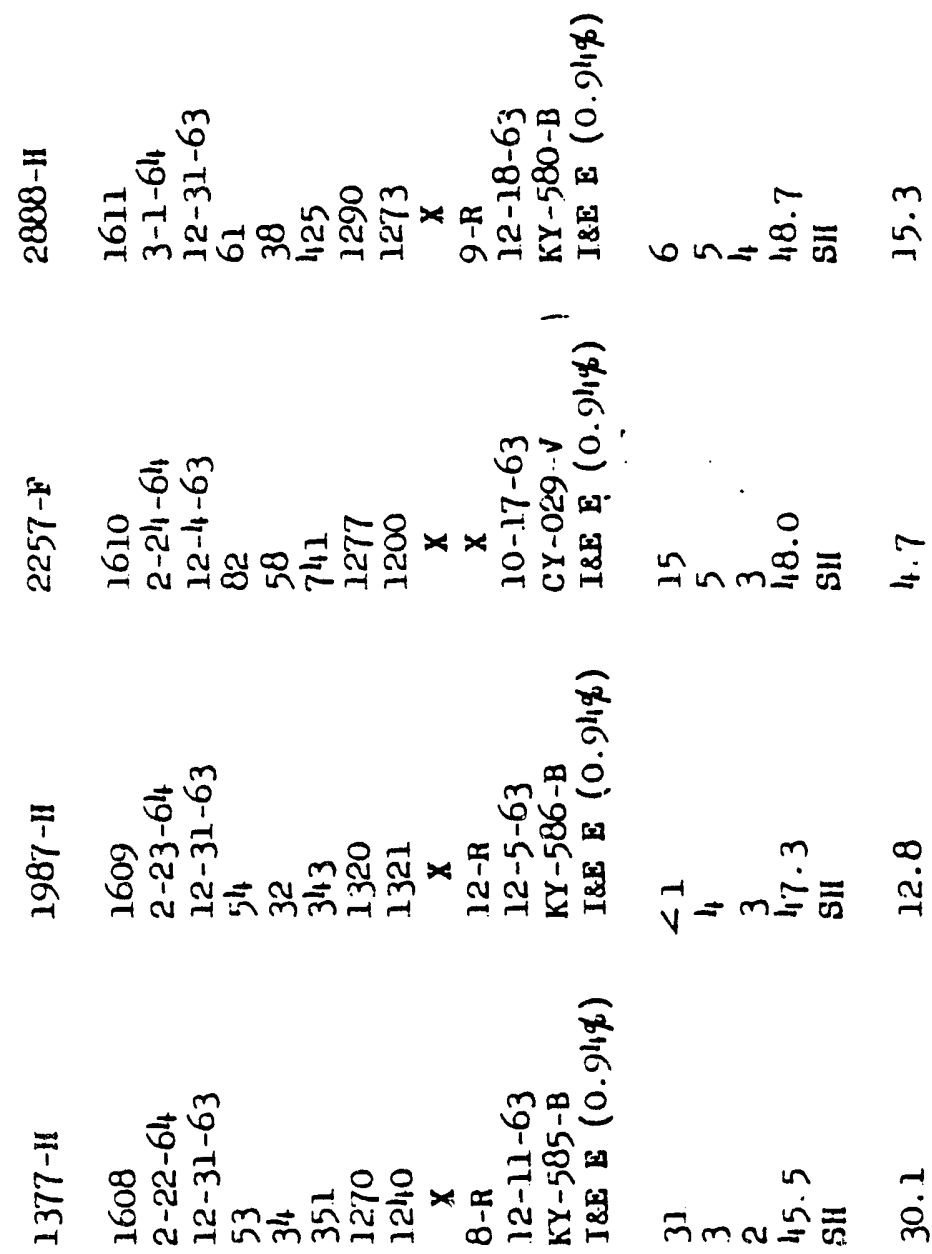

市

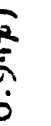

亗

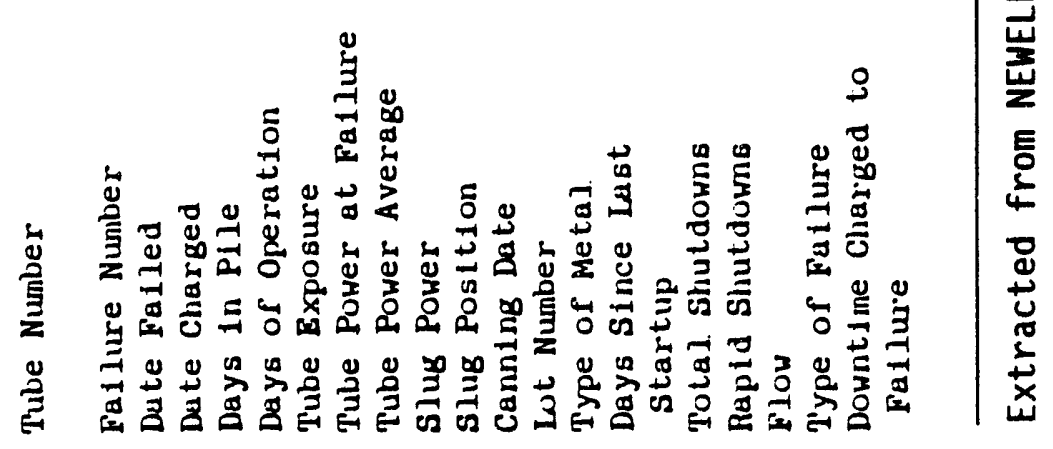




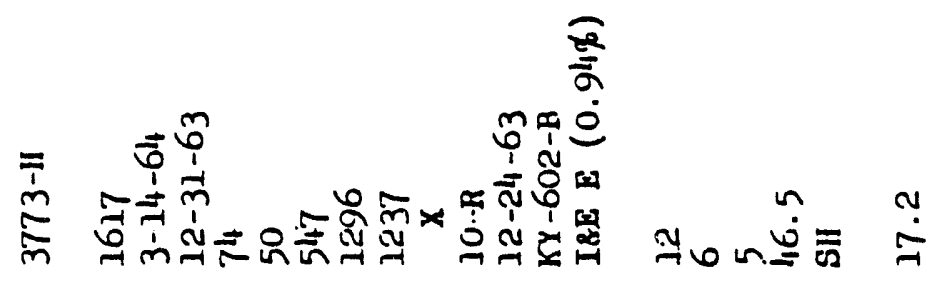

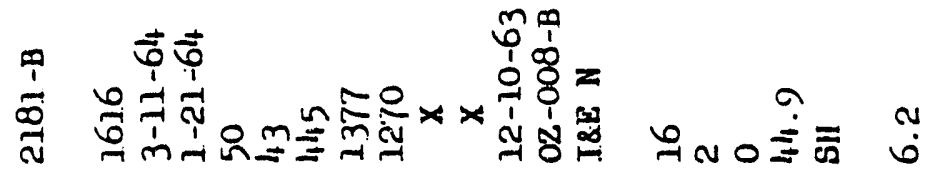

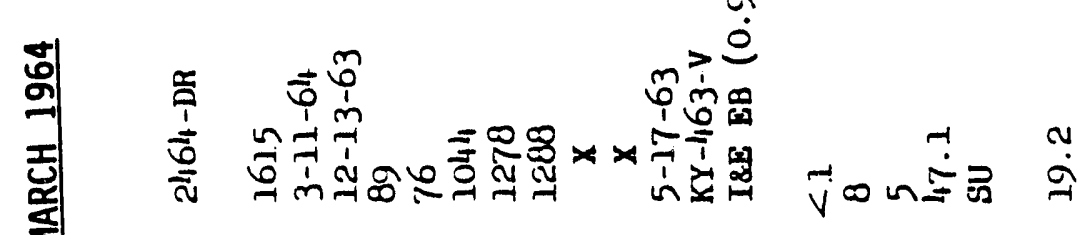

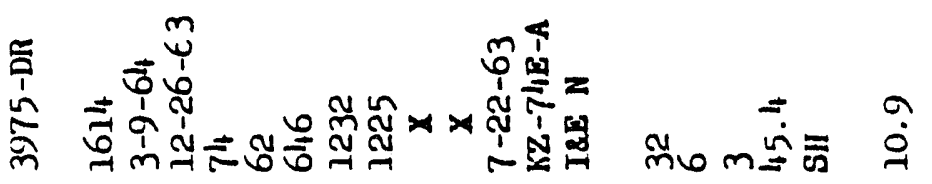

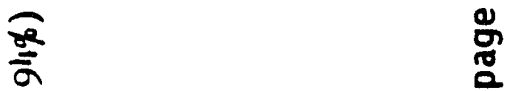

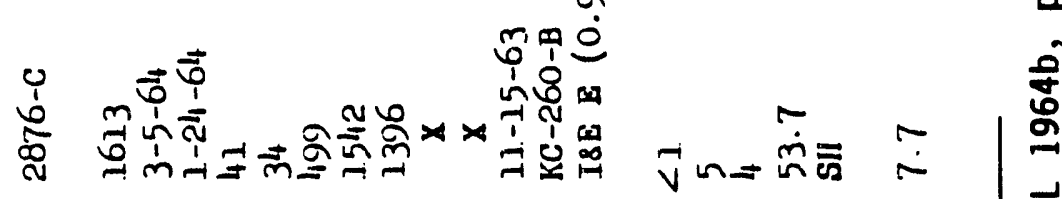

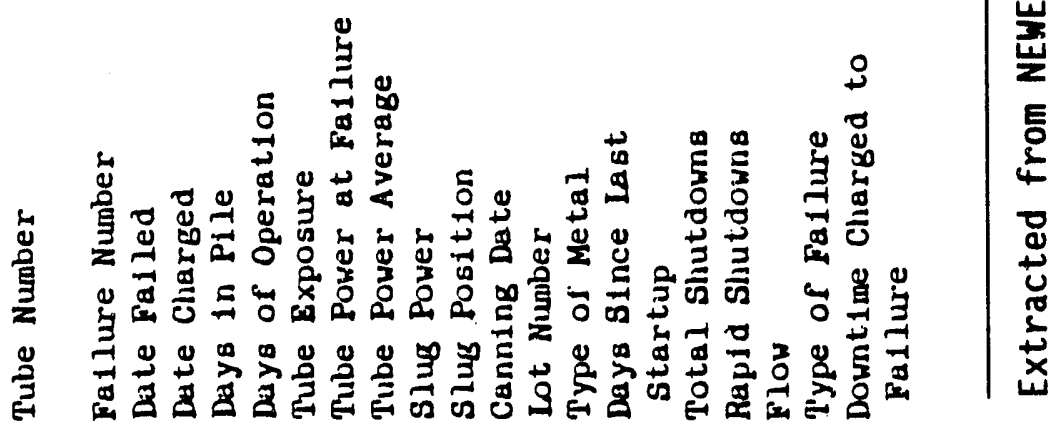




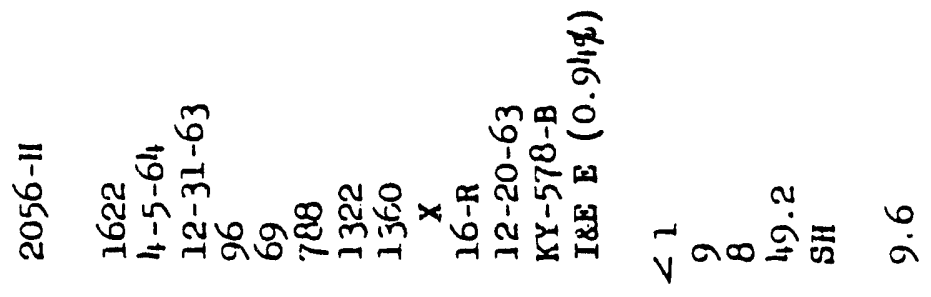

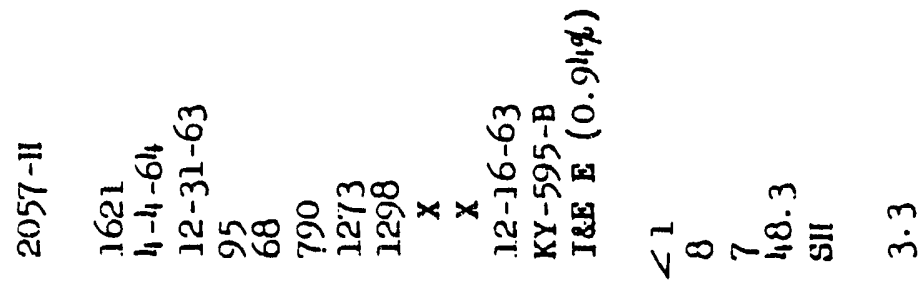

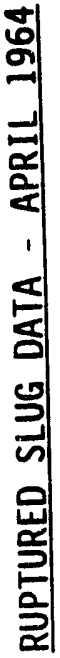
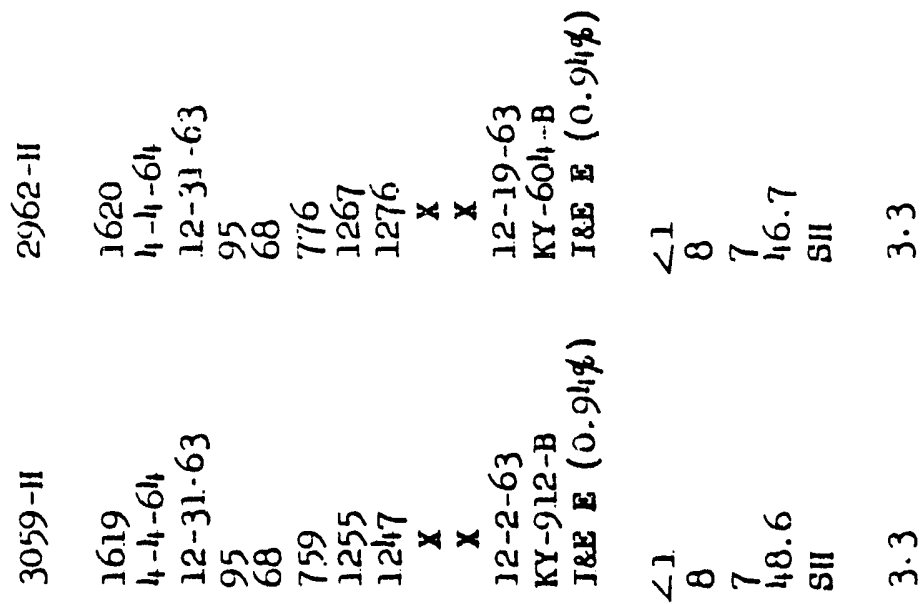

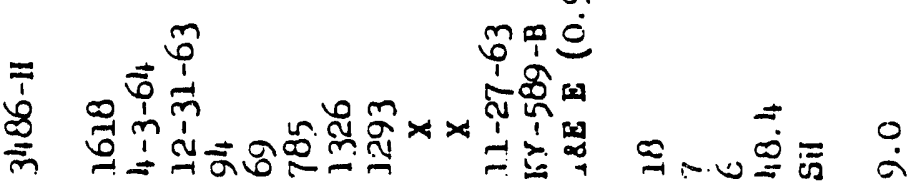

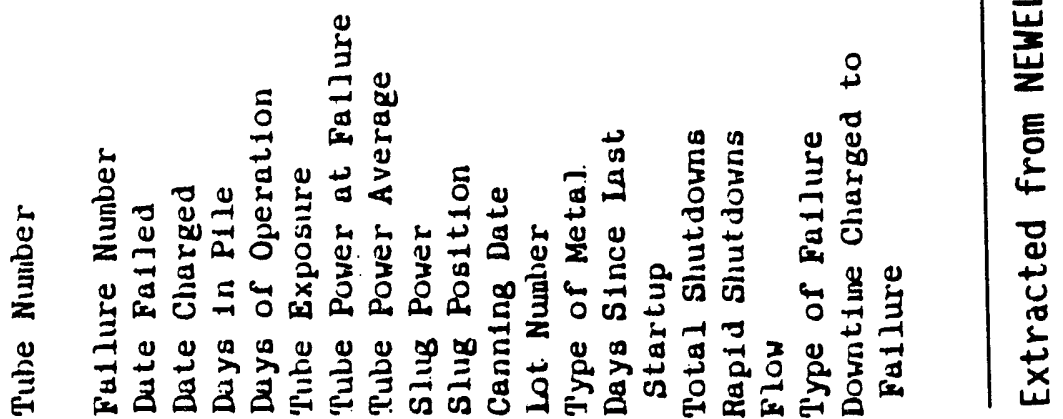

N. 5 


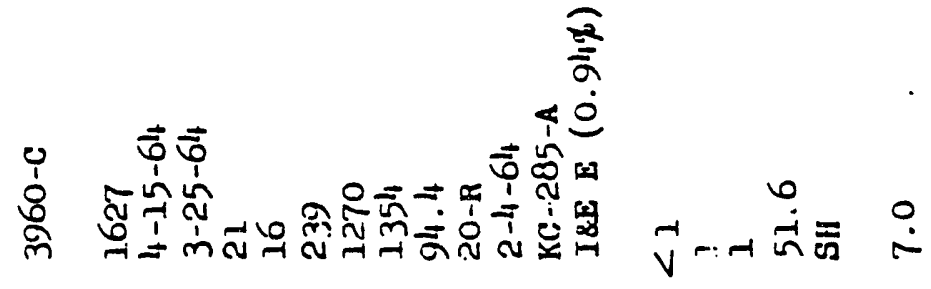

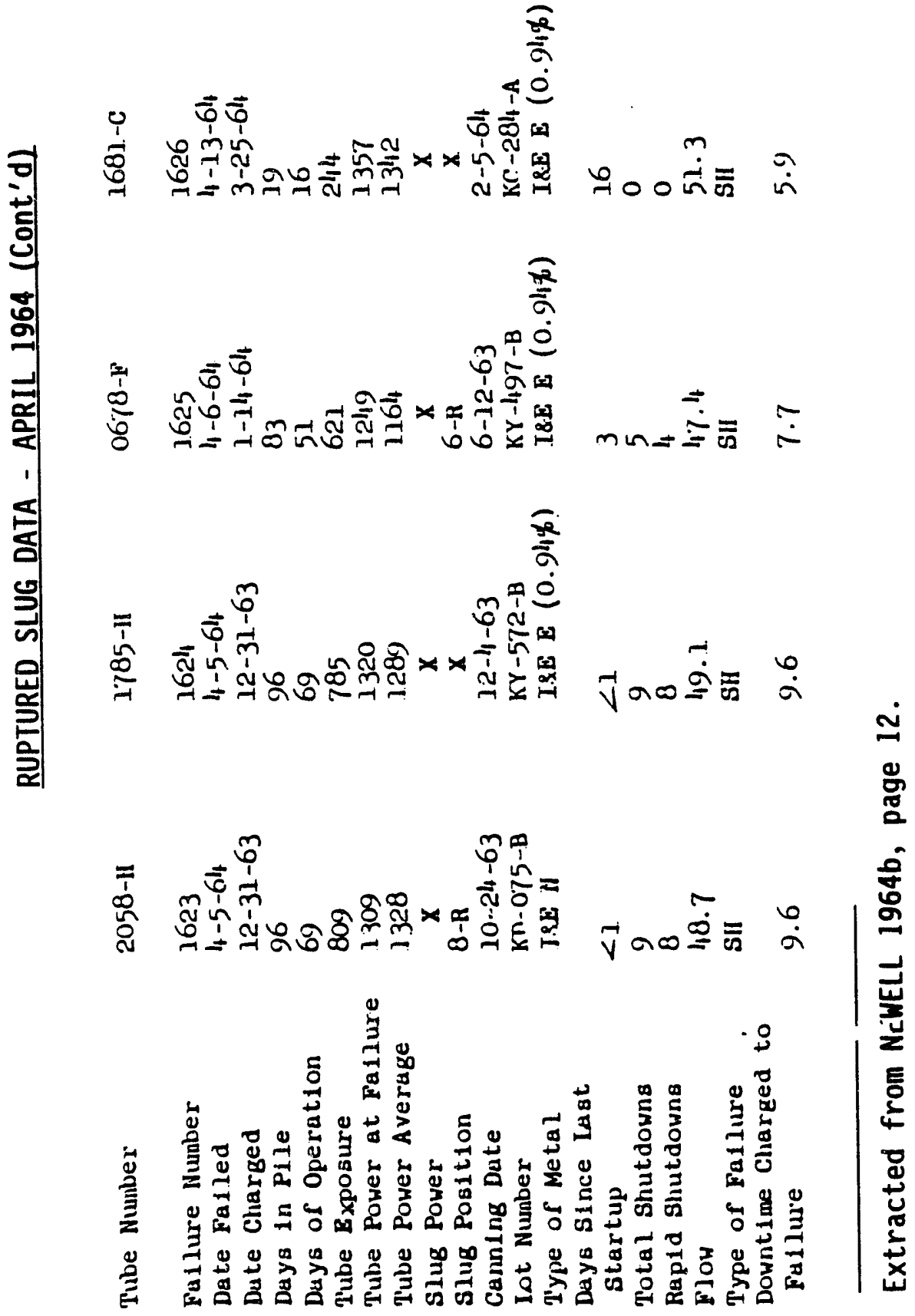




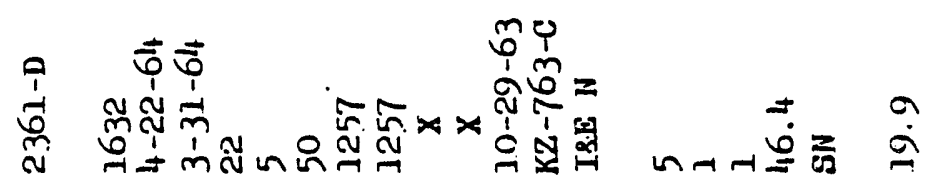

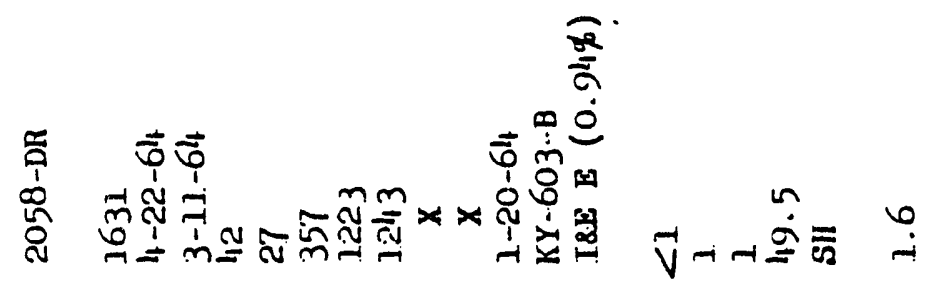

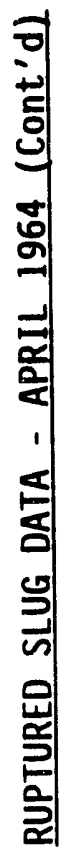
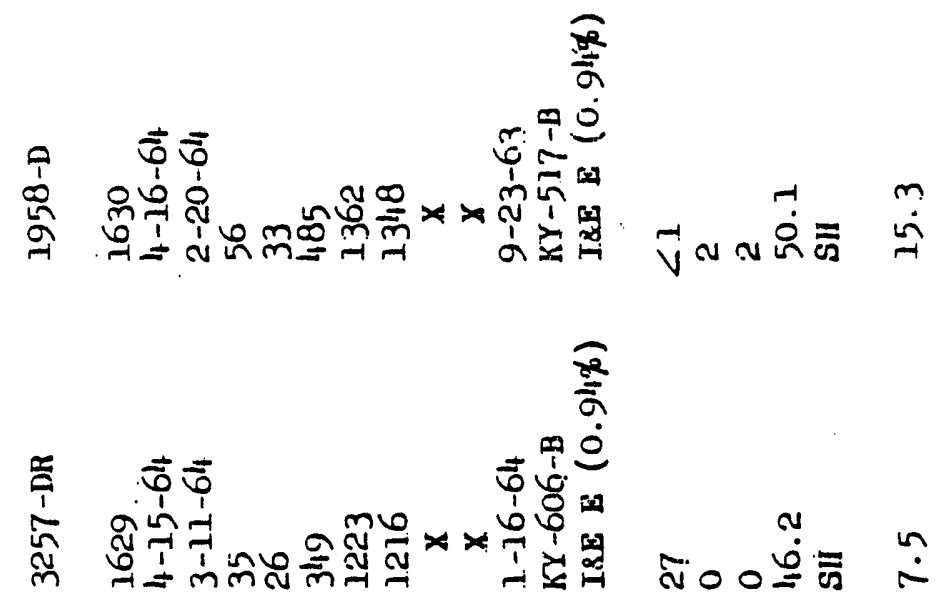

$\stackrel{\dot{m}}{\stackrel{\oplus}{\overparen{g}}}$

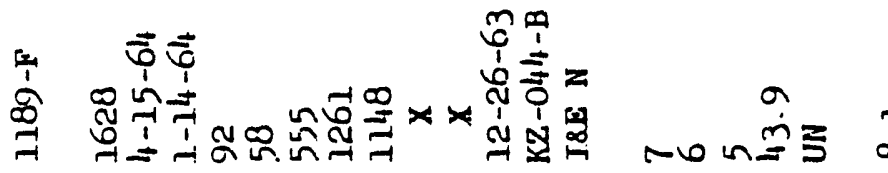

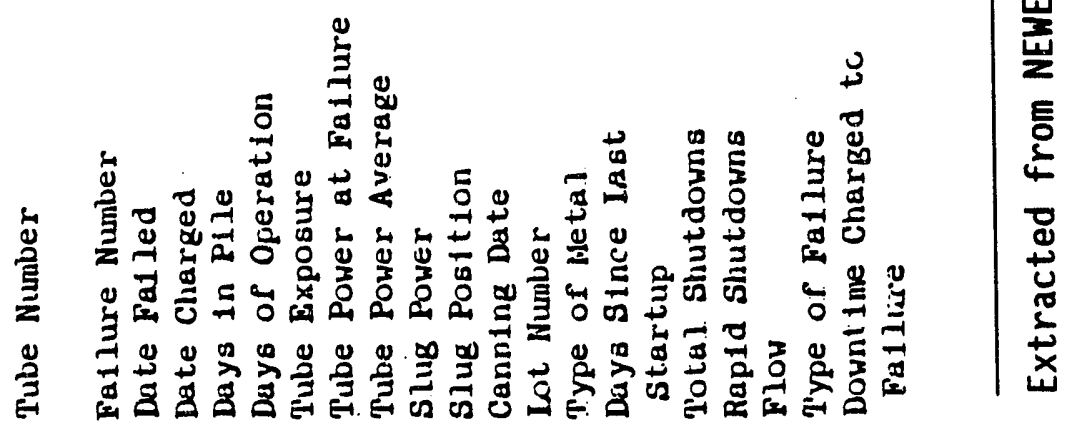




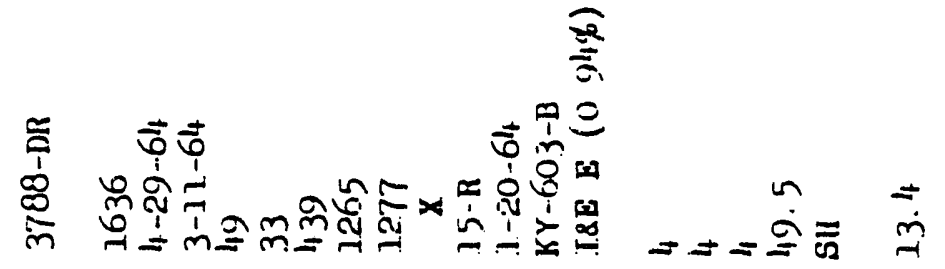

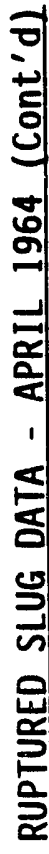
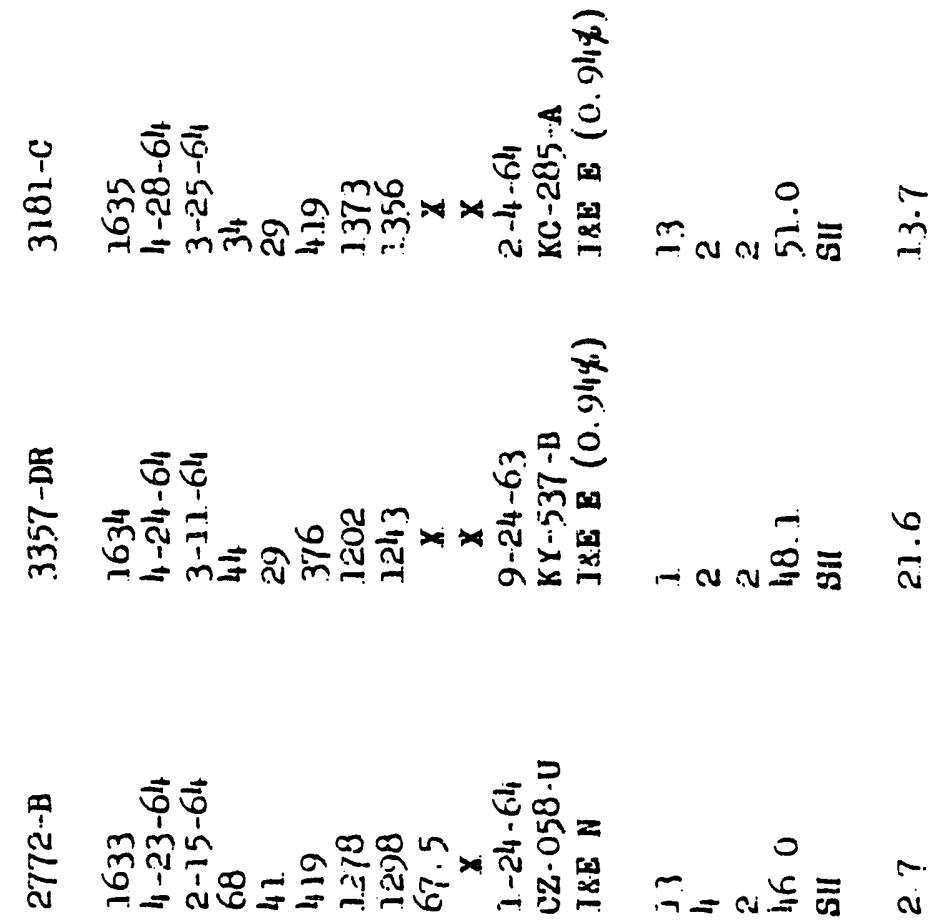

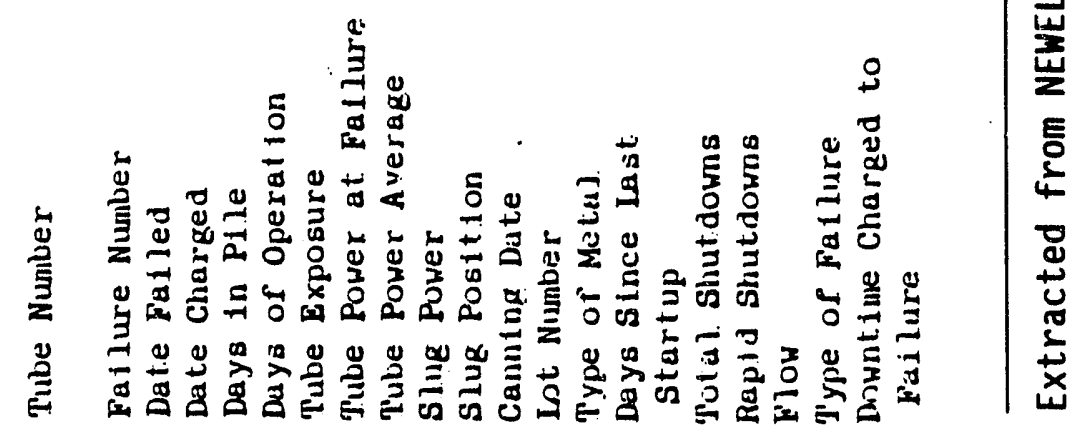




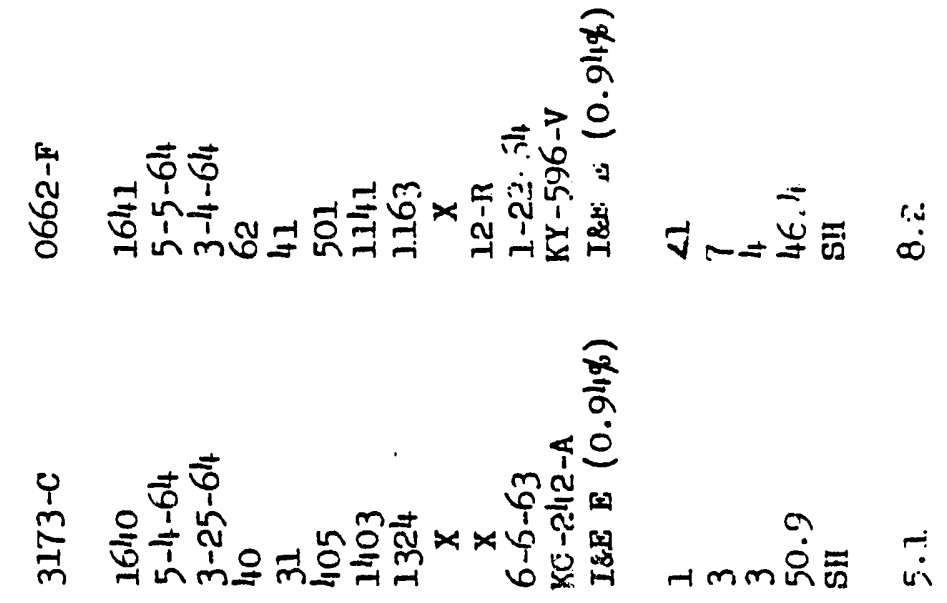

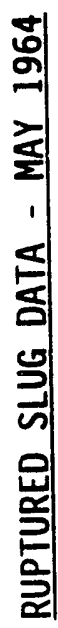

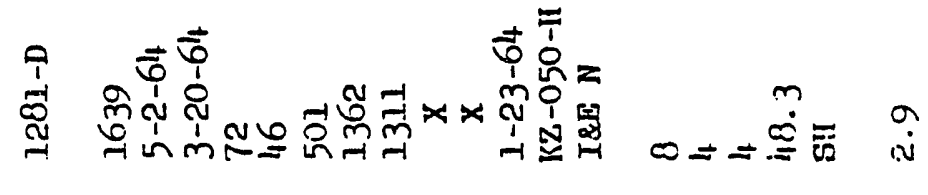

落

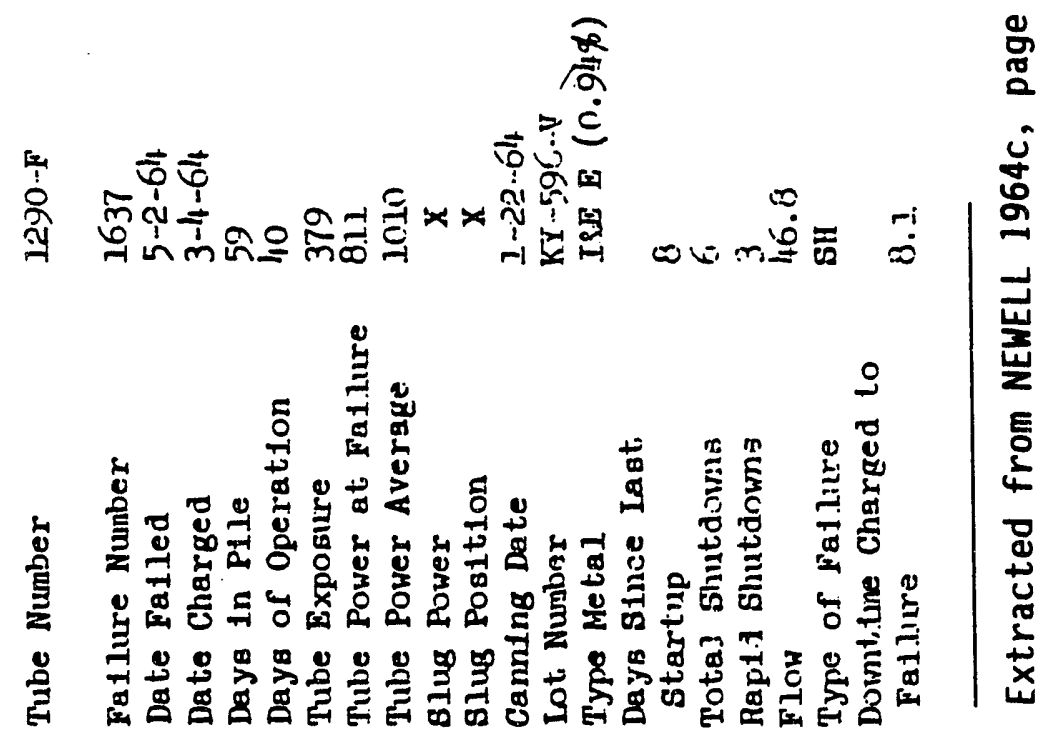




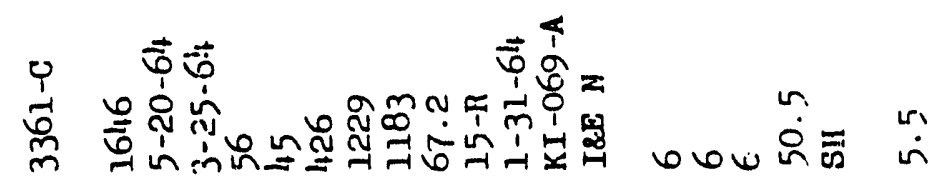

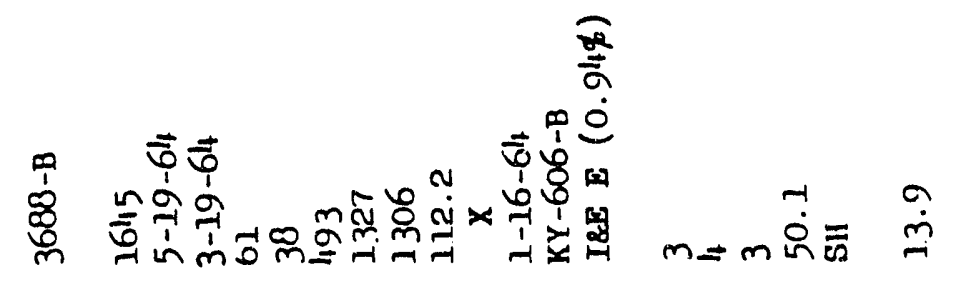

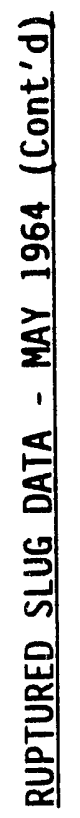

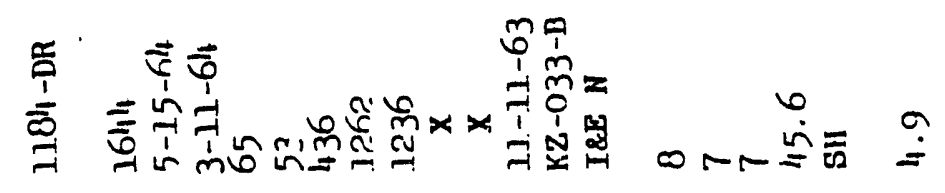

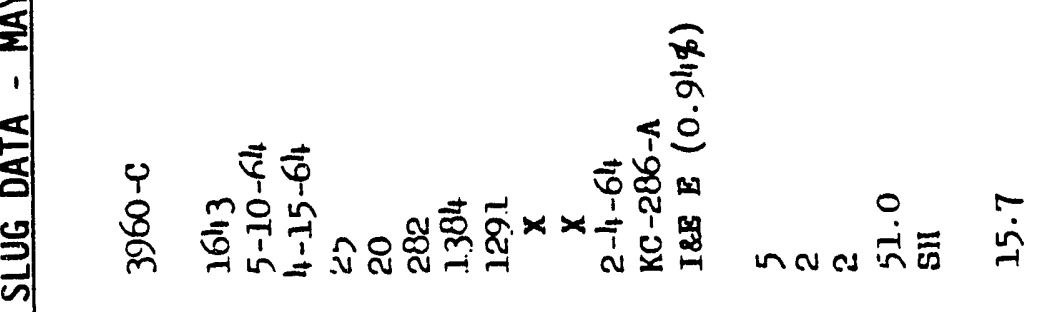

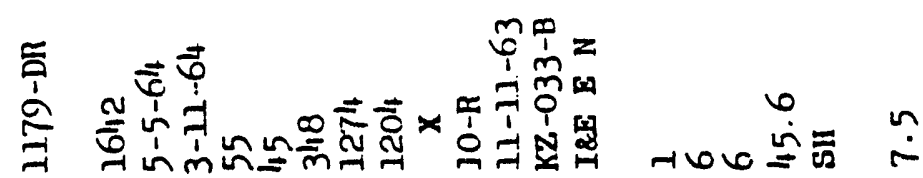

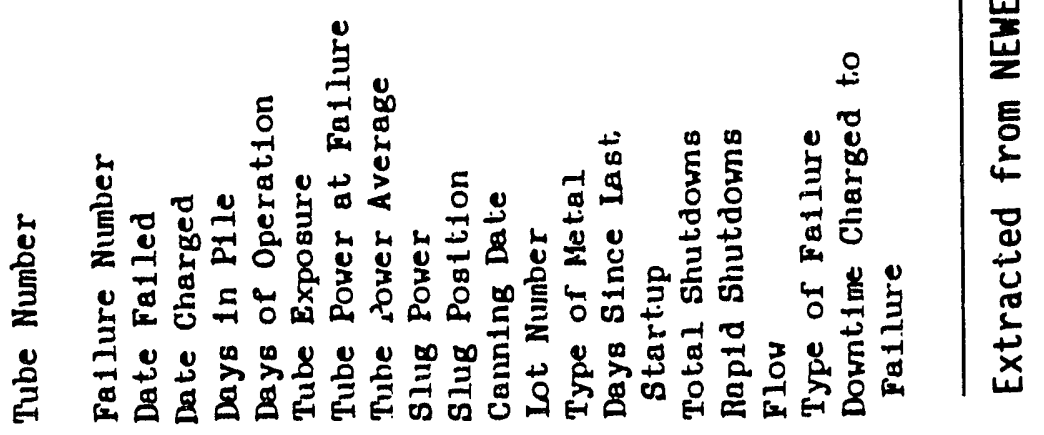




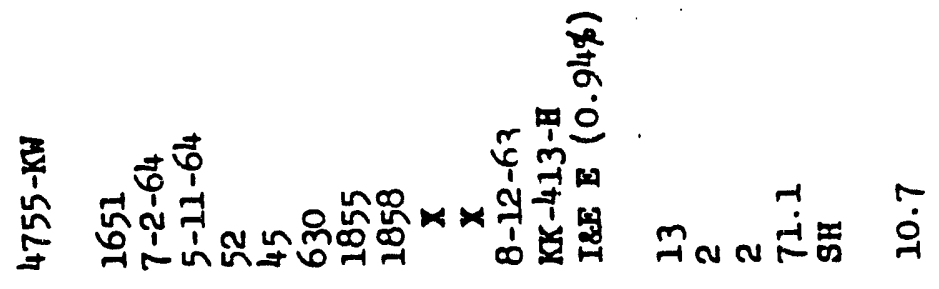

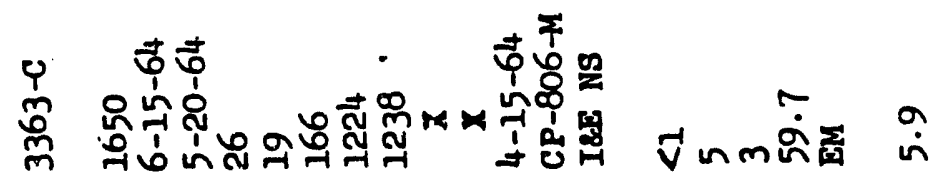

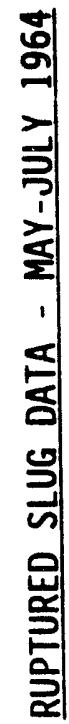
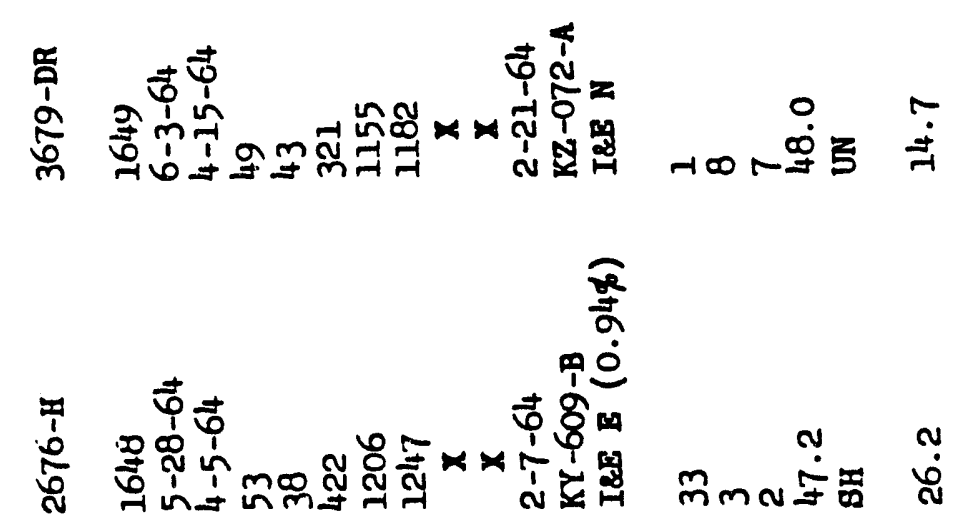

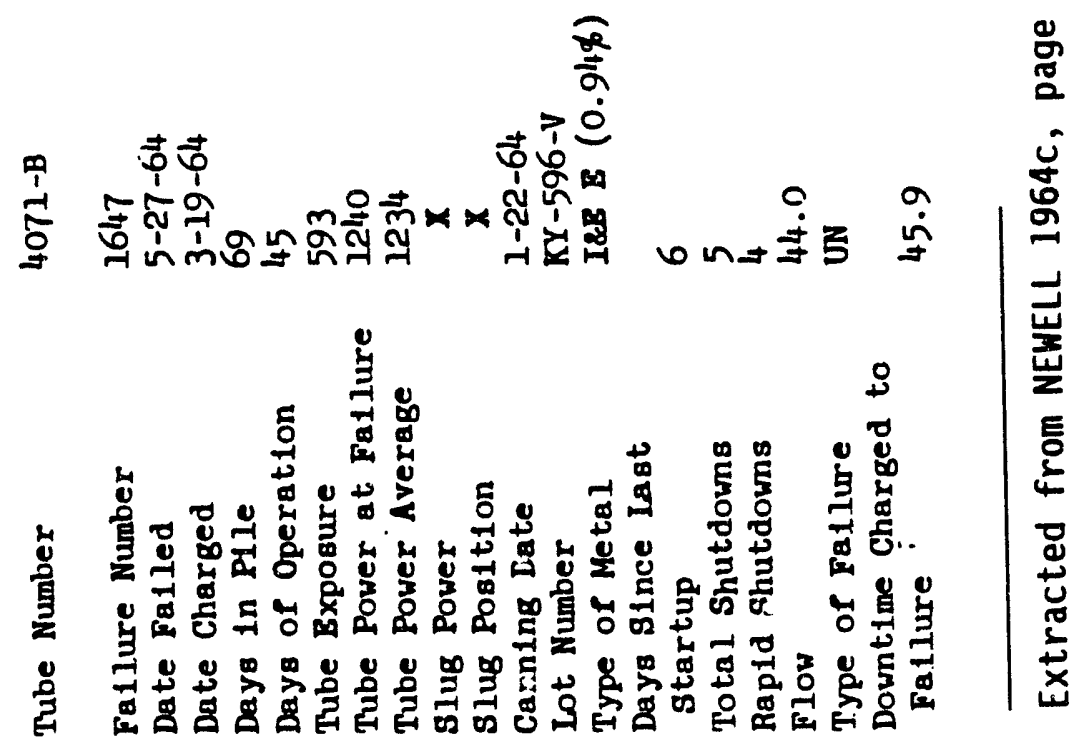




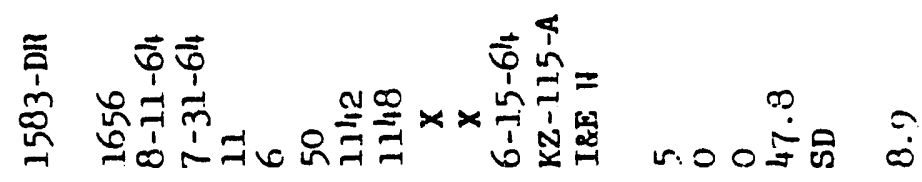

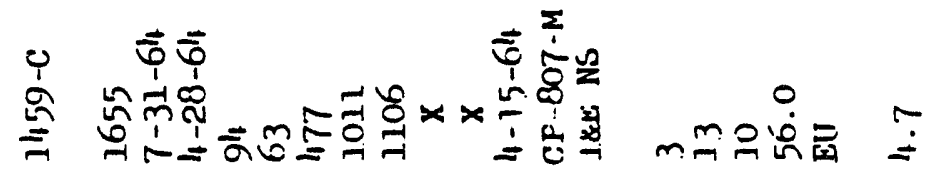

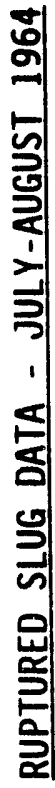

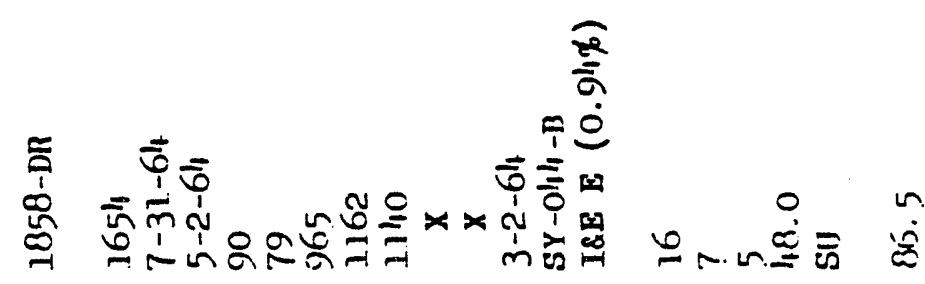

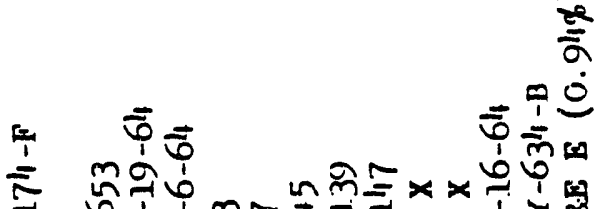

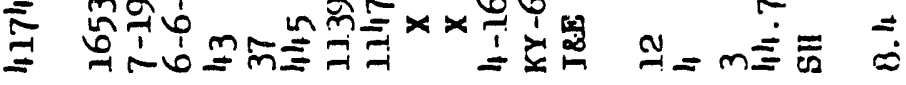

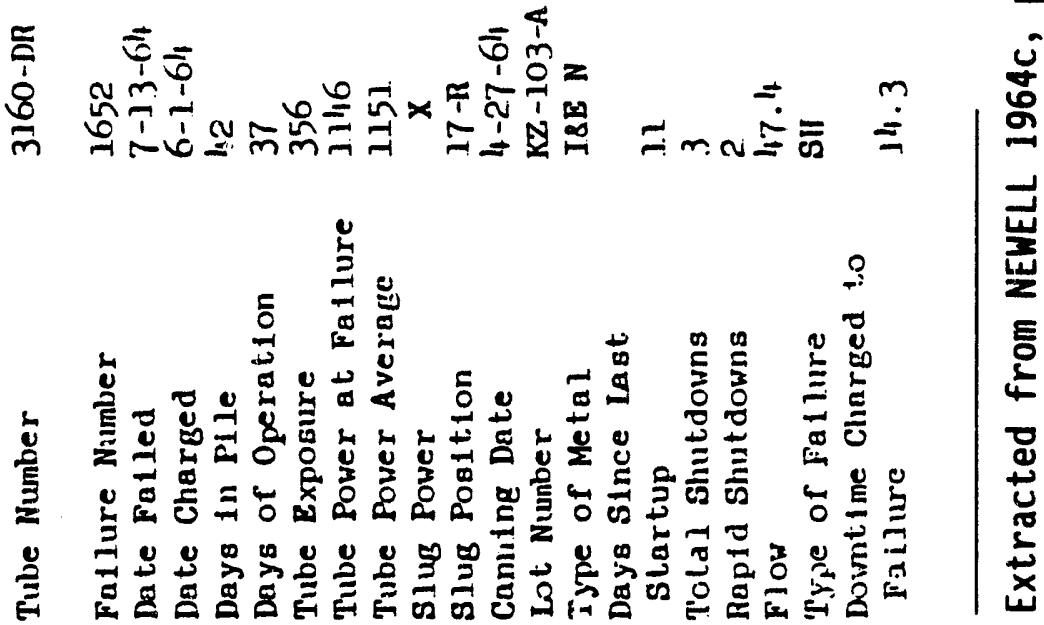




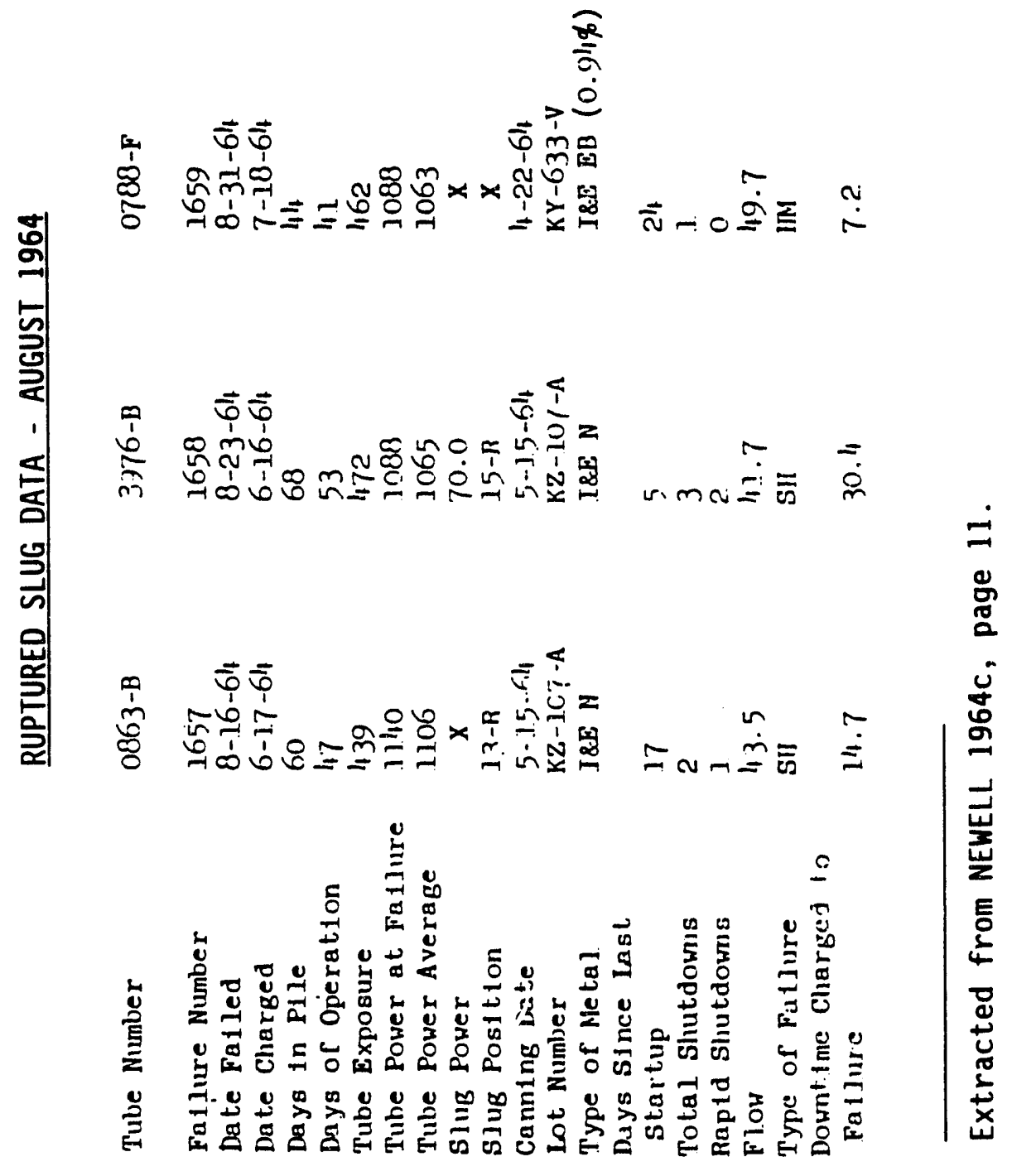




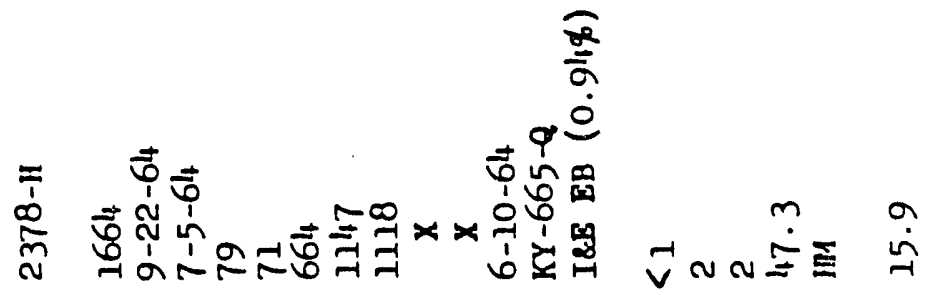

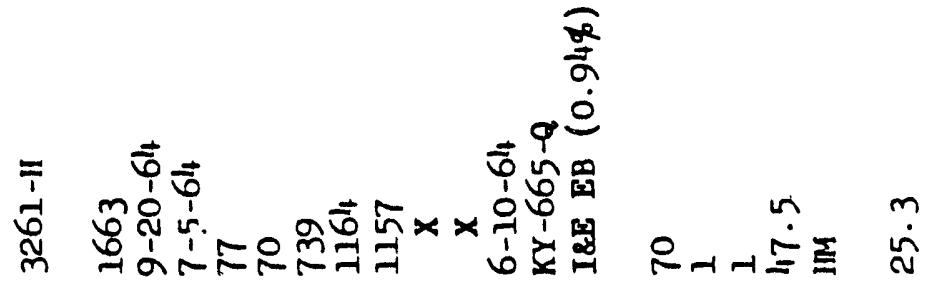

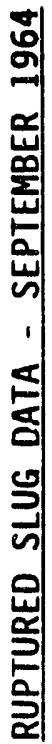

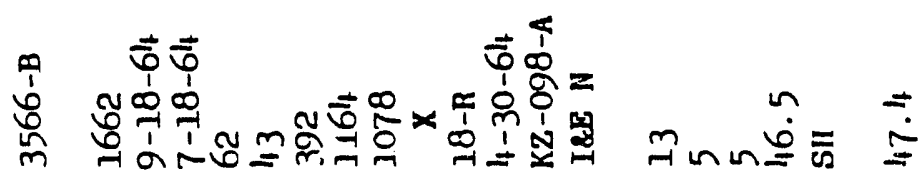

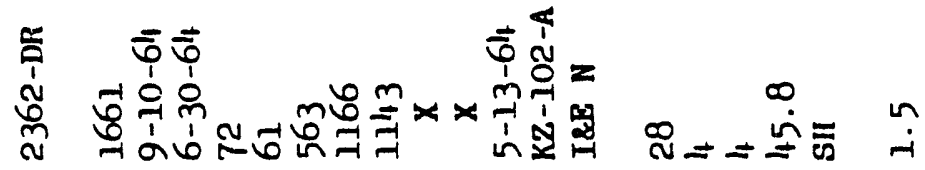

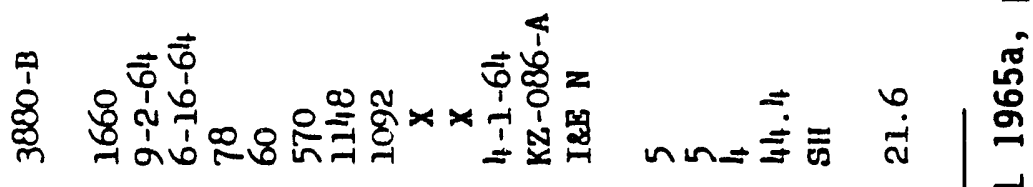

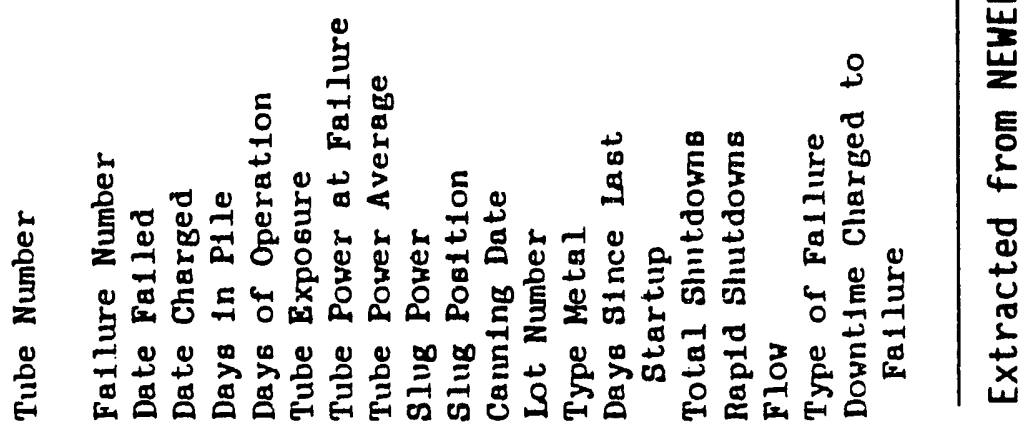




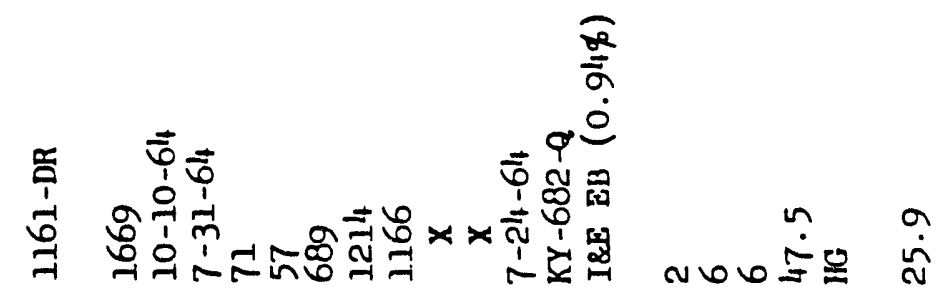

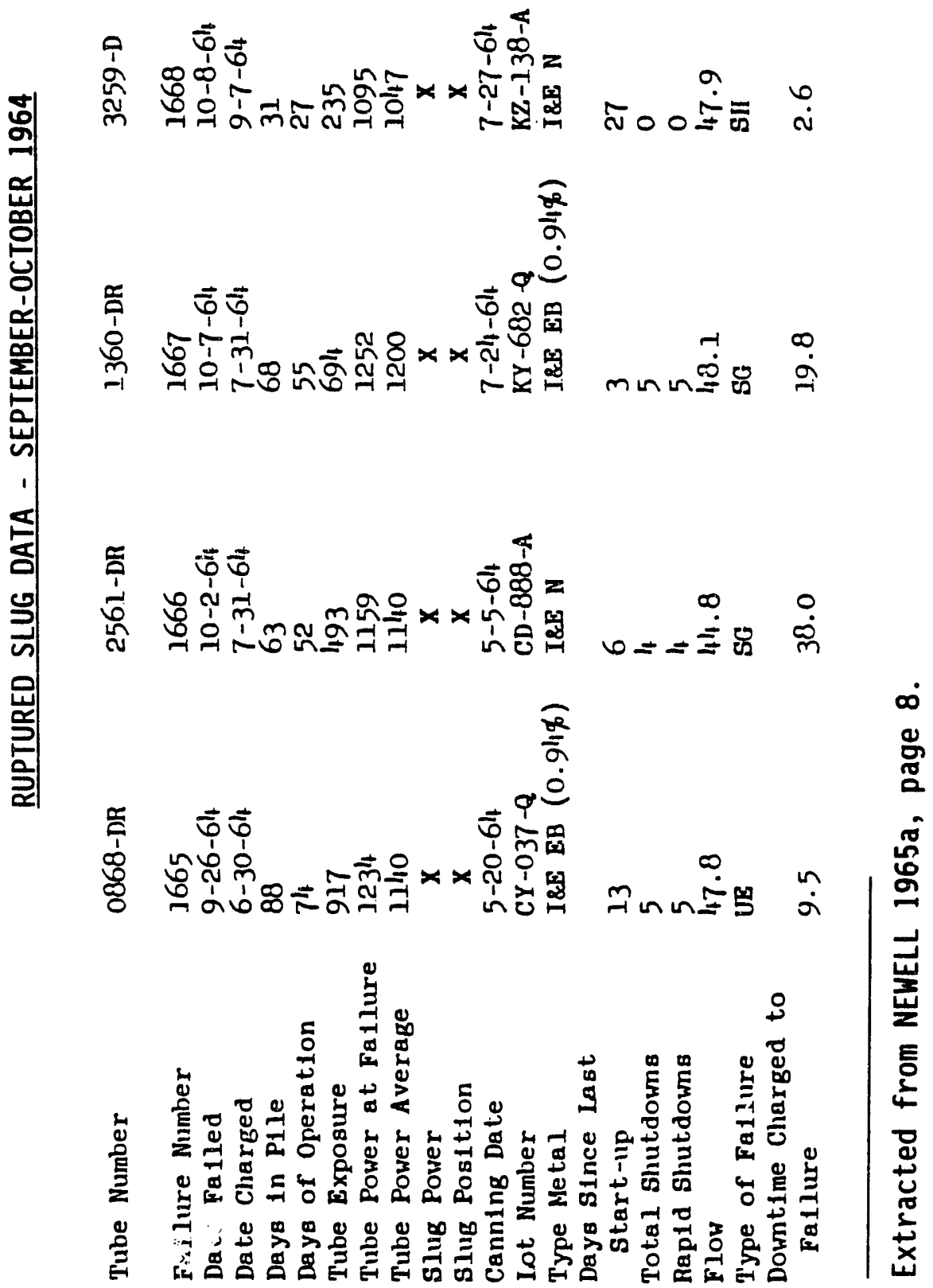




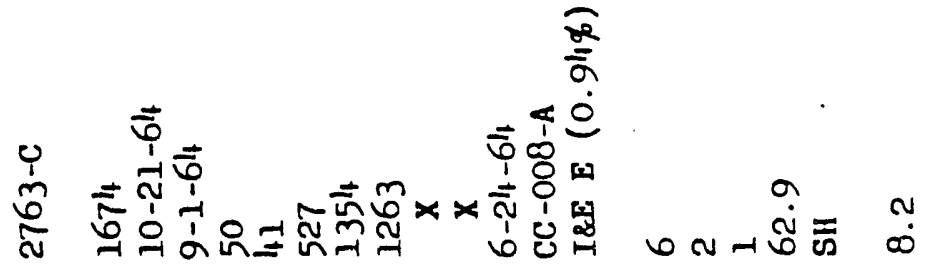

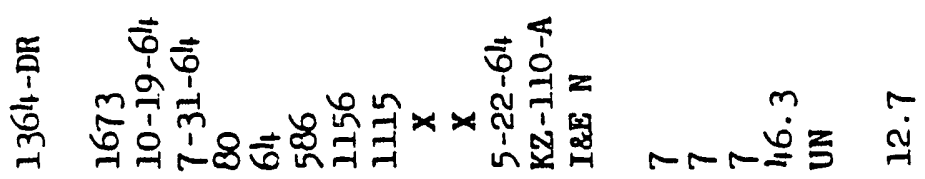

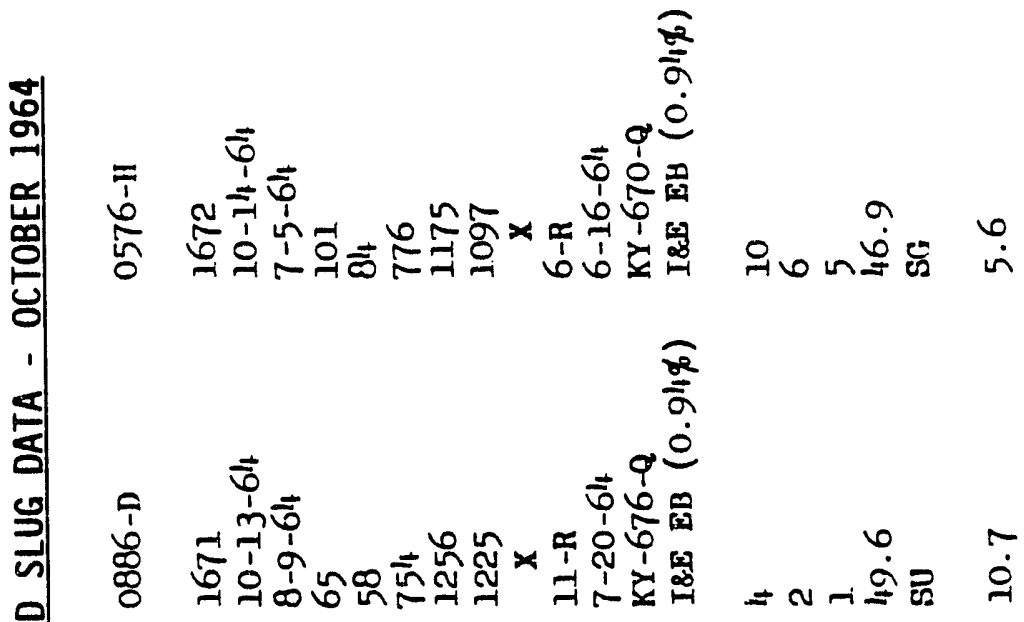

을

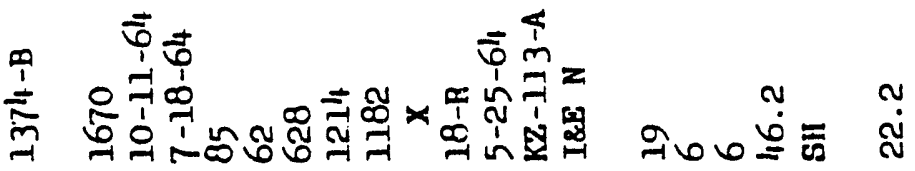

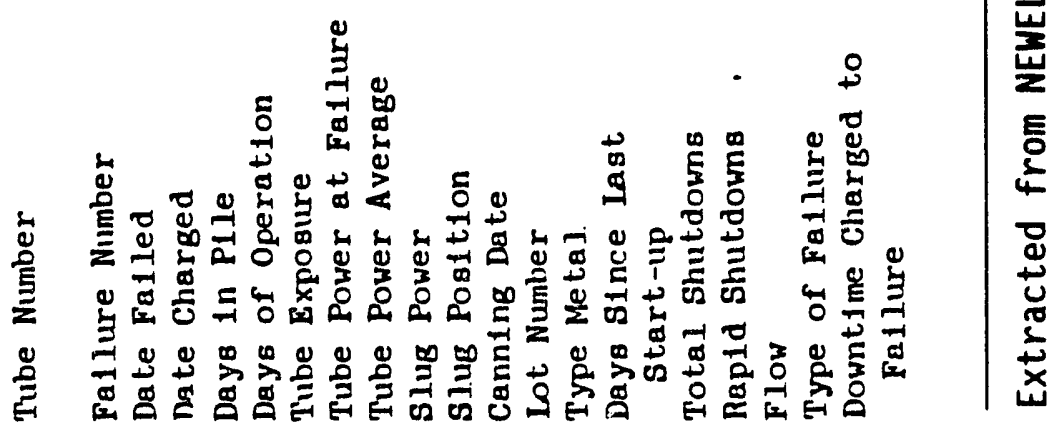




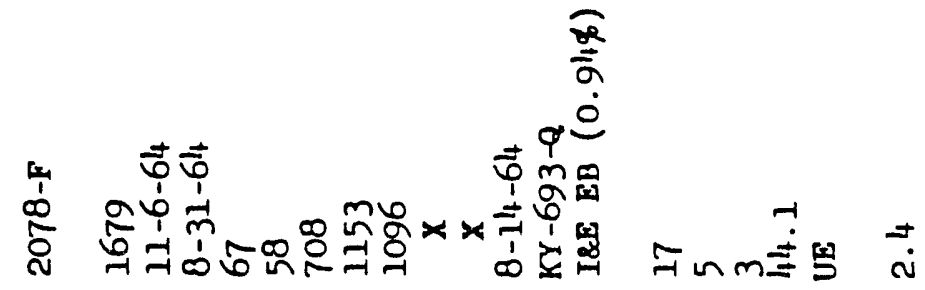

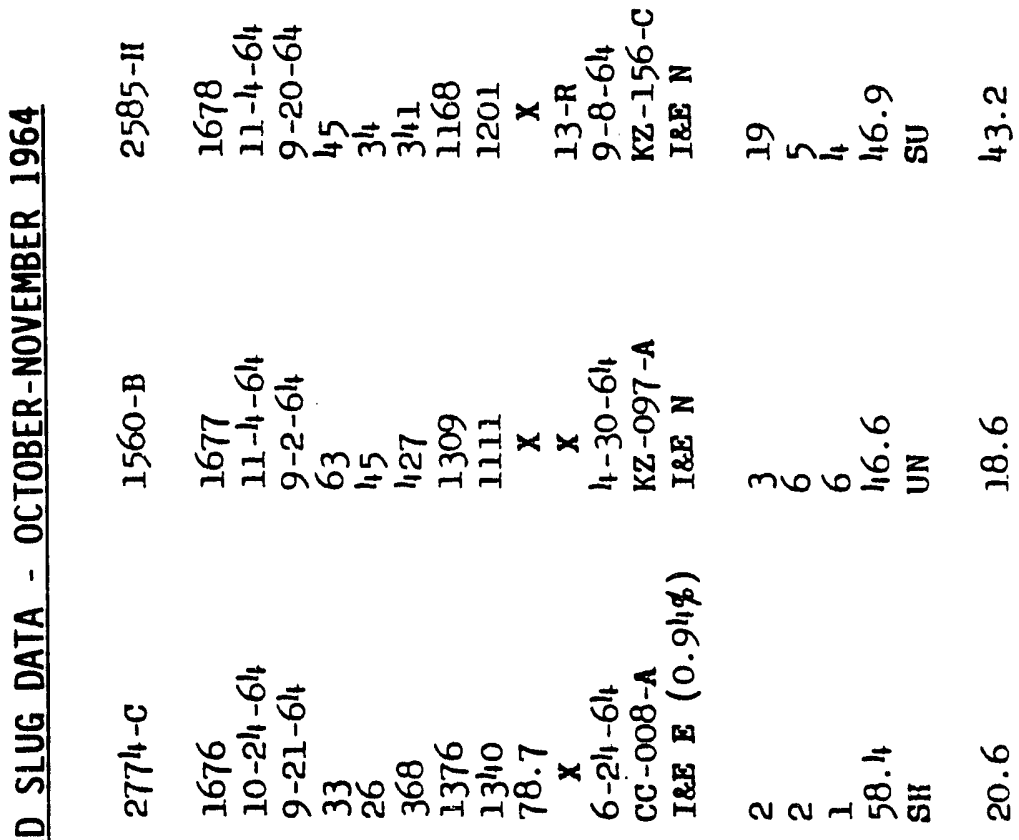

$\overrightarrow{0} \quad \overrightarrow{0} \quad \frac{1}{0}$

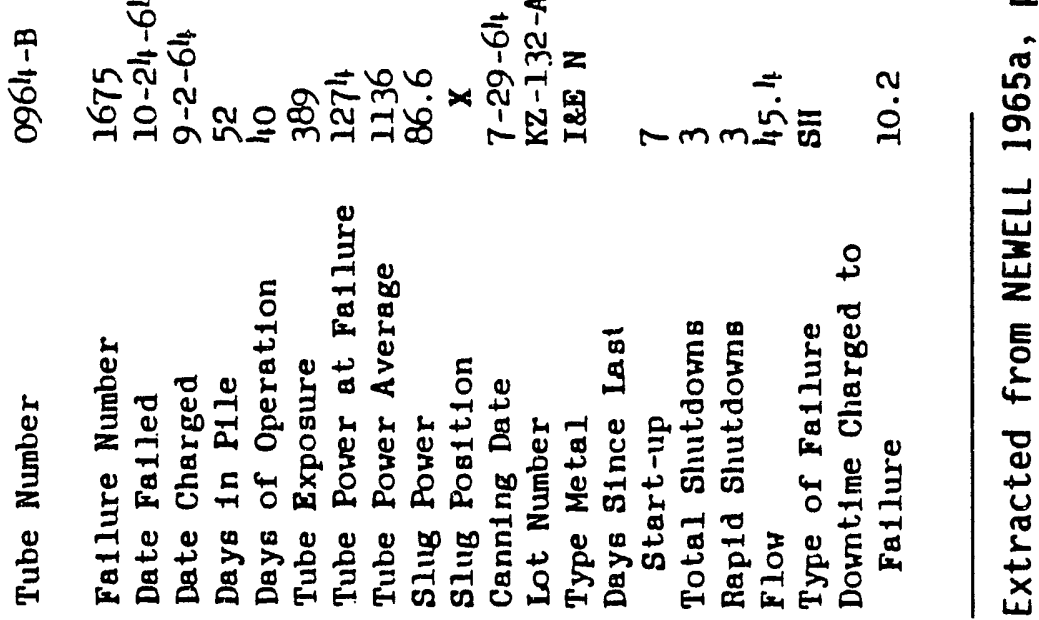




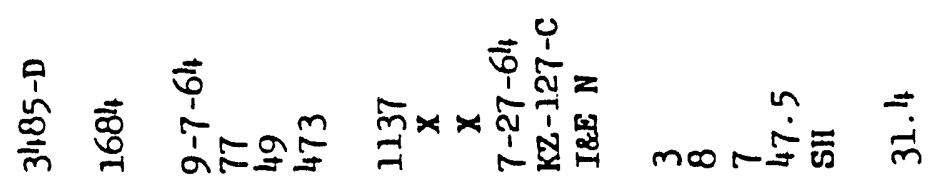

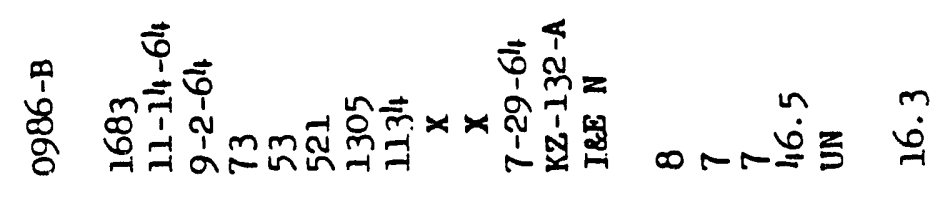

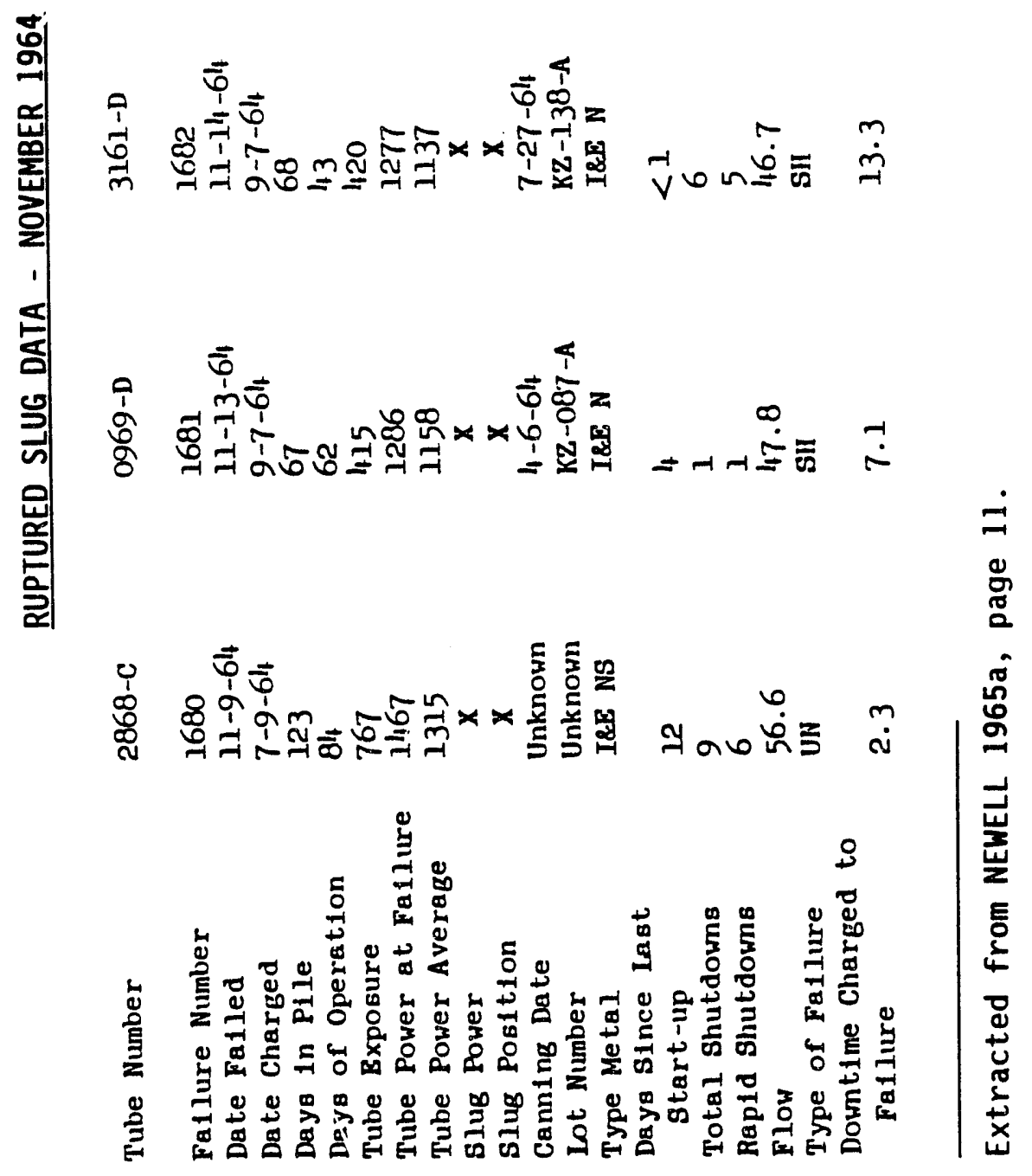




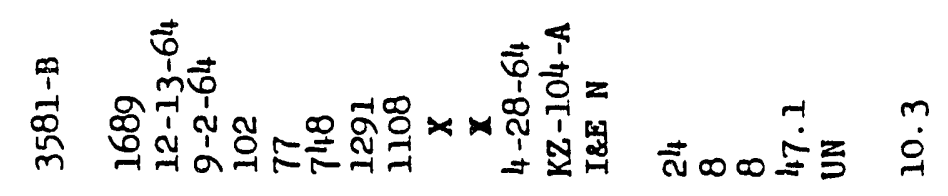

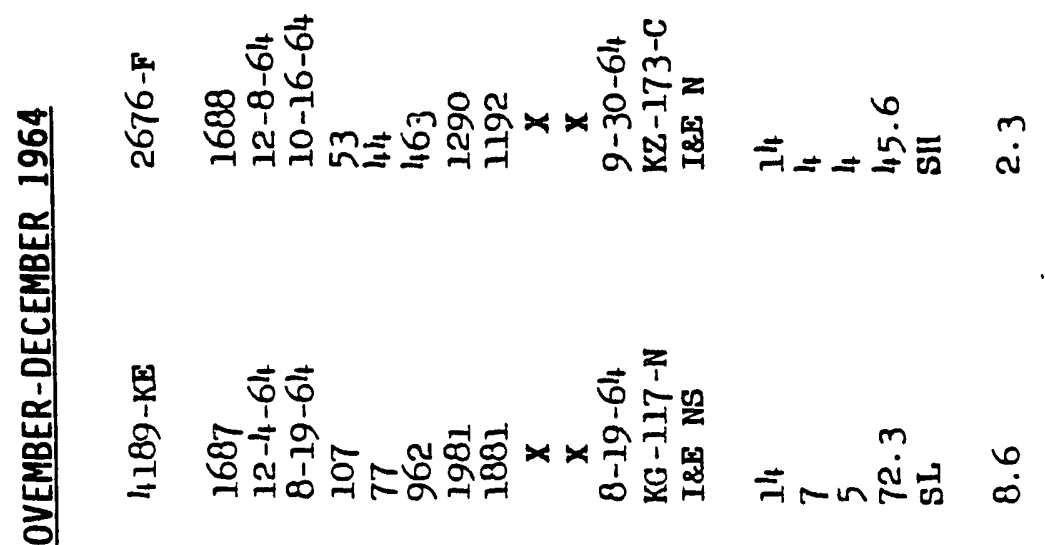

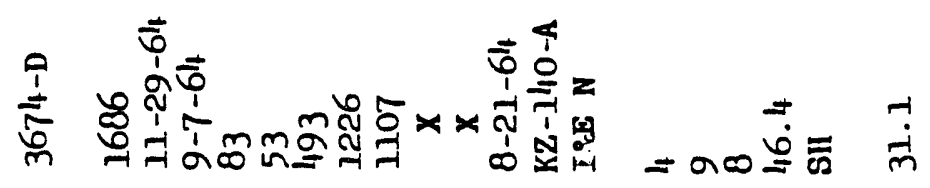

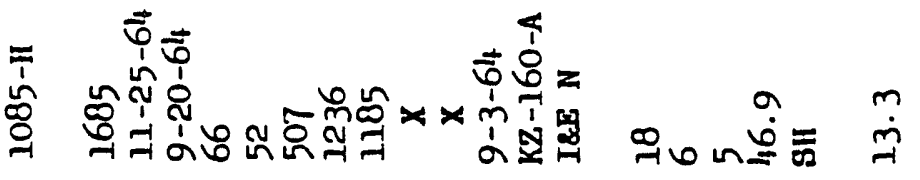

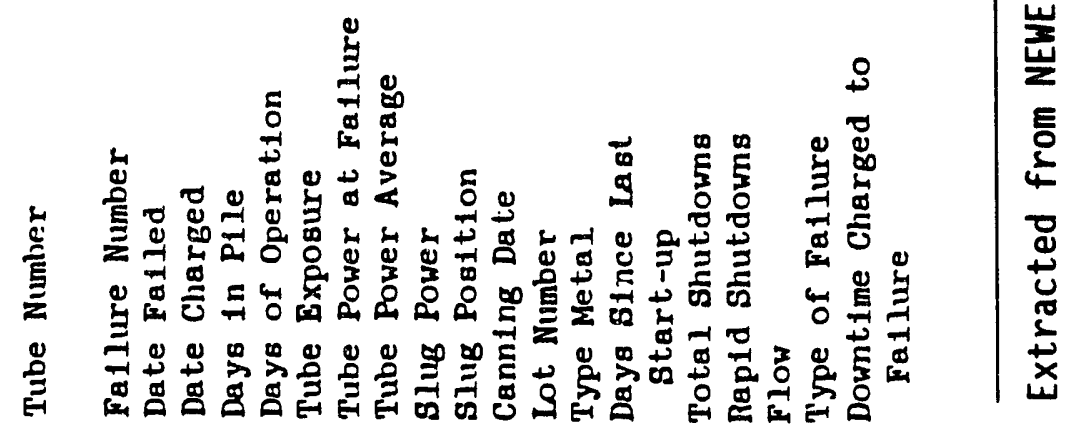

N. 19 

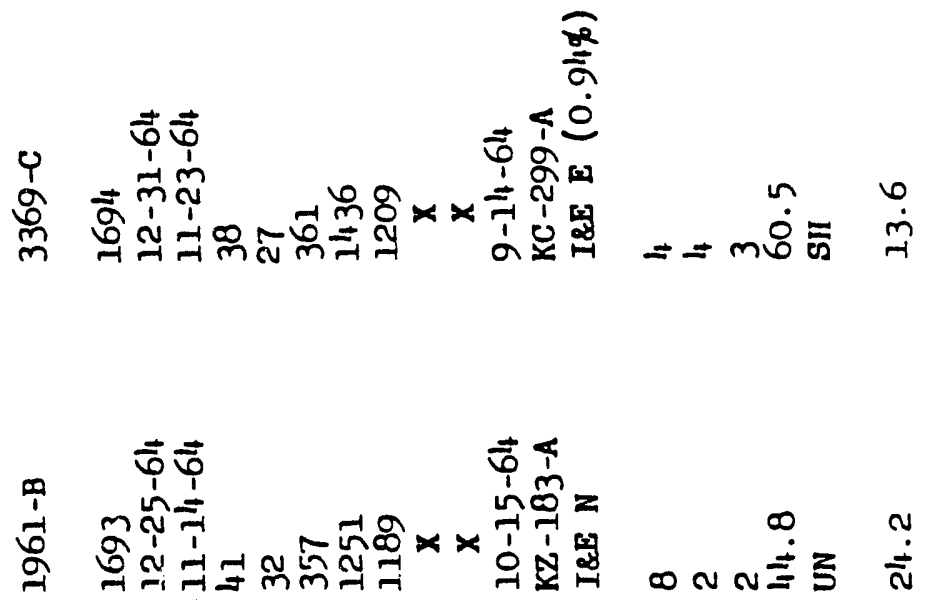

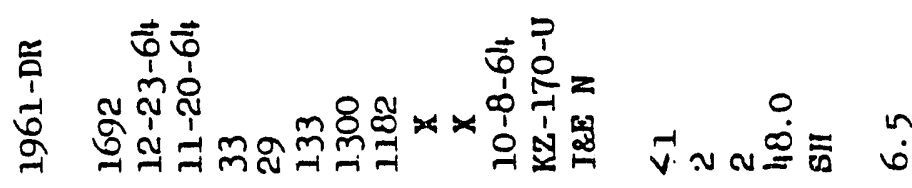

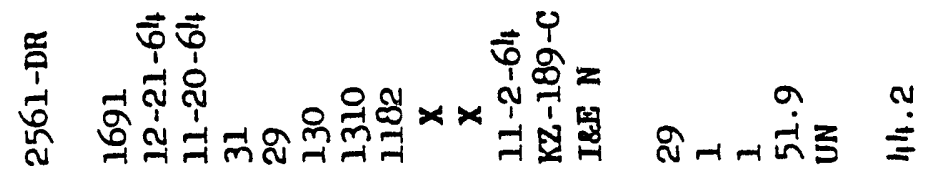

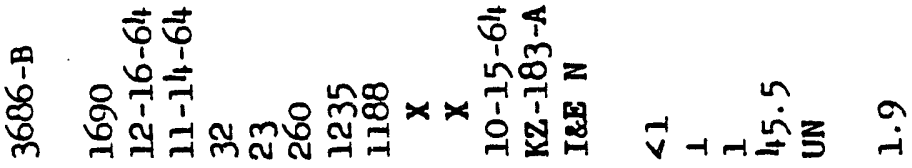

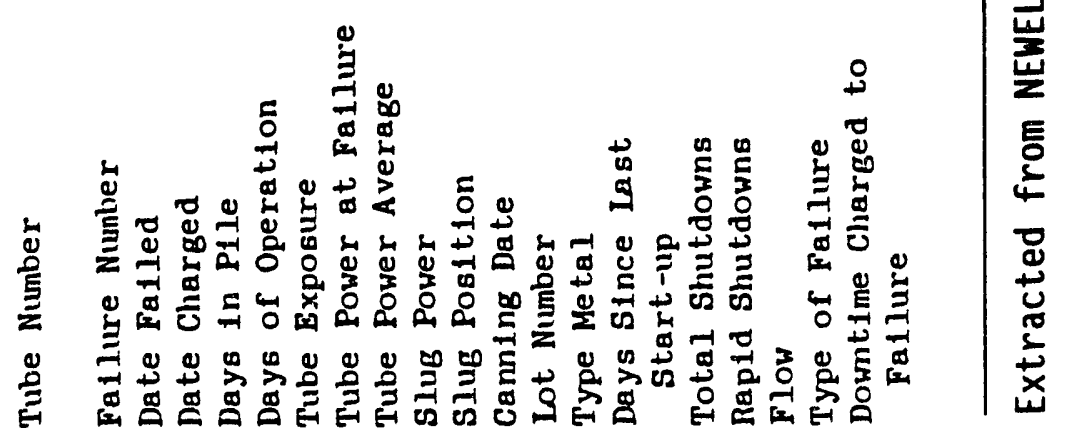




\section{APPENDIX 0}

FUEL-ELEMENT FAILURE DATA FOR 1965 


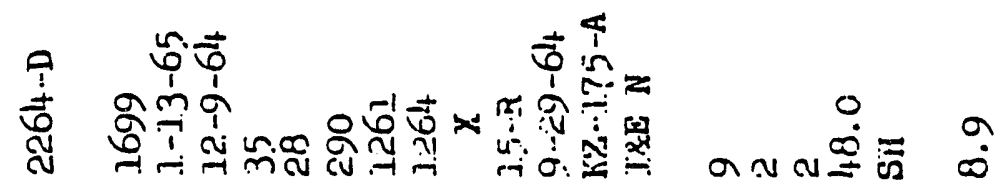

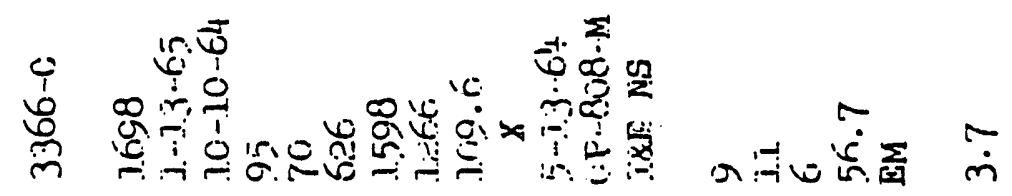

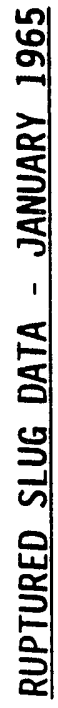

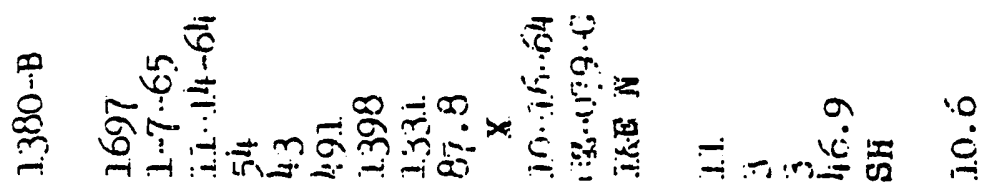

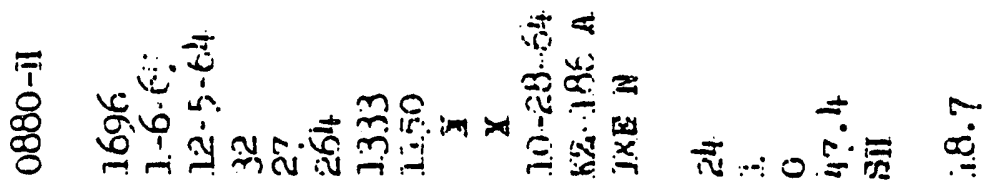

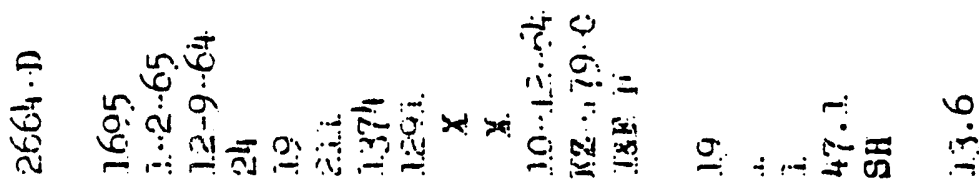

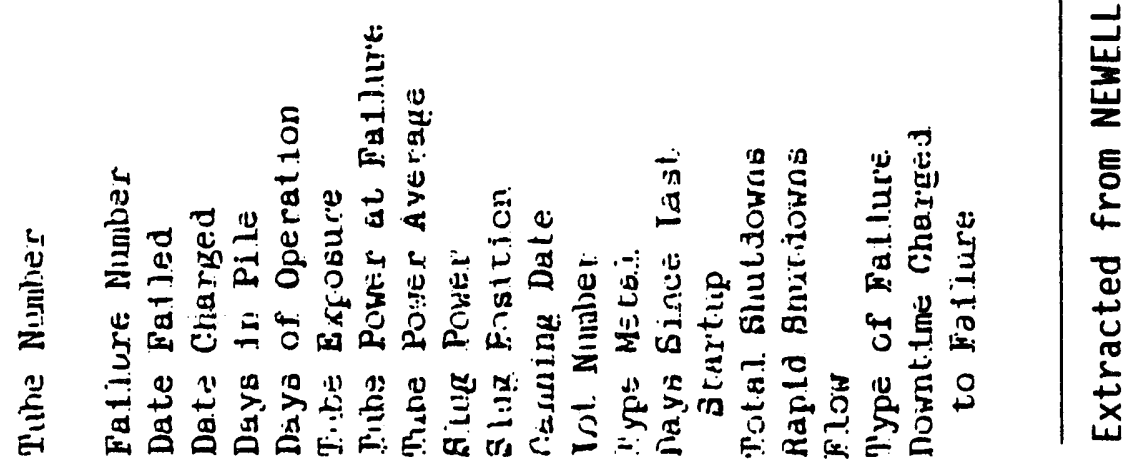




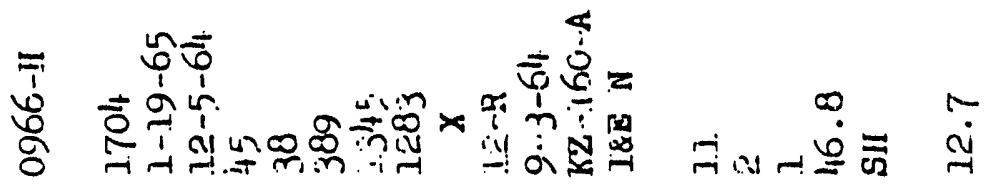

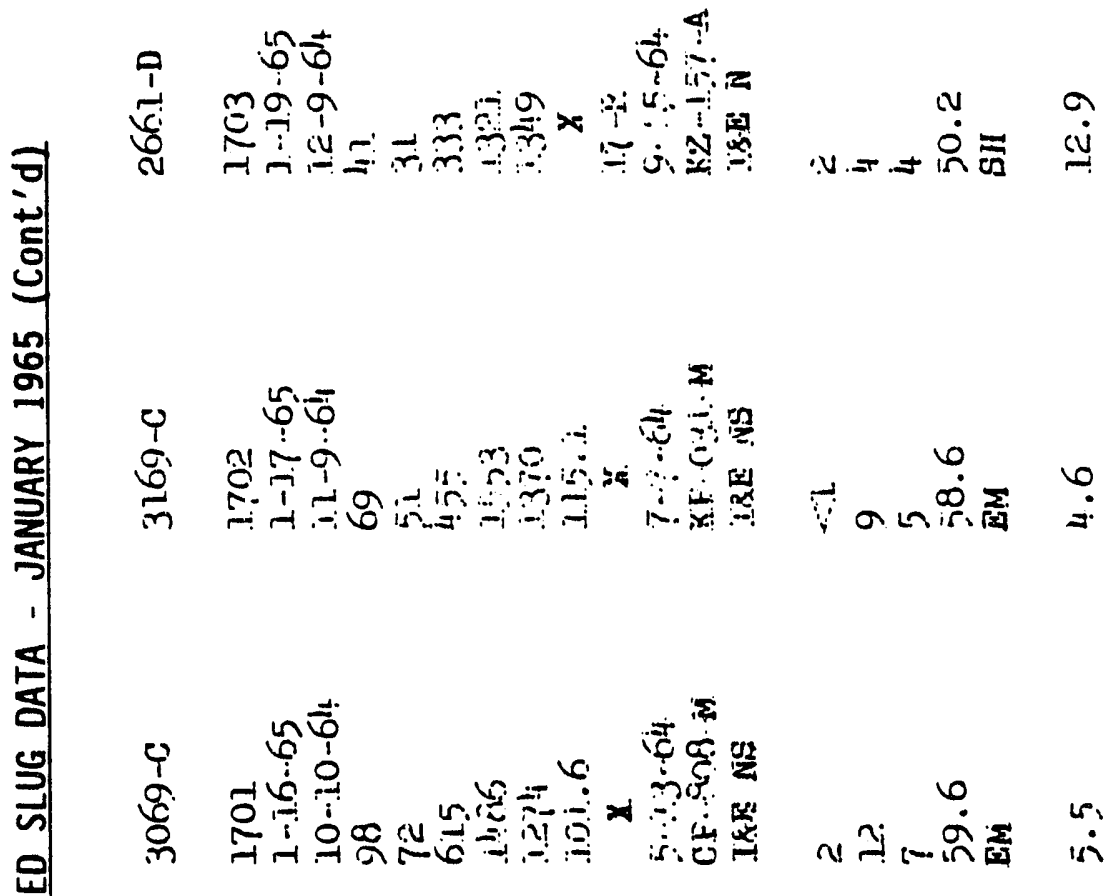

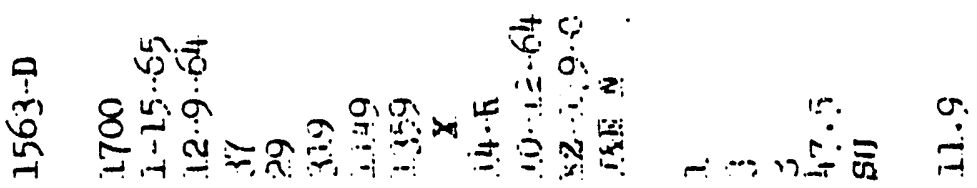

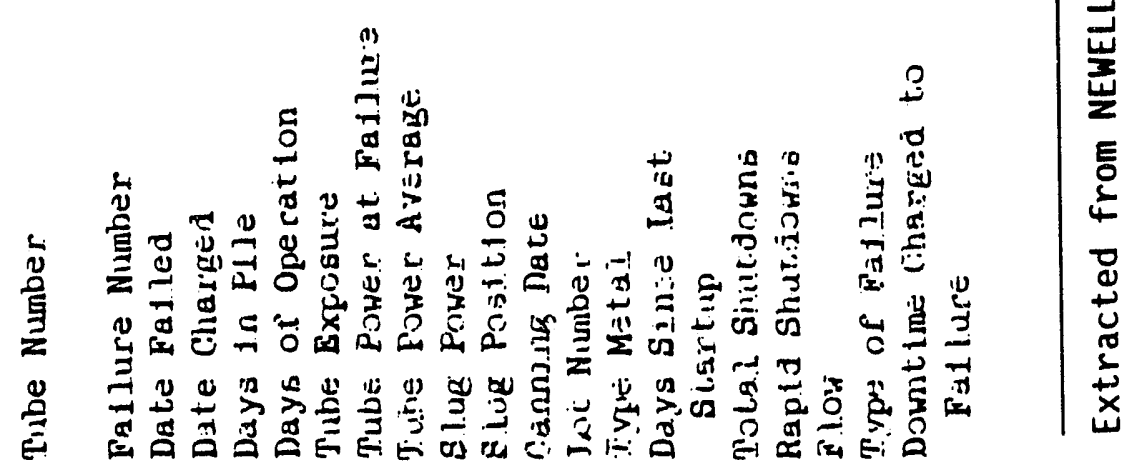




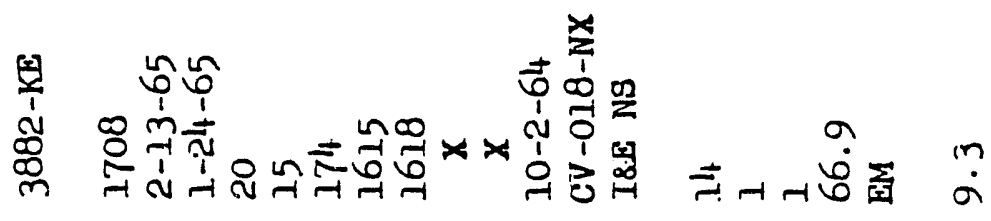

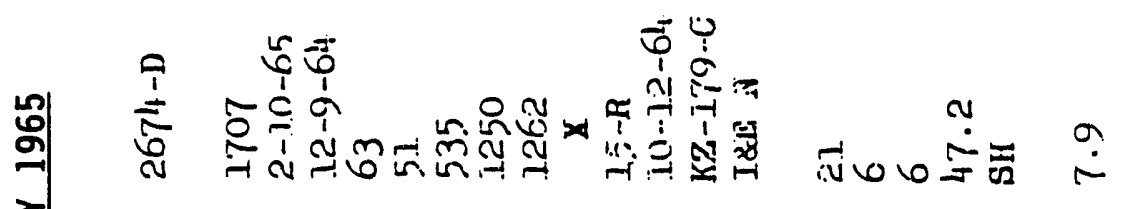

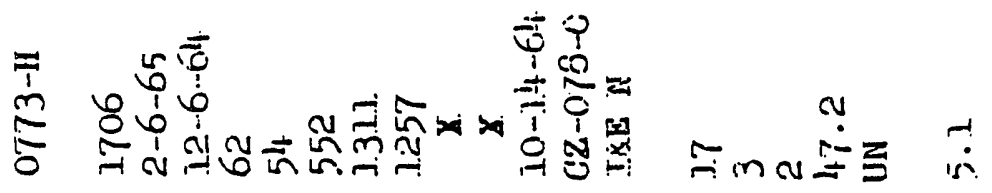

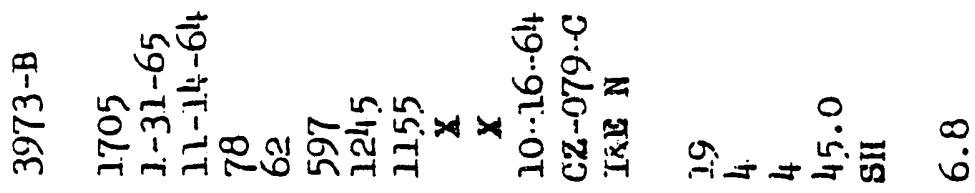

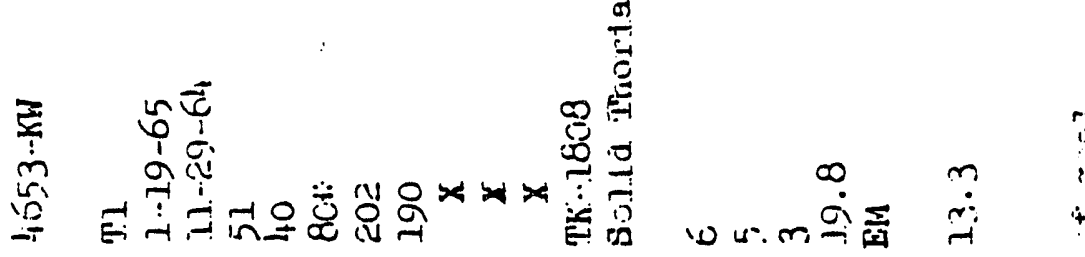

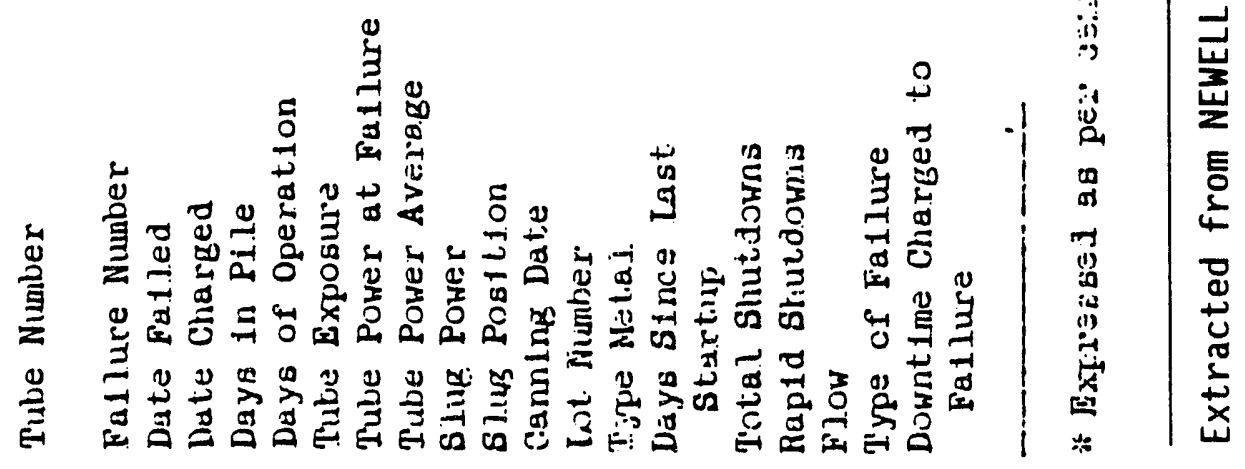


晃

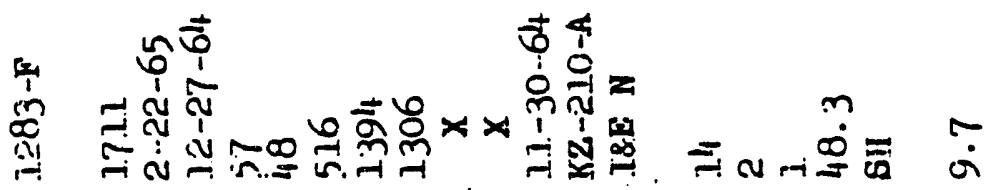

व̊.

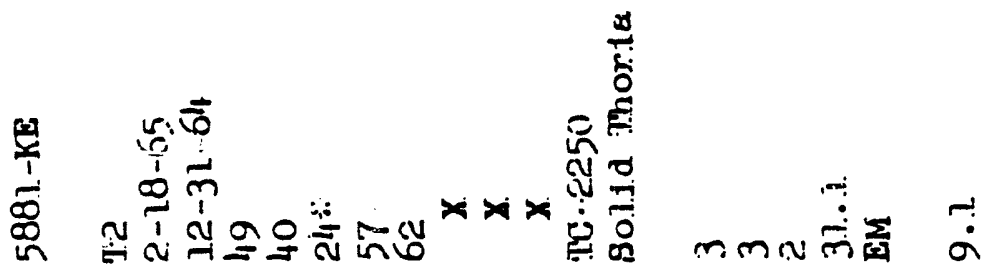

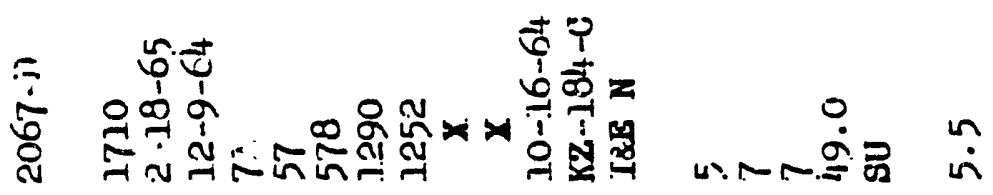

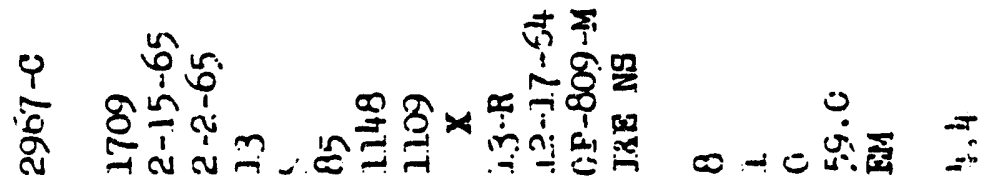

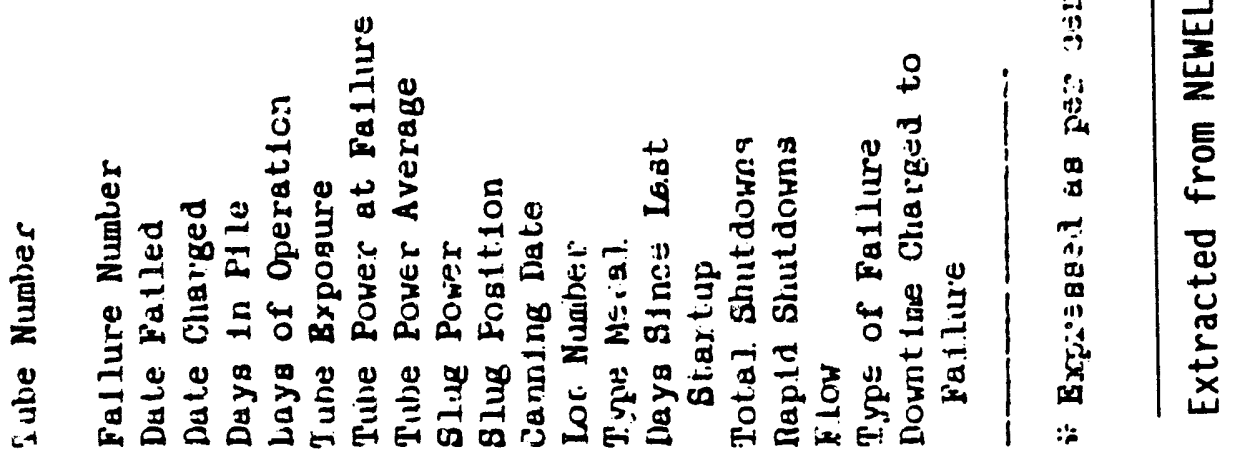

0.4 


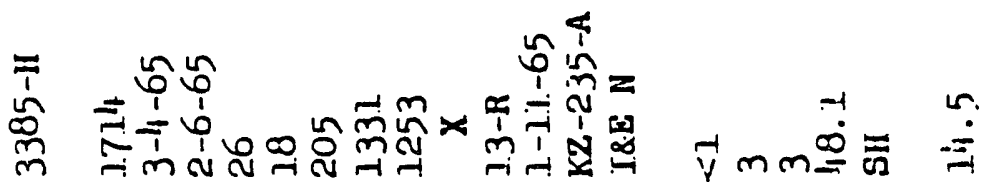

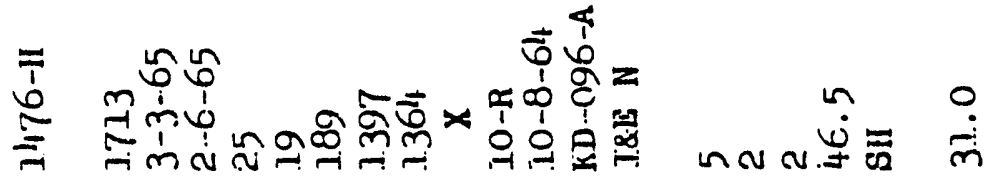

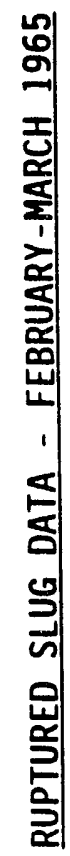

量

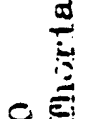

कृ.

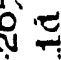

ᄋำ

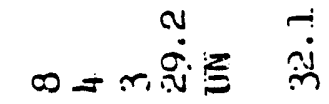
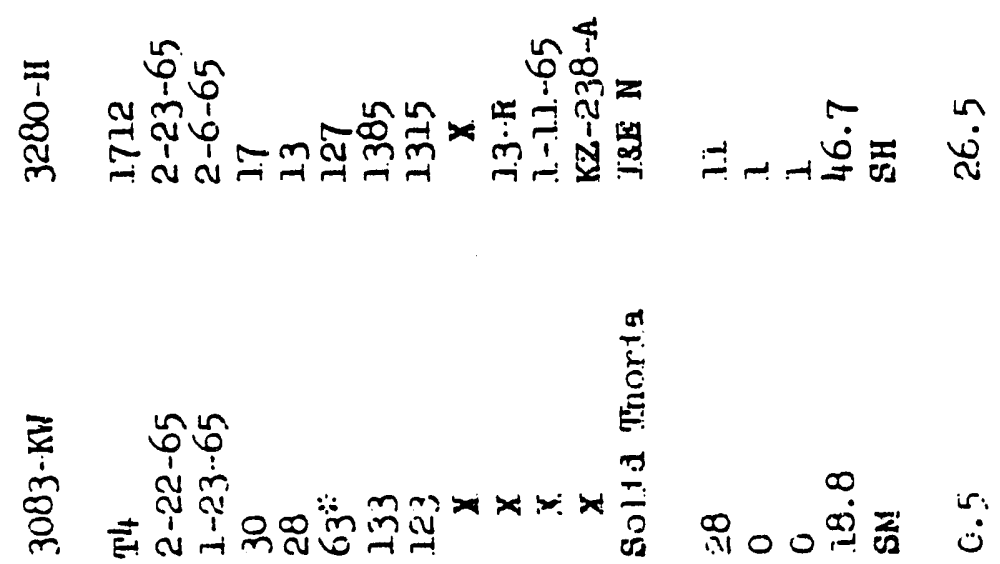

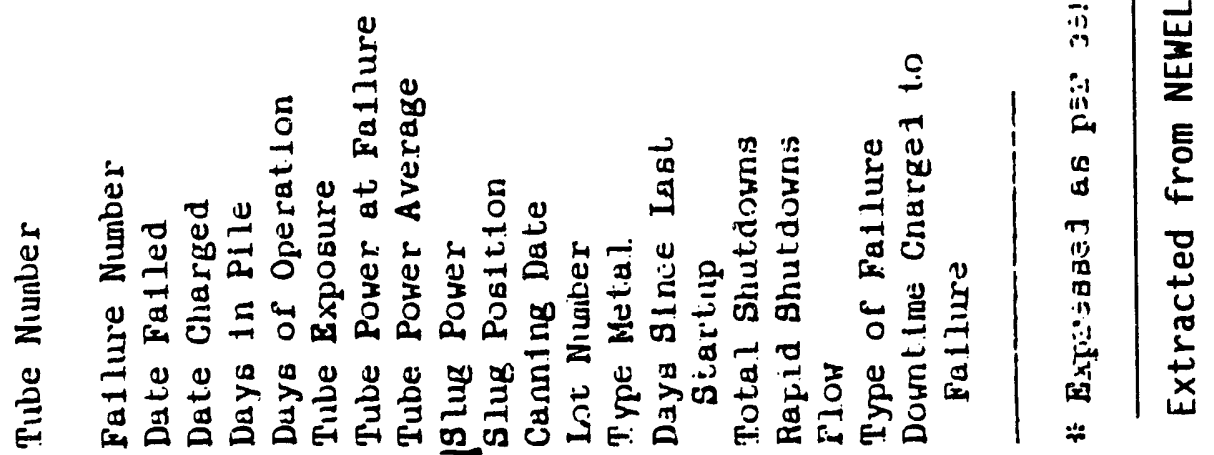




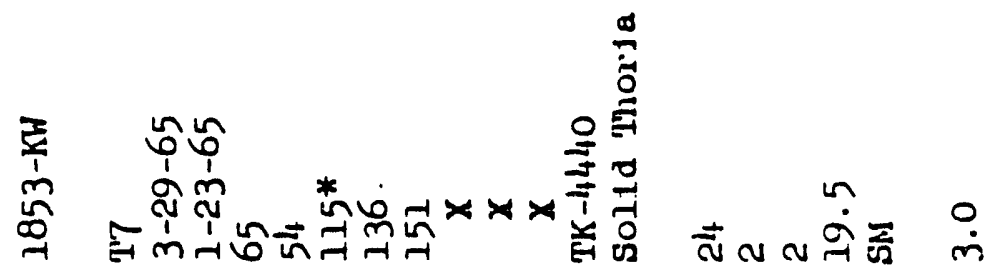

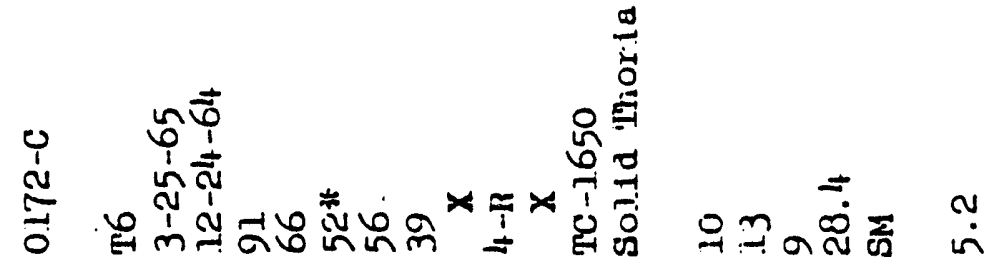

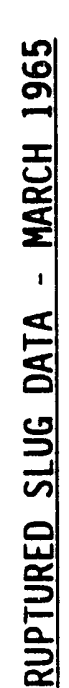

A.

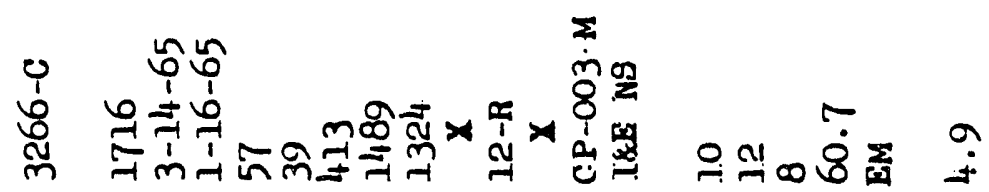

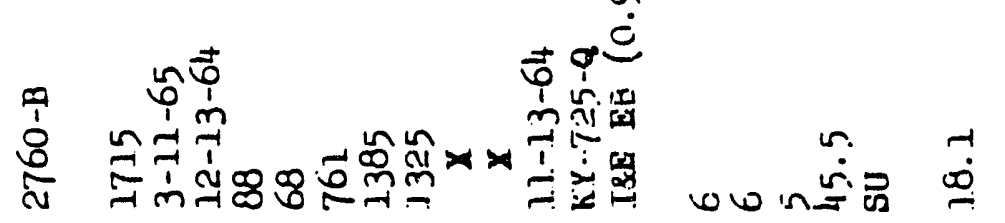

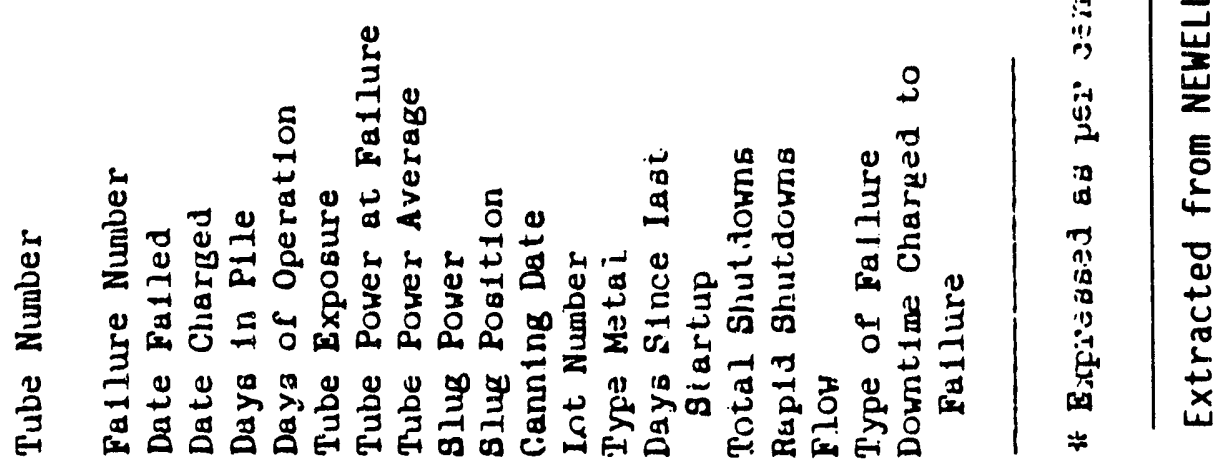

0.6 


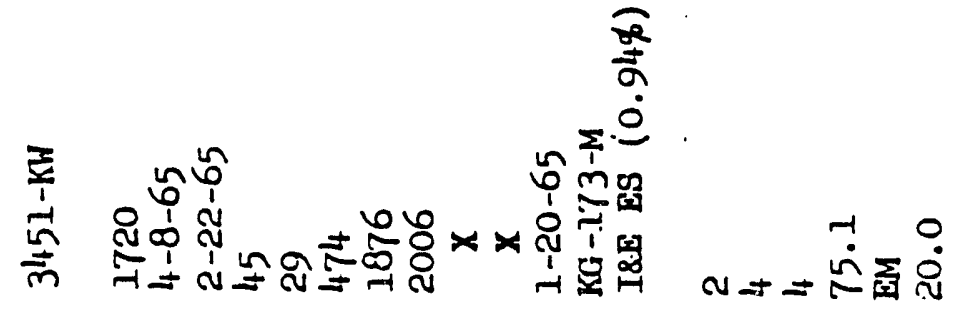

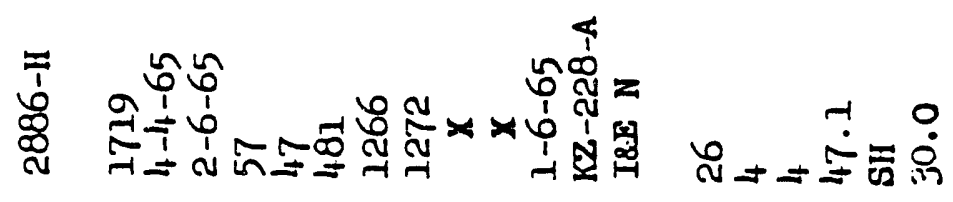

:

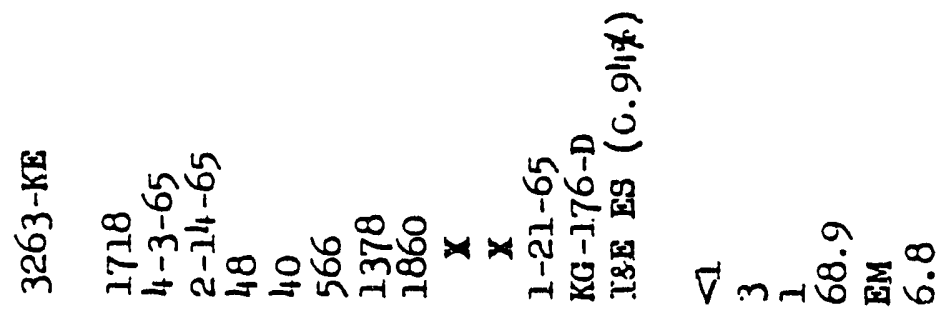

营

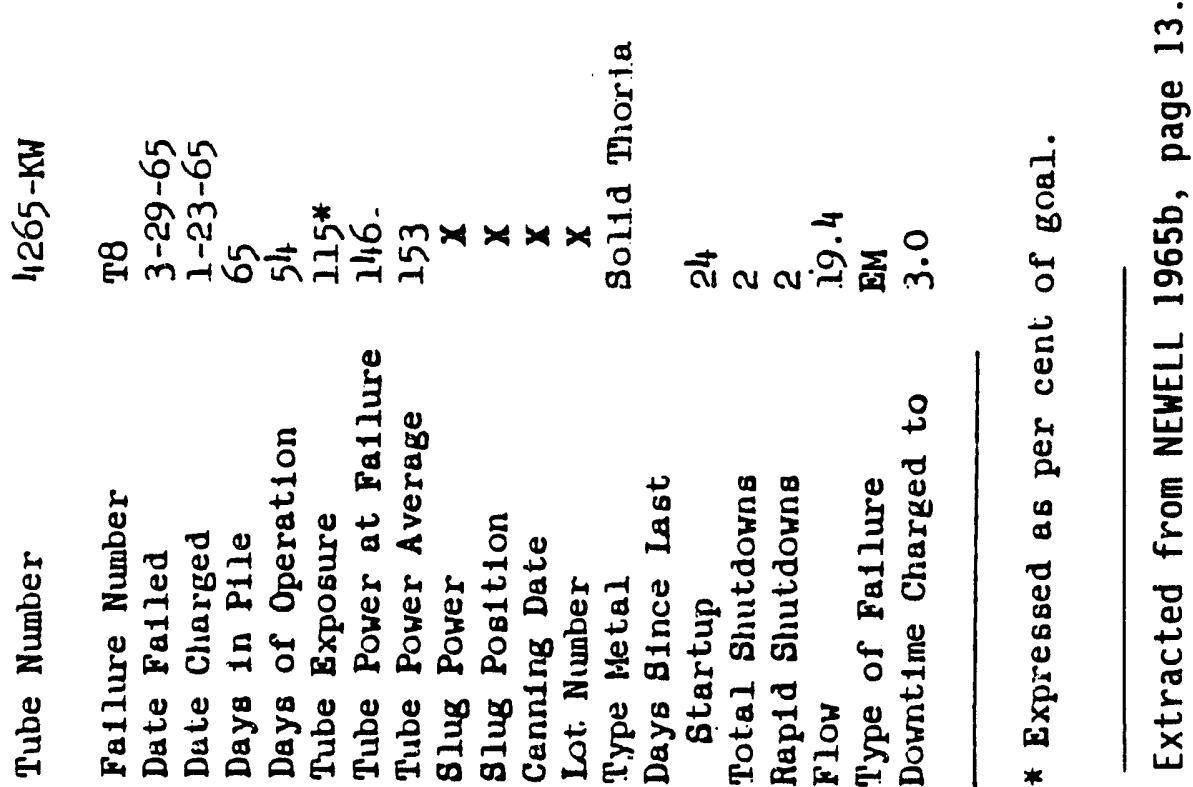

0.7 


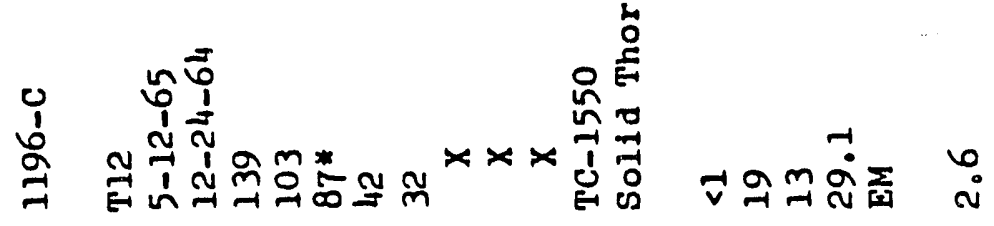

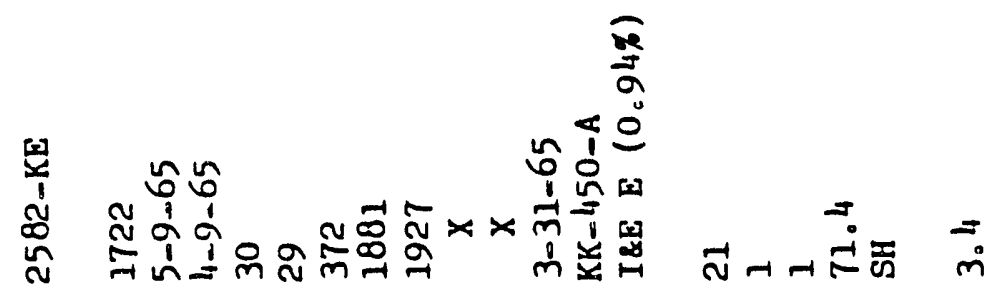

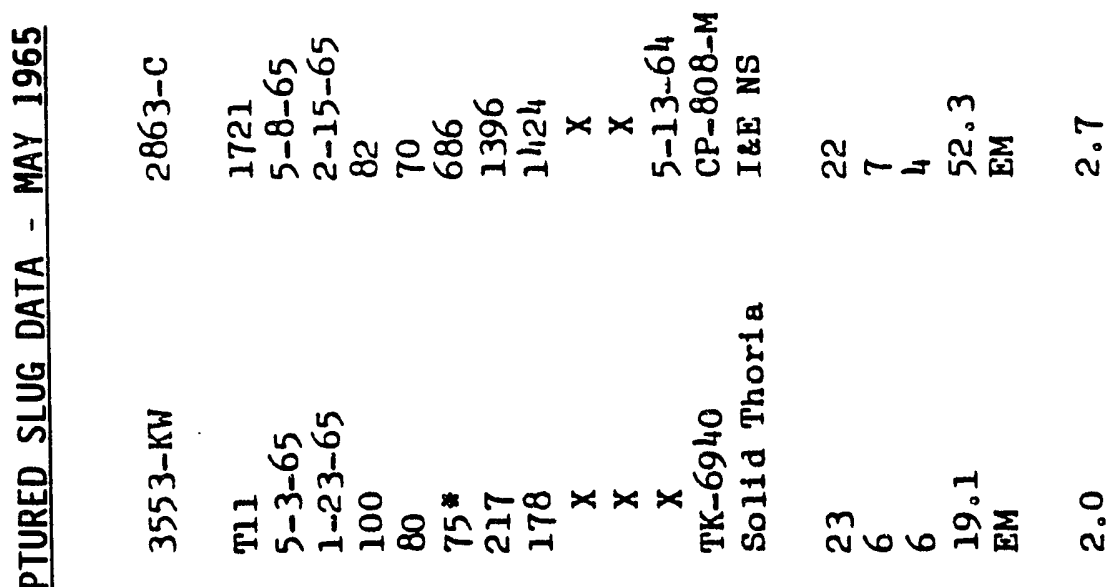

章

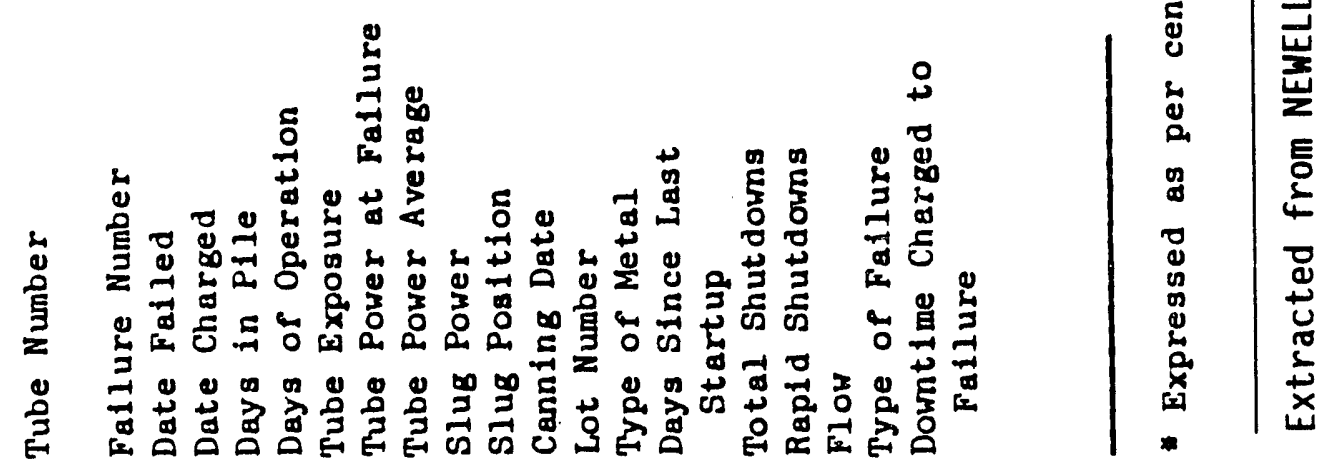

0.8 


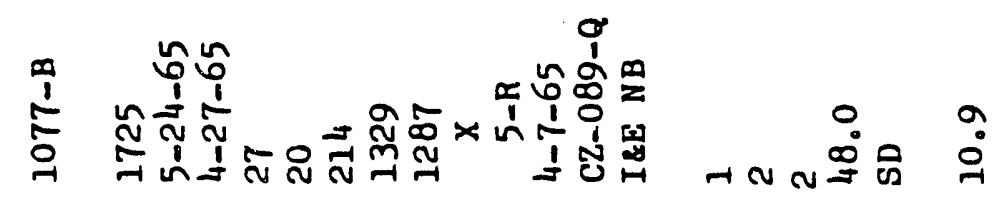

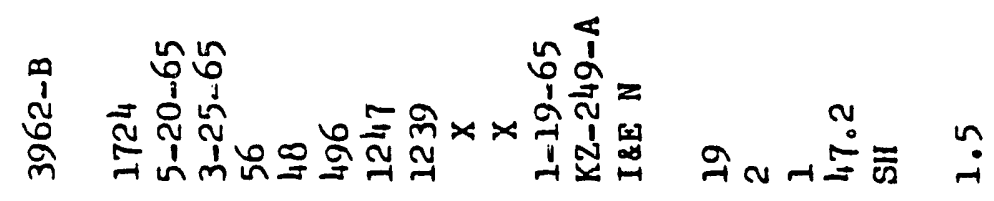

:

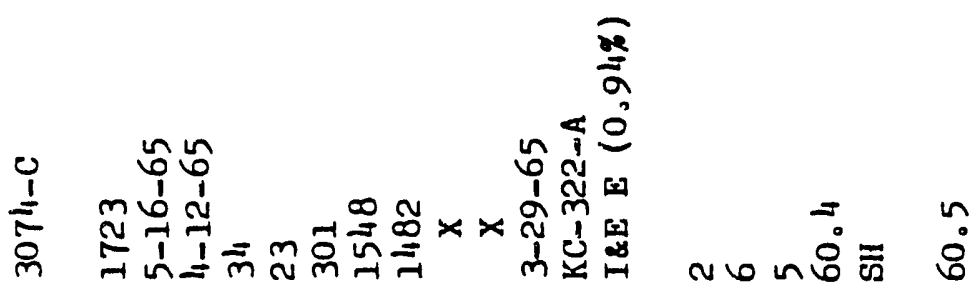

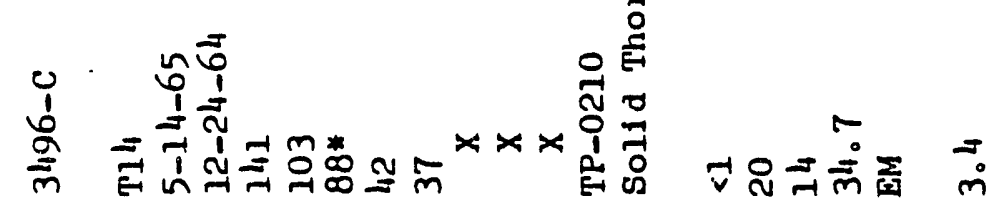

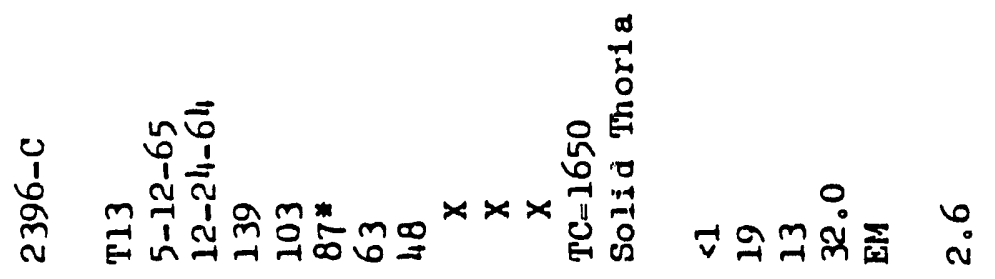

等

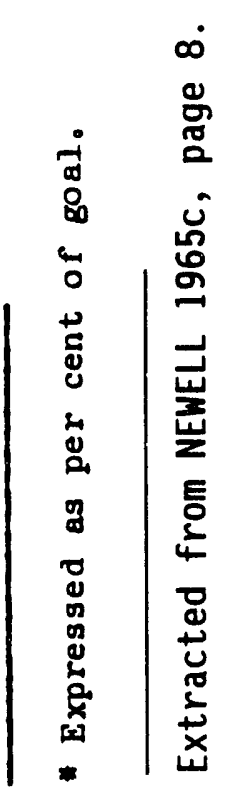




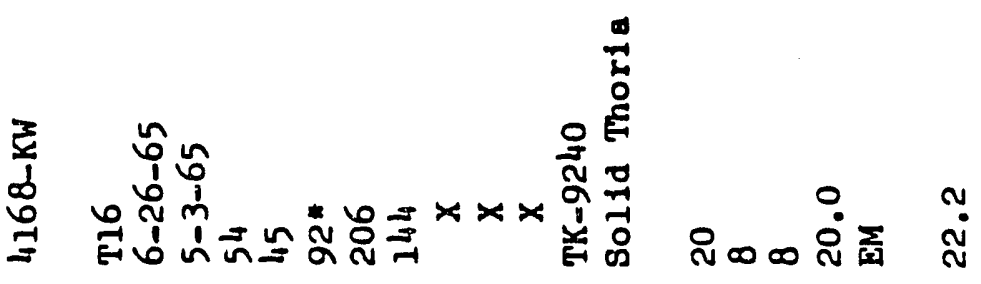

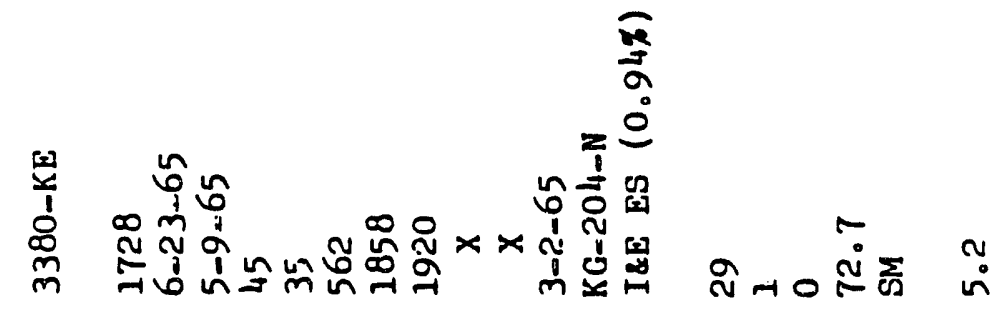

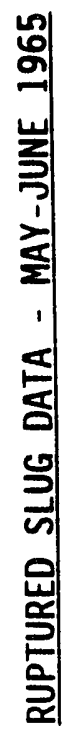
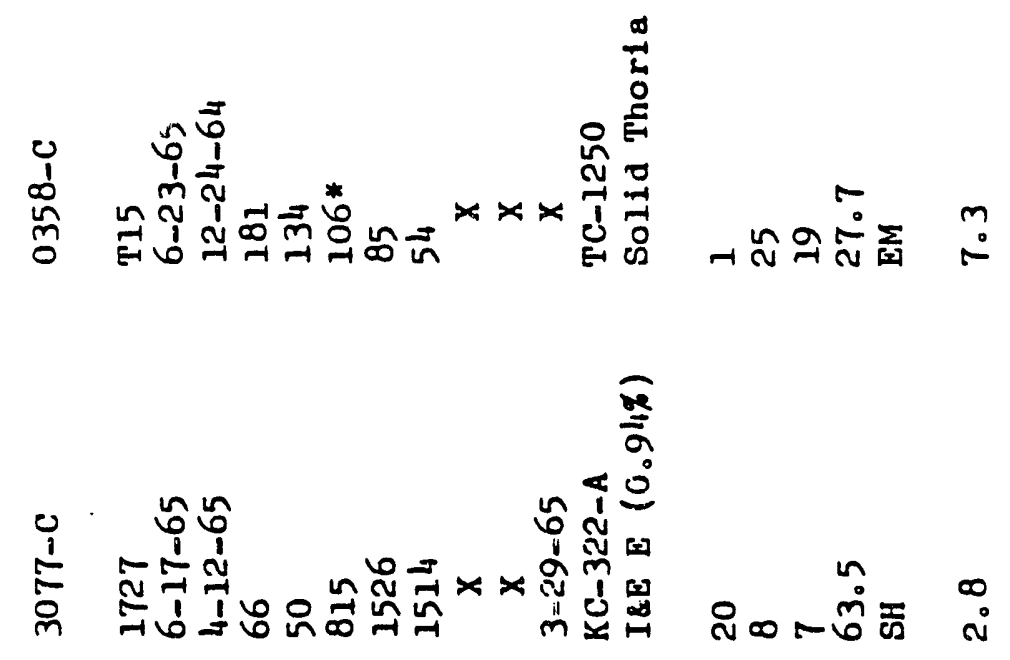

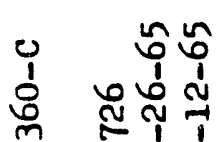

$n \leqslant 0^{\circ}$

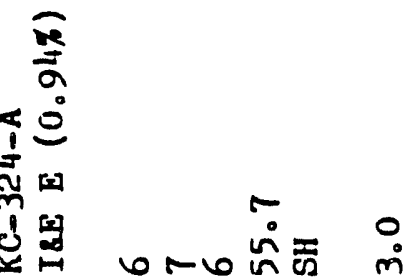

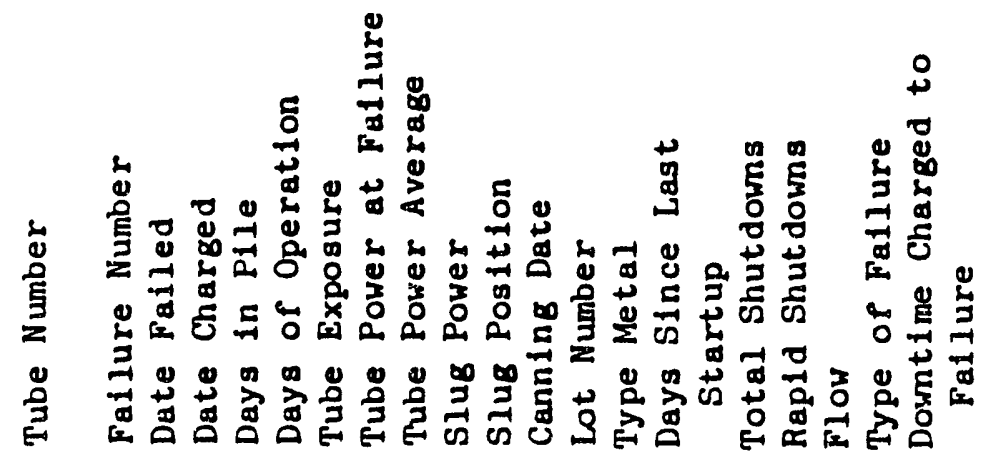

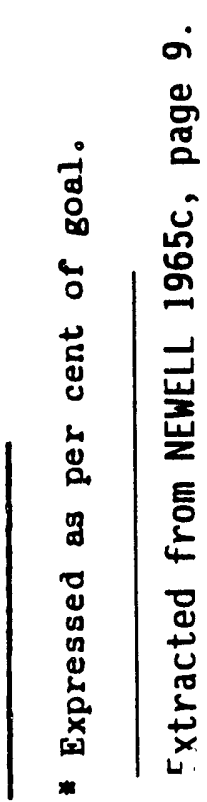


焉

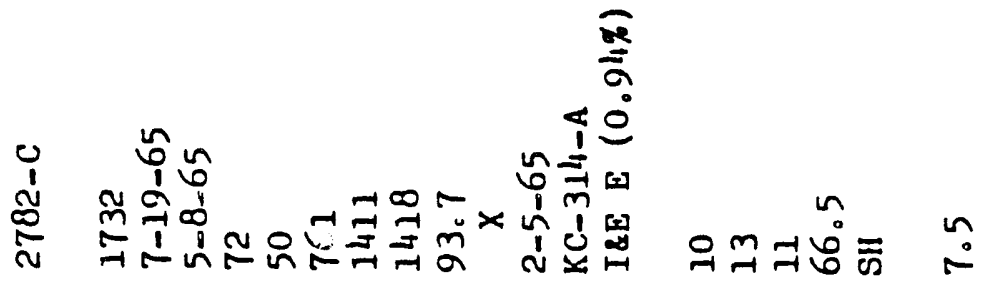

ป̊

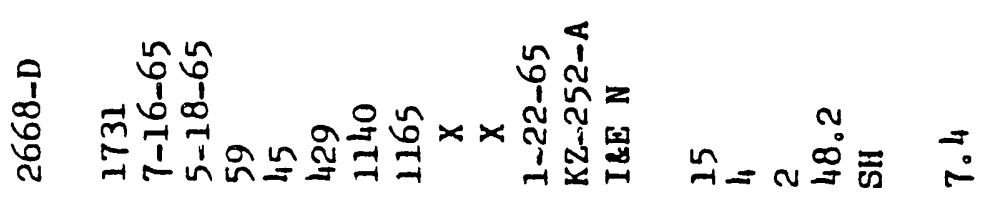

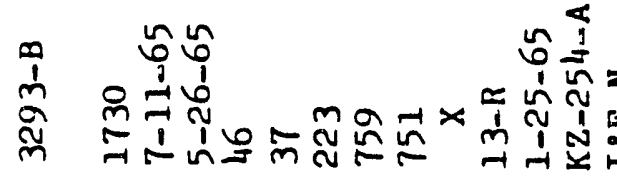

เ

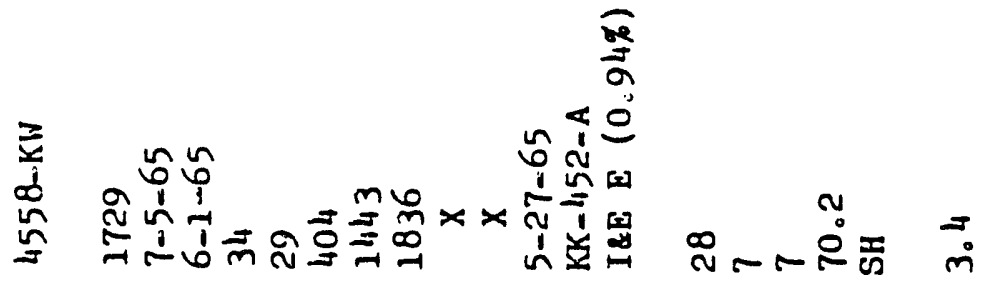

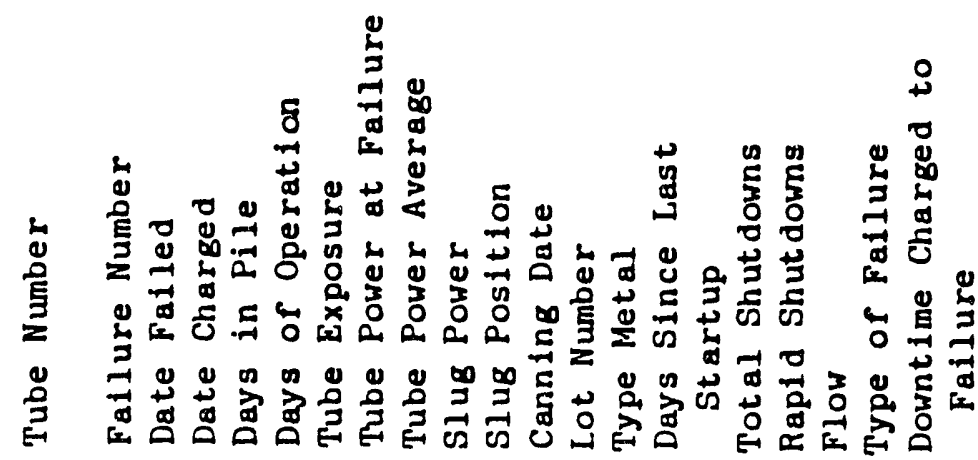

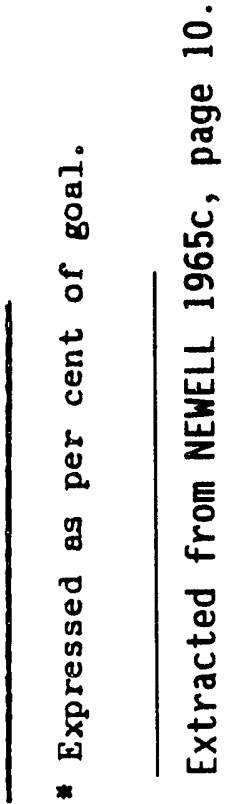

0.11 


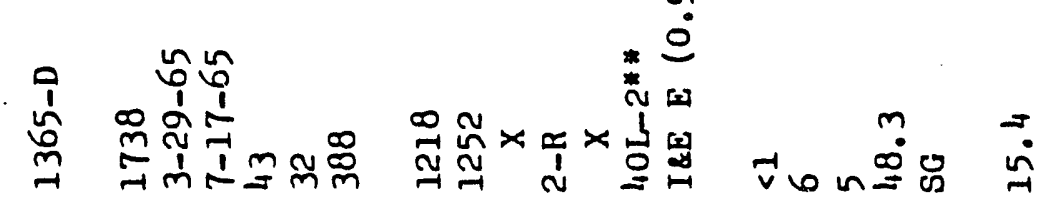

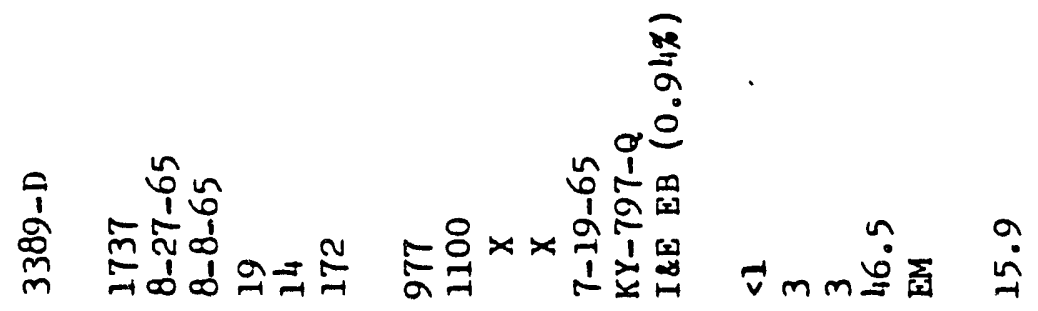

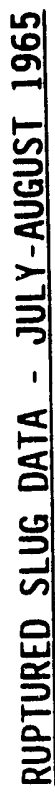

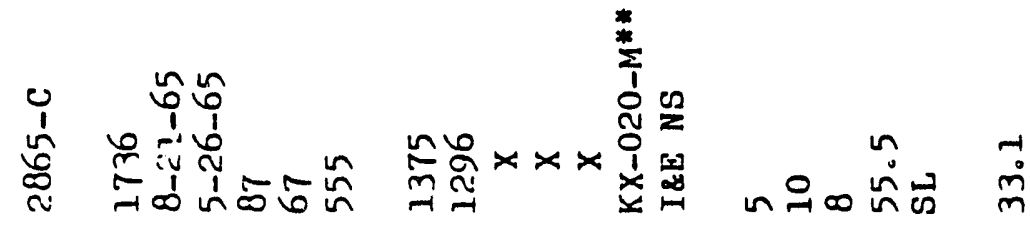

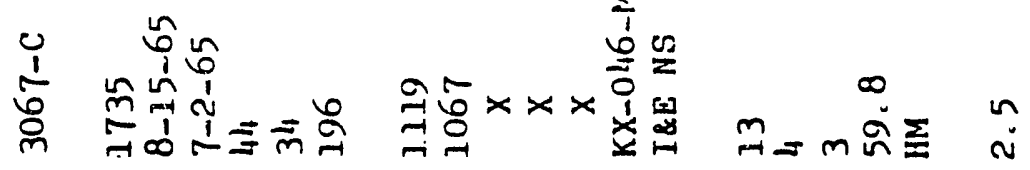

$\stackrel{*}{*}$

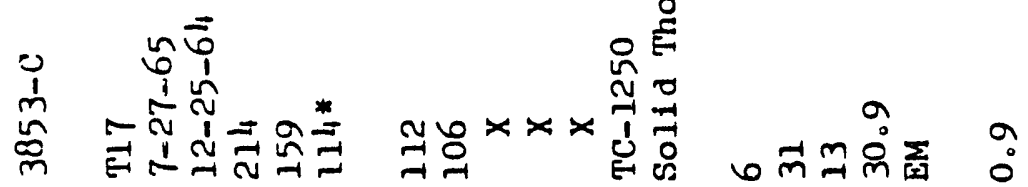

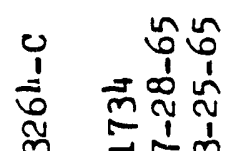

$\sum_{0}^{1}$

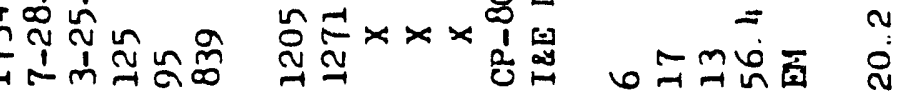

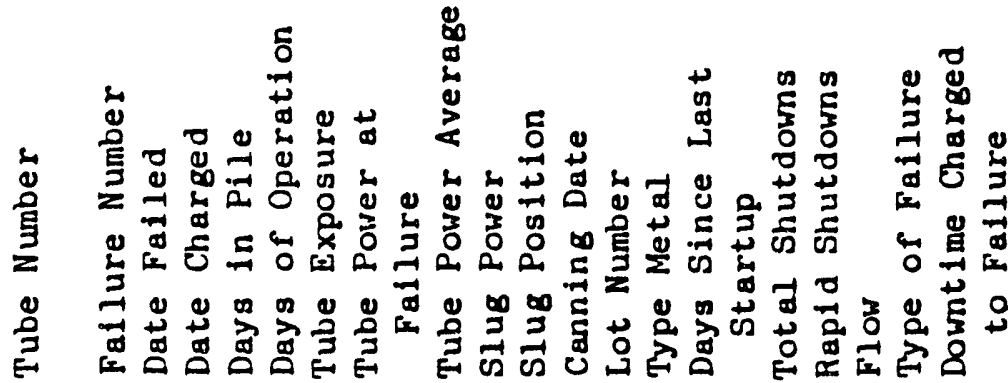




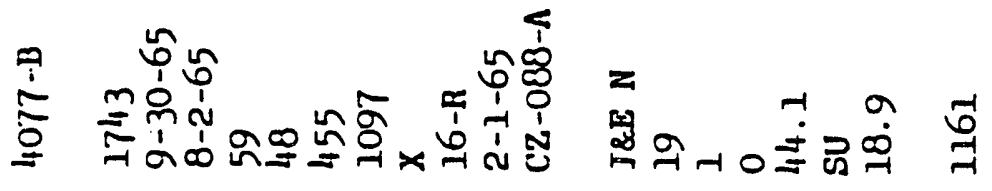

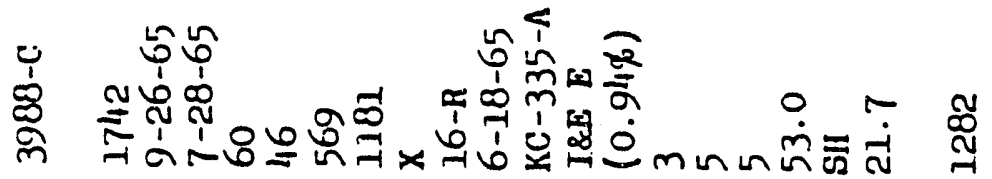

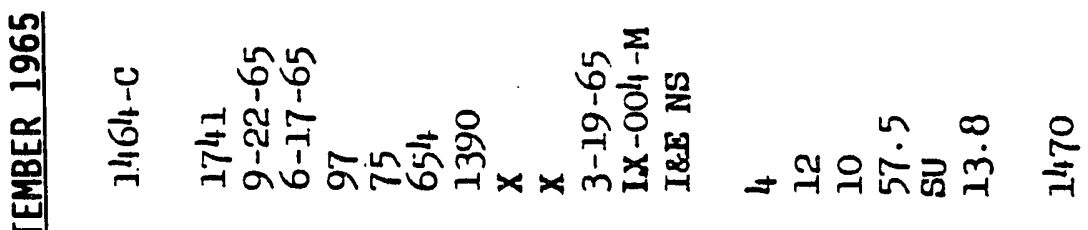

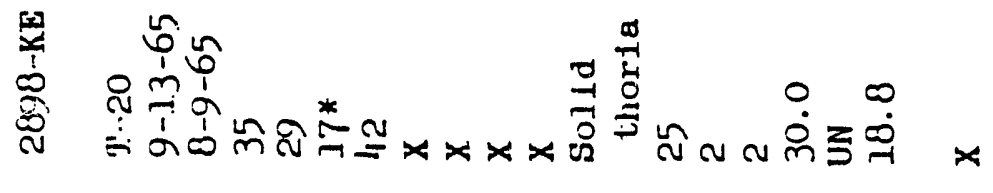

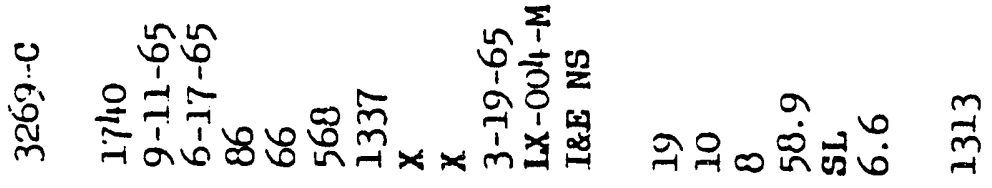

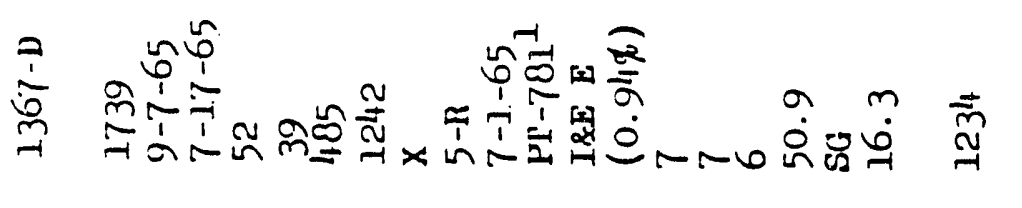

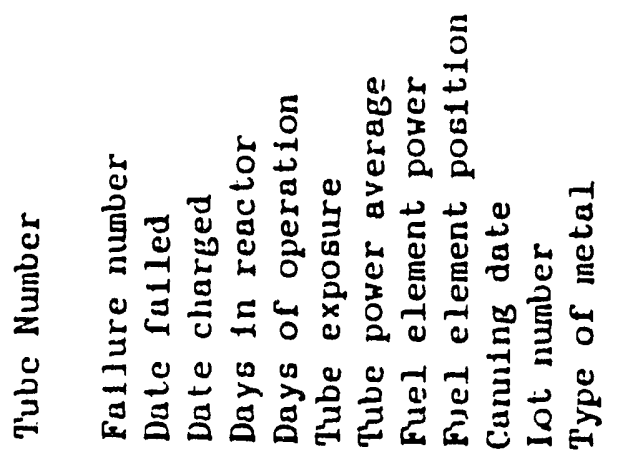

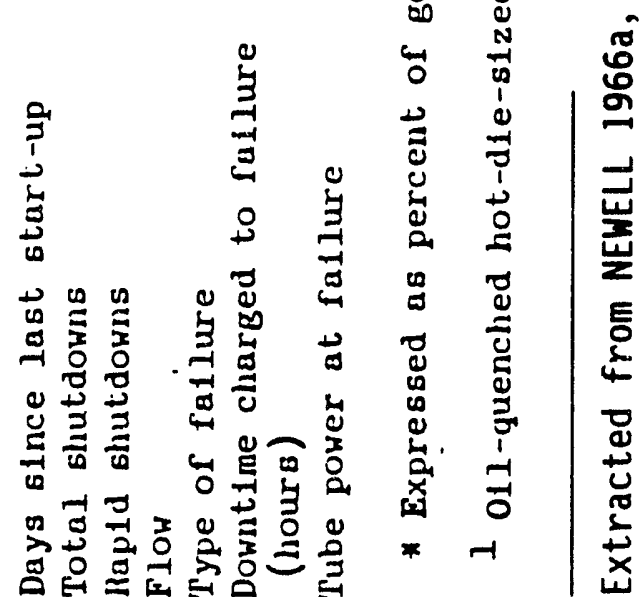




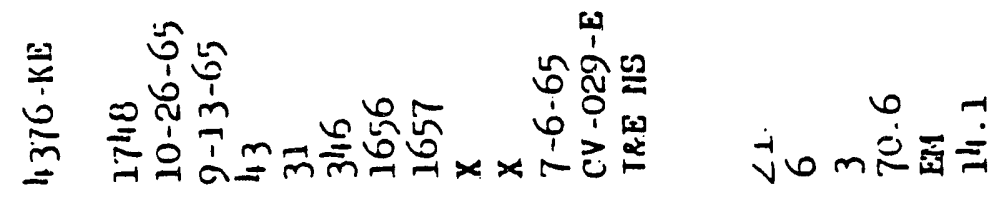

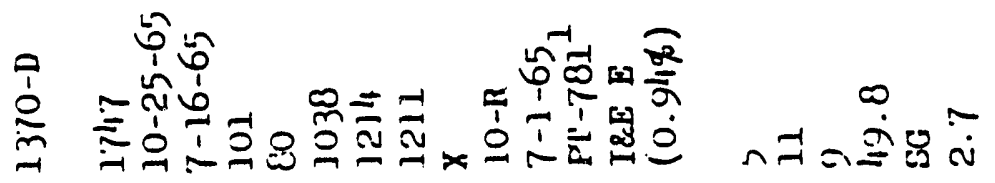

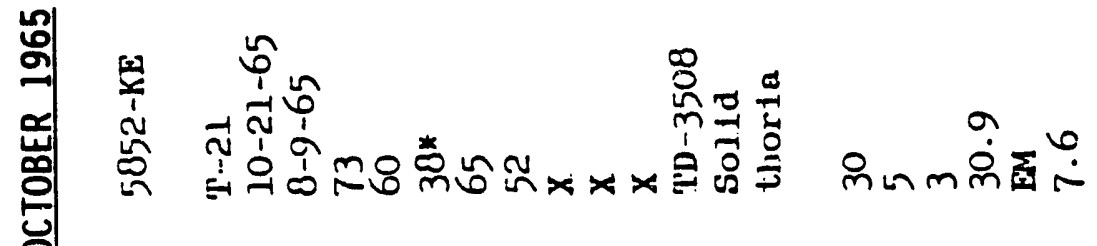

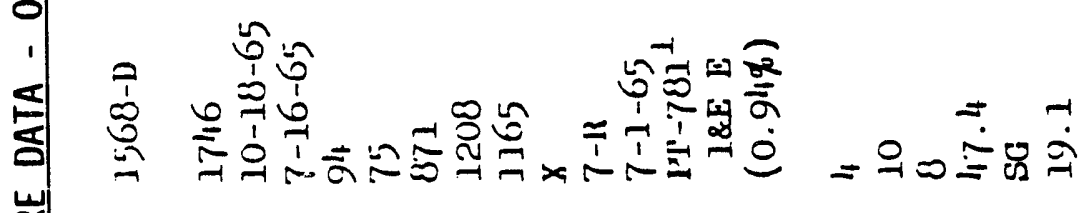

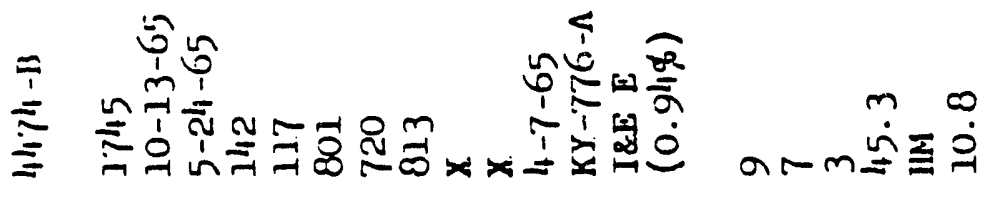

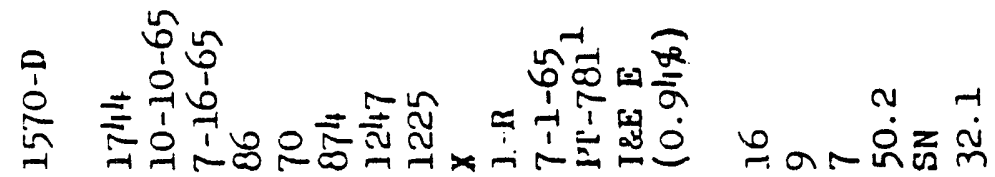
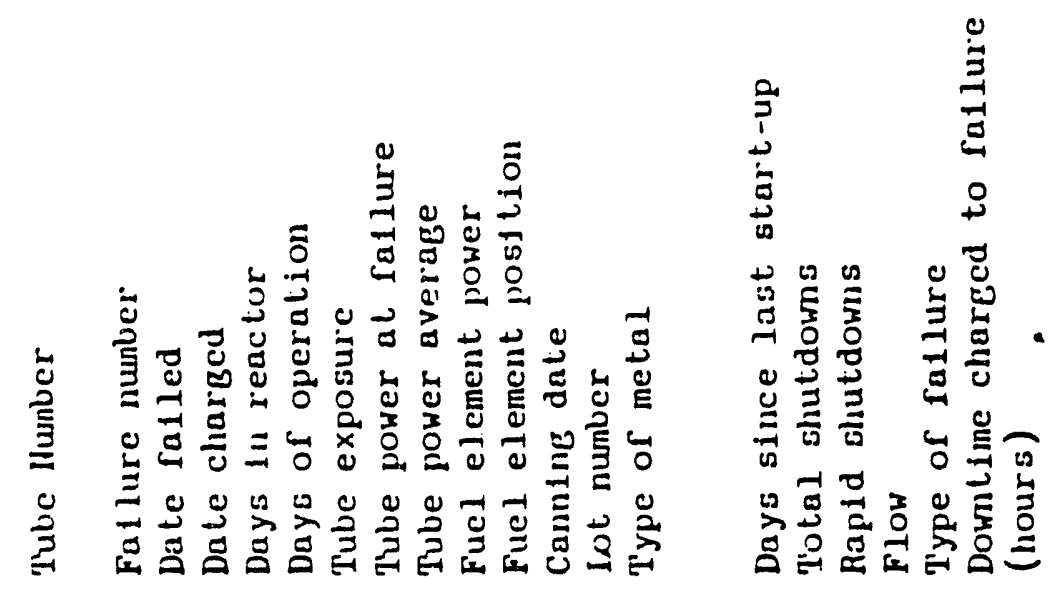

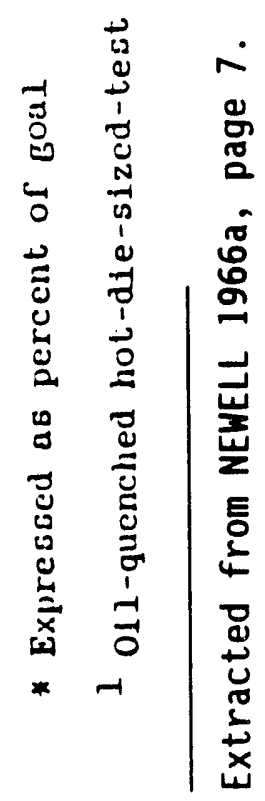




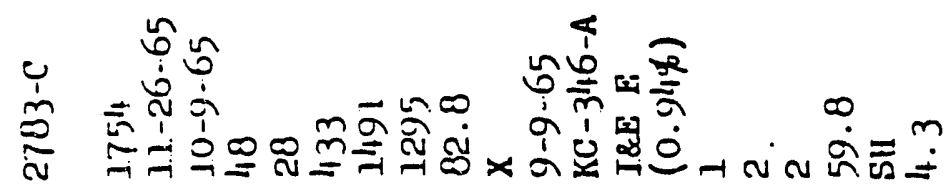

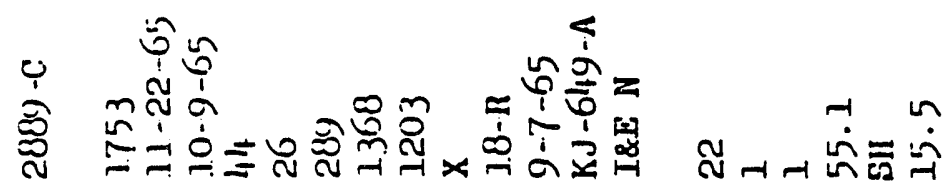

영

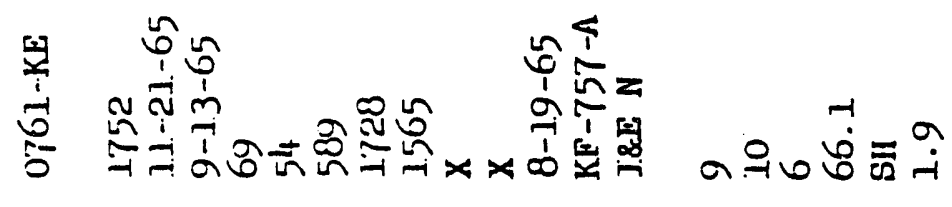

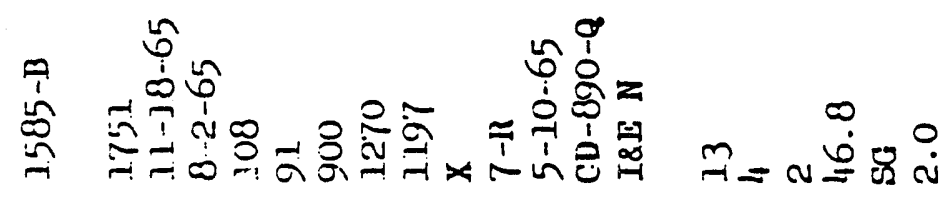

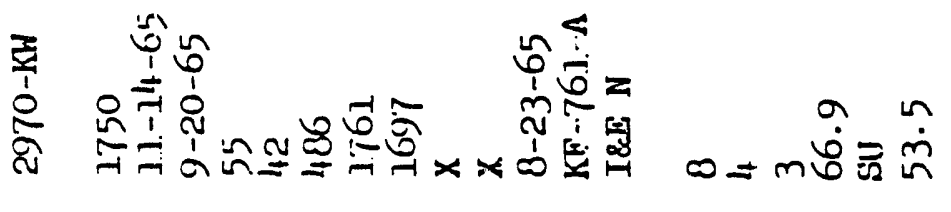

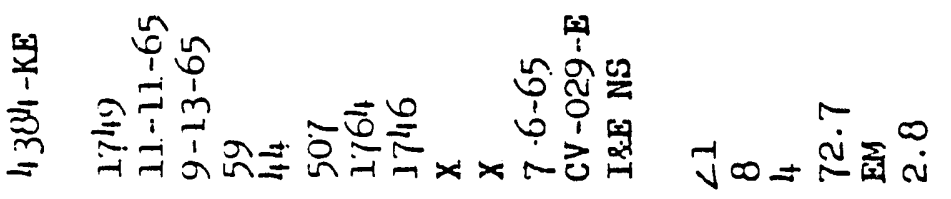

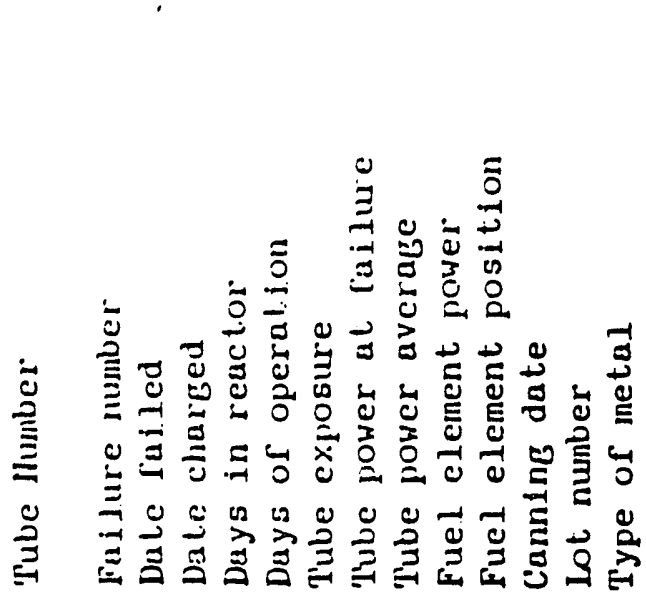

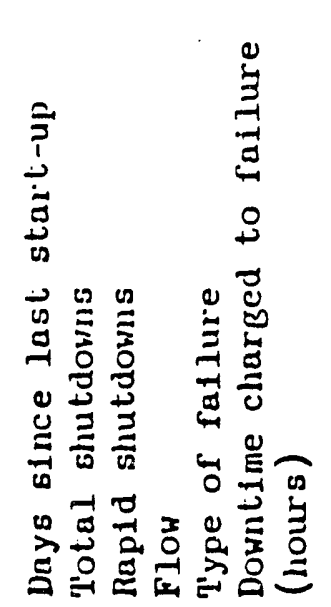

0.15 


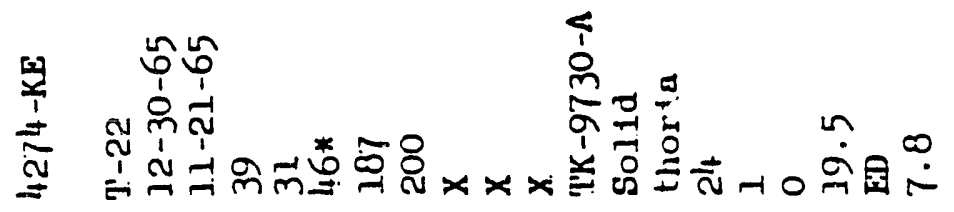

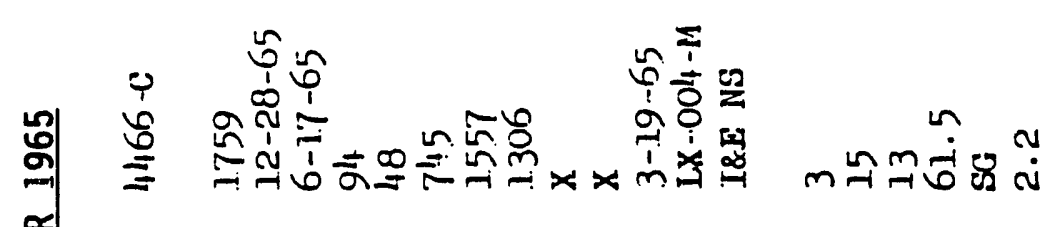

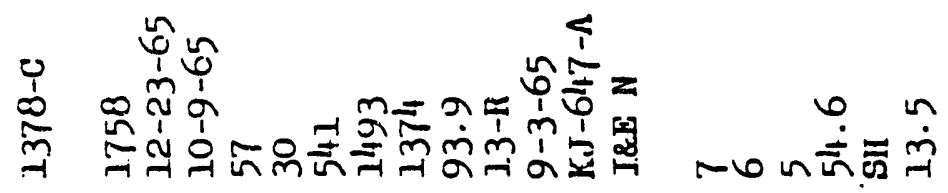

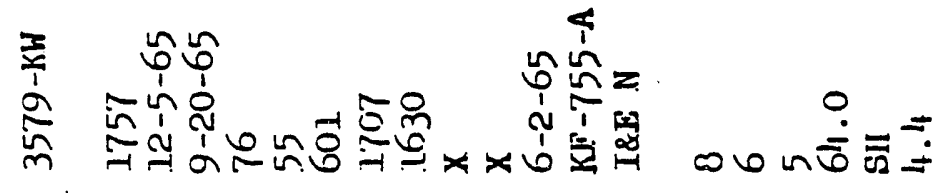

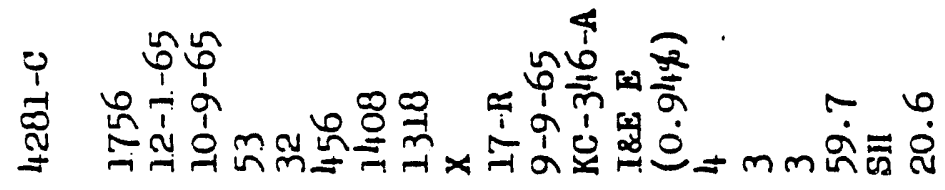

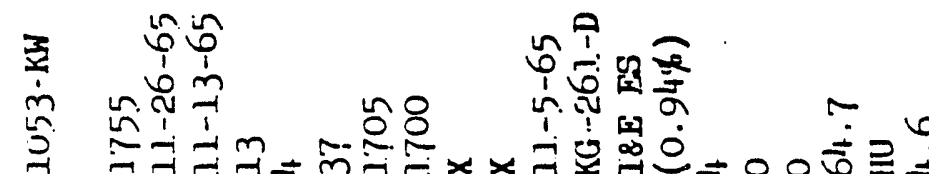

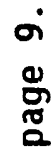

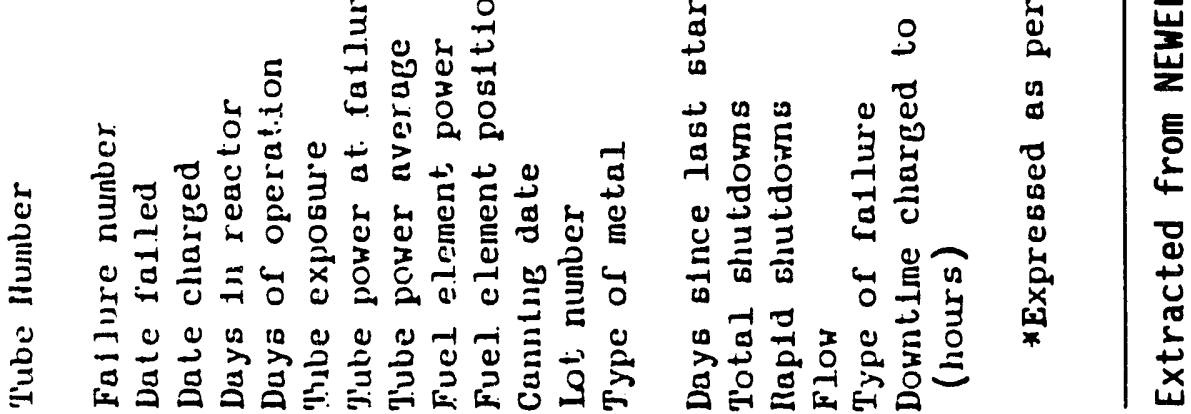


APPENDIX P

FUEL-ELEMENT FAILURE DATA FOR 1966 


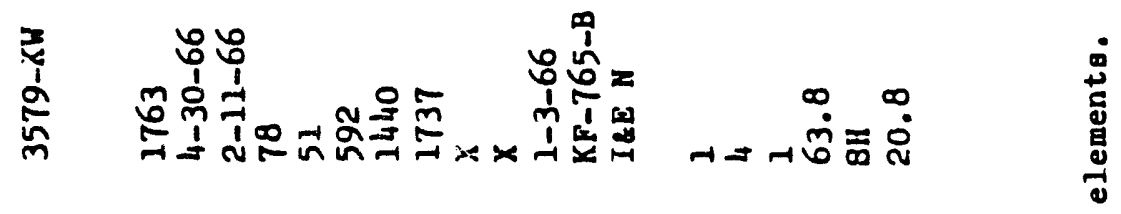

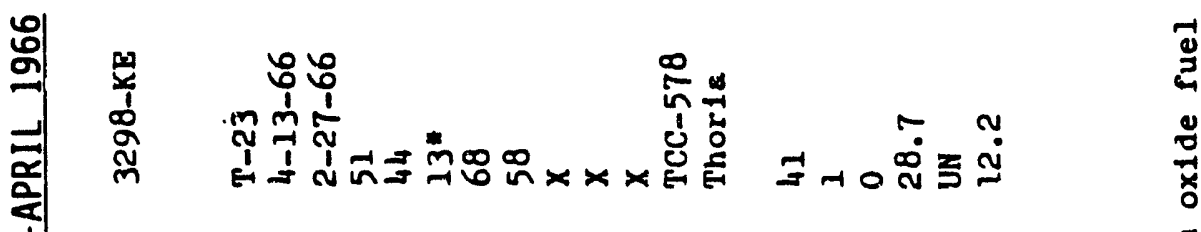

龱

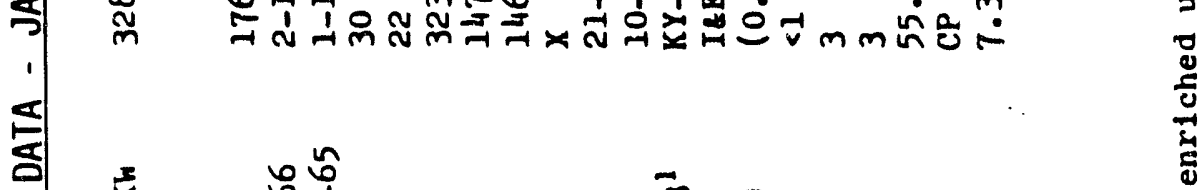

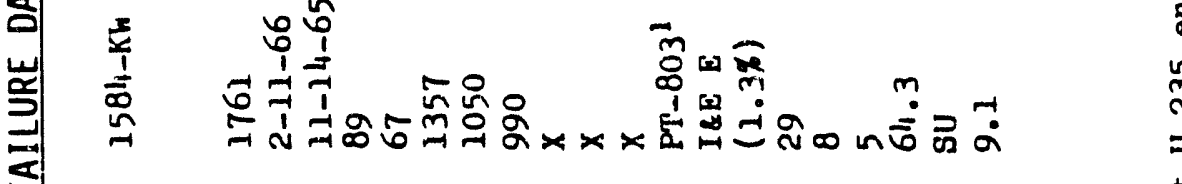

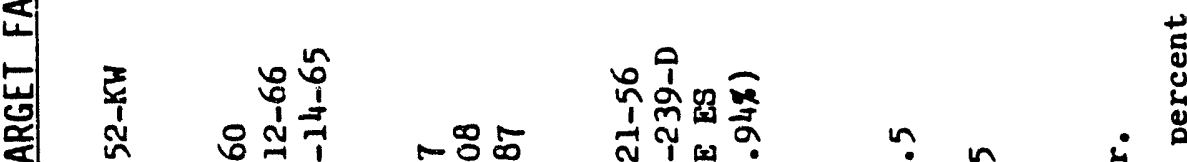

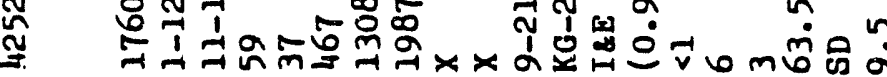

훈

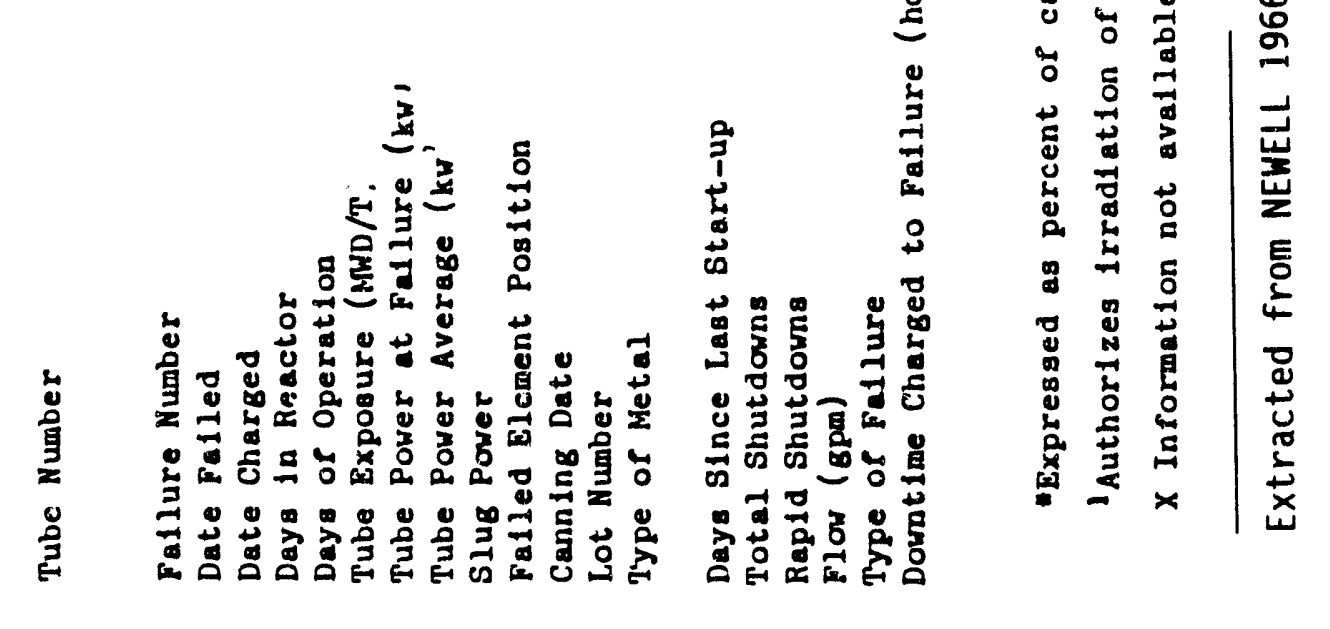

P.1 
อิำ:

ษ。ำ

도ำ

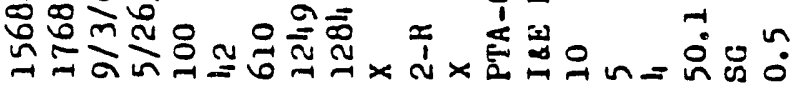

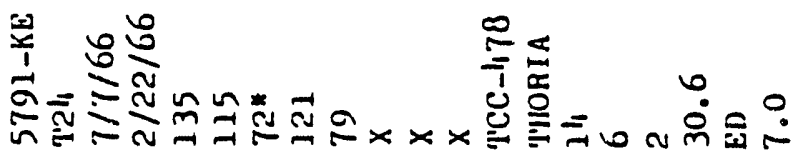

$\stackrel{\infty}{\Xi}$

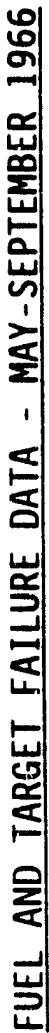

\section{है}

స

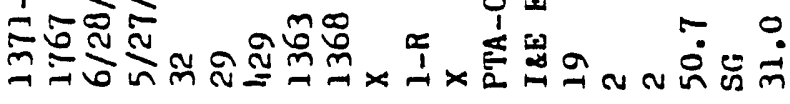

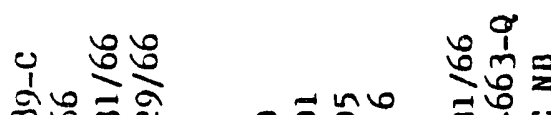

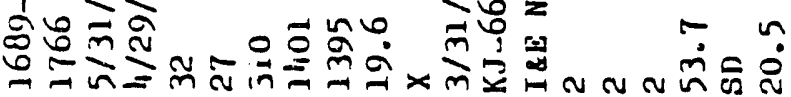

$\neg$ ニ

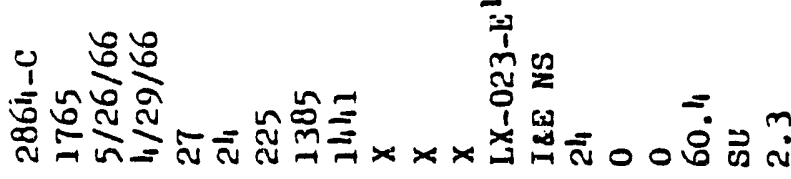

-

in

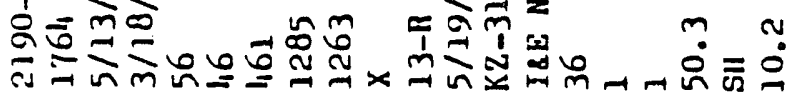

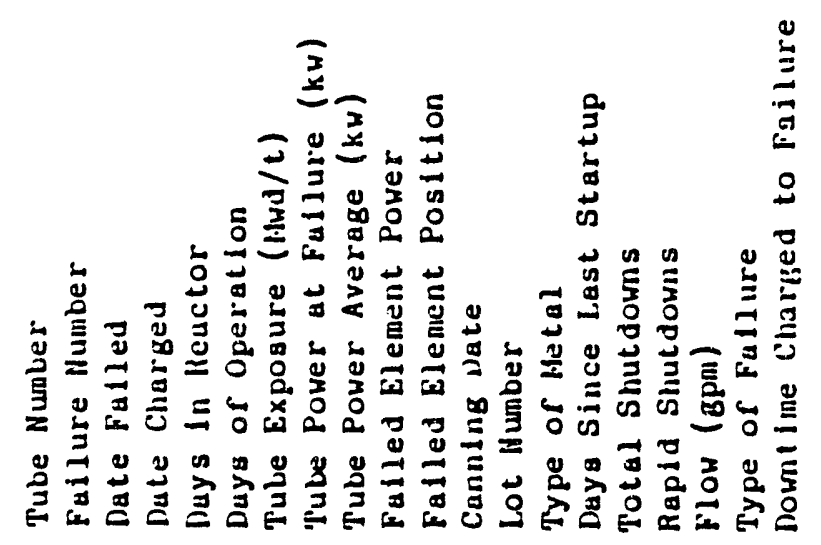

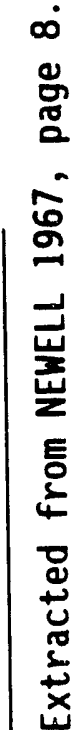




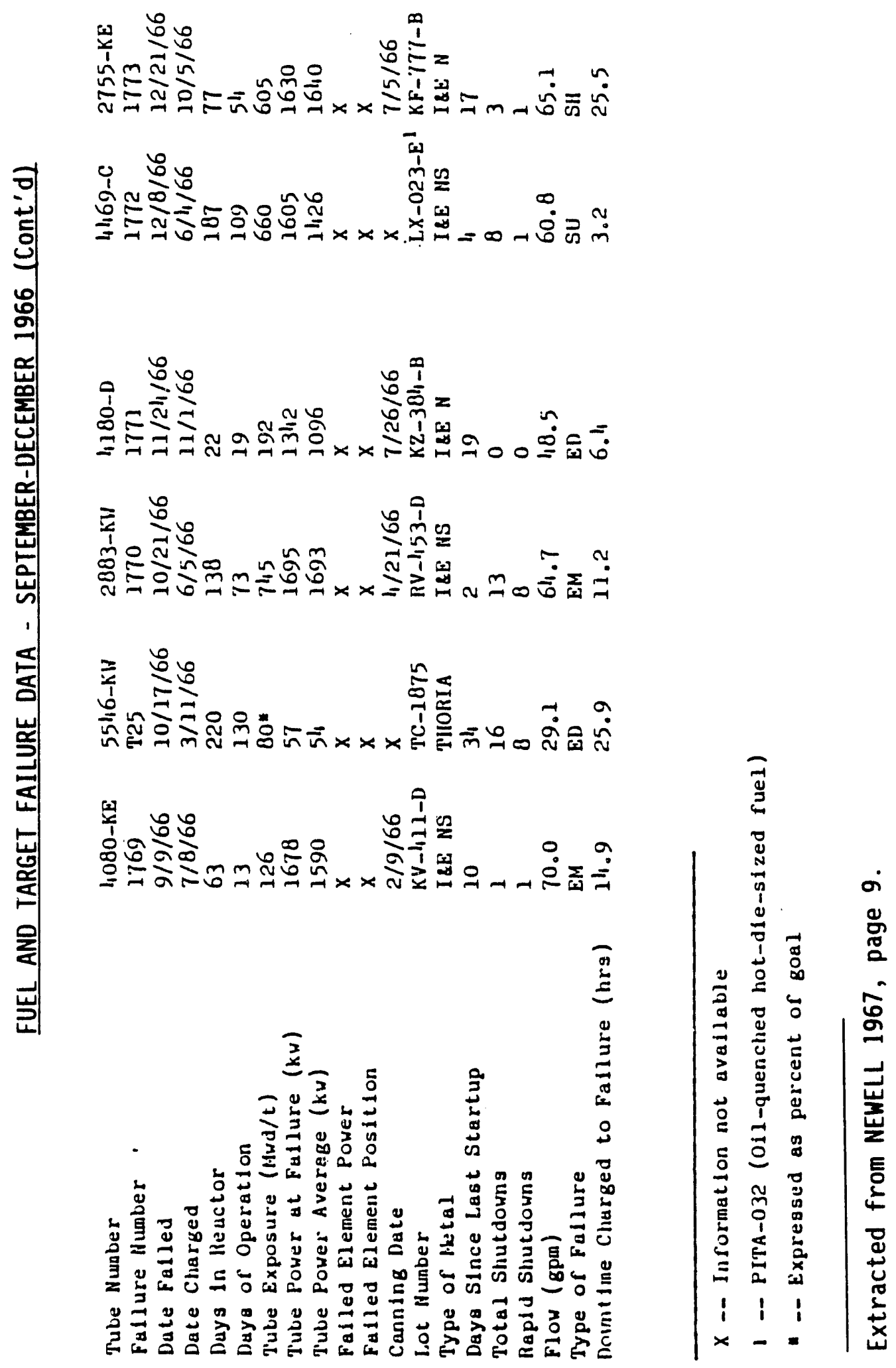


APPENDIX Q

FUEL-ELEMENT FAILURE DATA FOR 1967-1970 


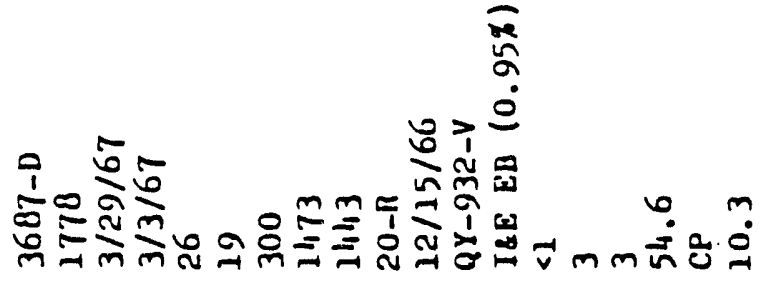

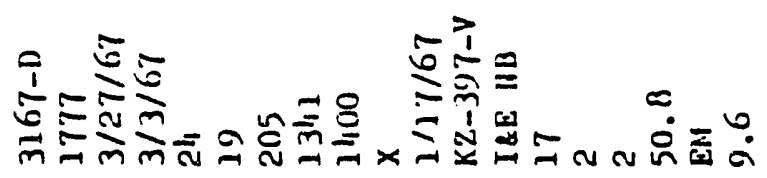

웜

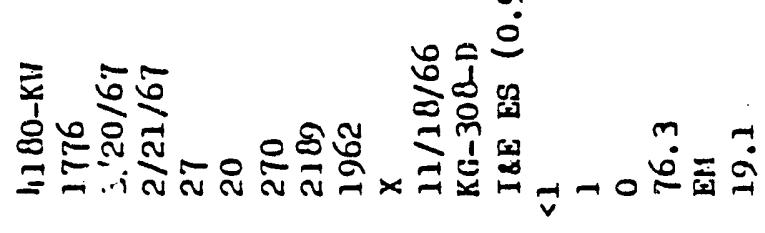

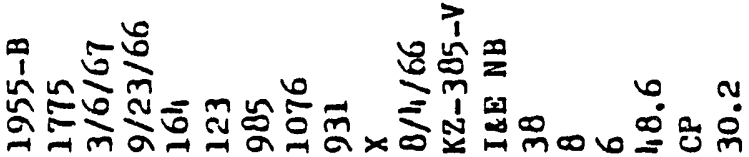

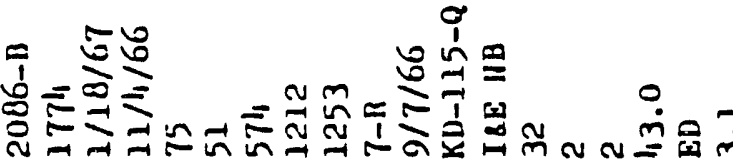

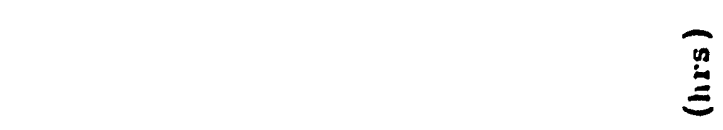

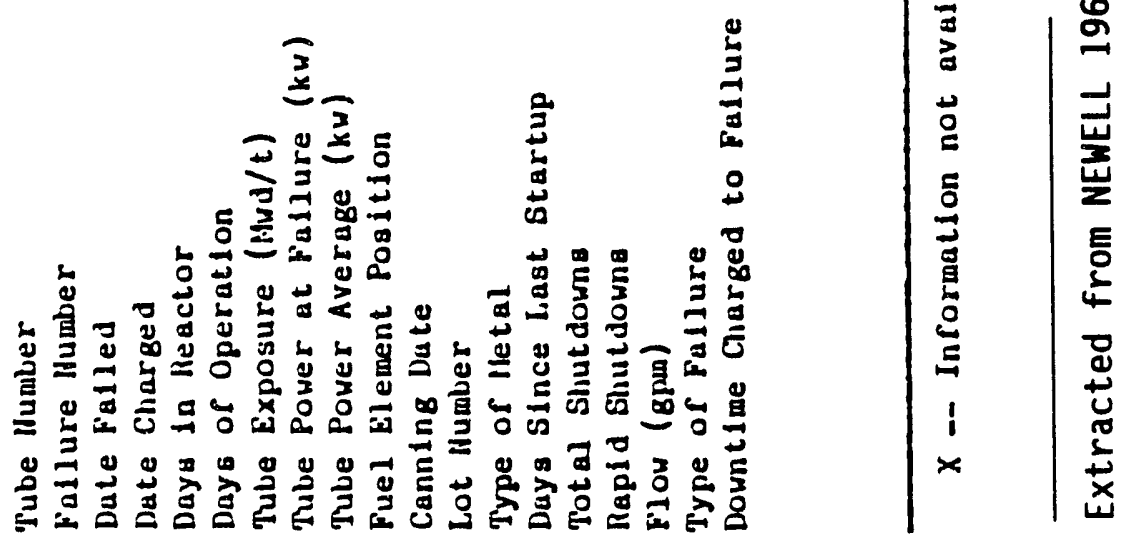




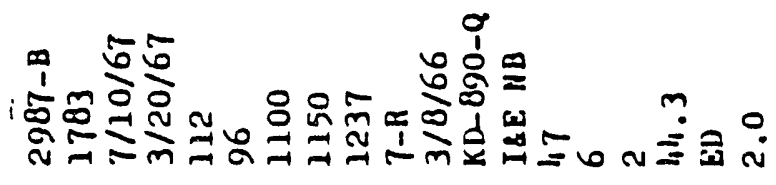

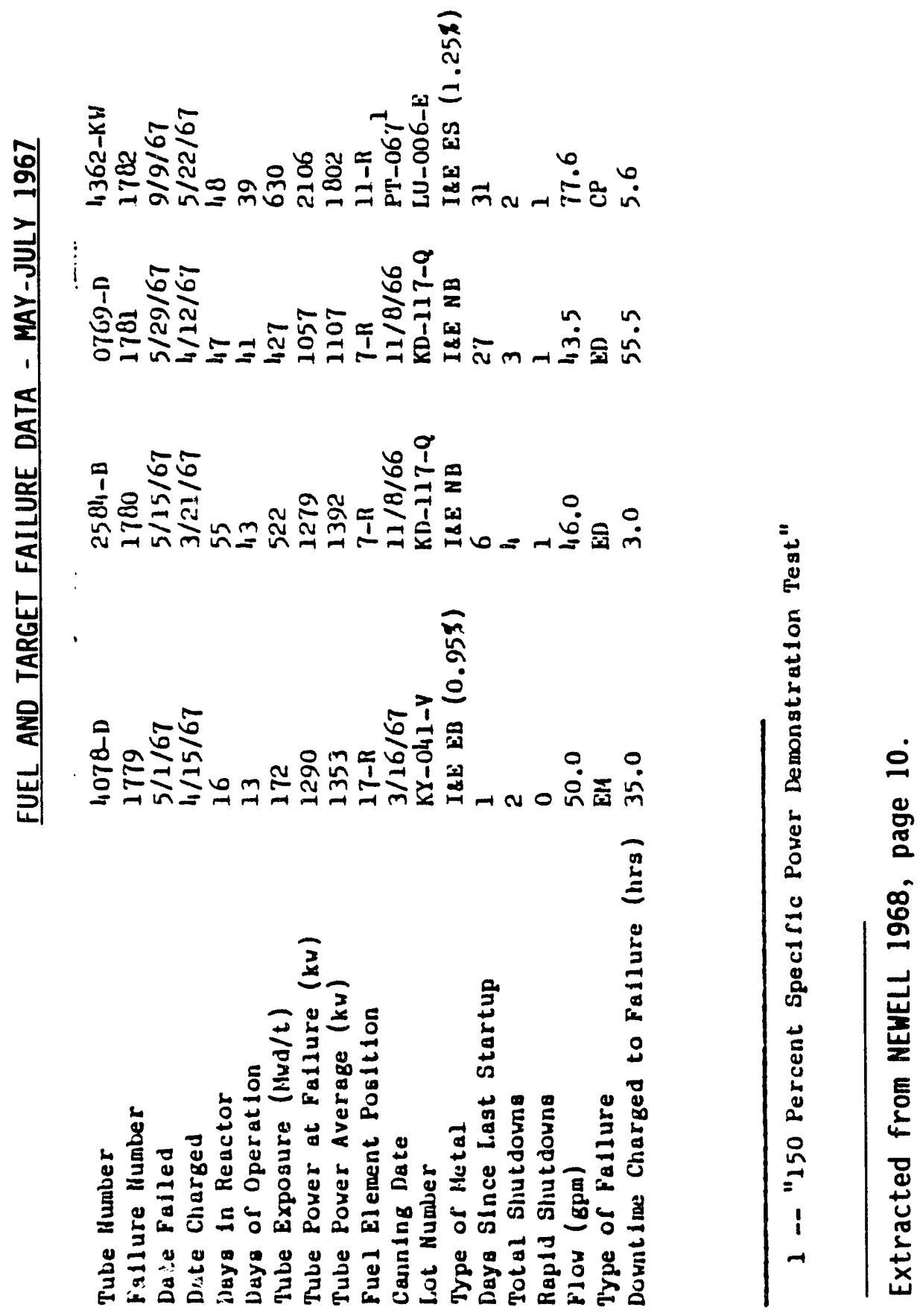




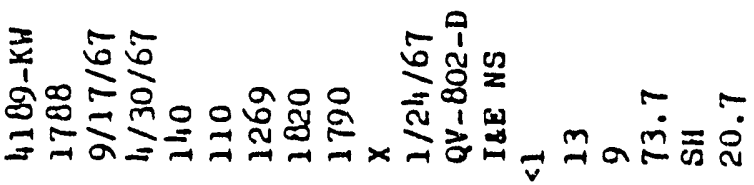

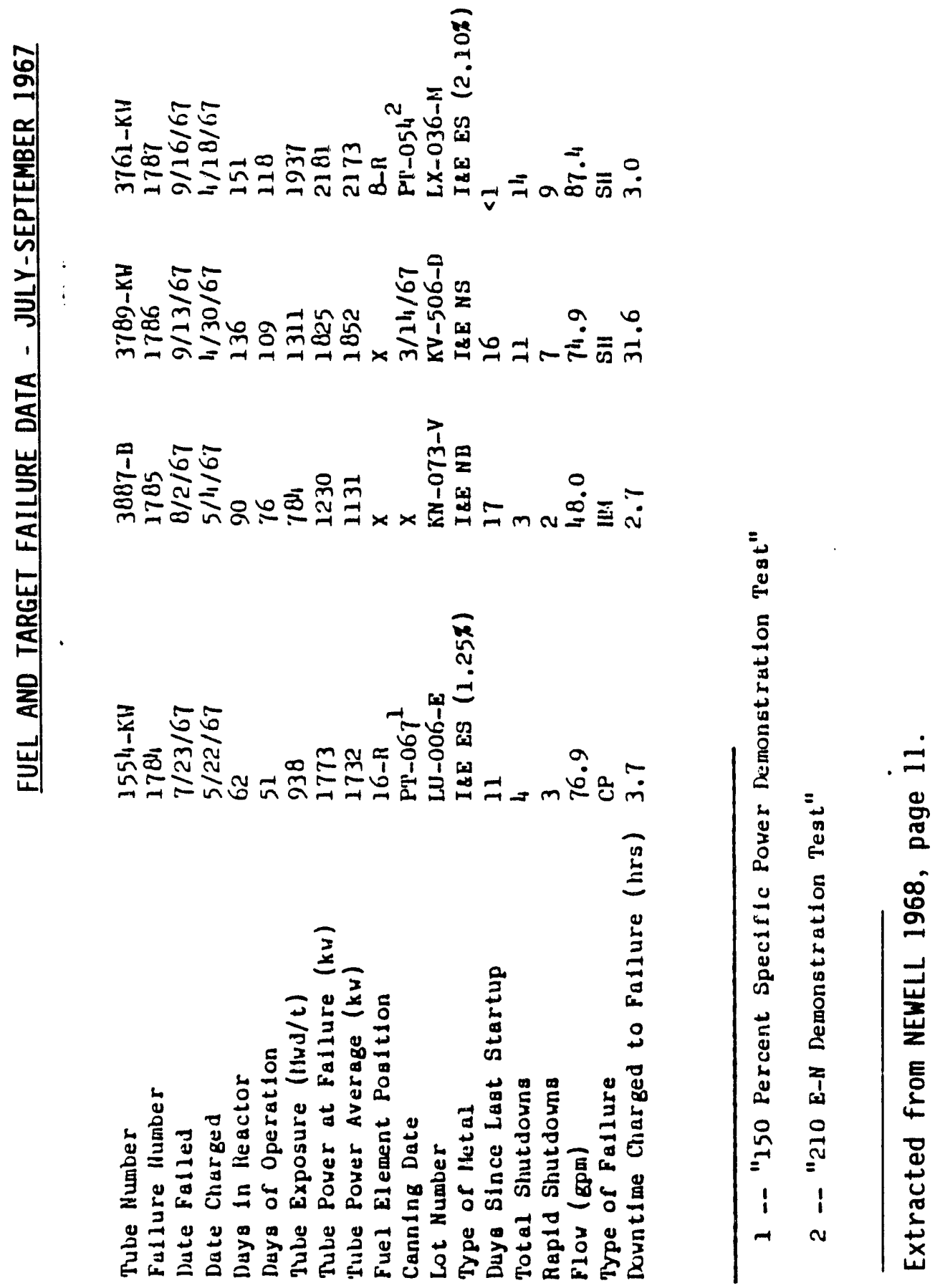




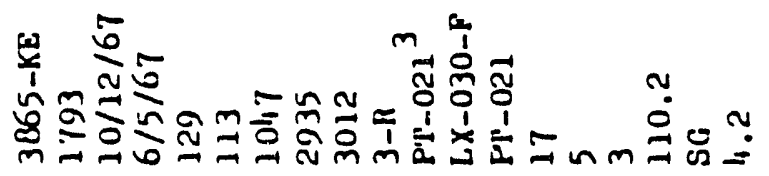

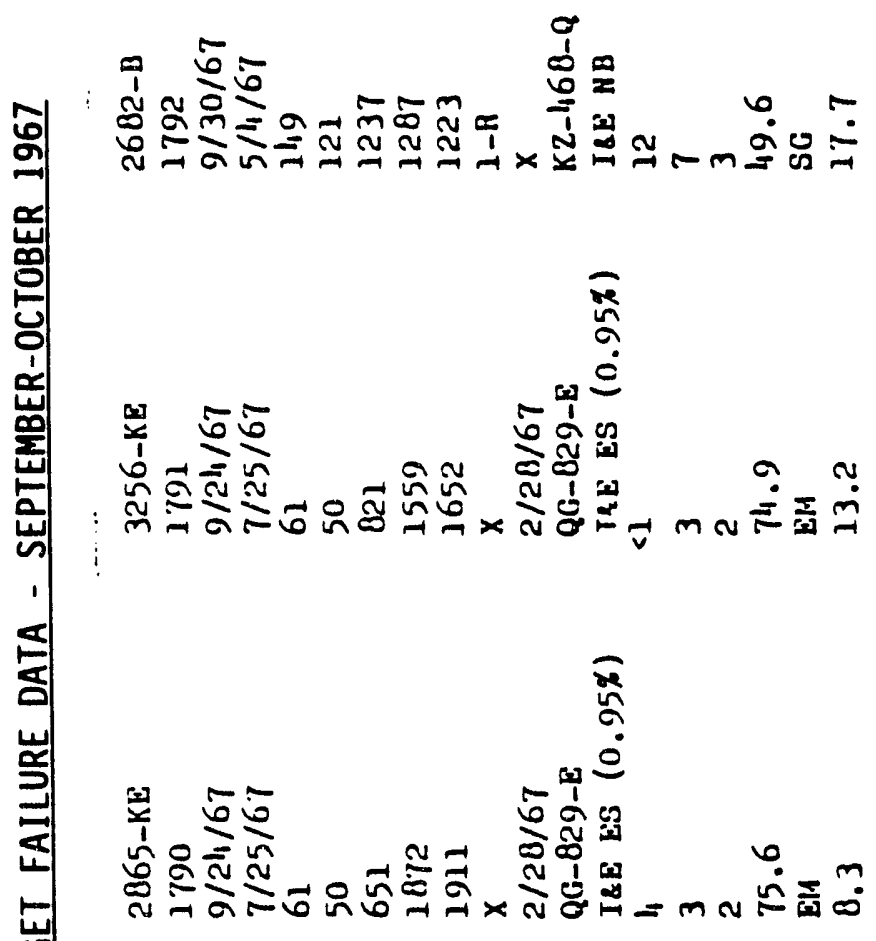

ปั.

월

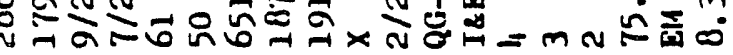

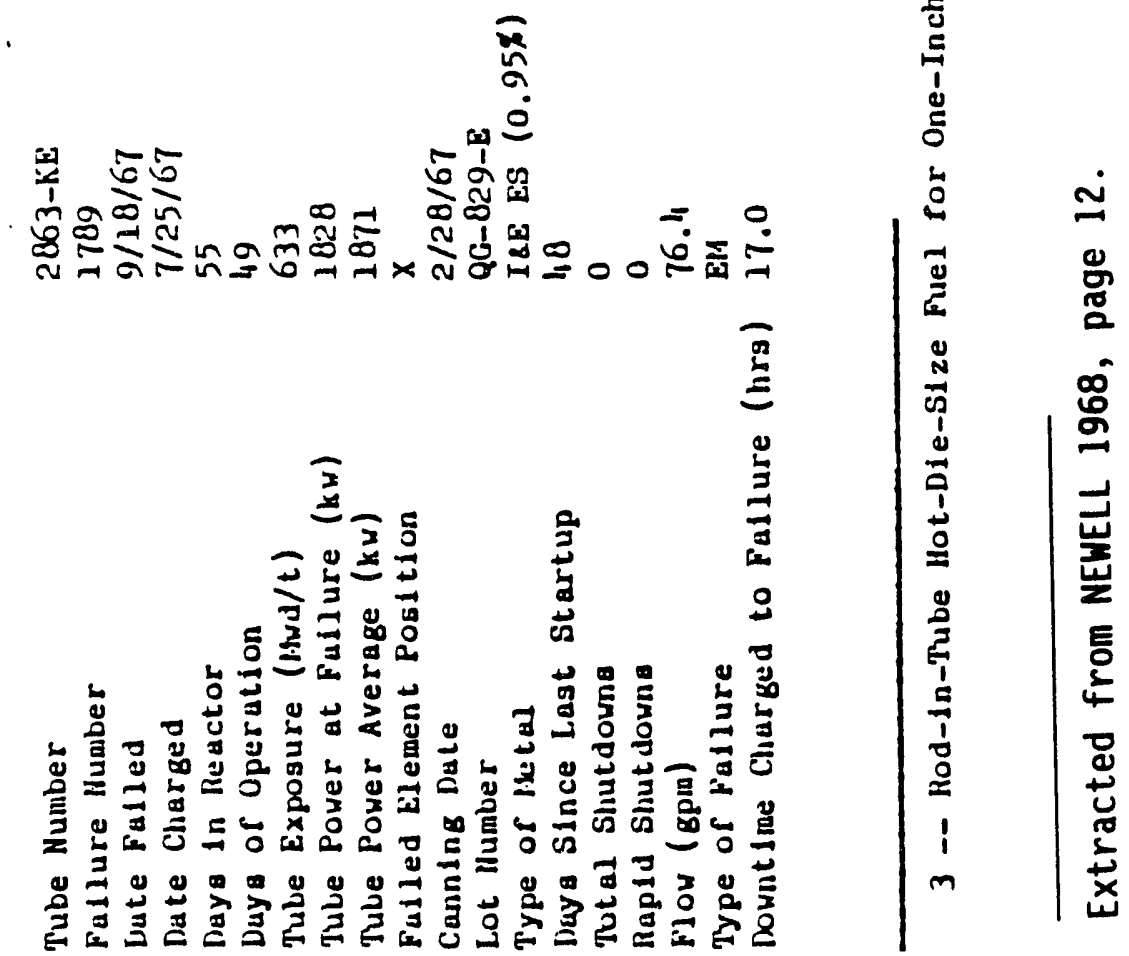




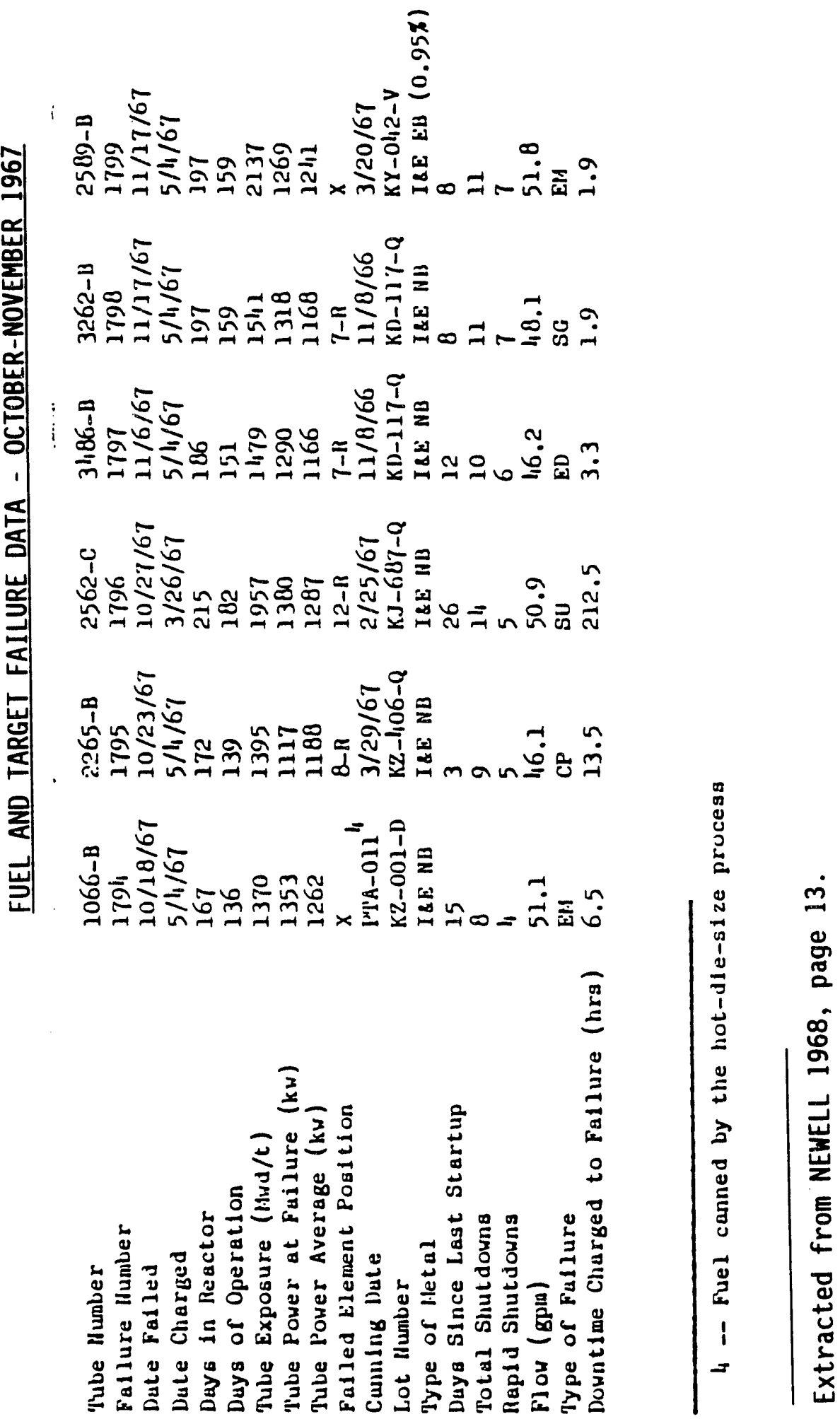




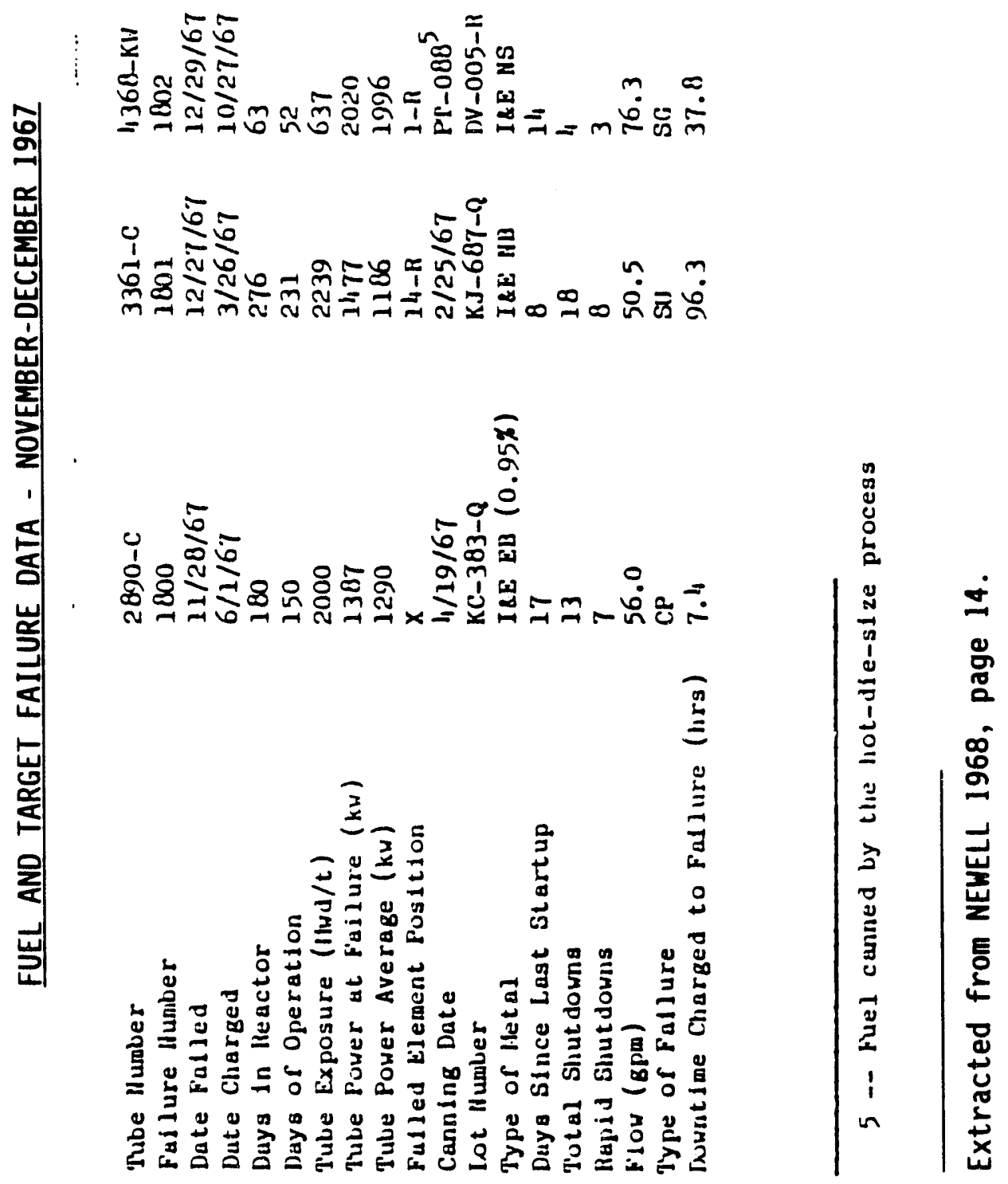




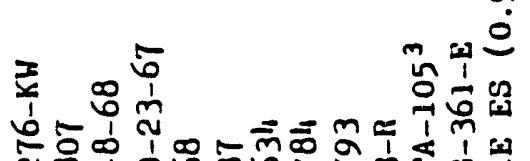

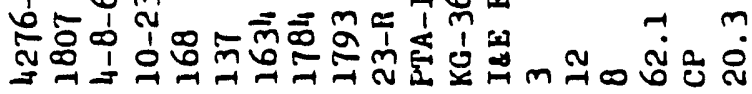

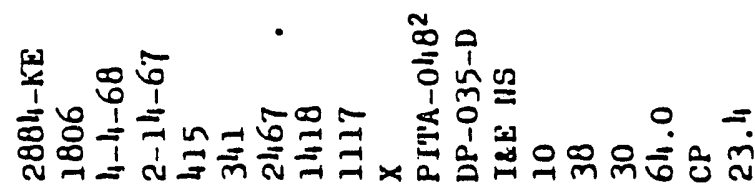

章|

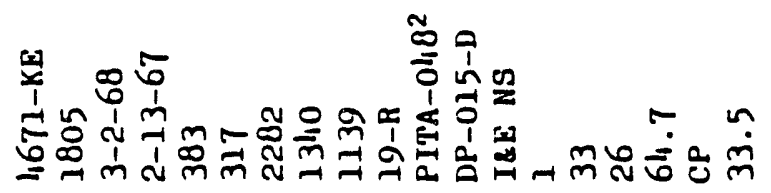

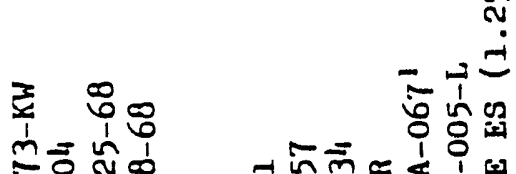

๙ู.

\section{(2)}

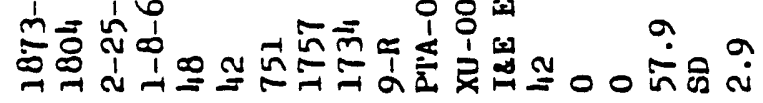

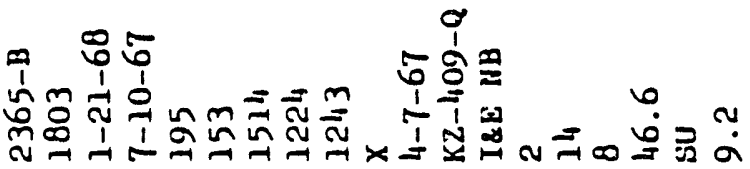

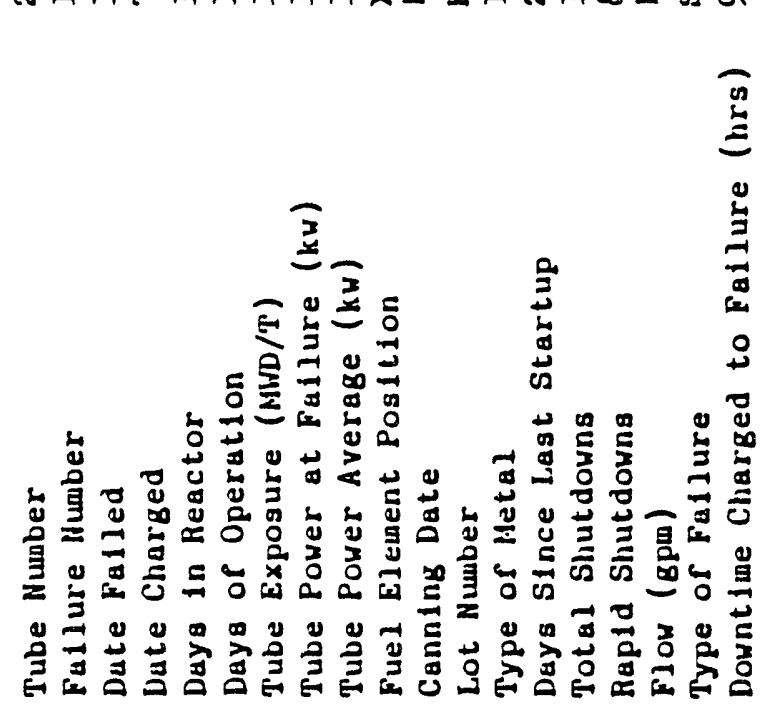

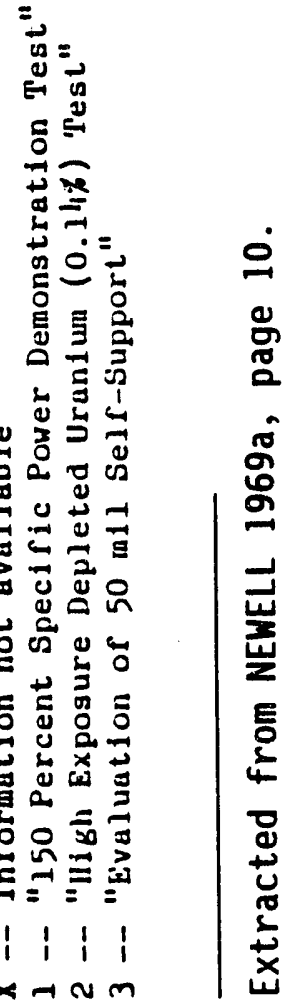




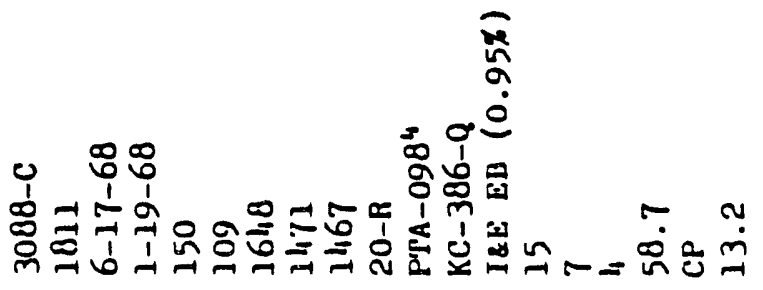

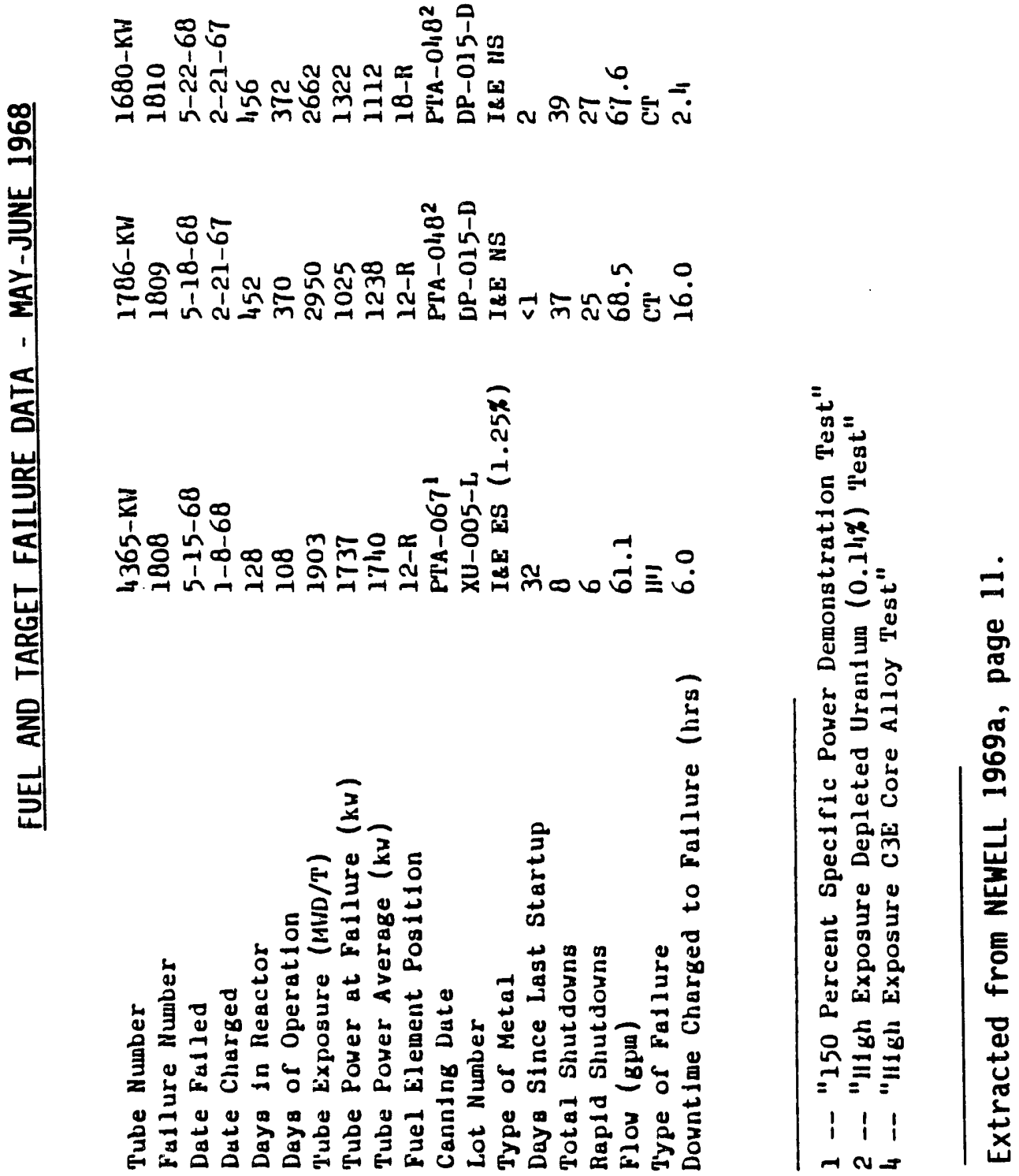



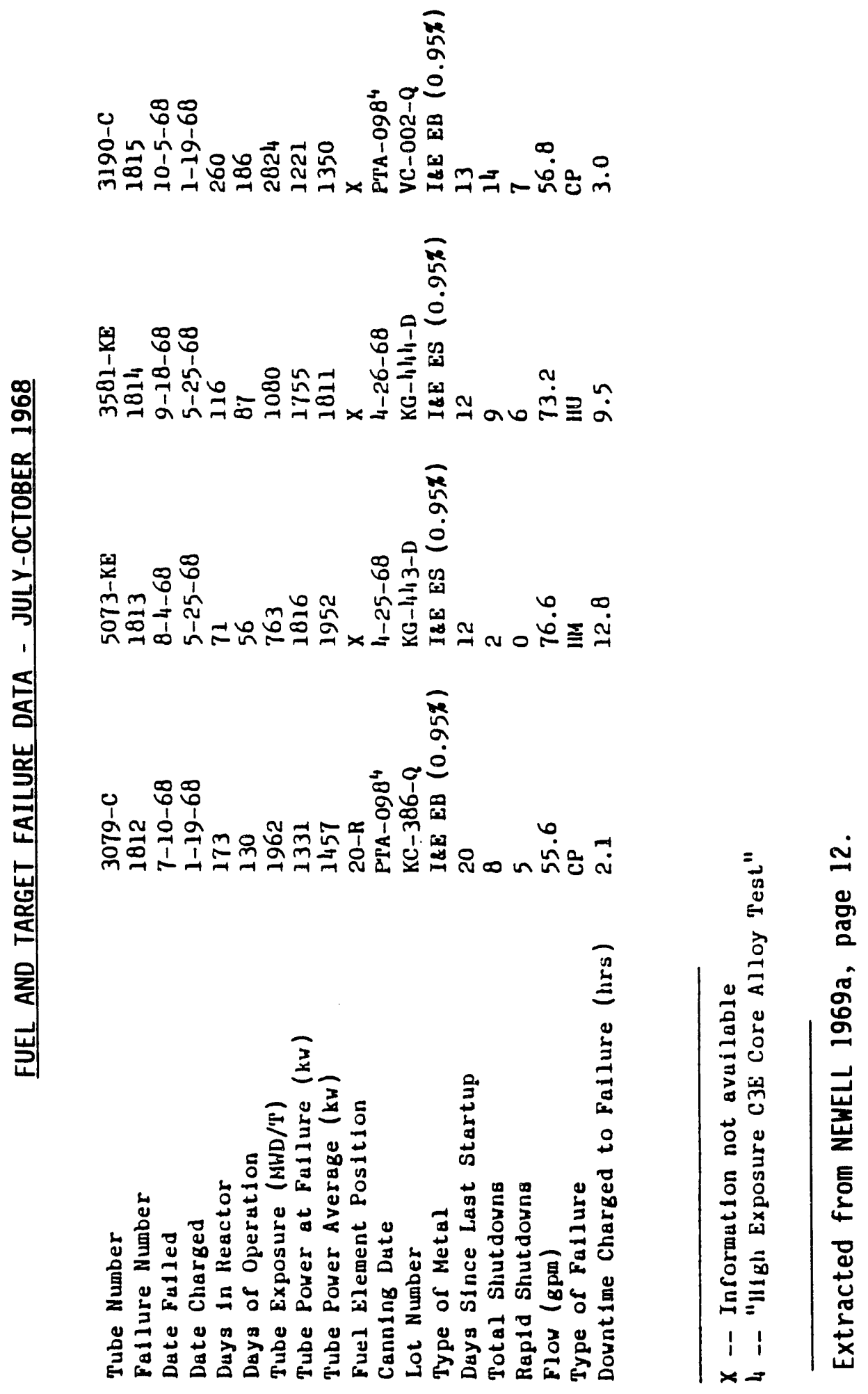


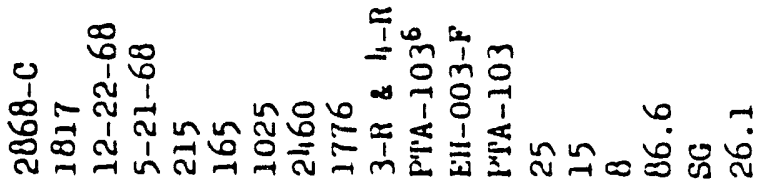

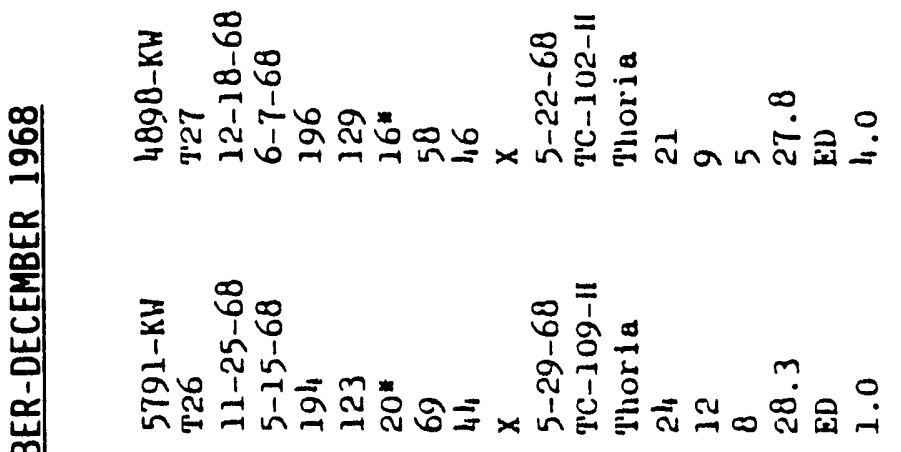

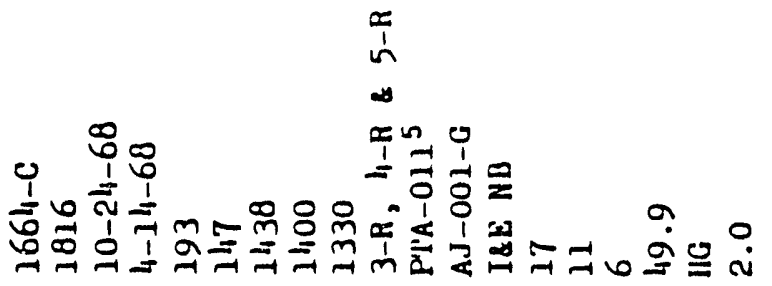

这

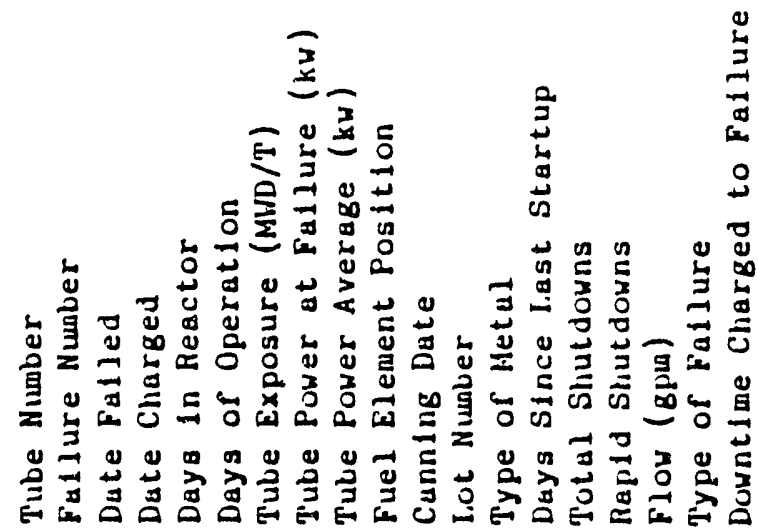

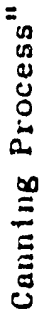

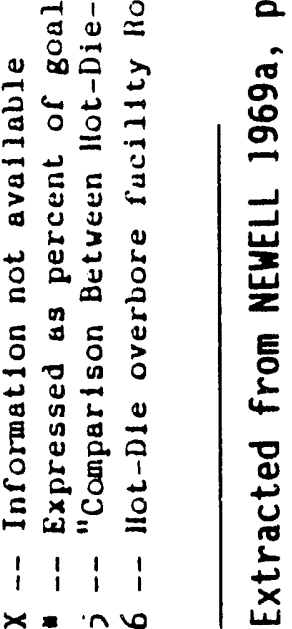


SLUG RUPTURE OR STUCK CHARGE REPORT - JANUARY 1969

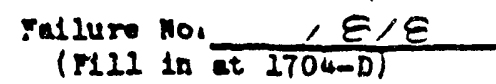

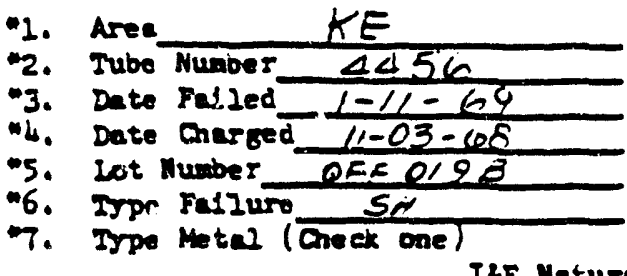

Rupiure Conslrmed stuei Charge Ondy (No Rupture) Bubbler

ILE Natural

IEE Enrtched

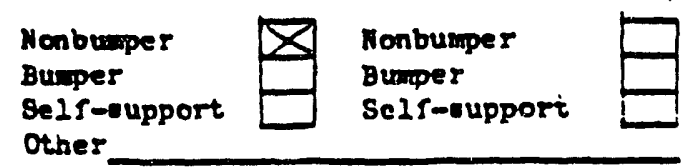

8. Cnerge Make Up $38 \mathrm{KIVN}$

(Pleces of each type metal, from iront end)

9. Production Teut No. DTA OSO Stuck

10. Slug Poaltion Not Stuck

10. Slus Poaltion $\ldots \ldots$

(From latent equilibriun or near-equilibriun operotink period)

i. Injet Temperature

-32. Tube $\Delta T$

3. Tube Row

14. Tube Ordsice zone

- . . . . . . . . . . . . . .

25. Dld rupture cause tube leak NO

26. DId tube contain a poison spline NT

17. Muxe= Locstion NONE

18. Date Tube inotariec $5-28-68$

20: How dotected (fam monltor, N1tered samle, Panellit Increase, etc.) Gumme manitor went full scale - Go-ce marensed 10 asi from 1st. indication on Gr M. wetil snutdown - 107 Inkt increasedtrij50 mR - 75 MR - Pijer Difenatial shourd ve change

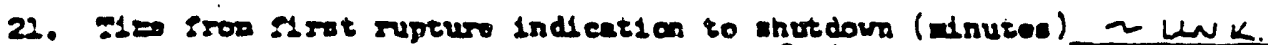

22. Rear plora1l readine (un1te) - Sz at rail

$\ldots \ldots \ldots \ldots \ldots \ldots \ldots$

Extracted from NEWELL 1969b, page 36. 


\section{SLUG RUPTURE OR STUCK CHARGE REPORT - FEBRUARY 1969}

\section{Fallure No. $\quad$ ig/9}

(F11 in at 1704-D)

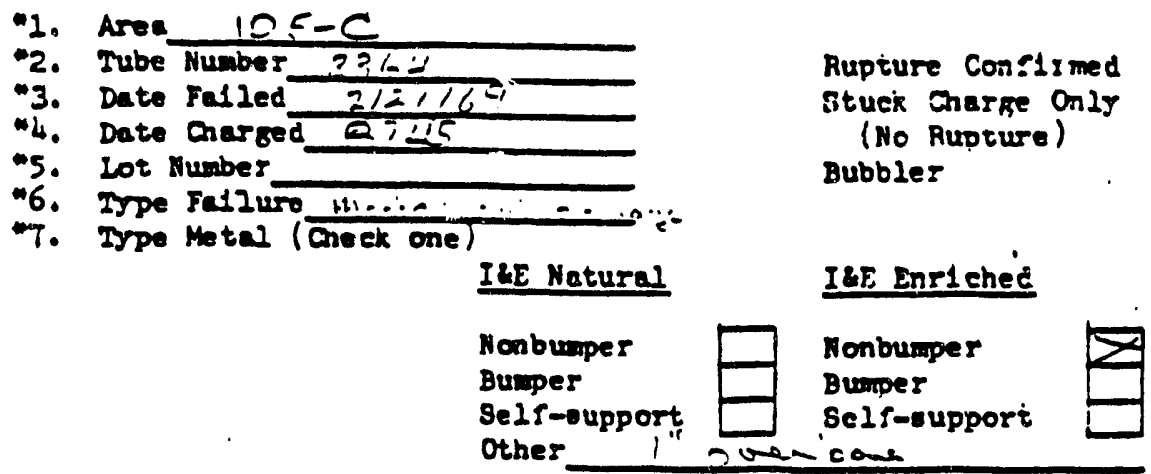

8. Charee Make up $\quad 27 \sim w=\equiv$

(Pleces of each type metal, from front end)

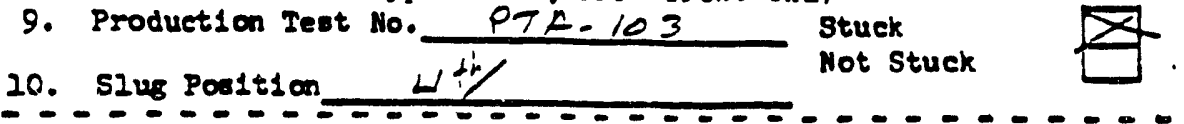

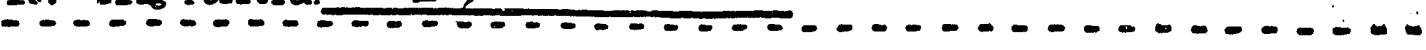

(From latant equilibrium or near-equilibrium operating period;

2. Inlet Temperature 3.2

32. Tube $\Delta T, 19 \%, ?$

13. Tube Flow ali.a

14. Tube ord Iice zone ?9

- _. . . . . . . . . . . . -

25. DId rupture cause a tube leak Jal

16. D1d tube contaln a poison opline $i i=$

27. MAxe: Ioestion

n)....

16. Vate Iube

Inotaied $1 / 19 / 68$

20: Hor detected (eamme monltor, r1tered sample, Panel11t Inerene, etc.) n .... a r.u. - ...

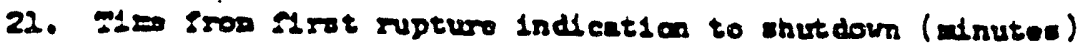

22. Rear p16tald feadine (unlts)

Extracted from NEWELL 1969b, page 38. 


\title{
SLUG RUPTURE OR STUCK CHARGE REPORT - MARCH 1969
}

\author{
powar lead - 2280

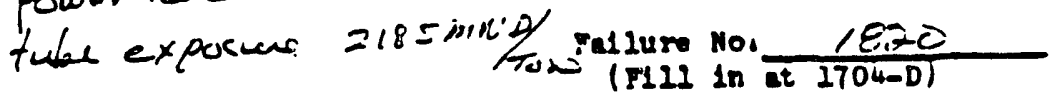

1. Area 105-C

2. Tube Number 2 he Rupture Consirmed

3. Date Folled $2124 / 10$ stucik Charke Ondy

4. Date Cnerged 619.16 C

5. Lot Number OT, Bubler

6. Trpe ratiuro

T. Type Matel (check one)

ILE Natural

I8E Enr1 thed

Nonbusper
Bumper
Self-oupport
Other

\section{Nonbusuper}

Bumper

Sels-ouppori $\square$

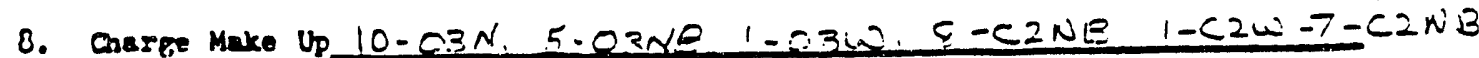
(Piecer of ecch type metal, from front end)

9. Production Test No. 127 Stuck

10. Slus Position

Not Stuck

10. S1us Porttion

(Frow Intest equildbriun or neer-equ121briun operating period)

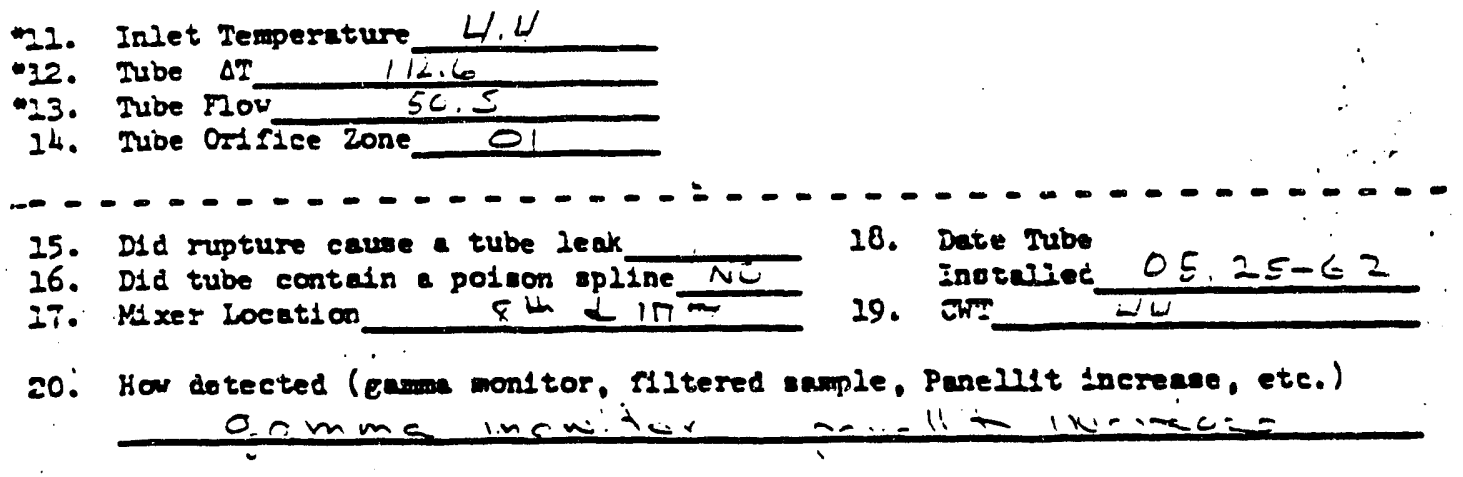

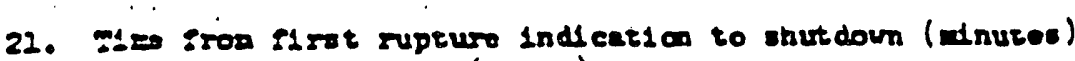

22. Rear plotell readine (undto)

Extracted from NEWELL 1969b, page 40. 


\title{
SLUG RUPTURE OR STUCK CHARGE REPORT - MARCH 1969 (Cont'd)
}

\author{
Ponis a level 1800 \\ tube pium 1789
}
rallure Nor $/ 821$
(F112 in at 1704-D)

1. Area 105-C

2. Tube Number 2 et-6

-3. Date Folled $3122 / 69$

44. Date Crarged $11,0 / 6 \%$

"5. Lot llumber $79 u$

"6. Irpe Fatluro

-T. Type Metal (Cneck one)

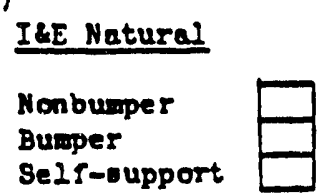

Rupture consirmed stuck Charse ondy

(No Rupture) Bubbler

\section{IEE Enrl ched}
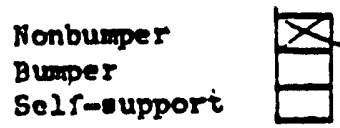
Other

8. Coarge Nake Up $32 \mathrm{CM} 2 \mathrm{E}$

(Pleces of ecch type metal, from front end)

9. Production Test Ho. $P T$ 103

20. S2us Pooltion Not Stuck

20.

(From latest equilibriw or near-equidibriun

-atink period)

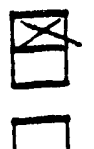

1. Inlet Temperature 52

32. Tube $\Delta T$

13. Tuibe Flov

14. Tube ordsice zone 04

25. D1d rupturo cause a tube 2 ak

26. D1d tube contadn a poison opline NC

IT. MAXe= Losation NONE

18. Dace Tube inotaried

29.

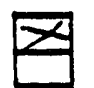

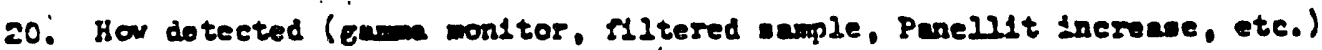
ormma man, for

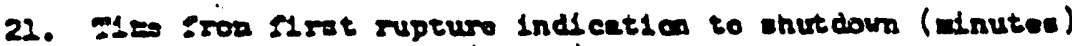

22. Rear plotall readine (un1ts)

Extracted from NEWELL 1969b, page 42. 


\section{SLUG RUPTURE OR STUCK CHARGE REPORT - APRIL 1969}

$$
\begin{aligned}
& \text { pouer levil } 2235 \\
& \text { tube power } 2280 \\
& \text { Exposum } 1600
\end{aligned}
$$

Falluro No. 1 EES

(F:11 in at 1704-D)

"1. Area $\frac{105-C}{2 E 63}$
"2. Tube Number
"3. Date Falled $\frac{4-25-69}{5-20-66}$
"4. Date Charged Lot Number
"6. Trpe Falluro
"7. Trpe Matal (Check one)

ItE Natural

Nonburaper

Buwper

Self-oupport

Other
Rupiure Cons1rmed stucik Oharae Only (No Ruprure)

Bubble:

I\&E Enrlehed

Nonbusaper

Bumper

SeIr-uppori
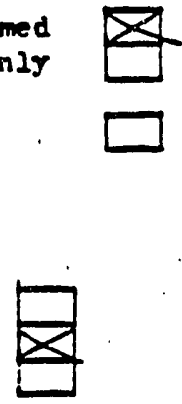

8. Cuare Make Up 32 CMIE

(Pleces of each type metal, from front end)

9. Production Teet Ho. 103

3tuek

20. S2u8 Posit100

Not Stuck

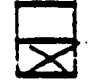

(From latent equdubrium or near-equ1l1brium operatine period)

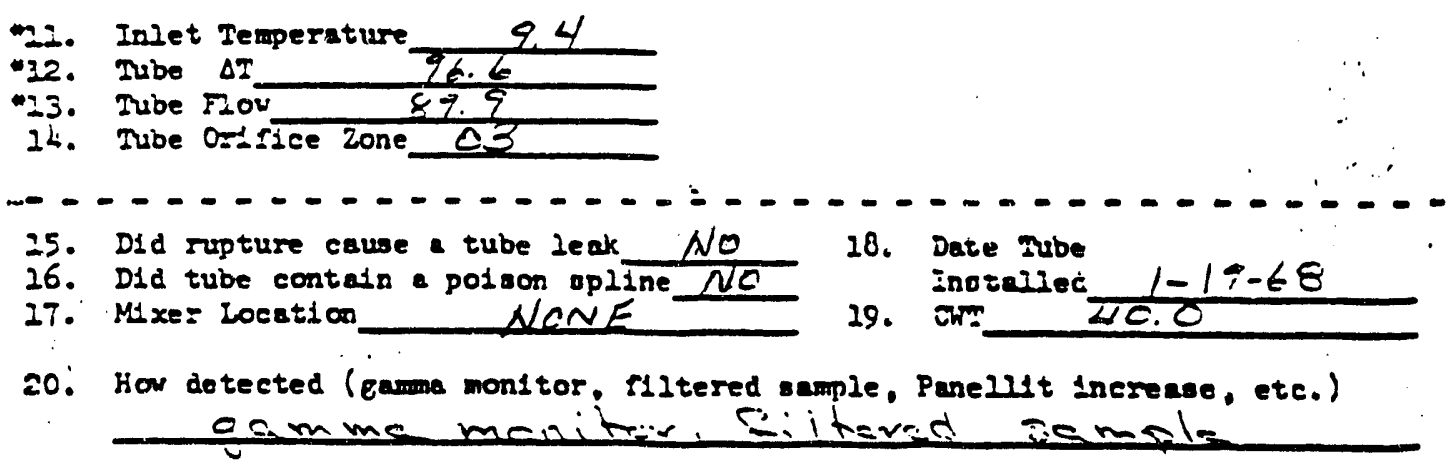

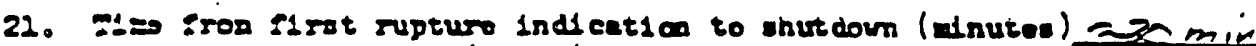
22. Rear p1gtald readine (undte)

Extracted from NEWELL 1969b, page 44. 


\section{SLUG RUPTURE OR STUCK CHARGE REPORT - JULY 1969}

Fallus No1 $10=3$

(T112 in at 1704-D)

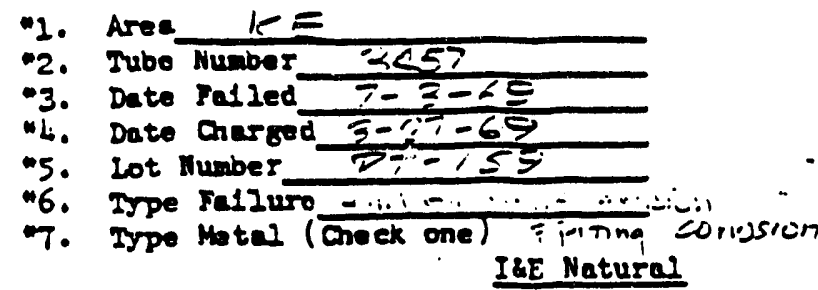

\section{Nonburper}

Burper

8elf-oupport Other
Rupiure Cons1 rmed stuek Charke Only

(No Ruprure)

Bubbler

I\&E Enrtched

Nonbumper

Bumper

Selr-euppori
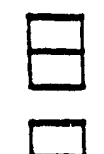

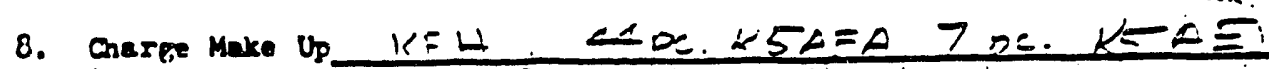
(Pleces of eech type metel, from front end)

9. Production Teet No. Stuck

10. SIus Position $C$ Not Stuck

(From lateot equilibriun or near-equ121briun operating period)

21. Inlet Temereture $17 \mathrm{ros}$

.32. Tube at 00 oc

13. Tube For $719 \sin$

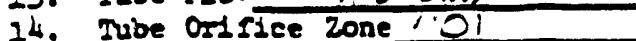

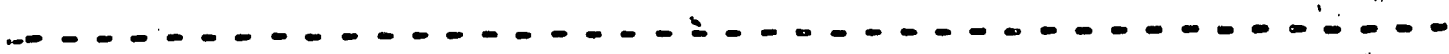

25. DId rupture cause a tube leak $=0$

16. D1d tube contain a poison opline "xo

17. Maxer Locetion no:

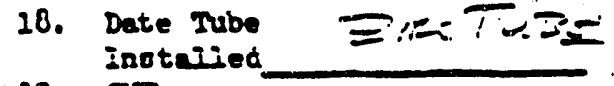

19. arr

20: How dotected (gam monltor, 11 tered sample, Panell1t Inereane, ete.) Samme Momige (R.jel :jo-r - i...nis

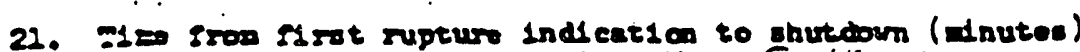

$\sim \geq<0$

22. Rear ploteld readine (und to) inn E lipeje

Extracted from NEWELL 1969b, page 46. 


\section{SLUG RUPTURE OR STUCK CHARGE REPORT - JULY 1969 (Cont'd)}

Fallure Nos

(F111 in at 1704-D)

-1. Area 100-kú

2. Tube Numider 2355

-3. Date Fatled $7-19-69$

4. Date Charged $t-13-6 ?$

"5. Lot Number

*6. Type Falzuro Edeos Joume

T. Type Matal (Check one)

\section{I\&E Natural}

Nonbusper Bumer Self-oupport
Rupture Constrmed Stuek Charge Only

(No Rupture) Bubbler

I\&E Enrlehed

Nonbumper Bumper

Sels-eupport
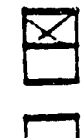

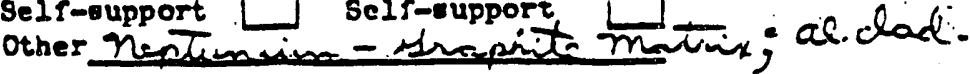

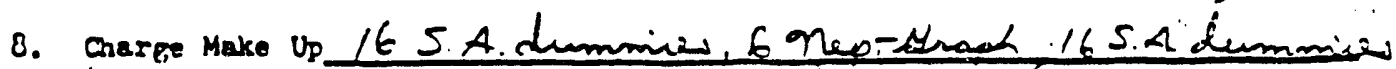
(Pleces of each type metal, from frorit end)

9. Production rest No. PT $A-163$ Stuck

10. Siug Position $54-22,-24-26$ Not Stuck

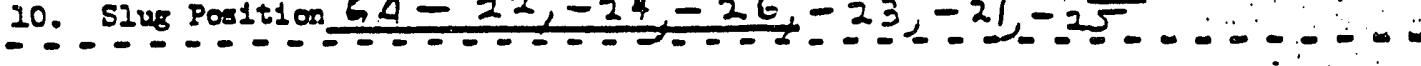

(From latest equilibrium or near-equ111briun opernting period)

12. Inlet Temperature 17.6

12. Tube $\Delta T$ I $S^{\prime} .4$

13. Tube Flow $10.5: 0 \mathrm{~m}$.

14. Tube ordise zone प/6

25. D1d rupture cause a tube leak w

26. D1d tube contain a poison opline $\ddot{\sim}$

17. MAxer Locetion me

10. Date Tube Zine Twhe

Inotariec

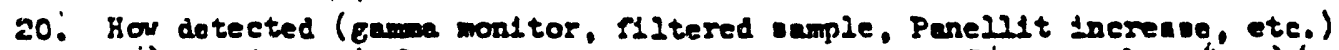

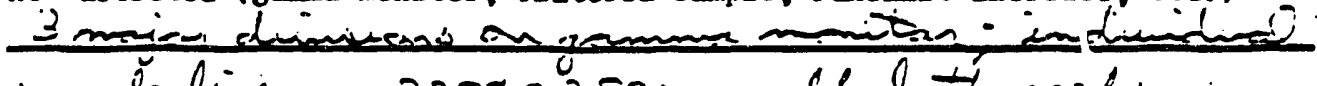

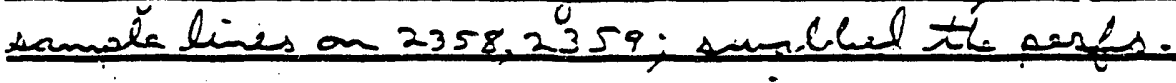

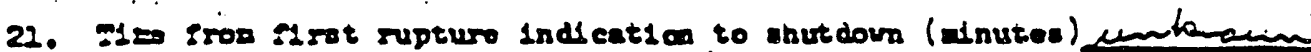

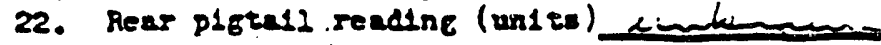

Extracted from NEWELL, 1969b, no page number. 


\section{SLUG RUPTURE OR STUCK CHARGE REPORT - JULY 1969 (Cont'd)}

Yatlure No.

(F11 in at 1704-D)

-1. Aree 100-kw

2. Tube Nuniber 2359

-3. Date Folled $\frac{239}{2-2 c-60}$

4. Date Charged $i-12-4$ ?

5. Lot Number

"6. Irpe Fas Iuro inicat hodurs

7. Typo Motal (Creck one)

ILE Natural

Aupture Cons1rmed

Stuck Charke Ondy

(No Rupture) Bubbler

\section{I\&E Enriched}

Nonburper

Bumper

Gel I-oupport

Other
Fonbuinper

Bumper

Sels-euppori $x$

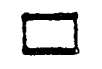

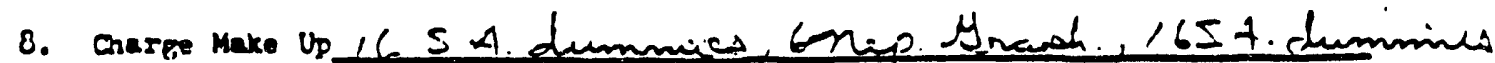
(Pleces of each type metel. from front end)

9. Production Teet No.PTA -163

10. S2us Position $G Z-36$ Stuck Not Stuck

(From latent equilibrium or near-quilibriun operating period)

21. Inlet Temperature 17.6

12. Tube $\Delta$ ?

13. Tube 16.4

14. Tube ord rice Zone

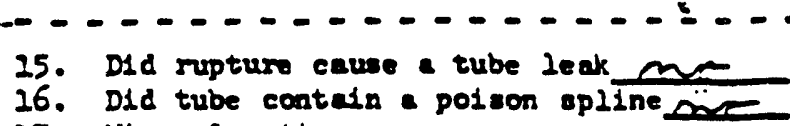

17. M1xer Locetion ma

10. Date Tube zive Tube. Invtried

19. ar.

20: How datected (eanm monltor. H1tered sample, Panell1t Inerane, ete.)

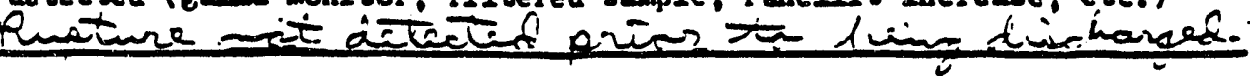

21. ads sron sist rupturo indleatien to ohutdown (adnutes)

22. Rear pletall readine (und to)

Extracted from NEWELL 1969b, no page number. 
SLUG RUPTURE OR STUCK CHARGE REPORT - AUGUST 1969

Yailure No. $128-$

(7112 in at 1704-D)

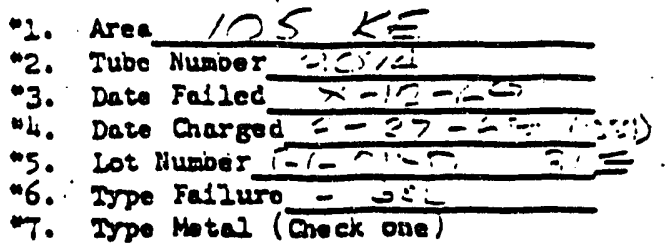

ILE Natural

Nonbumper

Busper

Self-oupport Other

\author{
Rupiuro Constrmed \\ Situck Enarge Only \\ (No Ruprure) \\ Bubbler

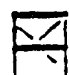 \\ $\square$
}

\section{ILE Enrlched}

Honoumpe:

Bumper

Selr-unpport

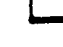

8. Carge Make up $51 \quad k \leq-1<=0$ (2)

(Pleces of each type netal, from front end

9. Production Test No. 0,1 Stuck

20. 52us Posit1on 6 Not Stuck

(Irom latest equidbriun or near-equilbrium operatiak period)

2. Inlet Tempersture 109

23. Mube $\Delta 2 \frac{-01}{07}$

14. Tube Orssice 2one_a!

- - - - - - - - - - - - - - - - - - - - - - - - - - - - -

25. DId rupture cause a rube $2 \mathrm{eak}$

16. D1d tube contaln a poicon opdine ....

27. Mrez Locaeton

28. Dato atube

Dotriect

20." How dotected (evme mondtor gltered sample, Panelut dnereane, etc.)

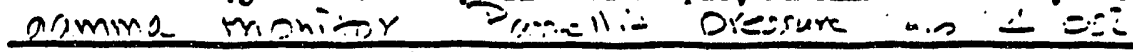

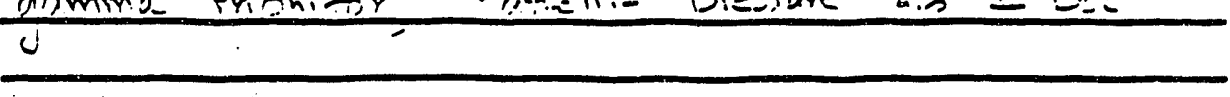

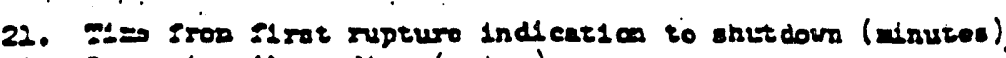

7 Snini:

22. Rear p1gteasd revalne (under)

Extracted from NEWELL 1969b, no page number. 


\section{SLUG RUPTURE OR STUCK CHARGE REPORT - AUGUST 1969 (Cont'd)}

Tasiuro No. 19:-

(7:I: in 22 1704-D)

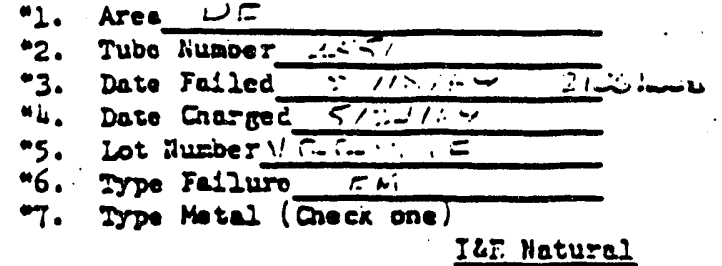

Nonbueper

Bureper

Sedr-oupport

Otr.er
Rupiure constrmed

stuck Cosage ondy

(No Ruprure)

Juible:

ILE Enrlché

Roribuane:-

Buener

Sclíoupport

0. Cuere Make Up Si KS:

(Pieces of each Eype meia), Iror. Sront eri

9. Production Test No. $=$

3tuck

Not Stuck

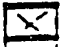

$x$

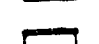

20. SIug Position

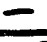

(5rom latast equilbrium or near-quilibrium operatian periodj

2. Inlet Temprature is 9

"i2. Tube $\Delta$ :

-3. Tube Fou

24. Trioe os sice 20

2ce 2one

$\ldots-\ldots \ldots-\ldots+\ldots \ldots+\ldots-\ldots$

25. .D1d rupture cause a tube leak ni:

26. Die tuibe contaln a poison apline

iT. Mixe = jocet1on . . . ....:=

18. Vast aube

inosaiect

29. ลพ:

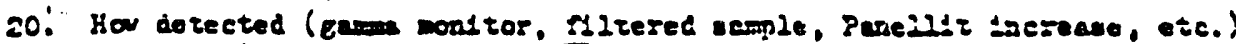

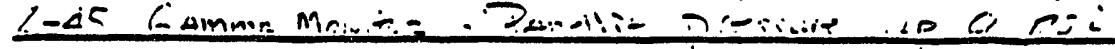

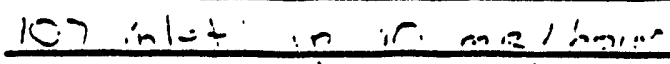

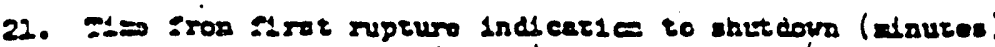

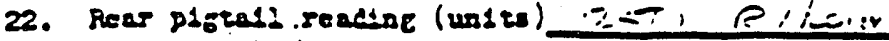

Extracted from NEWELL 1969b, no page number. 


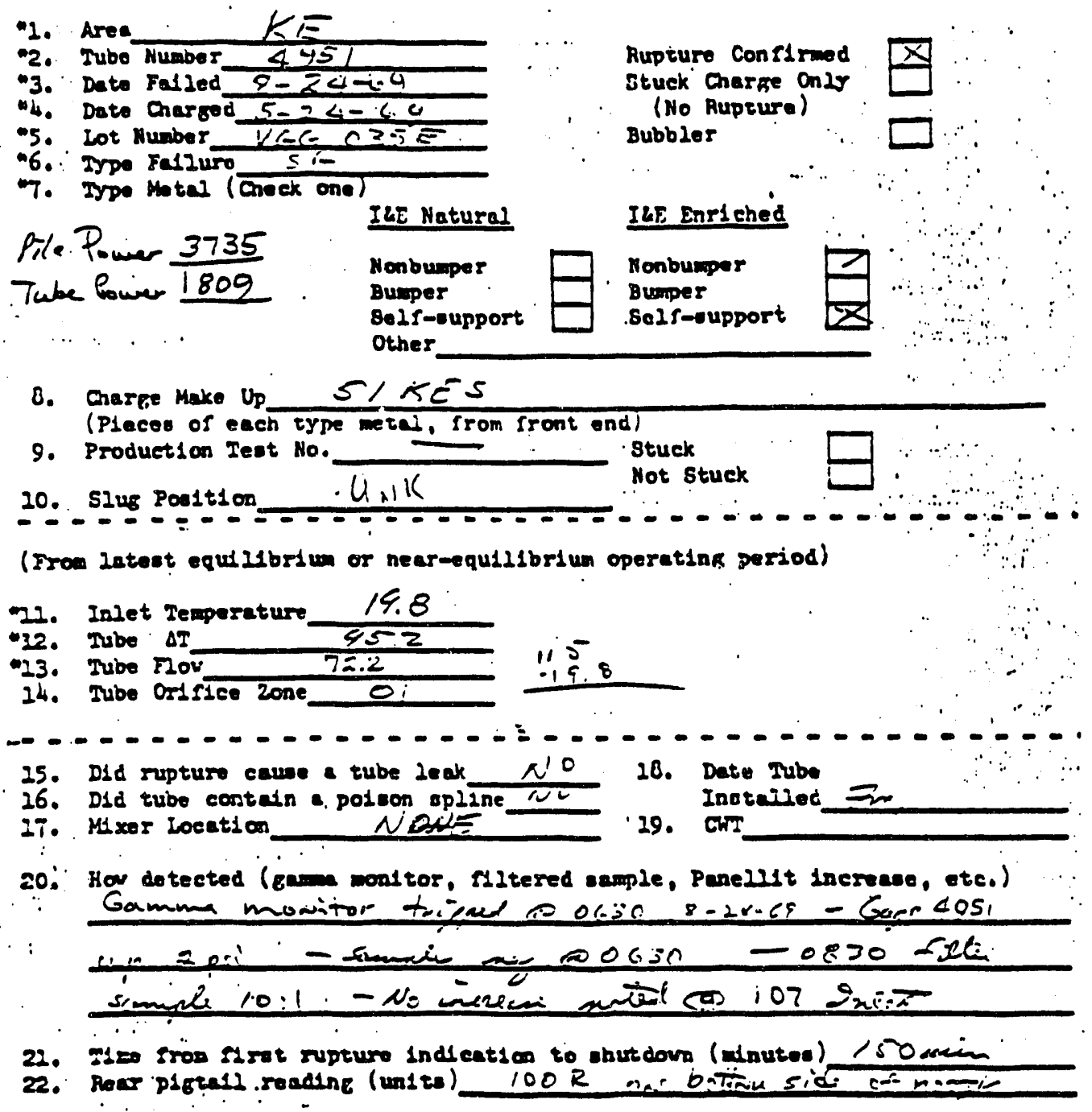

Extracted from NEWELL 1969b, no page number. 
Ind lure Nou

(F11 in at 1704-D)

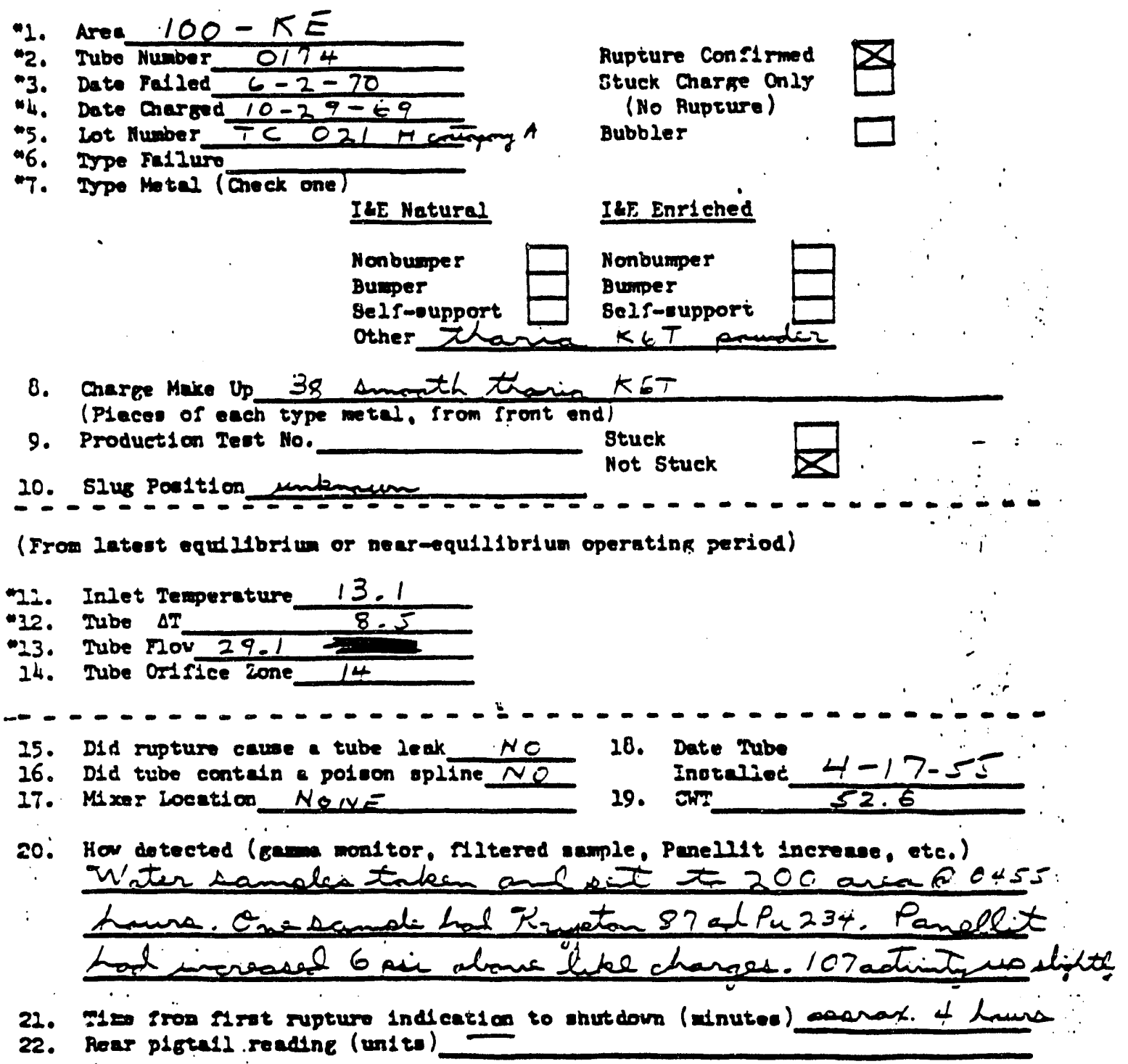

Extracted from NEWELL 1969b, no page number. 


\section{Distribution}

No. of

Copies

Offsite

\section{Technical Steering Panel}

D. S. Barth

University of Nevada

4505 Maryland Parkway

Las Vegas, NV 89154

W. A. Bishop

2503 Wedgewood Court S.E.

Olympia, WA 98501

M. L. Blazek

Oregon Department of Energy

625 Marion Street N.E.

Salem, OR 97310

G. G. Caldwell

Tulsa City-County Health Dept.

4616 East 15th Street

Tulsa, OK 74112

S. N. Davis

Dept. of Hydrology and Water Resources

Building 11

University of Arizona

Tucson, AZ 85721

N. J. Germond

224 Iron Mountain Blvd.

Lake Oswego, OR 97034

P. C. Klingeman

Civil Engineering Dept.

Apperson Hall 202

Oregon State University

Corvallis, OR 97331-2302
No. of

Copies

K. J. Kopecky

Fred Hutchinson Cancer Research

Center

1124 Columbia Street

Seattle, WA 98104

P. D. McGavran

Dept. of Health and Welfare

$450 \mathrm{~W}$. State Street, 4th Floor

Boise, ID 83720-5450

R. L. Morrill

Dept. of Geography, DP-10

University of Washington

Seattle, WA 98195

A. H. Murphy

3115 NW McKinley Drive

Corvallis, OR 97330

D. W. Price

Agricultural Economics

Hulbert Hall Room 211

Washington State University

Pullman, WA 99164-6210

M. A. Robkin

Radiological Sciences, SB-75

University of Washington

Seattle, WA 98195

G. S. Roessler

Route 1, Box 139H

Elysian, MN 56028

B. Shleien

2421 Homestead Drive

Silver Springs, MD 20902

Distr. 1 
No. of

Copies
A. P. Slickpoo, Sr.
P.O. Box 311
Kamiah, ID 83536
J. E. Till
Route 2 Box 122
Neeses, SC 29107
D. E. Walker, Jr.
P.O. Box 4147
Boulder, CO 80306

\section{Other}

2 DOE Office of Scientific and Technical Information

Technical Information Center

P.O. Box 62

Oak Ridge, TN 37830

B. G. Brooks, EH-421

Room J-112

Department of Energy

Germantown, MD 20545

18 K. CharLee

Office of Nuclear Waste Mgmt.

Department of Ecology

719 Sleater Kinney Road, S.E.

Suite 200

Olympia, WA 98504

L. E. Denham

Centers for Disease Control

and Prevention

4770 Buford Highway N.E., F-35

Atlanta, GA 30341-3724
No. of

Copies

M. R. Donnelly

Public Health Service

Centers for Disease Control

and Prevention

2201 Sixth Avenue, RX-22

Seattle, WA 98121

H. A. Haerer

Golder Associates, Inc.

4104 148th N.E.

Redmond, WA 98052

J. P. Thomas

HEAL

1720 N. Ash

Spokane, WA 99205

\section{Onsite}

\section{DOE Richland Operations Office}

R. F. Brich, TSD

A5-55

Public Reading Room (5)

A $1-65$

Battelle, Pacific Northwest

Laboratories

G. L. Black (5)

K1-25

S. D. Cannon

K1-25

D. H. Denham

K3-56

D. E. Deonigi

K6-54

P. W. Eslinger

K6-96

W. T. Farris

K3-54

M. D. Freshley

K6-77

R. O. Gilbert

K7-34

W. A. Glass (3)

K4-13

R. H. Gray

K1-33

S. P. Gydesen

P8-55 
No. of

Copies
G. L. Harvey (3)
C. M. Heeb
T. A. Ikenberry
B. A. Napier
J. V. Ramsdell
J. A. Shaw
D. B. Shipler
J. C. Simpson
K1-77
K6-42
K3-54
K3-54
K6-03
K1-25
K1-25
K7-34

No. of

Copies $\begin{array}{ll}\text { C. D. Taylor B } & \text { B }-40\end{array}$

W. L. Templeton K1-30

M. E. Thiede K6-13

W. H. Walters K6-09

Publishing Coordination K1-06

Records Center (2) K3-70

Technical Library (2) P8-55

Distr. 3 

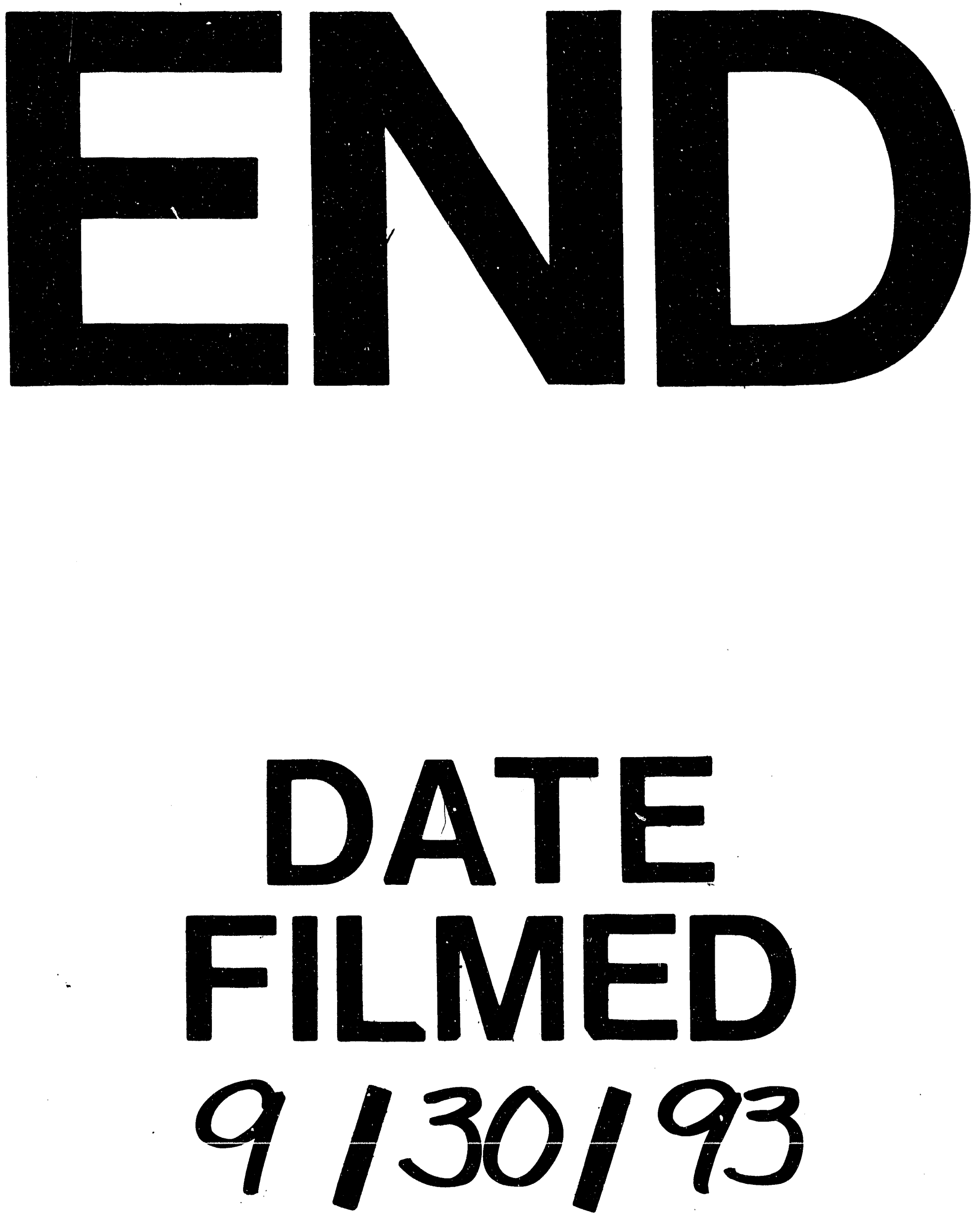

1 
